\title{
WestVirginiaUniversity
}

THE RESEARCH REPOSITORY @ WVU

Graduate Theses, Dissertations, and Problem Reports

2002

\section{Mode I fracture and durability of the CFRP -concrete interface bond}

David Moses Boyajian

West Virginia University

Follow this and additional works at: https://researchrepository.wvu.edu/etd

\section{Recommended Citation}

Boyajian, David Moses, "Mode I fracture and durability of the CFRP -concrete interface bond" (2002). Graduate Theses, Dissertations, and Problem Reports. 2459.

https://researchrepository.wvu.edu/etd/2459

This Dissertation is protected by copyright and/or related rights. It has been brought to you by the The Research Repository @ WVU with permission from the rights-holder(s). You are free to use this Dissertation in any way that is permitted by the copyright and related rights legislation that applies to your use. For other uses you must obtain permission from the rights-holder(s) directly, unless additional rights are indicated by a Creative Commons license in the record and/ or on the work itself. This Dissertation has been accepted for inclusion in WVU Graduate Theses, Dissertations, and Problem Reports collection by an authorized administrator of The Research Repository @ WVU.

For more information, please contact researchrepository@mail.wvu.edu. 


\title{
MODE I FRACTURE AND DURABILITY OF THE CFRP-CONCRETE INTERFACE BOND
}

\author{
David M. Boyajian \\ Dissertation submitted to the \\ College of Engineering and Mineral Resources \\ at West Virginia University \\ in partial fulfillment of the requirements \\ for the degree of
}

\author{
Doctor of Philosophy \\ in \\ Civil Engineering
}

\author{
Julio F. Davalos, Ph.D., Chair \\ Indrajit Ray, Ph.D., Co-Chair \\ Pizhong Qiao, Ph.D. \\ Xingbo Liu, Ph.D. \\ John Goldwasser, Ph.D.
}
Department of Civil and Environmental Engineering Morgantown, West Virginia 2002

Keywords: Fracture, Interface, DCB, Durability, CFRP-Concrete Copyright @2002 David M. Boyajian 


\title{
MODE I FRACTURE AND DURABILITY OF THE CFRP-CONCRETE INTERFACE BOND
}

\author{
David M. Boyajian \\ Advisor: Dr. Julio Davalos, and Co-Advisor: Dr. Indrajit Ray
}

\begin{abstract}
:
The continual degradation of civil infrastructures worldwide has prompted the need for effective solutions, posing major challenges to those involved with reinforced concrete structures. Externally bonded fiber reinforced polymer (FRP) composites have proven to be an efficient means of rehabilitation and enhancement to both existing and new structures alike. An important issue regarding this technology that needs further exploration, however, is the strength and integrity of the interface bond. This is evident, since, at best, an insufficient bond renders the external reinforcement as ineffective, thus defeating any contribution to have been gained by the placement of fibers there in the first place, and, at worst, may potentially result in catastrophic failure of the reinforced member altogether.

Until now, there were no available methods to rigorously evaluate the interface bond strength and integrity for concrete structures externally reinforced with FRP materials. Existing large-scale tests of structural components used for stiffness and strength evaluations, on the one hand, are ill suited for detecting delamination effects. On the other, small-scale tests only provide average interface strength properties that neither describe failure mechanisms nor provide fracture toughness data.

In an effort to resolve this dilemma, a new Fracture Mechanics test methodology, referred to as the Single Contoured-Cantilever Beam (SCCB) specimen, conceived and fashioned after the established double cantilever beam (DCB), is implemented.
\end{abstract}

(As a brief aside at this juncture, it is essential to understand that the solution to this seemingly intractable predicament must be approached from a Fracture Mechanics standpoint. The simple reason for this is that, in contrast to monolithic structures that may fail by a variety of modes, i.e. excessive elastic/inelastic deformations or fracture, adhesive joints, on the other hand, are restricted to one-namely, fracture (Ripling et al., p. 107, 1961).)

By the use of this newly developed experimental approach, then, insights have been gained into the elusive fracture toughness of the CFRP-concrete interface. Two points- 
one quantitative, the other qualitative-are implicated as a direct consequence of instituting this new test-methodology.

Quantitatively, the $G_{I c}$ value being sought here is unique in that it is a material constant; hence, it bears the distinguishing prospect of being incorporated as a useful parameter for both theoreticians and design engineers alike. Concerning the latter point, this energy-invariant term actually represents the lower-limiting fracture toughness value and therefore offers the assurance of conservatism in the practice of engineering design.

Of more immediate consequence, however, is the issue pertaining to quality. the SCCB specimen and fracture test methodology can be used as a qualification-tool for any number and type of fiber-resin systems. The drafting of standards based on qualitative assessments can then be undertaken to rate the performance of a variety of products subjected to a combination of weathering regimes. It is in this vein, that a rather comprehensive environmental aging scheme was devised and implemented here. Though only one system - that of the MBrace CFRP — was used for this study, a great deal was determined about its long-term effectiveness as a consequence of subjecting it to the carefully devised accelerated weathering-regimes described below:

(1) wet-dry cycling under sodium-sulfate $\left(\mathrm{Na}_{2} \mathrm{SO}_{4}-\right.$ simulating attack from ground water and wet-soil environments that lead to the formation of such harmful expansive-products as gypsum and ettringite, which, in turn, result in the loss of concrete mass)

(2) wet-dry cycling under sodium-hydroxide $(\mathrm{NaOH}-$ simulating a condition of naturally-occurring alkalinity that arises from the hydrated cement products and known to etch, spall, crack and dissolve the important resinmatrix)

(3) freeze-thaw cycling under calcium chloride $\left(\mathrm{CaCl}_{2}\right.$ - simulating harsh wintry conditions in many parts of the world wherein deicing agents are dispersed onto highways to safeguard against ice-formation)

It is interesting to note that considerable degradation of the interface bond integrity was found to have resulted in each case, when compared with companion specimens of the same age. The novel SCCB test method can therefore serve both, to qualify existing products, as well as develop new formulations of adhesives and FRP systems-both of which will necessarily boost consumer- and designer- confidence in the use of these materials as a viable means of strengthening and rehabilitating civil infrastructures worldwide. 
To my beloveds:

\section{Hasmig \& Christof Boyajian \\ Sam \& Vicky Boyajian}

Antranig \& Alice Der Artinian

...And, most of all, to my Beloved of beloveds:

Christ Jesus 
Books are always written as the flesh becomes

illumined with light, whencesoever words issue.

THE BOOK is the one and only exception to this rule-

for in this instance,

THE WORD of God

is LIGHT, (The unfolding of THE WORD is LIGHT (Psalm 119:130))

the very One (Jesus said, "I am THE LIGHT of the world" (John 9:5))

that illumined the darkened world

upon becoming FLESH.

(THE WORD became FLESH (John 1:14))

--D.MT. Boyajian

"Then spake Jesus again unto them, saying,

'I am THE LIGHT of the world:

he that followeth me shall not walk in darkness,

but shall have THE LIGHT of LIFE."' (John 8:12, KJV)

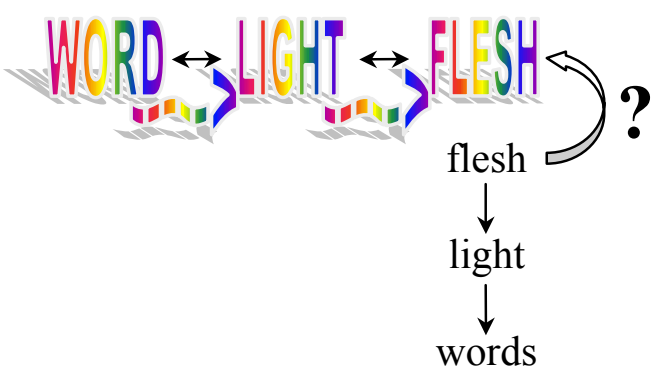




\section{ACKNOWLEDGEMENTS}

First, a general word of thanks to all my committee members for their willing participation to accommodate me amidst their busy schedules.

Words fail miserably, and at very best, fall short of capturing my feelings of gratitude towards the following two persons-truly my academic-Godsends! Dr. Julio F. Davalos, my dear advisor for the past $3 \frac{1}{2} 2$ years... THANK YOU for your uncanny ability of farsightedness to see and comprehend the fine-print that, tragically, was much too small and perplexing for other men to perceive. I always relish in sharing your intelligence and boundless creativity, your wit and your undying optimism. The multivaried fields of expertise that you've acquired are absolutely mind-boggling and greatly to be envied. I've matured much, both academically and personally as a result of being under your leadership and supervision. (¡Muchas Gracias!) And my dearest of personal friends, and co-advisor for the past 21/4 years, Dr. Indrajit Ray, the conceiver of the ingenious weathering regimes implemented in this study. Truly, the contents of Chapters 7 and 8 would not have been possible without your guidance. THANK YOU for all the help you gave me to better my understanding of concrete-both in theory and in the lab. As a person, I especially appreciate your genuine demeanor of selflessness, in the midst of a selfish world. Your ever-virtuous comport and sincere and kind consideration of others are ample proof of this. The world would do well to assume these, at present, nonpresent qualities. I am blessed because of our most-"durable" friendship.

I also want to thank Dr. Qiao for his friendship to me over the years-the longest (August 1996) in duration - and, in speaking of length, the one member of the committee who had to drive the farthest (from Akron, $\mathrm{OH}$ ). From the longest to the shortest, I extend a word of thanks to Dr. Liu whom I just had the pleasure of meeting (this October), and for his obliging to serve on this committee on such short notice. Moving now from engineering to my other passion, mathematics, I'm grateful for all the encouragement that Dr. Goldwasser (Prof. of Mathematics) has given me, both in and out of the classroom. Another passion that we both share is music-especially as it concerns the violin. On several occasions he has even invited me to participate in concerts given from his home.

I must also thank our Department Chairperson, Dr. David Martinelli, for all the help, advice, and encouragement he's given me over the years. I owe a special debt of gratitude to my dear "brother (see Proverbs 18:24b)", Dr. Shen-En Chen (University of Alabama, Birmingham), without whom my second-wind may not have materialized.

Thanks are owed to my dear friend, Ribal El-Amin, who gave me tremendous help in the laboratory-I would not have been able to meet the taxing demands of experimentation without his assistance. I recognize, too, the friendship given me by my colleague Junhui Jia in our travels to Montréal, Québec and West Lafayette, IN this past May and October, and the wonderful late-night talks that we shared in. 
It is only fitting here that I recognize the two brilliant men who imbued me with my deep-seated desire to succeed academically. First, is my Uncle Harry Kumjian, who personally took time out of his busy schedule to provide me with much needed tutoring during my high school years of study. I will always cherish our intellectually-steeped moments together. And second, is my undergraduate and then later on, graduate Professor of Civil Engineering that I most wanted to emulate as a teacher: Dr. Anjan K. Bhaumik (CSULA).

And now to my beloveds. To my beautiful wife, Hasmig-immeasurable blessings have descended upon me from Above because of Heaven's Supreme decision to send you into my life. Thank you, sweetheart, for all your encouragement, patience, support, love and prayers - this experience has been equally trying for you as it has me; also my dear son, Christof, who always manages to put a smile on my face and a glimmer in my heart, reminding me, daily, that the truly important things in life consist of those which require no acquiring. My parents, Sam and Vicky Boyajian, must be acknowledged for all their love, sacrifices, direction, support, encouragement, and most of all, prayers-you epitomize to me the best in parenting. In like fashion, the same must be said of my wife's parents, Antranig and Alice Der Artinian. MY LOVE TO YOU ALL! And God's richest blessings.

\section{Most importantly, I praise my Lord GOD, Jesus Christ, for bestowing upon me the Degree that will not perish one day, but instead will empower me with everlasting rights to eternal life in His presence.}

This research was funded in part by a National Science Foundation (NSF) grant-CMS9970008-under the program direction of Dr. Ken P. Chong, and also by the WVU Research Corp. Incentive Grant Program. A special word of thanks is extended to Mr. Steve Tysl, formerly of Master Builders, Inc., who generously supplied all the MBrace Primer/Saturant and CFRP materials. 


\section{TABLE OF CONTENTS}

ABSTRACT ii

ACKNOWLEDGEMENTS vi

LIST OF TABLES X xvi

LIST OF FIGURES - xix

ABBREVIATIONS X xxvii

NOTATIONS $\quad$ xxx

CHAPTER 1 INTRODUCTION 1

1.1 Introduction 1

The SCCB Specimen 5

$\begin{array}{ll}1.2 \text { Significance } & 7\end{array}$

Industrial Applications 9

Tonen's Log-Report...: $\quad \underline{9}$

Case Studies (Watson...): 11

1.3 Objectives

The Primary-, Pre-, and Post-Objectives 16

The Minor Objectives 18

1.4 Scope

1.5 Brief Synopses of Chapters to Follow 
CHAPTER 2 A REVIEW OF FRP AND CONCRETE DURABILITY

2.1 Introduction

2.2 Durability

Transport of Fluids in Concrete 24

\author{
Porosity: 24 \\ Permeability: 24 \\ Diffusion: $\underline{24}$ \\ Sorption: 25 \\ Coefficient of Permeability: 25 \\ Coefficient of Diffusion: $\underline{26}$
}

2.3 Sulfate Attack

Efflorescence 30

Salt Weathering 31

Sea Water Effects 32

2.4 Alkali Attack

Porewater Quantity 35

Porewater Mobility in Concrete 36

Porewater Mobility in FRP 36

2.5 Freeze-Thaw Attack

Scaling of Concrete 40

Mechanisms of Freeze-Thaw Deterioration 41

Hydraulic Pressures: $\underline{41}$

Osmotic Pressures: $\underline{43}$

Deicing Salts: $\underline{44}$

Influence of Aggregates: 45

A Summary of Protective Measures... 47

Sealants: $\underline{47}$

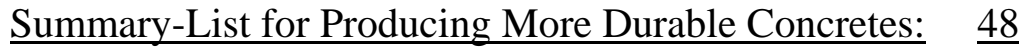

2.6 Experimental Case Studies

1. Chajes et al., $1995 \quad 51$

Threefold Conditioning Scheme...: $\quad \underline{51}$

Conclusions of Environmental Conditioning: $\quad \underline{52}$

2. Green et al., 199853

FT Conditioning Scheme...: $\quad \underline{53}$

Conclusions of FT Conditioning...: $\quad \underline{54}$

3. Karbhari et al., $1997 \quad 54$

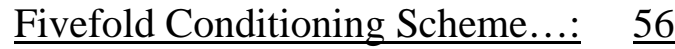

Conclusions of Peel/Durability Test...: $\quad \underline{56}$

4. Mukhopadhyaya et al., 199857

Threefold Conditioning Scheme...: $\quad \underline{59}$

Conclusions of Double-Lap Shear Tests...: $\quad \underline{59}$

5. Rahman et al., $1998 \quad 61$

Fourfold Conditioning Scheme...: $\quad \underline{61}$

Conclusions of Sustained Load/Durability Tests...: $\quad \underline{63}$ 


\section{Toutanji et al., $1997 \quad 65$}

Twofold Conditioning Scheme...: $\underline{65}$

Conclusions of Environmental Conditioning...: $\quad \underline{66}$

2.7 Summary

\section{CHAPTER 3 A REVIEW OF FRACTURE MECHANICS AND THE} DEVELOPMENT OF THE NOVEL SCCB TEST

73

73

Initial Attempts 75

3.2 Historical Background and Key Results of Fracture Mechanics

Studies on Wires 78

Theories of Griffith 80

Stresses Near Cracks 82

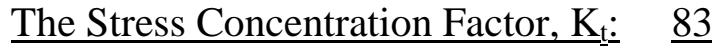

Griffith's Relationship: $\underline{84}$

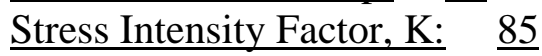

The Stress and Displacement Field Equations: $\quad \underline{87}$

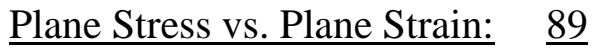

Liberty Ship Failures 97

Post War Progress 99

3.3 Three Modes of Fracture \&

the Derivation of the Irwin-Kies Relationship

Three Basic Modes of Fracture 101

Derivation of the Irwin-Kies Equation 104

The General Energy Balance: $\quad 104$

Elastic Potentials and

the Critical Strain Energy Release Rate $\quad \underline{109}$

3.4 Cracking Behavior from an

Experimental Fracture Mechanics Viewpoint

Different Crack Patterns: Qualitatively 116

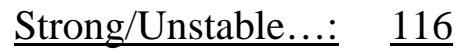

Strong/Stable...: 116

Strong/Moderately Unstable...: 117

Weak/Stable...: 117

Different Crack Patterns: Quantitatively 117

3.5 The Evolving DCB Test Specimen

120

The DCB (or UDCB) 121

The WTDCB 124

The $C D C B \quad 126$ 
3.6 A Novel Experimental Fracture Mechanics Specimen:

The SCCB \& Test Fixture

Requisite of a Single Contoured-Cantilever Beam Specimen 128

Three Differing Aspects between the DCB \& SCCB Specimens 129

Details of the SCCB Specimen and Test Fixture 132

The SCCB Specimen: 132

The Test Fixture: 136

3.7 Summary

\section{CHAPTER 4 COMPLIANCE CALIBRATIONS}

\section{OF THE CONTOURED-CANTILEVER}

4.1 Introduction

4.2 CDCB Calibrations

Calibration Equipment 159

The Experimental Calibration Scheme 161

Relocating Cracks Backwardly 162

The Actuator Speed 163

CDCB Calibration Results 164

Specimen 1: $\quad 164$

Specimen 2: 167

4.3 SCCB Calibrations

Background of the 3-Stage Calibration Scheme 169

Substrate Material Effects 173

Why Wood?: 173

The Substrate Material Effect Phenomenon: 175

Justification of Wood-Wood Assumption in both

SCCB Calibration Results 181

Modeling and Shape Optimization: 178

Specimen 1: $\quad \underline{182}$

Specimen 2: 183

Specimen 3...: 184

Specimen 4: $\quad \underline{185}$

Specimen 5: $\underline{186}$

4.4 Hinging Action-Limits of the FE and Analytical Approaches 
CHAPTER 5 TEST SPECIMEN FABRICATION

5.1 Introduction

5.2 Concreting

5.3 Contouring

205

5.4 Bonding

207

The MBrace Adhesive System 207

Surface Preparation 209

Priming 211

Saturating 215

5.5 Summary Remarks

CHAPTER 6 DRY FRACTURE

6.1 Introduction 224

6.2 Pristine Dry Fracture Specimens 226

The "Head" and "Set" Critical Loads 228

The Statistical Reduction of Raw Loads 233

A Step-by-Step Example 236

\begin{tabular}{|c|}
\hline$\frac{\text { Step 1: }}{\text { Step 2: }}$ \\
\hline Step 3: \\
\hline
\end{tabular}

6.3 Representative Dry Fracture Specimens 237

6.4 Dry Weights and Strains 243

Weight Changes of the Companion (i.e. Dry) Specimens 244

Strain Changes of the Companion (i.e. Dry) Specimens 246

6.5 Conclusions

CHAPTER 7 WET-DRY FRACTURE 256

7.1 Introduction 256

7.2 Sodium Sulfate $\left(\mathrm{Na}_{2} \mathrm{SO}_{4}\right.$-abbreviated: SS) Attack 257

Preliminaries (SS) 257

SCCB Fracture (SS) 261

Patterns of Fracture (SS) 267

Quantitatively (SS): 267

Qualitatively (SS): 268

Pre-Fracture Changes (SS) 274

Visual Changes (SS): 274

Weight Changes (SS): 278

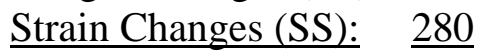


7.3 Sodium Hydroxide ( $\mathrm{NaOH}-$ abbreviated: $\mathrm{SH})$ Attack

Preliminaries $(\mathrm{SH}) \quad 282$

SCCB Fracture (SH) 284

Patterns of Fracture (SH) 289

Quantitatively (SH): $\quad 289$

Qualitatively (SH): 289

Pre-Fracture Changes (SH) 292

Visual Changes (SH): 292

Weight Changes (SH): 294

Strain Changes (SH): 296

7.4 Conclusions

CHAPTER 8 FREEZE-THAW FRACTURE

8.1 Introduction

8.2 Calcium Chloride $\left(\mathrm{CaCl}_{2}\right.$-abbreviated: $\left.\mathrm{CC}\right)$ Attack

Preliminaries $(C C) \quad 309$

SCCB Fracture $(C C) \quad 313$

Patterns of Fracture (CC) 320

Quantitatively (CC): $\quad \underline{320}$

Qualitatively (CC): 321

Pre-Fracture Changes (CC) 323

Visual Changes (CC): $\quad \underline{323}$

Weight Changes (CC): $\quad 326$

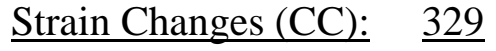

8.3 Conclusions

CHAPTER 9 CONCLUSIONS $\quad 337$

9.1 Introduction... of the End

Organization of the End 338

9.2 Summary of SCCB Relevancy and Appraisal of its Viability

Path of Relevancy 338

Appraisal of SCCB Viability 339

Why Mode I?: 341

9.3 Summary of Foremost Relevant Concepts and Relations

Relevant Concepts 343

Pertaining to Durability: $\underline{343}$

Pertaining to Griffith: 345

Pertaining to Material Constancy of Fracture Toughness: $\quad \underline{345}$

Pertaining to Cracking Behavior: 347

Pertaining to the Contoured-Cantilever: $\quad \underline{348}$

$\underline{\text { Pertaining to the Critical Load: } \underline{348}}$

Relevant Relations 349 
9.4 Summary of Secondary Interface Characterizations

Visual-Based Pre-Fracture Inspections 351

Sodium Sulfate (SS)/PRE-VISUAL (30 Cycles): 351

Sodium Hydroxide(SH)/PRE-VISUAL (30 Cycles): 351

Calcium Chloride (CC)/PRE-VISUAL (300 Cycles): $\quad \underline{552}$

Visual-Based Post-Fracture Inspections 352

Dry Companion Beams (WD and FT)/POST-FRACTURE: $\quad 352$

Sodium Sulfate (SS)/POST-FRACTURE...: $\quad \underline{352}$

Sodium Hydroxide (SH)/POST-FRACTURE...: 353

Calcium Chloride (CC)/POST-FRACTURE...: 35

Weight Changes 355

Dry Weights...: $\underline{355}$

Wet-Dry Weights...: $\quad \underline{355}$

Freeze-Thaw Weights...: $\underline{356}$

Strain Changes 357

Dry Strains...: 357

Wet-Dry Strains...: 357

Freeze-Thaw Strains...: $\underline{358}$

9.5 Summary of Primary Interface Characterizations

Fracture of Dry (Pris. and Rep.) Specimens 358

Fracture of Wet-Dry (SS and SH) Specimens 360

Fracture of Freeze-Thaw (CC) Specimens 362

Conclusive Magnitudes of Fracture: Dry - Weathered 362

9.6 Future Recommendations

For the Enhancement of CFRP-Strengthened

Concrete Structures 364

Subject to Sulfate Attack: $\quad \underline{364}$

Subject to Alkaline Attack: $\quad \underline{364}$

Subject to Freezing and Thawing in the

Presence of Deicing Chemicals: $\quad \underline{365}$

For Further Experimental Enhancement and Study 366

Test-Fixture Improvement: $\quad \underline{366}$

Specimen Fabrication and Experimentation: $\quad \underline{366}$

Qualification of Systems: $\underline{366}$

Other Characterization-Tools: $\quad \underline{366}$

Parameters and Correlations: $\underline{367}$

"Personal Communication by

M.F. Kanninen to D.M. Boyajian 367 
$\begin{array}{lr}\text { REFERENCES } & \mathbf{3 6 9}\end{array}$

$\begin{array}{lll}\text { APPENDIX A } & 384\end{array}$

APPENDIX B RELEVANT PROPERTIES OF MATERIALS USED 386

APPENDIX C INSTRUMENTATION (DEMEC, ZH-16, \& MTS) 388

$\begin{array}{lll}\text { APPENDIX D } & 396\end{array}$ 


\section{LIST OF TABLES}

Table 2.1 Classification of Sulfate Severity 29

Table 2.2 Main (+90\%, by weight) compounds of portland cement 34

Table 2.3 Peel test composite properties (Karbhari et al., p. 150, 1997) 55

Table 2.4 Values of critical energy release rates

(Karbhari et al., p. 155, 1997) 57

Table 2.5 Concrete cube strengths (Mukhopadhyaya et al., p. 381, 1998) 58

Table 2.6 FRP properties (Toutanji et al., p. 352, 1997) 65

Table 2.7 Resin properties (Toutanji et al., p. 352, 1997) 65

Table 4.1 Experimentally Calibrated Contour Shapes 156

Table 4.2 Experimental and FE compliance values of specimen S1 165

Table 4.3 Experimental and FE compliance values of specimen S2 167

$\begin{array}{lll}\text { Table 4.4 Summary of CDCB calibration results } & 168\end{array}$

Table 4.5 Wood-wood results for each of the five contour shapes: $a$-range $=101.6-355.6 \mathrm{~mm}\left(4^{\prime \prime}-14 "\right)$ throughout $\quad 174$

Table 4.6 Compliance and $d C / d a$ values for the different classes of calibrations for shape S3 using the non-sawn, crack length positions 177

Table 4.7 A summary of all the calibration results 194

Table 5.1 Relevant concrete data (no. of batches, w/c ratio, air, strength and std. dev.)

Table 5.2 Concrete mixture proportions based on a $0.026 \mathrm{~m}^{3}\left(0.917 \mathrm{ft}^{3}\right)$ volume 201

Table 5.3 Mean, 28-day, compressive strengths of concrete 204

Table 5.4 MBrace primer material properties 209

Table 5.5 MBrace saturant material properties 209

Table 5.6 Color, mix, coverage, thickness and working time of MBrace resins

Table 6.1 Pristine dry fracture loads_-initiations (i) and arrestments (a) (unit: N)

Table 6.2 Raw data results of pristine dry fracture specimens (see Fig. 6.1 and Table 6.1) 230

Table 6.3 Reduced data results of pristine dry fracture test specimens (see Table 6.1) 230

Table 6.4 Pristine dry fracture results (unit: $\mathrm{J} / \mathrm{m}^{2}$ ) 230

Table 6.5 Raw head-initiation loads of pristine specimens (from Table 6.1) 236

Table 6.6 Representative dry fracture results from head-initiation loads 239 
Table 6.7 Representative dry fracture results from set-initiation loads

Table 6.8 Representative dry fracture results from head-arrestment loads

Table 7.1 (see also Tables 6.4 and 6.10)

Table 8.1 Reduced data results of freeze-thaw, CC50 fracture test specimens

Table 8.2 Reduced data results of freeze-thaw, CC100 fracture test specimens

Table 8.3 Reduced data results of freeze-thaw, CC150 fracture test specimens

Table 8.4 Reduced data results of freeze-thaw, CC200 fracture test specimens

Table 8.5 Reduced data results of freeze-thaw, CC300 fracture test specimens

Table 8.6

Calcium chloride, freeze-thaw fracture results (unit: $\mathrm{J} / \mathrm{m}^{2}$ ) (see also Tables 8.6 and 8.7)

Table 9.1 (repeated from Table 6.11) Averaged conclusive dry fracture results

Table 9.2 (repeated from Table 7.11) Averaged conclusive wet-dry fracture results (unit: $\mathrm{J} / \mathrm{m}^{2}$ )

Table 9.3 (repeated from Table 8.8) Averaged conclusive freeze-thaw fracture results (unit: $\mathrm{J} / \mathrm{m}^{2}$ )

Table A.1 Pristine specimens

Table A.2 Plain specimens (i.e. no CFRP — these were non-fracture specimens used to gauge wt. \& strain changes in the concrete throughout the duration of aging) 
Table A.5 Freeze-Thaw specimens 385

Table B.1 LVL (Laminated Veneer Lumber-used for the contours) 386

Table B.2 CFRP strip (MBrace CF130 high tensile, unidirectional carbon fiber)

Table B.3 MBrace primer (Repeated from Table 5.4) 386

Table B.4 MBrace saturant (Repeated from Table 5.5) 386

Table B.5 Concrete (made with locally available natural sand and \#8 pea-gravel aggregates; each beam furnished with a pair of \#2 steel reinforcing bars, Gr. 60, $\left.\mathrm{f}_{\mathrm{y}}=60,000 \mathrm{psi}=413.7 \mathrm{MPa}\right)$

(Repeated from Table 5.1) 


\section{LIST OF FIGURES}

Figure 2.1 Schematic of a four-point bending specimen over simple supports 50

Figure 2.2 Schematic of the peel test (Karbhari et al., p. 150, 1997) 55

Figure 2.3 Schematic drawings of: (a) double-lap shear specimen, and (b) test-rig (Mukhopadhyaya et al., p. 384, 1998) 58

Figure 2.4 Schematic drawings of the: (a) FRP tensile-test specimen, and (b) sustained loading/conditioning apparatus (Rahman et al., pp. 503, 505, 1998)

Figure 3.1 The arm-breakoff effect - unsuccessful initial attempts at studying the FRP-concrete interface bond using a CDCB approach (Mostovoy et al., 1967)

$\begin{array}{lll}\text { Figure 3.2 Side view of steel jacket test apparatus with SCCB specimen } & 77\end{array}$

Figure 3.3 Sketch from the notebook of da Vinci illustrating the tensile test apparatus he used in studying the strengths of wires of varying lengths but constant diameter (Hertzberg, p. 235, 1976)

Figure 3.4 Alan Arnold Griffith (1893-1963)

(Source: http://fpml.tuwien.ac.at/fpml/history/techfig10.html)

Figure 3.5 (a) Plate with elliptical hole - tip radius of curvature, $\rho=b^{2} / a$

(Hertzberg, p. 237, 1976); (b) Plate with circular holemaximum stress at tip $=3$ times the applied stress

Figure 3.6 3-D stress field ahead of a crack-coordinates $(\mathrm{x}, \mathrm{y}, \mathrm{z})$, normal stresses $\left(\sigma_{\mathrm{x}}, \sigma_{\mathrm{y}}, \sigma_{\mathrm{z}}\right)$ and shearing stresses $\left(\tau_{\mathrm{xy}}, \tau_{\mathrm{xz}}, \tau_{\mathrm{yz}}\right)$ (Paris and Sih, p. 31, 1964)

Figure 3.7 Illustration of a plane (ABC) cutting across a cubic element with corresponding stresses shown (Mendelson, p. 31, 1970)

Figure 3.8 Through-thickness stress $\sigma_{z}$ in (a) a thin sheet under plane stress state and (b) a thick plate under plane strain conditions (Hertzberg, p. 265, 1976)

Figure 3.9 Fracture toughness $\left(K_{c}\right)$ as a function of specimen thickness (a) - (d) (Kanninen and Popelar, p. 179, 1985)

Figure 3.10

(a) Fractured T-2 tanker, the S.S. Schenectady; (b) Fractured oil barge (Hertzberg, p. 232, 1976)

Figure 3.11 (a) Mode I (opening mode); (b) Mode II (edge-sliding mode); (c) Mode III (tearing mode) (Hertzberg, p. 262, 1976)

Figure 3.12 The incremental growth of a crack due to an elemental load, $\delta P \quad 105$

Figure 3.13 Equilibrium-path for the specimen-loading of Fig. 3.12 
Figure 3.14 Schematic load-displacement curves of (a) a rate insensitive material with a flat region of cracking indicating stable cracking and (b) a rate sensitive material with a region of saw-toothed cracking indicating unstable cracking

Figure 3.15 Qualitative crack behavior of adhesive joints - (a) strong/unstable,

(b) strong/stable, (c) strong/moderately unstable, and

(d) weak/stable (River, 2002)

Figure 3.16 Double Cantilever Beam (DCB) specimens - (a) specimen originally used for measuring fracture toughness of adhesive joints, (b) side-grooved specimen used for measuring fracture toughness of monolithic materials (Mostovoy, Crosley and Ripling, 1967) 122

Figure 3.17 The WTDCB specimen (Bascom, et al., p. 971, 1984) 125

Figure 3.18 CDCB specimens - (a) straight tapered, (b) convex tapered, and (c) concave tapered (River and Okkonen, pp. 24 $\& 25,1993)$

Figure 3.19 CFRP-bonded concrete specimen - (a) front view, (b) side view 130

Figure 3.20 The steel jacket-(a) cross-sectional view, (b) side view 132

Figure 3.21 The bulk SCCB specimen-(a) LVL wooden contouredcantilever, (b) CFRP strip, (c) side view of concrete substrate, (d) front view (enlarged from part (c)) of concrete substrate (NOTE: All dimensions in millimeters) 133

Figure 3.22 Pictures of (a) the LVL contours (pictured upside-down), and (b) the CFRP strip (with blown-up view)

Figure 3.23 Pictures of the concrete substrate - (a) from the front, and (b) from the side

Figure 3.24 Top view of base plate $(228.6 \times 279.4 \times 19)$

(NOTE: All dimensions in millimeters)

Figure 3.25 Angle $(76.2 \times 76.2 \times 9.5)-($ a) elevation view, (b) top view

Figure 3.26 Picture of angle as affixed atop the base plate (see Fig. 3.24)

Figure 3.27 Steel Jacket- (a) Cross section view, (b) side view with dimensions, (c) cross section view (enlarged from parts (a) \& (b)) with dimensions (NOTE: all dimensions in millimeters)

Figure 3.28 Aluminum wedge-shaped clamp - (a) end view, (b) photograph, (c) side view (NOTE: All dimensions in millimeters)

Figure 3.29 Load strap - (a) front view, (b) side view

Figure 3.30 Picture of load strap affixed by the hydraulic grips of MTS 140

Figure 3.31 Pictures of the test fixture- (a) Steel jacket, aluminum wedge-shaped clamps, base plate, and angles (note also the continuous neoprene rubber sheet inside jacket), (b) close up of wedge-shaped clamp, steel jacket and angled member

Figure 3.32 Pictures of the SCCB test-setup from the: (a) front, and (b) back 141 
Figure 4.1 Optimum single contoured-cantilever beam specimen S3;

$$
\text { width (not shown), } b=43.4 \mathrm{~mm} \text { (1.71") }
$$

Figure 4.2 CDCB specimen S2 with initial and final (symmetric) heights shown

Figure 4.3 Calibration and details of actual CDCB specimen

Figure 4.4 Compliance vs. crack-length of CDCB specimen S1 experimental $(C$ ave $)$ and $\mathrm{FE}$ results shown

Figure 4.5 Compliance vs. crack-length of CDCB specimen S2 experimental $\left(C \_\right.$ave) and $\mathrm{FE}$ results shown

Figure 4.6

The wood-wood specimen

Figure 4.7 SCCB calibration specimen S1; width (not shown), $\mathrm{b}=43.4 \mathrm{~mm}\left(1.71^{\prime \prime}\right)$

Figure 4.8 Compliance vs. crack length of SCCB specimen S1

Figure 4.9 SCCB calibration specimen S2; width (not shown),

$$
\mathrm{b}=43.4 \mathrm{~mm}\left(1.71^{\prime \prime}\right)
$$

Figure 4.10 Compliance vs. crack length of SCCB specimen S2

Figure 4.11 SCCB calibration specimen S3; width (not shown),

$$
\mathrm{b}=43.4 \mathrm{~mm}(1.71 ")
$$

Figure 4.12 Compliance vs. crack length of SCCB specimen S3

Figure 4.13 SCCB calibration specimen S4; width (not shown),

$$
\mathrm{b}=43.4 \mathrm{~mm}\left(1.71^{\prime \prime}\right)
$$

Figure 4.14 Compliance vs. crack length of SCCB specimen S4

Figure 4.15 SCCB calibration specimen S5; width (not shown),

$$
\mathrm{b}=43.4 \mathrm{~mm}\left(1.71^{\prime \prime}\right)
$$

Figure 4.16 Compliance vs. crack length of SCCB specimen S5

Figure 4.17 Compliance vs. full-crack length range of SCCB specimen S3

Figure 4.18 The optimum SCCB calibration specimen S3; width (not shown), $b=43.4 \mathrm{~mm}\left(1.71^{\prime \prime}\right)$; contour angle $=6.3^{\circ}$

Figure 5.1 (a) Front- (i.e. cross-sectional) and (b) side- views of concrete substrate (for dimensions refer to

Figure 3.21, parts $(c)$ and $(d)$ )

Figure 5.2 Pictures of: (a) the concrete substrate molds, and

(b) a close-up of one such cell-note the inverted trapezoidal cross-section with respect to the concrete substrate beam (Fig. 5.1(a))

Figure 5.3 Pictures of the steel reinforcing bars and chairs

Figure 5.7 Picture of a 3-day old beam being removed from the mold 203

Figure 5.8 Picture of stockpiled concrete-beams after undergoing the 28-day curing regime and before being coated with primer 204

Figure 5.9 Attainment of two contours from a single LVL board 205 
Figure 5.10 The finished LVL contour (a) elevation-view, and (b) back-view ( $\mathrm{NOTE}_{1}$ : All dimensions in millimeters) $\left(\mathrm{NOTE}_{2}\right.$ : LVL material properties given in Table B.1)

Figure 5.11 The MBrace adhesive system - (a) Primer, parts A/B,

(b) color of part A (primer), and in fact, that of mix: amber;

(c) Saturant, A/B, (d) color of part A (saturant), and in fact, that of mix: dark-blue

Figure 5.12 (a) Before-sanding, and (b) after-sanding, pictures of the same concrete beam; (c) A picture depicting the amount of loosened particles removed as a result of sanding

Figure 5.13 Priming of: (a) bonding surface, and (b) undersides of beams

Figure 5.14 (a) Priming process, and (b) contrast of primed/unprimed beams

Figure 5.15 Bottoms of LVL contours with light coating of primer-

(a) close-up view, and (b) undersides displaying bevel and joint details

Figure 5.16 (a) Close-up, and (b) overall view of the saturation of the concrete bonding surface; (c) process of laying-down the doubled-over wax paper, and (d) a view of the wax-paper in place

Figure 5.17 (a) Saturated CFRP-strip, and (b) the CFRP-fabric imprint as transferred to the underlying wax paper 218

Figure 5.18 (a) The steel ribbed-roller, and (b) another coating of saturant applied after 30 minutes 219

Figure 5.19 Belt-sanded CFRP strips

Figure 5.20 Methods to protect the concrete raised-step and ledge from becoming infiltrated with saturant-

(a) Mylar release tape, and (b) butcher's wax

Figure 6.1 Fracture behavior of beam 3B6

Figure 6.2 Pictures of the pristine-dry fracture surfaces of beam 3B6(a) extended- and (b) close- views

Figure 6.3 The statistical-based bandwidth for-(a) compact, and

(b) widespread data

Figure 6.4 Pictures of the representative-dry fracture surfaces of wet-dry companion beams-(WDC20):

(a) 9B11(WDC30): (b) 10B9 and (c) 9B12

Figure 6.5 Pictures of the representative-dry fracture surfaces of freeze-thaw companion beams - (FTC200): (a) 7B10 and (b) 8B10 (FTC300): (c) 4B12 and (d) 8B12

Figure 6.6 Percent average weight change of the wet-dry companion beams

Figure 6.7 Percent average weight change of the freeze-thaw companion beams 
Figure 6.8 The demountable mechanical strain gauge (DEMEC) -

(a) DEMEC with setting-out and invar bars

(b) DEMEC in use atop CFRP

Figure 6.9 Microstrains of the wet-dry companion beams in two locations:

(1) on the CFRP, and (2) on the adjacent concrete ledge (see Fig. 3.21(d)) - the differential movement between the two is shown as well

Figure 6.10 The differential movement between the CFRP strip and adjacent concrete ledge (see Fig. 3.21(d)) of the freeze-thaw companion beams

Figure 6.11 Averaged conclusive pristine- and representative- dry fracture results

Figure 7.1 CFRP-bonded concrete specimen-(a) cross-sectional view, and (b) side view (repeated from Fig. 3.19)

Figure 7.2 Pictures related to the wetting-phase-(a) CFRP-bonded concrete specimens inside plastic-lined tank filled with $10 \%$ sodium sulfate solution, (b) carboy and tank, and (c) pneumatic stirrer shown in front of carboy

Figure 7.3 Picture related to the drying-phase - the Cincinnati Sub-Zero (CSZ), ZH-16, environmental chamber

Figure 7.4 Pictures of the concrete beam-(a) the priming process (note contrast between the primed and unprimed beams

(b) an example of a fully-primed concrete beam

Figure 7.5 Pictures of the undersides of the LVL contours-

(a) stockpiled specimens, and (b) close-up views of primed bonding surfaces

Figure 7.6 Fracture behavior of select WD-(a) 10-, (b) 20-, and

(c) 30- cycled beams in sodium sulfate (SS)

Figure 7.7 Percentage reductions in (a) $G_{I c}^{i}$ and (b) $G_{I c}^{a}$ resulting from 10, 20, and 30 wet-dry cycles of sodium sulfate (SS) exposure

Figure 7.8 Pictures of the SS10/WD fracture surfaces showing beams(a) 2B3, (b) 3B4...see also Fig. 7.6(a), and (c) 10B6

Figure 7.9 Pictures of the SS20/WD fracture surfaces showing beams-

(a) 8B7, (b) 9B9, and (c) 9B10...see also Fig. 7.6(b)

Figure 7.10 Pictures of the SS30/WD fracture surfaces showing beams-

(a) 7B11, (b) 7B12, and (c) 8B13... see also

Figs. 7.6(a) and 7.12

Figure 7.11 Pictures of visual SS30/WD changes taking place in specimens-

(a) 6B11 (plain SS30), (b) 9B12 (companion 30), and

(c) 9B13 (actual SS30)

Figure 7.12 Pictures of pre-fracture SS30/WD changes in CFRP-bonded concrete specimen $8 \mathrm{~B} 13$, displaying effects on the(a) concrete ledge, (b) CFRP and concrete substrate, 
and (c) concrete raised step, ledge, and substrate

(see also Figs. 7.6(a) and 7.10)

Figure 7.13 Percent average weight changes of the SS/wet-dry plain, companion, and actual beams

Figure 7.14 Microstrains of the SS/wet-dry beams in two locations:

(1) on the CFRP, and (2) on the adjacent concrete

ledge (see Fig. 3.21(d)) - the differential movement

between the two is shown as well

Figure 7.15 Pictures related to the wetting-phase-CFRP-bonded concrete specimens inside plastic-lined galvanized steel tank filled with sodium hydroxide solution $(\mathrm{pH}=12)$

Figure 7.16 Picture related to the SH drying-phase-specimens racked inside the Cincinnati Sub-Zero (CSZ), ZH-16, environmental chamber

Figure 7.17 Fracture behavior of select WD-(a) 10-, (b) 20-, and

(c) 30- cycled beams in sodium hydroxide $(\mathrm{SH})$

Figure 7.18 Percentage reductions in (a) $G_{I c}^{i}$ and (b) $G_{I c}^{a}$ resulting from 10,20 , and 30 wet-dry cycles of sodium hydroxide $(\mathrm{SH})$ exposure

Figure 7.19 Fracture behavior of select SH10, 20, and 30/WD beams-

(a) 6B3, (b) 7B9, and (c) 6B12

Figure 7.20 Pictures of two pre-fracture, 30-cycled beams-

(a) SS30/WD, 8B13, and (b) SH30/WD, 3B13-

and (c) an unconditioned/unprimed concrete beam

Figure 7.21 Percent average weight changes of the SH/wet-dry plain, companion, and actual beams

Figure 7.22 Microstrains of the SH/wet-dry beams in two locations:

(1) on the CFRP, and (2) on the adjacent concrete ledge (see Fig. 3.21(d)) - the differential movement between the two is shown as well

Figure 7.23 Conclusive percentage reductions in $\boldsymbol{G}_{I \boldsymbol{c}}^{\boldsymbol{i}} \& \boldsymbol{G}_{\boldsymbol{I c}}^{\boldsymbol{a}}$ after 10,20 , and $30 \mathrm{WD}$ cycles in [(a) \& (c)] SS, and [(b) \& (d)] SH media

Figure 8.1 Freeze-thaw cycling scheme

Figure 8.2 Pictures of the two CSZ (ZH-16) environmental chambers- the chamber of the FT conditioning protocol is shown in the foreground with a view of the racked stainless steel trays 309

Figure 8.3 (a) Dimensions of stainless steel tray (not to scale), and (b) plastic tubing used to elevate the CFRP-bonded specimens (one on each end-see Fig. 8.10)

Figure 8.4 Picture of FT beams inverted in stainless steel trays

Figure 8.5 Fracture behavior of select FT-(a) 50-, (b) 100-, (c) 150-,

(d) 200-, and (e) 300- cycled beams in 
calcium chloride (CC)

Figure 8.6 Percentage reductions in (a) $G_{I c}^{i}$ and (b) $G_{I c}^{a}$ resulting from $50,100,150,200$, and 300 freeze-thaw cycles of calcium chloride $(\mathrm{CC})$ exposure

Figure 8.7 Pictures of CC/FT fracture surfaces displaying a specimen from each target-age (superscripted) - (a) $5 \mathrm{~B} 3^{50}$, (b) $1 \mathrm{~B} 9^{100}$, (c) $2 \mathrm{~B} 10^{150}$, (d) $5 \mathrm{~B} 9^{200}$, and (e) $8 \mathrm{~B} 11^{300}$ (NOTE: the fracture behavior of these specimens, with the exception of (b), are shown in Fig. 8.5)

Figure 8.8 Pictures displaying progressive changes occurring at the $\mathrm{CFRP}$-concrete interface of various $\mathrm{CC} /$ freeze-thaw specimens - (a) FT companion (300-cycled), (b) FT150, (c) FT200, and (d) FT300

Figure 8.9 Percent average weight changes of the $\mathrm{CC} /$ freeze-thaw plain, companion, and actual beams

Figure 8.10 Pictures of concrete mass residue left behind in $\mathrm{CC} /$ freeze-thaw trays - (a) mass lost for two specimens after 300 cycles was approx. $0.53 \mathrm{~N}(0.12 \mathrm{lb})$, (b) \& (c) shows two views of the dispersed residue left behind (NOTE: dimensions of tray given in Fig. 8.3(a))

Figure 8.11 Microstrains of the CC/freeze-thaw beams in two locations:

(1) on the CFRP, and (2) on the adjacent concrete ledge (see Fig. 3.21(d)) - the differential movement between the two is shown as well

Figure 8.12 Conclusive percentage reductions in (a) $G_{I c}^{i}$ and

(b) $G_{I c}^{a}$ resulting from $50,100,150,200$, and 300 freeze-thaw cycles of calcium chloride (CC) exposure (repeated from Fig. 8.6)

Figure 9.1 Conceptualized FRP-strengthened beam-structures showing delamination - (a) start (Karbhari et al. (1997)), and (b) progression (Green et al. (1998))

Figure 9.2 (repeated from Fig. 3.9) Fracture toughness $\left(K_{c}\right)$ as a function of specimen thickness (a) - (d) (Kanninen and Popelar, p. 179, 1985)

Figure 9.3 (repeated from Fig. 4.18) The optimum SCCB calibration specimen S3 — width (not shown), b = $43.4 \mathrm{~mm}(1.71 ")$; contour angle $=6.3^{\circ}$

Figure 9.4 (repeated from Fig. 6.3) The statistical-based bandwidth for(a) compact, and (b) widespread data

Figure 9.5 Conceptual fracture surfaces of beams undergoing:

(a) 10-, (b) 20-, and (c) 30- SS/wet-dry cycles

Figure 9.6 Conceptual fracture surfaces of beams undergoing:

(a) 10-, (b) 20-, and (c) 30- SH/wet-dry cycles

Figure 9.7 Conceptual fracture surfaces of beams undergoing: 
(a) 50-, (b) 100-, (c) 150-, (d) 200-, and (e) 300-

$\mathrm{CC} /$ freeze-thaw cycles

Figure 9.8 (repeated from Fig. 6.11) Averaged conclusive pristine- and representative- dry fracture results

Figure 9.9 Averaged conclusive SS and SH wet-dry $\% G_{I c}$ reductions (based on Table 7.11)

Figure 9.10 Averaged conclusive $\mathrm{CC}$ freeze-thaw $\% G_{I c}$ reductions (based on Table 8.8)

Figure 9.11 Conclusive magnitudes of fracture toughness:

Dry - Weathered (based on initiation energies)

Figure 9.12 (repeated from Fig. 8.1) Freeze-thaw cycling scheme

Figure C.1 The demountable mechanical strain gauge (DEMEC)-

(a) DEMEC with setting-out and invar bars

(b) DEMEC in use atop CFRP

Figure C.2 The CSZ ZH-16 environmental chamber-(a) front view of chamber with wet-dry beams, (b) close-up of computerized keypad, and (c) close-up of control panel

Figure C.3 Freeze-Thaw Cycling Scheme (repeated from Fig. 8.1) 391

Figure C.4 MTS 418.91 microprofiler and 458.10 microconsole 393

Figure C.5 MTS schematic (Unmarked dimensions in millimeters) 395 


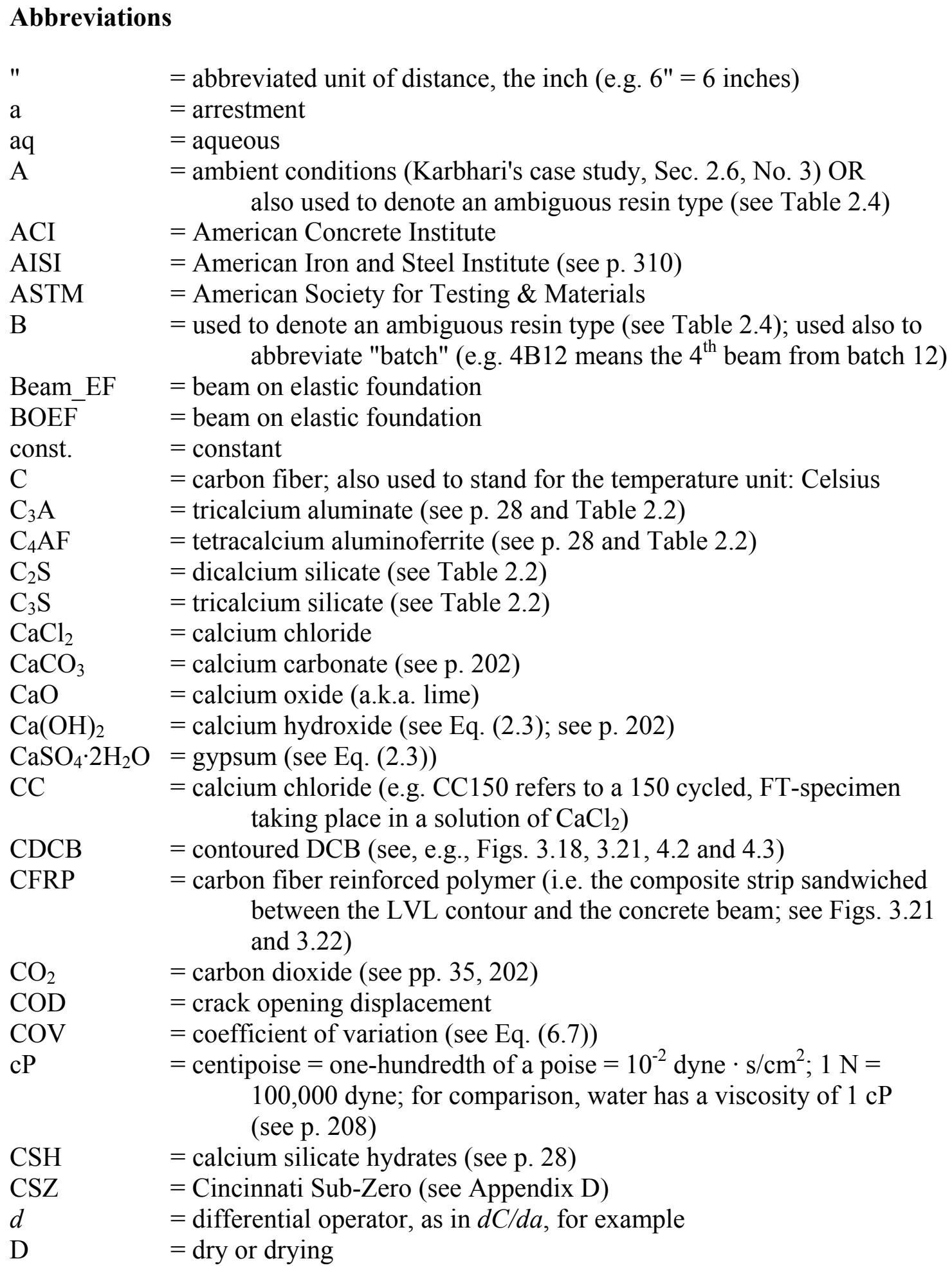




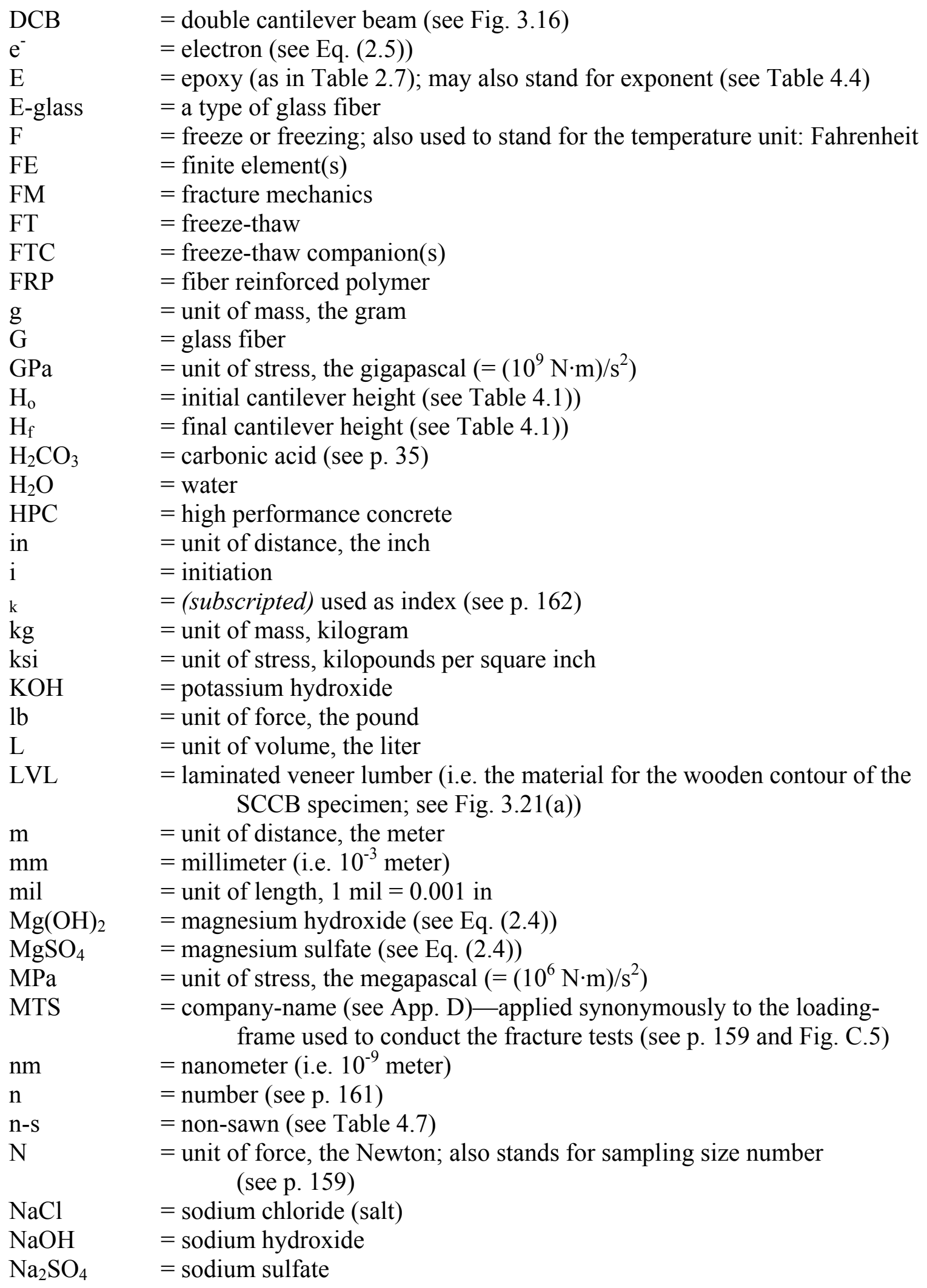




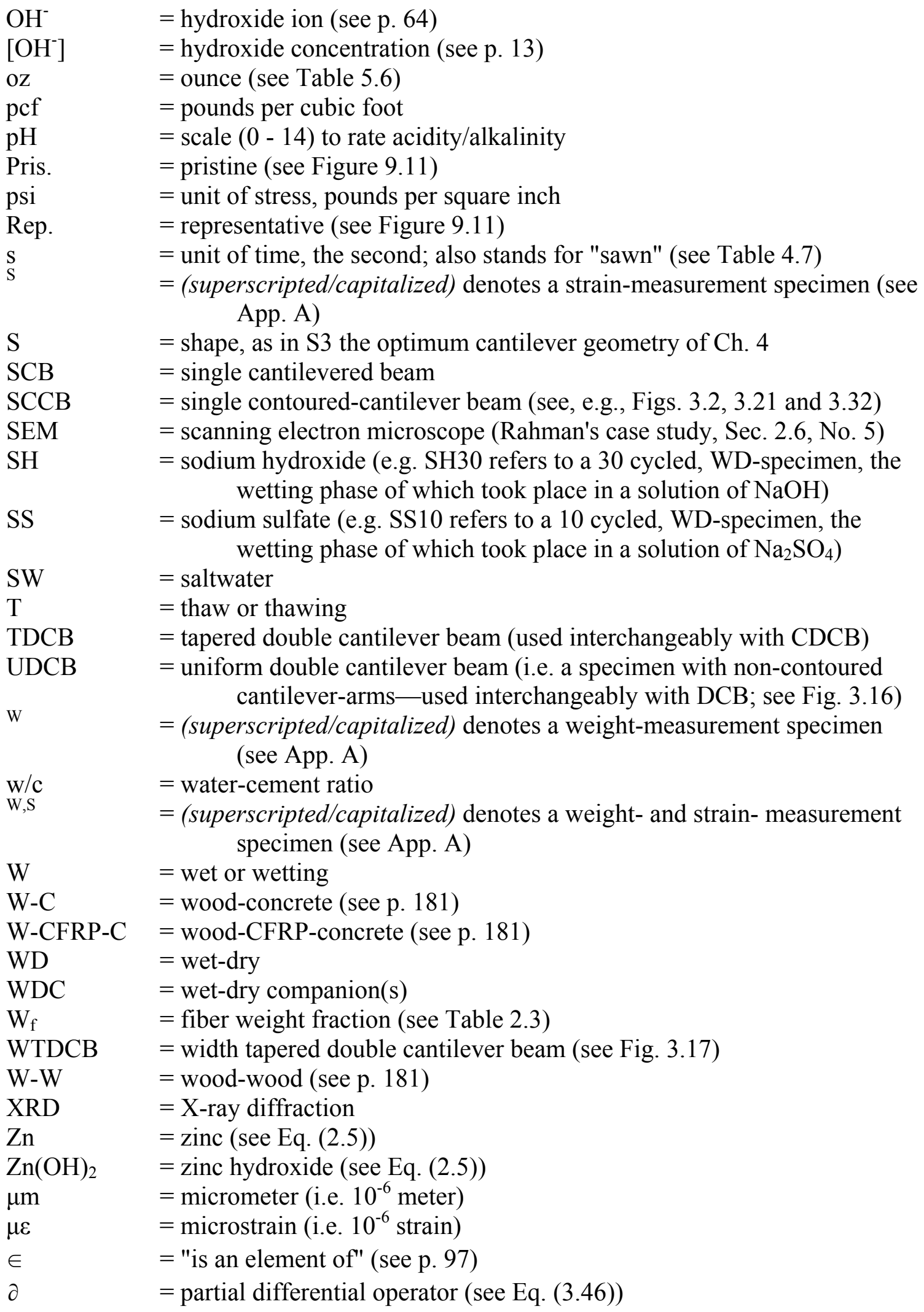




\section{Notation}

\begin{tabular}{|c|c|}
\hline$a$ & $\begin{array}{l}=\text { crack length position (see Fig. 3.12); also used for the semi-major axis } \\
\text { of an ellipse (see, e.g., Eq. (3.4)) }\end{array}$ \\
\hline$\left.a\right|^{i}$ & $=$ crack length at the instant that $G_{I c}^{i}$ is measured (see Eq. (3.75)) \\
\hline$\left.\Delta a\right|^{i-a}$ & $\begin{array}{l}=\text { incremental length of crack-growth measured between crack -initiation } \\
\text { and -arrest (see Eq. (3.75)) }\end{array}$ \\
\hline$A$ & $\begin{array}{l}=\text { area (in general); also strength of internal wire structure constant (see } \\
\text { Eq. (3.3)) }\end{array}$ \\
\hline$b$ & $\begin{array}{l}=\text { specimen width (e.g. see Eqs. (3.48) and (3.73)); also used for the semi- } \\
\text { minor axis of an ellipse (see, e.g., Eq. (3.4)) }\end{array}$ \\
\hline B & $=$ strength of surface wire structure constant (see Eq. (3.3)) \\
\hline$C$ & $=$ compliance $($ in this context, the ratio: $u / P$ ) \\
\hline$C_{i, f i n a l}$ & $=$ final compliance value $($ see $\mathrm{p} .161)$ \\
\hline$\left.C\right|^{i}$ & $=$ specimen compliance at the initial crack length, $a^{i}$ (see Eq. (3.76)) \\
\hline$\left.C\right|^{M}$ & $=$ compliance of the testing machine (see Eq. (3.76)) \\
\hline C_ave & $=$ average compliance \\
\hline $\mathrm{CO}^{\mathrm{a}}{ }_{\mathrm{Head}}$ & $=$ coefficient of variation of the head arrestment loads (see Eq. (6.8)) \\
\hline $\begin{array}{l}\mathrm{COV}_{\text {Head }}^{\mathrm{i}} \\
\mathrm{d}\end{array}$ & $\begin{array}{l}=\text { coefficient of variation of the head initiation loads (see Eq. (6.7)) } \\
=\text { diameter }\end{array}$ \\
\hline$d C / d a$ & $\begin{array}{l}=\text { compliance gradient, or technically, the rate change in compliance }(C) \\
\text { with respect to the crack length }(a)\end{array}$ \\
\hline $\mathrm{dc} / \mathrm{dL}$ & $=$ concentration gradient (see Eq. (2.2)) \\
\hline $\mathrm{dq} / \mathrm{dt}$ & $=$ flow-rate (see Eq. (2.1)) \\
\hline $\mathrm{D}$ & $=$ diffusion coefficient (see Eq. (2.2)) \\
\hline $\mathrm{E}$ & $=$ modulus of elasticity \\
\hline $\mathrm{E}_{\mathrm{c}}$ & $\begin{array}{l}=\text { modulus of elasticity of concrete (in this case: } 4.93 \times 10^{6} \text { psi; } \\
\text { see p. } 250,340 \text { ) }\end{array}$ \\
\hline$f_{c}^{\prime}$ & $=$ compressive strength of concrete \\
\hline$f_{\max }$ & $=$ maximum stress \\
\hline $\mathrm{f}_{\mathrm{sp}}^{\prime}$ & $=$ split-tensile strength of concrete (in this case: 640 psi; see Eq. (6.14)) \\
\hline $\mathrm{f}_{\mathrm{t}}$ & $=$ tensile strength of concrete (in this case: 598 psi; see Eq. (6.13)) \\
\hline $\mathrm{f}_{\mathrm{u}}$ & $=$ ultimate strength \\
\hline $\mathrm{F}$ & $=$ strength of wire $($ Eq. (3.3)) \\
\hline$F$ & $=$ available energy for fracture (e.g. see Eqs. (3.44) and (3.45)) \\
\hline$F_{y}$ & $=$ yield strength \\
\hline G & $=($ not italicized $)$ shear modulus \\
\hline$G$ & $=($ italicized) strain energy release rate (see Eq. (3.45)) \\
\hline$G_{c}$ & $=$ critical strain energy release rate \\
\hline
\end{tabular}


$G_{I c} \quad=$ Mode I critical strain energy release rate (see Eq. (3.73))

$G_{\text {Ic,companion(\#cycles) }}=$ Mode I critical strain energy release rate value for some companion specimen, with age corresponding to some number of cycles (see Eq. (7.1))

$G_{I c, \text { conditioned(\# cycles) }}=$ Mode I critical strain energy release rate value for some weathering scheme, conditioned for some number of cycles (see Eq. (7.1))

$G_{I c}^{a} \quad=$ arrestment-based critical strain energy release rate (see p. 115 and Eq. (3.74)

$G_{I c}^{i} \quad=$ initiation-based critical strain energy release rate (see p. 118 and Eq. (3.74)

$G_{I I c} \quad=$ Mode II critical strain energy release rate

$h \quad=$ height

$\Delta \mathrm{h} \quad=$ drop in hydraulic head (see Eq. (2.1))

$i \quad=$ index number (see p. 161)

I $\quad=$ brittleness index (see Eq. (3.74)); also used for moment of inertia (see Eq. (3.1))

$\mathrm{I}_{1} \quad=$ stress invariant (see Eq. (3.33))

$\mathrm{I}_{2} \quad=$ stress invariant (see Eq. (3.34))

$\mathrm{I}_{3}=$ stress invariant (see Eq. (3.35))

$\mathrm{J} \quad=$ mass transport rate (see Eq. (2.2))

$\mathrm{k}=$ coefficient of permeability

$K=$ = stress intensity factor; symbol also used to stand for: kinetic energy

$K_{c}=$ critical stress intensity factor, or simply fracture toughness

$K_{I c} \quad=$ Mode I fracture toughness (a.k.a. plain strain fracture toughness)

$K_{t} \quad=$ stress concentration factor (see Eq. (3.5))

$\Delta K \quad=$ increase in kinetic energy (e.g. see Eq. (3.44))

$\ell_{1} \quad=x$-direction cosine (see Fig. 3.7 and Eq. (3.23))

$\ell_{2} \quad=$ y-direction cosine (see Fig. 3.7 and Eq. (3.24))

$\ell_{3} \quad=$ z-direction cosine (see Fig. 3.7 and Eq. (3.25))

$\mathrm{L} \quad=$ thickness of sample (see Eq. (2.1))

$M \quad$ = bending moment

$\mathrm{n} \quad=$ number of specimens (i.e. sampling size)

$\mathbf{N} \quad=$ unit normal vector (see Fig. 3.7)

$P \quad=$ load

$P^{a} \quad=$ crack-arrest load (see p. 115)

$P_{c} \quad=$ critical load (read Sec. 6.2)

$P_{c}^{a} \quad=$ critical arrest-load (see p. 115)

$P_{c}^{i} \quad=$ critical initiation-load (see p. 115)

$P_{\text {Head }}^{a} \quad=$ the mean of the head arrestment loads (see Eq. (6.5))

$P_{\text {Head }}^{i} \quad=$ the mean of the head initiation loads (see Eq. (6.3))

$P_{n} \quad=$ nominal concentrated load (see Fig. 2.1)

$-Q \quad=$ amount of energy lost in the form of heat (e.g. see Eq. (3.44))

$r$

$=$ radial coordinate from crack-tip (see Fig. 3.6) 


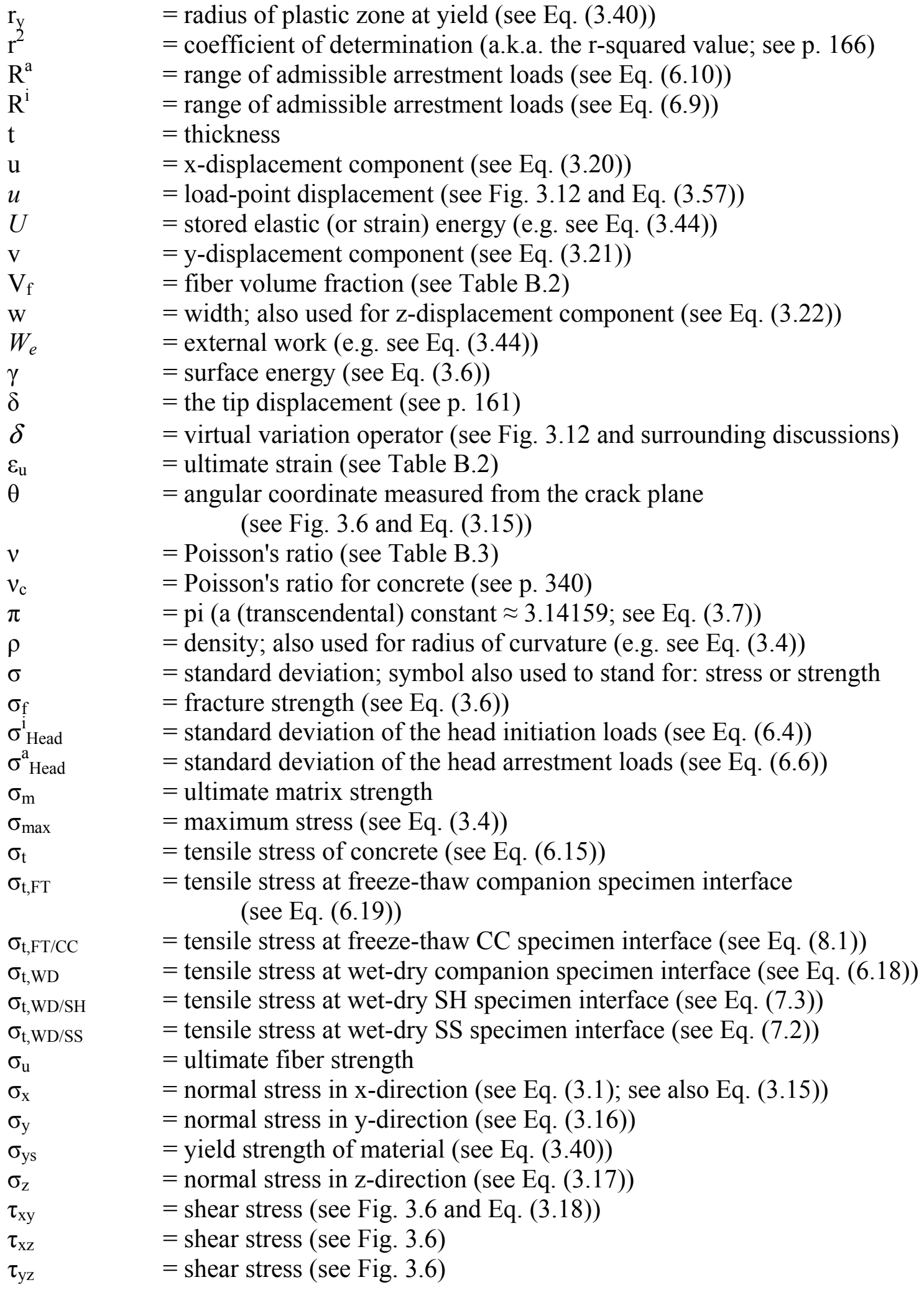




\section{CHAPTER 1}

\section{INTRODUCTION}

\subsection{Introduction}

The continual degradation of civil infrastructures worldwide has prompted the need for effective solutions, posing major challenges to those involved with reinforced concrete $(\mathrm{RC})$ structures and bridges. Various methods have been used to rehabilitate or upgrade deficient RC members. Traditionally, one such method, proving successful for a period spanning more than 20 years, involved the bonding of steel plates onto various structural components, e.g. as on the tension faces of RC beams. There are, however, some disadvantages associated with the use of steel plates, not least of which is the possibility of corrosion responsible for adversely affecting the bond between the plate and concrete (Quantrill et al., 1996). Other obvious disadvantages of using steel plates include the sheer weight of the unit itself - a significant problem, especially when longspanned structures are concerned - and the increased overall construction costs stemming from the need to use both heavy machinery and the skills of trained individuals to operate such equipment, during installation. 
In response to these concerns, the application of fiber reinforced polymer (FRP) plates and laminates has, in recent years, been demonstrated as an effective means in the strengthening and rehabilitating of RC structures. The many advantages associated with using FRPs include such qualities as: resistance to corrosion, i.e. tremendous durability against natural weathering processes, lightness in weight while exhibiting high strength, i.e. high strength-to-weight ratios, possessing nonmagnetic- and nonconductiveproperties, having desirably low maintenance-costs, and marked by both, simplicity and versatility of use regarding placement, which, as will be seen in the following section, contributes significantly to overall reductions in cost as well. Of the latter point concerning versatility, FRPs have been used to retrofit concrete members such as columns, smoke stacks, slabs, beams, and girders, as well as a variety of structuresbridges, parking decks, and buildings, just to name a few (Buyukozturk and Hearing, 1998; more about this in Section 1.2, under "Industrial Applications"). Hence, in succinctness, the external strengthening of concrete by FRP materials has the conjoined benefit of extending the service of the structure in terms of both capacity and longevity, while essentially lowering the associated life-cycle costs.

Though the potential exists for FRPs to enhance the overall strength and stiffness of concrete structures-yielding as much as a threefold increase in the flexural capacity of RC beams, for example (read Boyajian, 2002a, regarding flexural-enhancement guidelines in the design of FRP-strengthened concrete beams) - there are, however, concerns associated with the long-term performance and reliability of the interface bond that are principally-crucial to the successful application of this technology. This is 
evident, since, at best, an insufficient bond renders the external reinforcement as ineffective, thus defeating any contribution to have been gained by the placement of fibers there in the first place, and, at worst, may potentially result in catastrophic failure of the reinforced member altogether.

Unlike monolithic structures, which may collapse or be rendered inoperative by a variety of means, e.g. excessive elastic/inelastic deformations or fracture, failure of adhesive joints, on the other hand, is practically governed by fracture alone (Ripling et al., p. 107, 1971). The reason for this is that adhesive joints comprise such a small fraction of the total structural-volume that even large elastic or inelastic deformations are tolerable, globally-speaking. At this juncture, Ripling et al. (pp. 107-111, 1971) draw a lucid analogy between elementary design methods - the objectives of which are aimed at precluding excessive deformations - and that of fracture. In the former approach, the designer analyzes the part in question to determine whether the anticipated maximum stress encountered under service conditions, $f_{\max }$, is feasibly contained within the limits of the material's yield strength, $F_{y}$. Two quantities are analogously defined for fracture: one is a parameter that describes the stress field at a crack tip known as the stress intensity factor, $K$ (read Section 3.2, under the subhead titled "Stress Intensity Factor, $K$ ") and the other, referred to as the fracture toughness, identifies the critical value of this parameter at which a slow moving or stationary crack jumps ahead, denoted $K_{c}$. In this vein, Paul Paris and George Sih (1964) formed an excellent compilation of the stress fields existing at the crack-tips of a limited number of monolithic structures. The analyses that would be required to describe the stress fields surrounding crack-tips lying in a thin layer of 
neighboring low and high modulus materials - as with concrete-FRP interfaceshowever, would be much more involved. Consequently, it is preferable to define fracture toughness in terms of energy rather than by convention of stress intensity factors whenever heterogeneous conditions prevail—Eqs. (3.13) and (3.14) reveal the simple plane stress and strain relations, respectively, that can be used to transform between the two systems.

The strain energy release rate, $G$, is therefore defined as the energy required to extend a preexisting crack by an infinitesimal unit of area (see Figure 3.12 and Eq. (3.45)) - a definition that is a direct outgrowth of Griffith's (pictured in Figure 3.4) work on glass (1921; see also Sec. 3.2, under subsection titled "Theories of Griffith"). Returning now, momentarily, back to the analogy of the previous paragraph, inasmuch as a material can flow inelastically under the action of a tensile or shear force, fracture ensues in more than one mode- three to be exact (with, of course, any inter-mode combinations thereof) - denoted as Mode I, Mode II and Mode III (see Sec. 3.3). As is explained in great detail in Chapter 3, the first of these modes (a.k.a. the opening mode) relative to the critical strain energy release rate, $G_{I c}$, is of special importance, not only because it is encountered in the overwhelming majority of actual engineering situations concerning brittle fractures (Hertzberg, 1976), but also because it represents a practical lower limit which offers assurance of conservatism in design (see Figure 3.9 and the surrounding discussions).

Traditionally, Mode I fracture testing of materials such as metals, composites, adhesives, and wood was achieved with relative ease through standard experimental 
methods, e.g. the Double Cantilever Beam (DCB) or some variants thereof (see Section 3.5), owing to these materials' intrinsic ability to withstand tensile stresses. Unfortunately, attempts at extending this well-established technique to the study of FRPconcrete interfaces culminated without any success, resulting instead in the phenomenon of "arm-breakoff" (see Figure 3.1, Eqs. (3.1), (3.2), and the surrounding discussions) — a term that was coined by Mostovoy and associates (1967) while studying the fracture properties of plexiglas. In order to overcome this dilemma, a novel experimental Fracture Mechanics specimen conceived out of the DCB approach, was devised at West Virginia University (in the latter part of 1999) by the efforts of Dr. Julio F. Davalos and the present author which has now come to be known as the Single Contoured-Cantilever Beam (SCCB; see Figure 3.2).

\section{The SCCB Specimen}

As implied by the name, one of the novelties of this specimen is that it consists of only one cantilevered member in contrast to the more traditional, double-armed arrangement of its predecessor. Additionally, the height of this member is made to vary (linearly, in this case) along its longitudinal axis - hence, the descriptor, "Contoured", in its name. This is done, as with the CDCB specimen (see Sec. 3.5), to render a constant compliance gradient (i.e. $d C / d a=$ constant), thereby precluding any need for crack length (i.e. a) measurements during fracture-testing - a truly formidable undertaking, experimentally-speaking. In other words, by designing the cantilevered member with a specific contour-shape (the subject of Chapter 4), it is possible to reduce the number of 
independent variables in the governing critical strain energy equation (Eq. (3.73)—a.k.a. the Irwin-Kies relation) from 2 to 1 :

$$
G_{I c}=G_{I c}\left(P_{c}, a\right) \rightarrow G_{I c}=\left.G_{I c}\left(P_{c}\right)\right|_{d C / d a=\text { const. }}
$$

With this point in mind, a couple of vitally pertinent issues must be observed. First, that while parametric-reductions as this are a highly lucrative venture from a mathematicalstandpoint, they are, unfortunately, not achieved without an equalizing-price-in this case, the undertaking of extensive experimental compliance calibrations (see Ch. 4). Second, eliciting the surviving critical load parameter, $P_{c}$, from among the myriad of experimental data has traditionally been steeped in both ambiguity and controversy. In response to this, the author has devised a statistical approach to reduce the raw data (Boyajian et al., pp. 6-7, 2002b; see also Section 6.2) and thereby achieve a scientificallybased, representative value of critical load. The importance of procuring a truly representative $P_{c}$ cannot be overstated, as it is the square of this term that is directly proportional to the Mode I fracture energy $\left(G_{I c}\right.$; see Eq. $\left.(3.73)\right)$ - i.e. any error in $P_{c}$, digresses the value of $G_{I c}$ by the square of this amount - an especially baneful outcome provided, of course, that the absolute difference between the prescribed and actual $P_{c}$, results in a value greater than unity.

There are three other unique and noteworthy features of the SCCB specimen and test-setup. First, with respect to the specimen itself, the shape of the bulk concrete substrate is specially designed as a trapezoid in cross section (see Figure 3.19(a)); second, concerning the test-setup, a matching steel jacket (see Figures 3.2 and 3.27) is provided. The reasons for introducing such features as these are so that, during fracture-testing, on 
the one hand, the bulk concrete substrate will be sufficiently reinforced from without and kept intact; and on the other, so that the specimen will be constrained in the vertical direction, i.e. the action-line of the applied, Mode I tip-load. A final distinguishing attribute of the SCCB specimen to take note of, is the laterally-recessed, $3 \mathrm{~mm}$ concrete raised-step (see Fig. 3.19(a)). The width of this step was cast (discussed in Section 5.2) to accommodate the width of the LVL (laminated veneer lumber) section-see Figures 3.21, parts (a) and (d) — so as to not only aid in guiding proper placement of the contour during bonding, but more importantly, to ensure that the propagation of cracks during fracture-testing would remain true to the composite-concrete interface.

\subsection{Significance}

Prior to this research-effort, there were no methods available to rigorously evaluate the interface bond strength and integrity for concrete structures externally strengthened by FRP materials. Existing large-scale tests of structural components used for strength and stiffness evaluations, on the one hand, are ill suited for detecting delamination effects; on the other, small-scale tests render only average interface strength properties that neither describe failure mechanisms nor provide fracture toughness data. The SCCB specimen was designed to positively overcome these hindrances. Two points - one quantitative, the other qualitative — are implicated as a direct consequence of instituting this new Fracture Mechanics test-methodology.

First, from the quantitative perspective, it must be recognized that the $G_{I c}$ quantity being sought after here is unique, not only because adhesive joints are limited to a mode of failure that are governed by fracture, as was mentioned in the previous section, but 
more significantly, because this parameter is a material constant (see Fig. 3.9 and read the surrounding discussions) in just the same manner as are, for example, the Young's and shear moduli. The essentialness of these latter two quantities are well-understood by engineers universally; notwithstanding, the lesser understood role of the critical strain energy release rate (in this context, of FRP-concrete interfaces) bears the same distinguishing prospect of being eventually incorporated as a useful parameter for both theoreticians and design engineers, alike. (Reiterating a point made in the introductory section, the fact that this research-work revolves about the Mode I $G_{c}$ value bears with it the special benefit that this quantity represents a practical lower limit of fracture toughness; consequently, engineers would be assured of conservatism in their designs.)

Of more immediate importance, however, is the issue pertaining to quality. Given that the technology of FRP-strengthened concrete members is still in its infancy at this point in time, it can therefore safely be conjectured that there yet exists a number of years separating the approval of quantitatively-based, design-specifications (i.e. the subject of the last paragraph), and the unconstrained/widespread use of these materials in civil infrastructure applications. Contrarily, the SCCB specimen and fracture test methodology can be used as a qualification-tool for any number and type of fiber-resin systems. The drafting of standards based on qualitative assessments can then be undertaken to rate the performance of a variety of products subjected to a combination of weathering regimes. Of special value here will be the ability to determine the medley of fibers and resins bestsuited for use under diverse environmental settings. The potential for systemdevelopment in the R\&D sector is also tremendous, especially as it concerns the 
enhancing of coupling-agents and the formulation of more durable adhesives-the constituents most often cited as being the real culprits, or weak-links, in limiting the overall success of this technology. Finally, and pragmatically, results that are to be gained from qualitative SCCB fracture-testing may act to boost consumer- and designerconfidence in the use of FRP materials as a viable means of strengthening and rehabilitating civil infrastructure.

\section{Industrial Applications}

While the technology to strengthen structures using FRPs is still in its infancy at this point in time, there are nevertheless several documented reports chronicling the effective incorporation of these materials in industry. A concise citing of applications follows - taken from two sources: (1) the Tonen Corporation's log-report (inclusive through March 1994; FORCA TOW SHEET Technical Notes, 1995), and (2) several case studies as communicated by Ronald J. Watson (Watson, 1998 and 2002)-in demonstration of such instituted methods. By the consideration of these accounts, the virtually limitless range of possible FRP uses relative to infrastructure-assimilation should become evident, thus necessitating endorsement of the tremendous benefits to be gained from their widespread implementation into construction practice.

Tonen's Log-Report (as of March 1994; FORCA TOW SHEET Technical Notes, 1995): A total of seventeen different types of structures were externally strengthened using FRP materials (occurrence-frequencies are cited parenthetically) — in no particular order, these involved:

$>$ RC slabs (17) 
RC beams (14)

RC column (1)

$>$ RC walls (6)

$>\mathrm{RC}$ building repair (1)

$>\mathrm{RC}$ basement (1)

chimneys (7)

$>$ piers (4)

$>$ electric poles (19)

wastewater pits (2)

$>$ pipes (7)

$>$ tunnels (15)

$>$ bridges (8)

$>$ concrete foundations (4)

$>$ smokestacks (3)

$>$ general RC component (1)

$>$ general metallic components (6)

As can be seen, the top five structural entities to be retrofitted were:

(1) electric poles-19,

(2) RC slabs-17,

(3) tunnels-15,

(4) RC beams-14, and

(5) bridges- 8 
Case Studies (Watson, 1998 and 2002): Nine separate FRP -strengthening and -rehabilitating cases are reviewed in Watson's two papers-six from his earlier communication and three from the latter work. The retrofitted structures mentioned in these two reports range from bridge pilings and foundation-piers of beachfront homes, to smokestacks, floor beams, and RC columns. Following are synopses of each of these completed tasks in their order of presentation:

(1) $\underline{\boldsymbol{R} C \text { pilings }}$; Port Hueneme, CA-year not mentioned: The U.S. Navy at Port Hueneme has incorporated the use of carbon FRPs (or simply CFRPs) to repair RC pilings subjected to marine environments. The general problem facing structural engineers in these situations is the alternate cycles of wetting and drying (see also Chapters 2 and 7) as caused by varying storm- and tide- conditions which, subsequently, lead to the dissolution of the pilings (consisting, commonly, of: steel, concrete or wood).

(2) $\underline{\boldsymbol{R} C \text { foundation-piers; }}$; Malibu, CA-1995: A similar occurrence has been found with respect to the foundations of many beachfront homes that rest atop rectangular and circular RC piers. In these cases, the underlying concrete columns begin cracking and spalling due to internal corrosion of the reinforcing steel (read, for example, the subsection titled "Sea Water Effects" of Sec. 2.3). Previous attempts to rehabilitate these members ranged from simple patching techniques to extensive column encasements involving $152 \mathrm{~mm}(6 ")$ thick RC covers - both, of which, have been demonstrated as short term solutions to this universal problem. A better solution was achieved in 1995, when these piers were strengthened using glass and aramid FRP (or simply GFRP and AFRP) materials embedded in a chloride resistant epoxy matrix. Watson (p. 117, 1998) 
reports that after two years of service - the point at which conventional repairs show signs of deterioration - there was no evidence of continued decay and/or spalling.

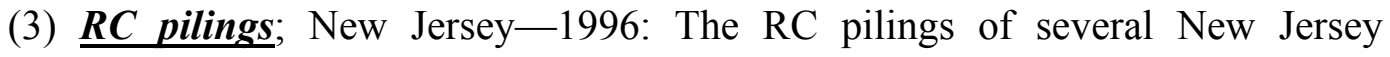
Turnpike bridges are also exhibiting problems associated with wetting and drying (see case (1) above) as a result of being located in tidal flood plane regions containing brackish waters. In 1996, the bridge engineers decided to perform a pilot-study on a severely deteriorated pile that had previously been encased by a $61 \mathrm{~cm}\left(2^{\prime}\right)$ diameter $\mathrm{RC}$ shell, by wrapping it with two layers of FRP — one ply with GFRP, the other with AFRP. Cited among the benefits gained from using this approach are the expeditiousness with which the entire procedure was accomplished - reported to have taken less than a day!and, relatedly, the disburdening procured from not having to bother with extensive traffic control matters.

(4) Concrete smokestacks; Manhattan, NY_-1997: In the spring of 1997, a storm tore pieces of concrete cladding from two $53 \mathrm{~m}\left(175^{\prime}\right)$ high, 90 year old smokestacks located atop an Amtrak power control station in midtown Manhattan. Concerns were raised that future storms might topple the towers onto adjacent structures - one of them being the renowned Madison Square Gardens. In an effort to stabilize the towers, engineers decided to reduce the heights of the stacks; this, however, raised new concerns of whether the deteriorated sections of the tower would be capable of withstanding the forces generated during dismantlement. A new solution was therefore proposed which recommended the wrapping of the smokestacks with FRP materials. The repairs were 
performed from a basket on top of a boom in the matter of days, and as such, resulted in a cost-effective answer to a potentially hazardous problem.

(5) Concrete Floor beams; Albany, NY-1995: In 1989, the Agway Corporation decided to upgrade the storage-capacity in one of its animal feed manufacturing facilities. This plan, however, was abandoned when it learned of the estimated project costs$\$ 250,000$ - and the further loss in revenue resulting from 4 - 6 weeks of plant-downtime. Five years later, engineers suggested the more cost-effective possibility of using FRP materials to retrofit the floor-system. When inspecting the concrete, they determined that the floor beams could adequately bear the demands of the added loads in flexure but not in shear. Due to the fact that shear cracking occurs at nearly $45^{\circ}$ angles, they therefore decided to strengthen the beams with a double bias $+45 /-45$ woven glass fabric. The retrofit was finally performed in 1995 at a total cost of $\$ 60,000-$ a savings of $\$ 190,000$ as compared to the conventional technique! Furthermore, the work was completed in less than two weeks with no downtime, resulting, therefore, in even greater savings for the owner.

(6) $\underline{R}$ columns (rehabilitation); Oakville, Ontario-1995: In 1993, the Petro Canada Refinery, serving the Metro-Toronto area, fabricated a new structural steel heating vessel to be supported atop 8 steel RC columns (each $194 \mathrm{~cm}$ sq. $=30$ inches sq. in cross section, and $2.7 \mathrm{~m}=9^{\prime}$ in height). During installation, however, it was discovered that the prefabricated slots in the vessel-connection were not aligned properly with the cast-in-place anchor bolts of the columns. To remedy this problem, the construction crew decided to hammer the bolts into position, and as a result, unwittingly induced cracks to 
form in the adjoining concrete member. Over the next two years, as these cracks grew, certain areas of the column became vulnerable to both spalling and internal steel corrosion. Consequently, engineers decided to rectify the situation by prescribing that the columns be rehabilitated with a blend of GFRP/AFRP composite sheets. The work, completed in a matter of only 3 days, proceeded without incident to the refinery's operating-schedule, and as such, translated to tremendous reductions in overall costs for the company.

(7) $\underline{R}$ C columns (seismic-retrofit); California-1991: In the fall of 1991, Caltrans bridge engineers decided to implement FRP materials on actual bridge columns. One particular project involved the strengthening of the columns supporting the I-5/Highway2 Interchange in Los Angeles, with GFRP sheets. This section of the freeway consists of 12-1.8 $\mathrm{m}\left(6^{\prime}\right)$ and 3-1.2 $\mathrm{m}\left(4^{\prime}\right)$ diameter columns ranging in heights from $5.5 \mathrm{~m}\left(18^{\prime}\right)$ to $15.2 \mathrm{~m}\left(50^{\prime}\right)$. The entire project was completed in 3 weeks at a cost that was significantly less than a comparable steel jacket retrofit. These columns, however, were really put to the test when, in the early morning hours of January 17, 1994, the Northridge Earthquake, measuring 6.7 on the Richter Scale, rocked Southern California. The massive temblor resulted in the collapse of the I-5/Highway-14 Interchange, located just northwest of downtown Los Angeles, killing a California Highway Patrol officer. Additionally, columns supporting sections of the Santa Monica Freeway (west of downtown) which had not yet been retrofitted, sustained extensive damage due to inadequate confinement. In contrast, the retrofitted-columns were perfectly left intact. 


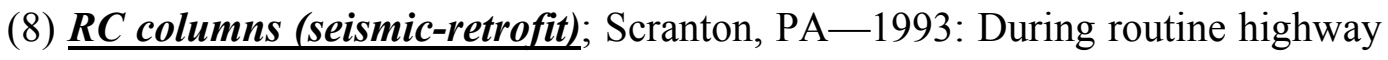
inspections, PennDOT officials determined that in the event of an earthquake, the RC columns supporting the I-84 bridge located just east of town, did not have adequate internal steel confinement. Previous retrofitting measures involved the tightening of threaded steel bars with turnbuckles around the base and top of columns, followed by a gunited-finish. While this seismic-retrofit approach is effective, it is also very costly. Alternatively, when the use of FRP materials was proposed, it received not only stronger consideration, but also the quick approval of department engineers; especially alluring, here, were the potential benefits to be gained: cost-effectiveness, installation-ease, and the feature of non-corrodibility. The project, which began in the month of July, 1993, took about a week to complete and cost the State of Pennsylvania a total of $\$ 78,000$ (or approximately, $\$ 84 / \mathrm{m}^{2}=\$ 8 / \mathrm{ft}^{2}$ ) - a savings-factor of about 3 with respect to the gunite method. Five years later, PennDOT officials thoroughly inspected the columns of the bridge to evaluate its overall performance. The engineers found that, apart from the FRPsurface being free of any indication of weathering, the bond-i.e. the most critical feature of this technology — was still very sound. Assuredly, the PennDOT study resulting from this investigation, as reported by Sukley and Howrylak (1998), went on to recommend the continued use of FRP materials to structurally-retrofit deficient bridge members.

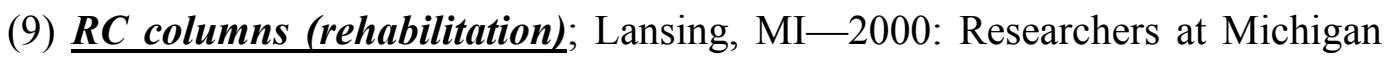
State University (Harichandran and Baiyasi, 2000) initiated a study on the repair of corrosion damaged columns of a bridge located in Lansing, MI, using FRP wraps. A total of 6 columns will be continually studied over the course of the next ten years - two of 
which are wrapped with 3 layers of GFRP, two with 2 layers of CFRP, and the remaining two, left unwrapped to serve as controls. As this study is being extended a whole decade, it should serve as an excellent barometer for gauging the overall long-term performance of FRP-enhanced bridge members. Some of the issues that are currently being explored in this investigation include the effects of: wet-dry cycling (see also Ch. 7), freeze-thaw durability (see also Ch. 8), rates of internal steel-corrodibility, impact resistance, and high temperature effects. Though it is yet too early to conclude anything decisively, no significant form of deterioration has been found to have occurred, thus far.

\subsection{Objectives}

The Primary, Pre-, and Post- Objectives

Specifically, the primary objective of this research is to use an experimental Fracture Mechanics approach to characterize the FRP-concrete interface bond for both unweathered (Chapter 6) as well as environmentally conditioned (Chapters 7 and 8) specimens.

As it was already explained in Section 1.2, since, until now, there were no widelyaccepted experimental methods to achieve this objective, a pre-objective to this research was to develop such a methodology, culminating in what has now come to be known as

the Single Contoured-Cantilever Beam (SCCB) specimen (see Figure 3.2; also read Section 3.6). Incidental to this pre-objective is the special-contouring of the cantilevered member to render a constant value of $d C / d a-$ see, Eq. (1.1) and the surrounding discussions. Fulfillment of this aim was achieved through the use of a beam on elastic 
foundation model (Qiao et al., 2001) and the verifications elicited by both a Finite Element analysis and the undertaking of extensive experimental compliance calibrations (see Chapter 4).

At the other extreme, a post-objective was revealed as an outgrowth of attempting to assimilate the multitudinous streams of fracture data and arrive at a representative critical value of load, $P_{c}$ (refer back to the discussions on p. 6). Surprisingly, the experimenters of Fracture Mechanics in the last half-century, i.e. beginning in the 1950's until just recently, never arrived at a universal-resolution of this crucially-important issue. Attempting to remedy this problem, then, the author devised a statistical approach to reduce the raw data (Boyajian et al., pp. 6-7, 2002b; see also Section 6.2) and thereby achieve a scientifically-based, representative value of critical load.

Having thus established an unbiased means by which the critical load and, hence, the critical strain energy release rate, $G_{I c}$ (see Eq. (3.73)), are to be determined, some details of the primary objective may now logically follow. Recall, first, that two types of specimens are to be studied: (1) unweathered, and (2) environmentally conditioned. The former, in turn, consists of two denominations of beams: (a) pristine (or virgin), and (b) representative (or virgin + companion) dry samples (see Ch. 6). From these two types of specimens, it is therefore possible to establish magnitudes of $d r y$ FRP-concrete fracture toughness. Another use for these values is realized in considering the environmentally conditioned samples. Here, it is not so much the magnitudes of the aged beams' fracture toughness values that are of immediate consequence, but rather their relative loss when compared with a companion (i.e. dry) specimen of the same age. Three weathering 
regimes are implemented based on commonly occurring types of actual field exposureconditions: alternate wet-dry cycling in (a) $\mathrm{Na}_{2} \mathrm{SO}_{4}$, and (b) $\mathrm{NaOH}$; and freeze-thaw cycling in (c) $\mathrm{CaCl}_{2}$ (see Chapters 7 and 8 ).

\section{The Minor Objectives}

Although the dominant means of interface characterization is achieved by using the experimental SCCB approach to obtain values of fracture energy (read pp. 3, 4 of Section 1.1, for example; the governing equation being given in (3.73)), two other minor schemes are also employed. These include: (1) patterns of fracture, and (2) pre-fracture changes. With regard to the former, fracture patterns can be investigated from both quantitative and qualitative standpoints. Quantitatively, it is possible to study cracking behavior through the convention of the brittleness index (Eq. (3.74)) - a normalization of the energy being released (i.e. lost) during a period of rapid crack growth with respect to the energy that was stored in the joint just at the onset of crack-initiation (Ebewele, River and Koutsky (1986); read also Section 3.4). Qualitatively, on the other hand, the postfracture surfaces may be analyzed visually to see what, if any, changes are ascertainable over the course of the various aging regimes.

As for the latter, "pre-fracture changes" scheme, the following three methods are implemented. First, a qualitative visual procedure, like the one just described above, is used to inspect the specimen prior to it being fractured. The remaining second and third methods are quantitative in constitution. Respectively, these include the periodic recording of weight and strain measurements (see Section 6.4; also see the subsections titled "Pre-Fracture Changes" in Sections 7.2, 7.3, and 8.2). 


\subsection{Scope}

A generic referencing-convention was assigned to the interface bond in this opening chapter, such as FRP-concrete. The reason for this was to emphasize the fact that the newly devised experimental Fracture Mechanics SCCB test-methodology is not limited to a particular kind of FRP (or for that matter, concrete-e.g. Type I, II, etc...or High Performance Concrete (HPC)). However, for this exploratory-research work, it was decided that only the carbon variety, i.e. the CFRP, be used (also, only normal concrete was prescribed for the substrate) - the subsequent research to stem from this one, will investigate the performance of GFRP-concrete and GFRP-HPC interfaces.

For reasons already explained on pp. 3 and 4, and Section 1.2 (p. 7), the interface bond, here, is to be studied from the viewpoint of Fracture Mechanics (Ch. 3 provides a self-contained review of this subject), and not from the widely used, large-scale, fourpoint bending approach (see, for example Figure 2.1 and, from Sec. 2.6, cases 1, 2, and 6); nor is it to be studied from the small-scale, double-lap shear or tensile-test specimens (see Figures 2.3 and 2.4, respectively, of Sec. 2.6, cases 4 and 5). The parameters to be determined and reported, include: the critical strain energy release rates, $G_{I c}$, and the corresponding brittleness indices, $I$. Additional parameters to be studied are: the weights and mechanical strains of the specimens, along with visual-based, pre- and post- fracture characterizations. Strains will be recorded in two locations: (1) on the CFRP strip itself, and (2) on the adjacent concrete ledge (see Figures 5.1(a) and 6.8(b), for example), in order to detect any resulting differential movement (or slippage) that may occur at the interface during the various aging protocols. The three protocols to be used for this study, 
based on commonly occurring types of actual field exposure-conditions, are: alternate wet-dry cycling in (1) $\mathrm{Na}_{2} \mathrm{SO}_{4}$, and (2) $\mathrm{NaOH}$; and (3) freeze-thaw cycling in $\mathrm{CaCl}_{2}$ (see Chapters 7 and 8); unweathered interface fracture results are the subject of Chapter 6.

\subsection{Brief Synopses of Chapters to Follow}

The layout of this volume, with chapter, title, and brief description, is as follows:

\section{CHAPTER 2 A REVIEW OF FRP AND CONCRETE DURABILITY}

$>$ Durability defined

$>$ Synthesis of a basis for the durability studies of Chapters 7 and 8

$>$ Citations of 6 experimental case studies

\section{CHAPTER 3 A REVIEW OF FRACTURE MECHANICS \& THE DEVELOPMENT OF} THE NOVEL SCCB TEST

$>$ Self-contained review on the subject of Fracture Mechanics

$>$ Derivation of the governing Irwin-Kies Relation

$>$ Significance of cracking behavior

$>$ Review on the subject of Double Cantilever Beams, and the variants thereof

$>$ Specifics concerning the SCCB and test fixture

\section{CHAPTER 4 COMPLIANCE CALIBRATIONS OF THE CONTOURED- CANTILEVER}

$>$ Specifics pertaining to experimental calibration of the contoured-cantilever

$>$ Equipment details

$>$ Investigation into the influence of the actuator speed (during calibrations)

$>$ Effects of the substrate material

$>$ Limitations of the FE and explicit approaches

\section{CHAPTER 5 TEST SPECIMEN FABRICATION}

$>$ Details of concreting, contouring, and bonding 


\section{CHAPTER 6 DRY FRACTURE}

$>G_{I c}$ and $I$ values of pristine and representative dry specimens

$>$ Visual inspections of dry fracture surfaces

$>$ Discussion of "head" and "set" loads

$>$ Determination of the critical load, $P_{c}$ - a statistical approach with example

$>$ Weight and strain changes of the dry specimens

\section{CHAPTER 7 WET-DRY FRACTURE}

$>$ Preliminary information on the sodium -sulfate and -hydroxide (i.e. SS and SH, respectively) wet-dry protocol

$>G_{I c}$ and $I$ values of SS and SH specimens

$>$ Visual-based pre- and post- fracture surface characterizations of SS and SH specimens

$>$ Weight and strain changes of SS and SH specimens

\section{CHAPTER 8 FREEZE-THAW FRACTURE}

$>$ Preliminary information on the calcium chloride (i.e. CC) freeze-thaw protocol

$>G_{I c}$ and $I$ values of CC specimens

$>$ Visual-based pre- and post- fracture surface characterizations of CC specimens

$>$ Weight and strain changes of CC specimens 


\section{CHAPTER 2}

\section{A REVIEW OF FRP AND CONCRETE DURABILITY}

\subsection{Introduction}

Two constituent-elements most dominantly define the importance of this research work. First, and foremost, is the actual development of a Fracture Mechanics methodology to characterize the fiber reinforced polymer (FRP) -and- concrete interface bond - an obstacle, which up until this point, had not been overcome. This is the subject of the following chapter (read, especially, Section 3.6) with both, the methodological application demonstrated, as well as the corresponding CFRP-concrete interface bond results cited, in Chapter 6.

Secondly, is the extension of this novel approach in studying the longevity of this utilitarian, external reinforcement technology, as it is subjected to commonly encountered actual field-exposure conditions. Included in this context are the following two prescribed regimes: (1) alternate wet-dry cycling, the wetting phase of which comprises soaking of the specimens in one of two solutions: (a) a weak-acidic mixture of sodium sulfate $\left(\mathrm{Na}_{2} \mathrm{SO}_{4}\right)$, and (b) an alkaline bath of sodium hydroxide $(\mathrm{NaOH})$, and (2) freeze-thaw cycling under a solution of calcium chloride $\left(\mathrm{CaCl}_{2}\right)$ - the subjects of Chapters 7 and 8 , 
respectively. On that account, the focus of the present chapter is to review both the mechanisms of such attack as well as to explain the origins and rationale for choosing to study the durability of the CFRP-concrete interface bond as disposed to such degradative environments. In this vein, then, Section 2.2 reviews the theme of general durability, Sections 2.3 and 2.4 consider matters of sulfate and alkali attack, respectively, and Section 2.5 examines the significant issues pertaining to freezing and thawing, listing, in summary-form at the end of the section, preventive measures to take to produce more enduring grades of concrete. The body of the chapter culminates in Sec. 2.6 by surveying six separate experimental case studies, each of which, in some fashion, involve overlapping-features with the aforementioned themes of Sections $2.2-2.5$.

\subsection{Durability}

The word, durability, in this context, describes the ability of a structural system to maintain its initial performance-properties over time (Cousins et al., p. 401, 1998). This vitally important characteristic of structural quality may be affected by repeated loadings, e.g. vehicular traffic loads, alternating environmental conditions, e.g. wet-dry and freezethaw cycling effects, general temperature and climate changes, a whole host of exposuresituations involving deteriorating chemicals, and a variety of combinations thereof (see also Chapters 7 and 8). With the exception of the kind of damage that is initiated mechanically, i.e. as a result of impact, abrasion or erosion, all of the adverse influences on structural-durability, just mentioned, involve the transport of fluids. In particular, focus is here given to the concrete medium of the interface, since the CFRP-matrix-layer may be thought of as being impermeable, for the most part. 


\section{Transport of Fluids in Concrete}

The durability of concrete largely depends on the ease by which fluids are able to move into and throughout the concrete-referred to as the permeability of concrete. The permeability, in turn, depends on the nature of the pore system (between $120 \mathrm{~nm}-160$ $\mathrm{nm}$ in size) within the bulk of the hardened cement paste and also in the zone near the interface between the cement paste and the aggregate. The presence of aggregates makes the path that a fluid has to move through longer and more tortuous, thus reducing the effective area of flow. Pores become ineffective for transport when they are either discontinuous, contain adsorbed water, or have a narrow entrance, even if the associated pore itself is large. Some relevant definitions follow (after Neville, Ch. 10, p. 482, 1996): Porosity: a measure of the proportion of the total volume of concrete occupied by pores, and expressed in per cent.

Permeability: the flow of a fluid under a pressure differential through a porous medium. (Note: if the porosity of concrete is high and the pores are interconnected, then the permeability of this concrete is said to be high. On the other hand, it is possible to have concrete with high porosity but possessing a discontinuous pore network so that the resulting permeability of the concrete is low.)

Diffusion: the process under which fluids move due to a concentration gradient. Carbon dioxide and oxygen are of primary interest relative to the diffusion of gases concerning concrete - the former leads to carbonation of the hydrated cement paste (mentioned also in Ch. 5, following the description of the curing scheme in Sec. 5.2), and the latter causes 
the embedded steel to corrode (discussed briefly in Sec. 8.2 in the "Weight Changes (CC)" subhead).

Sorption: the result of capillary movement in the pores of concrete that is open to the atmosphere. It follows that such capillary action can only take place in partially dry concrete - that is, sorption of water is not possible in completely dry concrete or in saturated concrete.

Coefficient of Permeability: the flow equation can be written as

$$
\frac{\mathrm{dq}}{\mathrm{dt}} \cdot \frac{1}{\mathrm{~A}}=\mathrm{k} \frac{\Delta \mathrm{h}}{\mathrm{L}}
$$

following Darcy's Law for laminar flow through a porous medium. Now with water as the fluid and a steady state of flow (dq/dt), the coefficient of permeability, $k$, can be determined directly. Here, $\mathrm{A}=$ cross-sectional area of the sample $\left[\mathrm{m}^{2}\right], \mathrm{dq} / \mathrm{dt}=$ flow-rate of water $\left[\mathrm{m}^{3} / \mathrm{s}\right], \Delta \mathrm{h}=$ drop in hydraulic head through the sample $[\mathrm{m}], \mathrm{L}=$ thickness of the sample $[\mathrm{m}]$, and $\mathrm{k}=$ coefficient of permeability $[\mathrm{m} / \mathrm{s}]$. (Note: the permeability of hardened cement paste is controlled by the capillary porosity, as the capillary pores are one to two orders of magnitude larger than the gel pores. Also, the permeability decreases with the decrease in water-cement ratio. For a water-cement ratio of $0.45, \mathrm{k}$ is typically in the order of $10^{-11}-10^{-12}[\mathrm{~m} / \mathrm{s}]$. Coefficients of permeability that are an order of magnitude lower than $10^{-12}[\mathrm{~m} / \mathrm{s}]$ are considered to represent concretes with low k-values. In general, the higher the strength of the hardened cement paste, the lower its permeability. This is expected, since strength is a function of the relative volume of gel in the space that is available. 
Coefficient of Diffusion: the diffusion equation applicable to water vapor and air can be expressed by Fick's First Law as

$$
\mathrm{J}=-\mathrm{D} \frac{\mathrm{dc}}{\mathrm{dL}}
$$

where, $\mathrm{J}=$ mass transport rate $\left[\mathrm{kg} /\left(\mathrm{m}^{2} \cdot \mathrm{s}\right)\right], \mathrm{dc} / \mathrm{dL}=$ concentration gradient $\left[\mathrm{kg} / \mathrm{m}^{4}\right], \mathrm{L}=$ thickness of sample $[\mathrm{m}]$, and $\mathrm{D}=$ diffusion coefficient $\left[\mathrm{m}^{2} / \mathrm{s}\right]$. The diffusion of water is 4 orders of magnitude slower than through air. It should be noted that the diffusion coefficient changes with age because the pore system in concrete changes over time, especially with the continual hydration process.

In addition to the diffusion of gases, ions of aggressive character, notably sulfates (Ch. 7) and chlorides (Ch. 8), move by diffusion in the porewater. It is in the porewater that reactions with hydrated cement paste take place so that ionic diffusion is of importance with respect to sulfate attack of concrete and chloride attack of embedded steel. Ionic diffusion is most effective when the pores in the hardened cement paste are saturated, but it can also occur in partially saturated concrete. As already discussed in the case of permeability, though to a lesser extent, there is lower diffusion at lower watercement ratios.

\subsection{Sulfate Attack*}

"(Though no actual sulfate case study is cited, see Sec. 2.6, cases 1, and 3 - 6 for studies involving general effects of salt-attacks)

Solid salts do not attack concrete but, when present in solution, they can react with hydrated cement paste. Particularly common are sulfates of sodium (used in Ch. 7), potassium, magnesium, and calcium occurring naturally in soil or groundwater. Sulfates 
in groundwater can also arise from the use of fertilizers or from industrial effluents. These sometimes contain ammonium sulfate, which attacks hydrated cement paste by producing gypsum $\left(\mathrm{CaSO}_{4} \cdot 2 \mathrm{H}_{2} \mathrm{O}\right)$. Soil in some disused industrial sites, particularly gas works, may contain sulfates and often other aggressive substances. Various reactions of sulfates with hardened cement paste include (after Neville, pp. 508, 509, 1996):

Sodium sulfate attacks the $\mathrm{Ca}(\mathrm{OH})_{2}$ :

$\mathrm{Ca}(\mathrm{OH})_{2}+\mathrm{Na}_{2} \mathrm{SO}_{4} \cdot 10 \mathrm{H}_{2} \mathrm{O} \rightarrow \mathrm{CaSO}_{4} \cdot 2 \mathrm{H}_{2} \mathrm{O}+2 \mathrm{NaOH}+8 \mathrm{H}_{2} \mathrm{O}$

This is an acid-type of attack. In flowing water, $\mathrm{Ca}(\mathrm{OH})_{2}$ can be leached out completely but if $\mathrm{NaOH}$ accumulates, equilibrium is reached with only a part of $\mathrm{SO}_{3}$ being deposited as gypsum.

Calcium sulfate attacks only the calcium aluminate hydrate $\left(3 \mathrm{CaO} \cdot \mathrm{Al}_{2} \mathrm{O}_{3} \cdot 12 \mathrm{H}_{2} \mathrm{O}\right)$ forming calcium sulfoaluminate $\left(3 \mathrm{CaO} \cdot \mathrm{Al}_{2} \mathrm{O}_{3} \cdot 3 \mathrm{CaSO}_{4} \cdot 32 \mathrm{H}_{2} \mathrm{O}\right)$ which is known as ettringite. (Depending on the ambient vapor pressure, the number of water molecules can vary between 31 and 32.$)$

Magnesium sulfate attacks the calcium silicate hydrates in addition to $\mathrm{Ca}(\mathrm{OH})_{2}$ and the calcium aluminate hydrate. The pattern of the reaction is:

$$
\begin{aligned}
& 3 \mathrm{CaO} \cdot 2 \mathrm{SiO}_{2(\mathrm{aq})}+3 \mathrm{MgSO}_{4} \cdot 7 \mathrm{H}_{2} \mathrm{O} \rightarrow \\
& \quad 3 \mathrm{CaSO}_{4} \cdot 2 \mathrm{H}_{2} \mathrm{O}+3 \mathrm{Mg}(\mathrm{OH})_{2}+2 \mathrm{SiO}_{2(\mathrm{aq})}+\mathrm{xH}_{2} \mathrm{O}
\end{aligned}
$$


Because of the very low solubility of $\mathrm{Mg}(\mathrm{OH})_{2}$, this reaction proceeds to completion so that, under certain conditions, the attack by magnesium sulfate is more severe than by other sulfates. A further reaction between $\mathrm{Mg}(\mathrm{OH})_{2}$ and silica gel is possible and may also cause deterioration. The critical consequence of the attack by magnesium sulfate is the destruction of the C-S-H (i.e. calciumsilicate-hydrates).

Ettringite can also form from the reaction between the sulfate and the $\mathrm{C}_{4} \mathrm{AF}$, but as this ettringite is nearly amorphous, no expansive damage has been reported. Nevertheless, ASTM C150-02a ("Standard Specification for Portland Cement", 1994) prescribes a limit on the combined content of $\mathrm{C}_{3} \mathrm{~A}$ and $\mathrm{C}_{4} \mathrm{AF}$ when sulfate resistance is required. It is also worth noting that, because of changes in solubility with temperature, expansion due to the formation of ettringite is very low at temperatures above $30 \mathrm{C}(86 \mathrm{~F})$.

In addition to disruptive expansion and cracking by sulfate attack, there is also a loss of strength of concrete due to the loss of cohesion in the hydrated cement paste and of adhesion between it and the aggregate particles. Concrete attacked by sulfates characteristically has a whitish appearance termed as efflorescence (discussed in the next subsection — see also Fig. 7.12(b), for example), with the damage usually beginning at edges and corners followed by progressive cracking and spalling. The attack occurs only when the concentration of the sulfates exceeds a certain threshold. Above that, the rate of sulfate attack increases with an increase in the strength of the solution, but beyond a concentration of about $0.5 \%$ of $\mathrm{MgSO}_{4}$ or $1 \%$ of $\mathrm{Na}_{2} \mathrm{SO}_{4}$ the rate of increase of the 
solution becomes smaller. A saturated solution of $\mathrm{MgSO}_{4}$ leads to serious deterioration of concrete, although with concretes having low water-cement ratios this takes place only after 2 to 3 years.

The classification of the severity of exposure recommended by ACI 201.2R-92 ("Guide to Durable Concrete") is given in Table 2.1 below. Two preventive measures are suggested as a result of this classification. In the first, the $\mathrm{C}_{3} \mathrm{~A}$ content in the cement should be minimized, thus resulting in sulfate-resisting cement. The second approach is to reduce the quantity of $\mathrm{Ca}(\mathrm{OH})_{2}$ in hydrated cement paste by using blended cements containing blast furnace slag or pozzolana with low calcium oxide content. The effect of pozzolana is twofold. First, it reacts with $\mathrm{Ca}(\mathrm{OH})_{2}$ so that it is no longer available to react with the sulfates. Second, compared with portland cement only, the same content of blended cement per volume of concrete results in less $\mathrm{Ca}(\mathrm{OH})_{2}$. While these measures are important, it is even more important to prevent the ingress of sulfates into the concrete. For this reason, the concrete should be made as dense as possible and with as low a permeability as possible. This can be achieved in one of three ways: (1) specify a maximum water-cement ratio, (2) a minimum-strength, or (3) a minimum cement content.

Table 2.1 Classification of Sulfate Severity (ACI 201.2R-92, p. 10, 1997))

\begin{tabular}{l|c|c}
\hline \multirow{2}{*}{ Exposure } & \multicolumn{2}{|c}{ Concentration of Sulfates } \\
\cline { 2 - 3 } & In Soil, \% & In Water [ppm] \\
\hline Mild & $<0.1$ & $<150$ \\
\hline Moderate & $0.1-0.2$ & $150-1500$ \\
\hline Severe & $0.2-2.0$ & $1500-10,000$ \\
\hline Very Severe & $>2.0$ & $>10,000$ \\
\hline
\end{tabular}


For concretes having moderate sulfate exposure (see Table 2.1), ACI 201.2R-92 recommends using Type II cement (i.e. modified cement) or a blended cement containing blast furnace slag or pozzolana. If the exposure is expected to be severe, then sulfateresisting cement should be used. For very severe exposure, a blend of sulfate-resisting cement and pozzolana (between $25 \%$ and $40 \%$ by mass of total cementitious material) or blast furnace slag (not less than $70 \%$ by mass) is required.

The resistance of concrete to sulfate attack can be tested in the laboratory by storing specimens in a solution of sodium or magnesium sulfate, or in a mixture of the two. Alternate wetting and drying accelerates the damage due to the crystallization of salts in the pores of the concrete. The effects of damage can be estimated by the loss in strength of the specimen, by changes in its dynamic modulus of elasticity, by its loss of mass, by its expansion, or even by visual inspection (Neville, p. 512, 1996) - in this present interface study, the effects of sulfate attack are determined through the use of the novel SCCB fracture test method (see Section 7.2).

\section{Efflorescence}

Leaching of lime compounds may under some circumstances lead to the formation of salt deposits on the surface of the concrete, known as efflorescence (see Fig. 7.12(b)). This is found, for instance, when water percolates through poorly compacted concrete or through cracks or along badly made joints, and when evaporation can take place at the surface of the concrete. Calcium carbonate formed by the reaction of $\mathrm{Ca}(\mathrm{OH})_{2}$ with $\mathrm{CO}_{2}$ is left behind in the form of a white deposit. Calcium sulfate deposits are encountered as well (Neville, pp. 513, 514, 1996). 


\section{Salt Weathering}

When concrete is repeatedly wetted by sea water with alternating periods of drying - during which pure water evaporates — some of the salts dissolved in sea water are left behind in the form of crystals, mainly sulfates. These crystals rehydrate and grow upon subsequent wetting, thereby exerting an expansive force on the hardened cement paste. Such progressive surface weathering, termed as salt weathering, takes place at high temperatures and strong insolation (i.e. the amount of solar radiation that is applied to a horizontal surface; Neville, p. 516, 1996) resulting in a rapid drying-out of the pores over some depth. Intermittently wetted concrete surfaces, such as those found in tidal areas or structures located in splash zones, are particularly vulnerable.

Salt weathering can also rise by sorption (i.e. by capillary action-the process of taking up and holding by either adsorption (taking-on the surface) or absorption (takingon within); see also Section 2.2). Evaporation of pure water from the surface leaves behind salt crystals which, when rewetted, can cause disruption (Neville, p. 516, 1996).

Salt weathering can occur not only as a consequence of direct spray by sea water (see the next subsection), but also when airborne salt that has been deposited on the surface of the concrete is dissolved by the action of dew followed by evaporation. Such behavior has been observed in the desert areas where the large temperature drop in the evening and early morning hours reduces the relative humidity of the air to the point where condensation in the form of dew takes place. In cold climate regions where deicing agents are used, the salt weathering that results is known as salt scaling or simply scaling (Neville, pp. 516, 517, 1996; see also Section 2.5). 
These effects can extend to a depth of several millimeters. Hardened cement paste and the embedded fine aggregate particles are removed, leaving behind the protruding coarse aggregate particles. With time, these particles loosen, resulting in greater exposure of the hardened cement paste to salt weathering.

Sea Water Effects

Concrete exposed to sea water can be subjected to various chemical and physical actions. These include chemical attack, chloride-induced corrosion of steel reinforcement, freeze-thaw attack (discussed in Section 2.5), salt weathering (see previous subsection), and abrasion by sand and ice in suspension. Of course, the presence and intensity of these various forms of attack depend on the location of the concrete with respect to the sea level.

Sea water contains a number of dissolved salts from which this chemical action stems. The concentration of such salts is lower in the colder and temperate regions than in the warm seas, and especially high in shallow coastal areas with excessive diurnal evaporation rates (ACI 201.2R-92, p. 10, 1997). The total salinity is typically $3.5 \%$. Some representative values are as follows: 0.7\%-Baltic Sea, 3.3\%-North Sea, 3.6\%Atlantic and Indian Oceans, 3.9\%-Mediterranean Sea, 4\%-Red Sea, 4.3\%-PersianArabian Gulf (Neville, p. 515, 1996). In all seas, the ratio of the individual salts is nearly constant. For example, the ion concentration for the Atlantic Ocean (in \%) is as follows: chloride 2 , sulfate 0.28 , sodium 1.11 , magnesium 0.14 , calcium 0.05 , and potassium 0.04 . The $\mathrm{pH}$ of sea water varies between 7.5 and 8.4 , the average value in equilibrium with atmospheric $\mathrm{CO}_{2}$ being 8.2. 
Where concrete structures are placed on reclaimed coastal areas with the foundations below the saline ground water levels, capillary suction and evaporation may cause supersaturation and crystallization in the concrete above ground, resulting both in chemical attack on the cement paste (sulfate) and in aggravated corrosion of the internal steel (chlorides). Concretes with low permeability-i.e. ones with low water-cement ratios, good consolidation, and proper curing - are essential in these cases, not only to delay the effects of sulfate attack, but also to afford adequate protection to the internal reinforcement. Careful attention should also be given to structural aspects, e.g. properly designed and constructed joints, so as to minimize the incidence of cracking. Furthermore, concrete structures that are expected to function under full seawaterexposure conditions should possess 28-day compressive strengths of at least $34.5 \mathrm{MPa}$ (5000 psi; ACI 201.2R-92, pp. 10, 11, 1997).

\subsection{Alkali Attack*}

*(See also Sec. 2.6, case 5)

The porewater environment of ordinary portland cement concrete is one of high humidity and $\mathrm{pH}-\mathrm{in}$ the range of $10-13.5$ (Andersson, K. et al., 1989). The sodium hydroxide $(\mathrm{NaOH})$ solution used in this research $(\mathrm{pH}=12)$ was chosen to simulate this naturally present alkaline-condition within the hydrated cement paste (see Section 7.3).

The composition of dry ordinary portland cement primarily consists of calcium and silicon oxides $\left(\mathrm{CaO}\right.$ and $\left.\mathrm{SiO}_{2}\right)$-also known as lime and silica, respectively - with smaller quantities of oxides of aluminum, iron, magnesium and potassium. The main compounds of portland cement are listed in Table 2.2. The two calcium silicates $\left(\mathrm{C}_{3} \mathrm{~S}\right.$ and 
$\mathrm{C}_{2} \mathrm{~S}$ - see Table 2.2), which constitute about $75 \%$ of the weight of portland cement, react with water in a process known as hydration to form two new compounds: calcium hydroxide and calcium silicate hydrate (abbreviated CSH). The latter is by far the most important cementing component in concrete-it is the very heart of concrete. The CSH particles are so minute (surface area $=300 \mathrm{~m}^{2} / \mathrm{g}$ ) that they can only be seen in an electron microscope. In hardened cement paste, these particles form dense, bonded aggregations between the other crystalline phases and the remaining unhydrated cement grains, adhering to both sand and coarse aggregate and cementing everything together (Kosmatka and Panarese, p. 3, 1990).

Table 2.2 Main (+90\%, by weight) compounds of portland cement

\begin{tabular}{l|c|c}
\hline \hline \multicolumn{1}{c|}{ Compound } & Oxide Composition & Abbreviation \\
\hline Tricalcium silicate & $3 \mathrm{CaO} \cdot \mathrm{SiO}_{2}$ & $\mathrm{C}_{3} \mathrm{~S}$ \\
\hline Dicalcium silicate & $2 \mathrm{CaO} \cdot \mathrm{SiO}_{2}$ & $\mathrm{C}_{2} \mathrm{~S}$ \\
\hline Tricalcium aluminate & $3 \mathrm{CaO} \cdot \mathrm{Al}_{2} \mathrm{O}_{3}$ & $\mathrm{C}_{3} \mathrm{~A}$ \\
\hline Tetracalcium aluminoferrite & $4 \mathrm{CaO} \cdot \mathrm{Al}_{2} \mathrm{O}_{3} \cdot \mathrm{Fe}_{2} \mathrm{O}_{3}$ & $\mathrm{C}_{4} \mathrm{AF}$ \\
\hline
\end{tabular}

During hydration, the cement hardens into its gel phase - the cohesive mass of hydrated cement in its densest paste-state - with byproducts of calcium hydroxide $\left(\mathrm{Ca}(\mathrm{OH})_{2}\right)$, sodium hydroxide $(\mathrm{NaOH})$, and potassium hydroxide $(\mathrm{KOH})$. Most of these hydroxides remain mixed with the concrete porewater and contribute to the high $\mathrm{pH}$ of the resulting solution (Diamond, 1981). The $\mathrm{pH}$ is directly defined by the hydroxide ion concentration, with a one point rise representing a tenfold increase in the $\left[\mathrm{OH}^{-}\right]$. It is important to recognize that the $\left[\mathrm{OH}^{-}\right]$(and thus the $\mathrm{pH}$ ) that results is that of the cement 
porewater-not that of the concrete porewater. The $\mathrm{pH}$ of concrete porewater may be substantially lower than this level due to effects causing it to be diluted. Other complicating factors must also be considered with respect to the $\mathrm{pH}$ of concrete porewater. For example, carbonation, i.e. the reaction of $\mathrm{Ca}(\mathrm{OH})_{2}$ within the concrete porewater and carbonic acid $\left(\mathrm{H}_{2} \mathrm{CO}_{3}\right.$ - stemming from environmental carbon dioxide, $\mathrm{CO}_{2}$ ) in forming calcium carbonate, $\mathrm{CaCO}_{3}$, can lead to a significant drop in porewater $\mathrm{pH}$ over time. Complete carbonation of a concrete reduces the $\mathrm{pH}$ to a level of 9 or lower (Padadakis, V. et al., 1992). On the other hand, Gentry (p. 84, 1999) observed that carbonation cannot be relied upon in reducing the $\mathrm{pH}$ level of the concrete porewater.

He goes on to say that the quantity and mobility of the porewater inside the concrete must also be addressed. The porewater solution must move from the pores of the concrete and diffuse through the polymer matrix, if the alkaline attack is to initiate. To understand the accelerating mechanism that is initiated when a FRP reinforcement is soaked in solution, three factors must be quantified: (1) the quantity of mobile porewater available in the concrete, (2) the rate at which this porewater is to move through the concrete to the surface of the reinforcement, and (3) its diffusion-rate through the polymer matrix so that it comes into contact with the fibers.

\section{Porewater Quantity}

The mobility of porewater depends on the state of water within the hydrated cement complex. The water in concrete may be thought of as existing in three phases. The first is chemically bound water that is locked into cement gel via hydration. The second is absorbed water that exists as films on pore walls within the concrete and is held 
in place due to surface forces. The final phase is capillary or free water that exists in the larger pores and is thus beyond the effect of surface forces (Horrigmoe, G., 1985Periera et al., 1989, present a concise introduction to this topic).

\section{Porewater Mobility in Concrete}

If the concrete in question is mature and isothermal conditions are assumed, then the only driving force for moisture migration is the concentration gradient, dc/dL of Eq. (2.2). Therefore, the physical transport phenomenon of interest is diffusion (see the subsection titled "Transport of Fluids in Concrete" in Section 2.2). Diffusion coefficients, D (Eq. (2.2)), of moisture in concrete have been measured to be in the range of $10^{-10}$ to $10^{-12}\left[\mathrm{~m}^{2} / \mathrm{s}\right]$ (Horrigmoe, G., 1985). Using accepted models of Fickian diffusion, it is possible to model the transport of porewater through the concrete to the locations of the FRP reinforcements.

Porewater Mobility in FRP

The diffusion coefficients of FRPs have been found to be less than that for concrete (Springer, G.S., 1988), and so it will be easier for the porewater to migrate through the concrete than for it to diffuse into the FRP reinforcement. One aspect of the accelerating environment, however, that has found to increase the diffusion coefficients, is the effect of temperature. For FRPs, both the diffusion-rate as well as the amount of moisture that is to be accommodated, increase as a function of temperature. These accelerating factors must be considered when an elevated temperature is used to condition FRP reinforcements (Gentry, p. 86, 1999). 


\subsection{Freeze-Thaw Attack*}

*(See also Sec. 2.6, cases $1-4)$

The severity of deterioration expected for different types of concrete structures depends largely on the varying levels of exposure conditions present during the life of the structure. The constituent most vulnerable to damage by the action of freezing is the concrete-particularly when the moisture within reaches the critical saturation point (Cordon, p. 17, 1967). This situation may be created when cold concrete is exposed to warmer, moist air on one side and evaporation is insufficient or restricted on the cold side, or when the concrete is subjected to a head of water for a period of time prior to freezing (ACI 201.2R-92, p. 3, 1997). As the water in moist concrete freezes, it produces osmotic and hydraulic pressures in the capillaries and pores of the cement paste and aggregate. If the pressure exceeds the tensile strength of the paste or aggregate, the cavity will dilate and rupture. The accumulative effect of successive freeze-thaw cycles and disruption of paste and aggregate eventually cause significant expansion and deterioration of the concrete. Deterioration is visible in the form of cracking, scaling (see following subsection) and crumbling (Kosmatka and Panarese, p. 48, 1990).

Hydraulic pressures are caused by the $9 \%$ volume-expansion of water that results upon freezing, in which growing ice crystals displace unfrozen water. If a capillary is above critical saturation (91.7\% filled with water), hydraulic pressures result as freezing progresses. At lower water contents, hydraulic pressures do not exist. At critical saturation, all of the capillary void space becomes filled with ice upon freezing, theoretically, with no development of pressure. Osmotic pressures develop from 
differential concentrations of alkali solutions in the paste. (Osmosis is the movement of a solvent through a semipermeable membrane into a solution of higher solute concentration that tends to equalize the concentrations of solute on the two sides of the membrane (Webster's Ninth New Collegiate Dictionary, 1987).) As ice develops it creates an adjacent high-alkali solution, which, through the mechanism of osmosis, draws water from lower alkali solutions in the pores. This osmotic transfer of water continues until equilibrium in the fluids' alkali concentration is achieved, and is a major factor for consideration concerning salt-scaling (Kosmatka and Panarese, pp. 48, 49, 1990). Both the effects of hydraulic and osmotic pressures will be further elaborated on in the subsection titled "Mechanisms of Freeze-Thaw Deterioration". Examples of structures most susceptible to such degradation include: dams, spillways, waste-ways, tunnel inlets and outlets, valve houses, canal structures, sidewalks, pavements and, especially highway bridges.; other structural components include: the tops of walls, piers, parapets, curb sills and ledges, copings, corners, and cornices.

Highway bridges also present a notable problem when exposed to freezing and thawing. Most bridges span water courses, and the abutments and piers are in areas of fluctuating water levels. This presents the same problem that exists with other hydraulic structures, i.e. the saturation of concrete at the water line. Also of concern here are particles of sand or gravel in flowing water that can erode surfaces through abrasion and wear.

Most concrete used in highway work is in the form of pavements where severe scaling (see following subsection) may occur. This is particularly the case for pavement 
slabs located in cold climates where it is common practice to use salts for snow and ice removal. (Note: it is for this reason that the current protocol prescribed for use in this research study is comprised of a combination of freeze-thaw cycling under a salt solution-see Chapter 8.) Specifications usually require low water-cement ratios and airentrainment of about $5 \%$ or $6 \%$ of the volume of concrete. (Entrained-air voids act as empty chambers in the paste for the freezing and migrating water to enter, thus relieving the build-up of pressures and preventing damage to the concrete (Kosmatka and Panarese, p. 49, 1990).) Neville (p. 553, 1996) reports, however, that the assuring of an adequate percentage of air in fresh concrete is not sufficient — the air voids must be stable so that they remain in position when the concrete hardens. In fact, he states that the total air content is not what is crucial here; instead, it is the spacing of the small air bubbles. A suggestion he offers in conformance to this criteria is the use of rigid-foam particles in lieu of air bubbles. Sommer (1997) describes the use of these so-called microspheres (modeled on medication microcapsules) in greater detail. The microspheres (density: 45 $\left.\mathrm{kg} / \mathrm{m}^{3}=2.8 \mathrm{pcf}\right)$ have a diameter of $10 \mu \mathrm{m}-60 \mu \mathrm{m}(0.4$ mil -2 mils $)$, which is a narrower range of sizes than is the case with the entrained air bubbles. Using $2.8 \%$ of microspheres by volume of hardened cement paste gives a spacing factor of $70 \mu \mathrm{m}$ ( 3 mils), which is well below the value normally recommended with entrained air (i.e. $250 \mu \mathrm{m}=10$ mils). The pressure developed by water as it expands during freezing depends largely upon the distance the water must travel to the nearest air void for relief. Therefore, the voids must be spaced close enough to reduce the pressure below that which would exceed the tensile strength of the concrete (Kosmatka and Panarese, p. 49, 1990). 
Scaling of Concrete

Scaling of concrete is one of the most insidious types of deterioration. (Scaling is the flaking or peeling off of surface mortar or concrete that is usually caused by freezing and thawing of concrete in damp or water-saturated environments (Kosmatka and Panarese, p. 48, 1990).) Several theories have been advanced to explain scaling (Cordon, pp. $10-12$, 1967; read also the following subsection, under the subheads titled "Osmotic Pressures" and "Deicing Salts"):

1. Pressure developed by the expulsion of water from saturated aggregate particles.

2. Hydraulic pressure developed in more numerous capillary cavities just below the concrete surface.

3. Accretion (i.e. accumulation) of moisture to ice crystals in capillary voids below the surface.

4. Osmotic pressures caused by concentration of salt in capillaries immediately beneath a concrete surface.

5. Finishing operations which may create a surface condition where the finished surface is dissimilar to the underlying concrete. Improper finishing may also densify the surface and destroy the effectiveness of air entrainment.

6. Compaction of the surface by the capillary force of receding water in the surface capillaries of drying plastic concrete and the subsequent formation of a plane of weakness.

7. Additional freezing of subsurface ice crystals caused by melting snow and ice with deicing salts.

8. Replenishment of surface moisture during freezing by melting snow and ice with deicing salts.

9. Cracking and crazing (i.e. the development of a mesh of fine surface cracks) that provide channels for moisture to reach underlying capillary ice. 


\section{Mechanisms of Freeze-Thaw Deterioration}

Hydraulic Pressures: The deterioration of concrete exposed to freezing and thawing is caused by the expansion of freezing water in the void system of the cement paste or the concrete aggregates. Pores in absorptive rock particles are usually large as compared with those in the cement paste. Freezing may damage the paste, leaving the aggregate undamaged or, conversely, freezing may damage the aggregate particles while the paste is only indirectly harmed (Cordon, p. 23, 1967). In a water-soaked paste the capillary cavities and the gel pores - the two types of voids within the hydrated cement paste (capillary cavities have nominal diameters of between $10 \mathrm{~nm}-100 \mathrm{~nm}$, whereas gel pores are only about $3 \mathrm{~nm}$ in diameter, and as such, too small for water to freeze unless the temperature drops far below the normal freezing range) - are full or nearly full of water. When the temperature falls to the point where freezing begins, ice crystals appear in the largest capillary cavities. When water in the larger cavities begins to change to ice, the volume of water plus ice exceeds the original capacity of the cavity. Therefore, during the time when the water in the capillaries is changing to ice, the cavity must either dilate or expel the excess water.

Ice in the capillaries of frozen cement paste is surrounded by unfrozen water in the gel pores. If the gel is saturated, the gel water has the same free energy as that of ordinary water in bulk. There is thermodynamic equilibrium between the gel water and the ice in the capillary at $0 \mathrm{C}(32 \mathrm{~F})$, assuming that both the ice and gel water are under a pressure of one atmosphere and the capillary is large enough to have negligible surface energy (Cordon, pp. 31, 33, 1967). If the temperature drops below the temperature at 
which the water in the capillary freezes, the gel water is then no longer in thermodynamic equilibrium with the ice-its free energy is higher than that of the ice. The gel water thus acquires an energy potential enabling it to move into the capillary cavity where it freezes and causes the ice crystal to grow and enlarge, and in turn, results in the shrinking of the gel pore, since water is being diffused from it.

On the other hand, the growth of the ice body in the capillary places the ice and the film around the ice under pressure. The swelling pressure in the ice film is enough to produce dilation and expansion of the paste. (Consider, for example, a gel structure that is saturated with the capillary cavities containing ice at -5 C (23 F)-under these circumstances the pressure in the film between the ice and the solid gel can get to be as much as 8.3 MPa (1200 psi).) This explains why continued expansion occurs in non-airentrained paste after freezing water in the capillary cavities has expelled the excess water. It should be pointed out that outside sources of moisture can also replenish the gel water during a thaw-cycle through cracks or fractures, and thereby provide moisture for the unlimited growth of ice crystals.

With cements possessing air-entrainment, however, the amount of ice present is usually not equal to the sum-capacity of the voids. Consequently, the ice in the air void grows but may be under no significant pressure. Volume increase of the air-void-ice either results in no expansive force at all or, at most, only one of negligible effect. The amount of gel water that can enter the capillary cavities by diffusion is therefore a maximum if the paste contains no air voids and if the boundaries of the specimen are located too far from the cavities. In this case the capillary ice grows as long as necessary 
to reach equilibrium with the gel water. If the stress, whether from hydraulic pressure or from subsequent growth of capillary ice, ruptures the gel and thus releases the pressure from the ice, the growth is then limited only by the amount of freezable water in the system. On the other hand, if the paste contains air voids, the period of diffusion to the capillary cavities is correspondingly brief (Cordon, p. 34, 1967). Cordon (p. 30, 1967) analogizes the expanding ice within the capillary as a pump forcing water through the paste toward the void-boundary. He explains that the pumping of water in this fashion involves the generation of pressure. Factors affecting this pressure include: (1) the coefficient of permeability, k (see Eq. (2.1)), of the material through which the water is forced, (2) the distance from the capillary to the void boundary, and (3) the rate at which freezing occurs.

Osmotic Pressures: When solutions of different concentrations of soluble materials are separated by a semipermeable barrier, the solvent particles move through the barrier toward the solution of greater concentration and a differential head is set up between the two solutions. This osmotic action increases the hydraulic pressure in the cement paste as freezable water is forced from the capillaries into the gel pores.

Consider the water in the capillary cavities as containing a concentration of salts or alkalies. This concentration increases as the water begins to freeze, and the water being forced from the capillaries will have a higher concentration of salt than the surrounding gel porewater. A pressure differential develops in the direction toward the capillary pore and opposite to the flow of water. The pressure required to overcome osmotic pressure in the capillary is a combination of the hydraulic pressure required to force capillary water 
into the gel pore structure plus the osmotic pressure which resists this flow. This mechanism has been suggested as a possible cause of scaling of concrete pavements where salt is used. Placing salt on pavements for ice removal will increase the concentration of salt in the capillary voids near the surface of the pavement. As the salt solution freezes, a greater concentration of salt results and osmotic pressure is developed in the capillary cavities. This increase in pressure may be sufficient to cause rupture of the cement gel near the surface of the pavement, and consequently, cause scaling (see the previous subsection).

Verbeck and Klieger (1957) found that an increase in the concentration of salt in freezing and thawing tests increased the effects of disruption, with concentrations from $2 \%-4 \%$ causing the greatest deterioration of concrete due to an optimum combination of freezable water and osmotic pressure. If, however, the concentration were to be increased above $4 \%$, they found a decrease in deterioration to result. The higher concentration of salt may lower the freezing temperature of the salt solution, which would give the capillary-water an antifreeze effect and reduce the freezable water. This antifreeze-effect was also observed for the pore structures of the surface concrete, where the availability of moisture for saturation of the concrete was also found to increase.

Deicing Salts: In addition to the osmotic forces created, deicing salts also provide a source of surface moisture in freezing weather by melting the ice and snow. In this case, the temperature immediately below the surface is reduced significantly because of the comparatively large heat of fusion of ice. This may cause a damaging temperature drop in 
the saturated zone immediately beneath the surface. Thus, deicing salts may cause concrete to scale by any combination of the following:

$>$ By providing moisture from the melting of ice and snow in freezing weather.

By causing additional freezing through the lowering of temperature in the subsurface zone.

By creating a system which develops osmotic pressures.

By a buildup of salt crystals in subsurface voids.

Influence of Aggregates: Concrete may deteriorate when exposed to freezing and thawing because of mechanisms within the aggregate particles. Lewis and Dolch (1956) found that the behavior of aggregate particles when exposed to freezing and thawing depends primarily upon their pore structure, permeability, and degree of saturation. The pressure exerted by freezing water, if no expansion or escape of water is possible, ranges up to 20 $\mathrm{MPa}(2900 \mathrm{psi})$ at $-20 \mathrm{C}(-4 \mathrm{~F})$. Pressures in this range may develop in aggregates when the saturation is critical. To avoid developing pressures that exceed the tensile strength of either the aggregate or the surrounding mortar, the porewater must be able to flow into unfilled pores or escape from the particle. Hindrance from the latter may be due to a blocked-frozen-zone around the outside of the aggregate resulting in the development of high pressure in the interior. Even when flow away from this zone is possible, hydraulic pressure is necessary to cause movement through small voids and may be so high as to cause fracture of the material. Actually, it is not even necessary for the aggregate to fail when the surrounding concrete suffers damage from freezing and thawing. Aggregates may have enough strength and elasticity to withstand such stresses without failure. The 
same is not true of the surrounding mortar, which may be damaged by the expansion of such particles. Three dominant mechanisms explain deterioration of aggregates and the concrete (Cordon, pp. 44 - 49, 1967-Note: the "accretion" mechanism has been omitted from the presentation below). These are: (1) elastic accommodation, (2) critical size, and (3) water expulsion, as explained below.

Elastic Accommodation: If the porosity of a rock is such that it can accommodate the expansion of water upon freezing, it then neither expands nor fractures. In cases where the saturation of the aggregate exceeds the critical value, the elastic strain within the rock structure itself may possibly accommodate the expansion as water freezes. Since most aggregates have greater tensile strengths than that of ordinary portland cement, the expansion-accommodating aggregates of the latter type will deteriorate the paste by causing it to fracture. Obviously, in this context, everything is dependent on the size and number of pores of the aggregate, as well as its strength and the value of its elastic-modulus.

Critical Size: The pore structure of some rocks is such that water is absorbed by the aggregates and when frozen, this water is forced to the outside surface. Other rocks have pore spaces within the rock itself, similar to entrained air bubbles. Verbeck and Landgren (1960) noted that the size of the aggregate becomes critical when water is being forced out the voids during freezing. Water forced for short distances develops low hydraulic pressures that increase with distance. The critical size of the aggregate depends on the: (1) freezing rate, (2) degree of saturation, and (3) permeability. The latter determines the critical size in the following two ways. First, it allows more water to be absorbed in a given period of time. Second, it permits water to be expelled from the aggregate more rapidly without developing high pressures. Experience has shown that very porous rocks do not deteriorate when exposed to freezing and thawing; they allow the ingress and egress of water rapidly enough to prevent the development of internal pressures. Rocks with relatively fine pores, on the other hand, are more susceptible to fracture unless the rocks are small enough that their size is not critical. Rocks such as cherts, shales, and some limestones are susceptible to fracturing when frozen in a saturated state, and can culminate in popouts in the concrete surface.

Water Expulsion: When saturated aggregate particles are frozen, the increase in the volume during the formation of ice must be accommodated either within the aggregate itself or forced into the surrounding cement paste. Because of this, it is necessary for there to be enough air entrained bubbles in the paste not only to contain the expelled capillary-water, but also to accommodate the water being 
driven out of the aggregate. The degree of saturation of the aggregate is critical because water cannot be forced out unless the saturation is greater than $91.7 \%$. Thus, the aggregates can be classified into three groups, each of which will behave differently under the freeze-thaw action: (1) highly porous rocks, such as reef rocks or porous sandstones - here the pore sizes are so large that there is no danger of deterioration from freezing and thawing - water is absorbed readily and the permeability is sufficient to prevent the pressure from building-up within the rock during freezing, (2) medium porous rocks - these also absorb water well and, again, have sufficient permeability-here, whatever water is expelled, however, is disseminated into the surrounding paste which can lead to popouts or expansion cracks, and (3) fine porous rocks, such as claystones, siltstones, cherts, and shales - these aggregates do not saturate rapidly but can absorb a lot of water over time-upon freezing, excessive pressures are developed within the rock to cause it to expand and fracture the surrounding concrete.

\section{A Summary of Protective Measures to Produce more Durable Concretes}

Before coming to the summary-list, a word is mentioned about one of the measures that was used in this research with tremendous success in protecting the bulk concrete substrate (see Figure 3.19(b)) from becoming pulverized throughout the harsh freeze-thaw protocol (see, for example, the progression of damage displayed in Figure 8.8 - notice that the unprotected areas, i.e. those of the concrete ledge and raised-step (see Fig. 3.21, parts (c) and (d)), were most susceptible).

Sealants: If the surface of dry concrete is effectively sealed to prevent the ingress of water, the damaging effects precipitated by the freeze-thaw mechanisms will be retarded since only the saturated concretes are susceptible to damage as caused by such actions. For this reason, it has been found that the application of waterproofing compounds, or sealants, significantly reduce the absorption of water while increasing the number of freeze-thaw cycles that are to be endured by the concrete structure. In this research study, the MBrace epoxy-based primer (see Table 5.4, also repeated in Table B.3) was used to 
seal the entire concrete beam - and this with surprisingly good results despite the fact that the primer was not designed with the express purpose of being a sealant (read Section 5.4) - except for the interface regions, constituted by the raised-step and ledge areas (see Figure 5.14).

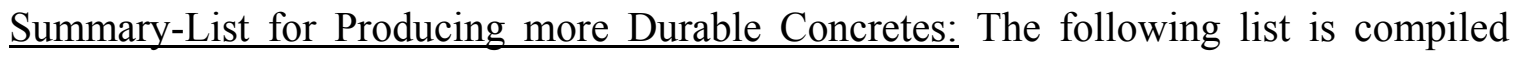
after Cordon's work (pp. 78 - 80, 1967):

1. Entrain 4\% - 6\% air in all exposed concrete that may be saturated in freezing weather. Entrained air relieves hydraulic pressures in the freezing gel structure and competes with capillary ice for available moisture, thus limiting two of the most important deterioration mechanisms. Ensuring the stability of the air voids, i.e. making sure that they remain in position when the concrete hardens, is critical. Neville (1996) and Sommer (1997) suggest the use of rigid-foam particles, called microspheres, in lieu of the more traditional air-entrainment admixtures. Apart from stability, these particles significantly reduce the spacing factor by more than a third, and as such, enhance the overall pressure-relieving performance of the concrete structure while being subjected to freezing and thawing.

2. Avoid use of concrete aggregates having high absorption, because the increase in the volume during the formation of ice must either be accommodated within the aggregate itself or forced into the surrounding cement paste. The degree of saturation of the aggregate is critical here because water cannot be forced out unless more than $91.7 \%$ of the pores are filled. Also, upon freezing, excessive pressures are developed within the rock causing it to expand and fracture the surrounding concrete and, thereby, disposing the structure to the deleterious effects of freeze-thaw cycling.

3. Use the minimum amount of mixing water possible as commensurate with good construction practices. Excess water in fresh concrete results in an increase in capillary voids in the hardened cement paste. Capillary voids are the starting point for several deterioration mechanisms.

4. Avoid saturation of exposed concrete in freezing weather. Most freezing and thawing deterioration mechanisms will not take place unless sufficient moisture is available to produce critical saturation.

5. Do not permit the concrete to undergo freezing and thawing until the hydration of cement is well advanced. Concrete which contains unhydrated portland cement is weak and will contain more capillary voids than the same concrete that has been 
more completely hydrated. Accretion (i.e. a gradual buildup) of water from unhydrated cement paste in cold weather may cause crazing (i.e. the production of minute surface-cracks).

6. Prevent the rapid drying of the surface of exposed concrete before the bleedingprocess is complete. The drying surface of fresh concrete is compacted by capillary forces. This compacted surface may trap bleed-water below the surface, thereby producing a weak, porous zone immediately beneath the top layer, and as such, creating a structure more prone to scaling.

7. Do not finish the surface of exposed concrete until the bleed-water has disappeared. Finishing operations densify the surface of the concrete and thereby prevent the escape of subsequent bleeding, thus making the structure prone to scaling (as just described in point 6 above).

8. Avoid the use of salts for ice or snow removal — of course this measure is, for the most part, left to the discretion of those in charge of the municipality. The problem with using salt to melt snow and ice is that it increases the supply of moisture to the capillary voids. Salts also lower the temperature of the concrete beneath the surface and thereby create the potential for additional freezing to occur. The degradative phenomenon of scaling increases as well due to the initiation of osmotic pressures, resulting from salt concentrations in the capillary voids.

9. Apply a sealant to the concrete once it has cured and dried - especially one that is thin enough to optimally penetrate and fill surface capillaries and thereby reduce the concrete's absorbency to water. This should then result in a concrete that is more resistant to becoming saturated and, as such, deteriorating from freeze-thaw cycling, provided that subsequent water channels are not developed from cracks or crazes. In the event that cracks or crazes develop, additional applications of the sealant should be provided. 


\subsection{Experimental Case Studies}

Six contemporary research studies on matters of FRP-concrete durability are considered in this section. In alphabetical order, these are:

1. Chajes, Michael J., Thomson, Jr., Theodore A. and Farschman, Cory A. "Durability of Concrete Beams Externally Reinforced with Composite Fabrics," Construction and Building Materials, v9, n3, pp. 141-148 (1995).

2. Green, Mark F., Bisby, Luke A., Beaudoin, Yves and Labossiere, Pierre. "Effects of Freeze-Thaw Action on the Bond of FRP Sheets to Concrete," Proceedings of the $1^{\text {st }}$ Intl. Conference on the Durability of Fibre Reinforced Polymer (FRP) Composites for Construction (CDCC), pp. 179-190 (1998).

3. Karbhari, V.M., Engineer, M. and Eckel II, D.A. "On the Durability of Composite Rehabilitation Schemes for Concrete: Use of a Peel Test," J. of Materials Science, v32, pp. 147-156 (1997).

4. Mukhopadhyaya, P., Swamy, R.N. and Lynsdale, C.J. "Durability of Adhesive Bonded Concrete-GFRP Joints," Proceedings of the First International Conference on the Durability of Fibre Reinforced Polymer (FRP) Composites for Construction (CDCC), pp. 373-389 (1998).

5. Rahman, A.H., Lauzier, C., Kingsley, C., Richard, J. and Crimi, J. "Experimental Investigation of the Mechanism of FRP Reinforcement for Concrete," Fiber Composites in Infrastructure, Proceedings of the Second International Conference on Composites in Infrastructure (ICCI), $\mathrm{H}$. Saadatmanesh and M.R. Ehsani (editors), volume II, pp. 501-511 (1998).

6. Toutanji, Houssam A. and Gómez, William. "Durability Characteristics of Concrete Beams Externally Bonded with FRP Composite Sheets," Cement and Concrete Composites, v19, pp. 351-358 (1997).

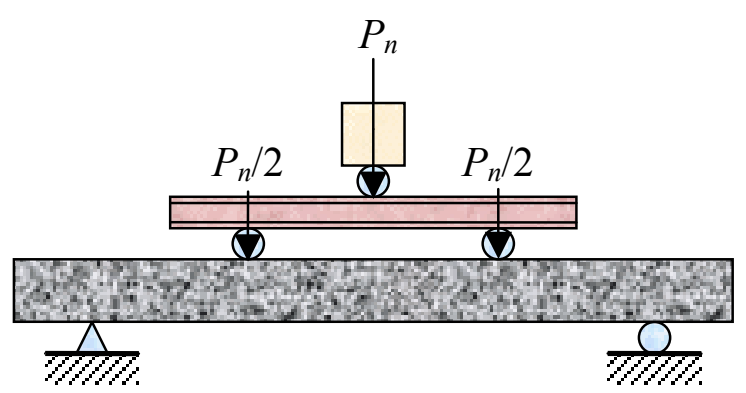

Figure 2.1 Schematic of a four-point bending specimen over simple supports 


\section{Chajes et al., 1995}

A total of 60 small-scale beams $(\mathrm{mm}: 38.1 \times 28.6 \times 330=$ in: $1.5 \times 1.125 \times 13)$ were cast in 5 batches (i.e. 12 beams/batch) and tested to failure in four-point bending (see Figure 2.1). Midspan deflections were measured at $44.5 \mathrm{~N}$ (10 lb) increments using a dial gauge. Three types of composite-wraps were studied: (1) aramid, (2) graphite, and (3) E-glass. Of the 60 beams, 15 were wrapped with aramid fibers, 15 with graphite, 15 with E-glass, and 15 were not wrapped. As for the durability scheme, 48 of the beams were exposed to wet-dry or freeze-thaw cycling (read the WD and FT subheads below for further details) in a solution of calcium chloride $\left(\mathrm{CaCl}_{2}\right)$, while the remaining 12 were left as control specimens (i.e. 3 aramid, 3 graphite, 3 E-glass, and 3 unwrapped). The concrete mix used consisted of a water-cement ratio of 0.50 (by weight) made of Type I portland cement and a $3.2 \mathrm{~mm}\left(1 / 8^{\prime \prime}\right)$ maximum size of aggregates; the average 28-day compressive strength of the concrete was found to be $31.3 \mathrm{MPa}$ (4540 psi), as determined through $50.8 \mathrm{~mm} \times 101.6 \mathrm{~mm}\left(2^{\prime \prime} \times 4 "\right)$ cylinder tests. Furthermore, each beam was internally reinforced with a single $2.38 \mathrm{~mm}(0.0937 ")$ diameter low-carbon threaded rod having a yield strength of $658.5 \mathrm{MPa}(95.5 \mathrm{ksi})$.

Threefold Conditioning Scheme (Chajes et al., 1995):

(1) Control: The batch 1 beams (i.e. 12 beams) did not undergo any cycling, whatsoever. These specimens were placed in the laboratory under ambient conditions for the same duration of time as their aged counterparts.

(2) WD: 1 wet-dry (abbreviated as WD) cycle $=16$ hours of $\mathrm{W}+8$ hours of $\mathrm{D}$, achieved with a fan. 24 beams underwent WD cycling-batch 4 beams subjected to 50 
and batch 5 beams to $100 \mathrm{WD}$ cycles—at room temperature. The conditioning solution was prepared by mixing in $4 \mathrm{~g}$ of anhydrous $\mathrm{CaCl}_{2}$ in $100 \mathrm{ml}$ of water.

(3) FT: 1 freeze-thaw (abbreviated as FT) cycle $=16$ hours at $-17 \mathrm{C}(1.4 \mathrm{~F})+8$ hours at room temperature. The FT scheme used followed the general procedures of ASTM C672 ("Standard Test Method for Scaling Resistance of Concrete Surfaces Exposed to Deicing Chemicals"). 24 beams were placed in airtight containers upside down (i.e. compression side down, fabric side up), and immersed in the same $\mathrm{CaCl}_{2}$ solution (see preceding WD subhead) while undergoing FT conditioning — batch 2 beams subjected to 50 and batch 3 beams to 100 FT cycles.

Conclusions of Environmental Conditioning (Chajes et al., 1995): Of the two exposure schemes, WD and FT cycling, the WD conditions had a slightly more severe effect on the ultimate strength of the externally reinforced beams, with a failure mode in all of the graphite-exposed cases resulting in tensile failure/partial debonding of the fabric. By the same token, the E-glass and aramid reinforced beams failed by tensile failure and debonding of the fabric, respectively; the control specimens failed through a mode of ductile flexural failure.

The most significant effect concerning the FT exposure scheme occurred in the unwrapped beams cycled for 100 days. These beams lost approximately $17 \%$ of their original strength — qualitative observations revealed some spalling of the concrete on the exposed tension. In contrast, the beams with external reinforcement were found to have been spared this sort of deterioration. 
Of the three fabric types under consideration, the E-glass fibers were observed to have degraded the most. The graphite fibers, on the other hand, seemed to be affected the least. On this basis, the general conclusions made by the authors are that, of the three fiber types studied, the graphite-variety is best suited for externally reinforcing concrete structures that are expected to be exposed to salt solutions (in this context, $\mathrm{CaCl}_{2}$ ) while undergoing wet-dry or freeze-thaw cycling.

\section{Green et al., 1998}

The effects of FT cycling on the bond of CFRP sheets (manufactured by SIKAthe web-address in the United States is: http://www.sikausa.com/) bonded to the tensionfaces of 45 concrete beams - the concrete was entrained with 7\% air and had an average 28-day compressive strength of $31 \mathrm{MPa}$ (4500 psi)—were tested in four-point bending (Fig. 2.1). The dimension of each beam was $100 \mathrm{~mm} \times 150 \mathrm{~mm} \times 1200 \mathrm{~mm}(4 " \times 6 " \times$ 48"). All the specimens were tested to failure, with Midspan displacements recorded by a displacement transducer (i.e. a linear variable differential transformer, LVDT) and the collection of loads was achieved through the use of a load cell.

Three potential concerns were cited concerning the use of this technology in cold regions: (1) differences in thermal expansion rates between the FRP and concrete (differing by a factor of 10 for the CFRP) that can lead to the generation of shear stresses at the interface and eventual bond deterioration, (2) embrittlement of the FRP at lowered temperatures, and (3) the combined effects of freezing-and-thawing in water-bath.

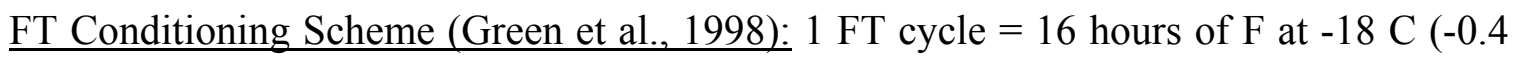
F) and 8 hours of T in water $\left(\mathrm{H}_{2} \mathrm{O}\right)$ at $15 \mathrm{C}(59 \mathrm{~F})$. 
Conclusions of FT Conditioning (Green et al., 1998): After 50 and 150 cycles of freezing-and-thawing exposure, it was discovered that all the beams behaved, for the most part, similarly. No significant differences were found to exist in the failure loads or deflections, except that these effects were slightly higher in the beams aged for 150 days. The authors mentioned, however, that this result is within the anticipated-range of experimental error, and as such, felt their rendering of a meaningful conclusion as being obscured. After qualitatively inspecting the interface, they found that this region had not sustained any damage as a result of the FT conditioning scheme, either. Finally, the characterizing failure-mode for all the specimens is described as a cohesive-debonding of the CFRP member.

\section{Karbhari et al., 1997}

Peel tests (see Figure 2.2) were performed in this study on both E-glass and carbon composites adhered to concrete substrates by means of two different resin epoxy systems, designated, without identity to the manufacturer(s), as resins A and B. The concrete substrates used were actually made of cement-mortar, mixed in a cement-sand proportion of $1: 3$ and a water-cement ratio of 0.45 . The average 28-day compressive strength and elastic-modulus of these substrates were found to be: $25.91 \mathrm{MPa}(3757 \mathrm{psi})$ and $21.53 \mathrm{GPa}(3120 \mathrm{ksi})$, respectively. As for the resins, matrix A is reported as having a nominal tensile strength and elastic-modulus of $46 \mathrm{MPa}$ (6670 psi) and $3.19 \mathrm{GPa}(462.7$ ksi), respectively; likewise, the values for system B are: $60 \mathrm{MPa}$ (8700 psi) and $3 \mathrm{GPa}$ (435.1 ksi). Another distinction between the two systems is that resin A is noted as being more water-absorbent. Composite properties are listed in Table 2.3. 


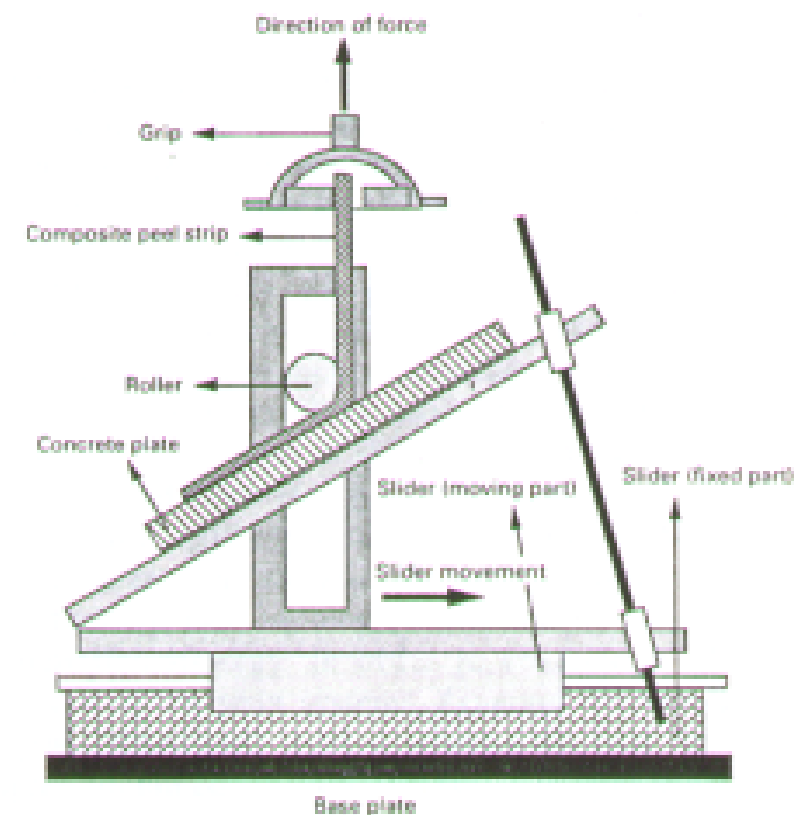

Figure 2.2 Schematic of the peel test (Karbhari et al., p. 150, 1997)

Table 2.3 Peel test composite properties (Karbhari et al., p. 150, 1997)

\begin{tabular}{c|c|c|c|c|c}
\hline \hline Resin & Fiber & $\mathbf{W}_{\mathbf{f}}{ }^{*}$ & $\mathbf{E}[\mathbf{G P a}]$ & $\boldsymbol{\sigma}_{\mathbf{u}}[\mathbf{M P a}]$ & $\boldsymbol{\varepsilon}_{\mathbf{u}}$ \\
\hline \multirow{2}{*}{$\mathbf{A}-$} & $\mathbf{G}$ & $57.36 \%$ & 22.68 & 532.15 & $2.5 \%$ \\
\cline { 2 - 6 } & $\mathbf{C}$ & $54.82 \%$ & 74.76 & 1241.67 & $1.7 \%$ \\
\hline \multirow{2}{*}{$\mathbf{B}-$} & $\mathbf{G}$ & $55.41 \%$ & 23.43 & 560.41 & $2.4 \%$ \\
\hline & $\mathbf{C}$ & $50.79 \%$ & 70.67 & 1254.32 & $1.8 \%$ \\
\hline
\end{tabular}

${ }^{*} \mathbf{W}_{\mathbf{f}}=$ fiber weight fraction 
Fivefold Conditioning Scheme (Karbhari et al., 1997): The specimens were subjected to the following five different environments for a conditioning period of two months:

$>\mathrm{A} \quad-\quad$ Ambient conditions at $20 \mathrm{C}(68 \mathrm{~F})$

$>\mathrm{W}-$ Water at $20 \mathrm{C}(68 \mathrm{~F})$

$>\mathrm{SW}-$ Saltwater at $20 \mathrm{C}(68 \mathrm{~F})$, in accordance with ASTM D1141

"Standard Practice for Substitute Ocean Water"

$>\mathrm{F} \quad-\quad$ Freezing at $-15.5 \mathrm{C}(4.1 \mathrm{~F})$

$>$ FT - Freeze-Thaw in alternate 1 day $\mathrm{F}(-15.5 \mathrm{C}=4.1 \mathrm{~F})$ and 1 day $\mathrm{T}(20$ $\mathrm{C}=68 \mathrm{~F})$ cycles

Conclusions of Peel/Durability Tests (Karbhari et al., 1997): The W and SW conditioning cases did not show a great percent change with respect to the ambient conditions for the Mode I strain energy release rate $\left(G_{I c}\right)$ values (see Table 2.4$)$. The Mode II values $\left(G_{I I c}\right)$, in contrast, were found to be more significant. It should be noted that the $G_{I c}$ values are primarily dependent on the properties of the interface, whereas the Mode II rates $\left(G_{I I c}\right)$ are subject to the attributes of the matrix. The cases involving $\mathrm{W}$ or $\mathrm{SW}$ immersion degraded both the matrix as well as the glass fibers.

The F and FT schemes increased the plasticization of the FRP material, which may have lead to misleading levels of toughness (again, see Table 2.4). At these temperatures, stable brittle crack growth is achieved, resulting in apparent increases in toughness due to a brittle/ductile transition.

As expected, of the two fiber systems, the carbon variety was found to yield higher interfacial fracture energies while being the least affected from the different 
exposure regimes. The Mode I/II ratios of these energies, on the other hand, remained approximately the same at each level.

The Mode II fracture toughness values were predominant in the case of the glass fiber reinforced specimens, thus correlating with an epoxy-based failure path. This may be due to the susceptibility of the glass-fibers to deteriorate, hence resulting in a lowered stiffness of the composite.

Table 2.4 Values of critical energy release rates (Karbhari et al., p. 155, 1997)

\begin{tabular}{|c|c|c|c|c|c|c|}
\hline \multicolumn{2}{|c|}{ Resin - Fiber } & $\mathbf{A}$ & $\overline{\mathbf{W}}$ & $\overline{\text { SW }}$ & $\mathbf{F}^{*}$ & FT $^{*}$ \\
\hline \multirow{2}{*}{$\mathbf{A}-\mathbf{G}$} & $G_{I c}\left[\mathrm{~J} / \mathrm{m}^{2}\right]$ & 563 & 553 & 558 & 1025 & 940 \\
\hline & $G_{I I C}\left[\mathrm{~J} / \mathrm{m}^{2}\right]$ & 1656 & 1407 & 1360 & 2073 & 3543 \\
\hline \multirow{2}{*}{$\mathbf{B}-\mathbf{G}$} & $G_{I c}\left[\mathrm{~J} / \mathrm{m}^{2}\right]$ & 406 & 409 & 384 & 714 & 686 \\
\hline & $G_{I I c}\left[\mathrm{~J} / \mathrm{m}^{2}\right]$ & 1580 & 481 & 448 & 2329 & 2454 \\
\hline \multirow{2}{*}{$\mathbf{A}-\mathbf{C}$} & $G_{I c}\left[\mathrm{~J} / \mathrm{m}^{2}\right]$ & 904 & 888 & 926 & 897 & 915 \\
\hline & $G_{I I c}\left[\mathrm{~J} / \mathrm{m}^{2}\right]$ & 1903 & 1554 & 1420 & 2305 & 2187 \\
\hline \multirow{2}{*}{ B - C } & $G_{I c}\left[\mathrm{~J} / \mathrm{m}^{2}\right]$ & 563 & $5 \overline{53}$ & 543 & 689 & 724 \\
\hline & $G_{I I c}\left[\mathrm{~J} / \mathrm{m}^{2}\right]$ & 558 & 485 & 402 & 840 & 724 \\
\hline
\end{tabular}

"Some of the F and FT toughness values may be incorrect—read "Conclusions..." above

\section{Mukhopadhyaya et al., 1998}

The significance of this investigation was to study the durability of the joint between epoxy-adhered GFRP plates and concrete substrates. A total of 24 specimens were tested by double-lap shear (see Figure 2.3). Two concrete mixtures, mix A and mix 
B, were prepared consisting of ordinary portland cement, the details of which are presented in Table 2.5.
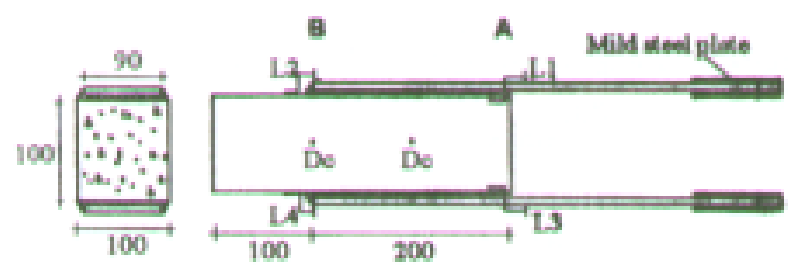

(a)

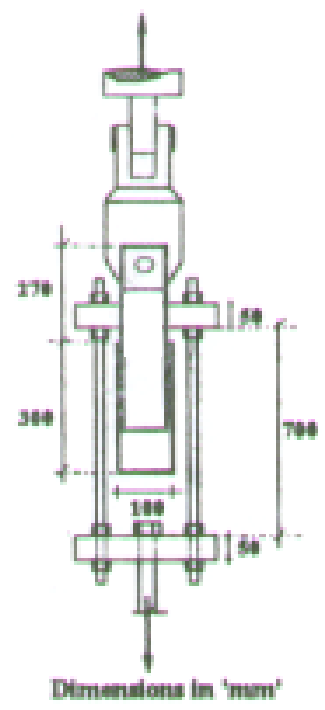

(b)

Figure 2.3 Schematic drawings of: (a) double-lap shear specimen, and (b) test-rig (Mukhopadhyaya et al., p. 384, 1998)

Table 2.5 Concrete cube strengths (Mukhopadhyaya et al., p. 381, 1998)

\begin{tabular}{l|c|c}
\cline { 2 - 3 } (unit: MPa) & Mix A (w/c = 0.57) & Mix B (w/c = 0.32) \\
\hline 28-day (in air) & 37.1 & 48.6 \\
\hline Control (in air) & 41.0 & 50.3 \\
\hline FT (in air) & 39.5 & 46.5 \\
\hline WD (in 5\% NaCl) & 55.0 & 69.7 \\
\hline Dual Exposure $^{*}$ & 54.5 & 64.6 \\
\hline "Ty
\end{tabular}

"Tested at end of exposure-at about 1 year 
The GFRP plate used in these tests contained both a mat of random continuous filaments and a layer of unidirectional roving. The longitudinal tensile strength and elastic-modulus is reported as being $328 \mathrm{MPa}(47.6 \mathrm{ksi})$ and $22.9 \mathrm{GPa}$ (3321.4 ksi), respectively. The fiber volume fraction of the plate was also determined and found to be $41.4 \%$. As for the two part (resin and hardener) epoxy adhesive, the tensile, flexural, and shear strengths are reported as: $24 \mathrm{MPa}$ (3481 psi), $55 \mathrm{MPa}$ (7977 psi), and $21 \mathrm{MPa}$ (3046 psi), respectively.

Threefold Conditioning Scheme (Mukhopadhyaya et al., 1998): In addition to 6 control specimens -3 from mix A, and 3 from mix B - a threefold aging regime was undertaken, consisting of 6 specimens, each, in the same fashion, i.e. $3+3$ from mixes $A$ and $B$, respectively.

$>\mathrm{WD}-18$ cycles in $\mathrm{NaCl}$ solution $(1$ cycle $=1$ week $\mathrm{W}$ and 1 week $\mathrm{D})$

FT - $\quad 450$ cycles in air (2 cycles/day; ramping between $20 \mathrm{C}(68 \mathrm{~F})$ and $-17.8 \mathrm{C}(-0.04 \mathrm{~F}))$

$>$ Dual - A combination of the WD and FT schemes - WD in $\mathrm{NaCl}$ for a week followed by FT cycling in air for a total of 36 weeks (i.e. a total of 18 weeks of saltwater exposure and 252 cycles of freezing and thawing)

Conclusions of Double-Lap Shear Tests (Mukhopadhyaya et al., 1998): The average shear stresses at failure, for mixes $\mathrm{A}$ and $\mathrm{B}$, respectively, ranged from, in $\mathrm{MPa}^{\mathrm{A}, \text { ave }}: 1.19$ 1.36 (in psi $^{\mathrm{A} \text {,ave }}: 173$ - 197), and, in $\mathrm{MPa}^{\mathrm{B} \text {,ave }}: 1.40$ - 1.57 (in $\mathrm{psi}^{\mathrm{B} \text {,ave }}: 203$ - 228). Likewise, the peak shear stresses ranged from, in $\mathrm{MPa}^{\mathrm{A} \text {,peak }}: 2.50$ - 6.11 (in $\mathrm{psi}^{\mathrm{A}, \text { peak }}: 362$ - 886), and, in $\mathrm{MPa}^{\mathrm{B} \text {,peak }}: 2.77$ - 5.63 (in $\mathrm{psi}^{\text {B,peak. }} 402$ - 816). 
The specimens subjected to WD cycling, of both mixes, registered strains between $200 \mu \varepsilon-400 \mu \varepsilon$, but the plate and concrete strains were almost identical. This was explained to mean that while constituent changes between the plate and concrete occurred dimensionally, practically no differential changes were induced. The specimens undergoing FT and dual exposure schemes, on the other hand, resulted in both dimensional and differential movements. The FT and dual aging conditions were therefore concluded to be more detrimental to the adhesive bonded joints than the WD cycling regime alone.

The failure patterns displayed by the control specimens culminated in the shearing-off of the concrete, indicating that the adhesive used in these cases was sufficiently strong. The same phenomenon was revealed for both the WD and FT specimens, with the exception that in a few places, the sheared-surfaces here exhibited a more mixed adhesive-cohesive pattern of failure. The most visible failure-mode change, relative to those just described, was observed in the specimens exposed to the dual conditioning regime.

In general, then, of the three exposure schemes, the most detrimental scenario was presented by the dual case, i.e. weekly alternations of WD cycling in $\mathrm{NaCl}$ solution followed by FT conditioning in air. A retrospective-point stressed by the authors, however, is that the duration of the aging program that they chose was not long enough to adequately gauge the overall strength of the adhesive joint. 


\section{Rahman et al., 1998}

One hundred and twenty FRP tensile-test specimens (see Figure 2.4(a))—96 made of carbon and 24 of glass fibers, embedded in a vinyl-ester resin-matrix-were tested under four separate exposure conditions (see following subhead) to determine the mechanisms by which these materials would deteriorate. The ultimate tensile strengths of the carbon and glass fibers were found to be $750 \mathrm{MPa}(108.8 \mathrm{ksi})$ and $225 \mathrm{MPa}(32.6$ ksi), respectively. The research program conducted adhered to ASTM E632-82 ("Standard Practice for Developing Accelerated Tests to Aid Prediction of the Service Life of Building Components and Materials", 1996) and consisted of the following tasks: (1) experimental work to study the causes and mechanisms of deterioration, (2) accelerated testing, (3) development of a provisional formula to predict the service life of FRP materials, and (4) field-verifications corresponding to the rates of deterioration of such materials, as predicted by the formula of step (3). In this paper, the authors achieved the first two of these objectives.

Fourfold Conditioning Scheme (Rahman et al., 1998): The specimens-four per loadapparatus (see Fig. 2.4(b)) —were exposed to the following environments under sustained-loads of $50 \%$, for the carbon fibers, and $30 \%$, for the glass, of their ultimate tensile values:

Air at room temperature, i.e. $24 \mathrm{C}(75.2 \mathrm{~F})$

Water at $70 \mathrm{C}(158 \mathrm{~F})$

Saline solution $(\mathrm{NaCl})$ at $30 \mathrm{C}, 50 \mathrm{C}$, and $70 \mathrm{C}$ (i.e. in F: 86, 122, and 158)

Alkaline solution $(\mathrm{NaOH})$ at $30 \mathrm{C}, 50 \mathrm{C}$, and $70 \mathrm{C}$ (i.e. in F: 86, 122, and 158) 
Specimen-assessments were then carried out by the use of a scanning electron microscope (SEM) at 45, 122, and 370 days. The breakdown of specimen-testing was as follows: the carbon-fiber samples were exposed to each of the four environments mentioned; the glass, on the other hand, were only subjected to the air and alkaline, at 50 $\mathrm{C}(122 \mathrm{~F})$, regimes.

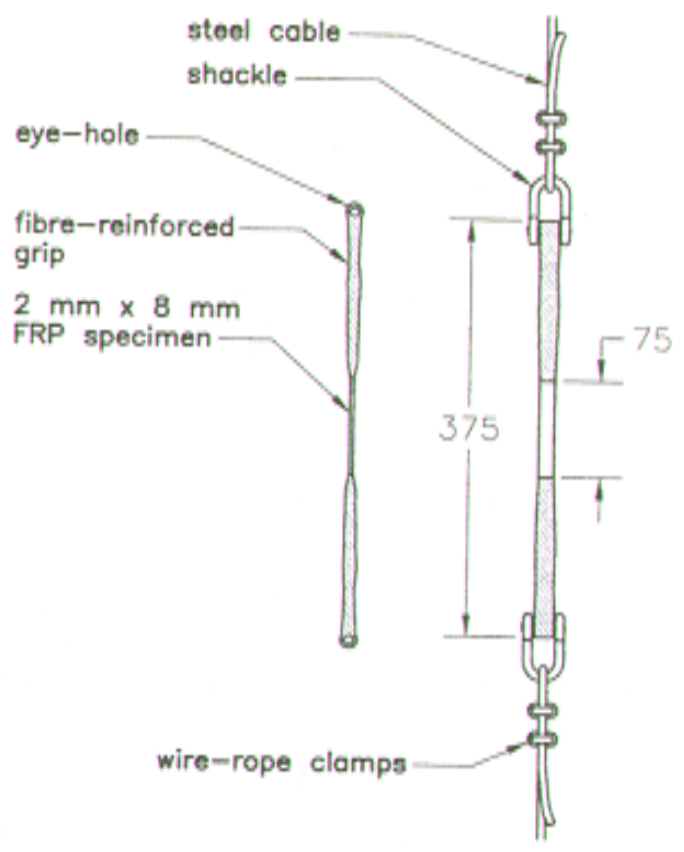

(a)

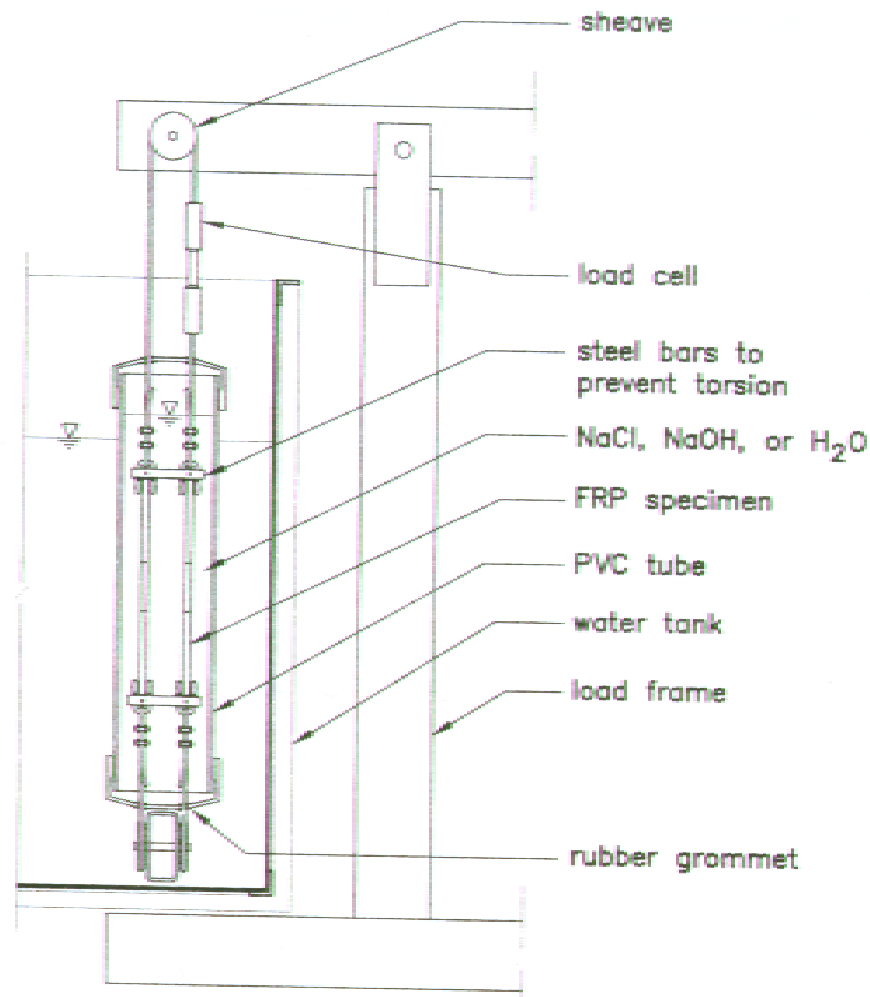

(b)

Figure 2.4 Schematic drawings of the: (a) FRP tensile-test specimen, and (b) sustained loading/conditioning apparatus (Rahman et al., pp. 503, 505, 1998) 
Conclusions of Sustained Load/Durability Tests (Rahman et al., 1998):

(1) Control: All of these specimens fared well, though the one kept under a sustained load for 370 days, developed very fine and regularly spaced cracks at about 200 $\mu \mathrm{m}(7.87$ mils). It was speculated that this may have been the case due to a thin resin cover at that location.

(2) Carbon Fiber: A pitting-type of degradation was discovered on the bottom surface of the specimen exposed for 370 days of wetting at $70 \mathrm{C}(158 \mathrm{~F})$. As for those aged under the saline $(\mathrm{NaCl})$ solution, all but two displayed cracking after 45 days irrespective of the temperature. (Incidentally, the authors mention that the saline solution may have behaved more characteristically alkaline, due to a chemical reaction resulting from the stainless steel cable, acting as a cathode, and the surface of the zinc-coated steel shackles, functioning as the anode (see Eq. (2.5)—for this reason, they believe the degradation observed in the $\mathrm{NaCl}$ solution to have stemmed from the same chemical transformations associated with the $\mathrm{NaOH}$ conditioning-scheme.)

$$
\begin{gathered}
2 \mathrm{O}_{2}+2 \mathrm{H}_{2} \mathrm{O}+4 \mathrm{e}^{-} \rightarrow 4 \mathrm{OH}^{-} \\
\mathrm{Zn} \rightarrow \mathrm{Zn}^{2+}+2 \mathrm{e}^{-} \\
\mathrm{Zn}^{2+}+2\left(\mathrm{OH}^{-}\right)=\mathrm{Zn}(\mathrm{OH})_{2}
\end{gathered}
$$

The alkaline $(\mathrm{NaOH})$ solution was found to be detrimental to all but one of the specimens. The resulting crack formation, however, did not adhere to a definite pattern and was best described as being random or alligator-skinned in appearance. For a temperature of $50 \mathrm{C}(122 \mathrm{~F})$ and a time of 122 days, the resin in some areas was discovered to have etched away, thus leaving behind a hole. After 370 days at $70 \mathrm{C}$ (158 
F), the entire surface of the sample (in susceptible-regions) had become severely chipped and splintered, with the average crack-width measuring about $2 \mu \mathrm{m}(0.0787$ mil). Again, regions of greatest susceptibility were those with thin resin covers.

(3) Glass Fiber: The only specimen to have remained intact, was the air-exposed room temperature $(24 \mathrm{C}=75.2 \mathrm{~F}$ ) case - all of the other specimens that were exposed to the alkaline $(\mathrm{NaOH})$ solution, i.e. at $50 \mathrm{C}(122 \mathrm{~F})$, broke within the first month of exposure. This signifies two points of observation: (1) the rapidity with which the hydrated hydroxyl ions $\left(\mathrm{OH}^{-}\right)$diffused through the resin, and (2) the vulnerability of the glass fibers to such an environment.

Based on the aforementioned SEM results, Rahman et al. (p. 510, 1998), formed the following qualitative exposure-scale, in the order of declining severity:

$$
\begin{aligned}
\mathrm{NaOH}_{(70 \mathrm{C})} & >\mathrm{NaOH}_{(50 \mathrm{C})}>\mathrm{NaOH}_{(30 \mathrm{C})}> \\
\mathrm{NaCl}_{(50 \mathrm{C})} & >\mathrm{NaCl}_{(30 \mathrm{C})}>\mathrm{H}_{2} \mathrm{O}_{(70 \mathrm{C})}>\operatorname{Air}_{(23 \mathrm{C})}
\end{aligned}
$$

Hence, as might be expected, the degree of deterioration was found to be the most severe at higher temperatures and longer durations of exposure. As a final point of significance, the authors mention that the danger concerning situations of resin-degradation, is that, unlike the corrosion that so clearly accompanies steel-members, damage is induced in these cases without the yielding of any external signs or warnings (Note: this same conclusion is independently drawn in this research as described in Section 7.3 and reiterated in the "Conclusions" of Section 7.4.) 
6. Toutanji et al., 1997

A total of $56,50.8 \mathrm{~mm} \times 50.8 \mathrm{~mm} \times 355.6 \mathrm{~mm}\left(2^{\prime \prime} \times 2^{\prime \prime} \times 14^{\prime \prime}\right)$ concrete beams were tested in four-point bending (see Fig. 2.1) after undergoing wet-dry cycling. The concrete consisted of Type II portland cement, with a cement-sand-gravel-water mixture ratio of $1: 2: 3: 0.5$. Four types of FRP sheets -2 of carbon fibers and 2 of glass (see Table 2.6) — and three varieties of epoxies (see Table 2.7) were used in this study.

Table 2.6 FRP properties (Toutanji et al., p. 352, 1997)

\begin{tabular}{c|c|c|c}
\hline \hline FRP Type & E [GPa] & $\boldsymbol{\sigma}_{\mathbf{u}}[\mathbf{M P a}]$ & $\boldsymbol{\varepsilon}_{\mathbf{u}}$ \\
\hline $\mathrm{C} 1{ }^{*}$ & 228 & 3485 & $1.5 \%$ \\
\hline $\mathrm{C} 2^{*}$ & 373 & 2940 & $0.8 \%$ \\
\hline $\mathrm{G} 1^{*}$ & 69 & 1518 & $2.1 \%$ \\
\hline $\mathrm{G} 2^{*}$ & 72 & 2270 & $3.2 \%$ \\
\hline $\mathrm{C}=$ carbon fiber; $\mathrm{G}=$ glass fiber
\end{tabular}

Table 2.7 Resin properties (Toutanji et al., p. 352, 1997)

\begin{tabular}{c|c|c|c}
\cline { 2 - 4 } & $\boldsymbol{\rho}[\mathbf{g} / \mathbf{c c}]$ & $\mathbf{E}_{\mathbf{m}}[\mathbf{G P a}]$ & $\boldsymbol{\sigma}_{\mathbf{m}}[\mathbf{M P a}]$ \\
\hline E1 $^{*}$ & 1.10 & 2.35 & 55.9 \\
\hline $\mathbf{E 2}^{*}$ & 1.11 & 3.06 & 69.7 \\
\hline $\mathbf{E 3}^{*}$ & N/A & 2.30 & 54.0 \\
\hline \multirow{2}{*}{$\mathrm{E}=$ epoxy } &
\end{tabular}

Twofold Conditioning Scheme (Toutanji et al., 1997): Of the 56 total beams, half were kept at constant room temperature environments, i.e. $20 \mathrm{C}$ (68 F), for 75 days, while the remaining 28 were exposed to $300 \mathrm{WD}$ cycles $-1 \mathrm{WD}$ cycle $=4$ hours $\mathrm{W}$ in saltwater $(35$ 
$\mathrm{g}$ of $\mathrm{NaCl}$ per $1 \mathrm{~L}$ of water-approximating the salt content of the ocean) and 2 hours $\mathrm{D}$ at $35 \mathrm{C}(95 \mathrm{~F})$ with $90 \%$ relative humidity.

Conclusions of Environmental Conditioning (Toutanji et al., 1997): After cycling, the beams underwent four-point bending tests at which time load and displacement curves were recorded. Using these plots, the strength, stiffness, and ductility of the beams were evaluated. These results were then compared with those of the control specimens, and subsequently, ratios of ultimate flexural loads were determined, i.e. the value computed by dividing the failure loads of the wrapped sections by those of the unwrapped members. The range of ratios for the two conditioning cases considered, i.e. room temperature and WD cycling, varied between $2.2-5.1$ and $2.1-4.4$, respectively. Obviously, these differences are a consequence of using four different fiber types and three distinct epoxies.

Beams bonded using epoxy 1 (E1) experienced the highest reduction in strength, between 19\%- 33\%; those adhered with E2, between 10\% - 24\%; finally, E3 was reduced between 3\% and 8\%. Midspan deflections were found to be highest for specimens bonded with E3 in the WD cases, while adhesive E2 exhibited the greatest reductions. Resin E2 also demonstrated the highest load carrying capacity as compared with the other two epoxies. In general, while it was observed that the load carrying capacities were improved by strengthening the beams, as expected, the WD conditioned specimens showed less improvements than those kept at room temperature. The authors comment that this reduction in strength improvement may be attributed to the degradation of the epoxy which led to the weakening of the bond between the concrete specimens and the 
FRP sheets. There is some speculation surrounding this conclusion, however, since the failure culminated in sheet-debonding, i.e. with fibers still intact at ultimate loads, thereby making it difficult to effectively assess the source of the strength reductions.

In conclusion, the authors stress the importance of selecting appropriate FRP systems, placing special emphasis on the choice of epoxy, when concerning the strengthening of concrete-members in marine, or otherwise, saline environments.

\subsection{Summary}

The objective of this chapter was to synthesize a basis for the durability studies performed in this research work - the results, of which, are reported in Chapters 7 and 8. To begin with, the concept of durability was defined in Section 2.2 as entailing a structural system to maintain its initial performance-properties over time. In this particular context, the "structural system" in question pertains to the interface-bond, consisting largely of the FRP-strip and the underlying concrete substrate to which it is adhered. It is for this reason that these two constituent materials have been considered separately, at times, throughout this chapter; also, this is the rationale for choosing the title of this chapter with the conjunctive "and" as opposed to hyphenating the words FRP and concrete, as is commonly done throughout the remainder of this study.

Section 2.2, next, defines three particular modes of fluid-transport in concrete. These are: permeability (i.e. movement due to a pressure differential in a porous medium), diffusion (i.e. fluid-flow as a result of a concentration gradient), and sorption (i.e. capillary action in pores subject to the atmosphere). The relationships associated 
with the first two of these phenomena are given in Eqs. (2.1) and (2.2), and are referred to as Darcy's Law for laminar flow and Fick's First Law, respectively.

Sections 2.3 and 2.4 dealt with the two separate conditions of immersion used in the wet-dry cycling protocol of Chapter 7-sulfate and alkaline (or, specifically in this context, hydroxide) attack. Preventive measures to reduce the effects stemming from the first of these conditions recommended minimizing the $\mathrm{C}_{3} \mathrm{~A}$ content of the cement, using blended cements containing blast furnace slag or pozzolanic materials, and taking measures to prevent the ingress of such harmful ions into the concrete in the first place. The mechanisms of sodium-, calcium-, and magnesium- sulfate attack were outlined, demonstrating how such adverse products as gypsum and ettringite might form causing concrete-deterioration. For these reasons, it was recommended that the concrete should be made as dense as possible and with as low a permeability as possible. This can be achieved in one of three ways: (1) specify a maximum water-cement ratio, (2) a minimum-strength, or (3) a minimum cement content.

Next, the phenomena of efflorescence, salt weathering, and sea water effects were treated in three separate subsections. The first of these was described as a leaching of lime compounds that leads to the formation of salt deposits. In such cases, a white powdery substance becomes visible, indicating the onset of damage. This action typically affects the edges and corners of the structure first, and then ultimately culminates in the cracking and spalling-off of the concrete. Salt weathering may occur under a variety of conditions such as: sea water splash, airborne salt deposits arising from marine or desert environments, and from applications of deicing agents in cold climate regions commonly 
referred to as scaling. Essentially, as pure water evaporates from the salt solution, a crystallized salt deposit remains which, upon subsequent wetting, grows and exerts an expansive force on the hardened cement paste. Lastly, general attacks that stem from sea water effects were described. These include chemical and physical actions, such as: sulfate attack, chloride-induced corrosion of steel reinforcement, freeze thaw attack, salt weathering, and abrasion by sand and ice in suspension.

Coming now to the subject of alkaline attack (i.e. Sec. 2.4), since the porewater environment of ordinary portland cement concrete is one of high humidity and $\mathrm{pH}-\mathrm{in}$ the range of $10-13.5$ - one of the two wet-dry conditioning regimes used for gauging the performance of the interface-bond in this study, prescribed the use of a sodium hydroxide solution $(\mathrm{pH}=12$; see Sec. 7.3). The porewater solution must move from the pores of the concrete and diffuse through the polymer matrix, if the alkaline attack is to initiate. To understand the accelerating mechanism that is initiated when a FRP reinforcement is soaked in solution, three factors must be quantified: (1) the quantity of mobile porewater available in the concrete, (2) the rate at which this porewater is to move through the concrete to the surface of the reinforcement, and (3) its diffusion-rate through the polymer matrix so that it comes into contact with the fibers. For this reason, the three subsections that were considered dealt with these issues under the following headings: porewater quantity, porewater mobility in concrete, and porewater mobility in FRP.

With respect to the first of these topics, it was explained that porewater is stored within the concrete in one of three ways: (1) chemically bound water, (2) absorbed water subject to surface forces, and (3) capillary water (a.k.a. free water) that exists in the larger 
pores, i.e. beyond the effects of surface forces. The mobility of porewater through both concrete and FRP is generally manifested by diffusion, and as such, may be modeled using Fickian behavior. Of course, in the latter case, the diffusion coefficient is much less than in the former, but interestingly, this has been found to be largely dependent on temperature, i.e. diffusivity is enhanced with increased temperatures.

Section 2.5 described the effects of freezing and thawing on concrete structures. The following subsections were considered: scaling of concrete, mechanisms of freezethaw deterioration, and a summary of protective measures. The first of these was already briefly mentioned in Section 2.3 under the general heading of "Salt Weathering", and for purposes of brevity, will not be repeated here. As for the second, in succinct fashion, when moisture in concrete freezes, it expands in volume by approximately $9 \%$. This then leads to the development of osmotic and hydraulic pressures and ultimately culminates in cracking and rupture of both the hardened cement paste as well as the aggregate particles. The last subsection listed a number of suggestions for producing concretes to better endure the harmful effects of freeze-thaw cycling. The most significant points are recounted here, these being: air-entrainment of $4 \%-6 \%$ with stable bubbles having low spacing factors, use of aggregates with low-absorption, minimization of the amount of mixing-water, and the application of a surface-sealant, once the concrete has properly cured and dried.

Finally, Section 2.6 presented the results of six different durability-studies, conducted between the years of 1995 - 1998, on FRP-bonded concrete members. The most common test methodology used was the four-point bending setup; others included 
the peel, double-lap shear, and tensile-testing approaches (see Figures $2.1-2.4$, respectively). 1. Chajes et al. (1995) performed four-point bending tests (see Fig. 2.1) on 60 small-scale beams under wet-dry (WD) and freeze-thaw (FT) cycling conditions while immersing the specimens in a $\mathrm{CaCl}_{2}$ solution. They reported a greater degradation, overall, occurring on the WD beams-especially those reinforced with the E-glass variety of fibers. 2. Green et al. (1998) also conducted four-point bending experiments, but did not find any significant damage to have been sustained by the beams after 150 FT cycles in plain water. 3. Karbhari et al. (1997) studied the interface fracture of FRP-concrete members using a peel test (see Fig. 2.2). After 2 months of aging under ambient (A), wetting (W), saltwater wetting (SW), freezing (F), and freeze-thaw cycling (FT), they did not find any great changes to have occurred for the W and SW specimens, as compared with the A samples. The F and FT regimes were believed to have produced an increase in the plasticization of the FRP material and, consequently, led to erroneous values in fracture toughness. The authors commented that more investigations are needed - the inference here is most likely due to the overall duration of experimentation - as well as to the role of both the type and thickness of the resin that is to be used. 4. Mukhopadhyaya et al. (1998) researched the effects of aging through double-lap shear tests (see Fig. 2.3) of specimens undergoing WD (18 cycles), FT (450 cycles), and dual (i.e. a combination of WD and FT) exposure conditions in salt solutions $(\mathrm{NaCl})$. Little or no differential movements, via strain measurements, were found to have occurred for the WD specimens; slippage was found, however, for the FT samples, indicating that these and the dual-exposure members were more detrimentally affected. The average and peak 
shear stresses at failure, for the two mixes considered, A and B, ranged from (all in MPa): A,ave: 1.19 - 1.36 and B,ave: 1.40 - 1.57; likewise, A,peak: 2.50 - 6.11 and B,peak: 2.77 5.63. Here too, however, the authors emphasized the insufficient duration of the aging program that they chose as not being long enough to adequately gauge the overall strength of the adhesive joint. 5. Rahman et al. (1998) conducted tensile tests (see Fig. 2.4) on fiber specimens. A pitting-type of degradation resulted in the carbon fiber samples wetted in plain water for 370 days at $70 \mathrm{C}(158 \mathrm{~F})$. Resin cracking was found to have taken place for specimens immersed in the alkaline solution $(\mathrm{NaOH})$, culminating in etching after 122 days at a temperature of $50 \mathrm{C}(122 \mathrm{~F})$, and severe chipping and splintering after 370 days at $70 \mathrm{C}(158 \mathrm{~F})$. With the exception of one of the glass fiber samples, all the others terminated in failure after only the first month of alkaline weathering. Lastly, 6. Toutanji et al. (1997) aged beams for $300 \mathrm{WD}$ cycles in $\mathrm{NaCl}$ and then tested these specimens under four-point bending (see Fig. 2.1). In particular, they studied the effects of three different epoxies (see Table 2.7), and concluded that the overall performance of externally strengthened structures located in or near saline environments are, for the most part, dependent on the choice of resin that is being used. 


\section{CHAPTER 3}

\section{A REVIEW OF FRACTURE MECHANICS AND THE DEVELOPMENT OF THE NOVEL SCCB TEST}

\subsection{Introduction}

While the use of fiber reinforced polymer (FRP) materials may not be new from a general engineering standpoint - consider the aerospace industry, for instance - it is still in its infancy with regard to mainstream civil infrastructure applications. One especially appealing aspect of this technology is its far-reaching potential to externally rehabilitate and strengthen existing and newly built structures, respectively (Boyajian, 2002a). Ultimately, though, the capability of this reinforcing system is limited by the degree with which it remains intact.

A prevalent question has then become: How long is this technology expected to endure? This notion of permanence, contextually, invokes two possible meanings with regard to the expected service-life of: (a) the FRP material itself, and (b) the FRPconcrete interface bond. As it will be seen later (in chapters 7 and 8), the former matter is really a non-issue, particularly as the type of FRP under consideration herein is that of the carbon variety (abbreviated as CFRP). In contrast, researchers such as Chajes et al. (1995b), Karbhari and Engineer (1996a), Rahman et al. (1998), and Toutanji and El- 
Korchi (1998), just to name a few, all found the glass fiber variety, denoted as GFRP, to be susceptible to humid, and moreover, alkaline and saltwater (or marine) environments. The truly chief issue concerning the application of CFRP fabric materials as a form of external reinforcement rests in that of the latter point, point (b), made above, i.e. regarding the reliability of the FRP-concrete interface. As such, this research focuses attention on both unconditioned (see Chapter 6) as well as conditioned (i.e. wet-dry and/or freeze-thaw cycled—see Chapters 7 and 8) specimens. For general information pertaining to the harmful effects of weathering on concrete and FRP-strengthened concrete members, the reader is directed to the literature review provided in Chapter 2.

Unsurprisingly, establishing a unified guideline in response to the integrity of the FRP-concrete interface bond is a rather complex task, as evidenced by the scarcity of literature addressing this matter. Such an undertaking is further compounded by the material heterogeneity of concrete as well as its inability to withstand tensile stresses of any appreciable magnitude - the significance of which is central to this chapter and the development and emergence of the novel single contoured-cantilever beam (SCCB) specimen.

Unlike monolithic structures, adhesive joints are limited as to how they can fail. The former may fail because of excessive elastic- or permanent- deformations, or its demise may be a result of fracture. The interface bond, on the other hand, being limited to such a small fraction of the total volume of the structure, can generally tolerate sizable elastic or inelastic deformations sustainable by the member at large. Hence, the critical problem surrounding the interface bond is reduced to one involving the prevention of 
fracture, and as a result, the determination of a special material parameter known as the fracture toughness (Ripling, Mostovoy and Corten, 1971).

\section{Initial Attempts}

Traditionally, the Mode I (opening mode-see Section 3.3) fracture toughness testing of materials such as metals, wood and composites, and adhesive joints in general, was accomplished with relative ease through standard experimental methods (e.g. the double cantilever beam, DCB) owing to these materials' intrinsic ability to withstand tensile stresses. A short list of available literature pertaining to these materials is provided for the interested reader:

$>\underline{\text { Metals }}$-Petch (1954), Felbeck (1955), Krafft (1964), Van Der Sluys (1969) and Kargol and Albright (1975). Two other excellent sources replete with cross-references and good general information are those of Brown and Srawley (1966) and Kenny and Campbell (1967).

$>$ Wood-Ebewele et al. (1979), Davalos et al. (1997) and Scott et al. (1992). Another resource with excellent information and many crossreferences is that of Bryan H. River's chapter in the Handbook of Adhesive Technology titled "Fracture of Adhesive-Bonded Wood Joints".

$>$ Composites-Devitt et al. (1980), Han and Koutsky (1981), Russell and Street (1982) and Whitney et al. (1982). Consider also the wonderful compilation prepared by Davies and Benzeggagh (1989), "Interlaminar Mode-I Fracture Testing," in Chapter 3 of Application of Fracture Mechanics to Composite Materials, K. Friedrich, Ed., pp. 81-112.

Adhesive Joints - Ripling, Mostovoy and Patrick (1964), Flashner et al. (1985), Crosley and Ripling (1991), River and Okkonen (1993) and Guo and Weitsman (2001).

Unfortunately, the brittle nature of concrete prevents the direct implementation of experimental fracture techniques that were traditionally used with high rates of success, the most popular of which is, arguably, the double cantilever beam (DCB) or a variant 
thereof. For reasons to be discussed shortly, the first attempts made at West Virginia University for initiating study of the FRP-concrete interface bond, strived to use a preferred variant of the DCB - the contoured double cantilever beam (CDCB). Needless to say, all such attempts warily ended in disappointment due to what Mostovoy et al. (1967) termed as "arm-breakoff" (see Figure 3.1). To see why this should be, one need only consider the tensile stress, $\sigma_{x}$, arising from bending. According to beam theory:

$$
\sigma_{x}=\frac{M h / 2}{I}=\frac{6 P a}{b h^{2}}
$$

where $M=$ bending moment at a crack-length distance, $a$, away from the applied load, $P$, and $I=$ the respective moment of inertia of the member at the same location having a height, $h$, and a width, $b$, in cross-section. Solving for the load, then, gives

$$
P=\frac{\sigma_{x} b h^{2}}{6 a}
$$

Now, if the tensile strength of concrete is generically approximated as being one-tenth that of its compressive capacity, say for all intents and purposes a value of $(0.1)^{*}(45$ $\mathrm{MPa})=(0.1)^{*}(6500 \mathrm{psi})$, at an initial crack-length of $152 \mathrm{~mm}(6 ")$, for which the crosssectional dimensions are $b=44 \mathrm{~mm}\left(1.75^{\prime \prime}\right)$ and $h=28 \mathrm{~mm}\left(1.1^{\prime \prime}\right)$, such a member is then only capable of sustaining a mere $170 \mathrm{~N}(\approx 40 \mathrm{lb})$ of load—a value that is much too low (about 1/10 - 1/5 too low) for the levels of critical loading actually required for fracture, as cited in chapters 6,7 and 8 .

To overcome this hindrance, a novel experimental methodology conceived out of the DCB approach was born that consists of a single contoured-cantilever beam (SCCB) 
bonded atop a steel-encased concrete substrate, between which, is a sandwiched strip of unidirectional CFRP fabric tow (see Figure 3.2).

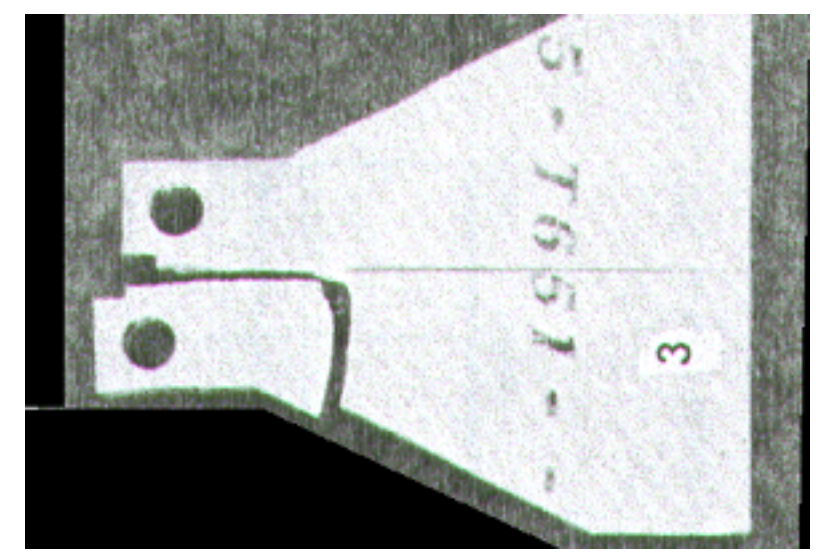

Figure 3.1 The arm-breakoff effect-unsuccessful initial attempts at studying the FRP-concrete interface bond using a CDCB approach (Mostovoy et al., 1967)

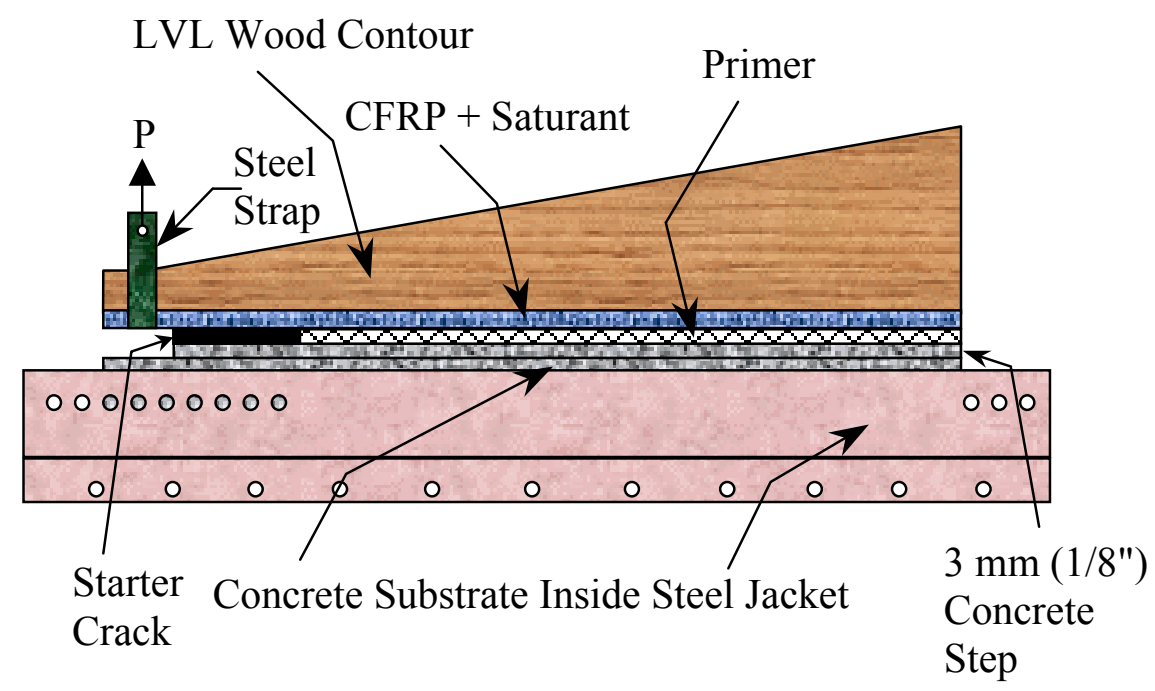

Figure 3.2 Side view of steel jacket test apparatus with SCCB specimen

More will be said about the novel SCCB specimen in section 3.6, including details of the test fixture. The remainder of this chapter is organized as follows: section 
3.2 will briefly recount the origins and history of Fracture Mechanics; the Irwin-Kies relation - the governing equation used in computing the magnitude of critical strain energy release rates in chapters 6,7 and 8 -is derived in section 3.3; experimental fracture mechanics concepts is the focus of section 3.4; section 3.5 will be a review of the literature concerning the evolving DCB specimen; finally, the chapter will conclude with a summary in section 3.7 .

\subsection{Historical Background and Key Results of Fracture Mechanics}

As the focus of this chapter, and in fact, that of the entire study, involves the topic of Fracture Mechanics, it is therefore only fitting that a brief historical review of the subject matter be presented.

\section{Studies on Wires}

Perhaps the earliest known fracture investigations were performed by the great Florentine artist and scientist of the Italian Renaissance, Leonardo da Vinci (1452-1519). He investigated the strengths of wires of differing lengths but constant diameter by loading a basket attached to an individual wire with sand from a hopper (see Figure 3.3) until the wire broke. Leonardo found, amazingly, that the short wires were stronger than their longer counterparts. The explanation of this size-effect phenomenon was ascribed, centuries after, to the greater number of microscopic defects present in the longer fibers. Griffith (p. 180, 1921—see also the following subsection) puts it this way: "In the limit, in fact, a fibre consisting of a single line of molecules must possess the theoretical molecular tensile strength." In other words, the greatest tensile strength that can be 
expected of any homogeneous material is that which is offered by its interatomic forces; optimality of this theoretical strength is approached as the material tends to smaller and smaller dimensions, thereby minimizing interatomic discontinuities.

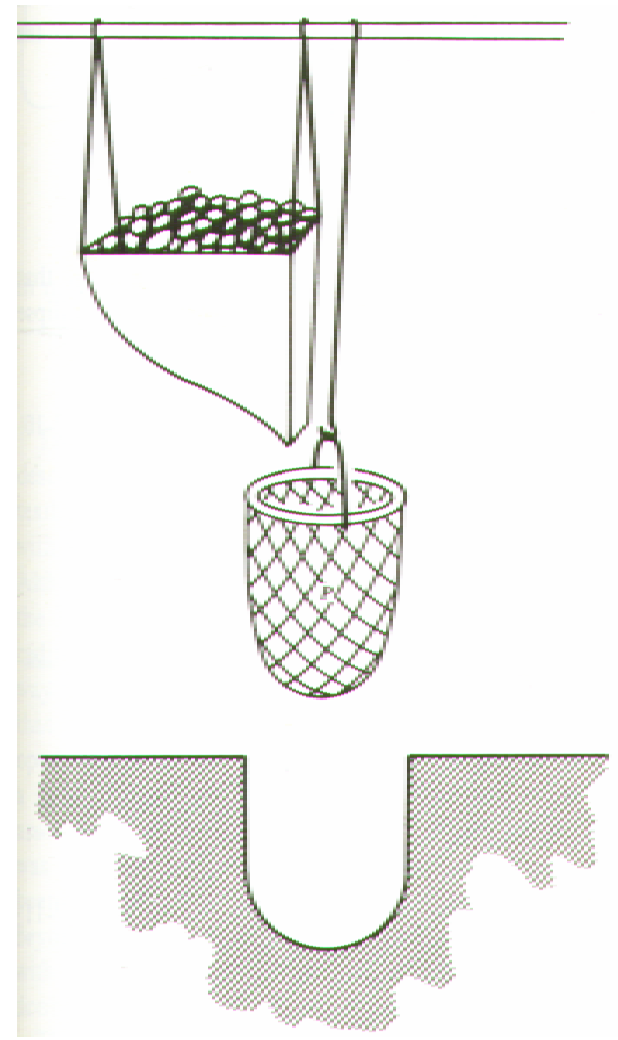

Figure 3.3 Sketch from the notebook of da Vinci illustrating the tensile test apparatus he used in studying the strengths of wires of varying lengths but constant diameter (Hertzberg, p. 235, 1976)

In the early-to-mid 1800 's, a similar type of study was undertaken by the German scientist, Karmarsch (1834, in German), who investigated the relationship between the strengths of wires of varying diameters. Like his predecessor da Vinci, Karmarsch found that an inverse relationship existed between the strength of the wire, F, and its diameter, d, and formulated the following expression in 1859 (also in German) which was later rephrased by Griffith (p. 181, 1921) as: 


$$
F=A+\frac{B}{d}
$$

where A and B are constants, later interpreted by Orowan (1933, in German as well) as being the strengths of the internal and surface structures, respectively. Greater insights into the analyses of such studies became available subsequently as the procedures of extreme value statistics were developed (Weibull, 1939). Consider the following short list of additional references for further reading on the subject of wires: Brenner $(1956,1958)$, Bateson (1958), Bokshtein et al. (1963), Bayer and Cooper (1967), Kelsey and Krock (1967), Noone (1967), Soltis (1967), Cook (1970), Jones (1971), Jones and Duncan (1971), and Perry et al. (1971).

\section{Theories of Griffith}

The present subject of Fracture Mechanics, however, undoubtedly began with the concepts that were presented by Alan Arnold Griffith (1893-1963; see Figure 3.4) in two of his renowned papers: "The Phenomena of Rupture and Flow in Solids" (1921) and "The Theory of Rupture" (1924). Two separate and fundamentally new ideas were contained in his work. One was the idea of "flaws" or minute defects which act as stress raisers and explain the difference between the observed, macroscopic and relatively low strength of solid materials versus the high values as expected from the known strengths of chemical bonds. In his paper, Griffith (p. 179, 1921) states that:

The general conclusion may be drawn that the weakness of isotropic solids, as ordinarily met with, is due to the presence of discontinuities, or flaws, as they may be more correctly called, whose ruling dimensions are large compared with molecular distances. The effective strength of technical materials might 
increase ten or twenty times at least if these flaws can be eliminated.

The other profound idea was that an energy balance is fundamentally responsible for governing the laws of crack propagation. Griffith assumed that this balance exists between the surface energy (i.e. surface tension) of the newly created fracture surface, on the one hand, and the elastic energy stored in the stress field, on the other (Benbow, 1961). F.C. Roesler (p. 981, 1956) describes it this way:

When a brittle body breaks its total surface area increases. There is energy associated with the new surface: in an atomic picture of matter it is the energy of the unsaturated bonds of the atoms in the surface layer, which have no neighbours in one direction. This additional surface energy must be balanced by the work of external forces or by a decrease of some form of energy in the system, for instance by a decrease of strain energy.

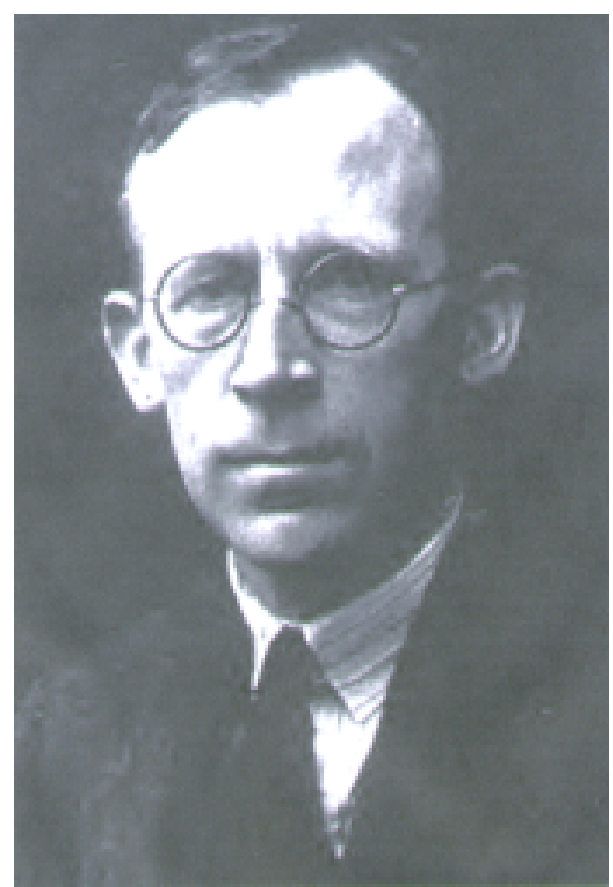

Figure 3.4 Alan Arnold Griffith (1893-1963)

(Source: http://fpml.tuwien.ac.at/fpml/history/techfig10.html) 
Roesler adds to his statement above saying:

But it is not the validity of the energy balance which is the central idea, since the first law of thermodynamics is not at issue. The point is in what might be called 'Griffith's hypothesis', that is the assumption that the balance of surface energy and mechanical energy controls the fracture process. This conception, confirmed empirically for the brittle fracture of glass, is striking because in many other fracture processes the surface energy is unimportant.

It is difficult to make a complete list of the conditions under which Griffith's hypothesis is likely to be true. One condition is that the material should be brittle, because when there is plastic deformation the energy that is dissipated is much larger than the surface energy. Another condition is that the fracture must grow slowly, because the energy of the stress waves that are associated with a running crack is also larger than the surface energy. Strictly, it might be argued that the concept of equilibrium between mechanical energy and surface energy demands infinitely slow fracturing, a quasi-reversible process. Such a quasiequilibrium can in most cases exist for a single moment only, because in most fracture processes the total load is fixed and as the crack develops this load is concentrated on a smaller and smaller cross section. In cases where Griffith's theory applies the stable or unstable behaviour of a fracture is connected with the dependence of the various forms of energy in the system on the crack length. The comparison of the first derivatives of the energies gives the Griffith balance condition. The second derivatives determine whether the crack will, once it is started, spread catastrophically; if with increasing crack length the supply of mechanical energy increases faster than the demand for surface energy then the crack is unstable and runs.

Stresses Near Cracks

In addition to these fundamental concepts, Griffith successfully calculated the stored elastic energy at the tip of a crack through a stress analysis based on the work of Inglis (1913). Seven years prior to him, Inglis had considered a flat plate subjected to uniform tension with an elliptical-shaped hole in its center, which, when degenerated, i.e. in the limit that the semi-minor axis tended to zero $(b \rightarrow 0)$, could be thought of as 
simulating a crack (see Figure 3.5(a)). He showed that the maximum stress, $\sigma_{\max }$, at the end of the major axis of the ellipse could be expressed as:

$$
\sigma_{\max }=\sigma\left[1+2 \sqrt{\frac{a}{\rho}}\right]
$$

where $\sigma=$ the applied stress acting normal to the major axis of the ellipse (see Figure 3.5(a)), $a=$ semi-major axis of ellipse, and $\rho=$ radius of curvature at the tip of the ellipse (i.e. $\rho=b^{2} / a$, where $\mathrm{b}=$ semi-minor axis of ellipse).

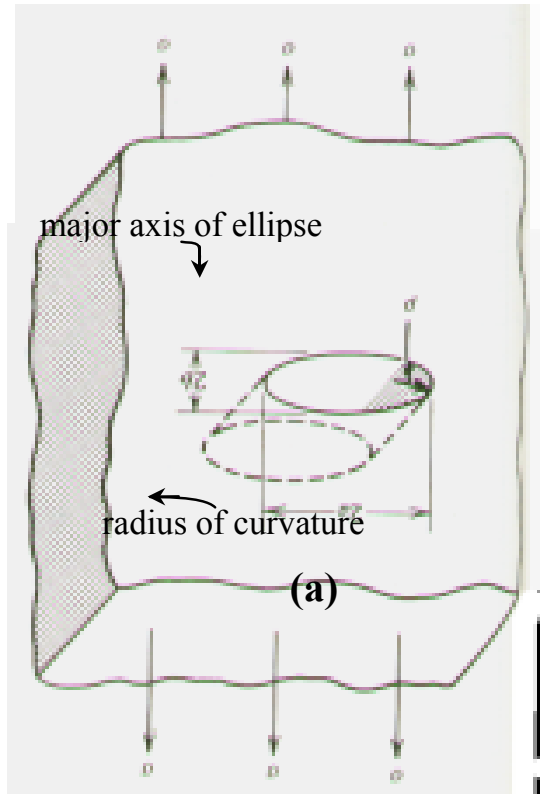

Figure 3.5 (a) Plate with elliptical hole - tip radius of curvature, $\rho=b^{2} / a$ (Hertzberg, p. 237, 1976); (b) Plate with circular holemaximum stress at tip $=3$ times the applied stress

The Stress Concentration Factor, $K_{t:}$ The ratio of maximum stress to applied stress is an important quantity in the study of Fracture Mechanics which describes the effect of crack geometry on the local crack tip stress level, and is known as the stress concentration factor, denoted $K_{t}$. Therefore, for the elliptical hole and Eq. (3.4): 


$$
K_{t}=\frac{\sigma_{\max }}{\sigma}=1+2 \sqrt{\frac{a}{\rho}}
$$

Since many geometrical holes, notches, flaws and cracks may be approximated by an elliptical hole, it is to be expected from (3.5) that as the radius of curvature tends to zero (i.e. $\rho \rightarrow 0)$, the stress concentration factor increases without bound $\left(K_{t} \rightarrow \infty\right)$-all tabulated solutions of the crack problem exhibit this behavior. Most of the studies of Fracture Mechanics are directed toward the behavior of the stress solution in the neighborhood of a crack tip as $\rho \rightarrow 0$ (Boresi, Schmidt and Sidebottom, p.617, 1993).

Note that the solution for a circular hole is readily determined from (3.4) or (3.5) since $a=b$ in this case, i.e. $\rho=a$. Thus, a threefold maximum tip stress is found relative to that of the applied stress, $\sigma$ (see Figure 3.5(b)), or equivalently, a stress concentration factor of $3\left(K_{t}=3\right)$.

Griffith's Relationship: By extension of this type of analysis, Griffith found the expression for fracture strength to be (Knott, p. 103, 1973; Hertzberg, p. 257, 1976; Kanninen and Popelar, p. 35, 1985):

$$
\begin{aligned}
& \sigma_{\mathrm{f}}=\sqrt{\frac{2 \mathrm{E} \gamma}{\pi a}} \quad \text { (in plane stress) } \\
& \sigma_{\mathrm{f}}=\sqrt{\frac{2 \mathrm{E} \gamma}{\pi a\left(1-v^{2}\right)}} \quad \text { (in plane strain) }
\end{aligned}
$$

where $\mathrm{E}=$ modulus of elasticity, $\gamma=$ surface energy, $a=$ half-crack length (i.e. the semimajor axis of the ellipse considered by Inglis above - see Figure 3.5(a)), and $v=$ Poisson's ratio. (The difference between plane stress and plane strain is explained in the subhead titled "Plane Stress vs. Plane Strain") Also, twice the unit surface energy is 
termed as the critical strain energy release rate, $G_{I c}$ (a.k.a. the crack extension force by Irwin (1957 and 1960)), where the subscript, $I$, refers to the first of three modes of cracking (see section 3.3) — thus, (3.6) and (3.7) may be rewritten as:

$$
\begin{array}{ll}
\sigma_{\mathrm{f}}=\sqrt{\frac{\mathrm{EG_{Ic }}}{\pi a}} & \text { (in plane stress) } \\
\sigma_{\mathrm{f}}=\sqrt{\frac{\mathrm{E} G I_{c}}{\pi a\left(1-v^{2}\right)}} \quad \text { (in plane strain) }
\end{array}
$$

Incidentally, because Griffith was chiefly responsible for introducing the concept of strain energy release rate, the representing quantity, $G$, used throughout the literature is denoted in honor of him.

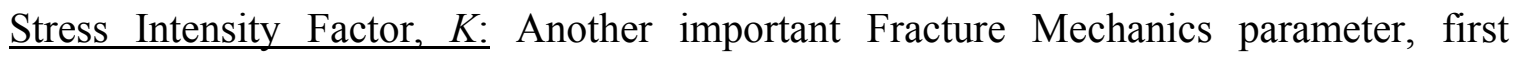
introduced by G.R. Irwin (1957) and related to the strain energy release rate, is the stress intensity factor, $K$-not to be confused with the stress concentration factor, $K_{t}$, of Eq. (3.5). The stress intensity factor provides more information than does the stress concentration factor since the former accounts for the stress level in addition to the incorporation of geometrical terms such as the crack length (Hertzberg, p. 269, 1976). This quantity, named in honor of one of Irwin's collaborators, Kies (appears also in the title of section 3.3), depends on the specimen dimensions, crack length, and loading conditions, and is proportional to the product of: [average stress $] \times[\text { crack length }]^{1 / 2}$. When a continuously increasing load is applied to a pre-cracked structure, $K$ increases, and at a critical value, the stationary or slow moving crack abruptly jumps ahead (Ripling, Mostovoy and Corten, 1971). This critical value of $K$, denoted $K_{c}$, is known as the fracture toughness. 
A useful analogy (Hertzberg, p. 269, 1976) may be drawn between stress and strength, and the stress intensity factor and fracture toughness. A component may experience many levels of stress, depending on the magnitude of load applied and the size of the component. However, there is a unique stress level that produces permanent plastic deformation and another stress level that causes failure. These stress levels are defined as the yield strength and fracture strength. Similarly, the stress intensity level at the crack tip will vary with the crack length (see Eq. (3.11) for example) and the level of load applied. That unique stress intensity level that causes failure is called the critical stress intensity level or the fracture toughness. Therefore, stress is to strength as the stress intensity factor is to fracture toughness (i.e. $\sigma: F \leftrightarrow K_{I}: K_{c}$ ).

The lower limiting value of $K_{c}$ for Mode I plane strain fracture is denoted as $K_{I c}$ in the literature (see Figure 3.9) and, similar to its energy counterpart, $G_{I c}$, is considered to be a material property in much the same manner as are the Young's and shear moduli. Strictly speaking, Irwin defined the stress intensity factor by means of the following limit (Boresi et al., p. 622, 1993; see also Irwin, 1957 and 1960):

$$
K_{I}=\lim _{\rho \rightarrow 0} \frac{\sqrt{\pi \rho}}{2} \sigma_{\max }
$$

Substituting $\sigma_{\max }$ from Eq. (3.4) into (3.10) and considering the line crack as the limiting case of the elliptical hole of Figure 3.5(a) as $b \rightarrow 0$ (i.e. as $\rho \rightarrow 0$, since $\rho=b^{2} / a$ ), gives:

$$
\begin{gathered}
K_{I}=\lim _{\rho \rightarrow 0} \frac{\sqrt{\pi \rho}}{2}\left[\sigma\left(1+2 \sqrt{\frac{a}{\rho}}\right)\right]=\lim _{\rho \rightarrow 0}\left[\frac{\sqrt{\pi \rho}}{2} \sigma+\frac{\sqrt{\pi \rho}}{2} \cdot 2 \sigma \sqrt{\frac{a}{\rho}}\right] \\
=\frac{\sigma \sqrt{\pi}}{2} \lim _{\rho \rightarrow 0}[\sqrt{\rho}]+\sigma \sqrt{\pi a} \lim _{\rho \rightarrow 0}[1]
\end{gathered}
$$


Therefore, for the case of the elliptical hole approximating a line crack:

$$
K_{I}=\sigma \sqrt{\pi a}
$$

Hence, crack propagation ensues if $K_{I}$ exceeds the fracture toughness of the material (Boresi et al., p. 620, 1993):

$$
K_{I}=\sigma \sqrt{\pi a} \geq K_{I c}
$$

The simple expressions relating $K$ with $G$ are (see, for example, Hertzberg, p. 273, 1976):

$$
\begin{array}{ll}
G=\frac{K^{2}}{\mathrm{E}} & \text { (in plane stress) } \\
G=\frac{K^{2}}{\mathrm{E}}\left(1-v^{2}\right) & \text { (in plane strain) }
\end{array}
$$

from which Eq. (3.11) can also be derived by substitution of the above into the fracture strength equations (3.8) and (3.9).

The Stress and Displacement Field Equations: The most direct approach to determining the stress and displacement fields (see Equations (3.15) - (3.22)) associated with each mode of fracture follows from the work of Irwin $(1957,1960)$ based on the method of Westergaard (1939). A full derivation of the equations below (see also Figure 3.6) is given in Appendix I of Paris and Sih's paper, "Stress Analysis of Cracks," pp. 63-65 (1964)—only the Mode I stresses and displacements are presented herein. 


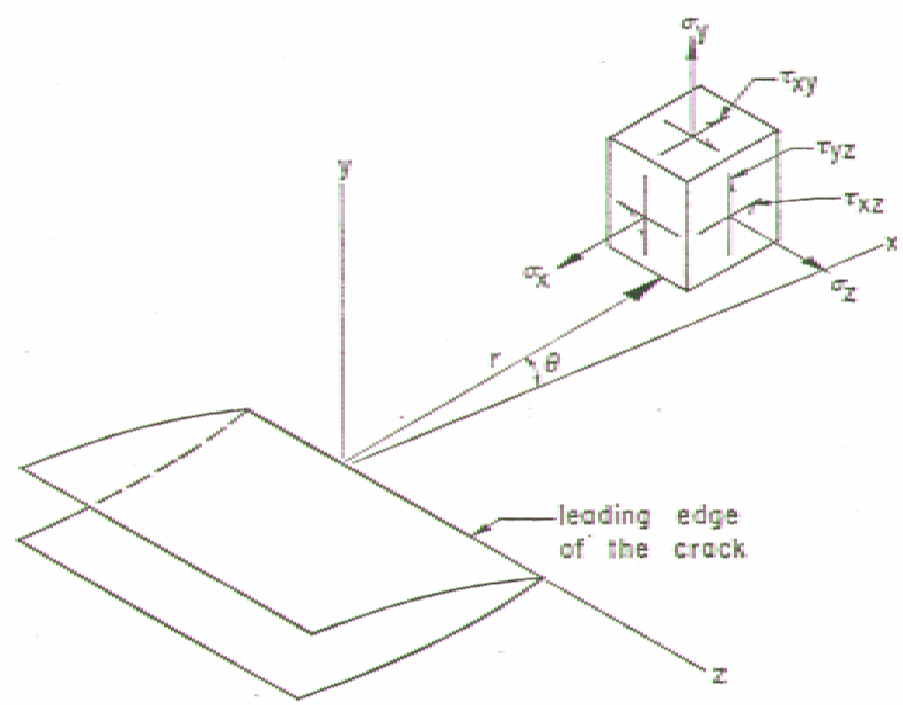

Figure 3.6 3-D stress field ahead of a crack-coordinates (x,y,z), normal stresses $\left(\sigma_{\mathrm{x}}, \sigma_{\mathrm{y}}, \sigma_{\mathrm{z}}\right)$ and shearing stresses $\left(\tau_{\mathrm{xy}}, \tau_{\mathrm{xz}}, \tau_{\mathrm{yz}}\right)$ (Paris and Sih, p. 31, 1964)

For Mode I:

$$
\begin{gathered}
\sigma_{\mathrm{x}}=\frac{K_{I}}{\sqrt{2 \pi \mathrm{r}}} \cos \frac{\theta}{2} \cdot\left[1-\sin \frac{\theta}{2} \sin \frac{3 \theta}{2}\right] \\
\sigma_{\mathrm{y}}=\frac{K_{I}}{\sqrt{2 \pi \mathrm{r}}} \cos \frac{\theta}{2} \cdot\left[1+\sin \frac{\theta}{2} \sin \frac{3 \theta}{2}\right] \\
\sigma_{\mathrm{z}}=v\left(\sigma_{\mathrm{x}}+\sigma_{\mathrm{y}}\right) \\
=\frac{K_{I}}{\sqrt{2 \pi \mathrm{r}}} \sin \frac{\theta}{2} \cos \frac{\theta}{2} \cos \frac{3 \theta}{2} \\
\mathrm{u}=\frac{K_{I}}{\mathrm{G}} \sqrt{\frac{\mathrm{r}}{2 \pi}} \cos \frac{\theta}{2} \cdot\left[1-2 v+\sin ^{2} \frac{\theta}{2}\right] \\
\mathrm{v}=\frac{K_{I}}{\mathrm{G}} \sqrt{\frac{\mathrm{r}}{2 \pi}} \sin \frac{\theta}{2} \cdot\left[2-2 v+\cos ^{2} \frac{\theta}{2}\right] \\
\mathrm{w}=0
\end{gathered}
$$


where $\left(\sigma_{\mathrm{x}}, \sigma_{\mathrm{y}}, \sigma_{\mathrm{z}}\right)=$ normal stresses, $\left(\tau_{\mathrm{xy}}, \tau_{\mathrm{xz}}, \tau_{\mathrm{yz}}\right)=$ shearing stresses, $\mathrm{r}=$ radial coordinate from the crack tip, $\theta=$ angular coordinate measured from the crack plane, $v=$ Poisson's ratio, $(\mathrm{u}, \mathrm{v}, \mathrm{w})=\mathrm{x}-, \mathrm{y}-$, and $\mathrm{z}-$ displacement components, respectively, and $\mathrm{G}=$ shear modulus of elasticity (this is not to be confused with the $G$ (italicized) used to denote the strain energy release rate of Equations (3.13) and (3.14)). Note also that a plane strain condition is assumed since, $\sigma_{z} \neq 0$ (3.17). To change the equation to one of plane stress, simply take $\sigma_{z}=0$ and appropriately change the Poisson's ratio, $v$, in (3.20) and (3.21).

For additional information regarding the development of stress-field expressions for crack tips see Inglis (1913), Phillips (1934), Westergaard (1939), Sneddon (1946), Irwin (1957) and Williams (1957) — see also the excellent paper written by Paris and Sih (1964) and the text by Boresi and Chong (1987).

Plane Stress vs. Plane Strain: It may be shown that for any general state of stress at any point $\mathrm{O}$ in a body (see Figure 3.7), there exist three mutually perpendicular planes at $\mathrm{O}$ on which the shear stresses vanish. The remaining normal stress components on these three planes are called principal stresses. Correspondingly, the three planes are called principal planes, and the three mutually perpendicular axes that are normal to the three planes are called principal axes (Boresi et al., 1993).

To determine the principal stresses $\left(\sigma_{1}, \sigma_{2}, \sigma_{3}\right)$, assume that the oblique plane, plane $\mathrm{ABC}$ (see Figure 3.7), is a principal plane - that is, the orientation of the resultant stress, $\sigma$, acting on it, coincides with the unit normal vector, $\mathbf{N}$, of the plane-and then sum the $x-, y-$ and z- direction forces of the free body diagram (Figure 3.7) to zero. For the $\mathrm{x}$-direction forces, this gives: 


$$
\sigma_{\mathrm{x}} \mathrm{A}_{1}+\tau_{\mathrm{yx}} \mathrm{A}_{2}+\tau_{\mathrm{zx}} \mathrm{A}_{3}-\left(\sigma \ell_{1}\right) \mathrm{A}=0
$$

where A1, A2, A3, and A are areas as defined in Figure 3.7 and $\ell_{1}$ is the $\mathrm{x}$-direction cosine (i.e. the x-component of the unit normal vector, $\mathbf{N}$ - see Figure 3.7). Dividing Eq. (3.23) throughout by A yields:

$$
\sigma_{\mathrm{x}} \ell_{1}+\tau_{\mathrm{yx}} \ell_{2}+\tau_{\mathrm{zx}} \ell_{3}-\sigma \ell_{1}=0
$$

or, rearranging:

$$
\left(\sigma_{\mathrm{x}}-\sigma\right) \ell_{1}+\tau_{\mathrm{yx}} \ell_{2}+\tau_{\mathrm{zx}} \ell_{3}=0
$$

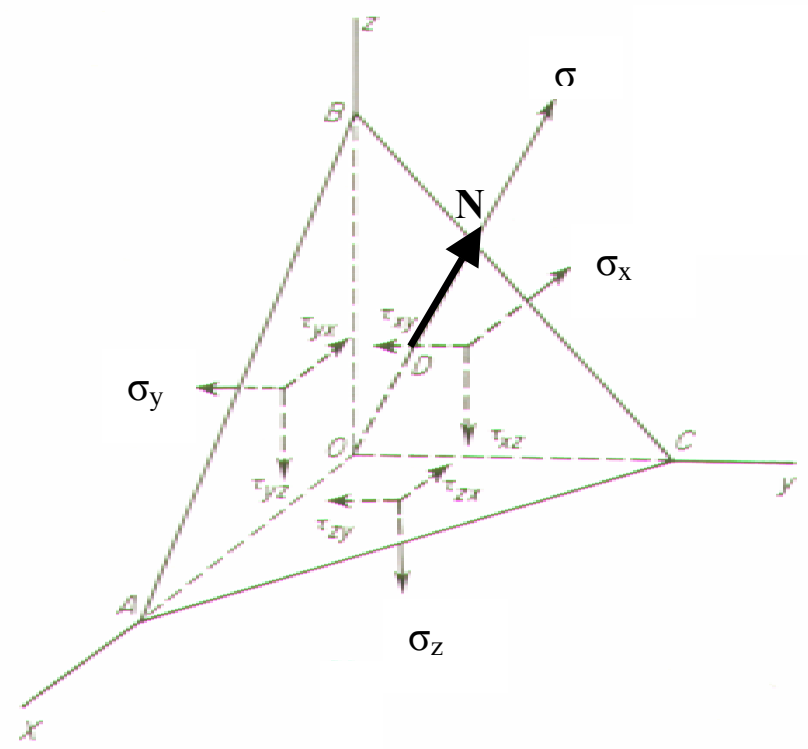

\section{Denote Areas as:}

$\mathrm{A}_{\mathrm{BOC}}: \mathrm{A}_{1}$

$\mathrm{A}_{\mathrm{BOA}}: \mathrm{A}_{2}$

$\mathrm{A}_{\mathrm{AOC}}: \mathrm{A}_{3}$

$\mathrm{A}_{\mathrm{ABC}}: \mathrm{A}$

\section{Corresponding}

Direction Cosines:

$$
\begin{aligned}
& \ell_{1}=\mathrm{A}_{1} / \mathrm{A} \\
& \ell_{2}=\mathrm{A}_{2} / \mathrm{A} \\
& \ell_{3}=\mathrm{A}_{3} / \mathrm{A}
\end{aligned}
$$

Figure 3.7 Illustration of a plane (ABC) cutting across a cubic element with corresponding stresses shown (Mendelson, p. 31, 1970)

Continuing in like fashion for the $y$ - and $z$ - direction forces gives the following system of linear equations (Eq. (3.25) is repeated for clarity): 


$$
\begin{aligned}
& \left(\sigma_{\mathrm{x}}-\sigma\right) \ell_{1}+\tau_{\mathrm{yx}} \ell_{2}+\tau_{\mathrm{zx}} \ell_{3}=0 \\
& \tau_{\mathrm{xy}} \ell_{1}+\left(\sigma_{\mathrm{y}}-\sigma\right) \ell_{2}+\tau_{\mathrm{zy}} \ell_{3}=0 \\
& \tau_{\mathrm{xz}} \ell_{1}+\tau_{\mathrm{yz}} \ell_{2}+\left(\sigma_{\mathrm{z}}-\sigma\right) \ell_{3}=0
\end{aligned}
$$

If only surface and body forces are involved then (Boresi et al., p. 32, 1993):

$$
\tau_{\mathrm{xy}}=\tau_{\mathrm{yx}} ; \tau_{\mathrm{xz}}=\tau_{\mathrm{zx}} ; \tau_{\mathrm{yz}}=\tau_{\mathrm{zy}}
$$

So the system of equations (3.25) - (3.27) may be written as:

$$
\begin{aligned}
& \left(\sigma_{\mathrm{x}}-\sigma\right) \ell_{1}+\tau_{\mathrm{xy}} \ell_{2}+\tau_{\mathrm{xz}} \ell_{3}=0 \\
& \tau_{\mathrm{xy}} \ell_{1}+\left(\sigma_{\mathrm{y}}-\sigma\right) \ell_{2}+\tau_{\mathrm{yz}} \ell_{3}=0 \\
& \tau_{\mathrm{xz}} \ell_{1}+\tau_{\mathrm{yz}} \ell_{2}+\left(\sigma_{\mathrm{z}}-\sigma\right) \ell_{3}=0
\end{aligned}
$$

Since (3.29)-(3.31) constitute a linear, homogeneous system of equations and the trivial solution (i.e. $l_{1}=\ell_{2}=\ell_{3}=0$ ) is impossible, because, by the law of direction cosines, $\ell_{1}^{2}+$ $\ell_{2}^{2}+\ell_{3}^{2}=1$, these equations are therefore consistent if and only if the determinant formed of the coefficients of $\ell_{1}, \ell_{2}$, and $\ell_{3}$ is made to vanish identically:

$$
\left|\begin{array}{ccc}
\sigma_{\mathrm{x}}-\sigma & \tau_{\mathrm{xy}} & \tau_{\mathrm{xz}} \\
\tau_{\mathrm{xy}} & \sigma_{\mathrm{y}}-\sigma & \tau_{\mathrm{yz}} \\
\tau_{\mathrm{xz}} & \tau_{\mathrm{yz}} & \sigma_{\mathrm{z}}-\sigma
\end{array}\right|=0
$$

By letting:

$$
\begin{gathered}
\mathrm{I}_{1}=\sigma_{\mathrm{x}}+\sigma_{\mathrm{y}}+\sigma_{\mathrm{z}} \\
\mathrm{I}_{2}=-\left|\begin{array}{ll}
\sigma_{\mathrm{x}} & \tau_{\mathrm{xy}} \\
\tau_{\mathrm{xy}} & \sigma_{\mathrm{y}}
\end{array}\right|-\left|\begin{array}{cc}
\sigma_{\mathrm{x}} & \tau_{\mathrm{xz}} \\
\tau_{\mathrm{xz}} & \sigma_{\mathrm{z}}
\end{array}\right|-\left|\begin{array}{cc}
\sigma_{\mathrm{y}} & \tau_{\mathrm{yz}} \\
\tau_{\mathrm{yz}} & \sigma_{\mathrm{z}}
\end{array}\right|
\end{gathered}
$$




$$
\mathrm{I}_{3}=\left|\begin{array}{ccc}
\sigma_{\mathrm{x}} & \tau_{\mathrm{xy}} & \tau_{\mathrm{xz}} \\
\tau_{\mathrm{xy}} & \sigma_{\mathrm{y}} & \tau_{\mathrm{yz}} \\
\tau_{\mathrm{xz}} & \tau_{\mathrm{yz}} & \sigma_{\mathrm{z}}
\end{array}\right|,
$$

equation (3.32) can therefore be expanded and written as a cubic in terms of $\sigma$ :

$$
\sigma^{3}-I_{1} \sigma^{2}-I_{2} \sigma-I_{3}=0
$$

the three roots $\left(\sigma_{1}, \sigma_{2}, \sigma_{3}\right)$ of which are the principal stresses at point $\mathrm{O}$ of Figure 3.7. The magnitudes and directions of $\sigma_{1}, \sigma_{2}, \sigma_{3}$ for a given member depend only on the loads being applied to the member and cannot be influenced by the choice of the coordinate axes $(x, y, z)$ used to specify the state of stress at point $O$. This means that $I_{1}, I_{2}$, and $I_{3}$ are invariants of stress and as such must have the same magnitudes for all choices of coordinate axes. Relative to the principal axes, the stress invariants (Equations (3.33) (3.35)) may be written in terms of the principal stresses as:

$$
\begin{aligned}
& I_{1}=\sigma_{1}+\sigma_{2}+\sigma_{3} \\
& I_{2}=-\sigma_{1} \sigma_{2}-\sigma_{2} \sigma_{3}-\sigma_{1} \sigma_{3} \\
& I_{3}=\sigma_{1} \sigma_{2} \sigma_{3}
\end{aligned}
$$

The visualization of the stress at a point is obviously simplified considerably if the stresses can be expressed with reference to principal axes. Various states of stress may be said to exist, depending on the values of $\sigma_{1}, \sigma_{2}, \sigma_{3}$ (Knott, p. 19, 1973).

$>$ In general, if the three principal stresses are unequal, the state of stress is then said to be triaxial

If all three are equal, it is hydrostatic

$>$ if two are equal, and the third is non-zero, it is cylindrical

$>$ if two are zero, it is uniaxial 
This last state is a situation commonly encountered in the loading of very thin sheets which do not develop any tensile stress through their thickness as illustrated by Figure 3.8(a). When a load is applied in the y-direction, both the $\mathrm{x}$ - and $\mathrm{z}$ - direction stresses, $\sigma_{\mathrm{x}}$ and $\sigma_{\mathrm{z}}$, act to restrict any contraction of the material in those directions. Since there can be no development of stress normal to a free surface, the through-thickness stress, $\sigma_{\mathrm{z}}$, must be zero at both of the outward faces of the specimen, but may attain a relatively large value at the mid-thickness plane as shown in Figure 3.8(b). The thinner specimen in part (a) of the figure, however, prevents any appreciable development of a z-direction stress, and as such, $\sigma_{\mathrm{z}} \approx 0$. Thus, only a biaxial state of stress exists, i.e. stresses confined to the (xy-) plane of the specimen, whence the name plane stress is given.
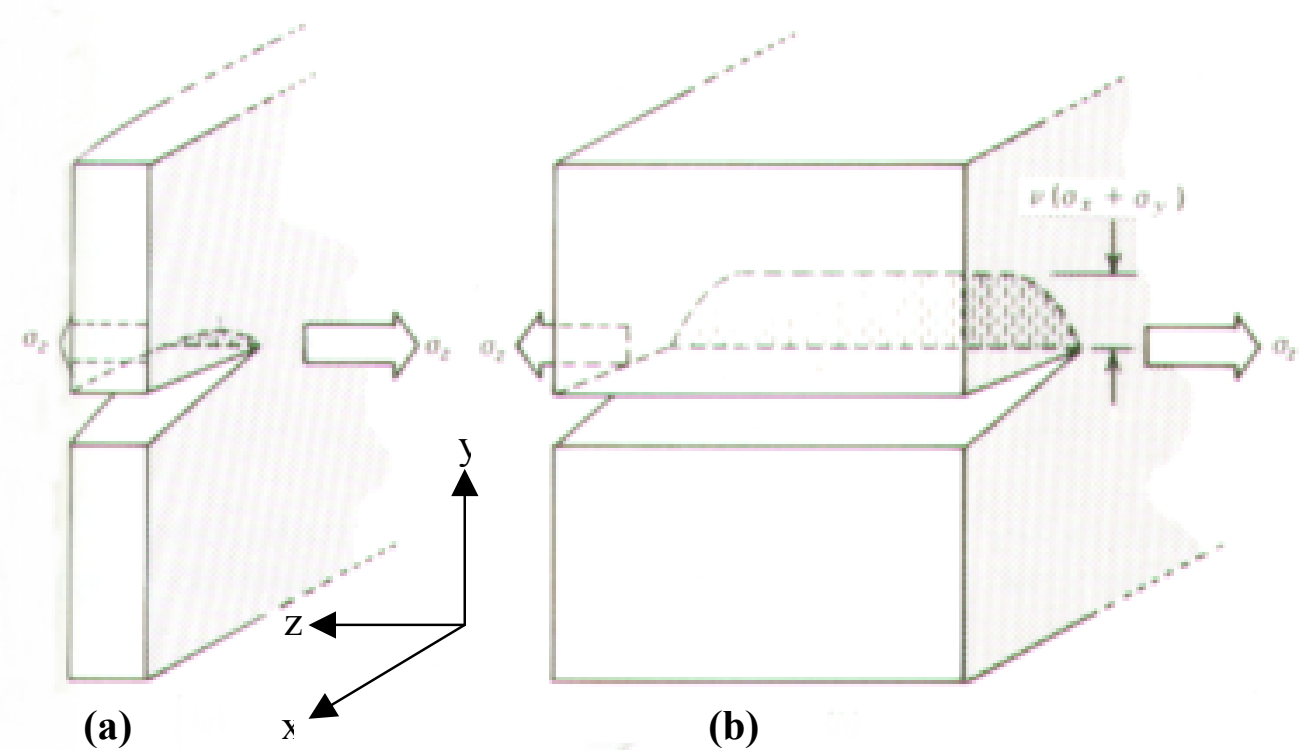

(b)

Figure 3.8 Through-thickness stress $\sigma_{z}$ in (a) a thin sheet under plane stress state and (b) a thick plate under plane strain conditions (Hertzberg, p. 265, 1976) 
In thick sections, however, a $\sigma_{z}$ stress is developed as shown in Figure 3.8(b), which creates a condition of triaxial tensile stresses acting at the crack tip and severely restricts the strains from developing in the z-direction. This condition of plane strain results in a through-thickness stress of $\sigma_{\mathrm{z}} \approx v\left(\sigma_{\mathrm{x}}+\sigma_{\mathrm{y}}\right)$ as given in Eq. (3.17).

It is important to note that the stress-field equations (3.15) - (3.22) are based on an elastic stress analysis of the crack tip and, as such, become violated anytime the yield strength of the material is exceeded, resulting in the development of a region, or zone, of plasticity. As a first approximation, the size of this region is found by solving for $r$ in (3.16) when $\theta=0$ and the magnitude of $\sigma_{\mathrm{y}}$ is assumed to have reached the yield strength of the material, $\sigma_{\mathrm{ys}}$ :

$$
\mathrm{r}_{\mathrm{y}}=\frac{K_{I}^{2}}{2 \pi \sigma_{\mathrm{ys}}^{2}} \quad \text { (in plane stress) }
$$

where $r$ is written as $r_{y}$ to denote the radius of the plastic zone region at yield. The apparent length of the crack is therefore slightly longer due to $r_{y}$. Consequently, the total crack length is adjusted as the sum of the actual crack length and some fraction pertaining to that of the plastic zone.

When the sample is thick, a large $\sigma_{\mathrm{z}}$ stress can be generated to restrict the region of plastic deformation from occurring in that direction. Estimates for the size of the plastic zone for plane strain conditions have been found to be approximately one-third that for plane stress:

$$
\mathrm{r}_{\mathrm{y}}=\frac{K_{I}^{2}}{6 \pi \sigma_{\mathrm{ys}}^{2}}=\left.\frac{1}{3} \mathrm{r}_{\mathrm{y}}\right|_{\text {plane stress }} \quad \text { (in plane strain) }
$$


It is also possible for plane strain conditions to prevail in thinner sheets for materials with higher yield strengths. For example, if the yield strength of a material were increased by a factor of two, the thickness necessary to achieve a plane strain condition for a given stress intensity level could be reduced by a factor of four. Conversely, very large sections of low yield strength materials may never bring about a fully plane strain condition (Hertzberg, p. 280, 1976).

The fracture toughness (i.e. $K_{c}$ ) of a material depends on the volume of the material that can deform plastically before fracture. Since this volume depends on the thickness, it follows that $K_{c}$ varies with thickness as shown in Figure 3.9. When the sample is thin (for example, with thickness $t_{1}$ of Figure 3.9 corresponding to parts (a) and (b)), plane stress conditions (i.e. $\sigma_{z}=0$; see also the aspect-ratio of Eq. (3.42)) prevail and the material exhibits maximum toughness. Note that if the sample were made any thinner, the toughness of the material would gradually decrease because less material would be available to absorb the energy of plastic deformation, as depicted by the slanted portions (a.k.a. shear lips) of the fractured surfaces in Figure 3.9 (a) and (b). 


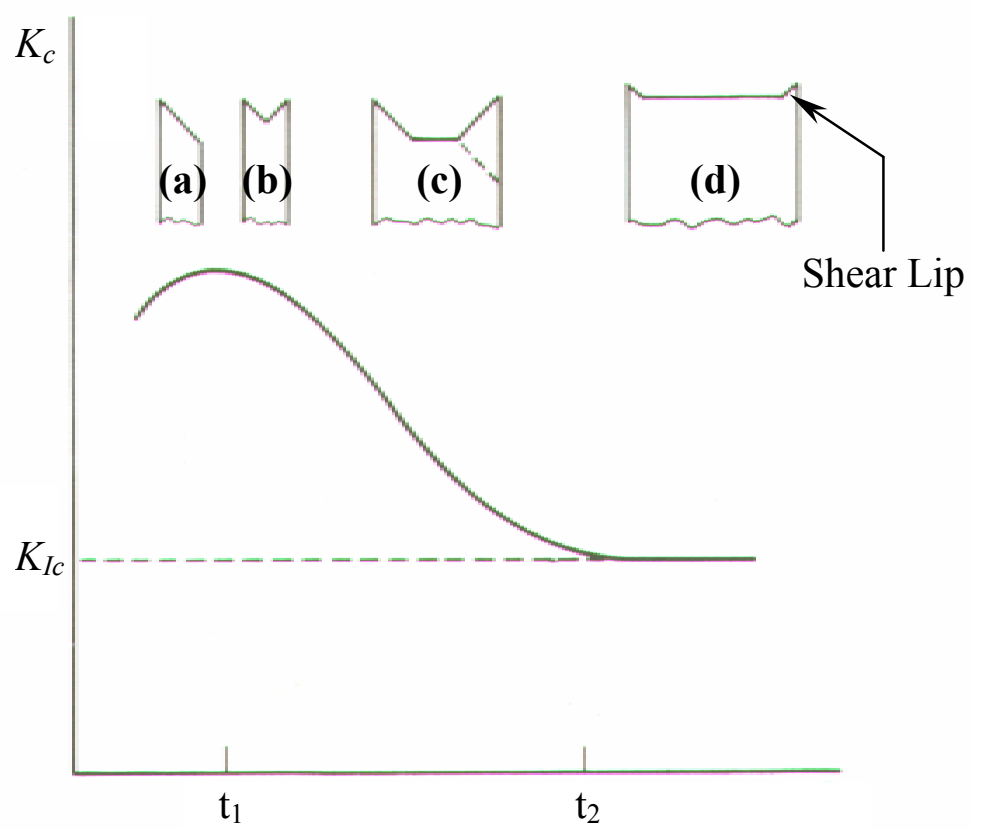

Figure 3.9 Fracture toughness $\left(K_{c}\right)$ as a function of specimen thickness (a) - (d) (Kanninen and Popelar, p. 179, 1985)

Alternately, when the thickness is increased ( $t_{2}$ corresponding to Figure 3.9(d)) to bring about plastic constraint with plane strain conditions prevailing $\left(\sigma_{\mathrm{z}} \neq 0\right.$ and $\mathrm{r}_{\mathrm{y}}<<\mathrm{t}$; see also the aspect-ratio of Eq. (3.43)), the toughness drops to a level that may be onethird (or less) than that of the plane stress value (Hertzberg, p. 279, 1976). The approximate aspect-ratios for plane stress and plane strain conditions are as follows:

$$
\begin{array}{ll}
\frac{\mathrm{r}_{\mathrm{y}}}{\mathrm{t}} \geq 1 \quad \text { (in plane stress) } \\
\frac{\mathrm{r}_{\mathrm{y}}}{\mathrm{t}}<\frac{1}{10} & \quad \text { (in plane strain) }
\end{array}
$$


For $\frac{\mathrm{r}_{\mathrm{y}}}{\mathrm{t}} \in\left[\frac{1}{10}, 1\right)$, a mixed-mode of plane stress/strain is otherwise exhibited.

One very important aspect of this lower level of toughness (i.e. the plane strain fracture toughness, $K_{I c}$ ) is that it does not decrease further with increasing thickness, thereby making it a conservative lower-limit and an especially useful parameter with regard to engineering applications. It is for this reason that $K_{I c}$ (or its energy counterpart, $G_{I c}($ Section 3.3)) is thought of as a material constant - a central issue to this dissertation, both, in the development and implementation of the novel experimental fracture mechanics approach that is to be introduced shortly and used throughout, i.e. the single contoured-cantilever beam specimen (see Section 3.6 for details).

\section{Liberty Ship Failures}

A couple decades after Griffith's work, ca. the Second World War, a major occurrence, responsible for further shaping the development of Fracture Mechanics, was the sudden, brittle failures of hundreds of Liberty ships and other vessels. Out of approximately 5000 welded ships constructed during the war, over 1000 suffered structural damage with 150 of these being seriously damaged (Kanninen and Popelar, p. 38, 1985). These fractures were noted as being extremely clean failures, essentially splitting the vessels into two parts (see Figure 3.10), and occurring without offering the slightest evidence or forewarning of impending danger to its occupants. The technicians who first investigated the accidents were particularly dumbfounded over two observations. First, they discovered that at the moment of failure, the hulls of the ships were subject to extremely low stresses. Their other counterintuitive finding was that the 


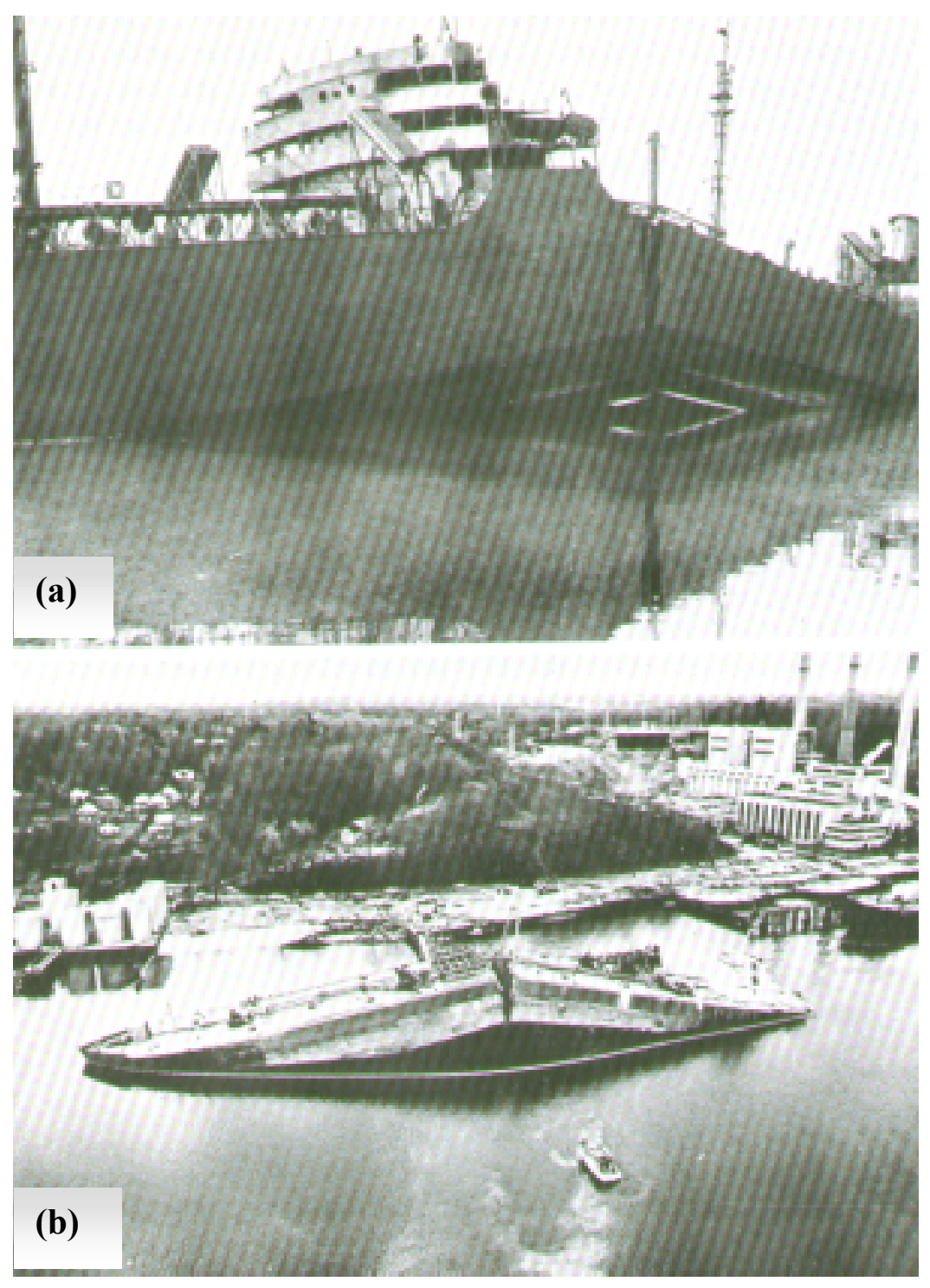

Figure 3.10 (a) Fractured T-2 tanker, the S.S. Schenectady; (b) Fractured oil barge (Hertzberg, p. 232, 1976)

actual failures were characteristically brittle, while the same steel, when tested in the laboratory, displayed ductility —an apparent contradiction (Carpinteri, p. 653, 1997). Once again, as with da Vinci's and Griffith's findings, these phenomena can be ascribed to the presence of flaws which afflict the larger archetypical structure more numerously. 
The result of these so-called "size-effects", as typified by the enormous steel structures, culminated in sudden, brittle failures dissimilar to their laboratory-sized counterparts which exhibited more prolonged, ductile failures.

Since World War II, large scale fractures were also found to occur in gas transmission lines, pressurized cabins of commercial jet airplanes, rotors of steam turbine-generators, and in varying types of pressure vessels (Irwin, p. 557, 1960). As with the ship failures, each incident occurred unexpectedly, in spite of the fact that the aforementioned structures were designed in accordance with accepted engineering conventions of the day.

\section{Post War Progress}

As might be expected, a great deal of progress was made in understanding fracture processes both in these decades and in the years following. During this time, the development of the subject was made in primarily two directions. First, it was realized, as alluded to by Roesler in the subsection titled "Theories of Griffith", that the quantity having the dimensions of surface tension appearing in Griffith's energy balance criterion may in practice exceed the true specific surface energy, $\gamma$, by a large factor. Secondly, attempts were made to include dynamic terms into the energy balance to take into account the stress waves created by fast moving cracks (Benbow, 1961). The former point was explained as the effect of a layer of plastically deformed material by George Rankin Irwin (1948) and Egon Orowan (1950), who independently arrived at the same conclusion. They demonstrated that the Griffith energy balance must not only be between the strain energy stored in the specimen and the surface energy, but the work done in 
plastic deformation as well, recognizing that for relatively ductile materials, the work done against surface tension is generally small as compared to the work done against plastic deformation. In 1955, Orowan further contributed to the understanding of brittle fractures by demonstrating that the modified Griffith condition is, in addition to being a necessary condition for crack propagation, a sufficient one as well. Also that same year Irwin indicated, and two years later, showed (Irwin, 1957) that the energy approach is equivalent to one of stress-intensity according to an occurrence of fracture whenever a critical stress distribution, characteristic of the material, is reached (Weiss and Yukawa, p. 3, 1964).

As for the latter issue, the conditions for the dynamics of a propagating crack were first formulated by Mott (1948) and then later by Yoffe (1951). Further developments were made by Krafft and Sullivan (1963) and Krafft and Irwin (1964) who studied the relation of dynamic loads to strain rate sensitive materials (see Section 3.4). For a good review see the work by Schardin (1959). More recently, Kanninen (1973, 1974) carried out dynamic analyses on run-arrest segments of crack extension and concluded that statically calculated arrested-plane strain fracture toughness values (see the previous subsection, particularly the subhead titled "Plane Stress vs. Plane Strain"), $K_{I a}$, were insufficient in describing crack arrest phenomena. Other researchers who have made contributions to the study of the dynamics of propagating cracks are Mostovoy, Crosley and Ripling (1976) and Ripling, Crosley and Wiersma (1986). More will be said about this subject in Section 3.4. 


\subsection{Three Modes of Fracture \& the Derivation of the Irwin-Kies Equation}

This section is subdivided into two parts. Typically, the first of these, i.e. the three basic modes of fracture, is discussed along with the subject of stresses near cracks which was introduced in the previous section. The purpose for delaying it until now is a simple matter of grouping. As the title of this dissertation begins: Mode I Fracture..., and the governing equation of the DCB specimen, i.e. the Irwin-Kies Equation, is central to the understanding of the material presented throughout, it was decided that the two should be delivered together for the purposes of easy comprehension and referencing.

\section{Three Basic Modes of Fracture}

Brittle fracture generally consists of two stages: crack-initiation and crack extension or propagation. Once a crack has been initiated, subsequent crack propagation may occur in several ways depending on the relative displacements of the particles in the two faces (surfaces) of the crack (Boresi et al., p. 610, 1993). The three basic modes of crack surface displacements are denoted as Mode I, Mode II and Mode III (see Figure 3.11) as distinguished by Irwin (1958).

In Mode I (see Figure 3.11(a)), the mating crack surfaces separate as the crack extends so that their relative displacement is normal to the fracture plane; hence, it is called the opening mode. It corresponds to the intuitive concept of cleavage separation, though it should not be confused with cleavage in a microcrystalline sense (Srawley and Brown, p. 145, 1964). Modes II and III are referred to as the edge-sliding and screwsliding (a.k.a. tearing) modes, respectively. 


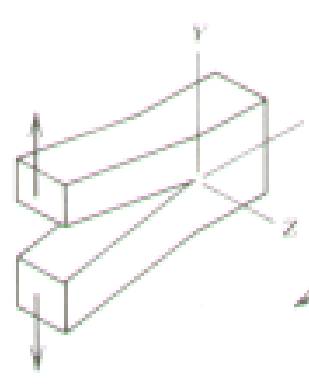

(a)

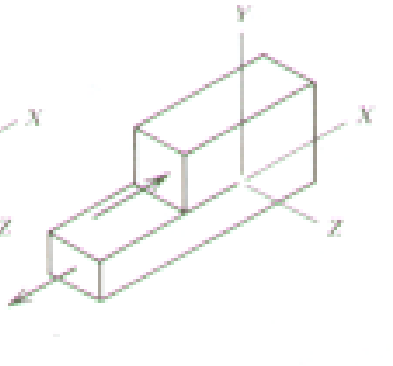

(b)

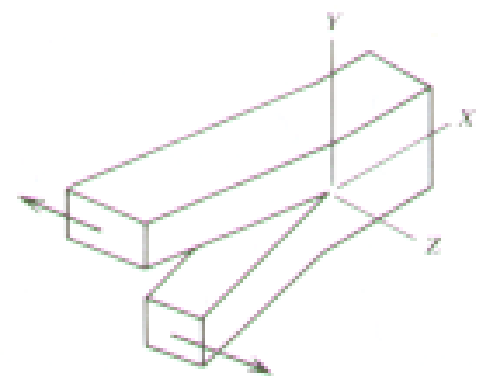

(c)

Figure 3.11 (a) Mode I (opening mode);

(b) Mode II (edge-sliding mode);

(c) Mode III (tearing mode)

(Hertzberg, p. 262, 1976)

In Mode II, i.e. the sliding or in-plane shear mode, the crack surfaces slide over one another in a direction perpendicular to the leading edge of the crack. With Mode III, i.e. the tearing or anti-plane shear mode, the crack surfaces move relative to one another and parallel to the leading edge of the crack. Note that in the two sliding-component modes, i.e. Modes II and III, there is no relative displacement of the mating crack surfaces in the direction of their normal. Note too that any arbitrary mode of crack extension can be represented as a linear combination of these three basic component modes. That is to say that the superposition of these three modes sufficiently describes the most general case of crack-tip deformation and the accompanying magnitudes of the stresses by the field equations (see Eqs. (3.15) - (3.19) for Mode I; similarly, for Modes II and III, see Paris and Sih's (1964) Eqs. (2) and (3), respectively). 
To reiterate what was already mentioned in Section 3.2 under the subhead titled "Plane Stress vs. Plane Strain", the Mode I (or plane strain) value of crack extension, i.e. $G_{I c}$, is of special importance in that it represents a practical lower limit to the fracture toughness of a material in a given condition and at a given temperature and rate of testing. This was seen diagrammatically in Figure 3.9 by gradually increasing the specimen thickness until a near-square fracture surface (see Fig. 3.9(d)) was achieved corresponding to a representative value of fracture toughness as a material constant. Srawley and Brown (p. 146, 1964) make the further point that even if the section of the load-bearing member were thin enough so that the fracture would be partly or entirely slanted (as in Fig. 3.9 (c), (b) and (a), respectively), the load-bearing capability of the member might still be governed by the value of $G_{I c}$ instead of its plane stress counterpart $G_{c}$, unless the ratio of $G_{c} / G_{I c}$ were to exceed some value dependent on the shape and size of the initial crack. To emphasize the importance of Mode I fracture over the other modes, consider some of the following assertions:

> Mode I loading is encountered in the overwhelming majority of actual engineering situations involving cracked components. Consequently, considerable attention has been given to both analytical and experimental methods designed to quantify Mode I stress-crack length relationships. Mode II is found less frequently and is of little engineering importance. ... Mode III may be regarded as a pure shear problem such as that involving a notched round bar in torsion. (Hertzberg, pp. $262 \& 263,1976$ )

$>$ In isotropic materials, brittle fracture usually occurs in Mode I. Consequently,...Mode I [loading is used] in establishing fracture criteria for sudden fracture of flawed members when the materials in these members are loaded in the brittle state. Although fractures induced by sliding (Mode II) and tearing (Mode III) do occur, their frequency is much less than the opening mode [of] fracture (Mode I). ... we do note that improvement of fracture resistance for Mode 
I usually results in improved resistance to mixed mode crack extension. (Boresi, et al., pp. $610 \& 611,1993$ )

> Since most materials are far less resistant to crack extension under the action of a normal load than under a shear load [or under a tearing load, for that matter,] Mode I fracturing has been of most interest in fracture mechanics. (Ripling, et al., p. 108, 1971)

Also from a pragmatic design perspective, if the Mode I fracture toughness value was to be used, the design engineer would have the assurance of a conservative design - that is to say that any other combination of fracturing (mixed-mode or Mode II, etc.) would yield higher values of toughness.

\section{Derivation of the Irwin-Kies Equation}

The governing equation used to determine the critical strain energy release rates of fracture, as applied herein in chapters 6,7 and 8, was first developed by G.R. Irwin and J.A. Kies in a paper they published back in 1954 titled: "Critical Energy Rate Analysis of Fracture Strength". Since then, different authors have chosen different explanations in deriving this important expression, e.g. see Carlsson and Pipes (pp. 1921, 1987), Polakowski and Ripling (pp. 409-412, 1966) and Bazant and Planas (pp. 2426, 1998), to name a few. The derivation of the Irwin-Kies equation given below most closely follows the development given by that of the latter authors, as some other important results, pertinent to structural mechanics, can be easily shown to arise as a consequence.

The General Energy Balance: Consider a plane structure of thickness, $b$, that contains a preexisting straight crack of length, $a$ (see Fig. 3.12). The First Law of Thermodynamics 


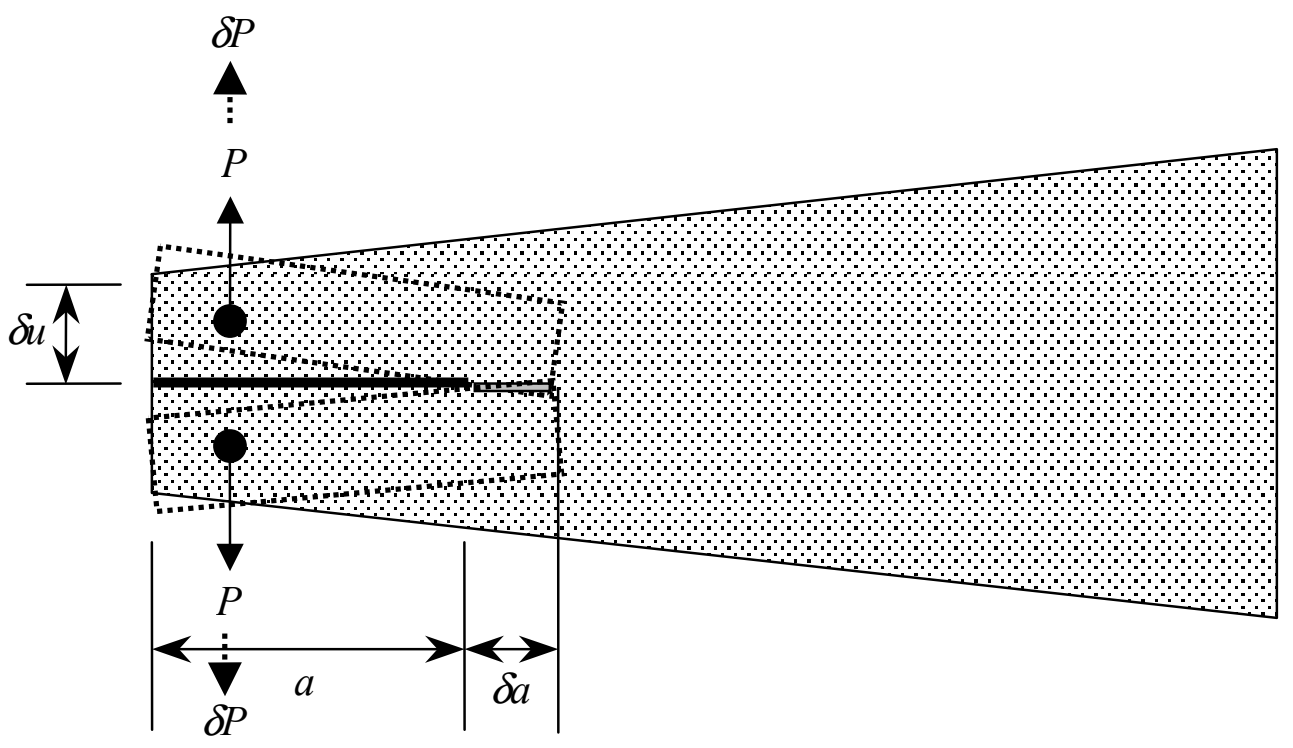

Figure 3.12 The incremental growth of a crack due to an elemental load, $\delta P$

is applied to the system when a virtual load, $\delta P$, causes the crack to grow by an incremental amount, $\delta a$. Thus, for the infinitesimal process under consideration, the total energy supplied to the structure arises from the virtual external work, denoted $\delta W_{e}$, that results from the action of the virtual load. This total supply of energy may be subdivided into four general portions: (1) that which is stored in the structure as elastic energy (a.k.a. strain energy), $\delta U,(2)$ a portion representing the increase in kinetic energy, $\delta(\Delta K),(3)$ the amount of energy lost in the form of heat, $-\delta Q$, and (4) the remainder of the energy in driving fracture, $\delta F$. Symbolically, then, the First Law of Thermodynamics may be written as 


$$
\delta W_{e}=\delta U+\delta(\Delta K)-\delta Q+\delta F
$$

By definition, the energy release rate, commonly denoted as $G$ in the literature, is, as the name implies, the rate at which the available fracture energy, $F$, changes with respect to the cracked area, $A$. Hence,

$$
G=\frac{\partial F}{\partial A}
$$

In Appendix D ("Principles and Concepts of Analytical Mechanics") of his fine book, under the subsection titled "Virtual Displacements", Holzer (see pp. $406 \& 407$, 1985) explains the concept of the virtual variation operator, $\delta$, in terms of displacements. He makes the point that virtual displacements are arbitrary variations in displacements consistent with constraints. He explains that a variation is an infinitesimal change; however, unlike the actual variation, $d$, of calculus, virtual variations can be imposed at will. An exceptional reference for further reading on the subject of virtual variations is provided in the book written by Cornelius Lanczos titled The Variational Principles of Mechanics (375 pp., 1966).

Now the variation in the fracture energy, $\delta F$, can be computed by the rules of elementary calculus (see, for example, p. 655, of the excellent textbook written by Leonard I. Holder titled Calculus with Analytic Geometry, 964 pp., 1988) as

$$
\delta F=\frac{\partial F}{\partial A} \delta A
$$

Substituting that: $\partial F=G \partial A$ from Eq. (3.45) into the above gives: $\delta F=\frac{G \partial A}{\partial A} \delta A$ which reduces to:

$$
\delta F=G \delta A
$$


Since the elemental crack area, $\delta A$, is nothing but the product of the specimen width, $b$, and the elemental crack length, $\delta a$, Eq. (3.47) may be rewritten as

$$
\delta F=G b \delta a
$$

and so the First Law of Thermodynamics (Eq. (3.44)) may be modified as

$$
\delta W_{e}=\delta U+\delta(\Delta K)-\delta Q+G b \delta a
$$

Equation (3.49) may be further modified since the loads are applied quasi-statically, i.e. $\delta(\Delta K)=0$, and the thermodynamical system under consideration is assumed to be adiabatic, i.e. $\delta Q=0$,

$$
\delta W_{e}=\delta U+G b \delta a
$$

The advantage of using $G$ is that it is a state function - this means that $G$ depends on the instantaneous geometry and boundary conditions, but not on how they vary in the actual fracture process, or on how they have been attained (Bazant and Planas, p. 25, 1998).

The quasi-statical growth of a crack requires the instantaneous available fracture energy, $\delta F$, to have attained a critical value. Thus,

$$
\delta F=\delta F_{c}
$$

or, by substitution of Eq. (3.48), that: $G b \delta a=G_{I c} b \delta a$ which reduces to

$$
G \delta a=G_{I c} \delta a
$$

Here, the subscript $I$ is used to denote a case of Mode I fracture, i.e. that of opening or cleavage (refer to Fig. 3.12), and the lowercase $c$ signifies the attainment of a critical energy-state. Technically, $G_{I c}$ is known as the critical strain energy release rate (see also the discussions of the previous section under the subheads: "Stress Intensity Factor, $K$ " and "Plane Stress vs. Plane Strain"). 
Equation (3.52) may be written more generally by reintroducing the kinetic energy term, and as such, accounting for the possibility of an incipiently dynamic situation (i.e. one in which the initial kinetic energy is zero, but thereafter, $\delta(\Delta K) \geq 0$ ) of crack growth, thus reading

$$
G \delta a=G_{I c} \delta a+\frac{1}{b} \delta(\Delta K)
$$

Consequently, three outcomes are possible:

(1) If $G<G_{I c}$ then $\delta a=0$ and $\delta(\Delta K)=0 \ldots$ No crack growth (stable)

(2) If $G=G_{I c}$ then $\delta a \geq 0$ and $\delta(\Delta K)=0 \ldots$ Quasi-static growth possible

(3) If $G>G_{I c}$ then $\delta a>0$ and $\delta(\Delta K)>0 \ldots$ Dynamic growth (unstable)

This system of conditions summarizes what seems obvious: (1) if the available energy is less than its ascribed critical value, crack-growth is precluded and the structure is said to be stable; (2) if, on the other hand, the available energy just equals its ascribed critical value, crack-growth may ensue statically, thus characterizing the structure as being either stable or unstable depending on the variation of the energy-level $\left(G-G_{I c}\right)$ with respect to the crack-displacements; and finally, (3) if the available energy exceeds its ascribed critical value, this excess amount becomes converted into the form of kinetic energy and cracking proceeds in a dynamic fashion; thus, the structure in this latter case is characterized as being unstable. More will be said about the subject of crack propagation and characterization in Section 3.4, but for now, it suffices to mention that the nature of cracking encountered in the laboratory (the results of which are presented in Chapters 6, 7, and 8) was typically quasi-static in nature, as described by the conditions of (3.55). 
Elastic Potentials and the Critical Strain Energy Release Rate: Once again, consider the plane elastic specimen of Figure 3.12 for which the crack length, $a$, may assume any value. Let $P$ denote the magnitude of the load that is being applied and $u$ the load-point displacement. By definition, the elemental external work is then

$$
\delta W_{e}=P \delta u
$$

In considering a situation of equilibrium, both the load and elastic strain energy are functions of the crack length and displacement:

$$
\begin{aligned}
& P=P(a, u) \\
& U=U(a, u)
\end{aligned}
$$

Just as in the derivation of Eq. (3.46), then, $\delta U$ may be expressed as

$$
\delta U=\frac{\partial U}{\partial a} \delta a+\frac{\partial U}{\partial u} \delta u
$$

Now if the elastic body (Fig. 3.12) that contains the preexisting crack of length, $a$, is statically displaced by the amount, $\delta u$, without resulting in any crack growth, i.e. $\delta a=0$, then all the work, as expressed by Eq. (3.50)- $\delta W_{e}=\delta U+G b \delta a$-is stored as strain energy and thus may be written

$$
\delta W_{e}-[\delta U]_{a}=0
$$

where the subscript, $a$, indicates that the crack length was held fixed. Equation (3.61), written as $\delta W_{e}=[\delta U]_{a}$, has the important result of being both a necessary and sufficient condition for equilibrium, according to the principle of virtual work (Weitsman, 1977).

In a more general process where both $u$ and $a$ are allowed to vary, Eq. (3.50)) $\delta W_{e}=\delta U+G b \delta a$-along with Eqs. (3.57) and (3.60), can then be expressed as 


$$
G b \delta b=P(a, u) \delta u-\left\{\left[\frac{\partial U(a, u)}{\partial u}\right]_{a} \delta u+\left[\frac{\partial U(a, u)}{\partial a}\right]_{u} \delta a\right\}
$$

Castigliano's second theorem is obtained as a direct consequence of (3.62) by considering the equilibrium variation $\delta a=0$ :

$$
P(a, u)=\left[\frac{\partial U(a, u)}{\partial u}\right]_{a}
$$

As a result of (3.63), then, the first two terms on the right-hand side of (3.62) cancel, yielding an expression for the energy release rate in an equilibrium process as

$$
G_{I c}(a, u)=-\frac{1}{b}\left[\frac{\partial U(a, u)}{\partial a}\right]_{u}
$$

and verifying that $G$ is indeed a state function, because it only depends on the instantaneous boundary conditions and geometry.

Revisiting once again the First Law of Thermodynamics yields that:

$$
W_{e}=U
$$

when the loading is quasi-static, the system is adiabatic and the preexisting crack has not yet extended. The external work can be found from the area underneath the equilibriumpath of the load vs. displacement diagram, as shown diagrammatically in Figure 
3.13 .

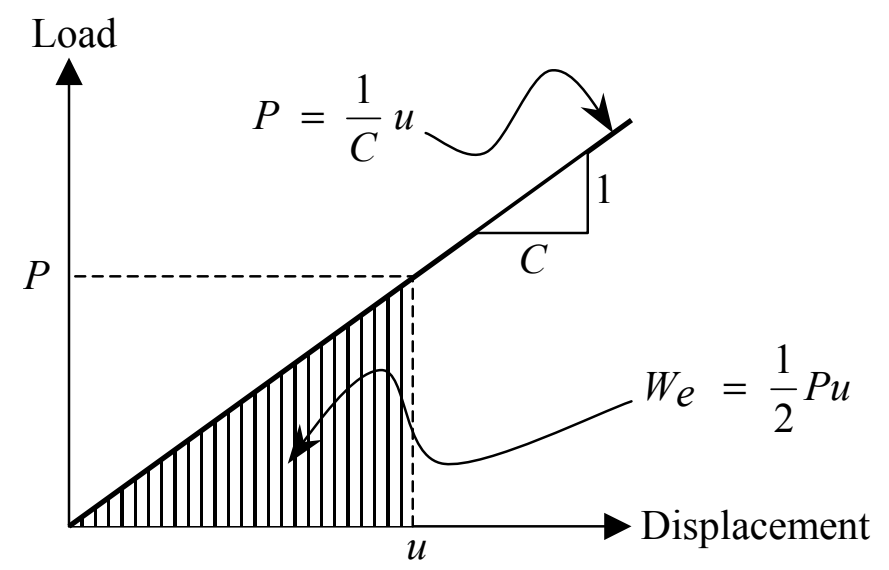

Figure 3.13 Equilibrium-path for the specimen-loading of Fig. 3.12

The external work is thus computed as:

$$
\left.W_{e}=\int_{0}^{u} \frac{w}{C} d w=\frac{w^{2}}{2 C}\right]_{0}^{u}=\frac{u^{2}}{2 C}=\frac{(P C)^{2}}{2 C}=\frac{P(P C)}{2}
$$

where $w$ is a dummy-variable representing the displacement in the integration and $C$ is the compliance of the specimen, i.e. the inverse-stiffness of the specimen. Note that the numerator of the last term in Eq. (3.66) was written as $P(P C)$ and not $P^{2} C$ so as to segregate the constant-displacement term, $u=$ constant $=P C$, in preparation for the taking of the derivative of the bracketed-term in Eq. (3.64): $G_{I c}(a, u)=-\frac{1}{b}\left[\frac{\partial U(a, u)}{\partial a}\right]_{u}$.

Now, since by Eq. (3.65), $W_{e}=U$, it was found that the external work and the internal strain energy are equal, Eq. (3.66) yields, then, that

$$
U=\frac{P(P C)}{2}
$$


Incidentally, partial derivatives are not needed in differentiating (3.67), $U=\frac{P(P C)}{2}$, since $u=$ constant $=P C$, and so $U=U(a$, constant $=u)=U(a)$, thus

$$
\frac{d U}{d a}=\frac{d}{d a}\left[\frac{P(P C)}{2}\right]=\frac{(P C)}{2} \frac{d P}{d a}
$$

To determine what $\frac{d P}{d a}$ is, the constant term, $u=$ constant $=P C$, is differentiated:

$$
\frac{d}{d a}[P C]=\frac{d}{d a}[\text { constant }]
$$

The right-hand side of (3.69) is simply zero, and the left-hand side is determined by the product-rule, thus giving

$$
C \frac{d P}{d a}+P \frac{d C}{d a}=0
$$

or, solving for $\frac{d P}{d a}$ :

$$
\frac{d P}{d a}=-\frac{P}{C} \frac{d C}{d a}
$$

Hence, Eq. (3.68) is written as

$$
\frac{d U}{d a}=\frac{(P C)}{2}\left\{-\frac{P}{C} \frac{d C}{d a}\right\}=-\frac{P^{2}}{2} \frac{d C}{d a}
$$

Substituting the above into (3.64): $G_{I c}(a, u)=-\frac{1}{b}\left[\frac{\partial U(a, u)}{\partial a}\right]_{u}$ gives, then, the famous Irwin-Kies relation that is the governing equation used in chapters 6,7 and 8 :

$$
G_{I c}=\frac{P_{c}^{2}}{2 b} \frac{d C}{d a}
$$

here $G_{I c}$ and $P_{c}$ are the respective critical values of the strain energy release rate and load. 


\subsection{Cracking Behavior from an Experimental Fracture Mechanics Viewpoint}

A great deal of experimental fracture mechanics investigations were carried out by a variety of researchers beginning in the decade of the 1950's with continuous progress being made until the present. While it is not feasible to highlight all of these major findings within this limited framework, not to mention that much of the context pertaining to these results may be immediately outside the scope of this work, it is at least appropriate to characterize the nature of crack-propagation that has been studied, especially as it relates to the experimental outcomes that are presented in subsequent chapters-for theoretical considerations concerning crack-stability, see Glucklich (1971).

Briefly touched upon in the previous section, Section 3.3, there are essentially two types of cracking associated with adhesively bonded, or jointed, structures (Mostovoy and Ripling, 1972). These are, stable and unstable cracks (see Eqs. (3.54)/(3.55) and (3.56)). Generally, all structural adhesive systems display a form of elastic behavior prior to crack-initiation. In other words, the crack remains stationary with no measurable inelastic flow until a critical value, $G_{I c}$ (see Eq. (3.73)), is reached. The differences that exist between adhesive systems are made evident once this critical value is exceeded. For some systems, the nature of cracking extends (very nearly) at a constant value of strain energy release rate, $G_{I c}$, so that materials displaying this sort of "cracking-stability" are classified as being strain rate insensitive. The rate of cracking exhibited by such materials is usually ascribed to the rate as dictated by that of the test actuator. This behavior is shown diagrammatically in Figure 3.14(a) by the approximately horizontal flat-branch of the load vs. displacement curve in the cracking-region. 
Other materials show a marked difference in the value of $G_{I c}$ responsible for initiating the stationary crack as compared with that required to drive the crack. Under a monotonically increasing load, these adhesives display a type of crack-behavior as shown in Figure 3.14(b). The high load at the first peak, also known as the (first) crack-initiation load, typically corresponds to the critical load, $P_{c}$, that is used in computing the critical strain energy release rate value of Eq. (3.73). In such cases where the crack-initiation load(s) is(are) taken as being critical, denoted generically as $P_{c}^{i}$, the corresponding critical strain energy release rate value is written as $G_{I c}^{i}$.
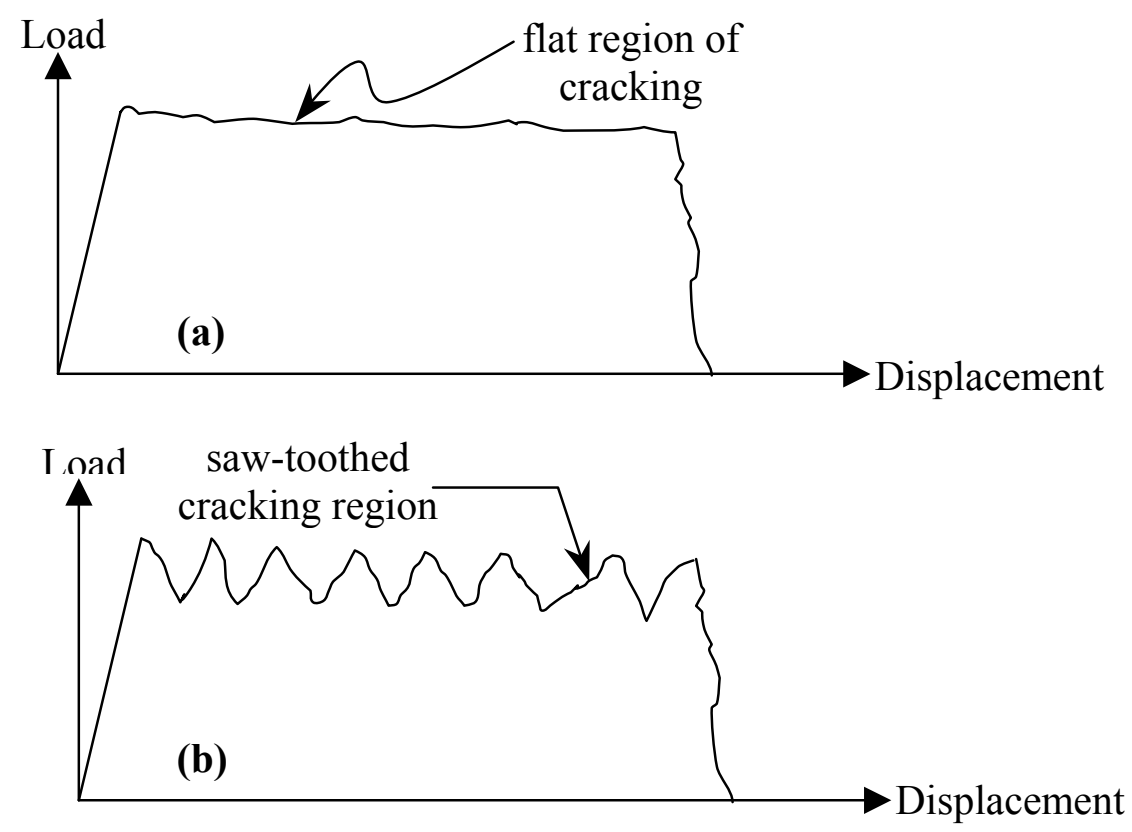

Figure 3.14 Schematic load-displacement curves of (a) a rate insensitive material with a flat region of cracking indicating stable cracking and (b) a rate sensitive material with a region of saw-toothed cracking indicating unstable cracking

Obviously, once the crack begins to move, the amount of crack extension force required to sustain crack-propagation decreases, thus accounting for the saw-toothed 
appearance of Fig. 3.14(b). At initiation, the crack "pops" ahead, running at a velocity higher than that as dictated by the rate of the actuator. At the end of the "pop", the crack stops from moving and is only reinitiated when the machine displacement increases sufficiently for the instantaneous load-level $(P)$ to once again reach a critical initiationvalue, $P_{c}^{i}$. The low-load representing the cessation of cracking and depicted by one of the "valley" regions, is known as a crack-arrest load, and is denoted, $P^{a}$. Following the convention in use with cracking-initiation, if the crack-arrest load(s) is(are) taken to be critical, then the corresponding value of fracture toughness is written as $G_{I c}^{a}$.

In actuality, fracturing occurs as a combination of unstable and stable crack growth, for if the "flat" horizontal branch of Fig. 3.14(a) were to be magnified, it too would display a saw-toothed behavior akin to that as shown in part (b) of the same figure. The real difference between the two is that, in part (a), the contrast that exists between the crack -initiation and -arrest loads, $P_{c}^{i}$ and $P_{c}^{a}$, is slight, whereas the differences are much more marked in part (b). The work of River (2002) helps in delineating such differences by not only categorizing the various types of fracture from a qualitative perspective, but from a quantitative sense, as well. First, from the qualitative viewpoint, he explains that, after initiation, a crack propagates in one of several ways. It may fracture completely and catastrophically, as in the case involving glass (see Figure 3.15(a)), or by several moderate increments associated with points of growth-and-arrest (Fig. 3.15(c)), or it may fracture by tearing or continuous small increments (Fig. 3.15, parts (b) and (d)). The preferred joint is the one requiring a high crack-initiation energy and producing stable crack growth, as depicted by the curve in part (b) of Fig. 3.15. 


\section{Different Crack Patterns: Qualitatively}

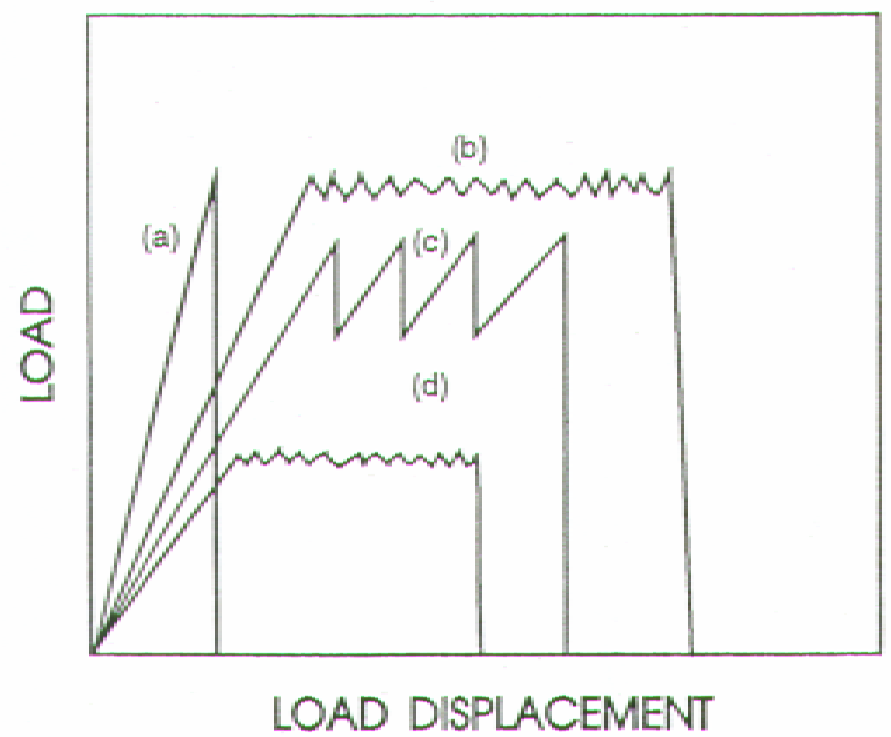

Figure 3.15 Qualitative crack behavior of adhesive joints-

(a) strong/unstable, (b) strong/stable,

(c) strong/moderately unstable, and (d) weak/stable (River, 2002)

Strong/Unstable (Fig. 3.15(a)): A dangerous situation of crack propagation may occur if the energy-storing potential of the crack-tip far outweighs the dissipatingcapability of the joint that lies ahead. Obviously, the use of such adhesives or, more broadly speaking, structural interfaces that display this type of abrupt failure, i.e. ones offering little or no forewarning of impending ruin, should absolutely be avoided.

Strong/Stable (Fig. 3.15(b)): This type of crack-growth occurs whenever a strong/ tough adhesive is used and applied properly in conjunction with a tough substrate. The crack, under these conditions, deviates into the substrate - thus the fracture toughness of the joint is essentially dictated by the toughness of this material. 
Strong/Moderately Unstable (Fig. 3.15(c)): This type of crack-growth (a.k.a. stick/slip) occurs when the adhesive is properly formulated, applied and cured, with a toughness that is greater than that of the substrate. The path of cracking in this case is directed superficially within the substrate and may occasionally cross back into the adhesive layer. The representative crack-initiation energy is moderately high to high, depending on the plasticity and strength of the adhesive and substrate. Once the crack initiates and extends, the energy that is stored in the joint gets consumed at a high ratethe crack becomes arrested once the level of energy drops below a certain level. The crack tip may remain fixed in this region or it may grow slowly by microcracking ahead of its primary location as additional energy is stored in the adhesive layer and the adherends. Rapid propagation resumes, once again, when the stored energy reaches the critical level.

Weak/Stable (Fig. 3.15(d)): If the adhesive is formulated, applied or cured improperly, its cohesive strength and toughness may be lower than that of the substrate material. In such cases, the crack travels preferentially in the weaker adhesive layer or joint interface. The crack-initiation energy is characteristically low and the crack-growth, stable, resulting in slight differences between the initiation and arrest energies.

\section{Different Crack Patterns: Quantitatively}

Apart from considering the attributes of a fracture test's load vs. displacement diagram to gain insight into the crack pattern of a joint, Ebewele, River and Koutsky (1986) defined a quantitative measure, known as the brittleness index, $I$, which is a normalization of the energy being released (i.e. lost) during a period of rapid crack 
growth with respect to the energy that was stored in the joint just at the onset of crackinitiation. Thus,

$$
I=\frac{G_{I c}^{i}-G_{I c}^{a}}{G_{I c}^{i}}
$$

Accordingly, an ideally brittle material that fails suddenly and completely as depicted in Figure 3.15(a) will have an index value of $I=1$, since $G_{I c}^{a}=0$. On the other hand, an ideally plastic material that fails by continuous tearing and having indistinct $G_{I c}^{i}$ and $G_{I c}^{a}$ energies will have an index value of $I=0$. Thus, larger $I$ values indicate abrupt and unstable crack growths whereas smaller ones signify cracks progressing more slowly and in slighter increments. In their wood fracture studies, River and Okkonen (1993) reported a value of $I=0.43$ as representing a strong/moderately unstable crack, while a value of $I$ $=0.06$ conveyed a more stable form of crack propagation. More will be said about the brittleness index in chapters 6,7 and 8 , as it pertains to actual experimental CFRPconcrete interface fracture results.

Nearly two decades earlier than the work of Ebewele, et al. (1986), Mostovoy, Crosley and Ripling (1967) had defined a concept to determine the crack-arresting capability of a joint. They found that the ratio of the crack-initiation energy with respect to its arrested value (for a specimen possessing a linear compliance vs. crack-length relationship) could be expressed as

$$
\frac{G_{I c}^{a}}{G_{I c}^{i}}=\left[1+\frac{\left.\Delta a\right|^{i-a}}{\left.a\right|^{i}}\right]^{2}
$$


where $\left.a\right|^{i}=$ crack length at the instant that $G_{I c}^{i}$ is measured, and $\left.\Delta a\right|^{i-a}=$ the incremental length of crack-growth measured between crack -initiation and -arrest. By plotting the ratio of energies given in Eq. (3.75) as a function of crack length, they were able to show that specimens with shorter initial crack lengths,$a^{i}$, resulted in smaller crack-arrest energies, $G_{I c}^{a}$. Consequently, the running cracks in these situations were more likely to halt, thereby enhancing the overall stability (see Fig. 3.15 parts (b) and (d)) of cracking. Another parameter that heightens the arresting capability of cracking, is the taper angle of the contoured-cantilever beam. Though this parameter is not implicit in Eq. (3.75), they do provide a derivation in the appendix of their paper of a modified expression involving the specimen compliance at the initial crack length, $\left.a\right|^{i}$, and the compliance of the testing machine, both of which are denoted below as $\left.C\right|^{i}$ and $\left.C\right|^{M}$, respectively. Thus, by taking into account the specimen and machine compliances, Eq. (3.75) is modified to

$$
\frac{G_{I c}^{a}}{G_{I c}^{i}}=\left[1+\frac{\left.\Delta a\right|^{i-a}}{\left.a\right|^{i}}\left(\frac{1}{1+\frac{\left.C\right|^{M}}{\left.C\right|^{i}}}\right)\right]^{2}
$$

For a perfectly rigid machine, i.e. $C^{M}=0$, the above expression reduces to Eq. (3.75). However, if the machine compliance is large enough to be a significant factor, it can be seen that specimens with higher values of $C^{i}$, i.e. specimens with lower taper angles, will offer greater crack stability. Thus by adhering to these two recommendations- 
namely, keeping the initial crack length, $\left.a\right|^{i}$, short and designing a contoured specimen with a lower taper angle - the SCCB specimen used in this study did in fact yield stable cracking, as will be seen in chapters 6,7 and 8 .

\subsection{The Evolving DCB Test Specimen}

The chief purpose of this section is to provide some perspective of the subject that follows in Section 3.6 with regard to the novel SCCB specimen by tracking the evolution of the Double Cantilever Beam (DCB) test. To achieve this objective in as succinct a manner as possible, the material of this section will be presented with little or no commentary. Instead, a general literature-outline of DCB testing is given, showcasing, first, the relevant ASTM standards, followed by the three varieties of specimens most commonly used in experimentation — namely, the DCB (or UDCB), WTDCB and CDCB (or TDCB). While the following listing is believed to consist of many dominant works pertaining to the DCB test, it is by no means an exhaustive compilation. It is rather hoped that cataloging the subsequent literature will: 1) sketch how the DCB specimen has evolved over the years, and 2) act as a handy assemblage of writings for further reading and cross-referencing (compare also with the listing that was presented back in Section $3.1)$.

Relevant ASTM Standards

The following are some of the existing standards published by the American Society for Testing and Materials (ASTM) that are relevant to fracture testing - the reader is directed to the website: http://www.astm.org/ for additional information. 
B645-98 Standard Practice for Plane-Strain Fracture Toughness Testing of Aluminum Alloys (4 pp.)

D907-00 Standard Terminology of Adhesives (11 pp.)

D3433-99 Standard Test Method for Fracture Strength in Cleavage of Adhesives in Bonded Metal Joints (7 pp.)

D3762-98 Standard Test Method for Adhesive-Bonded Surface Durability of Aluminum (Wedge Test) (4 pp.)

E399-90(1997) Standard Test Method for Plane-Strain Fracture Toughness of Metallic Materials (31 pp.)

E1290-99 Standard Test Method for Crack-Tip Opening Displacement (CTOD) Fracture Toughness Measurement (12 pp.)

E1820-01 Standard Test Method for Measurement of Fracture Toughness (46 pp.)

E1823-96e1 Standard Terminology Relating to Fatigue and Fracture Testing (20 pp.)

The DCB (or UDCB)

There are basically two divisions of Double Cantilever Beam (or Uniform DCB) tests outlined below-those used to determine the fracture energies of crystals as performed by physicists in the 1950's and 1960's, and those performed on engineeringmaterials. The former will be referred to as "Cleavage Tests" and the latter as "UDCB Tests" (see Figure 3.16 for illustrations of two typical DCB specimens).

\section{Cleavage Tests (arranged chronologically):}

Gilman, John J. "Propagation of Cleavage Cracks in Crystals," J. of Applied Physics, v27, n11, pp. 1262-1269 (1956).

Gilman, John J. "Direct Measurements of the Surface Energies of Crystals," J. of Applied Physics, v31, n12, pp. 2208-2218 (1960a). 
Gilman, John J. "Physical Nature of Plastic Flow and Fracture," Plasticity, Proceedings of the Second Symposium on Naval Structural Mechanics, pp. 43-96 (1960b).

Svensson, N.L. "The Variation of the Fracture Energy of Brittle Plastics with Temperature," Proc. Phys. Soc., v77, pp. 876-884 (1961).

Berry, J.P. "Determination of Fracture Surface Energies by the Cleavage Technique," J. of Applied Physics, v34, n1, pp. 62-68 (1963).

Gillis, Peter P. and Gilman, J.J. "Double-Cantilever Cleavage Mode of Crack Propagation," J. of Applied Physics, v35, n3, pp. 647-658 (1964).

Gillis, Peter P. "Surface-Energy Determinations by Cleavage," J. of Applied Physics, v36, n4, pp. 1374-1376 (1965).

Swanson, Gerald D. and Gross, Gordon E. "Range of Validity for Use of the Double Cantilever Fracture Energy Analysis," J. of Applied Physics, v40, n11, pp. $4684 \& 4685$ (1969).

(a)

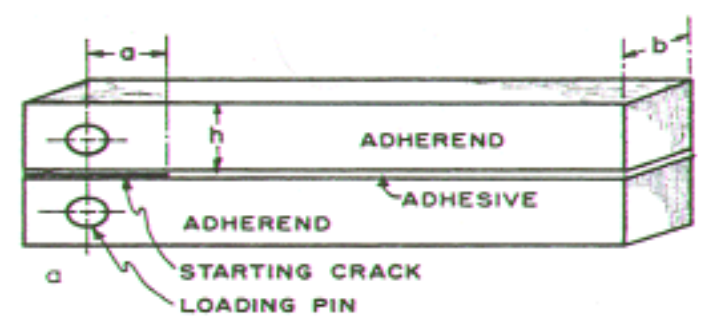

(b)

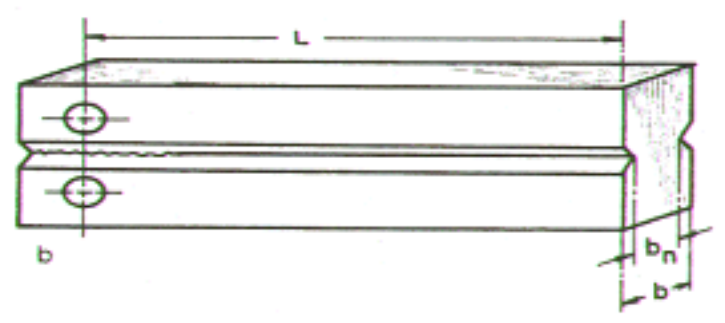

Figure 3.16 Double Cantilever Beam (DCB) specimens - (a) specimen originally used for measuring fracture toughness of adhesive joints, (b) side-grooved specimen used for measuring fracture toughness of monolithic materials (Mostovoy, Crosley and Ripling, 1967) 


\section{UDCB Tests (arranged chronologically):}

Gilman, John J. "Physical Nature of Plastic Flow and Fracture," Plasticity, Proceedings of the Second Symposium on Naval Structural Mechanics, pp. 43-96 (1960b).

Mostovoy, Sheldon, Crosley, P.B. and Ripling, E.J. "Use of Crack-Line-Loaded Specimens for Measuring Plane-Strain Fracture Toughness," J. of Materials, v2, n3, pp. 661-681 (1967).

Harrod, D.L., Hengstenberg, T.F. and Manjoine, M.J. "Fracture Toughness of Beryllium," J. of Materials, v4, n3, pp. 618-632 (1969).

Whitney, J.M., Browning, C.E. and Hoogsteden, W. "A Double Cantilever Beam Test for Characterizing Mode I Delamination of Composite Materials," J. Reinforced Plastics and Composites, v1, pp. 297-313 (1982).

Keary, Paul E., Ilcewicz, Larry B., Shaar, Casey and Trostle, Jess. "Mode I Interlaminar Fracture Toughness of Composites Using Slender Double Cantilevered Beam Specimens." J. of Composite Materials, v19, pp. 154-177 (1985).

Williams, J.G. "Large Displacement and End Block Effects in the 'DCB' Interlaminar Test in Modes I and II," J. of Composite Materials, v21, pp. 330-347 (1987).

Crosley, P.B. and Ripling, E.J. "A Thick Adherend, Instrumented DoubleCantilever-Beam Specimen for Measuring Debonding of Adhesive Joints," J. of Testing and Evaluation, v19, n1, pp. 24-28 (1991).

Sloan, Forrest. "A Constant- $G$ Double Cantilever Beam Fracture Specimen for Environmental Testing," J. of Composite Materials, v27, n16, pp. 1606-1615 (1993).

Nairn, John A. "Energy Release Rate Analysis for Adhesive and Laminate Double Cantilever Beam Specimens Emphasizing the Effect of Residual Stresses," Int. J. of Adhesion \& Adhesives, v20, pp. 59-70 (2000).

Guo, Ya-Jun and Weitsman, Y. Jack. "A Modified DCB Specimen to Determine Mixed Mode Fracture Toughness of Adhesives," Engineering Fracture Mechanics, v68, pp. 1647-1668 (2001). 
Tamuzs, V., Tarasovs, S. and Vilks, U. "Progressive Delamination and Fiber Bridging Modeling in Double Cantilever Beam Composite Specimens," Engineering Fracture Mechanics, v68, pp. 513-525 (2001).

The WTDCB

To obtain a constant compliance gradient (i.e. $d C / d a=$ constant) specimen, it is conventional to vary the specimen height as it is done for the CDCB specimen-the topic of the next subsection. Mostovoy, Crosley and Ripling (p. 665, 1967) raise the point, however, that for some cases, such specimens may not be practical. As an example, they suggest considering a thin-walled structure for which the delaminating toughness is sought. In this case, varying the specimen height would not only be impractical, but probably impossible as well. For this reason, it is best to taper the specimen width rather than its height (hence the name width-tapered DCB, or simply, WTDCB), whereby the shear contribution may be neglected (i.e. since $h$ is so small), and the linear compliance change is obtained by maintaining a constant $a / b$ ratio (see Figure 3.17). The corresponding critical strain energy release rate is therefore found as:

$$
G_{I c}=\frac{12 P_{c}^{2}}{E h^{3}}\left(\frac{a}{b}\right)^{2}
$$

As a footnote in their paper, they refer the reader to consult the work of D. Mulville of the Naval Research Laboratory (NRL) who used the WTDCB specimen in studying the delamination of fiber glass pressure vessels. Additionally, consider also the following five WTDCB-related references (arranged chronologically):

Brown, J.H. "Measuring the Fracture Toughness of Cement Paste and Mortar," Magazine of Concrete Research, v24, n81, pp.185-196 (1972). 
Miller, A.G., Hertzberg, P.E. and Rantala, V.W. "Toughness Testing of Composite Materials," $12^{\text {th }}$ National SAMPE (Soc. for the Advancement of Material and Process Engineering) Technical Conference, Materials, v12, pp. 279-293 (1980).

Han, Kyung S. and Koutsky, James. "The Interlaminar Fracture Energy of Glass Fiber Reinforced Polyester Composites," J. of Composite Materials, v15, pp. 371388 (1981).

Bascom, W.D., Bullman, G.W., Hunston, D.L. and Jensen, R.M. "The WidthTapered Double Cantilever Beam for Interlaminar Fracture Testing," 29th National SAMPE Symposium and Exhibition, v29, pp. 970-978 (1984).

Bascom, W.D., Boll, D.J., Hunston, D.L., Fuller, Bret and Phillips, P.J. "Fractographic Analysis of Interlaminar Fracture," Toughened Composites, ASTM STP 937, Norman J. Johnston, Ed., Am. Soc. For Testing and Materials, pp. 131-149 (1985).

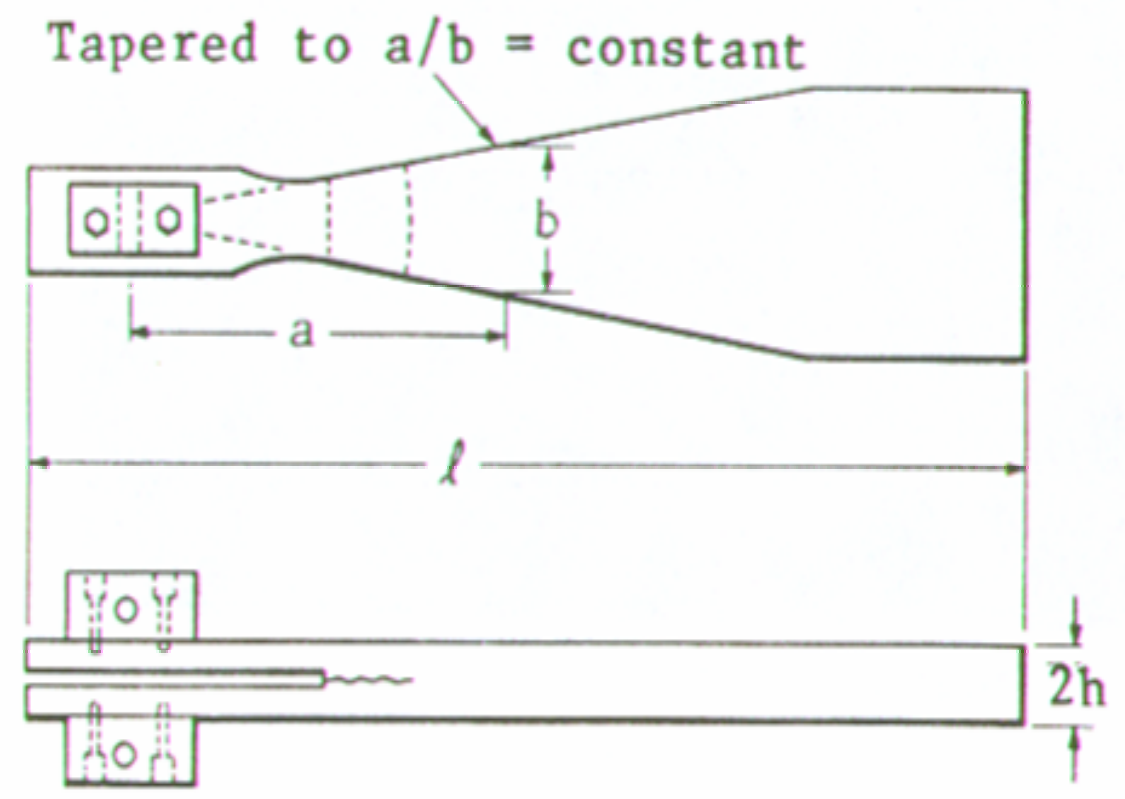

Figure 3.17 The WTDCB specimen (Bascom, et al., p. 971, 1984) 
The $C D C B$

By far the most popular variety of the DCB specimen is of the contoured type, abbreviated as $\mathrm{CDCB}$ or TDCB, for tapered DCB - the latter being used less frequently. The advantage gained by contouring the cantilevered-arms of a CDCB specimen (see Figure 3.18) is that its compliance changes accordingly with crack-extension, thus maintaining the gradient-parameter (i.e. $d C / d a$ ) of Eq. 3.73 a constant. This enables fracture testing to proceed without the need of having to periodically measure the location of the crack - an otherwise formidable criterion to achieve, experimentally. Parts (a), (b) and (c) of Figure 3.18 show different tapering geometries that have been used in CDCB tests, though the linear variety is most common.
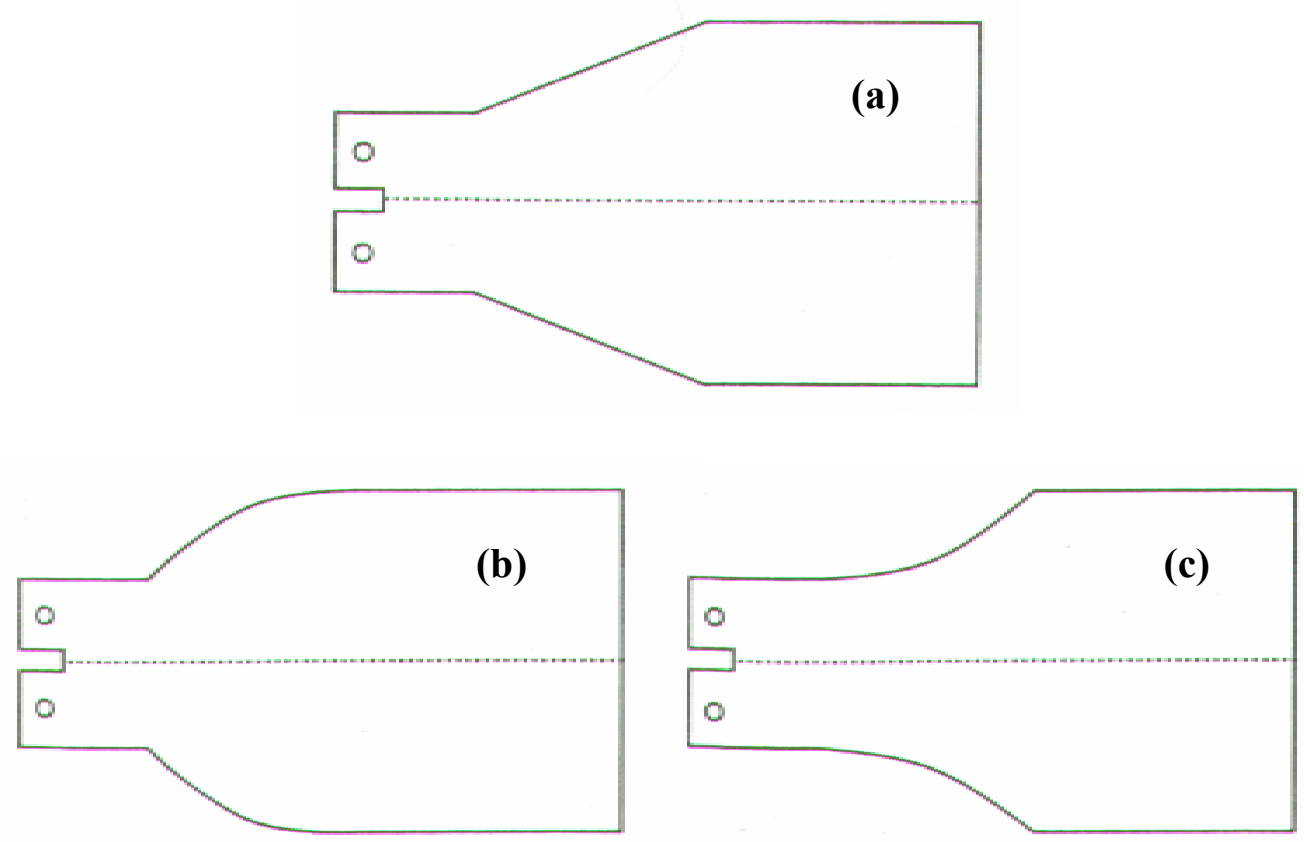

Figure 3.18 CDCB specimens - (a) straight tapered, (b) convex tapered, and (c) concave tapered (River and Okkonen, pp. 24 \& 25, 1993) 
The following thirteen works offer an excellent starting-point for readers interested in studies involving CDCB testing (arranged chronologically):

Mostovoy, Sheldon, Crosley, P.B. and Ripling, E.J. "Use of Crack-Line-Loaded Specimens for Measuring Plane-Strain Fracture Toughness," J. of Materials, v2, n3, pp. 661-681 (1967).

Van Der Sluys, W.A. "Mechanisms of Environment Induced Subcritical Flaw Growth in AISI 4340 Steel," Engineering Fracture Mechanics, v1, pp. 447-462 (1969).

Gallagher, J.P. "Experimentally Determined Stress Intensity Factors for Several Contoured Double Cantilever Beam Specimens," Engineering Fracture Mechanics, v3, pp. 27-43 (1971).

Marcus, H.L. and Sih, George C. "A Crackline-Loaded Edge-Crack Stress Corrosion Specimen," Engineering Fracture Mechanics, v3, pp.453-461 (1971).

Ripling, E.J., Mostovoy, S. and Corten, H.T. "Fracture Mechanics: A Tool for Evaluating Structural Adhesives," J. Adhesion, v3, pp. 107-123 (1971).

Dull, D.L., Buch, J.D. and Raymond, L. "Compliance Calibrations of a Contoured and Face Grooved Double Cantilever Beam Specimen," Engineering Fracture Mechanics, v4, pp. 523-531 (1972).

Schra, L., Boerema, P.J. and Van Leeuwen, H.P. "Experimental Determination of the Dependence of Compliance on Crack Tip Configuration of a Tapered Double Cantilever Beam Specimen," National Aerospace Institute of Amsterdam, Report TR 73025, 42 pp. (1973).

Kargol, J.A. and Albright, D.L. "A Fracture Mechanics Method for Determining the Crack Propagation Resistance of Embrittled Aluminum Bicrystals," J. of Testing and Evaluation, v3, n3, pp. 173-178 (1975).

Ebewele, Robert, River, Bryan and Koutsky, James. "Tapered Double Cantilever Beam Fracture Tests of Phenolic-Wood Adhesive Joints: Part I. Development of Specimen Geometry; Effects of Bondline Thickness, Wood Anisotropy and Cure Time on Fracture Energy," Wood and Fiber, v11, n3, pp. 197-213 (1979).

Ebewele, Robert O., River, Bryan H. and Koutsky, James A. "Tapered Double Cantilever Beam Fracture Tests of Phenolic-Wood Adhesive Joints: Part II. Effects of Surface Roughness, The Nature of Surface Roughness, and Surface Aging on Joint Fracture Energy," Wood and Fiber, v12, n1, pp. 40-65 (1980). 
Ting, R.Y. and Cottington, R.L. "Fracture Evaluation of High Performance Polymers as Adhesives," $12^{\text {th }}$ National SAMPE (Soc. for the Advancement of Material and Process Engineering) Technical Conference, Materials, v12, pp. 714-728 (1980).

Flashner, F., Kenig, S., Zewi, I.G. and Dodiuk, H. "Fracture Toughness of Adhesively Bonded Joints," Engineering Fracture Mechanics, v21, n5, pp. 9971004 (1985).

River, Bryan H. and Okkonen, E.A. "Contoured Wood Double Cantilever Beam Specimen for Adhesive Joint Fracture Tests," J. of Testing and Evaluation, v21, n1, pp. 21-28 (1993).

Davalos, Julio, Madabhusi-Raman, Prabhu and Qiao, Pizhong. "Characterization of Mode-I Fracture of Hybrid Material Interface Bonds by Contoured DCB Specimens," Engineering Fracture Mechanics, v58, n3, pp. 173-192 (1997).

\subsection{A Novel Experimental Fracture Mechanics Specimen: The SCCB \& Test Fixture}

Coming now to the heart of the matter as it pertains to this research study, a novel experimental Fracture Mechanics specimen was devised at West Virginia University in the latter part of 1999 by the efforts of Dr. Julio Davalos and the author to study the FRPconcrete interface bond. The author later set the name of this test-method as the Single Contoured-Cantilever Beam, which may simply be abbreviated as SCCB.

\section{Requisite of a Single Contoured-Cantilever Beam Specimen}

Previously, many researchers attempting to determine the fracture toughness of most engineering materials resorted to the established DCB technique of the previous section. As mentioned in Section 3.1, under the subsection titled "Initial Attempts", former studies relying on such a testing method had been successfully conducted on metals, wood, composites and adhesive joints, owing to a trait shared in common with all 
these materials - their inherent ability to sufficiently withstand tensile stresses. However, all attempts at extending this experimental Fracture Mechanics approach to the study involving the FRP-concrete interface bond culminated in failure-specifically, failure attributed to arm-breakoff (see Figure 3.1). To overcome this hindrance, a novel experimental methodology was conceived and born out of the DCB approach, differing by three aspects.

\section{Differing Aspects between the DCB and SCCB Specimens}

The most obvious difference of all is that this new method recast the DCB specimen by halving the pair of its symmetric constituents - thus the name Single Contoured-Cantilever Beam. This departure in symmetry roused a concern regarding methodological-viability that challenged the very essence of the device as it was intended to be used under an opening mode (Mode I) of loading. The concern, while completely not unfounded, asserted that the asymmetrical SCCB specimen would incur a large degree of shearing stresses (Mode II) at the interface, thus undermining the true value of the critical strain energy release rate being sought, $G_{I c}$. This, however, was found not to be the case and is explained in greater detail in the next chapter (read especially the "Introduction", Section 4.1; see also pp. 339-341).

Another difference between the DCB, or more specifically for the sake of comparison, the $\mathrm{CDCB}$, and the $\mathrm{SCCB}$, is that the specimen-portion of the latter minus the contoured-cantilever can be separated into two parts, though the concrete portion is cast monolithically: 1) the interface (see the dashed-region in Figure 3.19(a)), and 2) the bulk concrete substrate (see Figure 3.19(b)). The CDCB, on the other hand, traditionally 
consists of either a separate interface that is, in turn, bonded and sandwiched in between the pair of contoured-cantilever arms (as in the works of Ebewele et al., 1979, and Davalos et al., 1997), or is monolithic with the contoured-cantilever arms (as in the works of Mostovoy et al., 1967, and Marcus and Sih, 1971). This distinction is important based on the following three aspects: 1) function, 2) fabrication and 3) environmentalconditioning. The first of these is discussed in the next paragraph. As for fabrication (see also Chapter 5), a $3 \mathrm{~mm}\left(1 / 8^{\prime \prime}\right)$ raised-step of concrete (see Figure 3.19), recessed $51 \mathrm{~mm}$ (2") from the front-end (Figure 3.19(b)) to accommodate the load-strap, was cast with a width to match the thickness of the contoured-cantilever beam, thereby not only acting as

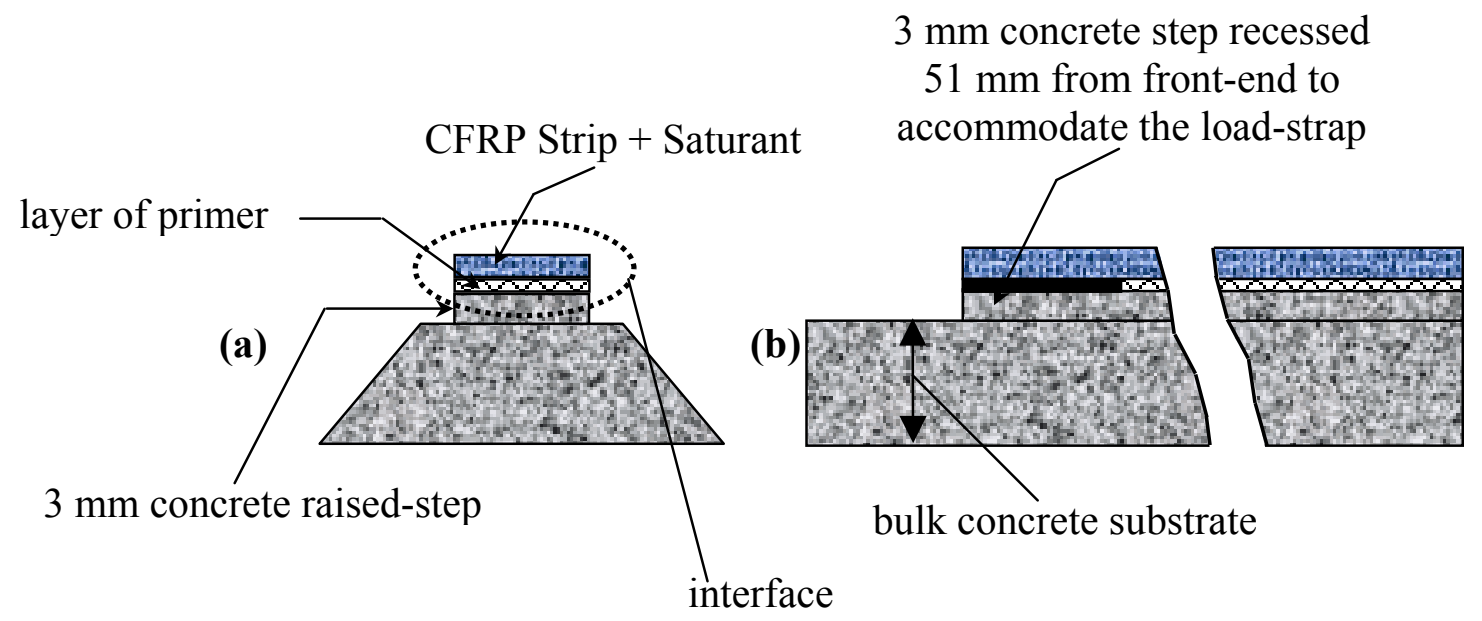

Figure 3.19 CFRP-bonded concrete specimen - (a) cross-section-, (b) side- view

a guide in promoting proper alignment of the contour during bonding, but more significantly, encouraging the propagation of cracks to proceed and remain at this interface-location. With respect to the matter concerning environmental-conditioning (see also Chapters 7 and 8), the bulk concrete substrate was coated with primer leaving only 
the interface vulnerable to the ingression of the degradative media- $-\mathrm{Na}_{2} \mathrm{SO}_{4}$ and $\mathrm{NaOH}$, used as the two separate cases of the wet-dry cycling protocol, and $\mathrm{CaCl}_{2}$ used for the case of freezing and thawing. In the latter scheme, the beams were actually inverted and only the interface was soaked in the $\mathrm{CaCl}_{2}$ to prevent the bulk substrate from the pulverizing action of the forming and expanding salt crystals. It should be understood that all of the environmental-conditioning schemes were performed on the CFRP-bonded concrete specimens in the absence of the contoured-cantilever beams. The cantilevers were, instead, bonded once the protocol of weathering in each case was completed, and only following the proper curing of the beams, would fracturing of the SCCB specimens be initiated.

The third distinguishing feature between the DCB and SCCB specimens is that of function. As already mentioned, the DCB was traditionally used on materials that could sufficiently withstand the tensile stresses responsible for causing arm-breakoff. Since concrete is an inherently weak material when subjected to tension, a special steel jacket (see Figure 3.20) was designed to provide confinement of the bulk concrete substrate (Fig. 3.19(b)) and, as such, offer a form of external reinforcement. In addition, a continuous sheet of neoprene rubber was placed in between the concrete and the steel jacket to ensure a fairly uniform distribution of confining-pressures upon the substrate. Moreover, to prevent the concrete substrate from becoming dislodged from its steel jacket under the action of the applied Mode I (i.e. opening) tip load, the concrete shape in cross-section (see Figs. 3.19(a) and 3.20(a)) was intentionally designed as a trapezoid, with the sides tapering inward from the bottom to the top. 


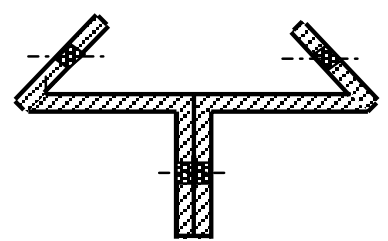

(a)

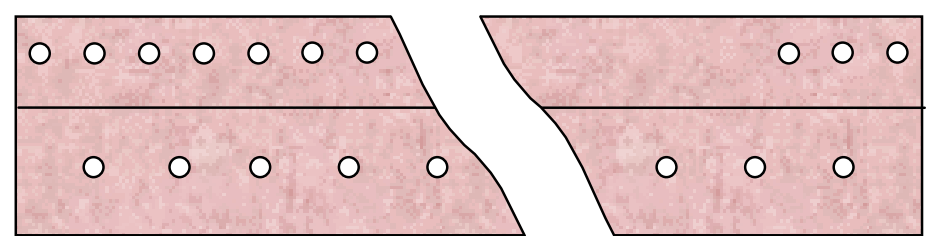

(b)

Figure 3.20 The steel jacket - (a) cross-sectional view, (b) side view

\section{Details of the SCCB Specimen and Test Fixture}

Since there are many components involved in constituting the SCCB specimen and test fixture, it is therefore fitting to give an account, as in an inventory-manner, of these bulk materials - that is, those materials that can be seen and which possess definite dimensions, with the exception of nuts, bolts and washers- thus, the internal steel reinforcement and aggregates are not included in this listing (as they cannot be seen), nor are the saturant and primer (as they do not possess definite dimensions). (The reader is referred to Chapter 5 for finer details of all the materials involved including specimen fabrication.) The section concludes with pictures of the SCCB test-setup (Fig. 3.32).

The SCCB Specimen: The specimen consists of 3 bulk materials (see Figures 3.21-3.23):

The wooden laminated veneer lumber (LVL) contoured-cantilever (Figs. 3.21(a) and 3.22(a))

The CFRP strip (Figs. 3.21(b) and 3.22(b))

$>$ The concrete substrate (Fig. 3.21 parts (c) and (d), and Fig. 3.23) 


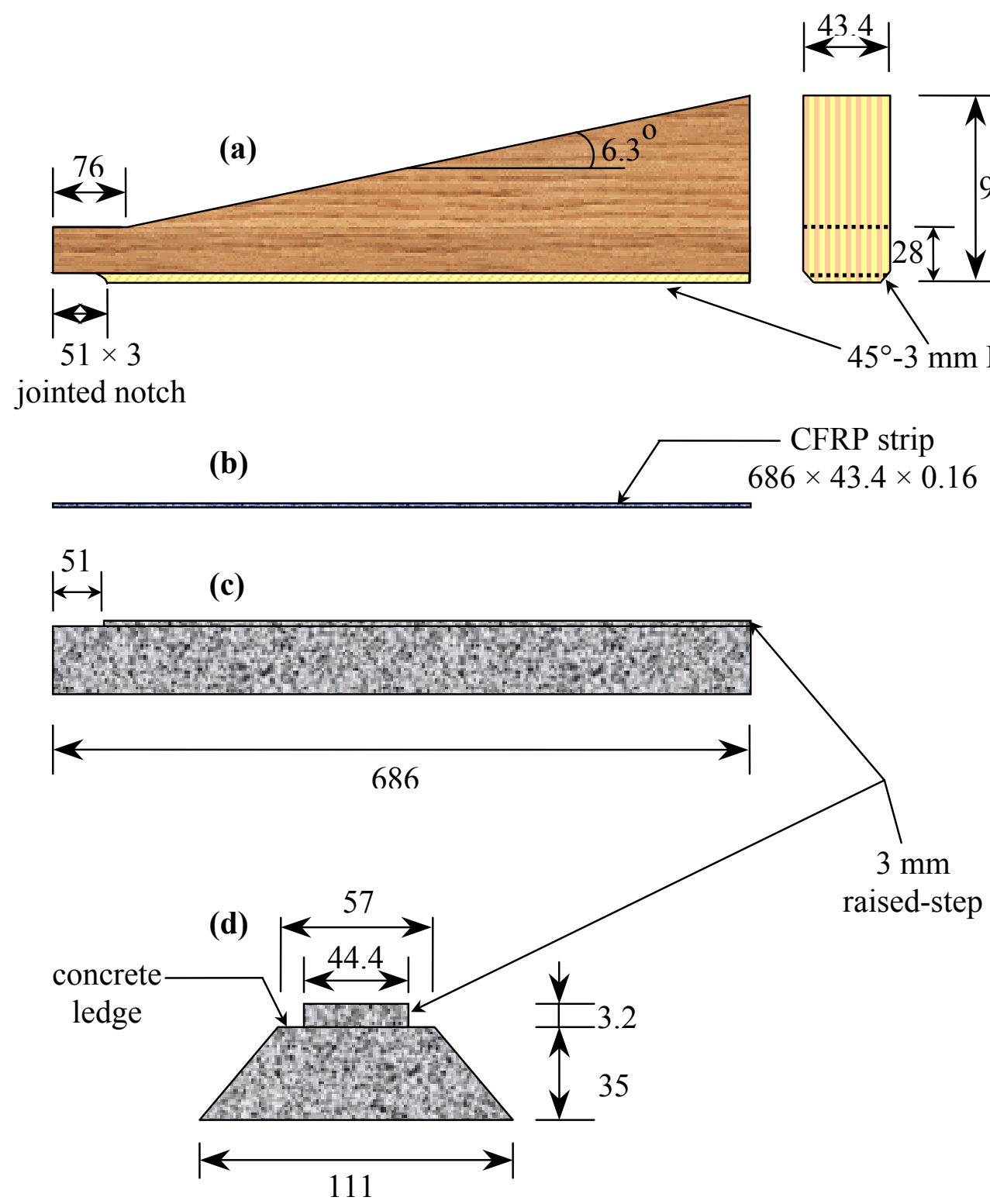

Figure 3.21 The bulk SCCB specimen-(a) LVL wooden contouredcantilever, (b) CFRP strip, (c) side view of concrete substrate, (d) front view (enlarged from part (c)) of concrete substrate (NOTE: All dimensions in millimeters) 


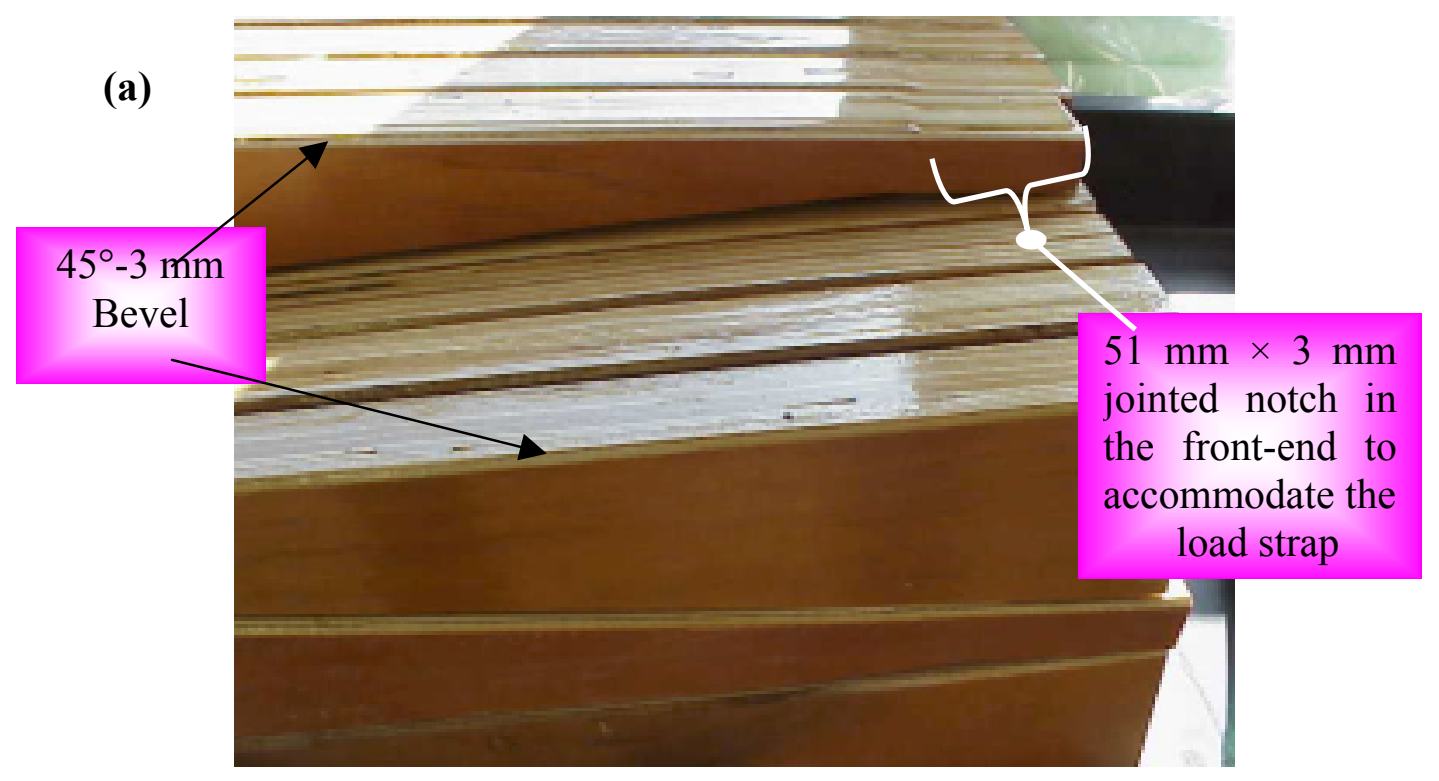

(b)

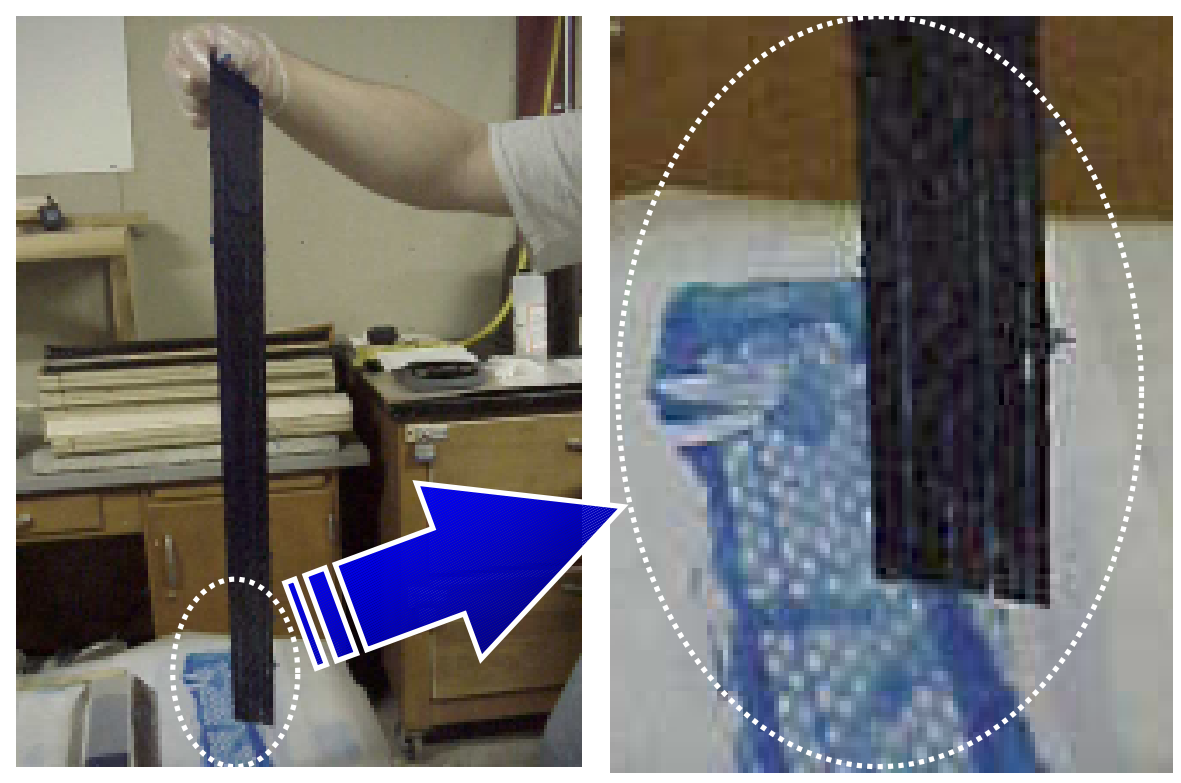

Figure 3.22 Pictures of (a) the LVL contours (pictured upside-down), and (b) the CFRP strip (with blown-up view) 


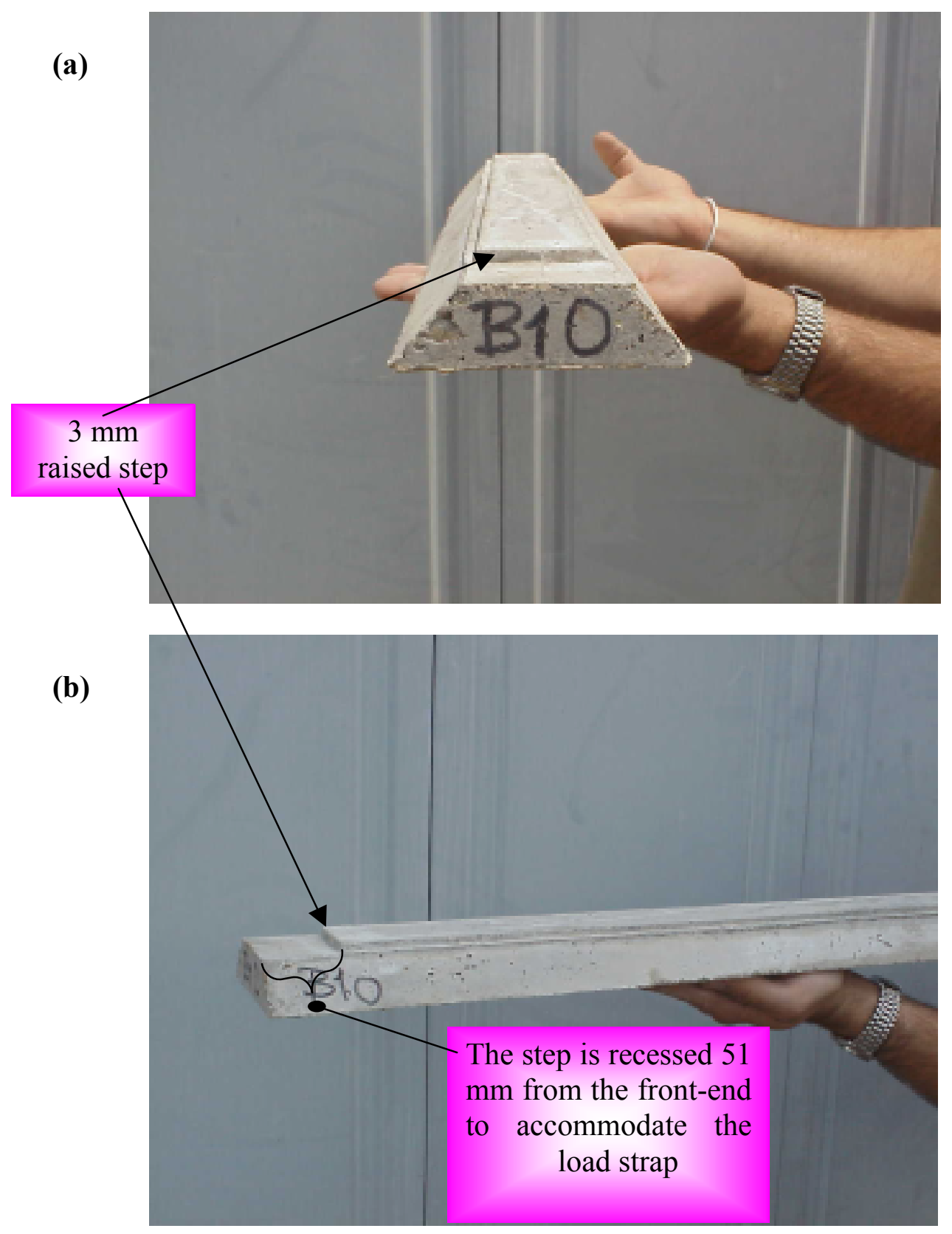

Figure 3.23 Pictures of the concrete substrate - (a) from the front, and (b) from the side 
The Test Fixture: A total of 10 bulk parts are involved in comprising the test fixture.

Beginning from the bottom and moving upward, these include the (see Figs. 3.24-3.31):

Base plate (1 piece; see Figs. 3.24, 3.26, and 3.31(a))

Angled members (2 pieces; see Figs. 3.25, 3.26, and 3.31(b))

Steel jacket (2 pieces; see Figs. 3.27 and 3.31(a))

Aluminum wedge-shaped clamps (4 pieces; see Figs. 3.28 and 3.31)

Load strap (1 piece; see Figs. 3.29 and 3.30)

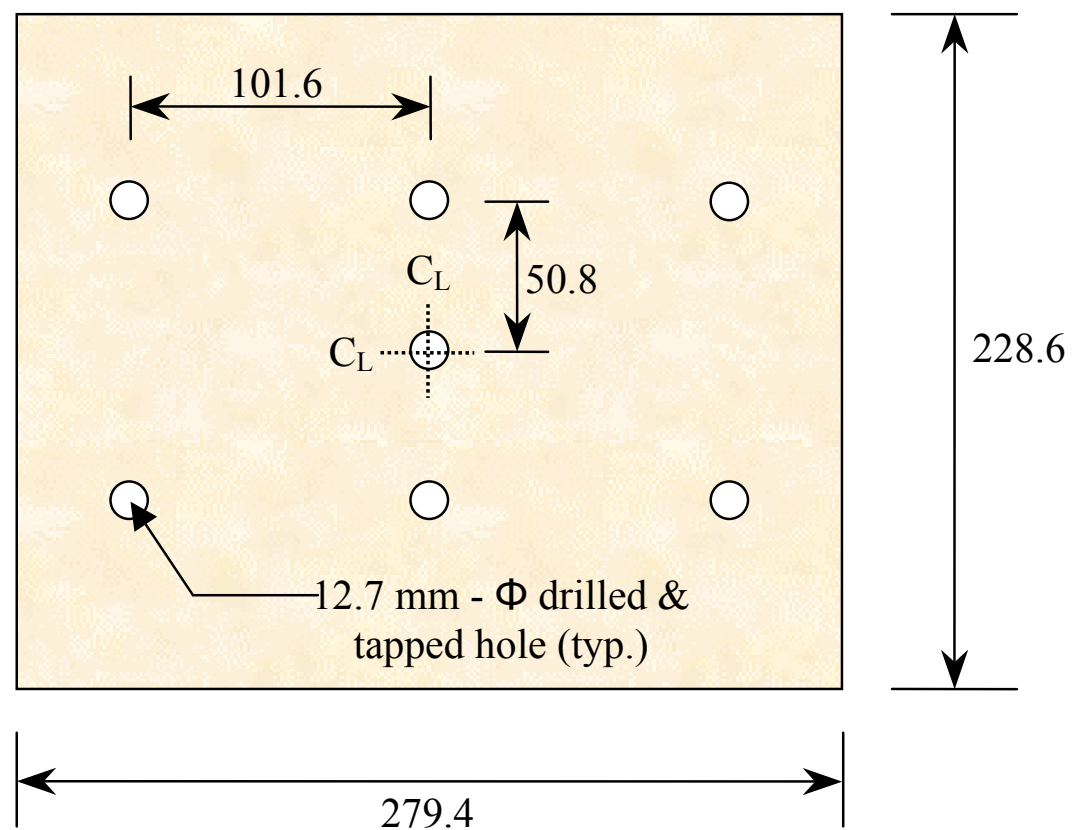

Figure 3.24 Top view of base plate $(228.6 \times 279.4 \times 19)$

(NOTE: All dimensions in millimeters) 


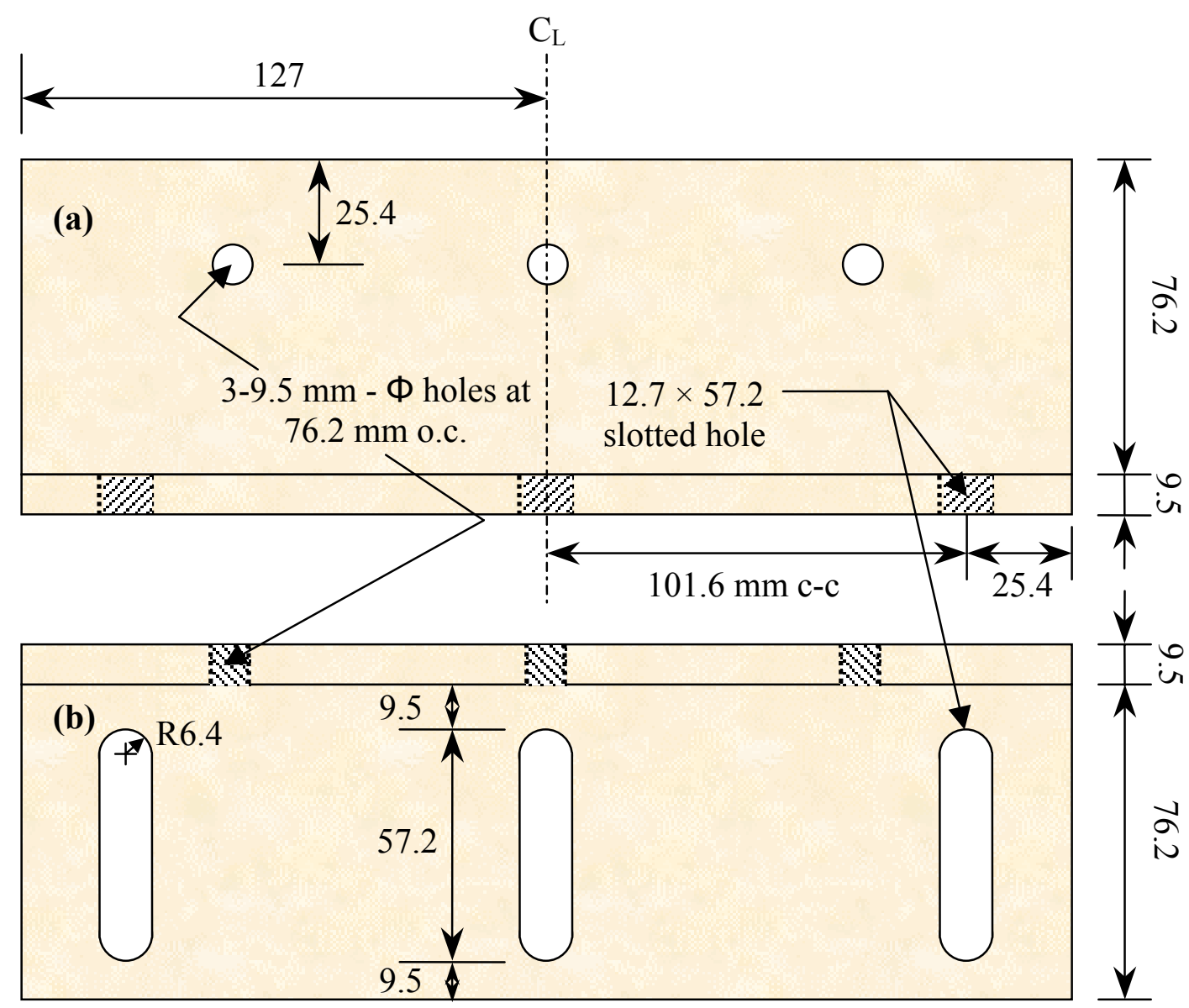

Figure 3.25 Angle $(76.2 \times 76.2 \times 9.5)-(a)$ elevation view, (b) top view

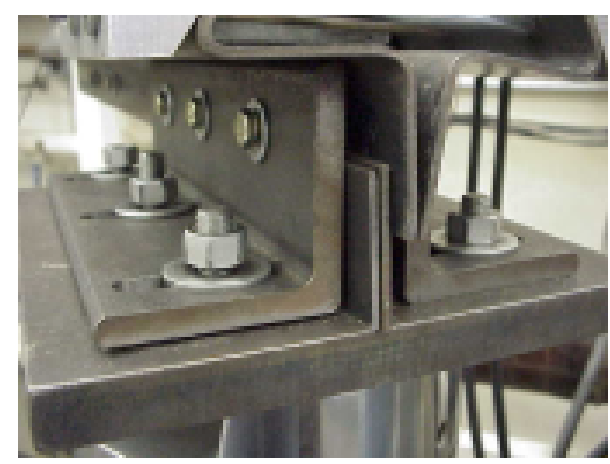

Figure 3.26 Picture of angle as affixed atop the base plate (see Fig. 3.24) 

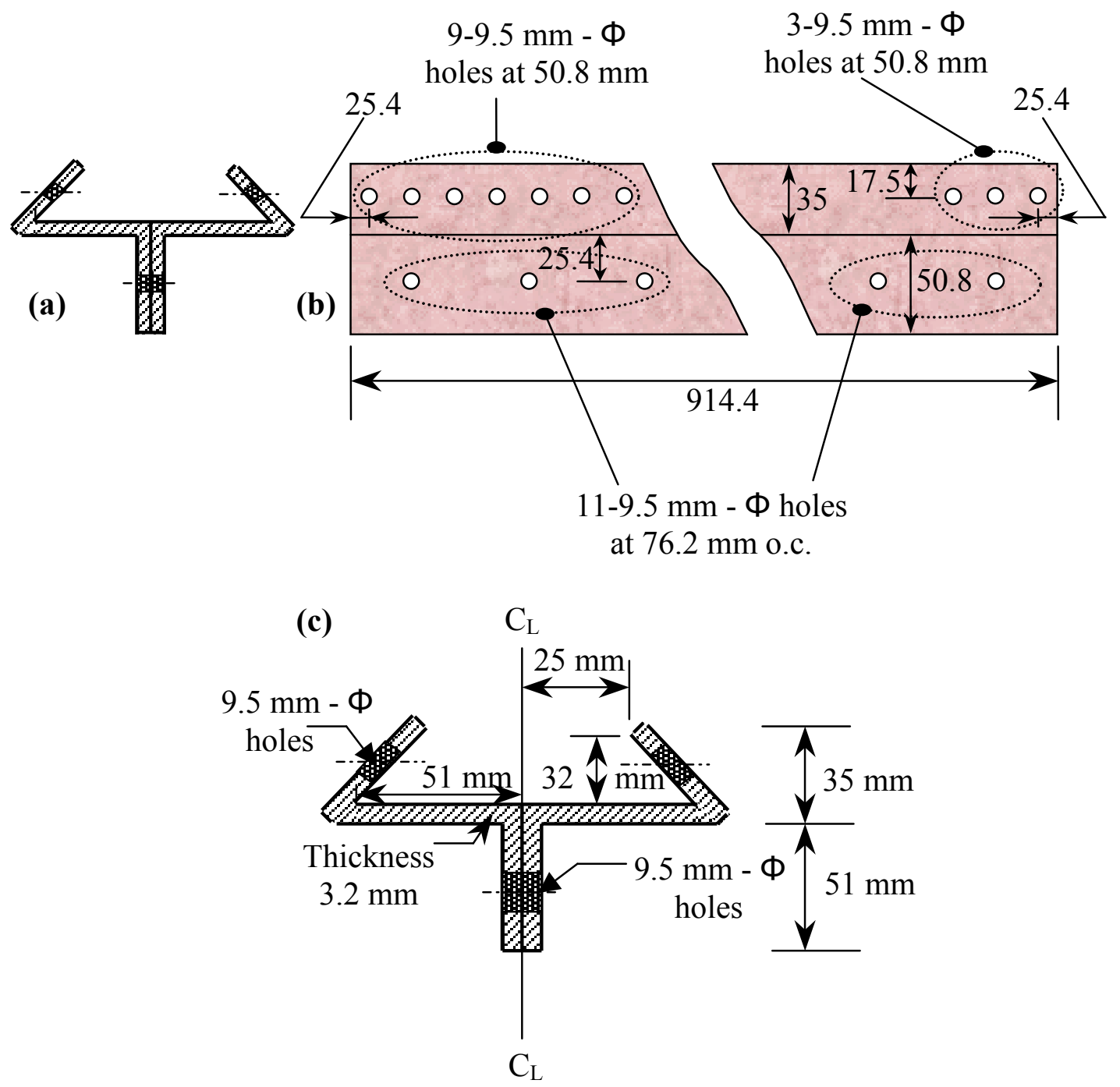

Figure 3.27 Steel Jacket - (a) Cross section view, (b) side view with dimensions, (c) cross section view (enlarged from parts (a) \& (b)) with dimensions (NOTE: all dimensions in millimeters) 


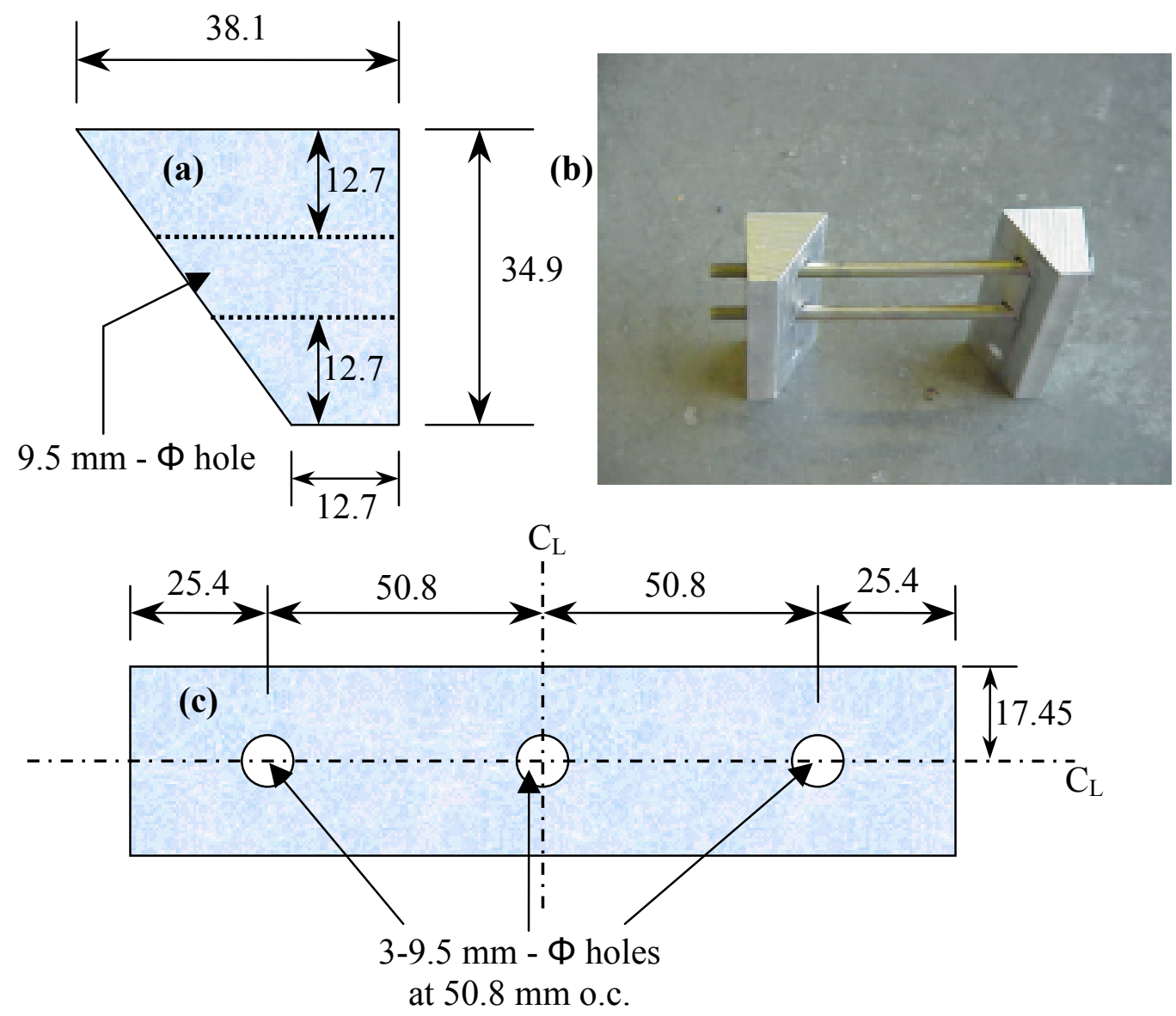

Figure 3.28 Aluminum wedge-shaped clamp - (a) end view, (b) photograph, (c) side view (NOTE: All dimensions in millimeters) 


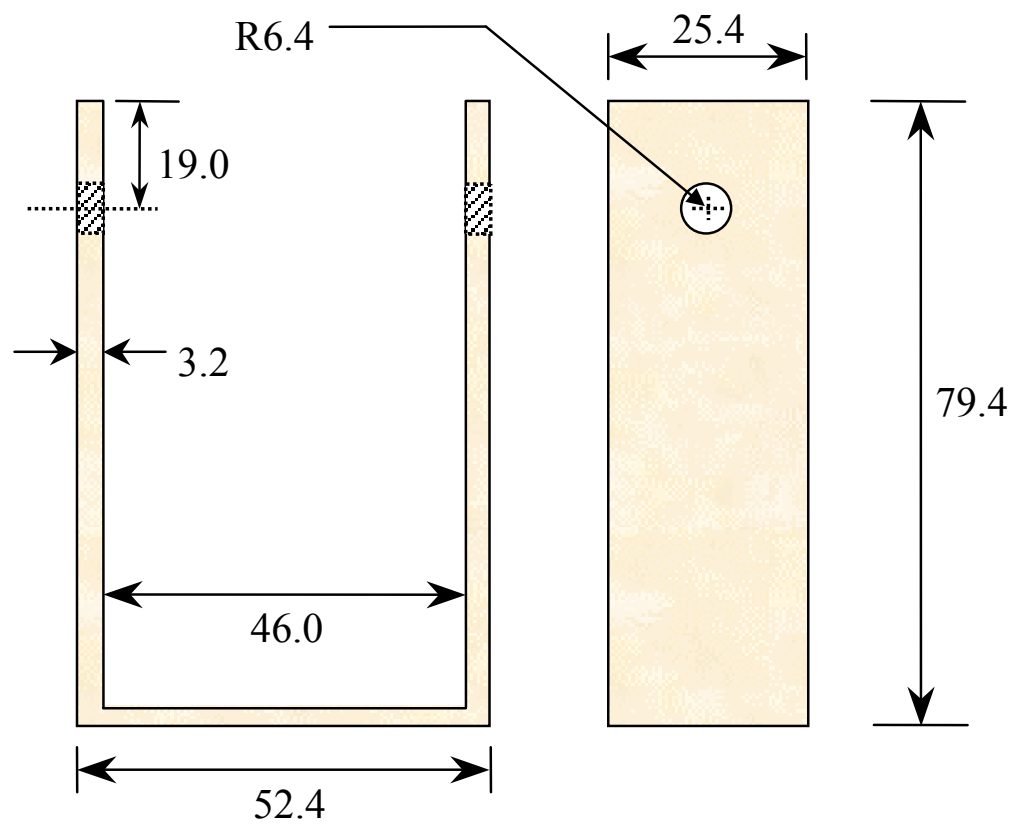

(a)

(b)

Figure 3.29 Load strap - (a) front view, (b) side view (unit: $\mathrm{mm}$ )

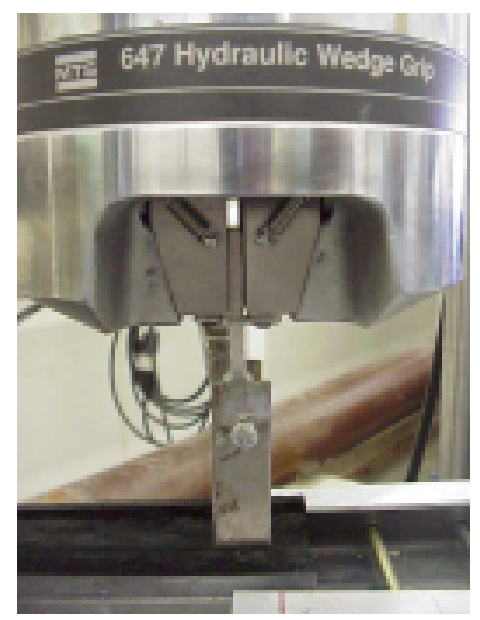

Figure 3.30 Picture of load strap affixed by the hydraulic grips of MTS 


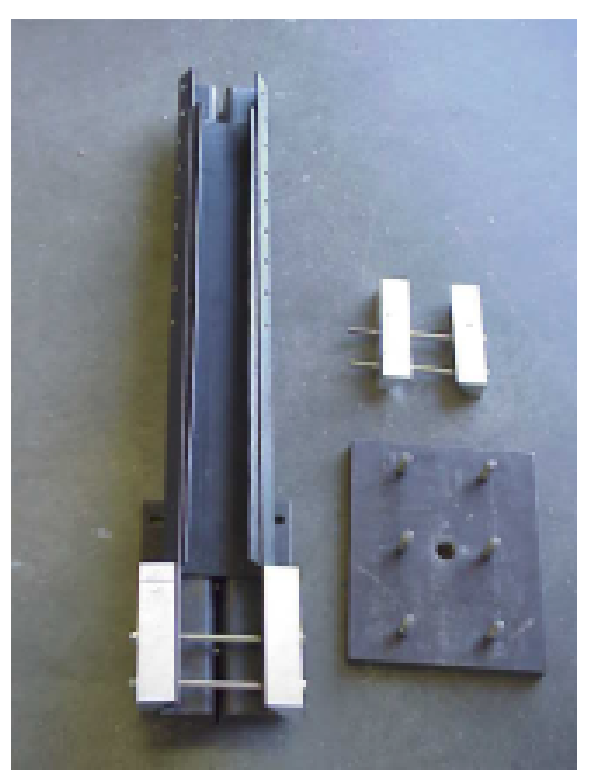

(a)

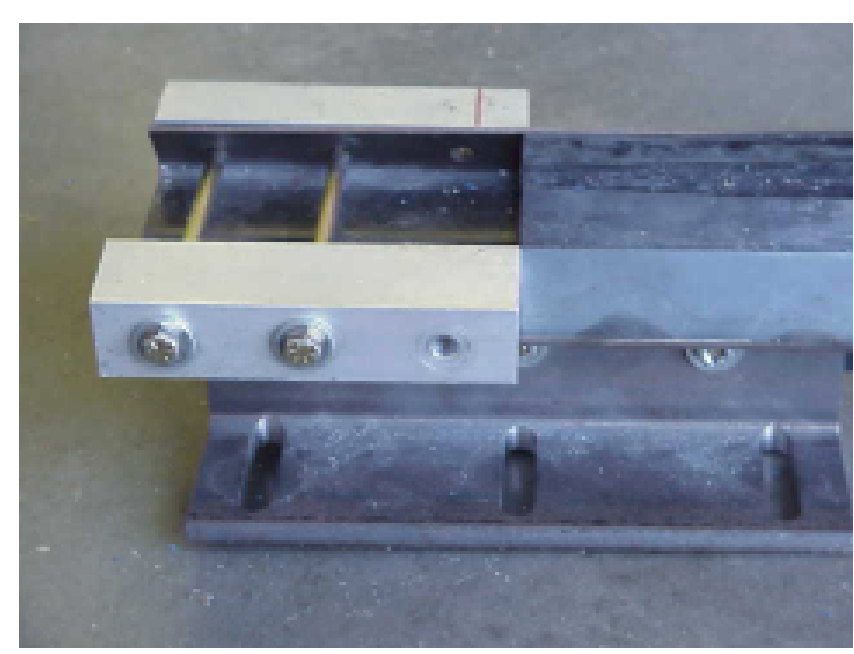

(b)

Figure 3.31 Pictures of the test fixture-(a) Steel jacket, aluminum wedgeshaped clamps, base plate, and angles (note also the continuous neoprene rubber sheet inside jacket), (b) close up of wedgeshaped clamp, steel jacket and angled member

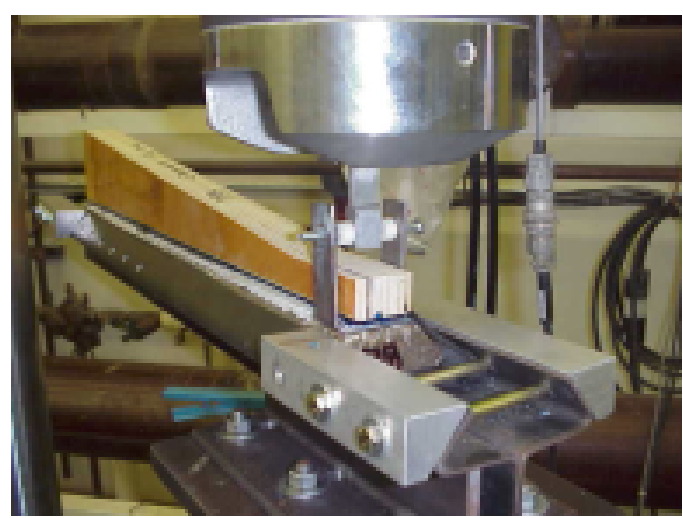

(a)

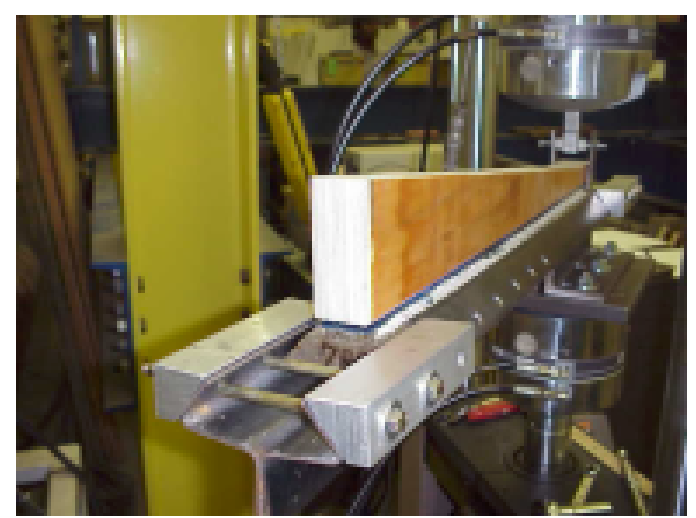

(b)

Figure 3.32 Pictures of the SCCB test-setup from the: (a) front, and (b) back 


\subsection{Summary}

This chapter began by posing a question central to the significance of this research, namely, that of permanence regarding the use of FRP materials as a means to externally reinforce concrete structures. It is for this very reason that the aspect of durability is addressed in chapters 7 and 8 . Additionally, since adhesive joints are limited in their mode of failure - that is, they predominantly fail by fracture - the characterizing parameter of the FRP-concrete interface bond that must be quantified is the critical strain energy release rate. This is the parameter, i.e. the $G_{I c}$, that is investigated for both conditioned (i.e. under wetting and drying, and freezing and thawing) as well as unconditioned specimens in chapters $6-8$. The difficulty in accomplishing this seemingly tenable objective, however, is that until now, there had been no way of measuring the critical strain energy release rate of the FRP-concrete interface. Thus, the focus of this chapter, as suggested by its title, has been an attempt to satisfy the following two objectives: 1) to sufficiently provide a review of the field that forms the basis for all that is to proceed, namely, that of Fracture Mechanics, and 2) to adequately introduce the novel experimental specimen and test fixture that is used in acquiring the characterizing FRP-concrete interface bond parameter.

Section 3.1 provided a brief review of past double cantilever beam (DCB) fracture toughness tests that were performed on traditional engineering materials (e.g. metals, wood, composites and adhesive joints). When the DCB method was initially attempted to study the FRP-concrete interface problem, one of the concrete arms would invariably breakoff (see Fig. 3.1) due to material insufficiency in withstanding tensile 
stresses. An example showed that for concrete possessing a strength of $f_{\mathrm{c}}{ }^{\prime}=45 \mathrm{MPa}=$ 6500 psi with an initial crack length of $152 \mathrm{~mm}(6 ")$ and cross-sectional dimensions of $b$ $=44 \mathrm{~mm}(1.75 ") \times h=28 \mathrm{~mm}(1.1 ")$, the arm-breakoff load is a mere $170 \mathrm{~N}(40 \mathrm{lb})$ - $\mathrm{a}$ value that is much too low (about 1/10 - 1/5 too low) for the levels of critical loading actually required for fracture, as cited in chapters 6,7 and 8 .

Section 3.2 tracked the origins of the study of Fracture Mechanics. In particular, it was explained that a size-effect phenomenon (described later in the section, as well, under the subsections titled "Liberty Ship Failures" and "Post War Progress") was responsible for initiating failure among materials, the source being attributed to the existence of interatomic discontinuities. The present subject of Fracture, however, began with two major concepts presented by Griffith (1893 - 1963; see portrait in Fig. 3.4). The first idea, just mentioned, is that of flaws or minute defects that act as stress raisers in a material and explain the difference between the observed low strengths of solid materials versus the high values as might be expected from the known strengths of their chemical bonds. Griffith's second idea postulated that an energy balance, responsible for governing the laws of crack propagation, existed between the surface energy (i.e. surface tension) of the newly created fracture surface and the elastic energy stored in the stress field (i.e. the balance of surface and mechanical energies). Griffith was able to calculate the stored elastic energy at a crack-tip by examining Inglis's (1913) groundbreaking work describing a method to model the stress field in the vicinity of cracks.

Later Roesler (1956) explained that the quantity having the dimensions of surface tension may in practice exceed the true specific energy, $\gamma$, by a large factor based on the 
independent works of G.R. Irwin (1948) and Orowan (1950), who demonstrated that the Griffith energy balance must not only be between the strain energy stored in the specimen and the surface energy, but the work done in plastic deformation as well. They arrived at these conclusions by recognizing that, for relatively ductile materials, the work done against surface tension is generally small as compared to the work done against plastic deformation. In 1955, Orowan further contributed to the understanding of brittle fractures by demonstrating that the modified Griffith condition is, in addition to being a necessary condition for crack propagation, a sufficient one as well. Also that same year Irwin indicated, and two years later, showed (Irwin, 1957) that the energy approach is equivalent to one of stress-intensity according to an occurrence of fracture whenever a critical stress distribution, characteristic of the material, is reached (Weiss and Yukawa, p. 3, 1964).

In addition, attempts were made to include dynamic terms into the energy balance to take into account the stress waves created by fast moving cracks (Benbow, 1961). The conditions for the dynamics of a propagating crack were first formulated by Mott (1948) and then later by Yoffe (1951). Further developments were made by Krafft and Sullivan (1963) and Krafft and Irwin (1964) who studied the relation of dynamic loads to strain rate sensitive materials (explained in Section 3.4). For a good review see the work by Schardin (1959).

Other key topics of Fracture Mechanics covered in section 3.2 are those of the stress concentration factor, $K_{t}$, the stress intensity factor, $K$, and its related energy term, $G$, i.e. the strain energy release rate, the stress and displacement field equations, the 
differences between plane stress and plane strain as related to fracture, and the size of the plastic zone (a.k.a. the fracture process zone). A brief description of each of these is given next:

Stress Concentration Factor, $K_{t}$ : The ratio of maximum stress to applied stress. $K_{t}$ describes the effect of crack geometry on the local crack tip stress level.

Stress Intensity Factor, K: A parameter that depends on the specimen dimensions, crack length, and loading conditions-not to be confused with the stress concentration factor, $K_{t}$. The stress intensity factor actually provides more information by incorporating geometrical parameters such as the crack length. When a continuously increasing load is applied to a pre-cracked structure, $K$ increases, and at a critical value, the stationary or slow moving crack jumps ahead. This critical value, denoted $K_{c}$, is known as the fracture toughness. The lower limiting value of $K_{c}$ for Mode I plane strain fracture is denoted as $K_{I c}$ and, to reiterate what was mentioned in the previous page, is related to its energy counterpart, $G_{I c}$-the critical strain energy release rate—as shown by Irwin in 1957. Both $K$ and $G$ are material properties in the same manner as are, for example, the Young's and shear moduli. The simple expressions relating the two were given in Eqs. (3.13) and (3.14):

$$
\begin{array}{ll}
G=\frac{K^{2}}{\mathrm{E}} & \text { (in plane stress) } \\
G=\frac{K^{2}}{\mathrm{E}}\left(1-v^{2}\right) & \text { (in plane strain) }
\end{array}
$$

The Stress and Displacement Field Equations: The most direct approach to determining the stresses and displacements surrounding a crack-tip is through the use of 
the field equations (e.g. Eqs. (3.15) - (3.22), valid for Mode I fracture). An excellent reference for further study on this topic is the paper of Paris and Sih (1964), "Stress Analysis of Cracks".

Plane Stress vs. Plane Strain: Various states of stress can be summarized with respect to their principal values $\left(\sigma_{1}, \sigma_{2}, \sigma_{3}\right)$ :

$>\operatorname{triaxial}\left(\right.$ if $\left.\sigma_{1} \neq \sigma_{2} \neq \sigma_{3}\right)$

$>$ hydrostatic (if $\left.\sigma_{1}=\sigma_{2}=\sigma_{3}\right)$

$>$ cylindrical (if any two of $\sigma_{1}, \sigma_{2}, \sigma_{3}$ are equal and the third is nonzero)

$>$ uniaxial (if any tow of $\sigma_{1}, \sigma_{2}, \sigma_{3}$ are zero)

$>$ biaxial (if any one of $\sigma_{1}, \sigma_{2}, \sigma_{3}$ is zero-a.k.a. a state of plane stress)

The last state is a situation that is commonly encountered in the loading of very thin sheets which do not develop any tensile stress through the thickness (see Fig. 3.8(a)). In thick sections, however, a through-thickness stress, $\sigma_{z}$ (see Fig. 3.8(b)), develops, creating a condition of triaxial tensile stresses at the crack-tip that severely restrict the strains from developing in the z-direction, thus yielding a condition of plane strain.

Plastic Zone Size: Whenever the yield strength of the crack-tip material is exceeded, thus rendering the stress field and displacement expressions (Eqs. (3.15) (3.22)) invalid, a region of plasticity develops, the size of which is approximately given by:

$$
\mathrm{r}_{\mathrm{y}}=\frac{K_{I}^{2}}{2 \pi \sigma_{\mathrm{ys}}^{2}} \quad \text { (in plane stress) }
$$


Consequently, the total crack length should be adjusted to include some fraction of the plastic zone length. Similar estimates have found the plastic zone length under plane strain conditions to be approximately one-third that for plane stress:

$$
\mathrm{r}_{\mathrm{y}}=\frac{K_{I}^{2}}{6 \pi \sigma_{\mathrm{ys}}^{2}}=\left.\frac{1}{3} \mathrm{r}_{\mathrm{y}}\right|_{\text {planestress }} \quad \text { (in plane strain) }
$$

Since the fracture toughness, $K_{c}$, of a material depends on the volume of the material that can deform plastically prior to fracture, and the volume, in turn, depends on the thickness, it follows that $K_{c}$ varies with the thickness as shown in Fig. 3.9. Here it was shown how the gradual increase of the specimen thickness brings about a near-square fracture surface (see especially Figure 3.9(d)) that corresponds to a representative value of fracture toughness as a material constant. Srawley and Brown (p. 146, 1964) made the further point that even if the section of the load-bearing member were thin enough so that the fracture would be partly or entirely slanted (as in Fig. 3.9 (c), (b) and (a), respectively), the load-bearing capability of the member might still be governed by the value of $G_{I c}$ instead of its plane stress counterpart $G_{c}$, unless the ratio of $G_{c} / G_{I c}$ were to exceed some value dependent on the shape and size of the initial crack. Along this line of thought, aspect ratios of thicknesses were examined to predetermine conditions responsible for plane stress or plane strain types of fracture, for example:

$$
\begin{array}{ll}
\frac{\mathrm{r}_{\mathrm{y}}}{\mathrm{t}} \geq 1 \quad \text { (in plane stress) } \\
\frac{\mathrm{r}_{\mathrm{y}}}{\mathrm{t}}<\frac{1}{10} & \quad \text { (in plane strain) }
\end{array}
$$


For aspect ratios not conforming to the above, a mixed-mode of plane stress/strain fracture is found to dictate. One very important feature discussed regarding this lower level of toughness (i.e. the plane strain fracture toughness, $K_{I c}$ ) was that it does not decrease further with increasing thickness, thereby making it a conservative lower-limit and an especially useful parameter for engineering applications. It is for this reason that $K_{I c}$ (or its energy counterpart, $G_{I c}$ (Section 3.3)) is thought of as a material constant-a central issue to this dissertation, both, in the development and implementation of the novel single contoured-cantilever beam (SCCB) experimental fracture mechanics approach, the details of which were given in section 3.6.

Section 3.3 began by explaining the three basic modes of fracture. These include the opening mode (Mode I) and two sliding modes (Modes II and III), known as the edge-sliding and screw-sliding (or tearing) modes, respectively (see Fig. 3.11). Here it was explained that Mode I is by far the most important case of fracture for engineering purposes (read the assertions made by Hertzberg, Boresi et al., and Ripling et al. in Sec. 3.3 under the subsection titled "Three Basic Modes of Fracture").

Next, the governing equation (named after Irwin and Kies (1954)) that is used in determining the critical strain energy release rate values of fracture, in chapters 6,7 and 8, was developed. The procedure outlined (after the explanation given by Bazant and Planas (1998)) begins with the first Law of Thermodynamics and relies on a Variational Mechanics approach. A direct advantage of introducing the development of the IrwinKies equation in this way is that Castigliano's first and second theorems materialize as by-products of the derivation, even though the second was only shown here- the first 
results in a similar manner by simply considering a fixed-load case (i.e. $P=$ constant) as opposed to a fixed-grip case (i.e. $u=$ constant) and introducing the dual strain energy elastic potential known as the complementary energy (see Bazant and Planas, p. 26, 1998, for the details). The resulting expression is found to be:

$$
G_{I c}=\frac{P_{c}^{2}}{2 b} \frac{d C}{d a}
$$

Section 3.4 dealt with the behavior of cracking — perhaps the foremost significant issue pertaining to the topic of experimental fracture mechanics. All forms of cracking were divided into two broad classes: those of stable and unstable cracks. Generally, all structural adhesive systems display a form of elastic behavior prior to crack-initiation. In other words, the crack remains stationary with no measurable inelastic flow until a critical value, $G_{I c}$ (see Eq. (3.73)), is reached. The differences that exist between adhesive systems are made evident once this critical value is exceeded. Cracking that proceeds with a nearly constant $G_{I c}$ value (i.e. stable crack growth) represents materials that are said to be strain rate insensitive (see Figs. 3.14(a) and 3.15, parts (b) and (d)). In contrast, strain rate sensitive materials display a marked difference in the values of $G_{I c}$ that are responsible for initiating the stationary crack and those that sustain propagation (see Figs. 3.14(b) and 3.15(c)). When the crack begins to move the amount of crack extension force required to sustain crack propagation decreases, thus accounting for the saw-toothed appearance of Fig. 3.14(b). The high load at the first peak of the saw-toothed behavior typically corresponds to the critical load, $P_{c}$, used to compute the value of $G_{I c}$ in Eq. (3.73). In such cases where the crack-initiation load(s) is(are) taken as being critical, 
denoted generically as $P_{c}^{i}$, the corresponding critical strain energy release rate value is written as $G_{I c}^{i}$ (see Ch. 6 for the statistical-based approach for $P_{c}$ determination).

At initiation, the crack "pops" ahead, running at a higher velocity than that as dictated by the rate of the actuator. At the end of the "pop", the crack stops from moving and is only reinitiated when the machine displacement increases sufficiently for the instantaneous load-level to once again reach a critical initiation-value, $P_{c}^{i}$. The low-load representing the cessation of cracking and depicted by one of the "valley" regions, is known as a crack-arrest load, and is denoted, $P^{a}$. Following the convention in use with cracking-initiation, if the crack-arrest load(s) is(are) taken to be critical, then the corresponding value of fracture toughness is written as $G_{I c}^{a}$.

In actuality, fracturing occurs as a combination of unstable and stable crack growth, for if the "flat" horizontal branch of Fig. 3.14(a) were to be magnified, it too would display a saw-toothed behavior akin to that as shown in part (b) of the same figure. The real difference between the two is that, in part (a), the contrast that exists between the crack -initiation and -arrest loads, $P_{c}^{i}$ and $P_{c}^{a}$, is slight, whereas the differences are much more marked in part (b). The work of River (2002) helps in delineating such differences by not only categorizing the various types of fracture from a qualitative perspective, but from a quantitative sense, as well. The four qualitative crack patterns (see Fig. 3.15) are: strong/unstable, strong/stable, strong/moderately unstable, and weak/stable.

Ebewele, Koutsky and River (1986) defined a quantitative measure known as the brittleness index, $I$, which is a normalization of the energy being released (i.e. lost) during 
a period of rapid crack growth with respect to the energy that was stored in the joint just at the onset of crack-initiation. This relation is thus expressed as

$$
I=\frac{G_{I c}^{i}-G_{I c}^{a}}{G_{I c}^{i}}
$$

Accordingly, an ideally brittle material that fails suddenly and completely as depicted in Figure 3.15(a) will have an index value of $I=1$, since $G_{I c}^{a}=0$. On the other hand, an ideally plastic material that fails by continuous tearing and having indistinct $G_{I c}^{i}$ and $G_{I c}^{a}$ energies will have an index value of $I=0$. Thus, larger $I$ values indicate abrupt and unstable crack growths whereas smaller ones signify cracks progressing more slowly and in slighter increments. In their wood fracture studies, River and Okkonen (1993) reported a value of $I=0.43$ as representing a strong/moderately unstable crack, while a value of $I$ $=0.06$ conveyed a more stable form of crack propagation. More will be said about the brittleness index in chapters 6,7 and 8 , as it pertains to actual experimental CFRPconcrete interface fracture results.

Prior to the work of Ebewele, et al. (1986), Mostovoy, Crosley and Ripling (1967) defined a concept to determine the crack-arresting capability of a joint (see Eqs. (3.75) and (3.76)). From their observations, they made the following two recommendations to enhance the stability of cracking during DCB testing: 1) maintain a short initial crack length, and 2) design the contoured-cantilever with a low taper angle. (Note: both of these guidelines were adhered to in the case of the SCCB tests.)

Section 3.5 listed a general literature-outline of DCB testing, showcasing first, relevant ASTM standards, and then, the three varieties of specimens most commonly 
used in experimentation - namely, 1) the double cantilever beam, DCB (or uniform DCB, UDCB), 2) the width tapered DCB, WTDCB and 3) the contoured DCB, CDCB (or tapered DCB, TDCB). By such a listing, an attempt was made to: 1) sketch how the DCB specimen evolved over the years, and 2) provide a handy assemblage of writings for further reading and cross-referencing (compare also with the listing presented back in Section 3.1).

Section 3.6, the final section of this chapter excluding, of course, this summary section, provided the details of the novel experimental fracture mechanics SCCB specimen and test fixture. Three differing aspects between the DCB and SCCB specimens were discussed. These include: 1) a recasting of the DCB specimen by halving the pair of its symmetric constituents, 2) the specimen-portion of the SCCB minus the contoured-cantilever can be separated into two parts, even though the concrete portion is cast monolithically: i) the interface (see the dashed-region in Figure 3.19(a)), and ii) the bulk concrete substrate (see Figure 3.19(b)), and 3) that of a functional difference-i) a special steel jacket (see Figure 3.20) was designed to provide confinement of the bulk concrete substrate (Fig. 3.19(b)) and, as such, offer a form of external reinforcement against the impairing tensile stresses, and ii) the concrete shape in cross-section (see Figs. 3.19(a) and 3.20(a)) was intentionally designed as a trapezoid, with the sides tapering inward from the bottom to the top to prevent the concrete substrate from becoming dislodged from its steel jacket under the action of the applied Mode I (i.e. opening) tip load. 
Next, details of the SCCB specimen and test fixture were furnished. Beginning with the SCCB specimen, the bulk materials (i.e. those materials that can be seen and which possess definite dimensions, with the exception of nuts, bolts and washers) are: 1) the wooden LVL contoured-cantilever (Figs. 3.21(a) and 3.22(a)), 2) the CFRP strip (Figs. 3.21(b) and 3.22(b)), and 3) the concrete substrate (Fig. 3.21 parts (c) and (d) and Fig. 3.23). As for the test fixture, these include: 1) a base plate (Figs. 3.24, 3.26, and 3.31(a)), 2) a pair of angled members (Figs. 3.25, 3.26, and 3.31(b)), 3) the steel jacket (2 pieces; see Figs. 3.27 and 3.31(a)), 4) aluminum wedge-shaped clamps (4 pieces; see Figs. 3.28 and 3.31), and 5) the load strap (Figs. 3.29 and 3.30). 


\section{CHAPTER 4}

\section{COMPLIANCE CALIBRATIONS OF THE CONTOURED-CANTILEVER}

\subsection{Introduction}

In considering the critical strain energy release rate expression of the last chapter, it becomes evident that the accuracy involved in establishing a representative $G_{I c}$ value of the interface is restricted by the precision with which the compliance gradient, $d C / d a$, is determined. Consequently, an extensive effort was undertaken to investigate this fundamentally important parameter, prior to any fracture testing, and is the focus of this chapter.

Analytical compliance expressions have been developed for both uniform- and contoured- double cantilever beam (DCB) specimens (see, for example, Mostovoy et al., 1967) that provide a sense of what an appropriate value of $d C / d a$ is for a given geometry. A point worth reemphasizing here, though previously mentioned in Chapter 3 , is the significance of contouring the specimen geometry. For the case of uniform DCB specimens, i.e. geometries that are un-contoured, the instantaneous compliance, $C$, of the cantilevered beam is ever-changing with the propagating crack location, $a$. Thus, the fracture toughness of the interface can only be determined by tracking this location, i.e. 
that of the crack tip, $a-$ which, experimentally speaking, is not easily accomplished. This experimental impediment is intrinsically circumvented by appropriately contouring the cantilevered-arm of the specimen, causing the compliance of the beam to change linearly with the extending crack, and as such, disencumbering the relation between the load, $P$, and the strain energy release rate, $G_{I}$, (developed in Chapter 3) of any dependence upon the parameter $a$ (see also Eq. (1.1) and the surrounding discussions).

Recently, a modified beam theory was developed (Qiao et al., 2001) to predict constant $d C / d a$ values of tapered DCB specimens involving hybrid interface bonds, e.g. polymer composite materials bonded to wood or concrete. In this analytical approach, the uncracked region of the specimen is modeled as a tapered beam on a generalized elastic foundation while taking into account relative translations and rotations of the crack tip. Using this explicit solution along with a subsequent finite element (FE) analysis, five initial geometries, designated as $\mathrm{S} 1-\mathrm{S} 5$, were arrived at as prospective single-contouredcantilever beam specimens. To simplify the modeling process, these shapes were designed by presupposing the symmetry of the double cantilever beam specimens. In fact, two of the proposed shapes, S1 and S2 (discussed in greater detail in section 4.2; see also Figure 4.2), were first calibrated as $\mathrm{CDCB}$ specimens to validate the theoretically predicted specimen compliance results. Table 4.1 lists the dimensions of shapes S1 - S5. The differing geometries of these shapes can be visualized by substituting the initial $\left(\mathrm{H}_{\mathrm{o}}\right)$ and final heights $\left(\mathrm{H}_{\mathrm{f}}\right)$ of Table 4.1 into Figure 4.1, while keeping the other two parameters, i.e. the initial uniform length $(76 \mathrm{~mm}=3 ")$ and overall specimen length $(686$ $\mathrm{mm}=27 ")$, fixed. 
Table 4.1 Experimentally Calibrated Contour Shapes

\begin{tabular}{c|cc}
\hline Shape & Initial Height, $\mathbf{H}_{\mathbf{0}} \mathbf{~ m m}$ (in) & Final Height, $\mathbf{H}_{\mathbf{f}} \mathbf{~ m m ~ ( i n )}$ \\
\hline S1 & $31.8(1.25)$ & $129.3(5.09)$ \\
\hline S2 & $31.8(1.25)$ & $104.9(4.13)$ \\
\hline S3 & $28.0(1.10)$ & $95.0(3.74)$ \\
\hline S4 & $28.0(1.10)$ & $88.9(3.50)$ \\
\hline S5 & $25.4(1.00)$ & $80.3(3.16)$ \\
\hline
\end{tabular}

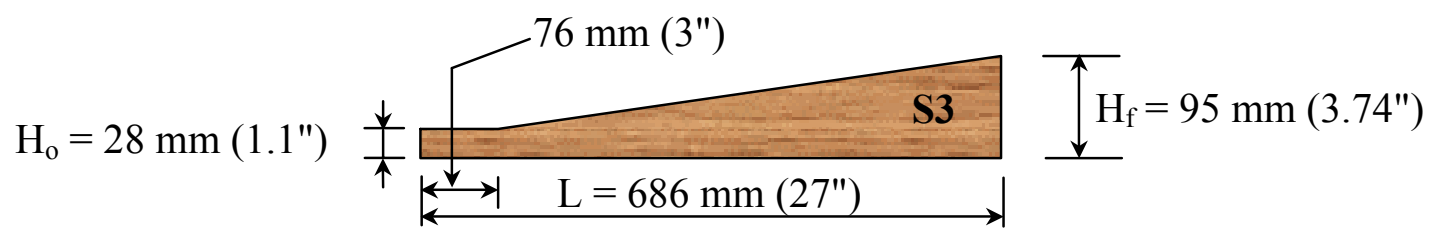

Figure 4.1 Optimum single contoured-cantilever beam specimen S3; width (not shown), $b=43.4 \mathrm{~mm}$ (1.71")

While both analytical and FE approaches offer a good reference point from which the contoured geometry might be arrived at, these methods are based on certain approximations (see section 4.4), and as such, should not be solely relied upon in the determination of the specimen $d C / d a$ value. Instead, the $d C / d a$ parameter should always be determined experimentally through compliance calibrations.

For this reason, a thorough experimental compliance calibration program involving four separate classes of specimens was sequentially conducted. Just mentioned, the first of these under consideration used the CDCB specimen (discussed in section 4.2) because of its simplicity and the fact that it is well-established; the latter point warranting a natural stepwise-progression to be made from what is easily determined and verified toward that which yet remains to be substantiated. Two of the shapes, S1 and S2, proved 
to be sufficient in not only corroborating the explicit solution results, but instrumental in validating both the proper performance of the equipment being used for data collection as well as the experimental methodology followed throughout.

Unlike the well-established CDCB specimen, the completely new concept of the single contoured-cantilever beam used for the remaining three classes of calibrations (discussed in section 4.3) was, from its very inception, shrouded in some confusion with respect to its viability. Particularly, it was speculated that the absence of symmetry accompanying this new specimen would impair its ability to behave purely under an opening, or cleavage, mode of loading due to a sizable degree of shearing stresses incurred at the interface. While this concern is not completely unfounded, it was discovered through the extensive course of calibrations conducted that this effect is much smaller than what was previously thought, and as such, can be neglected. As an interesting aside, even the $\mathrm{CDCB}$ specimen, in all its perfect symmetry, was found to contain small discrepancies. It is important to realize, however, that in all cases this variance is wholly seated within the realm of experimental error. That is to say, given the extent of skill involved in assembling the various constituents of the specimen (discussed with great detail in Chapter 5) it is not unreasonable to contract a trifling $10 \%-11 \%$ error due to human involvement alone without making any mention of the four widely disparate materials in use, not least of these being concrete - a grossly inhomogeneous material that undoubtedly could account for most, if not all, of the error itself. A short list of encountered human errors follows taken directly from the author's own experiences: a slight misalignment of the contour during bonding or of the specimen in the test fixture 
during loading, improper preparation of the saturant, insufficient impregnation of the fibers, and among a host of many others not attended to here, a deficient quantity of saturant used in adhering the contour to the substrate resulting in air voids.

Broadly speaking, each of the three remaining classes of calibrations that were investigated via the single contoured-cantilever beam setup corresponds to three different interface conditions: wood-wood, wood-concrete and wood-CFRP-concrete. The latter is an identical simulation of the actual fracture specimen interface and is what was used to finally obtain the value of $d C / d a$ that is used throughout. It should be mentioned here that this seemingly roundabout sequence of experimental calibration-progressions from woodwood to wood-concrete and finally to wood-CFRP-concrete interfaces was not proposed a priori, but rather stumbled upon through a trial-and-error process, that now, in retrospect, has proven indispensable in gaining insight into the effects that various substrate-material have on the $d C / d a$ parameter - a phenomenon that had neither been studied nor understood previously. Two significant consequences of this are worth mentioning. Firstly, it was discovered that the simplifying wood-wood assumptions used in modeling both the FE and explicit solutions are in fact comparable to the actual woodCFRP-concrete specimen substrate-material effects. The importance of this discovery is not to be underestimated, not only where it regards the obvious in which correlations between theoretical and experimental results would otherwise not have been possible, but in regarding the faulty conception perceived at the time, that a wood-concrete specimen would be sufficient in determining the actual $d C / d a$ parameter, as well. Secondly, with respect to the sawn specimens (to be described beginning in section 4.2), it was found 
that a small loss in contour depth did not have as pronounced an effect on the $d C / d a$ parameter as was formerly thought — this effect was more prominent, instead, on the compliance parameter, $C$. More will be said about these effects in the upcoming sections.

\subsection{CDCB Calibrations}

Two of the five shapes under consideration, S1 and S2, were calibrated as contoured double cantilever beams (see Table 4.1 and Figures 4.2 and 4.3). In both cases the CDCB specimen was cut out of a single board of Yellow Poplar laminated veneer lumber (LVL) possessing an experimentally-found mean modulus of elasticity of $\mathrm{E}_{\mathrm{LVL}}=$ 13.22 $\mathrm{GPa}(1.917 \mathrm{Msi})$ and a standard deviation of $\sigma_{\mathrm{LVL}}=1.21 \mathrm{GPa}(0.175 \mathrm{Msi})$ determined through a sampling size of $\mathrm{N}=20$.

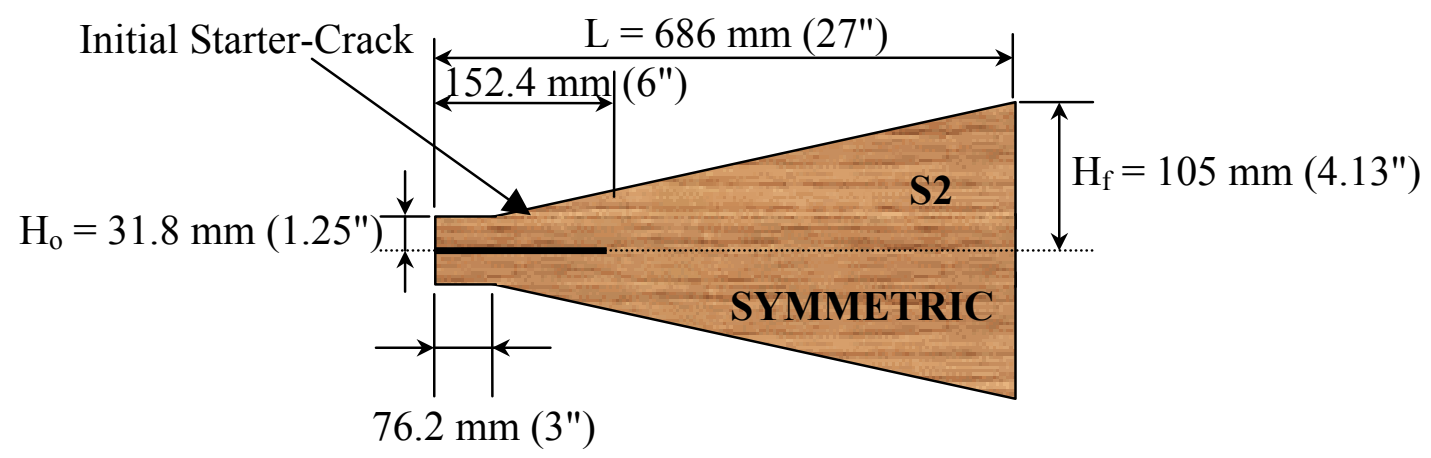

Figure 4.2 CDCB specimen S2 with initial and final (symmetric) heights shown

\section{Calibration Equipment}

All of the experimental calibrations conducted throughout were performed on a servo hydraulic MTS machine (458.10 MicroConsole/418.91 MicroProfiler) operating under a displacement-control mode (for a discussion of the displacement rates used, see 
the subsection titled "The Actuator Speed"). During the calibrations, a data acquisition system was used to collect three separate sets of data. Included among these were the displacement of the actuator, the applied load and the displacement at the load-points by the use of a highly sensitive extensometer (see Figure 4.3). The extensometer unit is an MTS model 632.02 clip-on gage, the electronic signals of which, when converted, gave accurate readings sensitive to 25 -one-hundred-thousandths of a millimeter (0.00001") and specifically designed to conform to ASTM E399-90 ("Standard Test Method for PlaneStrain Fracture Toughness of Metallic Materials"). It possesses a double cantilever configuration with slot-grooved free-ended deflecting arms that affix to the specimen through specially machined knife-edges (see Figure 4.3(b)).

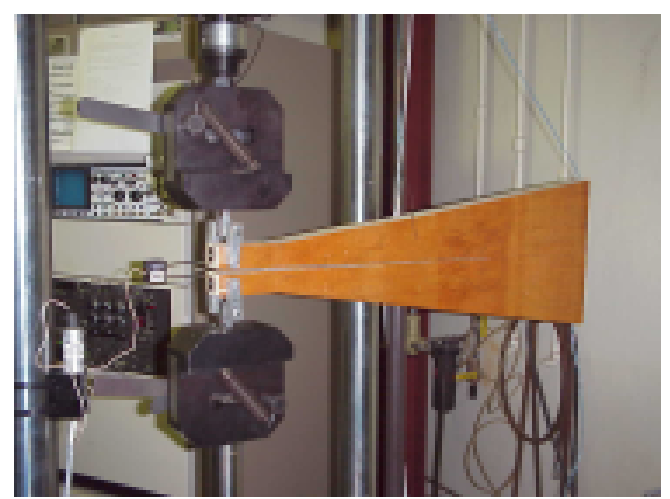

(a) Side-view of CDCB specimen

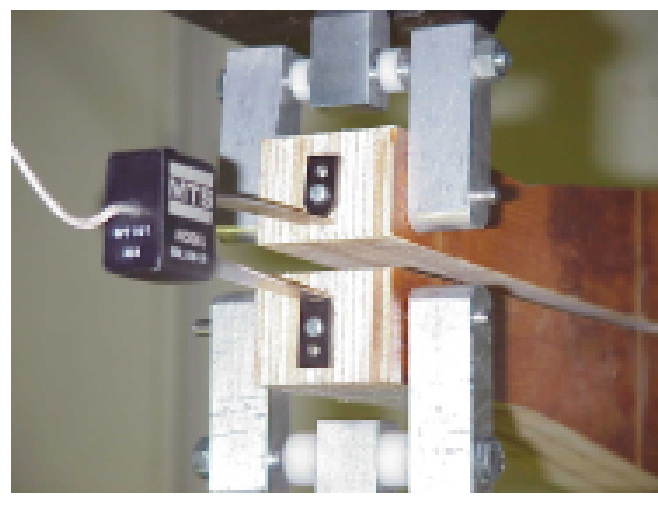

(b) Details of load-straps and MTS clip-on gage extensometer affixed to specimen by knifeedges

Figure 4.3 Calibration and details of actual CDCB specimen 


\section{The Experimental Calibration Scheme}

The first step in determining the $d C / d a$ parameter of a specimen is to determine the value of its compliance, $C$, at a particular location, $a$. The initial starter-crack (see Figure 4.2) typically constitutes the first $a$-location and, for the purposes of the discussion to follow, is denoted as $a_{1}$. In both of the CDCB specimens considered herein, this location was taken to be: $a_{l}=152.4 \mathrm{~mm}(6 ")$. By definition, the compliance is the inverse of the stiffness, and as such, can be found as the inverse of the ratio of a load and its corresponding displacement. Experimentally, this was determined by applying a monotonically increasing load within the linear elastic range-typically up to about 650 $\mathrm{N}$ (approximately $150 \mathrm{lb}$ ) —and simultaneously collecting the corresponding tip displacement. This results in a series of ordered pairs of the form $\left(\mathrm{P}_{\mathrm{n}}, \delta_{\mathrm{n}}\right)$, where $\mathrm{P}=$ applied load, $\delta=$ the corresponding tip displacement and $\mathrm{n}=$ the total number of such data collected. These data of ordered pairs are now plotted and a best-fit curve, i.e. a line in this context, is found through the displacement-load data using linear regression. The slope of this line is thus the experimental compliance of the beam, $C_{i}$, corresponding to position, $a_{i}$, where $i$ denotes the number of positions for which this process is to be repeated. For both of the CDCB specimens, $i=9$, beginning with $a_{1}=152.4 \mathrm{~mm}(6 ")$ and ending at $a_{9}=558.8 \mathrm{~mm}(22 ")$ with each successive location after the first being incremented in $50.8 \mathrm{~mm}(2 ")$ steps. The procedure just outlined was repeated for a minimum of three times at each particular location, so that the final compliance value, $C_{i, f i n a l}$, corresponding to any $a_{i}$, was found as the average of all the compliance values at that position. Finally, the $d C / d a$ parameter is found from the slope of the linear regression 
performed on the data of ordered pairs, $\left.\left(a_{i}, C_{i}\right)\right|_{\text {final }}$. It should be remarked that it is completely unnecessary to have as many as nine data points in order to effectively determine the slope of the regressed line in this context. As a matter of fact, while nine points were collected for each of the CDCB specimens S1 and S2, only six and seven, respectively, were used to finally determine the $d C / d a$ parameter. In fact, even six data points are somewhat excessive, and as it will be seen in the following section, as little as three data points are sufficient provided that they are amply spread out from each other and that their corresponding compliance values are nearly collinear. Logically, the decision to omit data arises primarily from one reason-namely, whenever a point is a clear outlier. There are two immediate circumstances for which this occurs. Firstly, any number of mistakes made in the collection of data at a particular location, human-based or otherwise, may result in a deviation of the corresponding value from the expected trend. The second reason, the one that is more likely to occur of the two and discussed in greater detail in section 4.4 , occurs whenever the crack location is extended back far enough to approach the specimen-end, resulting in greater flexibility of the specimen to displace upon loading due to the lack of material behind the crack-tip, and as such, yields a much larger value of compliance than what would have otherwise been obtained.

\section{Relocating Cracks Backwardly}

Two methods were used in extending a crack location, $a_{k}$, backwardly to its proceeding location, $a_{k+1}$, where $\mathrm{k} \in[1,9)$ in this context. The first of these used a bandsaw to physically advance the position of the crack. This was naturally the method of choice employed for all the wood-wood specimens and even used on some of the wood- 
concrete beams. Strictly speaking, the terms wood-wood or wood-concrete are used in reference to the single contoured-cantilever beam calibrations of section 4.3 , in which epoxy was used to bond the wooden LVL-cantilever to either the wooden Red Maple or the concrete substrate bases. It should be noted that the CDCB specimen can reasonably be thought of as a special variant of the wood-wood class of calibrations as well, because after all, it was monolithically cut out of a board of Yellow Poplar LVL, though lacking epoxy at its interface. The second method of relocating cracks borrowed its idea from the initial starter-crack of the actual fracture specimen (see Chapter 5). Here, a piece of wax paper acting as a barrier against the epoxy, was double-over to a length corresponding to the crack location and inserted at the interface during the bonding operation of the contour to the substrate beam. A drawback of this method is in the cost of concrete substrates required - one for every crack location. For this reason, only three crack locations spread apart (see the discussion of the previous page that justifies the use of only three positions) were studied using this technique on only two single contouredcantilever beam specimens (wood-concrete and wood-CFRP-concrete).

\section{The Actuator Speed}

As already mentioned, since the final compliance value at any one crack location consisted of no less than the average of three runs, a small investigation was carried out to see what, if any, effect varying the speed of the actuator would have on the compliance results. With respect to the $\mathrm{CDCB}$ specimens, an examination of displacement rates ranging between $0.00254 \mathrm{~mm} / \mathrm{s}(0.0001 \mathrm{in} / \mathrm{s})$ and $0.01016 \mathrm{~mm} / \mathrm{s}(0.0004 \mathrm{in} / \mathrm{s})$ were considered. Regarding, for example, the displacement rate between the second and third 
runs at the initial starter crack location of $152.4 \mathrm{~mm}\left(6^{\prime \prime}\right)$ for specimen $\mathrm{S} 1$, the rate was increased threefold from $0.00254 \mathrm{~mm} / \mathrm{s}(0.0001 \mathrm{in} / \mathrm{s})$ to $0.00762 \mathrm{~mm} / \mathrm{s}(0.0003 \mathrm{in} / \mathrm{s})$ but, for all intents and purposes, the respective compliance values remained comparable: $3.322 \times 10^{-3} \mathrm{~mm} / \mathrm{N}\left(5.819 \times 10^{-4} \mathrm{in} / \mathrm{lb}\right)$ and $3.308 \times 10^{-3} \mathrm{~mm} / \mathrm{N}\left(5.794 \times 10^{-4} \mathrm{in} / \mathrm{lb}\right)$, corresponding to a percent difference of only $0.42 \%$. The same exercise was repeated at the $203.2 \mathrm{~mm}\left(8^{\prime \prime}\right)$ crack location between the first and second runs. Once again, there was no major difference in the respective compliance values: $3.818 \times 10^{-3} \mathrm{~mm} / \mathrm{N}(6.686$ $\left.\times 10^{-4} \mathrm{in} / \mathrm{lb}\right)$ and $3.778 \times 10^{-3} \mathrm{~mm} / \mathrm{N}\left(6.616 \times 10^{-4} \mathrm{in} / \mathrm{lb}\right)-\mathrm{a} 1.05 \%$ difference.

\section{CDCB Calibration Results}

Specimen S1: The compliance values for this specimen were determined for crack lengths ranging between $152.4 \mathrm{~mm}\left(6^{\prime \prime}\right)$ and $558.8 \mathrm{~mm}(22 ")$ in $50.8 \mathrm{~mm}\left(2^{\prime \prime}\right)$ incremental steps (see Table 4.2). The $d C / d a$ value, however, was computed on the slightly truncated range of $203.2 \mathrm{~mm}\left(8^{\prime \prime}\right)-457.2 \mathrm{~mm}\left(18^{\prime \prime}\right)$ for both of the reasons cited in the section titled "The Experimental Calibration Scheme". Typically, this range is truncated for compliance values corresponding to crack locations near the end of the specimen, as done with the case for specimen S2 in which the crack positions $a=508 \mathrm{~mm}$ (20") and 558.8 $\mathrm{mm}$ (22") have been omitted, but in this instance, it was necessary to exclude the compliance value relative to the starter-crack position (i.e. $152.4 \mathrm{~mm}\left(6^{\prime \prime}\right)$ ) as well because this value is clearly an outlier (see Figure 4.4).

In order that comparisons might be made, all the $d C / d a$ values found and reported are normalized to those of single contoured-cantilever beam specimens. For this reason, 
the compliance gradients of specimens S1 and S2 are found by halving the values of the

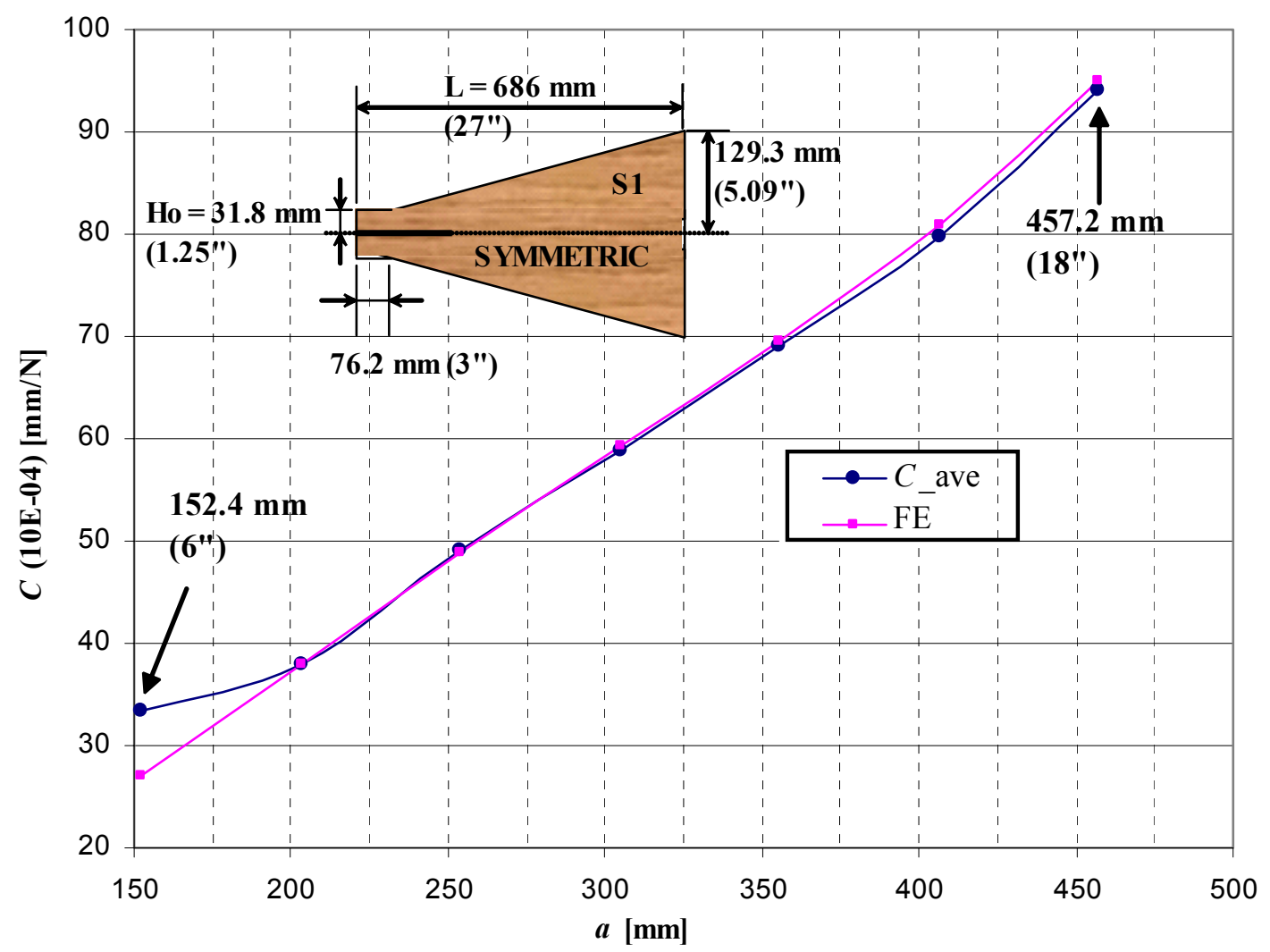

Figure 4.4 Compliance vs. crack-length of CDCB specimen S1 experimental $\left(C \_\right.$ave $)$and $\mathrm{FE}$ results shown

Table 4.2 Experimental and FE compliance values of specimen S1

\begin{tabular}{|c|c|c|}
\hline$a[\mathrm{~mm}]$ & $\begin{array}{c}C C_{-} \text {ave } \\
(10 \mathrm{E}-04) \\
{[\mathrm{mm} / \mathrm{N}]}\end{array}$ & $\begin{array}{c}\text { FE } \\
(10 \mathrm{E}-04) \\
{[\mathrm{mm} / \mathrm{N}]}\end{array}$ \\
\hline 152.4 & 33.34 & 26.96 \\
\hline 203.2 & 38.02 & 38.05 \\
\hline 254.0 & 49.01 & 48.80 \\
\hline 304.8 & 58.91 & 59.21 \\
\hline 355.6 & 69.06 & 69.63 \\
\hline 406.4 & 79.78 & 80.91 \\
\hline 457.2 & 94.04 & 95.03 \\
\hline 508.0 & 120.69 & 117.37 \\
\hline 558.8 & 186.54 & - \\
\hline
\end{tabular}


slopes of their corresponding best-fit lines (see Figures 4.4 and 4.5). With respect to specimen $\mathrm{S} 1$, these (respective) experimental and FE values are: $d C /\left.d a\right|_{C_{-} \text {ave }}=1.08 \times 10^{-5}$ $\mathrm{N}^{-1}\left(4.79 \times 10^{-5} \mathrm{lb}^{-1}\right)$ corresponding to an r-squared value (a.k.a. the coefficient of determination) of $\mathrm{r}^{2}=0.9962$ and $d C /\left.d a\right|_{\mathrm{FE}}=1.10 \times 10^{-5} \mathrm{~N}^{-1}\left(4.90 \times 10^{-5} \mathrm{lb}^{-1}\right)$ with $\mathrm{r}^{2}=$ 0.9969-a $1.82 \%$ departure from the predicted FE results. $C_{-}$ave denotes the experimental compliance findings resulting from the average of at least three compliance values per crack location (as previously mentioned). All of these results, as well as those of specimen S2, are summarized in Table 4.4 for easy referencing. Comparisons are also made in this table of the outcomes for both specimens through percentage differences computed relative to the respective FE case.

As a brief explanation, the statistical measure that is being used in conjunction with the presentation of the linear-regression discussions, namely, the r-squared valuealso referred to as the coefficient of determination-is an indicator $\left(0 \leq \mathrm{r}^{2} \leq 1\right)$ of how closely estimated values of a trend-line correspond to actual data. With respect to linear trends, the closer this parameter is with respect to unity, the greater is the degree to which the actual data matches that of a straight line. Obviously, an r-squared value of unity infers data lying perfectly on a straight line. In all cases, without exception, whether for the CDCB or the SCCB (see section 4.3) specimens, the $\mathrm{r}^{2}$ value was greater than 0.96 , with a range of $0.9699-0.9990$ for the experimentally calibrated specimens and as high as 0.9997 for both the FE and beam on elastic foundation results with respect to specimen geometry S5. 
Specimen S2: In a similar fashion, the findings involving specimen S2 (see Figure 4.5 and Table 4.3) are as follows: $d C /\left.d a\right|_{C_{-} \text {ave }}=1.35 \times 10^{-5} \mathrm{~N}^{-1}\left(5.99 \times 10^{-5} \mathrm{lb}^{-1}\right)$ with $\mathrm{r}^{2}=$ 0.9986 and $d C /\left.d a\right|_{\mathrm{FE}}=1.33 \times 10^{-5} \mathrm{~N}^{-1}\left(5.91 \times 10^{-5} \mathrm{lb}^{-1}\right)$ with $\mathrm{r}^{2}=0.9988$ - a percentage difference of $-1.50 \%$. Additionally, for this case a beam on elastic foundation value of $d C /\left.d a\right|_{\text {Beam_EF }}=1.23 \times 10^{-5} \mathrm{~N}^{-1}\left(5.48 \times 10^{-5} \mathrm{lb}^{-1}\right)$ with $\mathrm{r}^{2}=0.9992$ was also computed.

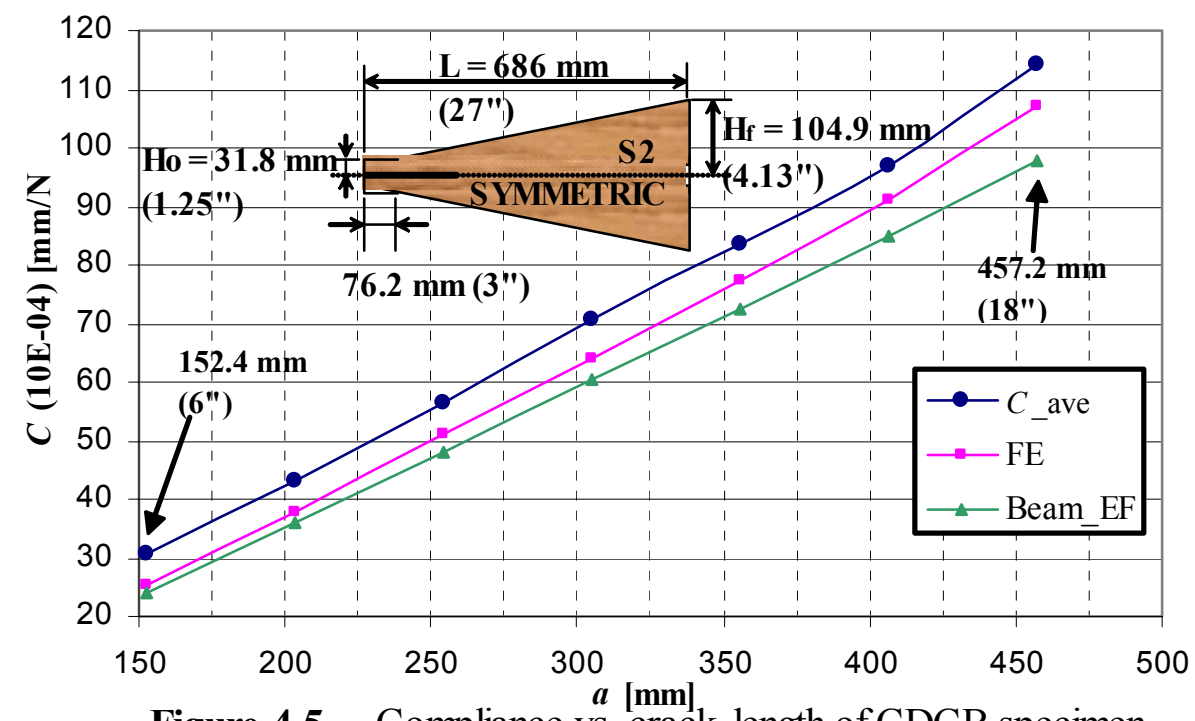

Figure 4.5 Compliance vs. crack-length of CDCB specimen S2 - experimental $\left(C_{-}\right.$ave $)$and FE results shown

Table 4.3 Experimental and FE compliance values of specimen S2

\begin{tabular}{c|ccc}
\hline $\boldsymbol{a}[\mathbf{m m}]$ & $\begin{array}{c}\boldsymbol{C}_{-} \text {ave } \\
(\mathbf{1 0 E - 0 4 )}\end{array}$ & $\begin{array}{c}\mathbf{F E} \\
{[\mathbf{1 0 E - 0 4})} \\
{[\mathbf{m m} / \mathbf{N}]}\end{array}$ & $\begin{array}{c}\text { Beam_EF } \\
(\mathbf{1 0 E - 0 4})\end{array}$ \\
{$[\mathbf{m m} / \mathbf{N}]$} \\
\hline 152.4 & 30.57 & 25.28 & 24.04 \\
\hline 203.2 & 43.28 & 37.82 & 35.84 \\
\hline 254.0 & 56.34 & 50.89 & 48.04 \\
\hline 304.8 & 70.70 & 64.04 & 60.33 \\
\hline 355.6 & 83.68 & 77.30 & 72.58 \\
\hline 406.4 & 97.04 & 91.10 & 84.90 \\
\hline 457.2 & 114.40 & 106.99 & 97.77 \\
\hline 508.0 & 142.68 & 129.62 & 112.50 \\
\hline
\end{tabular}


The $d C / d a$-percentage differences for both of the CDCB shapes, $\mathrm{S} 1$ and $\mathrm{S} 2$, were found to be less than $2 \%$ between the FE and experimental values. This conformity in results substantiates the goals mentioned at the outset of this chapter, placed with the intention of: (a) corroborating the theoretically predicted specimen compliance results by the use of a simple, well-established (i.e. CDCB) test methodology, (b) validating the proper performance of the equipment used in data collection, and (c) ensuring the viability of the hitherto unjustified SCCB experimental methodology.

Table 4.4 Summary of CDCB calibration results

\begin{tabular}{|c|c|c|c|c|}
\hline Specimen & $\begin{array}{c}\text { Symbolized } \\
\text { by: }\end{array}$ & $d C / d a\left[\mathrm{~N}^{-1}\right]^{*}$ & $\mathbf{r}^{2}$ & $\begin{array}{c}a \text {-range [mm] } \\
\text { (in) }\end{array}$ \\
\hline \multirow{3}{*}{$\begin{array}{c}\text { S1 } \\
\mathrm{H}_{\mathrm{o}}=31.75 \mathrm{~mm} \\
\left(=1.25^{\prime \prime}\right) \\
\mathrm{H}_{\mathrm{f}}=129.3 \mathrm{~mm} \\
\left(=5.09^{\prime \prime}\right)\end{array}$} & $\mathrm{FE}$ & 1.10 & 0.9969 & $\begin{array}{c}203.2-457.2 \\
(8-18)\end{array}$ \\
\hline & Beam EF & - & - & - \\
\hline & CDCB & $1.08(1.82 \%)^{+}$ & 0.9962 & $\begin{array}{c}203.2-457.2 \\
(8-18)\end{array}$ \\
\hline \multirow{3}{*}{$\begin{array}{c}\text { S2 } \\
\mathrm{H}_{\mathrm{o}}=31.75 \mathrm{~mm} \\
\left(=1.25^{\prime \prime}\right) \\
\mathrm{H}_{\mathrm{f}}=104.9 \mathrm{~mm} \\
\left(=4.13^{\prime \prime}\right)\end{array}$} & $\mathrm{FE}$ & 1.33 & 0.9988 & $\begin{array}{c}152.4-508 \\
(6-20)\end{array}$ \\
\hline & Beam_EF & $1.23(7.52 \%)^{+}$ & 0.9992 & $\begin{array}{c}152.4-508 \\
(6-20)\end{array}$ \\
\hline & CDCB & $1.35(-1.50 \%)^{+}$ & 0.9986 & $\begin{array}{c}152.4-457.2 \\
(6-18)\end{array}$ \\
\hline
\end{tabular}

${ }^{*} d C / d a$ values are in $10 \mathrm{E}-05$

${ }^{+}$Percent differences are computed against the FE results 


\subsection{SCCB Calibrations}

A total of ten single contoured-cantilever beam (SCCB) calibrations were performed spanning each of the five geometries, S1 - S5, under consideration (see Table 4.1 and Figure 4.1). As previously mentioned in section 4.1, three separate denominations of SCCB calibrations were undertaken in succession-beginning, progressing, and ending with the wood-wood, wood-concrete and finally the wood-CFRP-concrete specimens. In actuality, this sequence was happened upon as opposed to being designed in advance when, at the conclusion of each stage, it became evident that certain assumptions of the interface deemed valid at the outset were in question. As a result, each ensuing step of the sequence utilized a calibration specimen that more closely modeled the actual SCCB interface fracture specimen, culminating with an exact representation of the actual specimen in the third and final stage.

\section{Background of the 3-Stage Calibration Scheme}

There are several reasons why an exact replica of the actual fracture specimen was not used in the calibrations from the very beginning. The first of these reasons, just named, originated by falsely assuming that any substrate material would suffice in calibrating the upper LVL contoured-cantilever beam. Intimately connected to this was a desire to have a substrate material that would easily accommodate the backward relocation of the crack via a sawing operation; hence, wood was the natural material of choice. (It is worth mentioning that, at this point in time, no other method of backwardcrack-relocation had been realized.) Therefore, the resulting specimen from this first class 
of calibrations consisted of bonding the Yellow Poplar LVL contour to a Red Maple wooden substrate possessing the same cross-sectional shape and dimensions of its concrete counterpart, and as such, is what is being termed as the wood-wood specimen (see Figure 4.6). Another matter held to be important at this embryonic calibration-stage was a desire to reserve the concrete substrates for actual fracture testing because, at the time, it was not clear just how many specimens would be required to commit to each of the conditions being proposed, i.e. dry fracture, wetting-drying fracture, freezing-thawing fracture, etc.

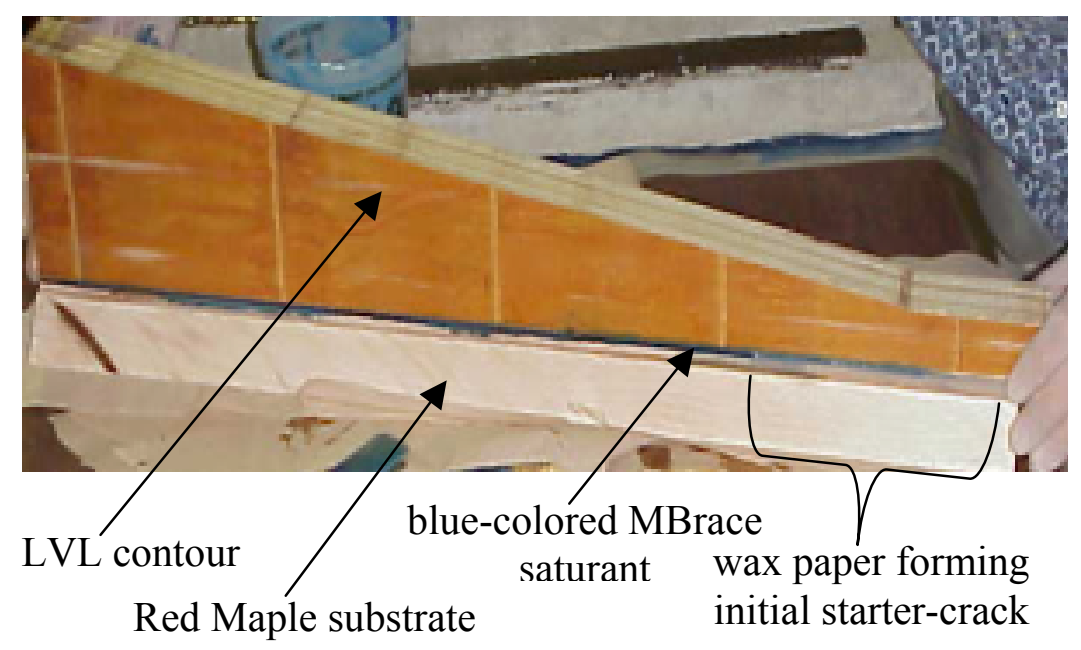

Figure 4.6 The wood-wood specimen

After this first stage, when it became apparent that the wood substrate might be responsible for some of the experimental discrepancies encountered, it was decided that the calibrations should be repeated using concrete. Consequently, the second class of calibrations, referred to as the wood-concrete specimens, involved bonding the LVL contour to the concrete substrates. The bonding agent used in the assembly of all the 
specimens is the blue-colored MBrace saturant (slightly evident in Figure 4.6), an epoxybased resin system manufactured by Master Builders Technologies (MBrace Composite Strengthening System, 1998). There were, however, two problems accompanying this intermediate stage of calibrations. Firstly, in all cases without exception, the $d C / d a$ values were found to be in even greater variance than those resulting from the wood-wood specimens - an outcome that seemed to be both confounding and contradictory at the time. Secondly, for obvious reasons emanating from the use of a concrete substrate, the process of relocating cracks backwardly in this class of calibrations via sawing was highly problematic. Inevitably, during the sawing process, it was found that the band saw would often freeze and become inoperable whenever the saw-blade became lodged at the wood-concrete interface. On the other hand, if, in an effort to avoid this from occurring, the saw blade was directed within the LVL contour near the interface boundary, the verity of the compliance data being collected due to a loss in contour-depth was in question (more will be discussed about this in the upcoming subsection titled "Substrate Material Effects"). These technical issues were overcome by the conception and implementation of the no-sawing method of backward-crack-relocation. In this technique, akin to forming initial starter-cracks, strips of wax paper are doubled-over and, prior to bonding, inserted at the prescribed crack length locations. Of course, one immediate disadvantage of the no-sawing technique is that it comes at a higher specimen-cost- that is, only one value of compliance can be obtained per specimen for a prescribed crack length. Recalling the discussion given under the subheading titled "The Experimental Calibration Scheme" of section 4.2, since three amply spread-out crack-locations are 
sufficient in establishing a representative $d C / d a$ value for a prescribed contour-geometry, this translates to a specimen-consumption requirement of no less than three concrete substrates. The utter significance of this technique, however, far outweighs this costrelated drawback as it enables calibrations to be performed on interfaces containing fibercomposite materials that simulate the actual fracture specimen. A sawing technique could, obviously, not be used on such a specimen as the process of backward-crackrelocation would invariably damage this seminal fiber interlayer.

This leads to a discussion of the third and final class of calibration within the SCCB sequence - the wood-CFRP-concrete specimen. After proceeding through the first two classes of calibrations, it became apparent that it would not be enough to simply calibrate the contoured-cantilever bonded to just any substrate. The substrate would, in fact, have to be an exact representation of the actual fracture test specimen. It may seem, then, that the results of the first two classes of calibrations are worthless and, as such, should be discarded. Nothing could be further from the truth, however, as the optimum contour-geometry, S3, was determined from the first, wood-wood, class of calibrations alone (refer to Table 4.5). The justification of this lies in the fact that both the FE and the beam on elastic foundation models were generated using this simplifying assumption. Furthermore, both the wood-wood and wood-concrete classes of calibrations were instrumental in exposing a little understood phenomenon with respect to the nature of the underlying substrate material (discussed in greater detail in the following subsection). Having now found the optimum shape, the compliance gradient of the wood-CFRPconcrete specimen was experimentally found using the no-sawing technique at three 
crack locations amply spread out from one another: $152.4 \mathrm{~mm}\left(6^{\prime \prime}\right), 254 \mathrm{~mm}\left(10^{\prime \prime}\right)$ and $355.6 \mathrm{~mm}(14 ")$. This value, to be used in all subsequent fracture testing as it resulted from the calibration of a specimen that identically represented the actual fracture-test beam, is: $1.78 \times 10^{-5} \mathrm{~N}^{-1}=7.92 \times 10^{-5} \mathrm{lb}^{-1}$.

\section{Substrate Material Effects}

Why Wood?: Already alluded to, the substrate material effects were taken for granted prior to commencing with the experimental calibration program. It was thought that since the compliance of the cantilevered-contour was all that was required, the use of any viable underlying material would suffice. Thus, wood was chosen, for several reasons, as the substrate material from the very start.

As discussed in the previous subsection, one reason for choosing wood was because a sawing technique was being used for the backward relocation of the crack during compliance testing. Also since at this time the details of the actual fracture tests involving dry and conditioned specimens had not yet been determined, it was deemed best to spare the concrete substrates, especially since such an option was granted. Besides, fabricating the wooden substrates is a relatively simple process that neither involves the same amount of attention (i.e. concrete curing) or time (i.e. weeks of concrete maturing) as required by that of freshly cast concrete. There was also a technical issue related to the ease of affixing the clip-on gage extensometer to the wood-wood specimen since the lower knife edge (refer back to Figure 4.3 (b)) could be simply screwed into place onto the Red Maple substrate. Later, when wood-concrete specimens 
and even the wood-CFRP-concrete specimen were used, this technical difficulty was overcome by bonding a small wooden tab onto the front face of the concrete substrate.

While all these cited reasons are compelling, the single-most important reason for choosing wood as a substrate from the beginning has to do with the fact that both the FE and beam on elastic foundation models were formulated with this simplifying assumption. In other words, as expressed in section 4.1, whatever the "shroud of confusion" might be surrounding this new, single contoured-cantilever beam specimen, it must, once and for all, be decisively mitigated with the calibrating of the wood-wood specimen. Thus, for the same reasons why two of the shapes were calibrated first as CDCB specimens, a wood substrate was chosen to induce a kind of moment-of-truth condition - either the experimental data would corroborate the viability of this new test methodology, or it would expose it as being flawed. As it can be seen, the experimental results presented in Table 4.5 auspiciously bespeak fulfillment of the former case, with a mere $11.23 \%$ average departure from the predicted FE values.

Table 4.5 Wood-wood results for each of the five contour shapes: $a$-range $=101.6-355.6 \mathrm{~mm}\left(4^{\prime \prime}-14 "\right)$ throughout

\begin{tabular}{|c|c|c|c|}
\hline Specimen & $\mathrm{FE} d C / d a\left[\mathrm{~N}^{-1}\right]^{*}$ & $d C / d a\left[\mathrm{~N}^{-1}\right]^{*}$ & $\mathbf{r}^{2}$ \\
\hline $\begin{array}{r}\text { S1: } \mathrm{H}_{\mathrm{o}} / \mathrm{H}_{\mathrm{f}}[\mathrm{mm}]: 31.75 / 129.3 \\
\text { (in): } 1.25 / 5.09\end{array}$ & 1.10 & $1.20(9.09 \%)^{+}$ & 0.9989 \\
\hline $\begin{array}{r}\text { S2: } \mathrm{H}_{\mathrm{o}} / \mathrm{H}_{\mathrm{f}}[\mathrm{mm}]: 31.75 / 104.9 \\
\text { (in): } 1.25 / 5.09\end{array}$ & 1.33 & $1.50(12.78 \%)^{+}$ & 0.9834 \\
\hline $\begin{array}{r}\text { S3: } \mathrm{H}_{\mathrm{o}} / \mathrm{H}_{\mathrm{f}}[\mathrm{mm}]: 27.94 / 95.0 \\
\text { (in): } 1.10 / 3.74\end{array}$ & 1.61 & $1.65(2.48 \%)^{+}$ & 0.9979 \\
\hline $\begin{array}{r}\mathbf{S 4 :} \mathrm{H}_{\mathrm{o}} / \mathrm{H}_{\mathrm{f}}[\mathrm{mm}]: 27.94 / 88.9 \\
\text { (in): } 1.10 / 3.50\end{array}$ & 1.84 & $2.08(13.04 \%)^{+}$ & 0.9962 \\
\hline $\begin{array}{r}\text { S5: } \mathrm{H}_{\mathrm{o}} / \mathrm{H}_{\mathrm{f}}[\mathrm{mm}]: 25.40 / 80.3 \\
\text { (in): } 1.00 / 3.16\end{array}$ & 2.40 & $2.85(18.75 \%)^{+}$ & 0.9920 \\
\hline
\end{tabular}

"dC/da values are in $10 \mathrm{E}-05$

${ }^{+}$Percent differences are computed against the FE results 
The Substrate Material Effect Phenomenon: The compliance of a material, from a nontechnical structural engineering viewpoint, can be thought of as its propensity to distort under the action of loading, i.e. its disposition to extend or flex or twist, etc. Consider, as an example, two vastly different materials, say rubber and steel. Keeping everything else the same, rubber is naturally the more compliant material of the two. Extending this line of reasoning to the first two calibration classes, it is sensible then how one might presume the wood-wood specimen to be more compliant than its wood-concrete companion. Counterintuitively, however, the exact opposite is true. It must first be understood that in each case, the measured compliance is not that of the substrate but rather of the LVL wooden contour. Of course, this is not to say that the effect of the substrate material is unimportant and therefore can be neglected. Instead, the significant role of the substrate material is realized by the extent to which it can absorb the energy resulting from the applied load - this is precisely what is being termed here as the substrate material effect. Between the cases of the wood-wood and wood-concrete specimens for example, the relatively more compliant Red Maple substrate of the former absorbs more energy resulting from the applied load and thereby is not as efficient in causing flexure of the LVL contour as compared with its latter counterpart. Diminishing the effect of contourbending translates to a smaller tip-deflection, i.e. crack opening displacement (COD), and since the compliance is directly proportional to the $\mathrm{COD}$, this essentially renders a greater decrease in the magnitude of contour-compliance than what would have otherwise resulted by using a stiffer substrate material. This inherent attribute of the underlying material to share in dissipating energy can be characterized using the analogy of 
constraints as well. In the case of a relatively stiff substrate material, for instance, the periphery of the bonded-contour in the vicinity of the crack front is represented by a greater degree of fixity, and thus, enhances bending-curvature of the bondless portion of the contour for a given unit of tip load. The constraint condition of a less stiff substrate, on the other hand, is more relaxed not only at the root of the crack but ahead of it to some extent as well. This means that for the same tip load, the degree of contour-arching is comparatively suppressed. Instead, the displacement of the cantilever beam is characterized more dominantly by rigid body translation, thus differing from that of the former, stiffer substrate which, in addition to undergoing some translation, experiences a greater degree of rotation and results in an overall larger tip displacement, i.e. magnitude of compliance.

This physical phenomenon was framed after a careful review of a large number of experimental observations. Interestingly, at a later date, the present author was pleasantly surprised to happen upon the published results of other researchers who, albeit, were engaged in a somewhat unrelated study, nevertheless faced in common with this research, the issue involving substrate material effects. In particular, Yu and Hutchinson (2002) analyzed the delamination effects of thin films subjected to more compliant substrate materials. They write that apart from their own study and another recent investigation performed by Cotterell and Chen (2000), all of the previous analyses performed on thin films only considered the influence of stiff substrates in which the blister-boundary is taken to be clamped. This statement gives credence to the one just made above regarding the constraint condition of greater fixity near the crack front in the case of the relatively 
stiffer substrate material. Another very pertinent result of their study is the determination of a zone wherein stresses are concentrated near the points of attachment of the adherend. They found that the length of this zone, which they denote as $\ell$, is larger with more compliant substrates. The meaning of this is further expounded on in their conclusions by writing, "The essential aspect of the substrate deformation is partial release of elastic energy in the regions of the film still bonded to the substrate along the edges of the delamination." This substantiates the claim made earlier asserting that more compliant substrates absorb a higher amount of energy from the applied load and are therefore not as efficient in causing flexure of the contour.

To consolidate the observations and assertions of these discussions, a look into the magnitudes of the compliances resulting from the various classes of calibrations would be helpful. For this purpose, the optimum geometry of shape S3 (see Figure 4.1) is chosen and the compliance values corresponding to the three, non-sawn, crack length positions are considered (see Table 4.6).

Table 4.6 Compliance and $d C / d a$ values for the different classes of calibrations for

\begin{tabular}{|c|c|c|c|c|c|}
\hline $\begin{array}{c}\text { Shape S3: } \\
\mathrm{H}_{\mathrm{o}} / \mathrm{H}_{\mathrm{f}}[\mathrm{mm}]: 27.94 / 95.0 \\
\text { (in): } 1.10 / 3.74 \\
\end{array}$ & \multicolumn{5}{|c|}{$\begin{array}{c}\text { Compliance, } C \\
(10 \mathrm{E}-04)[\mathrm{mm} / \mathrm{N}] \\
(10 \mathrm{E}-05)(\text { in//b) }\end{array}$} \\
\hline (1) $a \quad[\mathrm{~mm}]$ (in) & $\begin{array}{l}\text { (2) wood- } \\
\text { wood }^{+}\end{array}$ & $\begin{array}{l}\text { (3) wood- } \\
\text { concrete }^{+} \\
\text {sawn }\end{array}$ & $\begin{array}{l}\text { (4) wood- } \\
\text { concrete } \\
\text { non-sawn }\end{array}$ & $\begin{array}{l}\text { (5) wood- } \\
\text { CFRP- } \\
\text { concrete }\end{array}$ & (6) $\mathrm{FE}$ \\
\hline $152.4 \quad(6)$ & $17.8(31.1)$ & $23.3(40.8)$ & $12.8(22.4)$ & $14.7(25.7)$ & $16.2(28.3)$ \\
\hline $254.0(10)$ & $34.5(60.5)$ & $47.1(82.5)$ & $37.6(65.9)$ & $30.7(53.7)$ & $32.3(56.5)$ \\
\hline 355.6 (14) & $52.1(91.2)$ & 72.5 (127.0) & $59.8(105.0)$ & $50.8(89.0)$ & $48.6(85.1)$ \\
\hline $\begin{array}{c}d C / d a(10 \mathrm{E}-05) \\
{\left[\mathrm{N}^{-1}\right]} \\
\left(\mathrm{lb}^{-1}\right)\end{array}$ & $\begin{array}{r}1.65 \\
(7.36)\end{array}$ & $\begin{array}{c}2.35 \\
(10.47)\end{array}$ & $\begin{array}{c}2.32 \\
(10.31)\end{array}$ & $\begin{array}{c}1.78 \\
(7.92)\end{array}$ & $\begin{array}{c}1.61 \\
(7.16)\end{array}$ \\
\hline
\end{tabular}

${ }^{+}$Crack length positions achieved by sawing; $d C / d a$ values computed over the broader range of $101.6-355.6 \mathrm{~mm}(4 "$ - 14") in $50.8 \mathrm{~mm}(2 ")$ increments 
Observe that the compliance values (from Table 4.6) of the wood-concrete specimens in all cases, except for the one crack location at $a=152.4 \mathrm{~mm}(6 ")$ of the nonsawn group, is considerably higher than those of the wood-wood, as it should be, due to substrate material effects. The one exception may (or may not?-see the discussion below on generalizing the substrate material) be a spurious result due to some experimental error. On the average, it was found that the percent compliances of the sawn and non-sawn wood-concrete beams were, respectively, $35 \%$ and $12 \%$ larger in comparison than those of the wood-wood specimen. Also observe that while the compliance values from the non-sawn wood-concrete specimens were lower than their sawn counterparts by an average of $19 \%$ - as would be expected due to a slight privation in contour depth as a result of the sawing process, the $d C / d a$ values were, interestingly, nearly alike. This indicates that the substrate material effect has a more pronounced outcome on the $d C / d a$ parameter than does a small loss or discrepancy in contour depth. Thus it is reassuring to know, in the first place, that the element of human error involved in the manufacture of the contours should not become a major issue of concern.

Justification of Wood-Wood Assumption in both Modeling and Shape Optimization: While it is true that the sequence of events leading to a final contour shape was not planned in advance, the final conclusions that were made are nonetheless completely justifiable. Consider, first of all, the decision to use a wood-concrete specimen after the wood-wood calibrations had been completed. At that point in time, it appeared that of the two, the wood-concrete specimen would be the more likely candidate in representing the 
actual fracture specimen. This is not the case, however, owing to the now understood phenomenon of substrate material effects. Notwithstanding this lack of knowledge, the otherwise seemingly roundabout sequence of calibrations performed was vitally important in the substantiation of both analytical and experimental results. Had the intermediate class of wood-concrete calibrations been omitted, it is very probable that the connection between the wood-wood and wood-CFRP-concrete specimens might have been overlooked. This connection, as evidenced by the similarity in compliance values between columns (2), (5) and (6) of Table 4.6, is truly telling of the fracture specimen's complex substrate nature, as will now be explained.

In retrospect, this "connection" makes perfectly good sense, when considering the substrate conditions from a generalized viewpoint. During the manufacture of the sawn (non-sawn will be discussed shortly) wood-concrete (see column (3) of Table 4.6) specimen, for example, the LVL contour is bonded to the concrete base with a thin, single coating of saturant, and as such, the substrate material can be generalized as being concrete-dominated. The wood-CFRP-concrete specimen (column (5) of Table 4.6), on the other hand, requires approximately 3 - 5 times as much saturant (see Chapter 5) in its manufacture, resulting in a substrate material better characterized as dominantly epoxy/carbon-fiber based. By momentarily ignoring the complexity of this new metasubstrate, it becomes evident from Table 4.6 that there is justification in generalizing it to that of the simpler wood-wood case. As a matter of fact, it appears that this generalization may be made depending on the amount of resin used at the interface alone. Consider the non-sawn wood-concrete specimen (column (4) of Table 4.6). This specimen came 
toward the end of the sequence of calibrations by which time it had been learned that more resin was required in bonding the LVL contour to the concrete base. Previously, as with the sawn wood-concrete specimen, it was found in some specimens that an insufficient amount of saturant was applied (recall the descriptors "thin, single" in describing the coating layer) and that these specimens contained some finite voids, or airgaps, at the interface. To avoid this technical difficulty, all subsequent specimens were manufactured with a richer amount of resin at the interface. In other words, the compliance values of Table 4.6, column (4) should come as no surprise as having some similarity with those of the wood-wood case, because the resin-layer (more than 1 but less than two times the amount —not $3-5$ times as much, as was previously mentioned for the wood-CFRP-concrete beam) may also be generalized to that of the wood-wood specimen, albeit to a lesser degree now (the average, absolute percentage difference in compliance values from that of the wood-wood here is $17.33 \%$ ). An important observation that should not be neglected is that though the compliance values between the sawn and non-sawn concrete specimens were in variance between $27 \%-28 \%$, their $d C / d a$ values were quite similar- $2.35 \times 10^{-5}\left[\mathrm{~N}^{-1}\right]$ and $2.32 \times 10^{-5}\left[\mathrm{~N}^{-1}\right]$, respectively, a percentage difference of merely $1.3 \%$. Incidentally, the percentage differences in the values of $d C / d a$ between (1) the wood-CFRP-concrete and wood-wood, (2) the woodwood and FE and (3) the wood-CFRP-concrete and FE, are respectively: (1) 7.9\%, (2) $2.4 \%$ and (3) $9.6 \%$. These considerations therefore offer justification regarding use of the wood-wood assumption, both, for the simplification made in modeling, and in the determination of the optimized contour shape. 


\section{SCCB Calibration Results}

For the purposes of brevity, the SCCB results will be purveyed in a plain fashion without much commentary. The presentation of the material will adhere to the following convention: (1) the specimen shape with a corresponding diagram showing pertinent dimensions, (2) the FE and beam on elastic foundation results (abbreviated Beam_EF, where available), (3) the experimental results, beginning with the wood-wood (abbreviated as W-W), then on to the wood-concrete (i.e. W-C, where available) and ending with the wood-CFRP-concrete (i.e. W-CFRP-C, where available) specimenwhich, by the way, follows the actual sequence of testing, and finally (4) ending with a compliance versus crack length graph displaying the averaged values of compliance for each of the crack positions at which calibration testing was conducted. Also included will be the corresponding $r^{2}$ values (a.k.a. the coefficient of determination—see discussion in section 4.2 under the subsection titled "CDCB Calibration Results" for Specimen S1), the crack range considered ( $a$ typically ranged between 101.6 - $355.6 \mathrm{~mm}(4 "$ - 14") or 152.4 - $355.6 \mathrm{~mm}\left(6^{\prime \prime}\right.$ - 14") with calibrations being performed at every $50.8 \mathrm{~mm}(2 ")$ increments for the sawn specimens, and otherwise at the discrete locations of 152.4, 254 and $355.6 \mathrm{~mm}(6 ", 10 "$ and 14") for the non-sawn cases), and finally, a percentage difference comparison that is made relative to the FE results. Though mentioned before, it is worth repeating that the optimized shape among the five considered is specimen S3, yielding a value of $d C / d a=1.78 \times 10^{-5} \mathrm{~N}^{-1}\left(=7.92 \times 10^{-5} \mathrm{lb}^{-1}\right)$ that is to be used in all subsequent fracture testing. All of these results as well as those of the CDCB specimen calibrations are summarized in tabular form in section 4.5. 
Specimen 1:

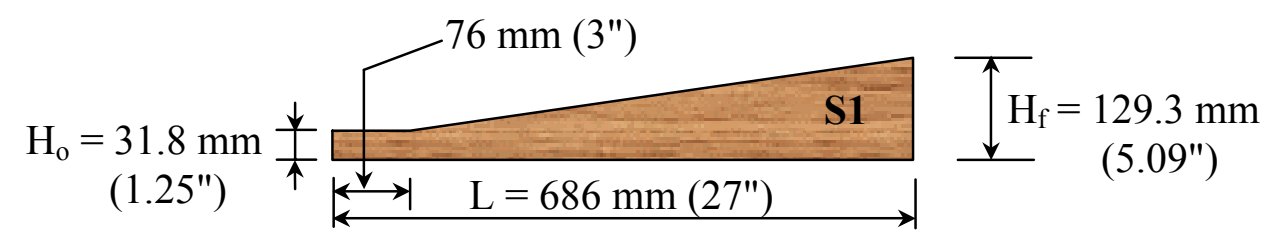

Figure 4.7 SCCB calibration specimen S1; width (not shown), $b=43.4 \mathrm{~mm}(1.71 ")$

FE: $\quad d C / d a=1.10 \times 10^{-5} \mathrm{~N}^{-1} ; \mathrm{r}^{2}=0.9969 ; a=203.2-457.2 \mathrm{~mm}$ $\left(=4.90 \times 10^{-5} \mathrm{lb}^{-1} ; \mathrm{r}^{2}=\uparrow \quad ; a=8^{\prime \prime}-18^{\prime \prime}\right)$

Beam_FE:

-N/A----------------

SCCB W-W: $d C / d a=1.20 \times 10^{-5} \mathrm{~N}^{-1} ; \mathrm{r}^{2}=0.9989 ; a=101.6-355.6 \mathrm{~mm} ; 9.1 \%$ $\left(=5.36 \times 10^{-5} \mathrm{lb}^{-1} ; \mathrm{r}^{2}=\uparrow ; a=4 "-14 "\right)$

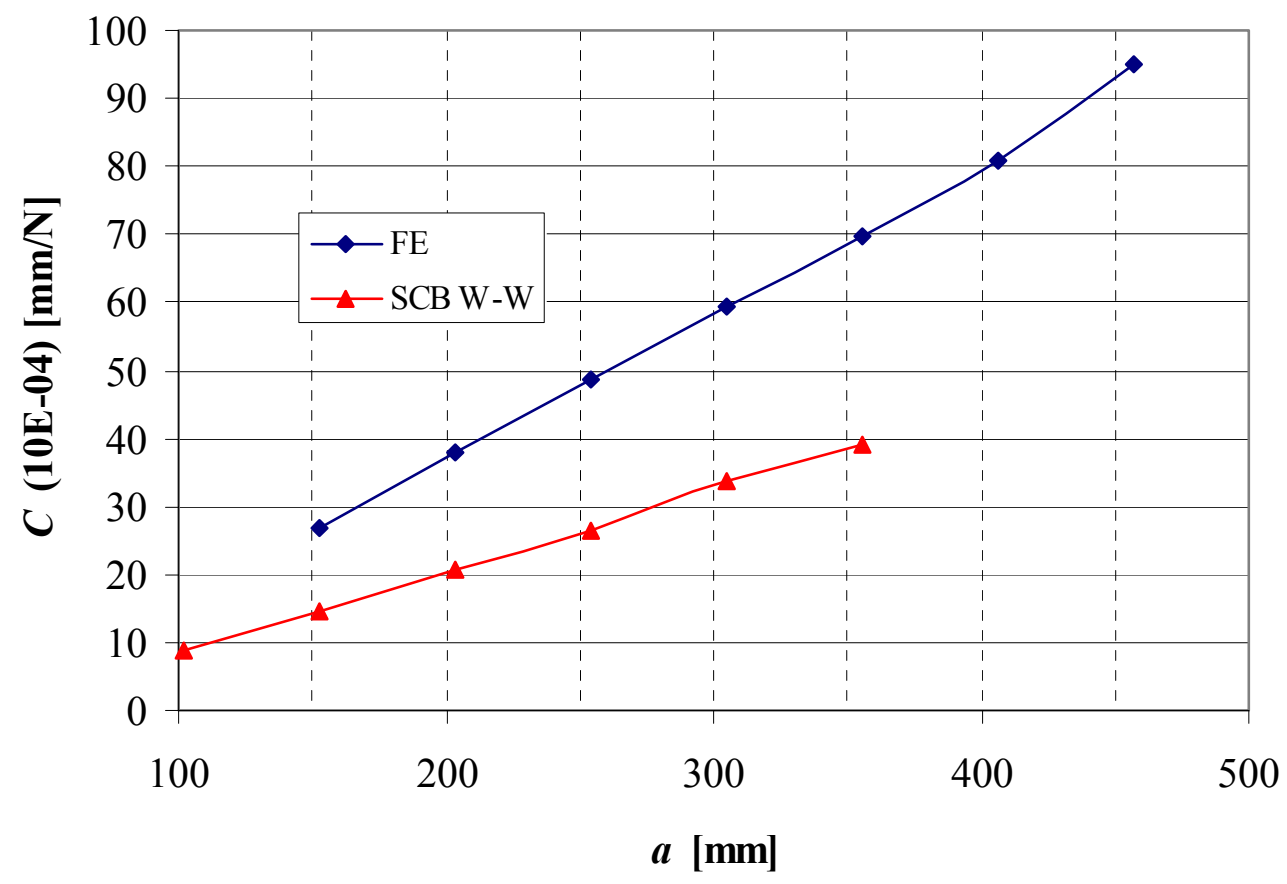

Figure 4.8 Compliance vs. crack length of SCCB specimen S1 
Specimen 2:

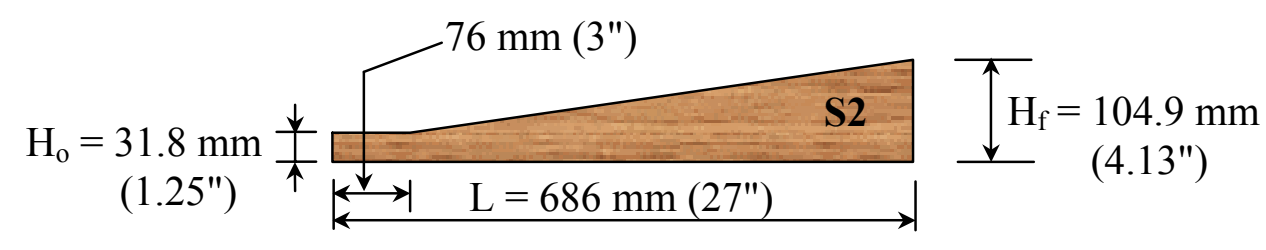

Figure 4.9 SCCB calibration specimen S2; width (not shown), $\mathrm{b}=43.4 \mathrm{~mm}(1.71 ")$

FE: $\quad d C / d a=1.33 \times 10^{-5} \mathrm{~N}^{-1} ; \mathrm{r}^{2}=0.9988 ; a=152.4-508 \mathrm{~mm}$

$$
\left(=5.91 \times 10^{-5} \mathrm{lb}^{-1} ; \mathrm{r}^{2}=\uparrow \quad ; a=6^{\prime \prime}-20^{\prime \prime}\right)
$$

Beam_FE: $\quad d C / d a=1.23 \times 10^{-5} \mathrm{~N}^{-1} ; \mathrm{r}^{2}=0.9992 ; a=152.4-508 \mathrm{~mm} ; \quad-7.5 \%$ $\left(=5.48 \times 10^{-5} \mathrm{lb}^{-1} ; \mathrm{r}^{2}=\uparrow ; a=6^{\prime \prime}-20^{\prime \prime}\right)$

SCCB W-W: $d C / d a=1.50 \times 10^{-5} \mathrm{~N}^{-1} ; \mathrm{r}^{2}=0.9834 ; a=101.6-355.6 \mathrm{~mm} ; 12.8 \%$ $\left(=6.67 \times 10^{-5} \mathrm{lb}^{-1} ; \mathrm{r}^{2}=\uparrow \quad ; a=4 "-14 "\right)$

W-C: $d C / d a=2.48 \times 10^{-5} \mathrm{~N}^{-1} ; \mathrm{r}^{2}=0.9968 ; a=152.4-406.4 \mathrm{~mm} ; 86.5 \%$ $\left(=11.02 \times 10^{-5} \mathrm{lb}^{-1} ; \mathrm{r}^{2}=\uparrow \quad ; a=6^{\prime \prime}-16^{\prime \prime}\right)$

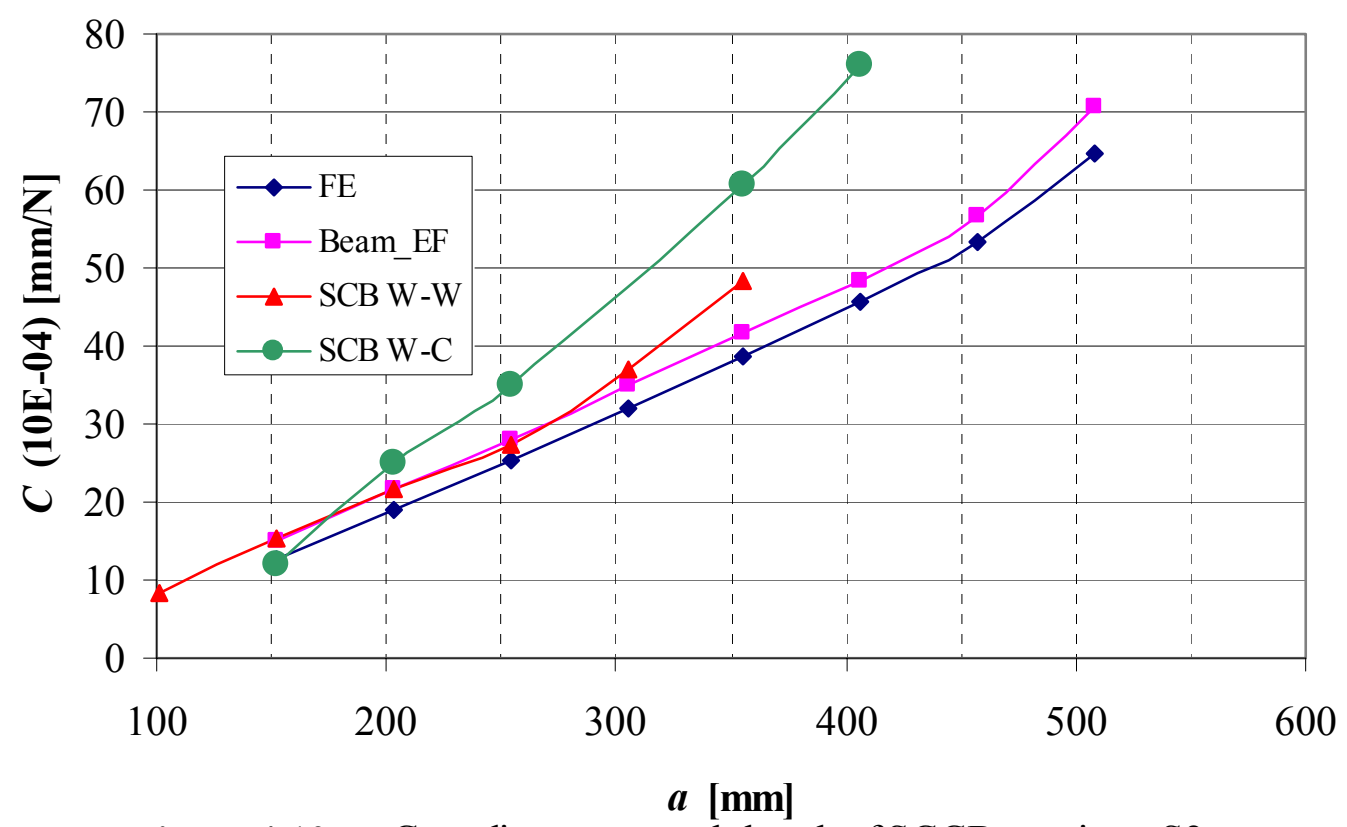

Figure 4.10 Compliance vs. crack length of SCCB specimen S2 
Specimen 3 (Note: Optimum Shape-actual fracture specimen results underlined below):

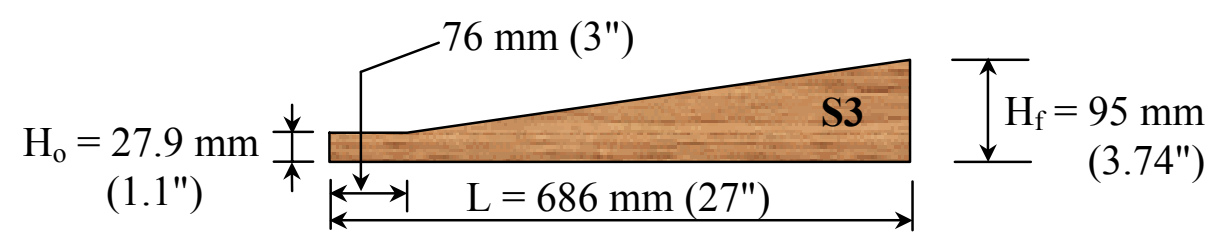

Figure 4.11 SCCB calibration specimen S3; width (not shown), $b=43.4 \mathrm{~mm}(1.71 ")$

FE: $\quad d C / d a=1.61 \times 10^{-5} \mathrm{~N}^{-1} ; \mathrm{r}^{2}=0.9988 ; a=152.4-508 \mathrm{~mm}$

$$
\left(=7.16 \times 10^{-5} \mathrm{lb}^{-1} ; \mathrm{r}^{2}=\uparrow ; a=6 "-20 "\right)
$$

Beam_FE: $\quad d C / d a=1.61 \times 10^{-5} \mathrm{~N}^{-1} ; \mathrm{r}^{2}=0.9992 ; a=152.4-508 \mathrm{~mm} ;-0.14 \%$ $\left(=7.15 \times 10^{-5} \mathrm{lb}^{-1} ; \mathrm{r}^{2}=\uparrow ; a=6 "-20^{\prime \prime}\right)$

SCCB W-W: $d C / d a=1.65 \times 10^{-5} \mathrm{~N}^{-1} ; \mathrm{r}^{2}=0.9979 ; a=101.6-355.6 \mathrm{~mm} ; 2.5 \%$

$$
\left(=7.36 \times 10^{-5} \mathrm{lb}^{-1} ; \mathrm{r}^{2}=\uparrow ; a=4 "-14 "\right)
$$

(sawn) W-C: $d C / d a=2.35 \times 10^{-5} \mathrm{~N}^{-1} ; \mathrm{r}^{2}=0.9933 ; a=101.6-355.6 \mathrm{~mm} ; 46.0 \%$ $\left(=10.47 \times 10^{-5} \mathrm{lb}^{-1} ; \mathrm{r}^{2}=\uparrow ; a=4 "-14 "\right)$

(non-sawn) $\quad d C / d a=2.32 \times 10^{-5} \mathrm{~N}^{-1} ; \mathrm{r}^{2}=0.9990 ; a=152.4,254,355.6 \mathrm{~mm} ; \quad 44.1 \%$ $\left(=10.31 \times 10^{-5} \mathrm{lb}^{-1} ; \mathrm{r}^{2}=\uparrow ; a=6 ", 10^{\prime \prime}, 14 "\right)$

(个)W-CFRP-C: $d C / d a=1.78 \times 10^{-5} \mathrm{~N}^{-1} ; \mathrm{r}^{2}=0.9956 ; a=152.4,254,355.6 \mathrm{~mm} ; 10.6 \%$ $\left(=7.92 \times 10^{-5} \mathrm{lb}^{-1} ; \mathrm{r}^{2}=\uparrow ; a=6 ", 10^{\prime \prime}, 14^{\prime \prime}\right)$

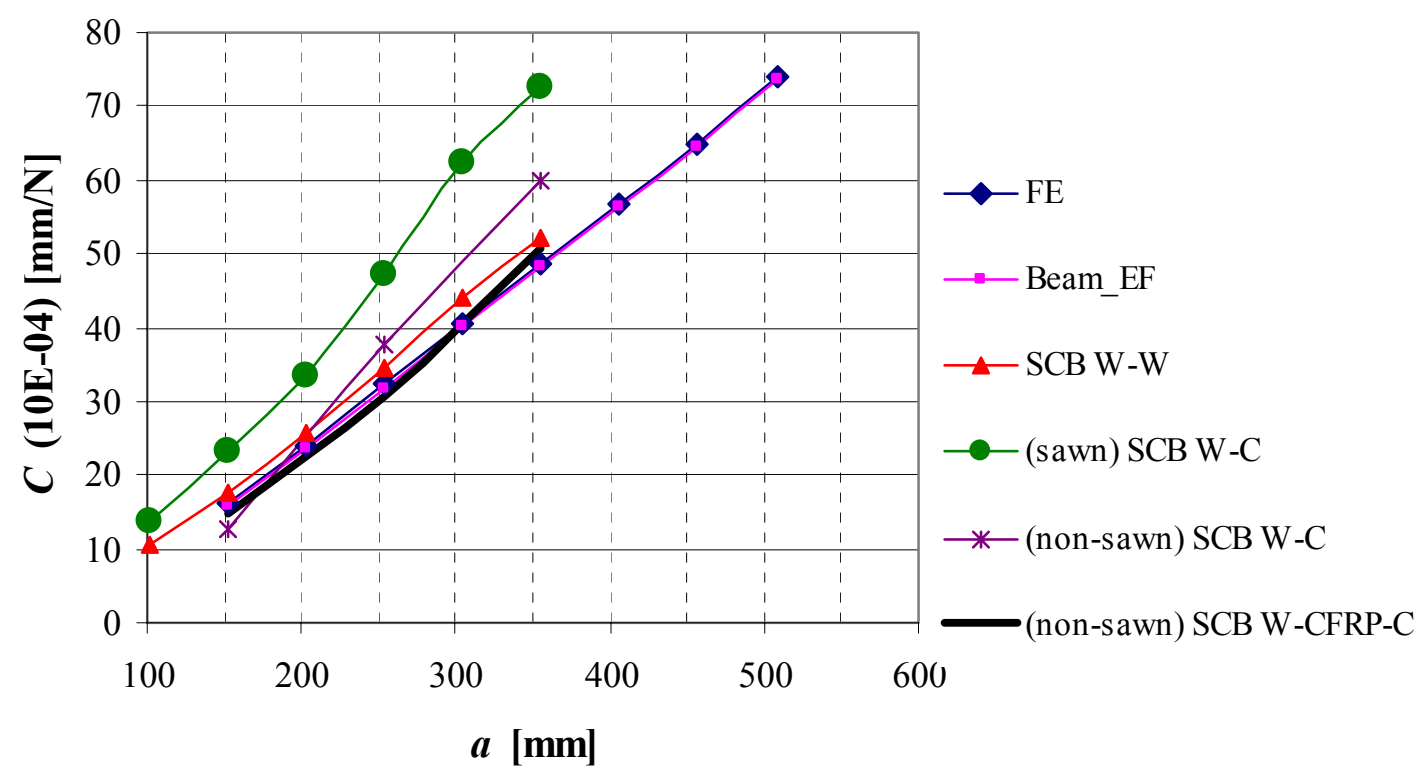

Figure 4.12 Compliance vs. crack length of SCCB specimen S3 
Specimen 4:

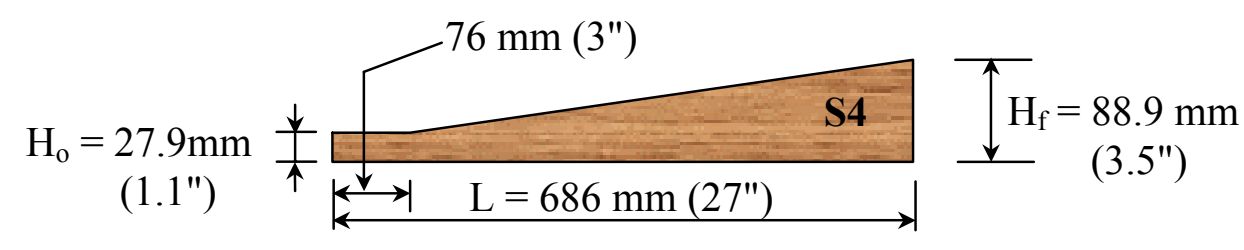

Figure 4.13 SCCB calibration specimen S4; width (not shown), $b=43.4 \mathrm{~mm}(1.71 ")$

FE: $\quad d C / d a=1.84 \times 10^{-5} \mathrm{~N}^{-1} ; \mathrm{r}^{2}=0.9996 ; a=152.4-508 \mathrm{~mm}$ $\left(=8.19 \times 10^{-5} \mathrm{lb}^{-1} ; \mathrm{r}^{2}=\uparrow ; a=6 "-20 "\right)$

Beam_FE: $\quad d C / d a=1.84 \times 10^{-5} \mathrm{~N}^{-1} ; \mathrm{r}^{2}=0.9996 ; a=152.4-508 \mathrm{~mm} ; \quad-0.24 \%$ $\left(=8.17 \times 10^{-5} \mathrm{lb}^{-1} ; \mathrm{r}^{2}=\uparrow \quad ; a=6^{\prime \prime}-20^{\prime \prime}\right)$

SCCB W-W: $d C / d a=2.08 \times 10^{-5} \mathrm{~N}^{-1} ; \mathrm{r}^{2}=0.9962 ; a=101.6-355.6 \mathrm{~mm} ; 13.0 \%$ $\left(=9.26 \times 10^{-5} \mathrm{lb}^{-1} ; \mathrm{r}^{2}=\uparrow \quad ; a=4^{\prime \prime}-14 "\right)$

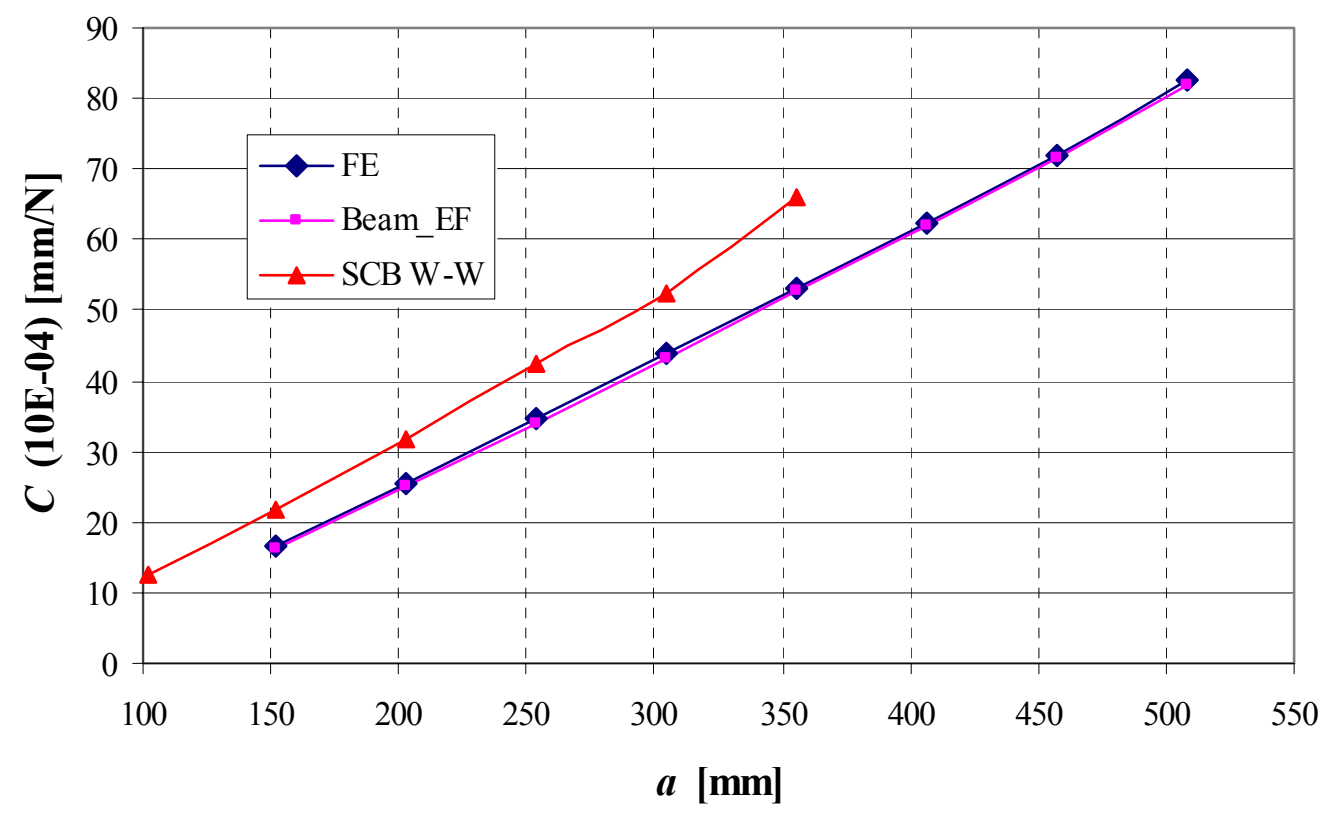

Figure 4.14 Compliance vs. crack length of SCCB specimen S4 
Specimen 5:

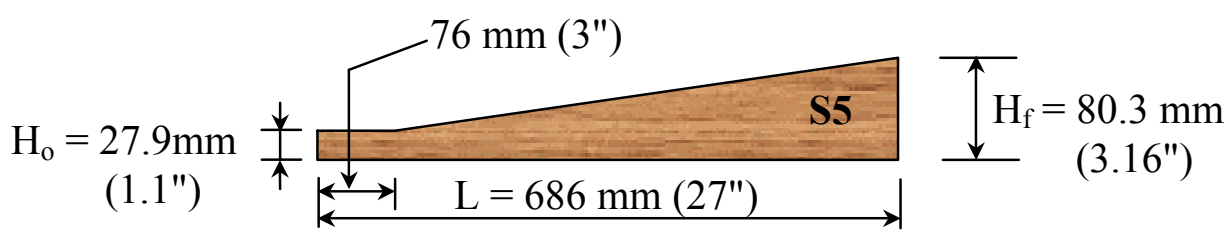

Figure 4.15 SCCB calibration specimen S5; width (not shown), $b=43.4 \mathrm{~mm}(1.71 ")$

FE: $\quad d C / d a=2.40 \times 10^{-5} \mathrm{~N}^{-1} ; \mathrm{r}^{2}=0.9997 ; a=152.4-508 \mathrm{~mm}$

$$
\left(=10.70 \times 10^{-5} \mathrm{lb}^{-1} ; \mathrm{r}^{2}=\quad \uparrow ; a=6^{\prime \prime}-20^{\prime \prime}\right)
$$

Beam_FE: $\quad d C / d a=2.40 \times 10^{-5} \mathrm{~N}^{-1} ; \mathrm{r}^{2}=0.9997 ; a=152.4-508 \mathrm{~mm} ; \quad-0.09 \%$ $\left(=10.69 \times 10^{-5} \mathrm{lb}^{-1} ; \mathrm{r}^{2}=\uparrow ; a=6 "-20 "\right)$

SCCB W-W: $d C / d a=2.85 \times 10^{-5} \mathrm{~N}^{-1} ; \mathrm{r}^{2}=0.9920 ; a=101.6-355.6 \mathrm{~mm} ; 18.8 \%$

$$
\left(=12.69 \times 10^{-5} \mathrm{lb}^{-1} ; \mathrm{r}^{2}=\uparrow \quad ; a=4 "-14 "\right)
$$

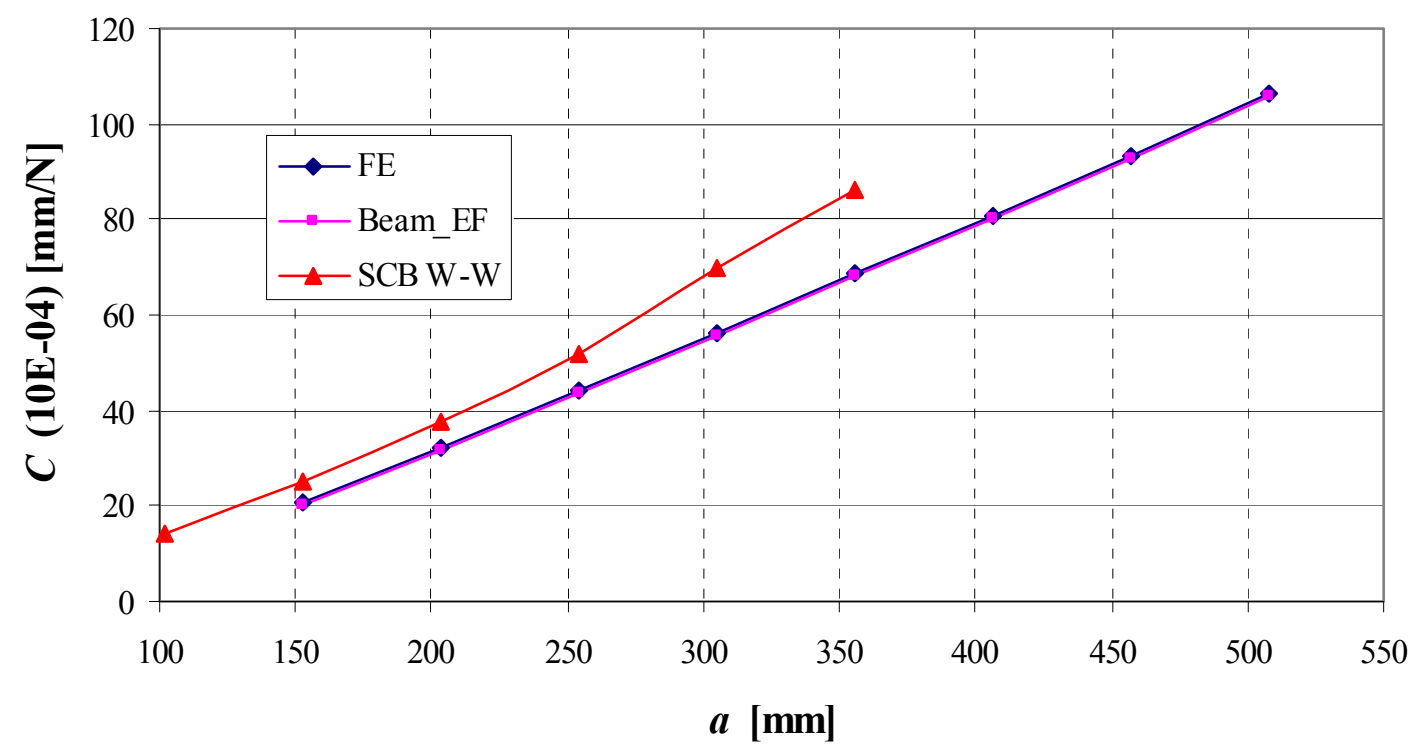

Figure 4.16 Compliance vs. crack length of SCCB specimen S5 


\subsection{Hinging Action-Limits of the FE and Analytical Approaches}

The range of crack extensions to consider is an important issue relevant to the experimental calibration of the single contoured-cantilever beam specimen. In practice, the compliance studies of the previous section were carried out over a broad range of locations, beginning at $101.6 \mathrm{~mm}(4 ")$ and ending at $508 \mathrm{~mm}(20 ")$, even though the experimental results were reported up to a crack length of about $355.6 \mathrm{~mm}$ (14"). Performing calibrations as far out in the specimen as $508 \mathrm{~mm}(20 ")$, however, revealed a phenomenon specific to the location of the crack that was not detected by the FE or the beam on elastic foundation model results. This length-effect, termed also as "hinging action" (Ripling, 1966, p.70), arises from a change in the constraint-condition as the location of the crack is extended farther and farther back near the end of the specimen. The presupposed condition of fixity for the contoured-cantilever beam is more closely achieved when the extension of the crack is yet small, i.e. when a larger portion of the contour is rigidly bonded to the substrate. In contrast, the condition of fixity becomes more and more relaxed as the position of the crack is extended back, thus shortening the bonded-segment of the contour. Berry (1963, p. 63) recognized this effect too when he wrote, "[the hinging action] is most apparent when the crack length becomes comparable to that of the sample [length]." This phenomenon is shown diagrammatically in Figure 4.17. The wood-concrete curve depicts a considerable increase in the specimen compliance after a certain value of crack-length, representing a departure from the otherwise expected linear trend. Brown and Srawley $(1966$, pp. 71, 72), in reply to E.J. Ripling's discussion, comment that the compliance values lie on a straight line for ratios 
of $a / \mathrm{L}$ up to 0.6 ( $\mathrm{L}$ is the specimen length) —note that in this case $a=406 \mathrm{~mm}$ and $\mathrm{L}=$ $686 \mathrm{~mm}$, representing a ratio of 0.59 (see Figure 4.17). They explain that this curved portion represents a solution of a semi-finite crack approaching the free edge of a half plane, as demonstrated by Paris and Sih (1964, equation 181). This hinging action is, however, nowhere present in the modeled FE and beam on elastic foundation plots which are linear throughout the full range of crack-extensions (see Figure 4.17).

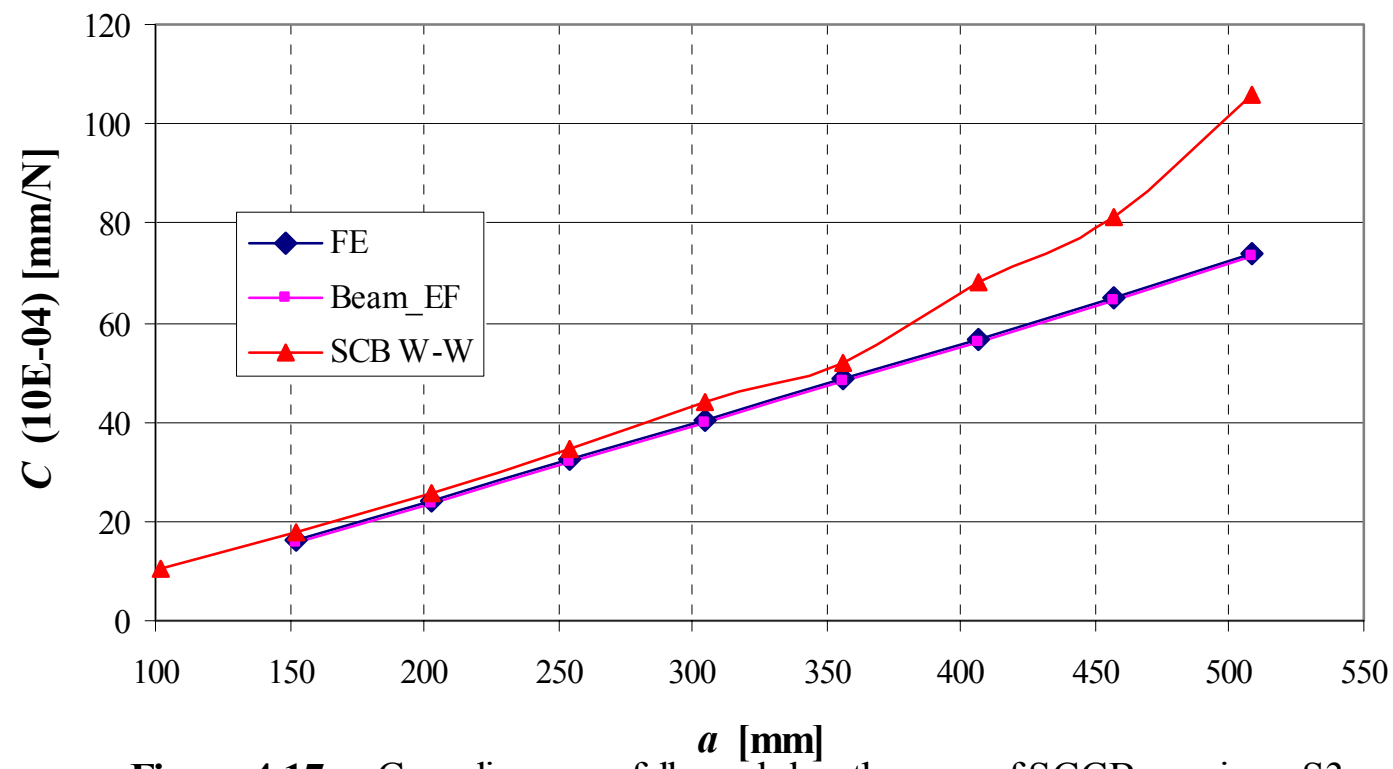

Figure 4.17 Compliance vs. full-crack length range of SCCB specimen S3

It is for this reason that the $d C / d a$ values of the calibrated specimens were computed by restricting the crack-extension range to the interval from $101.6 \mathrm{~mm}(4 ")-$ $355.6 \mathrm{~mm}(14 ")$, corresponding to the linear portion of the experimental curve. In all cases, the linear regression performed on the data in this interval yielded $r^{2}$ (a.k.a. the coefficient of determination—see discussion in section 4.2 under the subsection titled "CDCB Calibration Results" for Specimen S1) values in excess of 0.98, thus reinforcing confidence from a statistical standpoint, especially as it pertains to the experimental 
results. Significantly, this is the region in which major cracks were observed to occur during fracture testing. Such foregoing matters as the range wherein major fracture is to ensue and an adherence (approximately) to a constraint-condition of fixity at the location of the crack-front, dictate the choice of the specimen-length; hence, the rationale behind using a rather sizable specimen with a length of $686 \mathrm{~mm}(27 ")$, in lieu of one that would otherwise have been conveniently smaller and more compact.

\subsection{Summary and Conclusions}

The focus of this chapter has been to: (1) experimentally validate the newly conceived single contoured-cantilever beam (SCCB) specimen as a viable tool in evaluating the fracture toughness of FRP-concrete interfaces, and (2) account for the extensive calibration investigations performed to determine an optimum contour geometry —one for which the $d C / d a$-parameter would be most nearly constant during the onset and propagation of cracking. The small variance of $10.6 \%$ (see Table 4.7) existing between the FE and the actual fracture specimen (i.e. the wood-CFRP-concrete beam) $d C / d a$-results demonstrates fulfillment of the former objective. The significance of the latter focal-point is that the Irwin-Kies relation, developed in Chapter 3, can now be taken as being independent of the crack length, $a$-this means that the critical strain energy release rate, $G_{I c}$, can simply be determined from the critical load, $P_{c}$, itself, irrespective of the crack's position at any given instant of fracture testing (refer also to Eq. (1.1)).

In an attempt to satisfy the above mentioned objectives from a practical standpoint, the experimental calibration program undertaken was initiated by the 
consideration of five shapes from the start, labeled S1 - S5 (see Table 4.1 and Figure 4.1). An explicit beam on elastic foundation model (see Qiao et al., 2001) and a finite element (FE) solution were used in generating these shapes so that each might render a constant $d C / d a$ parameter. Two varieties of specimens were used in the experimental verifications.

Firstly, the well-established contoured double cantilever beam (CDCB) specimen was relied on to calibrate shapes S1 and S2 (see Table 4.1 and Figures 4.2 and 4.3). This enabled positive assertions to be made regarding the verity of the FE and explicit solutions, the proper functioning of the instrumentation, and an overall meriting of the experimental protocol being implemented. Secondly, the novel SCCB specimen was used to conduct sequential calibrations on three classes of samples, namely those of the woodwood, wood-concrete, and wood-CFRP-concrete-the latter being an exact representation of the actual fracture beam.

Owing to the inherent intricacy to model the SCCB specimen, especially the fact that it is asymmetric and involves hybrid interfaces, the FE and explicit solutions were formulated by assuming (1) the symmetry of the CDCB specimen and (2) the simpler wood-wood interface pairing-scheme. This explains part of the rational for performing two CDCB calibrations from the start—-both of which were found to have less than a $2 \%$ variance with the predicted FE results - and for beginning each of the SCCB sequence of calibrations first on the wood-wood specimens. On the other hand, the role played by the substrate material in the calibration scheme was not fully realized at the time and so it was erroneously assumed that the base material used would not greatly influence the compliance results of the (upper) contoured cantilever beam. Wood was therefore chosen 
as the base material for primarily two reasons: (1) to economize on the existing concrete substrates which, incidentally, came at a high price of labor and time (curing for one month), and (2) to facilitate the backward relocation of the crack by sawing (a task that was achieved with great hardship in the case of the wood-concrete specimens). It was not until after the wood-concrete calibrations were performed, however, that the effect of the substrate material became apparent (see "Substrate Material Effects" of Section 4.3).

Contrary to what was believed then, the wood-wood case was discovered to more closely represent the actual fracture specimen (i.e. the wood-CFRP-concrete case) than its wood-concrete counterpart. This was found to be due to the excess amount of saturant needed at the interface to fabricate the wood-CFRP-concrete specimen, which essentially had the effect of causing the substrate material to behave more like that of wood as opposed to concrete. Of course this discovery came as a welcomed-surprise since the FE and explicit model results were based on this simplifying assumption, even though it was done out of innocence and not by preplanning.

With this revelation, it was now possible to hone in on the most optimum geometry among the five being considered. This happened to be shape S3, with only a $2.5 \%$ discrepancy with its corresponding FE result — a rather significant outcome when considering that even the perfectly symmetric CDCB specimens, S1 and S2, were in variance with their predicted values by approximately the same degree.

One challenge still remained to overcome, however, before the $d C / d a$ parameter of the wood-CFRP-concrete specimen could be determined now that the optimum shape was realized. Just briefly mentioned, the technique that was relied on in extending the 
crack backwardly till now was that of sawing. Another method would have to be implemented since this actual fracture specimen contained the vital carbon fabric at its interface, and sawing would inevitably ravage this crucial layer. The no-sawing technique was thus conceived in answer to this dilemma. Here a piece of wax paper, acting as a barrier against the epoxy, is doubled-over to a length corresponding to the crack location desired and inserted at the interface during the bonding operation of the contour to the substrate beam. An obvious drawback of this technique is the costly requirement that one concrete substrate be sacrificed for every crack position tested. For this reason, testing of the wood-CFRP-concrete (similarly done for one of the two wood-concrete cases, see Table 4.7) specimen was limited to the three reasonably spaced (see "The Experimental Calibration" scheme of Section 4.2) crack locations of $152.4 \mathrm{~mm}(6 "), 254 \mathrm{~mm}$ (10") and $355.6 \mathrm{~mm}(14 ")$. The resulting $d C / d a$ value that was found by testing the wood-CFRPconcrete specimen using geometry S3 and the no-sawing technique - this value is to be used in all subsequent fracture testing herein-is $1.78 \times 10^{-5} \mathrm{~N}^{-1}=7.92 \times 10^{-5} \mathrm{lb}^{-1}$, with a coefficient of determination of $r^{2}=0.9956$ (see discussion in section 4.2 under the subsection titled "CDCB Calibration Results" for Specimen S1—see also Table 4.7 and Figure 4.18). This $d C / d a$ value corresponds to an overall $10.6 \%$ deviation from the expected FE result and a 7.9\% departure from the amount found for its SCCB woodwood counterpart.

The chapter concluded with a word about the second of two phenomena that was discovered while performing the experimental calibrations (the first being the "Substrate Material Effects" of Section 4.3). This so-called "hinging-action" (see Section 4.4), first 
coined perhaps by Ripling (1966, p. 70), is found to occur whenever the location of the crack is extended farther and farther back near the end of the specimen. A relaxation of the fixity-condition is subsequently found to dictate there as less and less of the bonded portion of the contour ahead of the crack-front becomes available in resisting the rotations being imposed at the joint due to the tip-load (see Figure 4.17). Brown and Srawley $(1966$, pp. 71, 72) observed that the compliance values remain linear for ratios of $a / \mathrm{L}(\mathrm{L}=$ the specimen length) up to 0.6 - this finding was, likewise, confirmed by the results reported herein. In contrast to the compliance behavior found experimentally, the hinging action could not be detected by the modeled FE and beam on elastic foundation plots, which remained linear throughout the full range of crack-extensions that were considered. For this reason, the $d C / d a$ values of the calibrated specimens were computed by restricting the crack-extension range to the interval from $101.6 \mathrm{~mm}(4 ")-355.6 \mathrm{~mm}$ (14"), corresponding to the linear portion of the experimental curve. Significantly, this is the region in which major cracking was observed to occur during fracture testing, resulting, therefore, in a rather large specimen of length $686 \mathrm{~mm}(27 ")$, in lieu of one that would otherwise have been conveniently smaller and more compact.

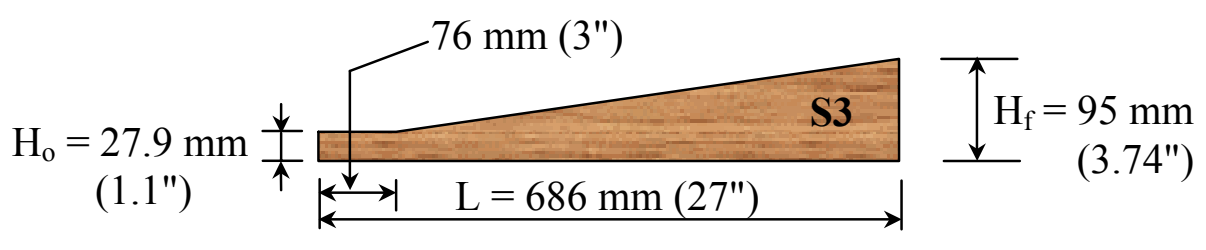

Figure 4.18 The optimum SCCB calibration specimen S3; width (not shown), $\mathrm{b}=43.4 \mathrm{~mm}(1.71 ")$; contour angle $=6.3^{\circ}$ 
Table 4.7 A summary of all the calibration results

\begin{tabular}{|c|c|c|c|c|}
\hline Geometry & Beam Type & $\begin{array}{r}d C / d a\left[\mathrm{~N}^{-1}\right]^{+} \\
(\%)^{\ddagger}\end{array}$ & $\mathbf{r}^{2}$ & $a[\mathrm{~mm}]$ \\
\hline \multirow{4}{*}{$\begin{array}{c}\mathbf{S 1} \\
\mathrm{H}_{\mathrm{o}} / \mathrm{H}_{\mathrm{f}} \\
{[\mathrm{mm}]: 31.75 / 129.3} \\
\text { (in): } 1.25 / 5.09\end{array}$} & $\mathrm{FE}$ & 1.10 & 0.9969 & $203.2-457.2$ \\
\hline & Beam_EF & $\overline{-}$ & - & \\
\hline & $\mathrm{CDCB}(\mathrm{s})^{*}$ & $1.08(-1.82 \%)$ & 0.9962 & $203.2-457.2$ \\
\hline & SCB_W-W (s) ${ }^{*}$ & $1.20(9.09 \%)$ & 0.9989 & $101.6-355.6$ \\
\hline \multirow{5}{*}{$\begin{array}{c}\text { S2 } \\
\mathrm{H}_{\mathrm{o}} / \mathrm{H}_{\mathrm{f}} \\
{[\mathrm{mm}]: 31.75 / 104.9} \\
\text { (in): } 1.25 / 5.09\end{array}$} & $\mathrm{FE}$ & 1.33 & 0.9988 & $152.4-508$ \\
\hline & Beam_EF & $1.23(-7.52 \%)$ & 0.9992 & $152.4-508$ \\
\hline & $\operatorname{CDCB}(\mathrm{s})^{*}$ & $1.35(1.50 \%)$ & 0.9986 & $152.4-457.2$ \\
\hline & SCB_W-W (s) ${ }^{*}$ & $1.50(12.78 \%)$ & 0.9834 & $101.6-355.6$ \\
\hline & $\mathrm{SCB} W-\mathrm{C}(\mathrm{s})^{*}$ & $2.48(86.47 \%)$ & 0.9968 & $152.4-406.4$ \\
\hline \multirow{6}{*}{$\begin{array}{c}\mathrm{H}_{\mathrm{o}} / \mathrm{H}_{\mathrm{f}} \\
\text { [mm]: } 27.94 / 95.0 \\
\text { (in): } 1.10 / 3.74\end{array}$} & FE & 1.61 & 0.9988 & $152.4-508$ \\
\hline & Beam_EF & $1.61(-0.14 \%)$ & 0.9992 & $152.4-508$ \\
\hline & SCB_W-W (s) ${ }^{*}$ & $1.65(-0.96 \%)$ & 0.9979 & $101.6-355.6$ \\
\hline & $\mathrm{SCB} \_\mathrm{W}-\mathrm{C}(\mathrm{s})^{*}$ & $2.35(45.96 \%)$ & 0.9933 & $101.6-355.6$ \\
\hline & SCB_W-C (n-s) ${ }^{*}$ & $2.32(44.10 \%)$ & 0.9990 & $152.4 ; 254 ; 355.6$ \\
\hline & ${ }^{*} S C B \_W-C F R P-C(n-s)^{*}$ & $1.78(10.56 \%)$ & 0.9956 & $152.4 ; 254 ; 355.6$ \\
\hline \multirow{3}{*}{$\begin{array}{c}\mathbf{S 4} \\
\mathrm{H}_{\mathrm{o}} / \mathrm{H}_{\mathrm{f}} \\
{[\mathrm{mm}]: 27.94 / 88.9} \\
\text { (in): } 1.10 / 3.50\end{array}$} & $\mathrm{FE}$ & 1.84 & 0.9996 & $152.4-508$ \\
\hline & Beam_EF & $1.84(-0.24 \%)$ & 0.9996 & $152.4-508$ \\
\hline & SCB_W-W (s) ${ }^{*}$ & $2.08(13.04 \%)$ & 0.9962 & $101.6-355.6$ \\
\hline \multirow{3}{*}{$\begin{array}{c}\text { S5 } \\
\mathrm{H}_{\mathrm{o}} / \mathrm{H}_{\mathrm{f}} \\
{[\mathrm{mm}]: 25.40 / 80.3} \\
\text { (in): } 1.00 / 3.16\end{array}$} & FE & 2.40 & 0.9997 & $152.4-508$ \\
\hline & Beam_EF & $2.40(-0.09 \%)$ & 0.9997 & $152.4-508$ \\
\hline & SCB_W-W (s) ${ }^{*}$ & $2.85(18.75 \%)$ & 0.9920 & $101.6-355.6$ \\
\hline
\end{tabular}

(s) = sawn; (n-s) = non-sawn

${ }^{+} d C / d a$ values are in $10 \mathrm{E}-05$

${ }^{\ddagger}$ Percentage differences are computed against the FE results

*Actual fracture specimen 


\section{CHAPTER 5}

\section{TEST SPECIMEN FABRICATION}

\subsection{Introduction}

Considering that this research work is chiefly experimental-based, it is therefore only appropriate to devote some attention to the pre-testing details of specimen fabrication. Actually, much of the post-experimental success can be directly attributed to the skill involved in the behind-the-scenes manufacture of the specimen. While the single contoured-cantilever beam (SCCB) with dimensions was already introduced in Section 3.6, the aim here is to provide insight into the various processes that were undertaken to successfully fashion the specimen into existence.

Broadly, the presentation of this material is divided into the following three topics: concreting, contouring, and bonding. Section 5.2 discusses the various aspects associated with concreting from which the substrate beam emerged. The design of an appropriately shaped LVL contour was the subject of the previous chapter wherein all of the painstaking efforts invested to determine such a configuration were described. Here, in Section 5.3, certain distinctive features of this eminent member are disclosed along with the technical measures implemented for their achievement. Finally, information 
pertaining to actual SCCB -prep and -assembly, as accomplished through a process of bonding, is illustrated in Section 5.4.

\subsection{Concreting}

The concrete substrates of the SCCB were intentionally designed with a trapezoidal cross-section (see Figure 5.1(a)) to prevent the specimens from becoming dislodged from the steel jacket during fracture testing, i.e. under the action of Mode I tiploading (i.e. opening mode of loading; see Section 3.3). These shapes were created by casting concrete into specially constructed molds (Figure 5.2(a)). A close-up view of one of the casting-cells, or troughs, is displayed in Fig. 5.2(b) with end-piece removed to illustrate that the shape is actually inverted to the cross-section of the concrete substrate depicted in Fig. 5.1(a). This ensures a perfectly level bonding surface-a critical feature if the specimen is to be fabricated successfully, i.e. if the LVL contour is to be uniformly bonded (later on) throughout its length without incurring air -gaps or -voids. Details, such

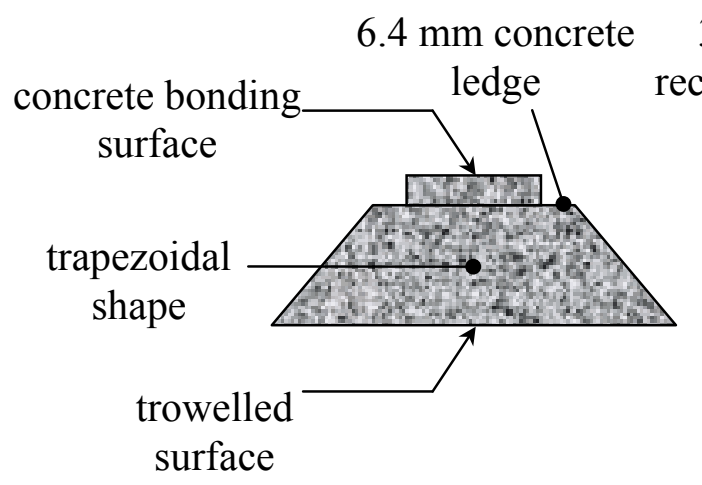

(a)

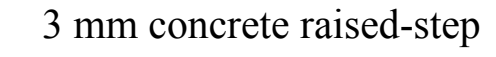
recessed $51 \mathrm{~mm}$ from front-end

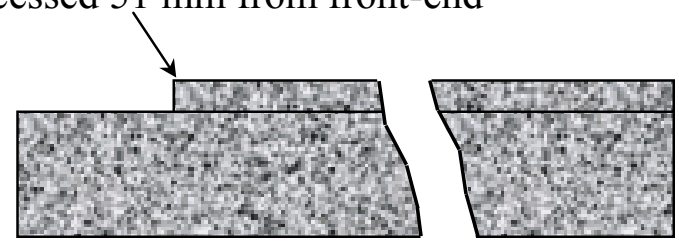

Figure $5.1 \quad$ (a) Front- (i.e. cross-sectional) and (b) side- views of concrete substrate (for dimensions refer to Figure 3.21, parts (c) and (d)) 
as the concrete ledge and raised-step (Fig. 5.1(a)) were achieved by stacking a pair of 1.6 $\mathrm{mm}\left(1 / 16^{\prime \prime}\right)$ thick, vinyl panels together underneath each of the tapered wooden slats of the mold with a $6.4 \mathrm{~mm}(1 / 4 ")$ offset; the $51 \mathrm{~mm}(2 ")$ front-end recess of the raised-step (Fig. 5.1(b)) - provided to easily accommodate the load strap during testing (see. Figs. 3.29 and 3.32(a)) — was made by nailing two $3.2 \mathrm{~mm}\left(1 / 8^{\prime \prime)}\right.$ thick pieces of wood (see

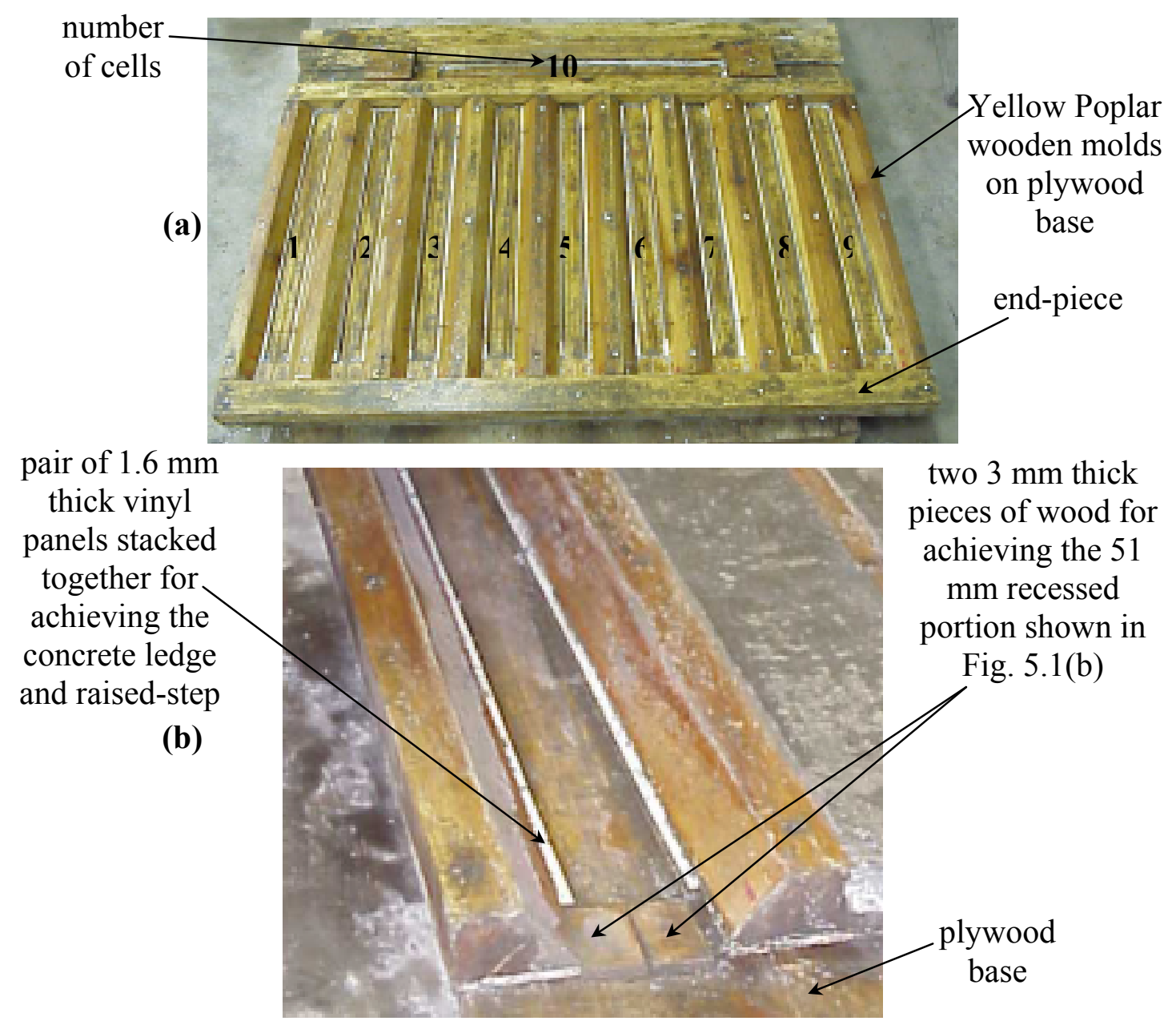

Figure 5.2 Pictures of: (a) the concrete substrate molds, and (b) a close-up of one such cell- note the inverted trapezoidal cross-section with respect to the concrete substrate beam (Fig. 5.1(a)) 
Fig. 5.2(b)). The width of the raised-step, incidentally, was cast to match the thickness of the LVL beam, not only in promoting proper alignment of the contour during the bonding-process, but more significantly, in encouraging the propagation of cracks to proceed and remain at this interface-location.

Altogether, a total of 14 batches of concrete were cast. Since 10 beams resulted from each batch (see Fig. 5.2(a)), this means that, in sum, 140 concrete substrates were produced. Of these 14 batches, though, 4 were rejected on the basis of: 1) an improper adherence to a uniform curing scheme - this for batches 1 and 2, as a curing-standard was devised after the second batch, and 2) the proximity of the 28-day compressive strengths of each batch $( \pm 3.45 \mathrm{MPa}= \pm 500 \mathrm{psi})$ about the mean value-here batches 5 and 14 were discarded. The 28-day compressive strengths of each batch were determined from averaged compression test results on three, $102 \mathrm{~mm} \times 203 \mathrm{~mm}\left(4^{\prime \prime} \times 8 "\right)$ cylinders. In all cases, ASTM Type I portland cement was used with a water-cement mixture proportioning ratio of $0.5,6 \%$ air (in accordance to ASTM C231), and locally available natural sand and \#8 pea-gravel aggregates. The mean 28-day compressive strength of the concrete was found to be 51.6 MPa (7480 psi) with a standard deviation of 2.1 MPa (310 psi)—see Table 5.1 (repeated, also in Appendix B as Table B.5).

Table 5.1 Relevant concrete data (no. of batches, w/c ratio, air, strength and std. dev.)

\begin{tabular}{c|c|c|c|c}
\hline \hline $\mathbf{n}$ & $\begin{array}{c}\text { water-cement ratio, by } \\
\text { weight }\end{array}$ & Air content & $\begin{array}{c}\mathbf{f}_{\mathbf{c}}{ }^{\prime}[\mathbf{M P a}] \\
\mathbf{( \mathbf { p s i } )}\end{array}$ & $\begin{array}{c}\boldsymbol{\sigma}[\mathbf{M P a}] \\
(\mathbf{p s i})\end{array}$ \\
\hline 10 & 0.5 & $6 \%$ & $\begin{array}{c}51.6 \\
(7480)\end{array}$ & $\begin{array}{c}2.1 \\
(300)\end{array}$ \\
\hline
\end{tabular}


To reduce the occurrence of cracking within the bulk concrete-substrate during fracture testing, each concrete beam was furnished with a pair of \#2, Grade 60 (i.e. 413.7 $\mathrm{MPa}=60,000 \mathrm{psi}$ ), steel reinforcing bars (see Figure 5.3 below). Prior to casting, a

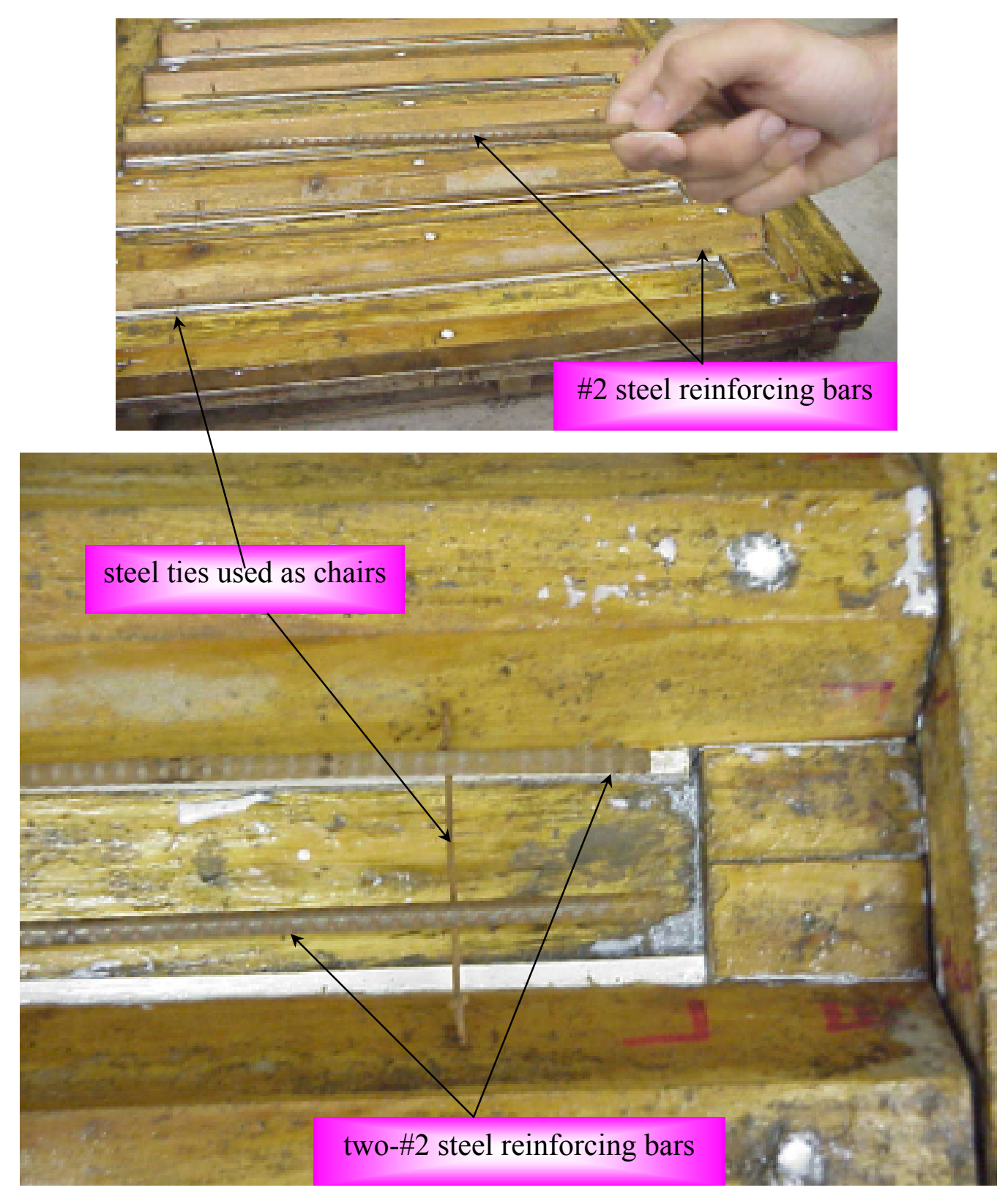

Figure 5.3 Pictures of the steel reinforcing bars and chairs 
water-based mold releasing agent (see Figure 5.4 below) was spread to the interior of the molds to ensure a bonding surface free of any oils that might otherwise impair the adhering-quality of the epoxy when applied to the concrete.

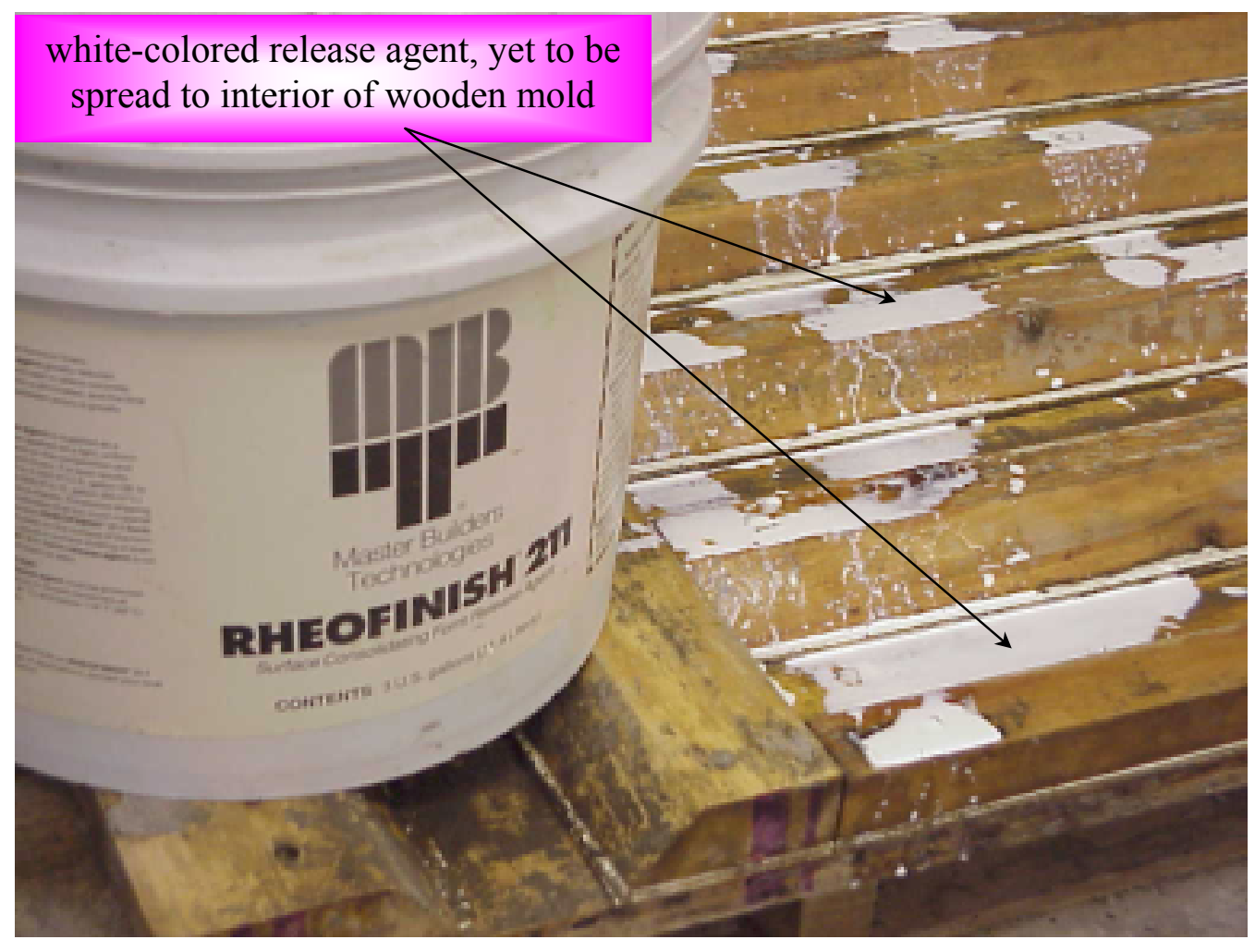

Figure 5.4 Water-based mold releasing agent

Typically, the volume of concrete that was mixed to cast 10 beams and 3 (102 $\left.\mathrm{mm} \times 203 \mathrm{~mm}=4^{\prime \prime} \times 8^{\prime \prime}\right)$ cylinders was about $0.026 \mathrm{~m}^{3}\left(0.917 \mathrm{ft}^{3}\right)$ - the batching-recipe for this blend is given in Table 5.2. After each cell was filled with concrete, a pokervibrator was used for consolidation (see Figure 5.5). Kosmatka and Panarese (p.104, 1990) explain that consolidation is the process of compacting fresh concrete to mold it 
within the forms and around embedded items and reinforcement and to eliminate stone pockets, possible honeycombing, and entrapped air.

Table 5.2 Concrete mixture proportions based on a $0.026 \mathrm{~m}^{3}\left(0.917 \mathrm{ft}^{3}\right)$ volume

\begin{tabular}{l|cc}
\hline \hline Cement & $9.70 \mathrm{~kg}$ & $(21.39 \mathrm{lb})$ \\
\hline Water & $4.89 \mathrm{~kg}$ & $(10.79 \mathrm{lb})$ \\
\hline Coarse Aggregates & $26.20 \mathrm{~kg}$ & $(57.77 \mathrm{lb})$ \\
\hline Fine Aggregates & $18.09 \mathrm{~kg}$ & $(39.89 \mathrm{lb})$ \\
\hline Air-Entraining Admixture & $11.46 \mathrm{~mL}$ & $\left(0.7 \mathrm{in}^{3}\right)$ \\
\hline
\end{tabular}

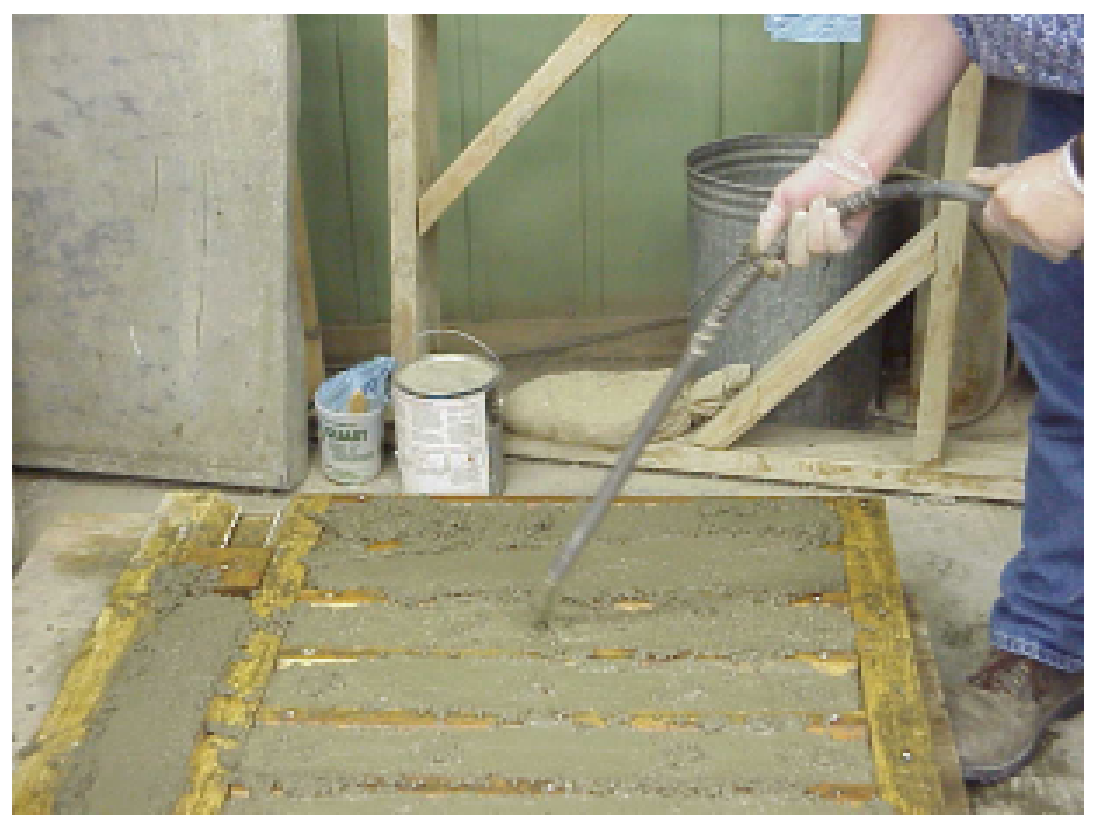

Figure 5.5 Picture of the poker vibrator used for concrete consolidation 
Following vibration, the concrete was trowelled to yield a smooth finish on the bottom of the specimen (see Figure 5.6(a)) - recall that the exposed portion of the concrete in the mold is actually the bottom of the beam. For the first $2-3$ days after casting, the specimens (i.e. 10 beams and 3 cylinders) were left within their molds and covered under wet burlap and thick plastic sheeting. On about the third day, the beams (Figure 5.7) and cylinders were then removed from their respective forms and transferred to a room for continuous curing (at $23 \mathrm{C}=73 \mathrm{~F}$ and approximately $75 \%$ relative humidity) under wet burlap (Fig. 5.6(b)) and thick plastic sheeting for an additional 11 days, followed by 14 days of open-racking within the same room. After the completion of curing, the cylinders were subsequently failed under compression to determine the batch's mean, 28-day, strength (denoted throughout as $\mathrm{f}_{\mathrm{c}}{ }^{\prime}$ ). All these results are presented in Table 5.3. At the same time, the beams, on the other hand, were stacked within the curing room (see Figure 5.8), where they were wrapped securely under thick sheets of plastic to artificially preserve a relative hydration-stage among the specimens cast at different times, and thus minimize the chance of carbonation (i.e. a process in which carbon dioxide $\left(\mathrm{CO}_{2}\right)$ from the air penetrates the concrete and reacts with the hydrates of the cement paste — calcium hydroxide, $\mathrm{Ca}(\mathrm{OH})_{2}$, being the one reacting most readily—to form carbonates (e.g. $\mathrm{CaCO}_{3}$ ). Among the other effects, carbonation increases shrinkage on drying and thereby promotes the development and formation of cracks (Kosmatka and Panarese, p. 72, 1990; and Neville, pp. 497 and 498, 1996). 
(a)

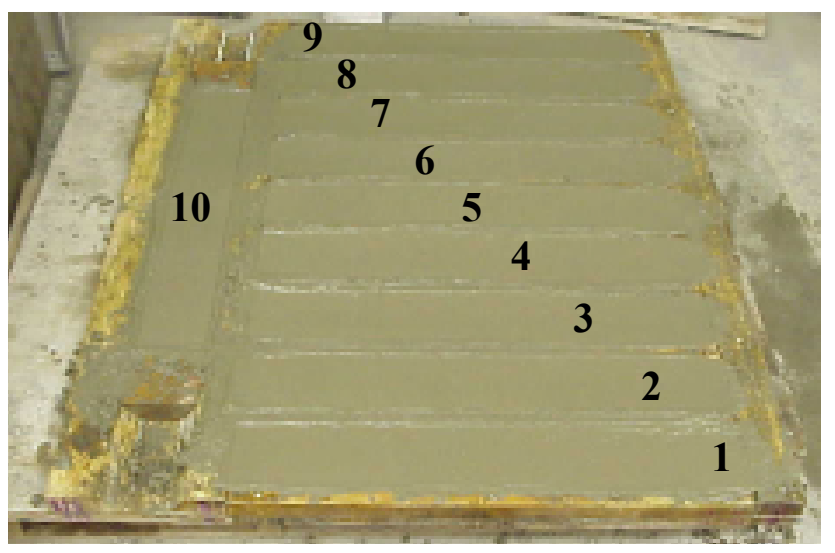

(b)

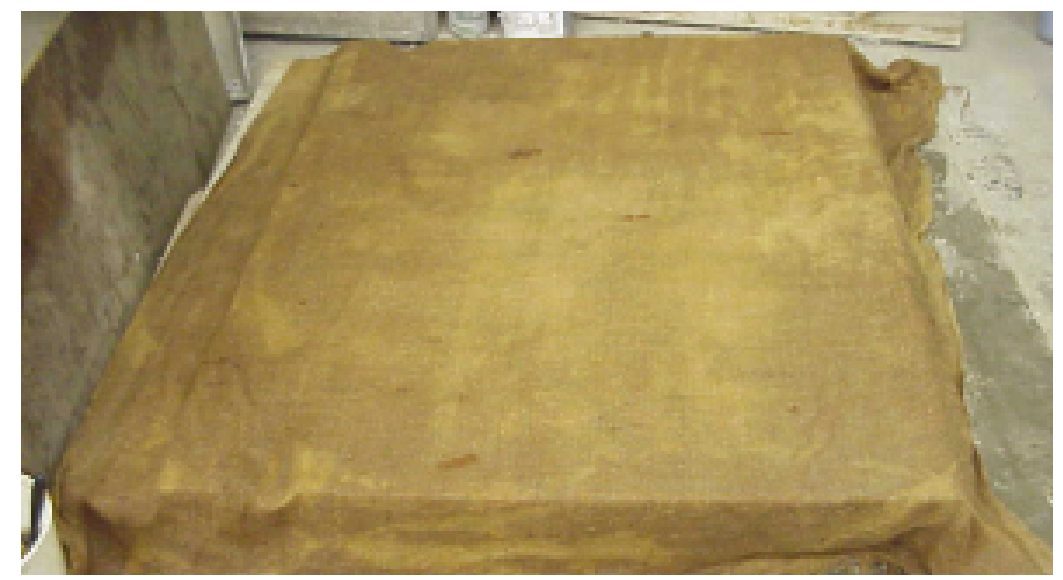

Figure 5.6 Pictures showing the: (a) freshly cast concrete after trowelling, and (b) wet burlap covering of the freshly cast concrete

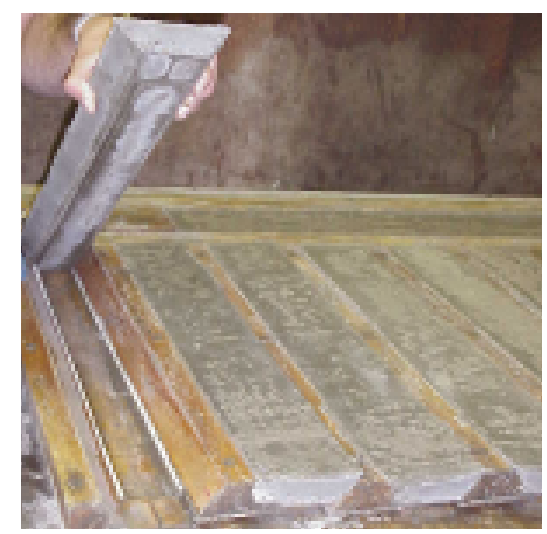

Figure 5.7 Picture of a 3-day old beam being removed from the mold 
Table 5.3 Mean, 28-day, compressive strengths of concrete

\begin{tabular}{l|c}
\cline { 2 - 2 } & $\mathbf{f}_{\mathbf{c}}{ }^{\prime}[\mathbf{M P a}] \mathbf{( p s i )}$ \\
\hline \hline Batch 3 & $50.0(7190)$ \\
\hline Batch 4 & $53.4(7750)$ \\
\hline Batch 6 & $51.3(7440)$ \\
\hline Batch 7 & $47.6(6900)$ \\
\hline Batch 8 & $51.0(7400)$ \\
\hline Batch 9 & $54.6(7920)$ \\
\hline Batch 10 & $50.4(7310)$ \\
\hline Batch 11 & $50.7(7350)$ \\
\hline Batch 12 & $53.2(7720)$ \\
\hline Batch 13 & $53.5(7760)$ \\
\hline Mean (approx.): & $\mathbf{5 1 . 6}(\mathbf{7 4 8 0})$ \\
\hline
\end{tabular}

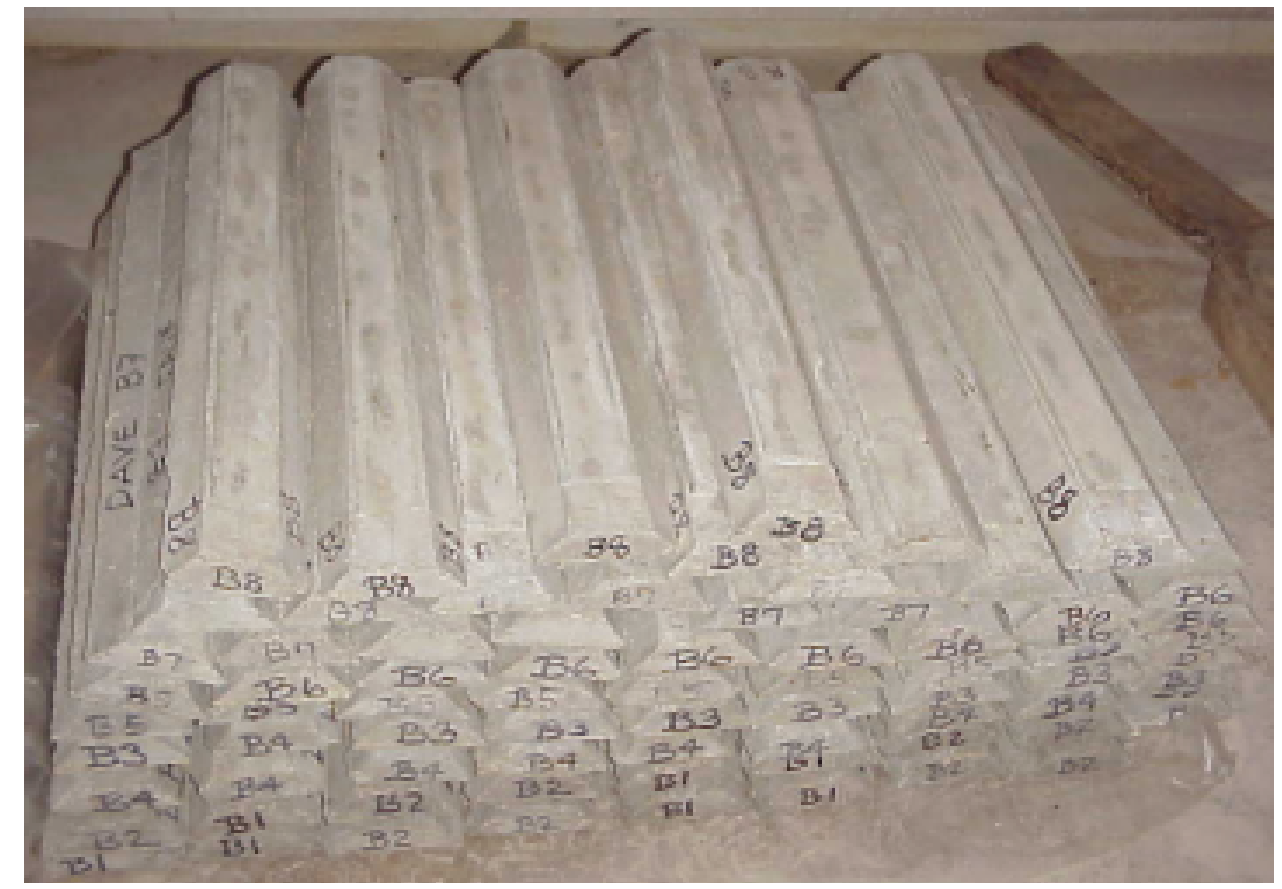

Figure 5.8 Picture of stockpiled concrete-beams after undergoing the 28-day curing regime and before being coated with primer 


\subsection{Contouring}

Preparation of the LVL (Laminated Veneer Lumber, supplied through: Trus Joist MacMillan—see Appendix D) contours entails four basic steps. First, the LVL boards are cut close to the length of the contour using a cross-cutting saw. The width of these boards is such that it was possible to cut two beams from a single section, one inverted from the other (see Figure 5.9). The two opposing longitudinal faces of the rectangular section, then, were conveniently taken as the bonding surface of each beam. Because of this, and the necessity for perfectly level bonding-faces, these two sides were, secondly, jointed until the desired effect was achieved. The degree of levelness was visually inspected by placing the jointed bonding-face on a level surface (e.g. the tabletop of the jointer) and checking that no light shone through from the other side.

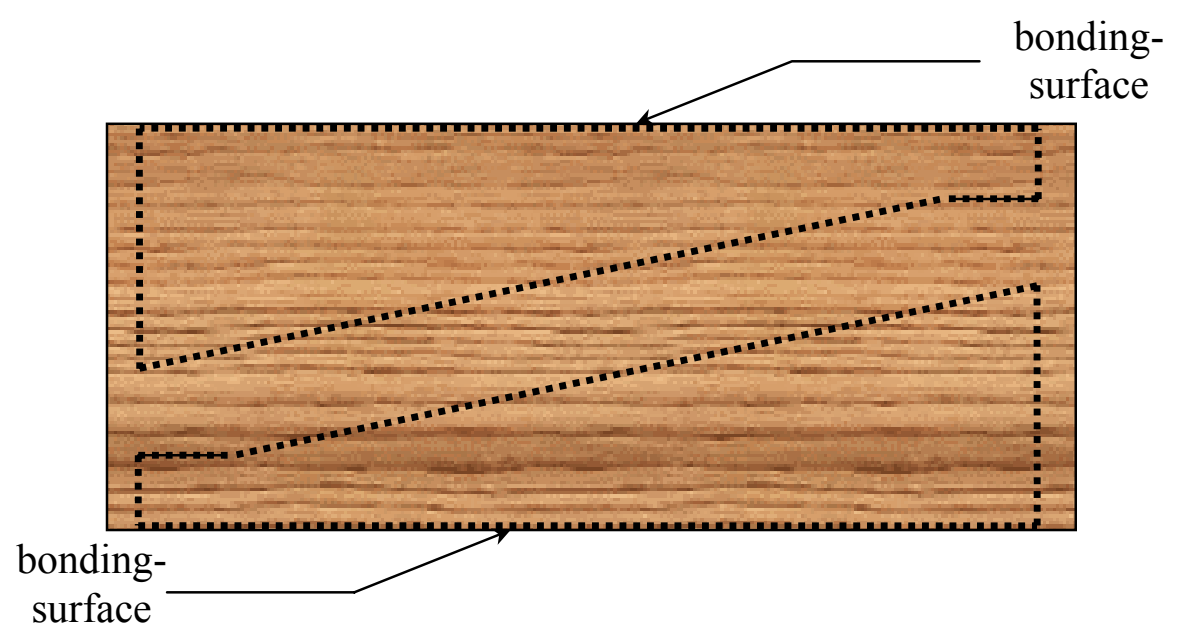

Figure 5.9 Attainment of two contours from a single LVL board

Thirdly, each of these shapes was cut using a band saw. To facilitate in this cutting process, the outline of each contour was traced on the board using a template 
made from cardstock. The template and board were placed upright on a level surface when tracing to ensure that the bottom of the template aligned properly with the jointed bonding surface. Lastly, each of the beams underwent jointing once more in three locations. The first and second of these places were on the two opposite edges of each bonding-face which were slightly chamfered at $45^{\circ}\left(3 \mathrm{~mm} \times 3 \mathrm{~mm}=1 / 8^{\prime \prime} \times 1 / 8^{\prime \prime}\right)$ by the jointer to induce higher concentrations of stress and, thereby, promote the issuing of cracks at the interface location (see Figure 5.10(b)). The third and final place to be jointed, involved the lead $51 \mathrm{~mm}(2 ")$ of the bonding surface of each contour (see Fig. $5.10(a))$. This area was slightly notched to a depth of $3 \mathrm{~mm}\left(1 / 8^{\prime \prime}\right)$ to both, accommodate the load strap and, more significantly, enhance the initiation of the starting crack during fracture testing.

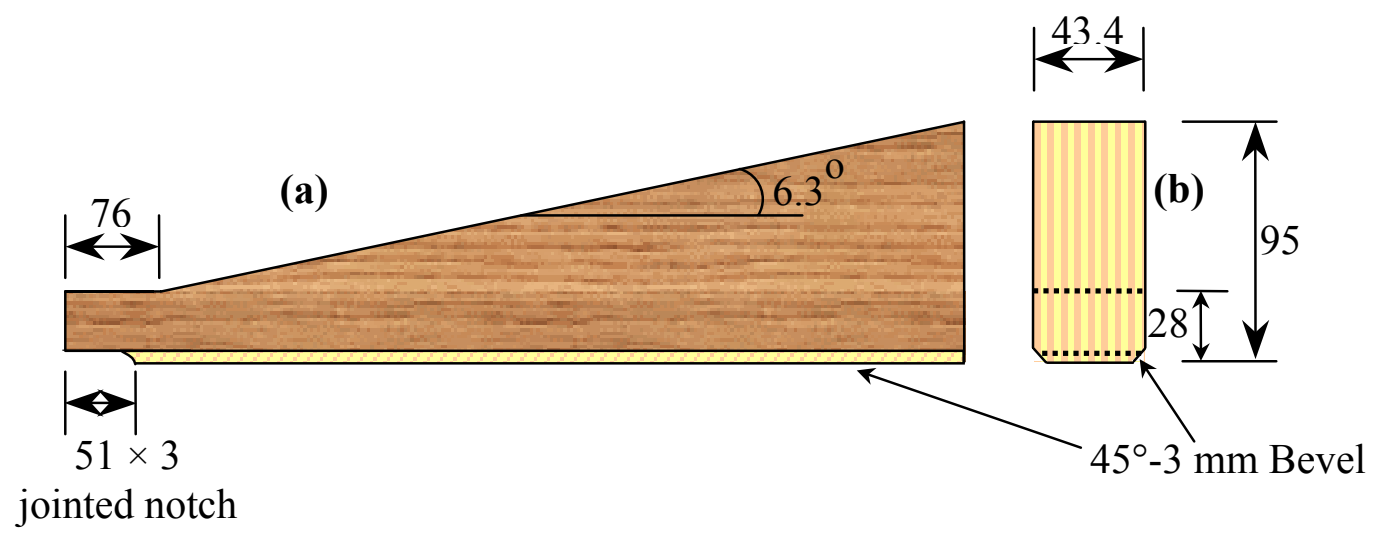

Figure 5.10 The finished LVL contour (a) elevation-view, and (b) back-view (NOTE ${ }_{1}$ : All dimensions in millimeters)

(NOTE 2 : LVL material properties given in Table B.1) 


\subsection{Bonding}

The MBrace Adhesive System

The adhesive system used for this study is an MBrace product consisting of two components: a primer and a saturant (see Figure 5.11, parts (a)/(b) and (c)/(d), respectively). Each of these components, in turn, comes in two parts: part A/part B (Fig. 5.11, parts (a) and (c)). While both the primer and saturant are basically epoxy resins,

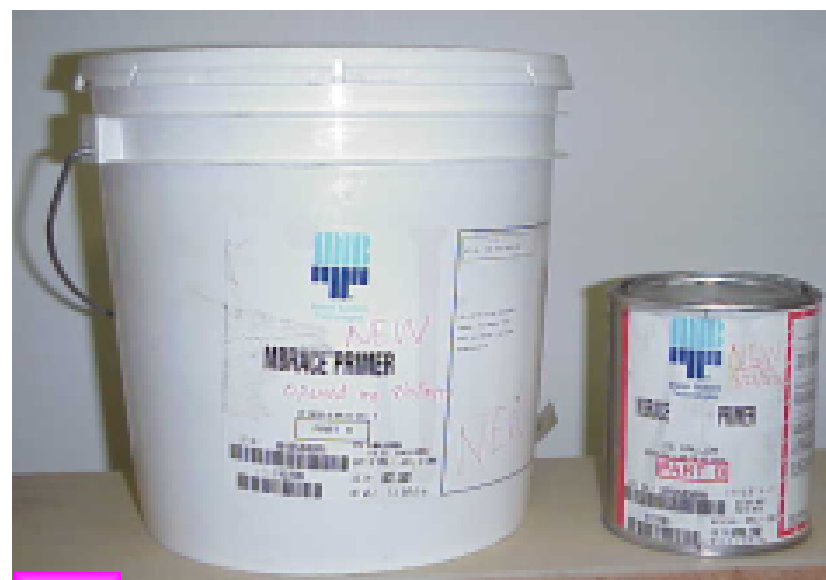

(a)
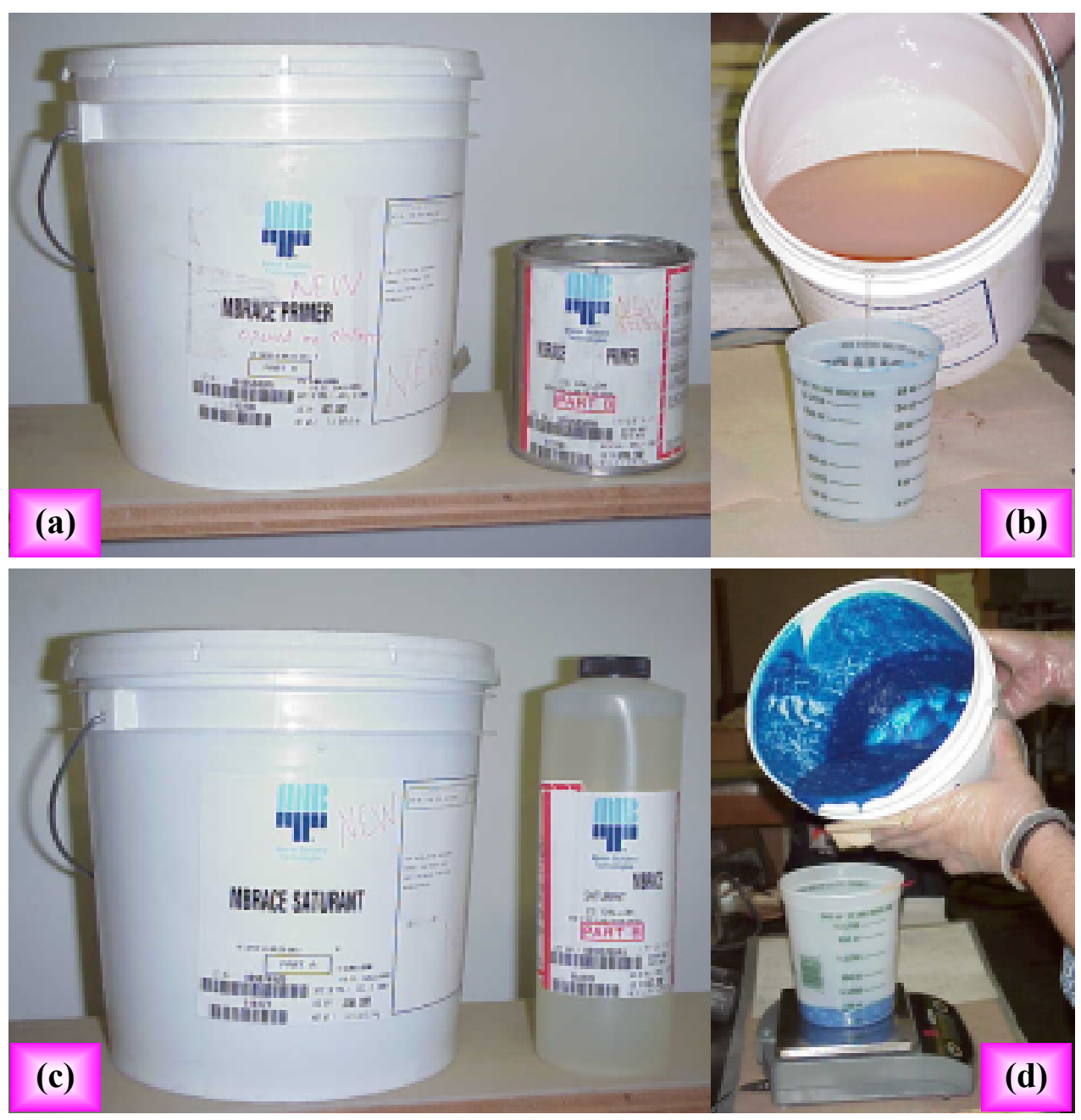

Figure 5.11 The MBrace adhesive system - (a) Primer, parts $A / B$, (b) color of part A (primer), and in fact, that of mix: amber; (c) Saturant, A/B, (d) color of part A (saturant), and in fact, that of mix: dark-blue 
they each serve different purposes. The former is applied to the concrete base material to penetrate pores and promote adequate bond (MBrace Composite Strengthening System, p. $3-2,1998)$. In other words, the primer is used as a coupler between the concrete-substrate and the saturant-impregnated carbon fiber tow overlay that is eventually applied. (Particular to this research, the primer was found to have two additional uses. First, it was discovered that its coupling-abilities were not restricted to just that of concrete and the wetted-CF material, but that it coupled the LVL wooden contours remarkably well to the CFRP-bonded concrete beams. The significance of this is one of convenience, since only a single adhesive system was used to bond the fabric to the concrete and the contours to the CFRP-bonded specimens. Second, except for the interface region, the primer was applied to all other surfaces of the concrete beam to protect the bulk-matter from chemical-impairment during subjection to the various environmental conditioning schemes called for in Chapters 7 and 8 . Both of these uses are also confirmed by ACI committee report 503, titled "Use of Epoxy Compounds with Concrete" - the first, in section 5.4 .5 (p. 13), and the second, in section 4.2.1 (p. 9), 1993). The saturant, on the other hand, is the glue that fastens the carbon-fiber fabric to the concrete, and thus establishes the external reinforcing composite system. Other distinguishing features between the primer and saturant include color, the former has an amber-hue while the latter is darkish-blue (see Fig. 5.11, parts (b) and (d)), viscosity, $400 \mathrm{cP}$ versus $1350 \mathrm{cP}$, respectively, and several differences in material properties, as displayed in Tables 5.4 and 5.5. $\left(\mathrm{A} \mathrm{cP}=\right.$ centipoise $=$ one-hundredth of a poise $=10^{-2} \mathrm{dyne} \cdot \mathrm{s} / \mathrm{cm}^{2} ; 1 \mathrm{~N}=100,000$ dyne; for comparison, water has a viscosity of $1 \mathrm{cP}$.) 
Table 5.4 MBrace primer material properties

\begin{tabular}{c|c|c|c}
\hline \hline E [GPa] (Msi) & $\begin{array}{c}\text { Yield Stress [MPa] } \\
\text { (psi) }\end{array}$ & Yield Strain & Poisson's Ratio, v \\
\hline $0.72(0.104)$ & $14(2100)$ & $4 \%$ & 0.48 \\
\hline
\end{tabular}

Table 5.5 MBrace saturant material properties

\begin{tabular}{c|c|c|c}
\hline \hline E [GPa] (Msi) & $\begin{array}{c}\text { Yield Stress [MPa] } \\
(\mathbf{p s i})\end{array}$ & Yield Strain & Poisson's Ratio, $v$ \\
\hline $3(0.440)$ & $54(7800)$ & $2.5 \%$ & 0.40 \\
\hline
\end{tabular}

\section{Surface Preparation}

An important measure that was taken, having a directly positive consequence on the performance of the adhesive system, was the preparatory-attention given to the concrete surfaces before the first dollop of primer was applied. In particular, these surfaces were thoroughly sanded to remove any local irregularities, defects, and laitance that might otherwise be clogging the pore structure within the concrete. Figure 5.12, parts (a) and (b), shows a before and after shot of a beam that was just sanded. Notice, especially, the white powdery film that is absent from the bonding surface after sanding. Fig. 5.12(c) shows just how much of this material is removed from a typical sanding operation. The purpose for uncovering these apertures is to enable the primer to 
sufficiently penetrate the substrate and, as such, effectively couple it with the saturant that is to be applied at a later stage.

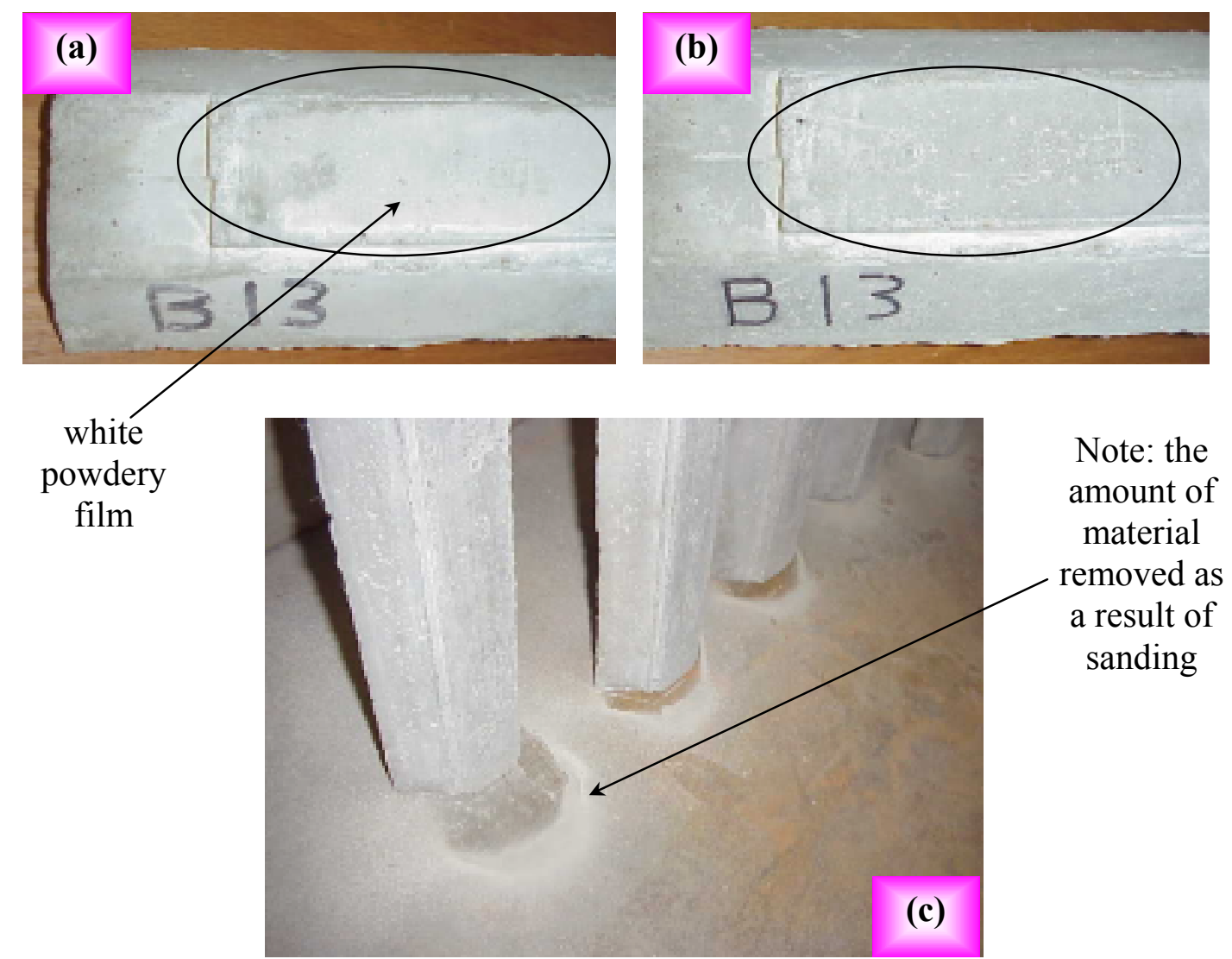

Figure 5.12 (a) Before-sanding, and (b) after-sanding, pictures of the same concrete beam; (c) A picture depicting the amount of loosened particles removed as a result of sanding

A pressurized air-hose was used to remove the bulk of the particles loosened as a result of sanding. The remaining residue was then cleaned by gently wiping the bonding surfaces with acetone-dampened paper-towels. Sand-blasting and grinding, the most 
common operations adopted during the repair of deteriorated surfaces, were not followed in this case because the specimens were molded under laboratory-controlled conditions with the bonding surfaces facing the mold, as explained in Section 5.2.

\section{Priming}

To begin the priming process, a long, flat surface, e.g. a banquet table, is lined with 2 - 3 layers of wax paper. (Note: Epoxies do not adhere to wax or Mylar surfaces. For this reason, wax paper is used in all of the priming and saturating processes to be discussed. This not only protects the underlying surface from messy spills but safeguards the edges of the beams from becoming fixed to the table when the resin collects at these locations.) Next, the beams are placed on this surface, leaving enough of a clearance in between each successive specimen so that the sides can be comfortably accessed with paintbrush in hand-say about $100 \mathrm{~mm}$ - $150 \mathrm{~mm}$ (approximately 4" - 6"). Due to the nature of the priming and saturating work, wherein the bristles of the paintbrush harden after approximately 24 hours of exposure - and this, when attempts have been made to clean the bristles, and the brush itself is bathed in a tub of acetone overnight - the most inexpensive types of brushes were therefore used to accomplish the work. For this purpose, disposable, wood chip paintbrushes were found to be most economical.

Also, the width of the brush used to paint the critical bonding surface was selected to be less than the width of the bonding surface itself (a $25 \mathrm{~mm}=1$ " brush was chosen; see Figure 5.13(a)). This measure was taken to reduce the occurrence of the primer and saturant from seeping over the edge of the bonding surface and onto the raised-step and ledge region when being brushed on the concrete - the only places on the entire beam 
that were not to be coated with resin, whatsoever. While the remainder of the beam was primed, this critical interface area was purposely left exposed to focus the attack of all the aggressive media (see Chapters 7 and 8) to this region of interest (see Figure 5.14). This way, the bulk matter of the concrete substrate is expected to remain intact, at least relatively speaking, after weathering the worst combinations of attack designed. A brush of triple the width $(75 \mathrm{~mm}=3 ")$ was used on the undersides of the beams (see Fig. 5.13(b)) to facilitate in the spreading of the primer to this wider surface.
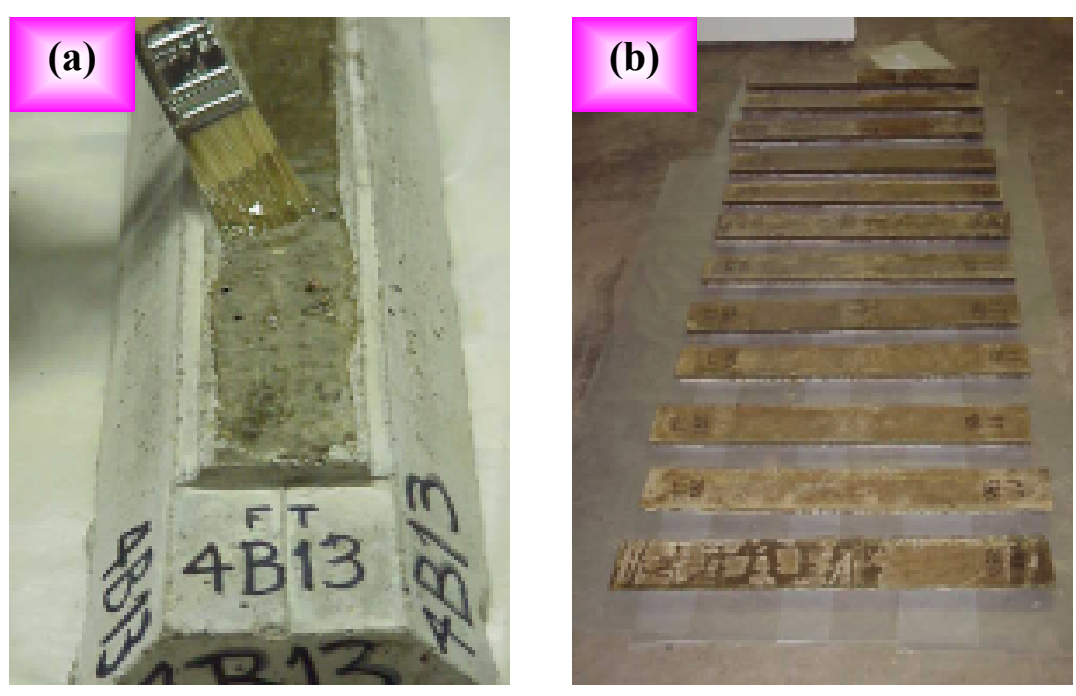

Figure 5.13 Priming of: (a) bonding surface, and (b) undersides of beams

As already mentioned in "The MBrace Adhesive System" subsection, the primer comes in two parts: part A/part B (see Fig. 5.11(a)). The mixture-ratio for these two parts is as follows: volumetrically, $\mathrm{A} / \mathrm{B}=3 / 1$, or by weight, $\mathrm{A} / \mathrm{B}=100 / 30$. While the manufacturer offers two approaches for measuring the part A/part B portions, the author 
believes, from personal experience, that for experimental purposes, only the weight measurement should be used. The reason for this is that experimental work often requires small quantities of resin to be mixed, and slight variations in these portions may be critical to the success and overall performance of the epoxy-field applications, on the other hand, usually involve vast amounts of resin-mixtures and so volumetric measurements may be more than adequate. For this reason, a digital balance, sensitive to a tenth of a gram, was used to measure each of the part A/part B portions (see Fig. 5.11(d)). This blend would then be vigorously hand-mixed for five minutes. The average measured coverage rate for the primer was found to be about $6 \mathrm{~m}^{2} / \mathrm{L}\left(275 \mathrm{in}^{2} / \mathrm{oz}\right)$ with a resulting cured thickness of approximately $0.0762 \mathrm{~mm}(3 \mathrm{mils} ; 1 \mathrm{mil}=$ a thousandth of an

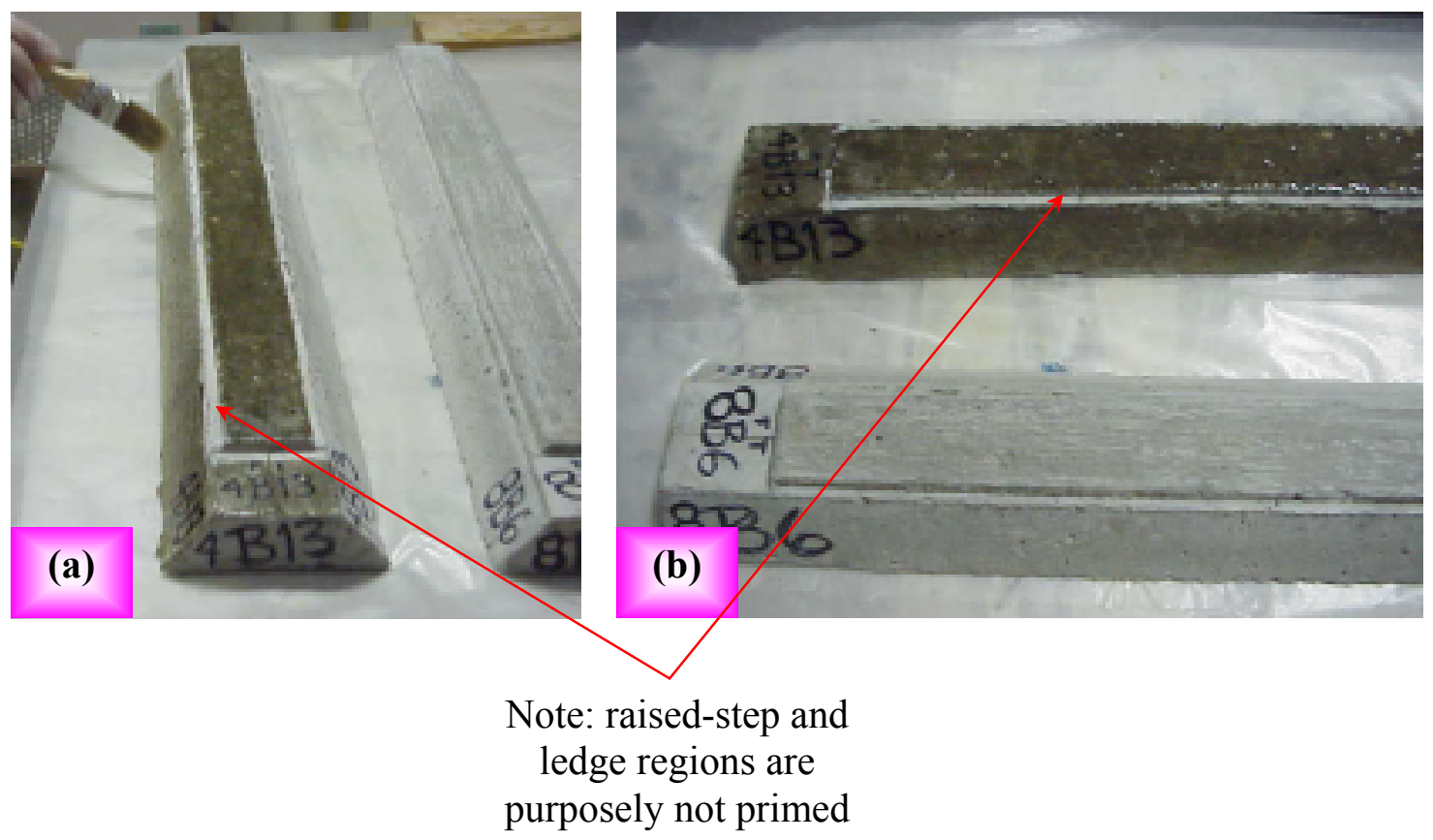

Figure 5.14 (a) Priming process, and (b) contrast of primed/unprimed beams 
inch). The working time of the primer was found to last approximately 20 - 30 minutes. All this information is presented in Table 5.6. To avoid major variations in quality and rates of coverage during the assembly of all the test specimens, the workmanship of only one individual was relied upon throughout. Pictures of the priming process are given in Fig. 5.14. Apart from the concrete beams, the only other material to receive priming

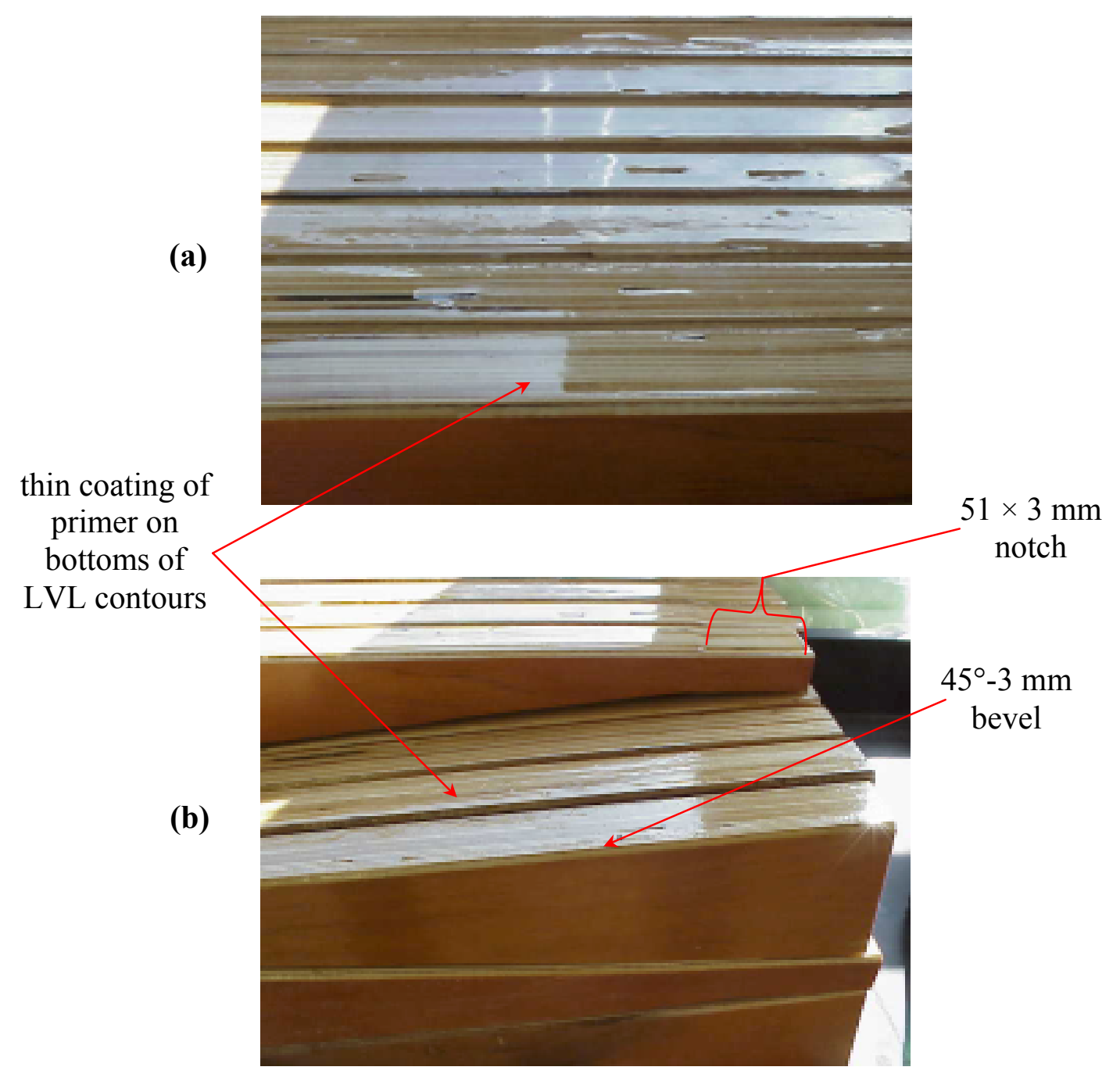

Figure 5.15 Bottoms of LVL contours with light coating of primer-(a) closeup view, and (b) undersides displaying bevel and joint details 
was the LVL wooden contour. The bottoms of the contours received a thin coating of primer (see Figure 5.15) as preparation to couple it to the saturant used during final SCCB specimen assembly (see next subsection).

\section{Saturating}

Typically, the priming process would involve two days wherein the top and bottom surfaces of the concrete beams would be coated, respectively. So the saturating step could not begin until the third day. At this time, the beams would first be freed from the underlying wax paper, and any excess primer that might have collected in certain locations throughout the night would be removed using a sharp utility knife. The knife was also used to scrape-off any primer that might have spilled over onto the restricted raised-step or ledge regions. If needed, new sheets of wax paper would be placed atop the old ones and the beams would be aligned, as before, with some space left in between each successive specimen $(100 \mathrm{~mm}-150 \mathrm{~mm}$, i.e. about $4 "-6 ")$ - the exception being the front part of the table $\left(50 \mathrm{~cm}\right.$, i.e. about $\left.1.5^{\prime}\right)$. This region would intentionally be left unoccupied to carry-out the saturating of the CFRP strips. Next, pre -measured and -cut CFRP strips and the doubled-over wax papers for the starter-cracks would be placed alongside each specimen.

The saturant, as with the primer, comes in two parts: part A/part B (see Fig. 5.11(c)). The mixture-ratio for these two parts is as follows: volumetrically, $\mathrm{A} / \mathrm{B}=3 / 1$, or by weight, $\mathrm{A} / \mathrm{B}=100 / 34$. Again, as per the author's experience, only the weight measure should be used for experimental purposes (read the explanation given in the previous 
subsection). The blend would then be vigorously hand-mixed for five minutes. The average measured coverage rate for the saturant was found to be about $1.2 \mathrm{~m}^{2} / \mathrm{L}$ (55 $\left.\mathrm{in}^{2} / \mathrm{oz}\right)$ with a resulting cured thickness of approximately $0.508 \mathrm{~mm}(20 \mathrm{mils} ; 1 \mathrm{mil}=\mathrm{a}$ thousandth of an inch). All this information is presented in Table 5.6. In the author's experiences, the working time of the saturant was found to last approximately $20-30$ minutes, as was the case with the primer, though the manufacturer states the working time of the saturant to be slightly longer at about 45 minutes. Of course these times can vary somewhat depending on the temperatures under which the epoxies are stored, the current temperature of the room, the quantity that is being used at any given time, the care with which each of the parts, A and B, are measured, and how thoroughly the two parts are mixed prior to application.

Table 5.6 Color, mix, coverage, thickness and working time of MBrace resins

\begin{tabular}{l|c|c}
\cline { 2 - 3 } \multicolumn{1}{c|}{} & Primer & Saturant \\
\hline Color & & \\
Part A & Amber & Blue \\
Part B & Clear & Clear \\
Mixed & Amber & Blue \\
\hline Volume Mixture Ratio, A/B & $3 / 1$ & $3 / 1$ \\
\hline Weight Mixture Ratio, A/B & $100 / 30$ & $100 / 34$ \\
\hline Average Coverage Rate & $6 \mathrm{~m}^{2} / \mathrm{L}\left(275 \mathrm{in}^{2} / \mathrm{oz}\right)$ & $1.2 \mathrm{~m}^{2} / \mathrm{L}\left(55 \mathrm{in}^{2} / \mathrm{oz}\right)$ \\
\hline Cured Thickness & $0.0762 \mathrm{~mm}(3 \mathrm{mils})$ & $0.508 \mathrm{~mm}(20 \mathrm{mils})$ \\
\hline Working Time & approx. $20-30 \mathrm{mins}$. & approx. $20-30 \mathrm{mins}$. \\
\hline
\end{tabular}

The saturation process would begin by painting a thin layer of the blue-colored adhesive onto the concrete bonding surface, as shown in Figure 5.16, parts (a) and (b). 

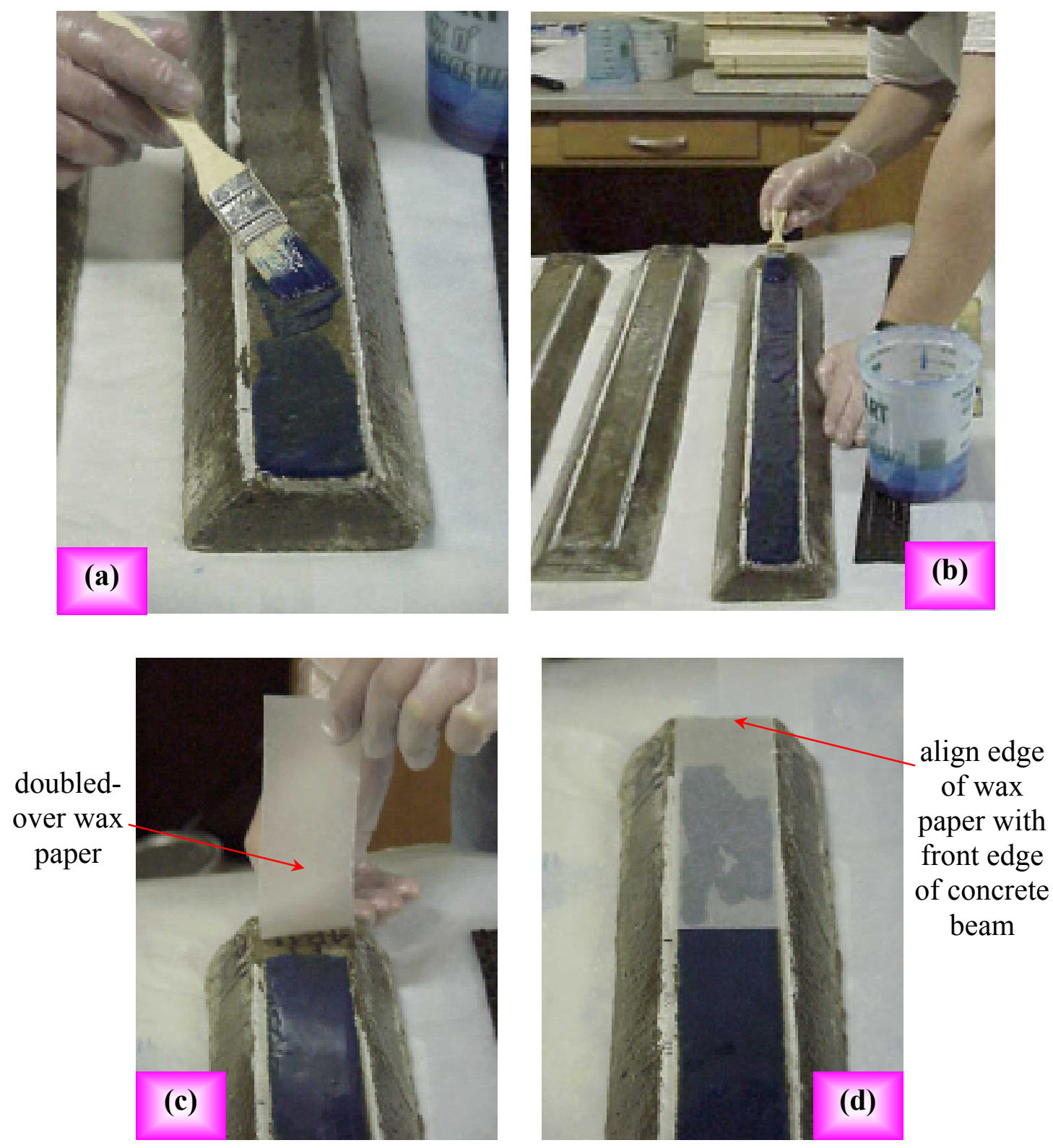

Figure 5.16 (a) Close-up, and (b) overall view of the saturation of the concrete bonding surface; (c) process of laying-down the doubled-over wax paper, and (d) a view of the wax-paper in place

Once again, a $25 \mathrm{~mm}(1 ")$ paintbrush was used and care was taken to prevent the resin from spilling over onto the raised-step and ledge regions. After the concrete bonding 
surfaces were covered, the doubled-over wax papers were placed atop this wetted region (see Fig. 5.16, parts (c) and (d)). These strips were pre-measured in such a way so as to preclude any need for measuring at the time of placement—all that was required was for the end of the paper to align with the front edge of the concrete beam (see Fig. 5.16(d)). After this, the CFRP strips were taken one-by-one from beside the beams and saturated, front and back (see Figure 5.17—see also the fabric pattern left behind on the wax paper after saturation, part (b)), and then carefully placed onto the concrete bonding surface and wax paper while checking for proper alignment. After a period of 30 minutes, a special
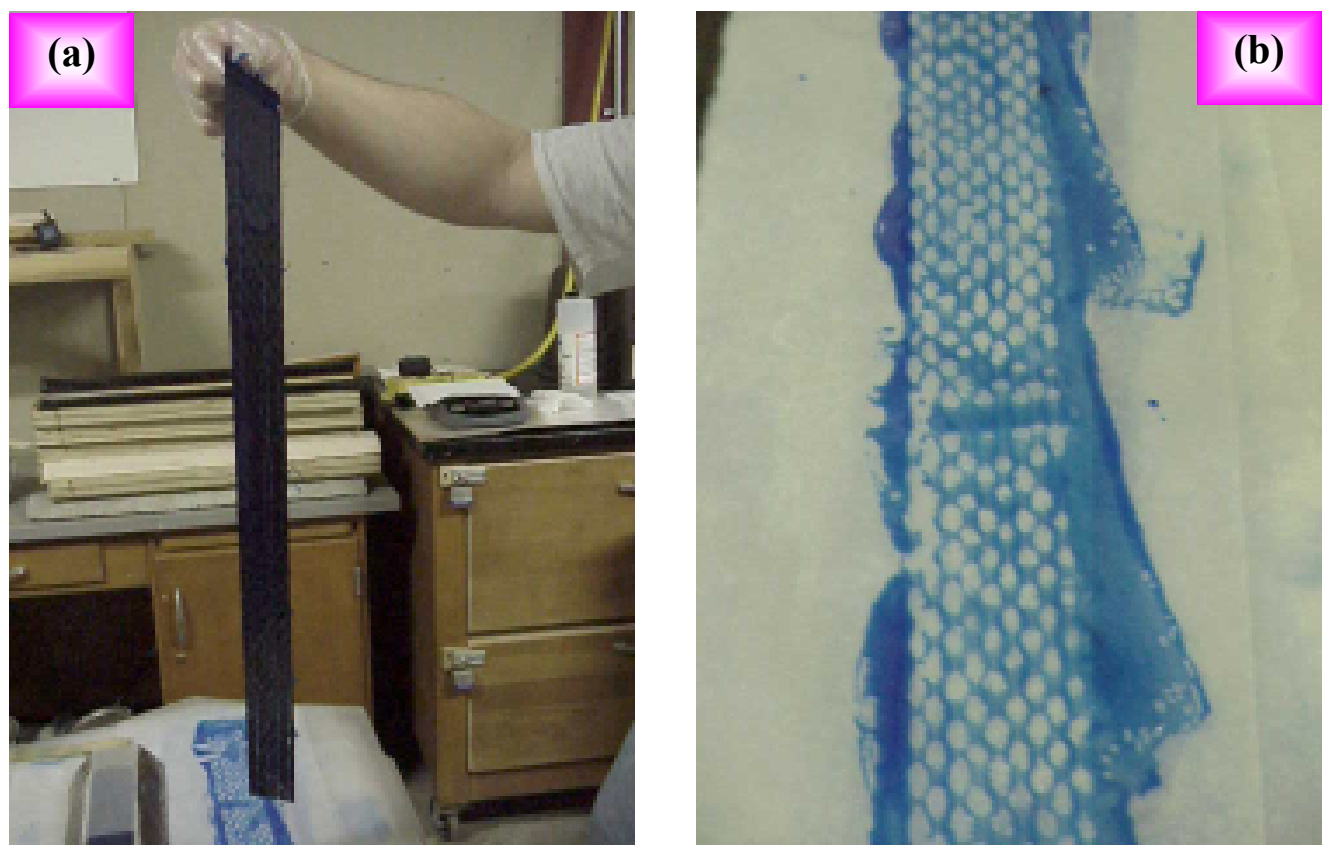

Figure 5.17 (a) Saturated CFRP-strip, and (b) the CFRP-fabric imprint as transferred to the underlying wax paper 
steel ribbed-roller (supplied by Master Builders, Inc.- - see Figure 5.18(a)) was applied over the semi-fixed CFRP strips in the longitudinal direction to separate the fibers and allow for better infiltration and impregnation by the saturant. Then a fresh batch of saturant was mixed and another coating was brushed on each beam (Fig. 5.18(b)). This completes the assembly of the CFRP-bonded concrete specimens. All that is required now is for the saturant to cure properly - this is usually achieved after about a 24-hour period. Thus, the following day, the CFRP-bonded concrete specimens can begin undergoing the battery of conditioning schemes planned, i.e. wetting-drying and freezingthawing (see Chapters 7 and 8) - this, of course, after initial readings have been taken.
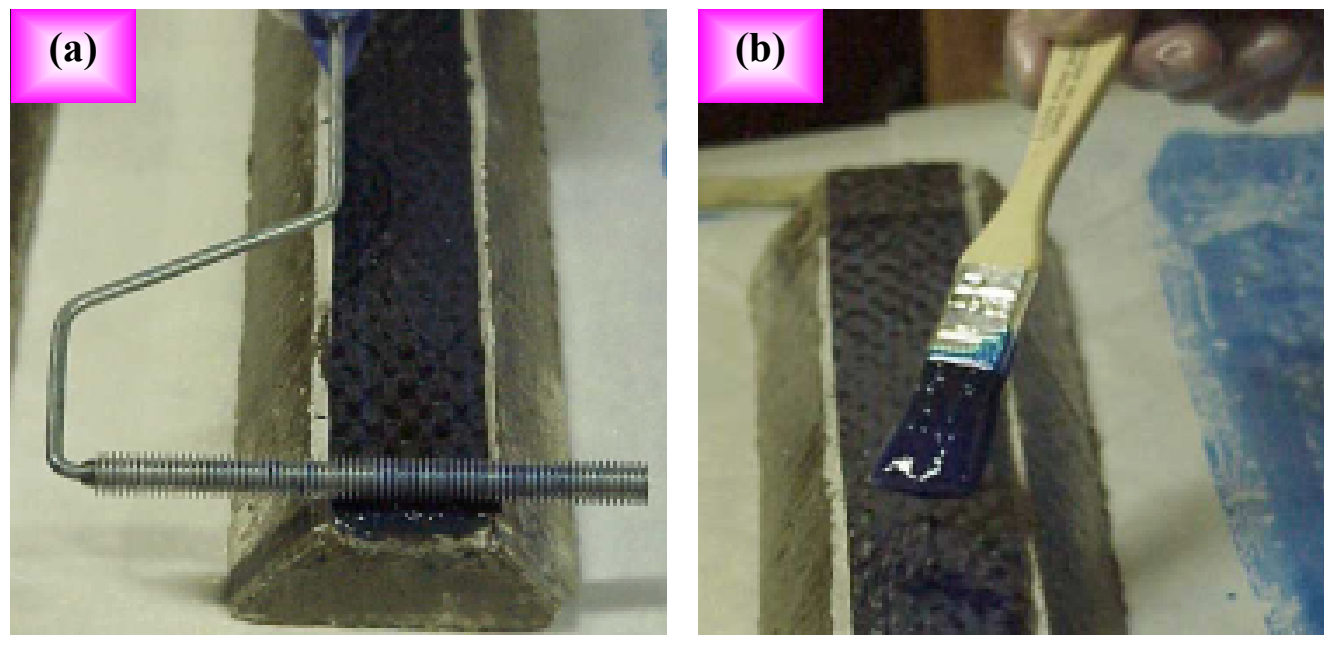

Figure 5.18 (a) The steel ribbed-roller, and (b) another coating of saturant applied after 30 minutes 
The actual SCCB specimen assembly does not take place until after the designed conditioning age has been attained and final readings have been taken. To begin this process, the CFRP-strips are first thoroughly belt-sanded (see Figure 5.19) — make sure that the depth of sanding, however, does not extend into the fibers themselves. Note, some of the beams have been outfitted with steel locating discs for the purpose of measuring strains (see Ch. 6, especially Fig. 6.8(b)) — these discs must first be removed. This is easily accomplished by placing the flathead portion of a screwdriver against the disc, laterally, and then gently tapping the handle with a hammer. A few gentle taps is usually all that is required to pop the disc off the CFRP-strip.

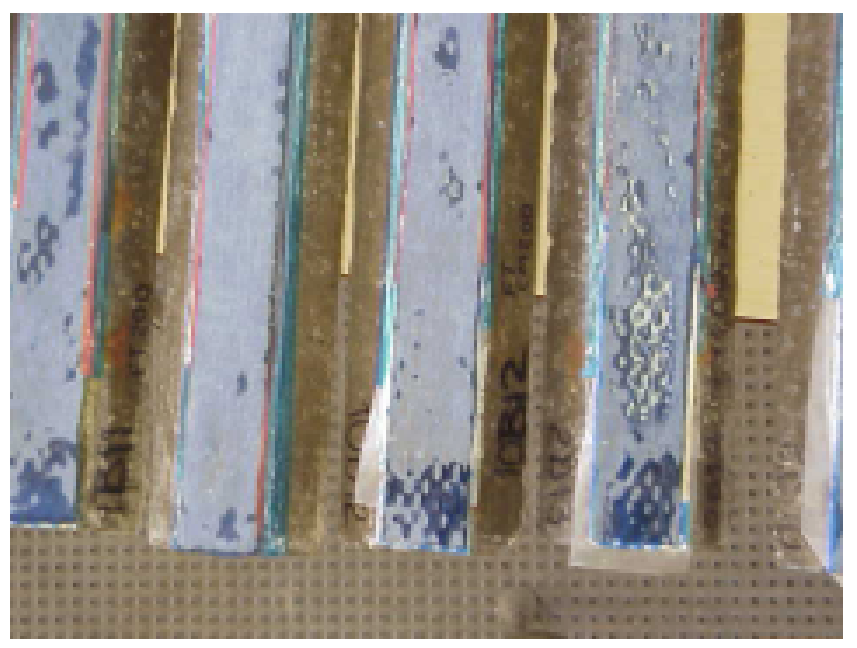

Figure 5.19 Belt-sanded CFRP strips

After the CFRP strips have been sanded, it is important to form some kind of barrier at the raised-step and ledge regions prior to the bonding of the LVL contours. The reason for doing this is to prevent any saturant from spilling over into these areas and, in 
a sense, re-strengthening the interface that had been conditioned and weathered for so long - this would truly defeat the purpose of the cycling regime in the first place. Two barrier methods were tried. The first involved the use of Mylar release tape (Figure 5.20(a)—supplied by Richmond Aircraft Products, see Appendix D). A couple of the problems experienced with using Mylar tape include: (1) the tape, at some points, starts detaching, and (2) since some of the bonding surface is used to secure the tape, the effective bonding width for the LVL slightly decreases. This latter point should not be a problem provided the LVL contour does not become detached during fracture testing. The second barrier method that was attempted made use of butcher's wax (Fig. 5.20(b) available at any hardware store). Special care must be taken, here, that none of the wax, or the oily residue that results from the spreading of the wax, gets onto the bonding surface.
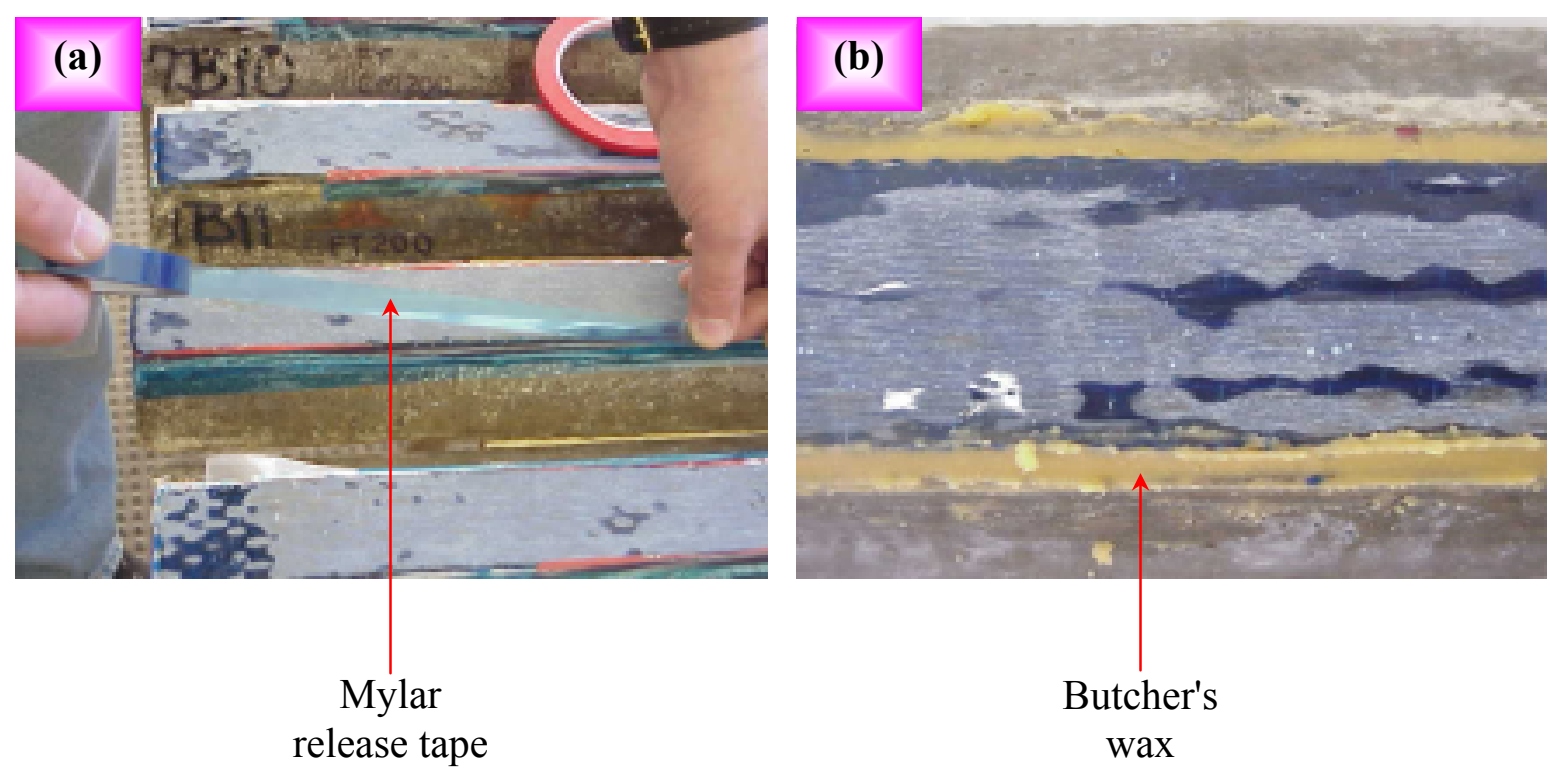

Figure 5.20 Methods to protect the concrete raised-step and ledge from becoming infiltrated with saturant-(a) Mylar release tape, and (b) butcher's wax 
An idea that was not attempted at the time, but may be worth trying, is to purchase Mylar release tape of the same width as the bonding surface and completely cover this area before applying the butcher's wax onto the raised-step and ledge regions. The tape can then be stripped-off before the LVL contours are to be bonded.

Once the concrete raised-step and ledge regions have been sufficiently secured against saturant infiltration, the LVL contours may then be bonded. Mix a batch of saturant in accord with the weight ratio given in Table 5.6- once again, vigorous handmixing for 5 minutes is adequate. Then with a $25 \mathrm{~mm}(1 ")$ wide paintbrush, spread the saturant onto the bonding surfaces of each beam and on the undersides of the LVL contours - use generous amounts of saturant this time so as to avoid places of inadequate wet-out. Lastly, place the contour onto the bonding surface, one at a time, and make sure that everything is aligned properly. It is not unusual to have to make slight readjustments to the contours several times until they align properly. The best way to ensure proper alignment is to visually sight along the juncture formed at the bottom of the contour and the top of the bonding surface - sighting from front to back, and side to side - to see that these two edges are evenly positioned. This, then, completes the assembly of the SCCB specimen. Fracture testing may therefore commence after a 24-hour period, at which time the LVL contour should have become fixed to the CFRP-bonded concrete beam. One way to ensure that the saturant has properly cured is to touch the adhesive at the interface. If it feels somewhat tacky to the touch, then more time should be given for the glue to cure. A saturant that has sufficiently set should feel hard and tough to the touch. 


\subsection{Summary Remarks}

Because this work is largely experimental-based, the importance of skill in assembling the specimens can not be overemphasized-it is precisely here that the crossroads of success and failure meet. Skill is a quality that often depends on experience and practice. It is therefore advisable that before the final experimental-specimens are assembled, some mock-beams be fabricated for practice. Going through such a couple of iterations will undoubtedly reveal some mistakes being made along the way as well as alert the individual to better techniques of craftsmanship to try in the future.

In this vein, then, a couple of observations made by the author over the course of this work, are offered as advice to those endeavoring to undertake a similar line of research. The first remark, also commented in Chapter 7 where attempts are made at comparing specimen-effects arising from the same concrete-batch, is to restrict the number of batches made in the first place. Concrete, in and of itself, is already a highly complex material to work with, and having 10 different batches in a research protocol, as this, only acts to further complicate matters. Elimination of this parameter is, therefore, something to be greatly desired. A second and final remark, is to give heed to the thickness of the glue-line. As mentioned earlier, the workmanship of only one individual (yours truly) was relied upon throughout to avoid major variations in quality and coverage-rates during assembly. It would be more advantageous if some type of jig could be devised to consistently spread the same amount of primer and saturant at the interface for each of the beams, and thereby exclude another factor known to confound studies of interface fracture toughness. 


\section{CHAPTER 6}

\section{DRY FRACTURE}

\subsection{Introduction}

Mode I CFRP-concrete interface fracture results, as achieved by use of the SCCB methodology (see 3.1 and 3.6), are presented in this and the following next two chapters. The objectives, herein, are to determine the critical strain energy release rates (Eq. (3.73)) of (a) pristine and (b) representative dry (i.e. unweathered) specimens. Chapter 7 extends the above objectives to investigate the durability of interfaces as subject to wet-dry cycling, the wetting-phase of which entails immersion of the specimens in two separately aggressive media-half in sodium-sulfate and the other half in sodium-hydroxide. Freezing and thawing in a mixture of calcium chloride is the subject of chapter 8 and the final conditioning scheme of interface-exposure considered in this study.

Four pristine, or virgin-state, specimens, just mentioned above, are considered first (Section 6.2), from which the derived interface fracture values are used to define the performance of the freshly-applied external CFRP material to an unadulterated concrete-

substrate. Another use of the values of these beams, as will be seen in the following chapter, is that they are actually substituted in lieu of the $10^{\text {th }}$ cycle companion values 
(see Ch. 7 for further details) when percentage reductions in fracture toughness are computed for the wet-dry specimens of that age.

Additionally, a representation of dry interface fracture is obtained (Section 6.3) from a pool of 20 separate specimens -4 from the pristine cases just mentioned, and 4 and 12 from the wet-dry and freeze-thaw companion beams, respectively. Companion specimens are those that have not been subjected to any aggressive media, but instead were fabricated at the same time as their weathered counterparts and isolated in a controlled environment for the duration of each target conditioning stage, at which time they were fractured alongside the aged beams. Thus, the percentage reductions in fracture toughness is computed relative to the companion sample values of the same age - the one exception being the $10^{\text {th }}$ cycle wet-dry companion results as mentioned in the previous paragraph.

During fracture testing, simultaneous load and crack opening displacements (COD) are recorded at every one-fifth of a second by a data acquisition system, with an actuator speed of $0.0254 \mathrm{~mm} / \mathrm{s}(0.001 \mathrm{in} / \mathrm{s})$, and as such, possibly tens-to-hundreds of loads are collected that correspond to a single specimen. When Eq. (3.73) is inspected, however (repeated here as (6.1) for convenience),

$$
G_{I c}=\frac{P_{c}^{2}}{2 b} \frac{d C}{d a}
$$

the fundamental question becomes: Which among these numerous loads is critical? A generic attempt at answering this significant question, based mostly on theory, was made in Section 3.4, although a specific and conclusive method was withheld until now, wherein actual raw data will be used to illustrate this procedure. Succinctly, this method 
is a statistical-approach that considers a one-standard deviation about the mean of the raw head loads (to be explained shortly) from which two separate analytical cases emerge for further investigation: the critical -head and -set loads needed in computing corresponding $G_{I c}$ values. As described in Section 3.4, cracking -initiation and -arrestment is delineated for each of these divisions and by the use of Eq. (3.74) — repeated below as (6.2):

$$
I=\frac{G_{I c}^{i}-G_{I c}^{a}}{G_{I c}^{i}}
$$

brittleness indices are computed to gain an understanding of the cracking behavior from a quantitative viewpoint, as fashioned after the work of Ebewele, River and Koutsky (1986). Pictures of the fractured surfaces are also studied to gain a qualitative understanding of the cracking behavior.

Finally, Section 6.4 presents the results of any weight- and strain- changes that may have taken place with respect to the companion specimens over their term of study.

\subsection{Pristine Dry Fracture Specimens}

Four fracture tests were performed on pristine specimens, designated as 3B3, 3B6, 4B8 and 3B9, by the use of a MTS machine operating under displacement-control at a rate of $0.00254 \mathrm{~cm} / \mathrm{s}(0.001 \mathrm{in} / \mathrm{s})$. Here, the lead number indicates the beam and the letter-and-number specify the concrete batch. Recall, from the previous chapter, that the mean 28-day compressive strength of the concrete was found to be $51.6 \mathrm{MPa}$ (7480 psi) with a standard deviation of $2.1 \mathrm{MPa}$ (310 psi).

As already mentioned, simultaneous load and crack opening displacements (COD) were recorded at every one-fifth of a second by a data acquisition system as each 
of the beams was tested to failure. The plotting of this data revealed features commonly associated with fracture toughness testing, e.g. see the results of beam 3B6 as displayed in Figure 6.1. First, there is the linear elastic region prior to the onset of cracking. Following this, are a series of regions in which cracking initiates (i1 - i4) and arrests (a1 a3) corresponding to the region of COD containing peaks and valleys approximately between 6 and $9 \mathrm{~mm}\left(0.24 "\right.$ and $\left.0.35^{\prime \prime}\right)$, with an average COD value of $1.7 \mathrm{~mm}\left(0.07^{\prime \prime}\right)$ for the four pristine specimens. Lastly, testing is terminated in an abrupt manner with the complete fracturing of the interface, as depicted graphically (Fig. 6.1) by the sudden drop in load.

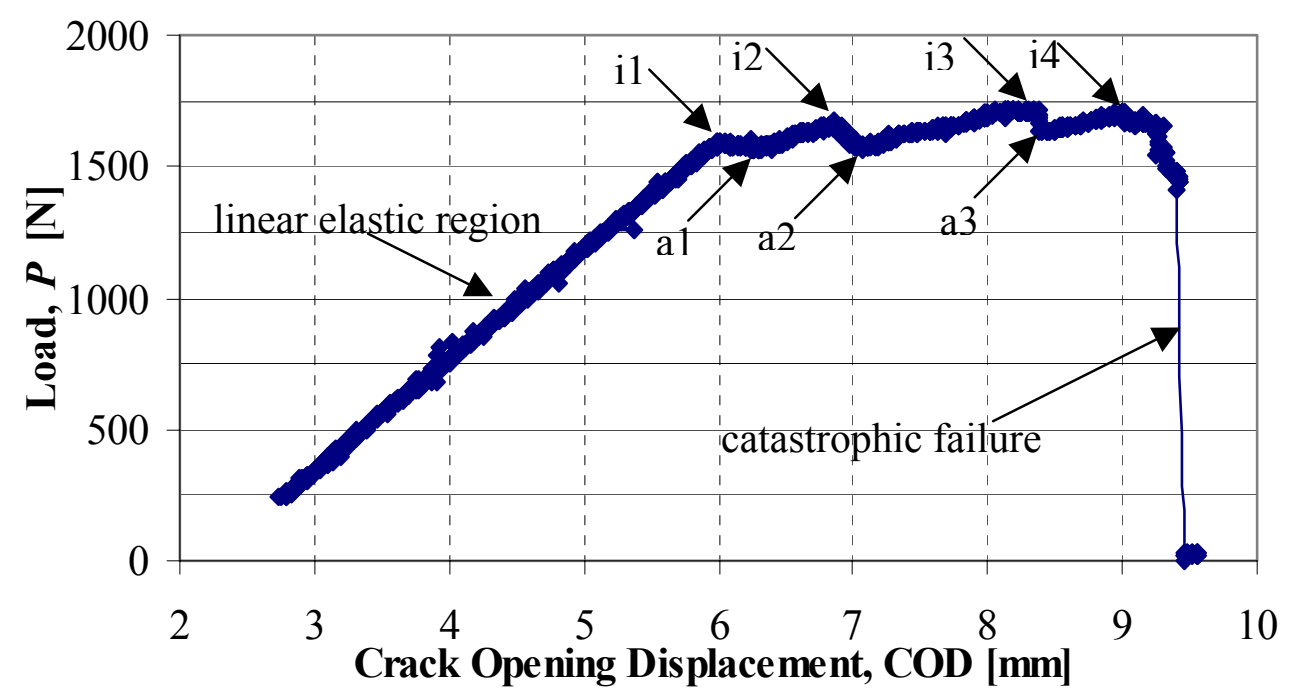

Figure 6.1 Fracture behavior of beam 3B6

To reiterate, the Mode I crack growth of an adhesive joint is characterized by a quantity known as the interface fracture toughness, or more specifically, the critical strain 
energy release rate, $G_{I c}$ (read Sections 3.1 and 3.3, and see Eq. (3.73)), repeated here, again as:

$$
G_{I c}=\frac{P_{c}^{2}}{2 b} \frac{d C}{d a}
$$

where, $P_{c}=$ critical load, $b=$ width of the specimen $=43.4 \mathrm{~mm}(1.71 ")$, and $d C / d a=$ rate change of compliance with respect to crack length $a$. The compliance gradient, a constant in this case due to the designed shape of the contour, was determined through experimental calibrations (see Ch. 4) as being $d C / d a=1.78 \times 10^{-5} \mathrm{~N}^{-1}\left(7.92 \times 10^{-5} \mathrm{lb}^{-1}\right)$ and verified by an explicit beam on elastic foundation solution as well as through a finite element analysis.

\section{The "Head" and "Set" Critical Loads}

Theoretically, delamination of the interface occurs whenever the instantaneous strain energy release rate, denoted as $G_{I}$, exceeds the critical value of Eq. (6.1), i.e. $G_{I}>$ $G_{I c}$. Since both $b$ and $d C / d a$ of Eq. (6.1) are known (see previous paragraph), establishing such a critical value reduces to determining the critical load, $P_{c}$. The following two approaches have been adopted to this end.

In the first, $P_{c}$ is taken to be the mean of the "head" -initiation and -arrestment, reduced loads (i.e. the non-stricken boldfaced numbers given in Table 6.1 - the procedure of reducing loads is explained in the following two subsections) of each class of beams tested, and is denoted as $P_{c, \text { Head }}^{\mathrm{i}}$ and $P_{c, \text { Head }}^{\mathrm{a}}$, respectively. For example, corresponding to the four initial dry fracture test specimens (see Table 6.1), the reduced head -initiation and -arrestment loads are $\left(\right.$ Head $\left.^{\mathrm{i}}: 1716,1720,1768\right)$ and $\left(\operatorname{Head}^{\mathrm{a}}: 1659,1712\right)$ as they are 
above all the other loads in their category and lie within the range of cited acceptable

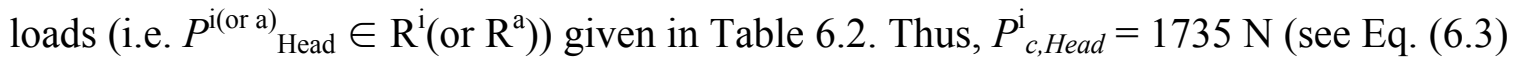
and the subsection titled "A Step-By-Step Example") and $P_{c, \text { Head }}^{\mathrm{a}}=1686 \mathrm{~N}$.

In the second, $P_{c}$ is found as the mean of the "set" of crack -initiation and -arrestment, reduced loads (i.e. the non-stricken numbers given in Table 6.1) for the same class of beams, i.e. loads (Seti: 1716, 1720, 1725, 1742, 1755, 1768, 1751, 1733) and $\left(\right.$ Set $\left.^{\mathrm{a}}: 1659,1690,1703,1712,1651\right)$, and is denoted as $P_{c, S e t}^{\mathrm{i}}$ and $P_{c, \text { Set }}^{\mathrm{a}}$, respectively. Hence, $P_{c, S e t}^{\mathrm{i}}=1736 \mathrm{~N}$ and $P_{c, \text { Set }}^{\mathrm{a}}=1683 \mathrm{~N}$. The corresponding critical strain energy release rates computed (using Eq. (6.1)) based on the first convention are thus denoted as $G_{I c, \text { Head }}^{\mathrm{i}}$ and $G_{I c, \text { Head }}^{\mathrm{a}}$, and for the latter case, as $G_{I c, S e t}^{\mathrm{i}}$ and $G_{I c, S e t}^{\mathrm{a}}$, the results of which are presented in Table 6.3.

Table 6.1 Pristine dry fracture loads_-initiations (i) and arrestments (a) (unit: N)

\begin{tabular}{|c|c|c|c|c|c|c|c|c|}
\hline \multirow[t]{2}{*}{$\mathbf{n}$} & \multicolumn{2}{|c|}{ 3B3 } & \multicolumn{2}{|c|}{ 3B6 } & \multicolumn{2}{|c|}{ 4B8 } & \multicolumn{2}{|c|}{ 3B9 } \\
\hline & i & $\mathbf{a}$ & i & $\mathbf{a}$ & i & $\mathbf{a}$ & i & $\mathbf{a}$ \\
\hline 1 & 1716 & 1659 & 1599 & 1568 & 1725 & 1690 & 1768 & 1712 \\
\hline 2 & $1668^{*}$ & 1581 & 1659 & 1568 & 1742 & 1703 & 1751 & 1651 \\
\hline 3 & 1590 & 1529 & 1720 & 1633 & 1798 & 1746 & 1733 & \\
\hline 4 & 1538 & 1477 & 1707 & & 1755 & 1516 & & \\
\hline 5 & 1516 & 1468 & (NC & & 1568 & 1503 & & \\
\hline 6 & 1499 & 1438 & see & & 1516 & & & \\
\hline 7 & 1490 & $146 \theta$ & Fig & & & & & \\
\hline 8 & 1533 & & & & & & & \\
\hline COD-range [mm] & 6.35 & 8.13 & 6.10 & 8.89 & 5.84 & 7.62 & 8.38 & 8.89 \\
\hline Abs.-COD [mm] & & & & & & & & \\
\hline
\end{tabular}

"Stricken numbers are those falling outside the initiation or arrestment ranges $\left(\mathrm{R}^{\mathrm{i}}, \mathrm{R}^{\mathrm{a}}\right)$ as cited in Table 6.2; see Eqs. (6.9) - (6.12)

For purposes of comparison, the raw fracture results are also furnished as given in Table 6.2. These values of fracture are computed indiscriminately_in other words, all of 
the loads from Table 6.1 are averaged and used in computing the critical strain energy release rates. Note especially the rather large discrepancies that are found to exist between the corresponding head and set values, i.e. between the initiation and arrestment fractures. Using the set values as reference, the head -initiation and -arrestment fractures are in variance by $13 \%$ and $14 \%$, respectively, as compared with only $-0.5 \%$ and $0.2 \%$, as obtained from the statistically reduced values given in Table 6.3 (see the next two subsections).

Table 6.2 Raw data results of pristine dry fracture specimens (see Fig. 6.1 and Table 6.1)

\begin{tabular}{c|cc|cc}
$\mathbf{R}^{\mathbf{i}:} \mathbf{1 7 1 1}-\mathbf{1 7 9 0}$ & \multicolumn{3}{|c|}{ Head } & \multicolumn{2}{c}{ Set } \\
\cline { 2 - 5 } $\mathbf{R}^{\mathbf{a}}: \mathbf{1 6 3 7}-\mathbf{1 7 3 8}$ & $\mathbf{i}$ & $\mathbf{a}$ & $\mathbf{i}$ & $\mathbf{a}$ \\
\hline $\mathbf{n}$ & 4 & 4 & 21 & 17 \\
\hline $\boldsymbol{G}_{\boldsymbol{I c}}\left[\mathbf{J} / \mathbf{m}^{2}\right]$ & 628 & 584 & 556 & 513 \\
\hline $\boldsymbol{\sigma}\left[\mathbf{J} / \mathbf{m}^{2}\right]$ & 0.32 & 0.53 & 2.20 & 2.01 \\
\hline $\mathbf{C O V}$ & $2.27 \%$ & $3.01 \%$ & $6.28 \%$ & $6.26 \%$ \\
\hline
\end{tabular}

*The determination of ranges is discussed in the following two subsections

Table 6.3 Reduced data results of pristine dry fracture test specimens (see Table 6.1)

\begin{tabular}{c|cc|cc}
\cline { 2 - 5 } & \multicolumn{3}{|c|}{ Head } & \multicolumn{2}{c}{ Set } \\
\hline $\mathbf{n}$ & $\mathbf{i}$ & $\mathbf{a}$ & $\mathbf{i}$ & $\mathbf{a}$ \\
\hline $\boldsymbol{G}_{\boldsymbol{I c}}\left[\mathbf{J} / \mathbf{m}^{2}\right]$ & 617 & $\mathbf{5 8 2}$ & $\mathbf{6 2 0}$ & $\mathbf{5 8 1}$ \\
\hline $\boldsymbol{\sigma}\left[\mathbf{J} / \mathbf{m}^{2}\right]$ & 0.17 & 0.28 & 0.07 & 0.15 \\
\hline $\mathbf{C O V}$ & $1.67 \%$ & $2.19 \%$ & $1.06 \%$ & $1.59 \%$ \\
\hline
\end{tabular}

Table 6.4 Pristine dry fracture results (unit: $\mathrm{J} / \mathrm{m}^{2}$ )

\begin{tabular}{|c|c|c|c|c|}
\hline & \multicolumn{2}{|c|}{ Initiation } & \multicolumn{2}{|c|}{ Arrestment } \\
\hline & $G_{I c, \text { Head }}$ & $G_{I c, S e t}$ & $G_{I c, \text { Head }}$ & $G_{I c, S e t}$ \\
\hline & 617 & 620 & 582 & 581 \\
\hline Mean: & \multicolumn{2}{|c|}{618} & \multicolumn{2}{|c|}{582} \\
\hline
\end{tabular}


The final pristine dry fracture results for each of the head- and set- initiations and arrestments are given in Table 6.4, with averaged corresponding head/set critical strain energy release rates of approximately $G_{I c}^{i}=618 \mathrm{~J} / \mathrm{m}^{2}$ and $G_{I c}^{a}=582 \mathrm{~J} / \mathrm{m}^{2}$. The only other credible source known to the author by which these values may be compared to is found in the work of Karbhari and Engineer (1996b) who used a peel test to study the fracture toughness of FRP-concrete interfaces. After conducting a number of tests to determine the optimum peel angle $\left(45^{\circ}\right)$ and actuator speed $(0.085 \mathrm{~mm} / \mathrm{s}=0.0033 \mathrm{in} / \mathrm{s})$, they found the Mode I CFRP-concrete value to be: $G_{I c}=478 \mathrm{~J} / \mathrm{m}^{2}$. The pristine SCCB initiation and arrestment values vary from this by $+29 \%$ and $+22 \%$, respectively. Of course, such differences arise chiefly due to the dissimilarity in material properties existing between the epoxy, carbon fiber tow and the concrete in use. Nevertheless, to a large extent, this demonstrates fulfillment of two vitally important issues regarding interface bond characterization, namely: (1) the ability to quantify this, hitherto seemingly elusive, fracture property with respect to FRP-concrete interfaces, and (2) verification that this property is indeed a material constant, as are, for example, the Young's and shear moduli-this latter point bears the distinguishing prospect of eventually being incorporated as a parameter useful to both theory and design.

It is also now possible to quantitatively characterize the behavior of cracking (see Section 3.4) by using the convention of Eq. (3.74), i.e. the brittleness index, $I$, repeated in this chapter as Eq. (6.2):

$$
I=\frac{G_{I c}^{i}-G_{I c}^{a}}{G_{I c}^{i}}
$$


which is a normalization of the energy being released (i.e. lost) during a period of rapid crack growth with respect to the energy that was stored in the joint just at the onset of crack-initiation (Ebewele, River and Koutsky (1986)). Thus, $I=0.06$ in this case, corresponding to a stable form of crack propagation as diagrammatically shown in Figures 3.14(a) and 3.15(b). Using the terminology of Section 3.4, then, the pristine CFRP-concrete interface may be characterized as being strain rate insensitive.

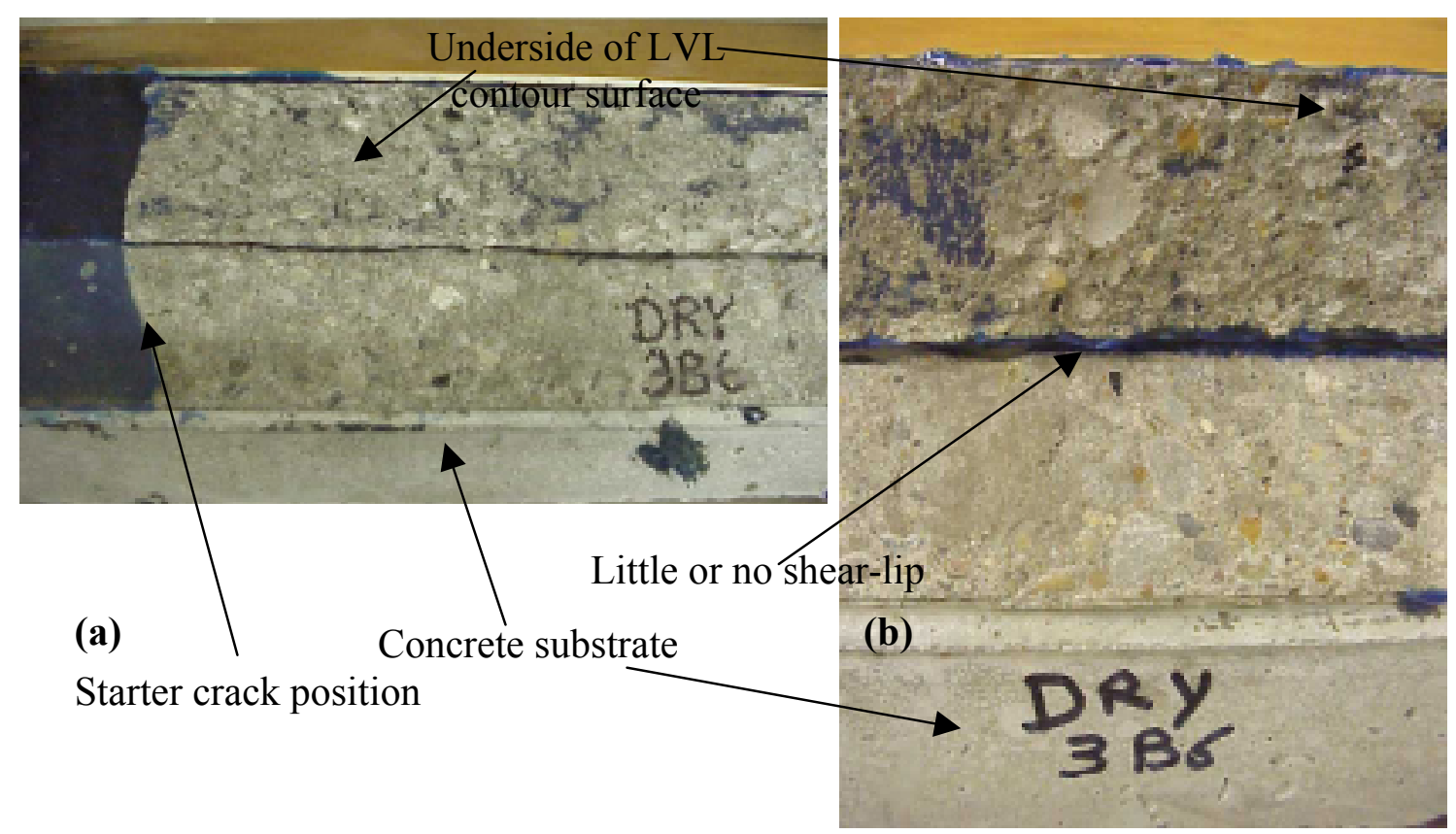

Figure 6.2 Pictures of the pristine-dry fracture surfaces of beam 3B6(a) extended- and (b) close- views

Inspection of the fractured surfaces (Fig. 6.2), lastly, reveals a couple of important features that are characteristic of the dry test specimens. A more protracted view of the surfaces is shown in Fig. 6.2(a) - the top portion displaying the underside of the LVL contour, and the bottom, exposing the mated surface of the concrete- the blue surfaces are those of the epoxy resin (i.e. the saturant). Note especially from the top view that the 
failure may be dominantly characterized as being cohesive, i.e. a form of separationfailure ensuing within a body of like-matter; in contrast, adhesive failures occur when the separation of dissimilarly bonded materials manifests itself at the bi-material interface (Anderson, Bennett and DeVries, 1977). The cohesive nature of failure is made evident by the very sparse bluish areas that are visible in Figure 6.2(a). Obviously, in order for the failure to be chiefly cohesive, the adhesive-joint must necessarily be soundly intact, and stronger than that of the underlying concrete substratum.

The other important observation to be made from Figure 6.2 is actually linked to the discussion of "Plane Stress vs. Plane Strain" back in Section 3.2. Referring especially to Fig. 3.9(d) in conjunction with Fig. 6.2(b) above, note that the failed surface has very little, if any, of a shear-lip. In other words, the failure is truly one of plane strain and the values of $G_{I c}$ being reported from these tests are distinctly the conservative, lower-limits (see Fig. 3.9) as claimed, thus affirming, once again, that these values are indeed material constants.

\section{The Statistical Reduction of Raw Loads}

Since the fracture toughness of a certain class of specimens is a material constant that depends solely on the critical load (see Eq. (6.1) and recall that $d C / d a$ was made to be a constant by the contouring of the cantilevered member-see Ch. 4), it is therefore reasonable to perform a statistical study of all the loads responsible for initiation, and likewise, though separately, those accounting for arrestment. Obviously, the head-loads of each beam within such a division of specimens are of special interest as they represent 
an upper-limiting case for the characterization of fracture. Thus to begin with, the mean and standard deviation of the head-loads for initiation and arrestment are found as

$$
\begin{array}{r}
P_{\text {Head }}^{\mathrm{i}}=\frac{\sum_{\mathrm{j}=1}^{\mathrm{n}} P_{\text {Head }}^{\mathrm{ij}}}{\mathrm{n}} \\
\sigma_{\text {Head }}^{\mathrm{i}}=\sqrt{\frac{\sum_{\mathrm{j}=1}^{\mathrm{n}}\left(P_{\text {Head }}^{\mathrm{ij}}-P_{\text {Head }}^{\mathrm{i}}\right)^{2}}{\mathrm{n}-1}} \\
P_{\text {Head }}^{\mathrm{a}}=\frac{\sum_{\mathrm{j}=1}^{\mathrm{n}} P_{\text {Head }}^{\mathrm{aj}}}{\mathrm{n}} \\
\sigma_{\text {Head }}^{\mathrm{a}}=\sqrt{\frac{\sum_{\mathrm{j}=1}^{\mathrm{n}}\left(P_{\text {Head }}^{\mathrm{aj}}-P_{\text {Head }}^{\mathrm{a}}\right)^{2}}{\mathrm{n}-1}}
\end{array}
$$

where $\mathrm{n}=$ the total number of initiation, or equally, arrestment, head-loads for a class of specimens. The coefficient of variation may then be computed from Equations (6.3) and (6.4), and likewise by Equations (6.5) and (6.6), as the ratio of the standard deviation to the mean, yielding

$$
\begin{aligned}
& \operatorname{COV}_{\text {Head }}^{\mathrm{i}}=\frac{\sigma_{\text {Head }}^{\mathrm{i}}}{P_{\text {Head }}^{\mathrm{i}}} \\
& \operatorname{COV}_{\text {Head }}^{\mathrm{a}}=\frac{\sigma_{\text {Head }}^{\mathrm{a}}}{P_{\text {Head }}^{\mathrm{a}}}
\end{aligned}
$$

A prescribed range of admissible loads is then defined as

$$
\begin{aligned}
& \mathrm{R}^{\mathrm{i}}=\left[P_{\text {Head }}^{\mathrm{i}} \cdot\left(1-\operatorname{COV}_{\text {Head }}^{\mathrm{i}}\right), P_{\text {Head }}^{\mathrm{i}} \cdot\left(1+\mathrm{COV}_{\text {Head }}^{\mathrm{i}}\right)\right] \\
& \mathrm{R}^{\mathrm{a}}=\left[P_{\text {Head }}^{\mathrm{a}} \cdot\left(1-\mathrm{COV}_{\text {Head }}^{\mathrm{a}}\right), P_{\text {Head }}^{\mathrm{a}} \cdot\left(1+\mathrm{COV}_{\text {Head }}^{\mathrm{a}}\right)\right]
\end{aligned}
$$


The ranges in Eqs. (6.9) and (6.10) essentially act to accommodate the various types of experimental scatter that may be obtained (see Figure 6.3). For example, if there is not much scatter about the mean of the head-loads, then the bandwidth of the corresponding range is narrower (Fig. 6.3(a)) than it would otherwise be if the loads were more widespread (Fig. 6.3(b)).
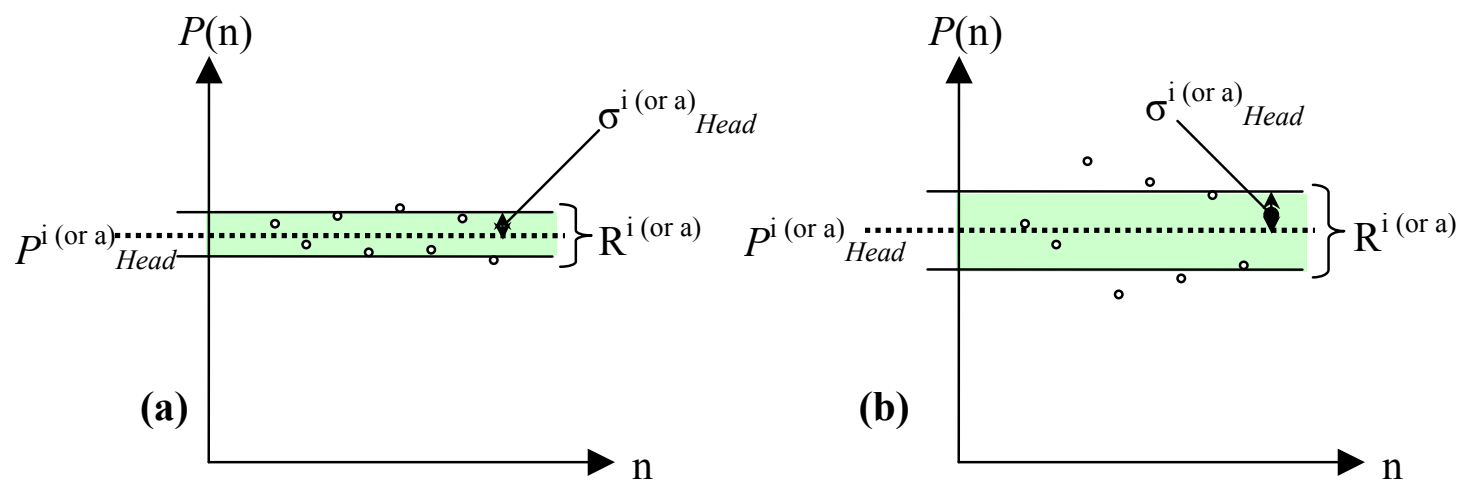

Figure 6.3 The statistical-based bandwidth for-(a) compact, and (b) widespread data

Note that if the mean of the head-loads is multiplicatively distributed throughout the parenthetical expressions of Eqs. (6.9) and (6.10) and the coefficient of variations are expressed in ratio-form (as in Eqs. (6.7) and (6.8)), the ranges are then equivalently expressed as

$$
\begin{aligned}
& \mathrm{R}^{\mathrm{i}}=\left[P_{\text {Head }}^{\mathrm{i}}-\sigma_{\text {Head }}^{\mathrm{i}}, P_{\text {Head }}^{\mathrm{i}}+\sigma_{\text {Head }}^{\mathrm{i}}\right] \\
& \mathrm{R}^{\mathrm{a}}=\left[P_{\text {Head }}^{\mathrm{a}}-\sigma_{\text {Head }}^{\mathrm{a}}, P_{\text {Head }}^{\mathrm{a}}+\sigma_{\text {Head }}^{\mathrm{a}}\right]
\end{aligned}
$$

where the significance of using the standard deviation, $\sigma$, in these expressions is illustrated diagrammatically in Figure 6.3. 
A Step-by-Step Example

The process given in the previous subsection is outlined using the case of the head-initiation loads of the pristine beams (see Table 6.1) as an example. The first step is to determine the mean of the raw head loads (listed in Table 6.5 below for easy referencing).

Table 6.5 Raw head-initiation loads of pristine specimens (from Table 6.1)

\begin{tabular}{c|cc|cc} 
& 3B3 & 3B6 & 4B8 & 3B9 \\
\hline $\mathbf{n}$ & 1 & 2 & 3 & 4 \\
\hline $\boldsymbol{P}_{\text {Head }}^{i}[\mathbf{N}]$ & $\mathbf{1 7 1 6}$ & $\mathbf{1 7 2 0}$ & $\mathbf{1 7 9 8}$ & $\mathbf{1 7 6 8}$ \\
\hline
\end{tabular}

Step 1: Thus, by Eq. (6.3): $\quad P_{\text {Head }}^{\mathrm{i}}=\frac{\sum_{\mathrm{j}=1}^{\mathrm{n}} P_{\text {Head }}^{\mathrm{ij}}}{\mathrm{n}}$ $=\frac{1716+1720+1798+1768}{4}=1750.5 \quad \rightarrow \quad P_{\text {Head }}^{i}=1750.5 \mathrm{~N}$

Step 2: Next, by Eq. (6.4): $\quad \sigma_{\text {Head }}^{\mathrm{i}}=\sqrt{\frac{\sum_{\mathrm{j}=1}^{\mathrm{n}}\left(P_{\text {Head }}^{\mathrm{ij}}-P_{\text {Head }}^{\mathrm{i}}\right)^{2}}{\mathrm{n}-1}}$

$$
\begin{aligned}
& =\sqrt{\frac{(1716-1750.5)^{2}+(1720-1750.5)^{2}+(1798-1750.5)^{2}+(1768-1750.5)^{2}}{(4)-1}} \\
& =\quad 39.5 \quad \rightarrow \quad \sigma_{\text {Head }}^{\mathrm{i}}=39.5 \mathrm{~N}
\end{aligned}
$$

Step 3: Hence, by Eq. (6.7): $\quad \operatorname{COV}_{\text {Head }}^{\mathrm{i}}=\frac{\sigma_{\text {Head }}^{\mathrm{i}}}{P_{\text {Head }}^{\mathrm{i}}}$ 


$$
=\frac{39.5}{1750.5}=0.02256 \quad \rightarrow \quad \mathrm{COV}_{\text {Head }}^{\mathrm{i}}=\mathbf{0 . 0 2 2 5 6}
$$

Step 4: Finally, by either Eq. (6.9):

$$
\mathrm{R}^{\mathrm{i}}=\left[P_{\text {Head }}^{\mathrm{i}} \cdot\left(1-\operatorname{COV}_{\text {Head }}^{\mathrm{i}}\right), P_{\text {Head }}^{\mathrm{i}} \cdot\left(1+\operatorname{COV}_{\text {Head }}^{\mathrm{i}}\right)\right]
$$

or by Eq. (6.11):

$$
\mathrm{R}^{\mathrm{i}}=\left[P_{\text {Head }}^{\mathrm{i}}-\sigma_{\text {Head }}^{\mathrm{i}}, P_{\text {Head }}^{\mathrm{i}}+\sigma_{\text {Head }}^{\mathrm{i}}\right]
$$

the admissible range of initiation loads is therefore found as:

$$
\begin{array}{ll} 
& =[1750.5 \cdot(1-0.02256), 1750.5 \cdot(1+0.02256)] \\
\text { or } & =[1750.5-39.5,1750.5+39.5] \\
& =\quad[1711,1790] \quad \rightarrow \quad R^{i}=[1711 \mathrm{~N}, 1790 \mathrm{~N}]
\end{array}
$$

where the square brackets indicate an inclusive-range of values (i.e. the mathematical closed-set notation). Recall that this is the initiation-range cited in Table 6.2 from which inadmissible values in Table 6.1 were stricken, the application, of which, is indiscriminately used for both cases of head and set initiation-loads.

\subsection{Representative Dry Fracture Specimens}

The preceding section presented pristine values of the CFRP-concrete interface though, in reality, the application of externally reinforcing structures by CFRP materials more realistically entails subjection to some degree of aging. It is with this objective, then, that an attempt is made here to determine a representative value of dry fracture.

Under dry conditions, the value of interface fracture has been observed to increase with time (see Figure 6.11). This follows reason, because both the epoxy and underlying cement matrices cure during this period and gain in strength. Of course, as with concrete, this progression is expected to be more pronounced in the beginning hours of curing with 
considerable retardation observed in the weeks, months, and years that follow (see, for example, Neville, Figs. 2.2 and 6.32).

A representation of dry fracture is obtained by considering all of the specimens that did not undergo either of the wet-dry or freeze-thaw regimes - here are included the four pristine beams, as well as a total of sixteen companion specimen results. Given the timeframe of this research, the extent of age-effects on interface fracture comes from the $50,100,150,200$ and 300 cycles of freeze-thaw companions, translating to $12.5,25$, 37.5, 50 and 75 days, respectively; and the 20 and 30 cycles of wet-dry companions corresponding to 140 and 210 days, respectively - recall that the $10^{\text {th }}$ cycle values are not considered, but instead, replaced by those of the pristine beams due to the inconsistent results obtained.

The representative dry fracture results are presented in Tables $6.6-6.10$ below. The data concerning the initiations and arrestments are each shown in pair-wise fashion per page, beginning with the head and set results in Tables 6.6 and 6.7 and ending with Tables 6.8 and 6.9, respectively; conclusive results are presented in Table 6.10 with head and set values averaged for the initiation and arrestment cases. These values are found to be: $G_{I c}^{i}=720 \mathrm{~J} / \mathrm{m}^{2}$ and $G_{I c}^{a}=694 \mathrm{~J} / \mathrm{m}^{2}$. When comparing these to the pristine values of the previous section, the corresponding initiation and arrestment fracture energies are found to be $+16 \%$ (i.e. $G_{I c}^{i}=618 \mathrm{~J} / \mathrm{m}^{2}$ ) and $+19 \%$ (i.e. $G_{I c}^{a}=582 \mathrm{~J} / \mathrm{m}^{2}$ ) higher which, as already explained, follows reason due to the curing of the epoxy and cement matrices when permitted to set over time (see also Figure 6.11). 
The representative-dry brittleness index (Eq. 6.2) is found to be, $I=0.04$ (i.e. $I=$ $(720-694) / 720$; see Table 6.10). Once again, this is indicative of stable crack propagation during fracture testing as diagrammatically shown in Figures 3.14(a) and 3.15(b) - thus, the representative-dry CFRP-concrete interface may also be characterized as being strain rate insensitive, as was the case with the pristine specimens.

Table 6.6 Representative dry fracture results from head-initiation loads

\begin{tabular}{|c|c|c|c|c|c|}
\hline & Beams & $\mathbf{n}$ & $G_{I c}\left[\mathrm{~J} / \mathrm{m}^{2}\right]$ & $\sigma\left[J / \mathbf{m}^{2}\right]$ & $\mathrm{COV}$ \\
\hline Pristine & 3B3, 3B6, 3B9 & 3 & 617 & 0.17 & $1.67 \%$ \\
\hline WDC20 & 1B7, 9B11 & 2 & 744 & 24.70 & $18.22 \%$ \\
\hline WDC30 & 10B9, 9B12 & 2 & 782 & 12.07 & $12.43 \%$ \\
\hline FTC50 & $7 \mathrm{~B} 3,8 \mathrm{~B} 3,4 \mathrm{~B} 9$ & 3 & 602 & 2.18 & $6.02 \%$ \\
\hline FTC100*** & 1B4, 6B7, 1B10 & 3 & 622 & 26.26 & $20.55 \%$ \\
\hline FTC150 ${ }^{* * *}$ & $5 \mathrm{~B} 8$ & 1 & 729 & ----- & ----- \\
\hline FTC200 $^{* * *}$ & $7 \mathrm{~B} 10,8 \mathrm{~B} 10$ & 2 & 785 & 4.64 & $7.69 \%$ \\
\hline FTC300 $^{* * *}$ & 4B12, 8B12 & 2 & 811 & 0.07 & $0.93 \%$ \\
\hline \multicolumn{2}{|c|}{ Mean Values: } & 2.25 & 712 & 10.01 & $9.64 \%$ \\
\hline
\end{tabular}

"WDC20 (or 30) = wet-dry companions at 20 (or 30) cycles

${ }^{* *}$ FTC50 (or 100, etc.) $=$ freeze-thaw companions at 50 (or 100 , etc.) cycles

Table 6.7 Representative dry fracture results from set-initiation loads

\begin{tabular}{|c|c|c|c|c|c|}
\hline & Beams & $\mathbf{n}$ & $G_{I c}\left[\mathrm{~J} / \mathrm{m}^{2}\right]$ & $\sigma\left[\mathrm{J} / \mathrm{m}^{2}\right]$ & $\mathrm{COV}$ \\
\hline Pristine & 3B3, 3B6, 3B9, 4B8 & 8 & 620 & 0.07 & $1.06 \%$ \\
\hline WDC20 & 1B7, 9B11 & 31 & 828 & 7.20 & $9.33 \%$ \\
\hline WDC30 & 10B9, 9B12 & 32 & 810 & 2.84 & $5.93 \%$ \\
\hline FTC50 & 7B3, 8B3, 4B9 & 14 & 605 & 0.77 & $3.57 \%$ \\
\hline FTC100** $^{* *}$ & $1 \mathrm{~B} 4,6 \mathrm{~B} 7,1 \mathrm{~B} 10$ & 29 & 643 & 17.27 & $16.39 \%$ \\
\hline FTC150** $^{* *}$ & 5B8, 6B9 & 51 & 746 & 0.44 & $2.42 \%$ \\
\hline FTC200 & 7B10, 8B10 & 17 & 766 & 1.31 & $4.13 \%$ \\
\hline FTC300 $^{* * *}$ & 4B12, 8B12 & 9 & 811 & 0.03 & $0.57 \%$ \\
\hline \multicolumn{2}{|c|}{ Mean Values: } & 23.88 & 729 & 3.74 & $5.42 \%$ \\
\hline
\end{tabular}

"WDC20 (or 30) = wet-dry companions at 20 (or 30) cycles

${ }^{* *}$ FTC50 (or 100, etc.) $=$ freeze-thaw companions at 50 (or 100 , etc.) cycles 
Table 6.8 Representative dry fracture results from head-arrestment loads

\begin{tabular}{c|c|c|c|c|c} 
& Beams & $\mathbf{n}$ & $\boldsymbol{G}_{\boldsymbol{I c}}\left[\mathbf{J} / \mathbf{m}^{2}\right]$ & $\boldsymbol{\sigma}\left[\mathbf{J} / \mathbf{m}^{2}\right]$ & $\mathbf{C O V}$ \\
\hline Pristine & 3B3, 3B6, 3B9 & 2 & 582 & 0.28 & $2.19 \%$ \\
\hline WDC20 $^{*}$ & 1B7, 9B11 & 2 & 704 & 31.20 & $21.06 \%$ \\
\hline WDC30 $^{* *}$ & $10 \mathrm{~B} 9,9 \mathrm{~B} 12$ & 2 & 764 & 10.31 & $11.61 \%$ \\
\hline FTC50 $^{* *}$ & 7B3, 8B3, 4B9 & 3 & 585 & 1.84 & $5.60 \%$ \\
\hline FTC100 $^{* *}$ & $1 \mathrm{~B} 4,6 \mathrm{~B} 7,1 \mathrm{~B} 10$ & 3 & 596 & 26.84 & $21.21 \%$ \\
\hline FTC150 $^{* * *}$ & $5 \mathrm{~B} 8$ & 1 & 712 & ----- & ---- \\
\hline FTC200 $^{* *}$ & 7B10, 8B10 & 2 & 757 & 2.65 & $5.91 \%$ \\
\hline FTC300 & 4B12, 8B12 & 2 & 775 & 0.85 & $3.32 \%$ \\
\hline \multicolumn{2}{l|}{ Mean Values: } & 2.12 & $\mathbf{6 8 4}$ & 10.57 & $10.13 \%$ \\
\hline
\end{tabular}

"WDC20 (or 30) = wet-dry companions at 20 (or 30) cycles

${ }^{* *}$ FTC50 $($ or 100, etc. $)=$ freeze-thaw companions at 50 (or 100, etc.) cycles

Table 6.9 Representative dry fracture results from set-arrestment loads

\begin{tabular}{|c|c|c|c|c|c|}
\hline & Beams & $\mathbf{n}$ & $G_{I C}\left[\mathbf{J} / \mathbf{m}^{2}\right]$ & $\sigma\left[\mathrm{J} / \mathbf{m}^{2}\right]$ & $\mathrm{COV}$ \\
\hline Pristine & $3 \mathrm{~B} 3,3 \mathrm{~B} 6,3 \mathrm{~B} 9,4 \mathrm{~B} 8$ & 5 & 581 & 0.15 & $1.59 \%$ \\
\hline WDC20 & 1B7, 9B11 & 30 & 804 & 8.10 & $10.04 \%$ \\
\hline WDC30 & 10B9, 9B12 & 30 & 787 & 2.34 & $5.46 \%$ \\
\hline FTC50 & 7B3, 8B3, 4B9 & 12 & 587 & 0.70 & $3.46 \%$ \\
\hline FTC100** & 1B4, 6B7, 1B10 & 28 & 614 & 16.20 & $16.24 \%$ \\
\hline FTC150 $^{* * *}$ & $5 \mathrm{~B} 8,6 \mathrm{~B} 9$ & 50 & 723 & 8.06 & $10.56 \%$ \\
\hline FTC200** & $7 \mathrm{~B} 10,8 \mathrm{~B} 10$ & 12 & 770 & 0.40 & $2.29 \%$ \\
\hline FTC300 $^{* * *}$ & 4B12, 8B12 & 14 & 770 & 0.31 & $2.00 \%$ \\
\hline \multicolumn{2}{|c|}{ Mean Values: } & 22.62 & 704 & 4.53 & $6.46 \%$ \\
\hline
\end{tabular}

"WDC20 (or 30) = wet-dry companions at 20 (or 30) cycles

${ }^{* * *}$ FTC50 (or 100, etc.) $=$ freeze-thaw companions at 50 (or 100, etc.) cycles

Table 6.10 Representative dry fracture results (unit: $\mathrm{J} / \mathrm{m}^{2}$ )

\begin{tabular}{|c|c|c|c|c|}
\hline & \multicolumn{2}{|c|}{ Initiation } & \multicolumn{2}{|c|}{ Arrestment } \\
\hline & $\boldsymbol{G}_{I c, \text { Head }}$ & $\boldsymbol{G}_{\boldsymbol{I}, \mathrm{Set}}$ & $\boldsymbol{G}_{\boldsymbol{I}, \mathrm{H} \text { Head }}$ & $\boldsymbol{G}_{I c, S e t}$ \\
\hline & 712 & 729 & 684 & 704 \\
\hline Mean: & \multicolumn{2}{|c|}{720} & \multicolumn{2}{|c|}{694} \\
\hline
\end{tabular}




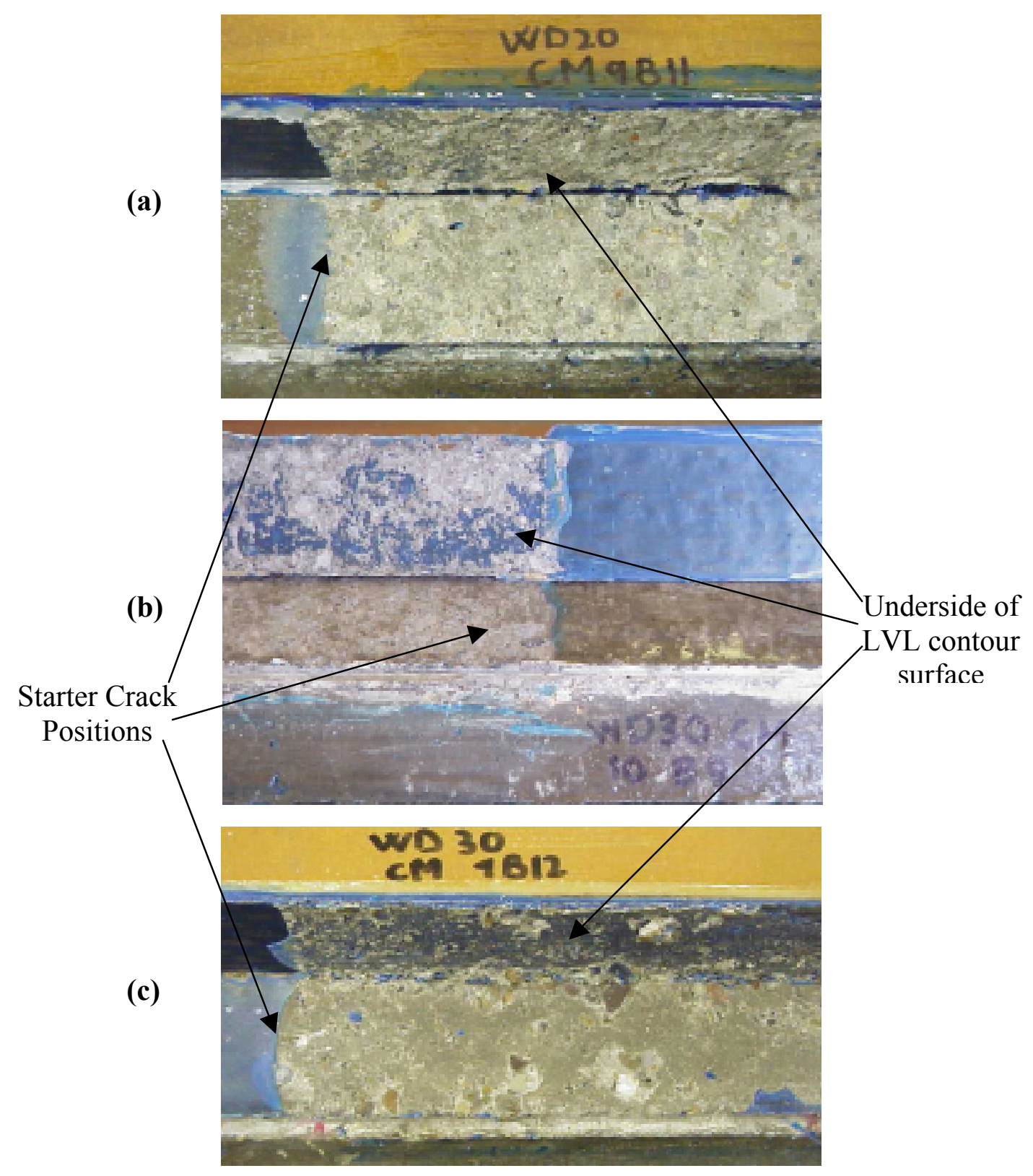

Figure 6.4 Pictures of the representative-dry fracture surfaces of wet-dry
companion beams -
(WDC20):
(a) 9B11
(b) $10 \mathrm{~B} 9$ and (c) $9 \mathrm{~B} 12$ 


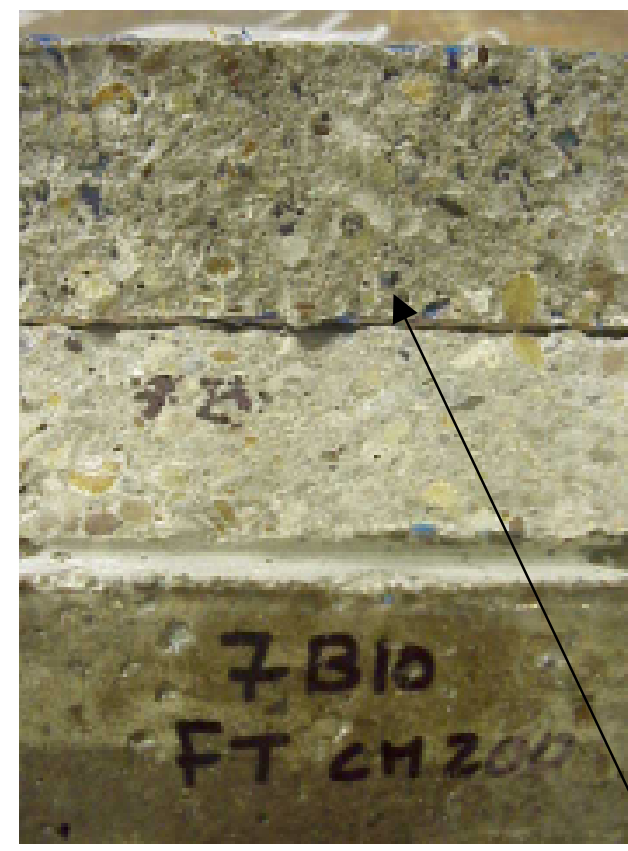

(a)

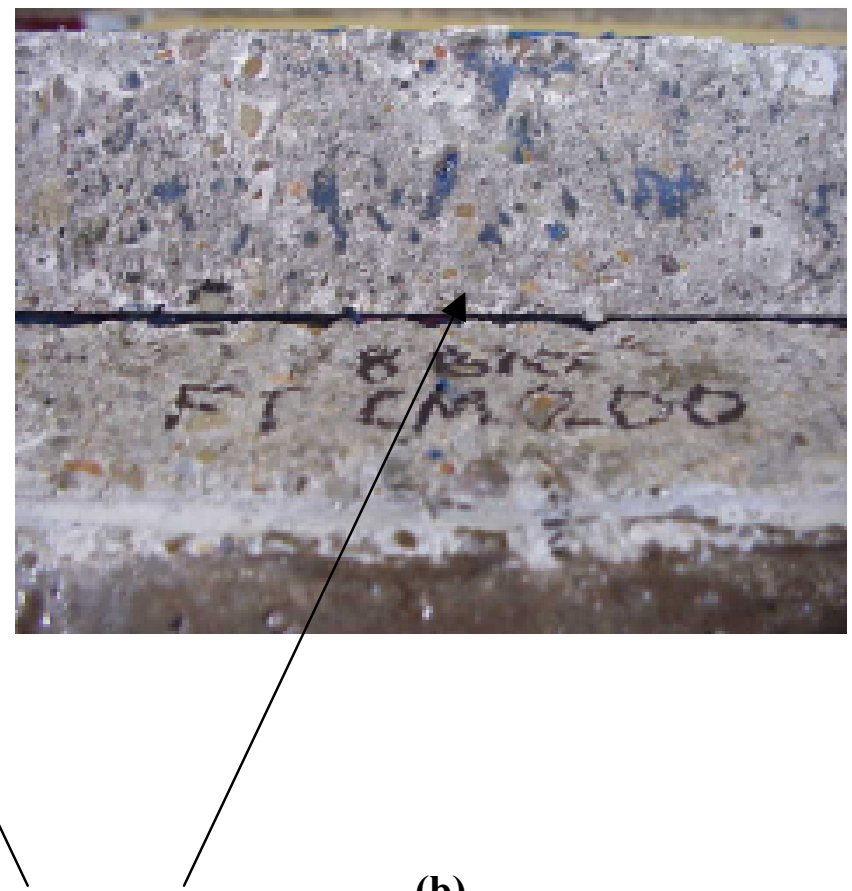

Underside of LVL

(b)

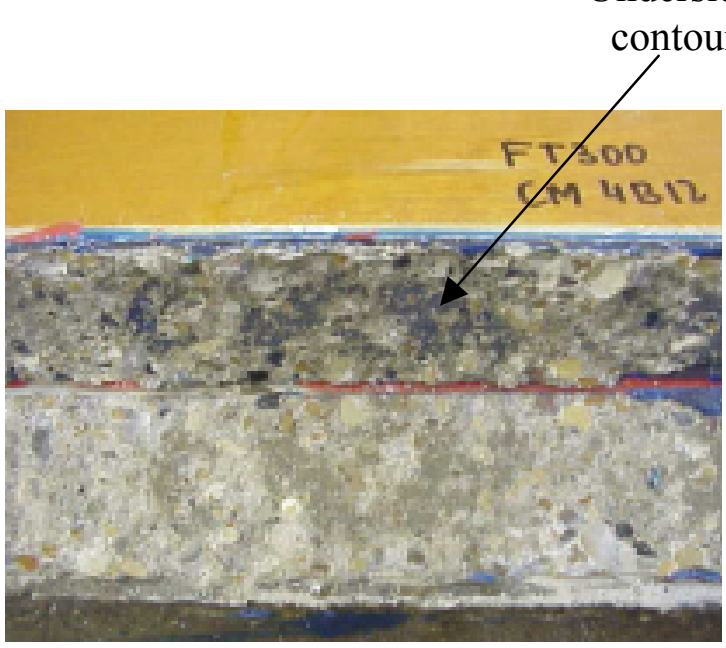

(c)

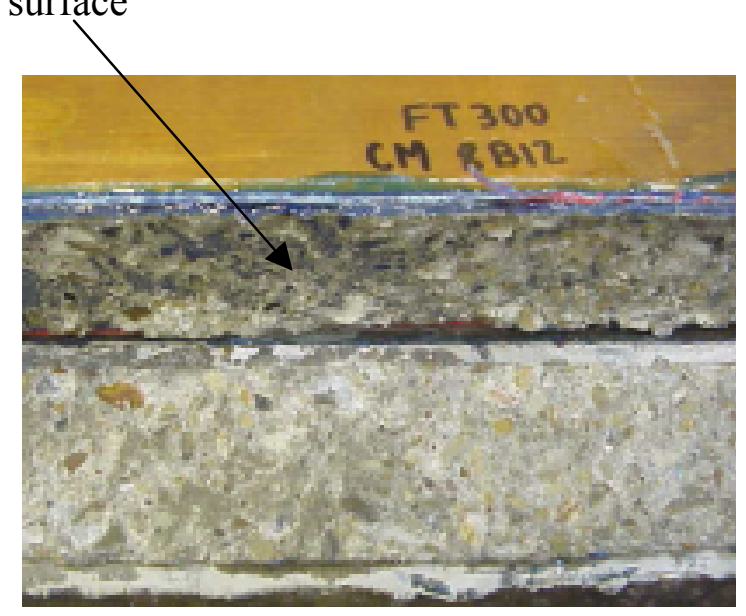

(d)

Figure 6.5 Pictures of the representative-dry fracture surfaces of freeze-thaw companion beams - (FTC200): $\quad$ (a) 7B10 and (b) 8B10

(FTC300): $\quad$ (c) $4 \mathrm{~B} 12$ and (d) $8 \mathrm{~B} 12$ 
Figures 6.4 and 6.5 show pictures of the representative-dry fracture surfaces of the wet-dry and freeze-thaw companion beams. Seven specimens are shown in all, three of which are wet-dry companions with the remaining four taken from among the freezethaw companions. Part (a) of Fig. 6.4 shows the surface of a 20-cycled wet-dry companion (the grouping of which is abbreviated as WDC20 in keeping with the conventions of the asterisked notes of Tables $6.6-6.9$ ), and parts (b) and (c) have pictures of two, 30-cycled wet-dry companions, 10B9 and 9B12. Also, parts (a) and (b) of Fig. 6.5 display the surfaces of two, 200-cycled freeze-thaw companions (abbreviated as FTC200, again see notes in Tables $6.6-6.9$ ), 7B10 and 8B10, and parts (c) and (d) have pictures of two, 300-cycled freeze-thaw companions, 4B12 and 8B12. Note especially from each of the top views, i.e. the undersides of the LVL contours, that the failure may once again be dominantly characterized as cohesive. The freeze-thaw specimens of Fig. 6.5 depict fracture surfaces that are highly cohesive as do those of the wet-dry specimens (Fig. 6.4), though the latter beams, particularly those of parts (b) and (c), appear to be somewhat moderately cohesive in comparison.

\subsection{Dry Weights and Strains}

In addition to fracture, several beams from among each of the representative groups (i.e. dry, wet-dry, and freeze-thaw) were periodically measured for any weight and strain changes. 
Weight Changes of the Companion (i.e. Dry) Specimens

Three companion beams (7B8, 9B11, and 9B12) for the wet-dry and ten (7B3, $8 \mathrm{~B} 3,1 \mathrm{~B} 4,6 \mathrm{~B} 7,5 \mathrm{~B} 8,6 \mathrm{~B} 9,7 \mathrm{~B} 10,8 \mathrm{~B} 10,4 \mathrm{~B} 12$, and $8 \mathrm{~B} 12)$ for the freeze-thaw cases were routinely checked for any changes in weight. Beam-weights typically ranged between 3.86 $\mathrm{kg}-4.31 \mathrm{~kg}(8.5 \mathrm{lb}-9.5 \mathrm{lb})$ as measured on an electronic bench scale (Gilson Company, Inc., Ohaus). The scale has a capacity of $91 \mathrm{~kg}(200 \mathrm{lb})$ with a platform size of $457 \mathrm{~mm} \mathrm{x}$ $457 \mathrm{~mm}(18 \mathrm{"} \mathrm{x} \mathrm{18")}$ and can measure weights accurate to $4.5 \mathrm{~g}(0.01 \mathrm{lb})$.

The weighing-schedule of the wet-dry beams was as follows: $0,2,5,10,15,20$, 25 , and 30 cycles - the $10^{\text {th }}$ cycle readings, however, were lost and so interpolated values are assumed in their stead; those of the freeze-thaw occurred at cycles: $0,50,100,150$, 200 , and 300 - the weighing of this latter group corresponded identically with that of the fracture schedule with the exception of the initial $\left(0^{\text {th }}\right)$ stage.

Not much weight change was expected to occur, especially concerning these dry specimens; moreover, this anticipation was only reinforced from the fact that all of the sides of the specimens had been sealed with a coating of primer (see Ch. 5) barring the sides of the concrete step and ledge (see Fig. 3.21 parts (c) and (d)). And, indeed, judging from Figures 6.6 and 6.7, the weight changes in the specimens during their term of study is truly negligible - the largest change of which, occurred in the $300^{\text {th }}$ cycle of the freezethaw specimens (Fig. 6.7) yielding only an averaged-percentage increase of $0.22 \%$.

Though there is not much to report concerning changes in weight, it is interesting to note the direction of these changes with respect to each of the two schemes. The wetdry companions show a loss of weight whereas the freeze-thaw specimens, in contrast, 


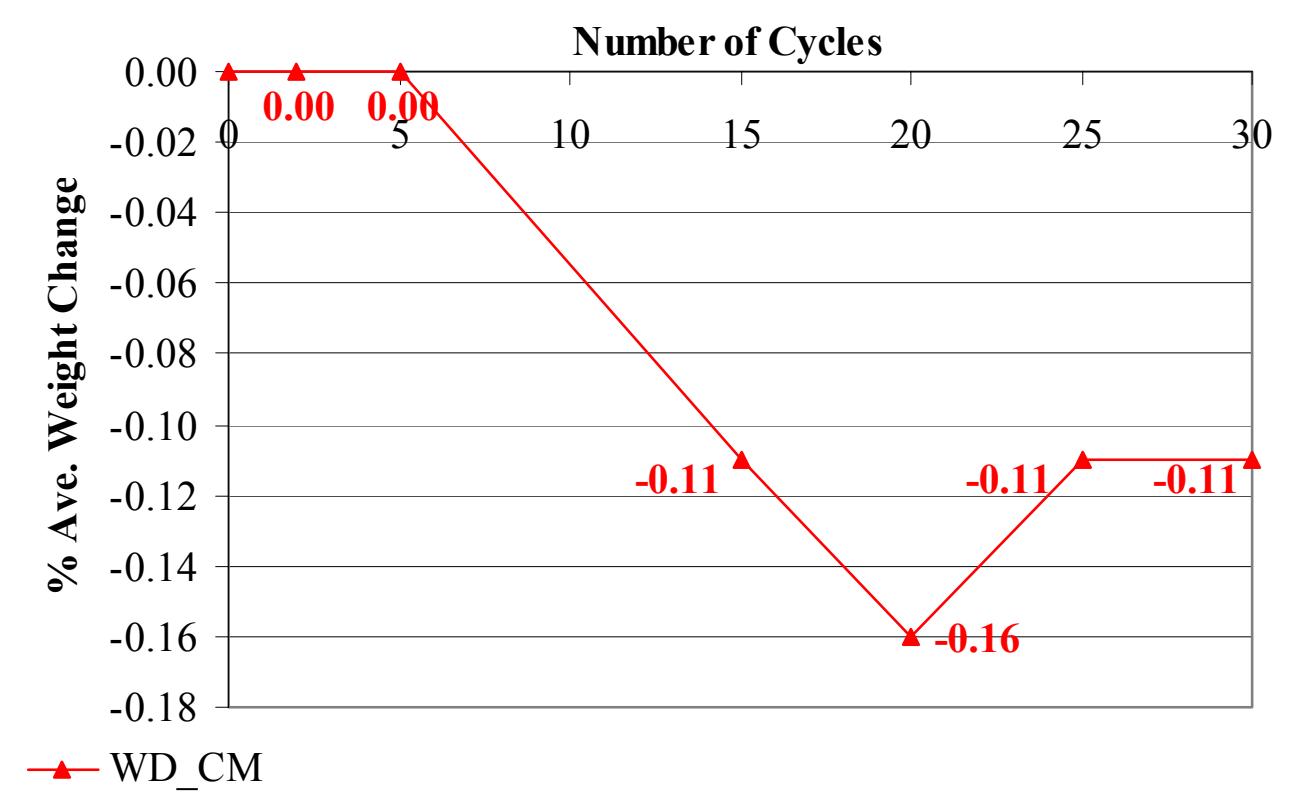

Figure 6.6 Percent average weight change of the wet-dry companion beams

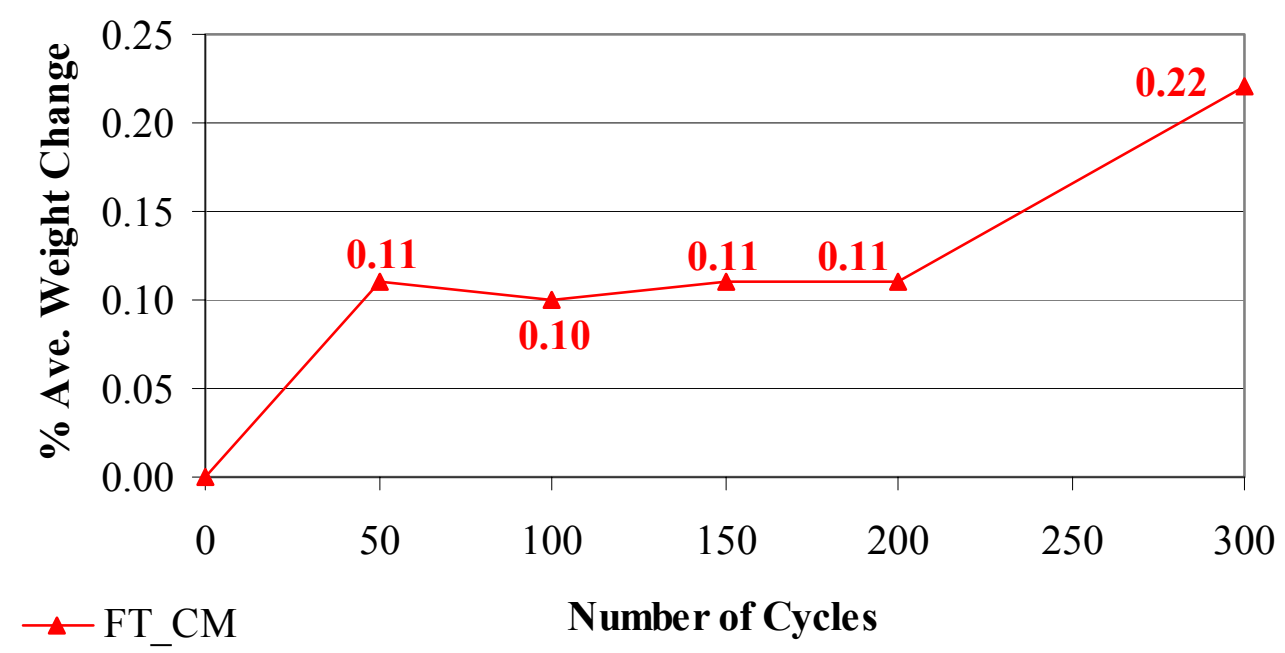

Figure 6.7 Percent average weight change of the freeze-thaw companion beams

demonstrate gains. This may be attributed to changes of temperature, humidity, or both, temperature and humidity, that occurred in the curing-room at the different stages of specimen-storage. For instance, the wet-dry scheme was begun at the end of September, 
2001, and so the environment of the curing-room was most probably different than at the time when the freeze-thaw regime began, approximately four months later, at the end of January, 2002. To reiterate, however, the magnitudes of these losses or gains are so slight that not much else may be commented concerning the dry-specimen weights.

\section{Strain Changes of the Companion (i.e. Dry) Specimens}

Six companion beams (4B3, 2B6, 4B6, 1B7, 10B9, and 10B10) for the wet-dry and five $(4 \mathrm{~B} 9,1 \mathrm{~B} 10,5 \mathrm{~B} 11,10 \mathrm{~B} 12$ and 5B13) for the freeze-thaw cases were also routinely checked for any changes in strains. Strain variations in both the CFRP and concrete were measured through the use of a demountable mechanical strain gauge (DEMEC) instrument (see Figure 6.8). The DEMEC (MASTRAD Quality and Test Systems, England) consists of a Mitutoyo digital dial gauge (sensitive to $0.01 \mathrm{~mm}=$

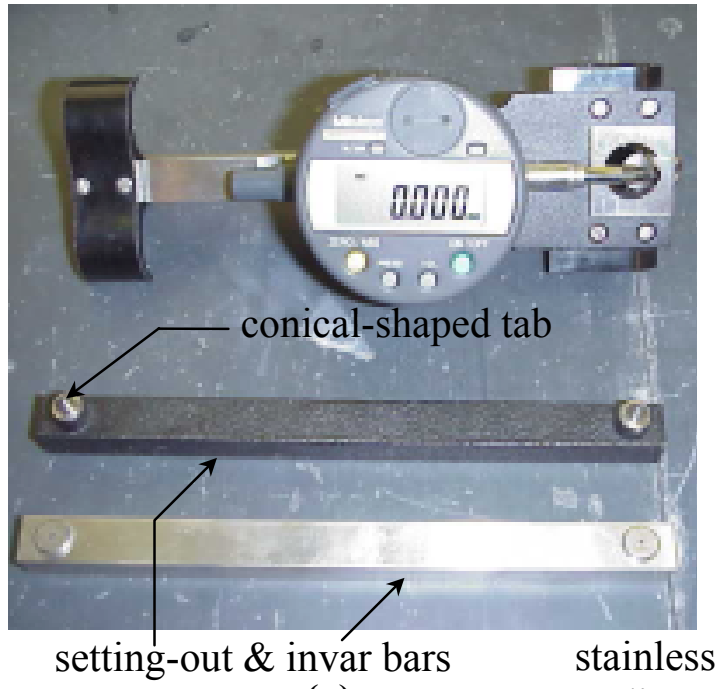

(a) discs on CFRP \& concrete ledge

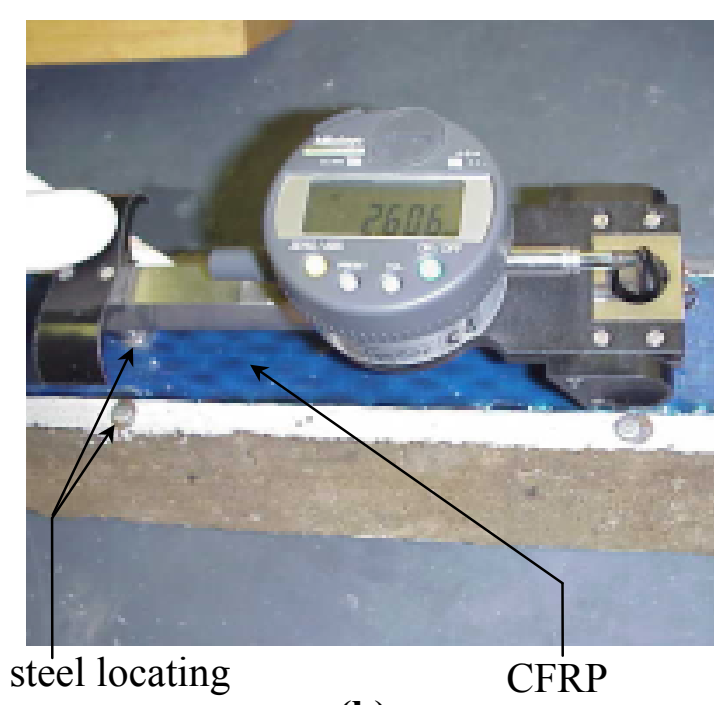

(b)

Figure 6.8 The demountable mechanical strain gauge (DEMEC) -

(a) DEMEC with setting-out and invar bars

(b) DEMEC in use atop CFRP 
$0.0005 ")$ that is attached to an invar (i.e. an alloy of iron-nickel that expands very little upon heating) bar (see Fig. 6.8(a)). Cylindrical stainless steel locating discs (dimensions: diameter $=6.4 \mathrm{~mm}\left(0.25^{\prime \prime}\right)$ and height $=1.5 \mathrm{~mm}(0.06 ")$ —see Fig. $\left.6.8(\mathrm{~b})\right)$ with predrilled holes are attached to the structure with a suitable adhesive. Maintaining the prescribed gauge length of these discs is accomplished by the use of a setting-out bar (Fig. 6.8(a)). All beams that were outfitted with locating discs, were done so with two pairs-one pair bonded by the MBrace Saturant and the other by the Micro-Measurements M-Bond AE15 adhesive, an epoxy-based glue sold by the Vishay Measurements Group. A decision was made to use two pairs of discs each attached with a different adhesive so that in the event a disc becomes dislodged during the course of the aging-program, there would be another pair from which strain measurements could be taken. A secondary reason for deciding to use two different adhesives was to study the durability of each adhesive under aggressive environments, both of which were found to perform satisfactorily. The DEMEC instrument contains two pointed tabs that are conical in shape and similar to what is shown on the setting-out bar of Fig. 6.8(a); one is stationary and the other, adjustable, with a maximum travel, in this case, of $100 \mathrm{~mm}$. Linear changes in length (i.e. strains) are measured by inserting these tabs, in turn, into the predrilled holes of the locating discs.

Strains of both the wet-dry and freeze-thaw beams were recorded alongside the weighing-schedule given above, i.e. corresponding to weeks: $0,2,5,10,15,20,25$, and 30 for the wet-dry beams — once again, the $10^{\text {th }}$-week readings are interpolated; and, at: 0 , $50,100,150,200$, and 300 cycles for the freeze-thaw specimens. 


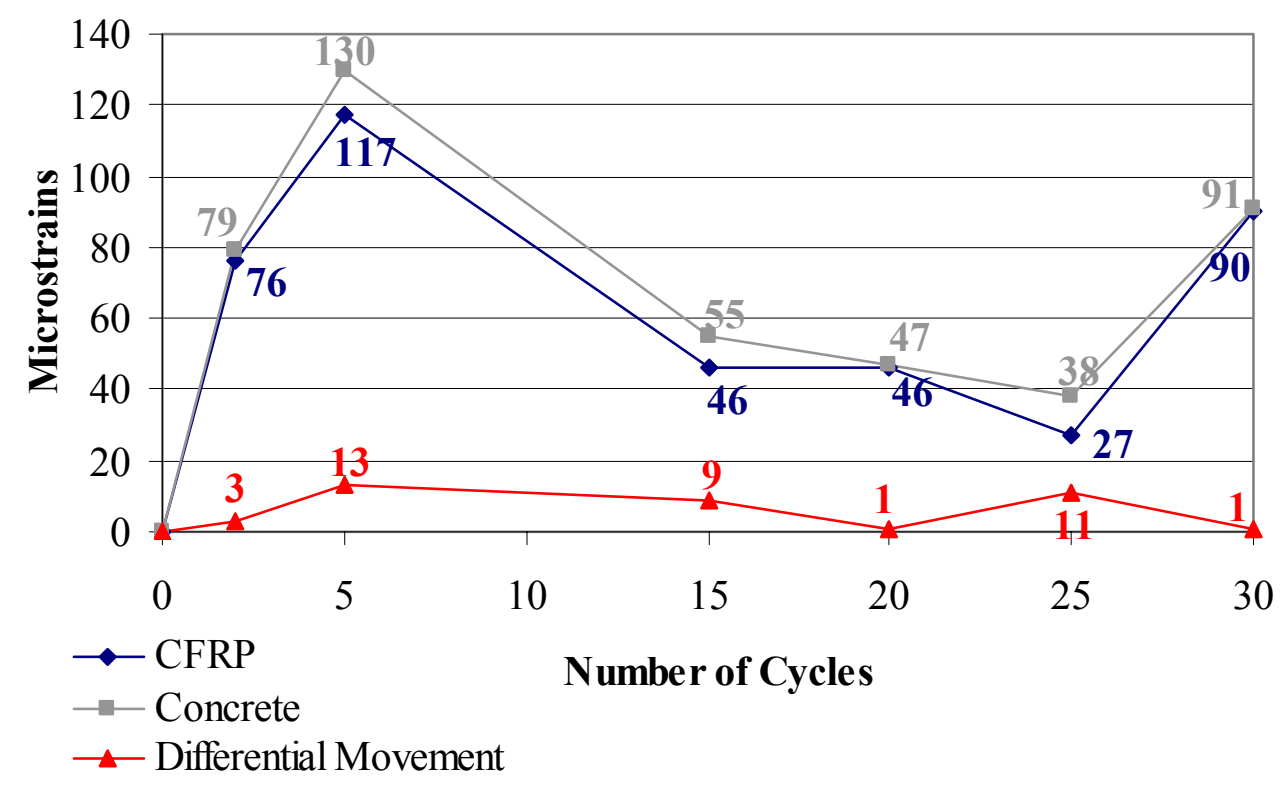

Figure 6.9 Microstrains of the wet-dry companion beams in two locations: (1) on the CFRP, and (2) on the adjacent concrete ledge (see Fig. 3.21(d)) - the differential movement between the two is shown as well

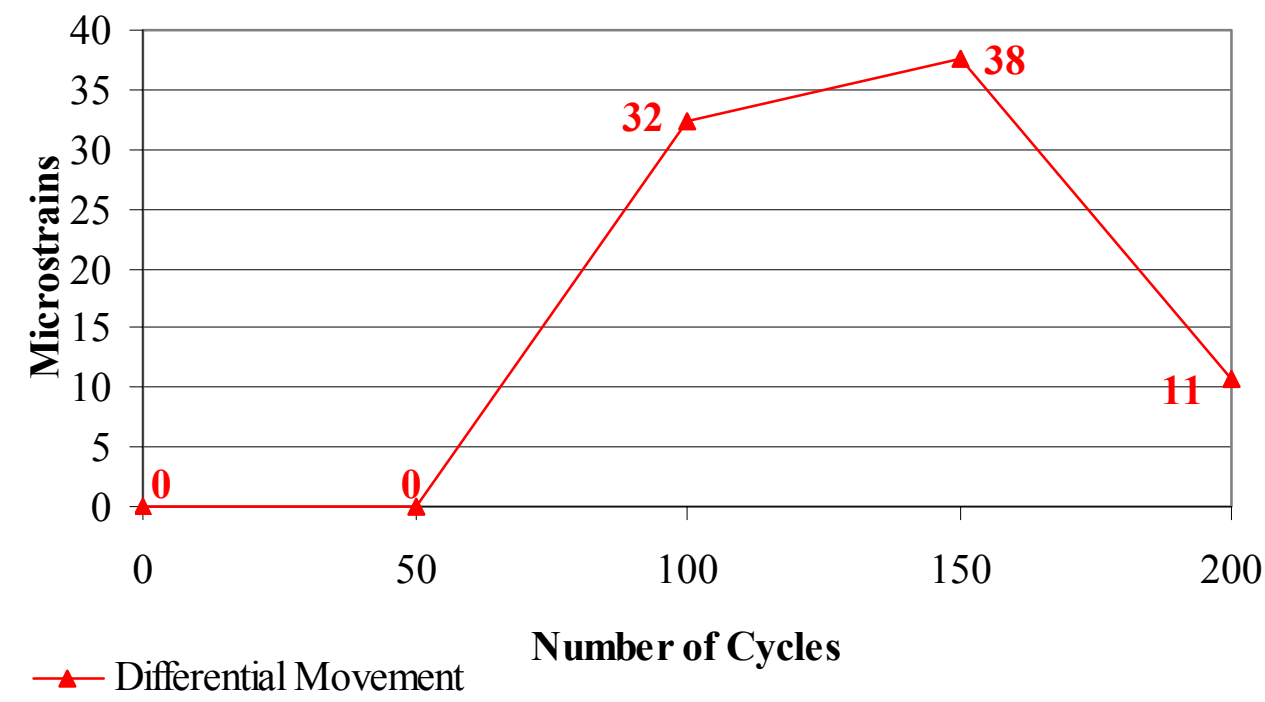

Figure 6.10 The differential movement between the CFRP strip and adjacent concrete ledge (see Fig. 3.21(d)) of the freeze-thaw companion beams 
Figures 6.9 and 6.10 show the microstrain results of the wet-dry and freeze-thaw beams, respectively. The former graph displays the microstrains recorded at the top of the CFRP strip and the adjacent ledge of concrete (see Figs. 3.21(d) and 6.8(b)) as well as any resulting differential movement that has taken place between the two. The latter graph only shows the differential movement between the CFRP and concrete since there were no beams from which continuous readings were taken from start to finish, as was done with the wet-dry specimens. Instead, initial strains were first taken on all five of the companions at the $0^{\text {th }}$ cycle, after which, the first strain readings were only taken on companion beam $4 \mathrm{~B} 9$ at the $50^{\text {th }}$ cycle. This specimen was then sacrificed to obtain data for fracture. The next readings of strain were taken on companion specimen 1B10 which was subsequently fractured at the $100^{\text {th }}$ cycle. This method was used for companion beams $5 \mathrm{~B} 11,10 \mathrm{~B} 12$ and $5 \mathrm{~B} 13$, corresponding to the $150^{\text {th }}, 200^{\text {th }}$ and $300^{\text {th }}$ cycles, respectively. Thus, while it is not possible to report on (freeze-thaw) absolute microstrain data, the relative (or differential) values can be cited and are the most decisive items of information to be gleaned from among the two.

In order to get a sense of what may be deemed as either significant or insignificant concerning the reported microstrains, the tensile strength of concrete is considered (or assumed) as a critical parameter. McCormac (p. 14, 1993) remarks that the tensile strength of concrete, $\mathrm{f}_{\mathrm{t}}$, varies between $8 \%-15 \%$ of its compressive value, $\mathrm{f}_{\mathrm{c}}{ }^{\prime}$. As a second means of corroboration, the American Concrete Institute (ACI 363R-92, Eq. 53 , p. 25, 1992) reports that the split-tensile strength (an indirect measure of tensile strength), $f_{s p}{ }^{\prime}$, can be taken as 7.4. $\left(f_{c^{\prime}}\right)^{1 / 2}$ - the value of $f_{c}{ }^{\prime}$ used here must be expressed in 
pounds per square inch (psi) and is valid for $3000 \leq \mathrm{f}_{\mathrm{c}}{ }^{\prime} \leq 12,000$ psi, or equivalently in MPa, $f_{s p}{ }^{\prime}=0.59 \cdot\left(f_{c}{ }^{\prime}\right)^{1 / 2}$, valid for $21 \leq f_{c}{ }^{\prime} \leq 83 \mathrm{MPa}$. Taking the more conservative value of the former along with the latter yields the following pair of equations:

$$
\begin{aligned}
& \mathrm{f}_{\mathrm{t}}=0.08 \cdot \mathrm{f}_{\mathrm{c}}{ }^{\prime} \\
& \mathrm{f}_{\mathrm{sp}}{ }^{\prime}=7.4 \cdot\left(\mathrm{f}_{\mathrm{c}}{ }^{\prime}\right)^{1 / 2} \quad \text { [in psi] }
\end{aligned}
$$

where the mean 28-day compressive strength of the concrete was found to be $f_{c}{ }^{\prime}=7480$ psi (see Chapter 5 or Table B.5). Therefore, with respect to this concrete, Eqs. (6.13) and (6.14) may consequently be written as:

$$
\begin{aligned}
& \mathrm{f}_{\mathrm{t}}=598 \mathrm{psi} \\
& \mathrm{f}_{\mathrm{sp}}{ }^{\prime}=640 \mathrm{psi}
\end{aligned}
$$

Correspondingly, the tensile stress of the concrete that has developed at the interface due to the differential strain, may then be computed from Hooke's law as:

$$
\sigma_{\mathrm{t}}=\mathrm{E}_{\mathrm{c}} \cdot \varepsilon
$$

where $\mathrm{E}_{\mathrm{c}}$ is given by the ACI code requirements (318R-92, Sec. 8.5.1, p. 79, 1992):

$$
E_{c}=57,000 \cdot\left(f_{c}{ }^{\prime}\right)^{1 / 2} \quad\left[f_{c}{ }^{\prime} \text { in psi }\right]
$$

and $\varepsilon=$ strain $=10^{6}$ microstrains (note: microstrains may be abbreviated as $\mu \varepsilon$ ). Thus,

$$
\mathrm{E}_{\mathrm{c}}=4.93 \times 10^{6} \mathrm{psi}
$$

for the specific concrete in use herein. By considering the maximum differential microstrains of both the wet-dry and freeze-thaw companions of Figures 6.9 and 6.10, i.e. $13 \mu \varepsilon$ and $38 \mu \varepsilon$, respectively, the resulting stresses by (6.15) are therefore found to be:

$$
\begin{aligned}
& \sigma_{\mathrm{t}, \mathrm{WD}}=64 \mathrm{psi} \\
& \sigma_{\mathrm{t}, \mathrm{FT}}=187 \mathrm{psi}
\end{aligned}
$$


Comparing Eq. (6.18) of the wet-dry companion specimens with (6.14') reveals that an order of magnitude exists between the developed stress of the interface and the underlying strength of the concrete; Eq. (6.13') exceeds this wet-dry companion stress by more than nine times. Also, a similar comparison of the freeze-thaw companion specimen-stress, Eq. (6.19), with the pair of strength equations $\left(\left(6.13^{\prime}\right)\right.$ and $\left.\left(6.14^{\prime}\right)\right)$ manifests a difference in excess of three times. Thus, it can safely be expected that, under dry conditions with temperatures near that of room and a variable relative humidity between approximately $50 \%-80 \%$, no appreciable development of strain will form at the CFRP-concrete interface.

\subsection{Conclusions}

As a foundation for the durability issues presented in the following two chapters, the present chapter's focus has been to unearth the hitherto evasive FRP-concrete interface fracture property, $G_{I c}=\mathrm{a}$ material-constant. This property was investigated for two varieties of specimens, namely: pristine and representative dry beams. The former class of samples was tested to attain early-age, or unadulterated, fracture toughness values; those of the latter kind, to characterize the performance of the same over the passage of time. It is interesting to note that, similar to the strength-gains realized in concrete with age, so it is with the CFRP-concrete interface fracture (see Figure 6.11). This is logical since both the epoxy and underlying cement matrices cure during this period and, thus, progress in acquiring strength. In total, conclusions were drawn from the consideration of 4-pristine and 20-representative dry specimens (see Table 6.11). 
A method was devised to answer the vitally important question, "Which load is critical?" in attempting to determine the CFRP-concrete value of fracture toughness, Eq. (3.73-repeated herein as 6.1). The prescribed approach is statistically-based on a one(may be higher, as deemed appropriate) standard deviation about the mean of the raw head loads from which the data is refined by the establishment of permissible load-ranges (Eqs. (6.9), (6.10); or similarly, (6.11), (6.12))—an attempt was made to elucidate this procedure by giving a step-by-step example in Section 6.2 , relying on the raw headinitiation loads of the pristine beams (see Table 6.1). Two types of fracture energies, designated head and set, may thus be computed for each of the initiation and arrestment cases. Finally, the head and set values are averaged to determine the conclusive critical strain energy release rates, as was done in Tables 6.4 and 6.10; these findings have been combined together for convenience and compactness in Table 6.11 below along with corresponding brittleness index, $I$, results.

The behavior of cracking was considered, as mentioned in Chapter 3, both from a quantitative and qualitative standpoint. The former measure, just mentioned in the previous paragraph, was formally introduced in Section 3.4 as Eq. (3.74-repeated herein for convenience as (6.2)), is known as the brittleness index, $I$; low $I$ values indicate a stable form of crack propagation (see Figs. 3.14(a) and 3.15(b)). Both the pristine and representative dry beam fractures were found to exhibit stable cracking $-I_{\text {pristine }}=0.06$ and $I_{\text {representative }}=0.04-$ and, as such, the interface-material may thus be characterized as being strain rate insensitive. 
The latter, qualitative, approach was achieved through visual inspection of the fracture surfaces of the beams (see Figs. 6.2, 6.4, and 6.5). Two important features were delineated:

(1) Failures throughout were largely cohesive as made evident by the very sparse bluish areas (i.e. representing the color of the saturant) visible on the undersides of the LVL contours. This is significant because it indicates that the adhesive-joint is soundly intact and stronger than that of the underlying concrete substratum.

(2) The failed surfaces showed very little, if any, of a shear-lip (see Fig. 3.9(d)). In other words, the failure is one that is truly dictated by plane strain conditions; thus, the reported $G_{I c}$ values are distinctly the conservative lower-limits as was depicted diagrammatically in Fig. 3.9. This affirms that the critical strain energy release rates, determined and reported herein for these specimens, are indeed material constants as claimed, in just the same manner as are, for example, the Young's and shear moduli.

Aside from fracture, dry weight and strain measurements were recorded on select specimens (Weights: 3-WDCs and 10-FTCs; Strains: 6-WDCs and 5-FTCs, where WDC $=$ wet-dry companions and FTC $=$ freeze-thaw companions-see also Table A.3 of 
Appendix A) as a secondary means of detecting any changes occurring in the beams over time. The case with the largest weight change occurred in the $300^{\text {th }}$ cycle of the freezethaw specimens (see Fig. 6.7) which yielded an averaged-percentage increase of $0.22 \%-$ a truly negligible difference. Not much could be stated with respect to the strains either, except that under normal dry conditions, no appreciable development of strain should be expected to form at the interface. This anticipation is based on the comparison of the longitudinal stresses, as determined by measurements made with the DEMEC strain gauge (see Fig. 6.8), with those of the underlying tensile strength of concrete- the latter being estimated by two approaches: i) a conservative percentage estimate (Eq. (6.13)), and ii) an ACI prescribed equation (Eq. (6.14)). The closest difference found to exist, using these methods, was in excess of a threefold disparity; an order of magnitude difference was determined at the other extreme. 


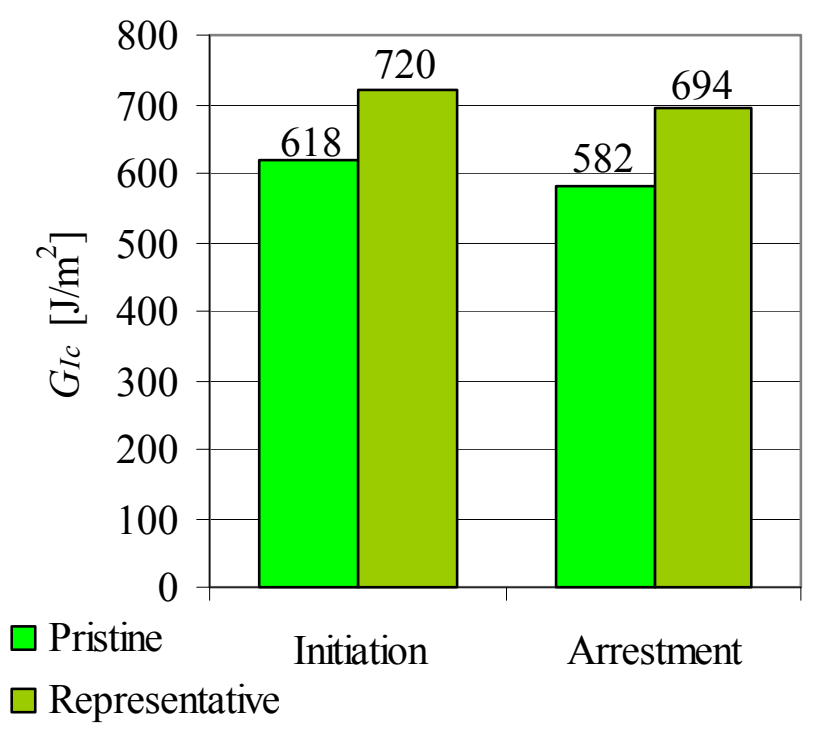

Figure 6.11 Averaged conclusive pristine- and representative- dry fracture results

Table 6.11 Averaged conclusive dry fracture results (see also Tables 6.4 and 6.10)

\begin{tabular}{c|c|c}
\cline { 2 - 3 } & Pristine & Representative \\
\hline $\boldsymbol{G}_{I c}^{\boldsymbol{i}}\left[\mathbf{J} / \mathbf{m}^{\mathbf{2}}\right]$ & 618 & 720 \\
\hline $\boldsymbol{G}_{\boldsymbol{I c}}^{\boldsymbol{a}}\left[\mathbf{J} / \mathbf{m}^{\mathbf{2}}\right]$ & 582 & 694 \\
\hline $\boldsymbol{I}$ & 0.06 & 0.04 \\
\hline
\end{tabular}




\section{CHAPTER 7}

\section{WET-DRY FRACTURE}

\subsection{Introduction}

Central to this and the following chapter is the question of permanence that was raised in Chapter 3, namely: How long is this (external-reinforcement of concrete) technology expected to endure? This thematic question elicits answers upon the figurative-stages of this and the upcoming chapter.

The focus herein is on CFRP-concrete interfaces having undergone subjection to a total of 30 alternate wet-dry cycles $(1$ cycle $=3$ days of wetting and 4 days of drying in an environmental chamber maintained at $40 \mathrm{C}$ and $50 \%$ relative humidity). The effects of two conditioning schemes - one that is slightly acidic (sodium sulfate, $\mathrm{Na}_{2} \mathrm{SO}_{4}, \mathrm{pH}=6.2$ - 6.5) and the other, alkaline (sodium hydroxide, $\mathrm{NaOH}, \mathrm{pH}=12$ ) - are examined in Sections 7.2 and 7.3, respectively. Just as in the previous chapter, the elements of presentation are comprised, first and foremost, with fracture data as obtained through the employment of the SCCB methodology, then the pattern of cracking is considered, both from a quantitative and qualitative standpoint, and lastly, any detectable visual, weight, and strain changes are analyzed. Degradative fracture results are computed at each of the 
three target ages, 10, 20, and 30 cycles, relative to their companion values (see Tables 6.6 - 6.9), and are charted as bar-graphs displaying percentage reductions in the values of $G_{I c}$ found with respect to the cycling-age. Also, interface fracture energies are computed using Eq. (3.73 — which, incidentally, was repeated again as (6.1)) by the two approaches discussed in Ch. 6, namely: the averaging, separately, of initiation and arrestment loads for the ascertaining of head- (i.e. topmost) and set- (i.e. all) loads. For a review of this procedure, read Section 6.2, and especially see the subsection titled "A Step-by-Step Example". Conclusive $G_{I c}$ values are then finally obtained by the averaging of such, correspondingly, admissible (see Eqs. $(6.9,6.10$; or 6.11, 6.12)) head and set results.

\subsection{Sodium Sulfate $\left(\mathrm{Na}_{2} \mathrm{SO}_{4}\right.$ - abbreviated: $\left.\mathrm{SS}\right)$ Attack}

Preliminaries (SS)

A $10 \%$ sodium sulfate solution was used to simulate attack from ground water and wet soils in which gypsum predominantly forms, causing softening of the concrete surface and the ultimate loss of mass (for more details, see Chapter 2). Twelve CFRPbonded concrete specimens (see Figure 7.1, i.e. the SCCB without the LVL contour; see

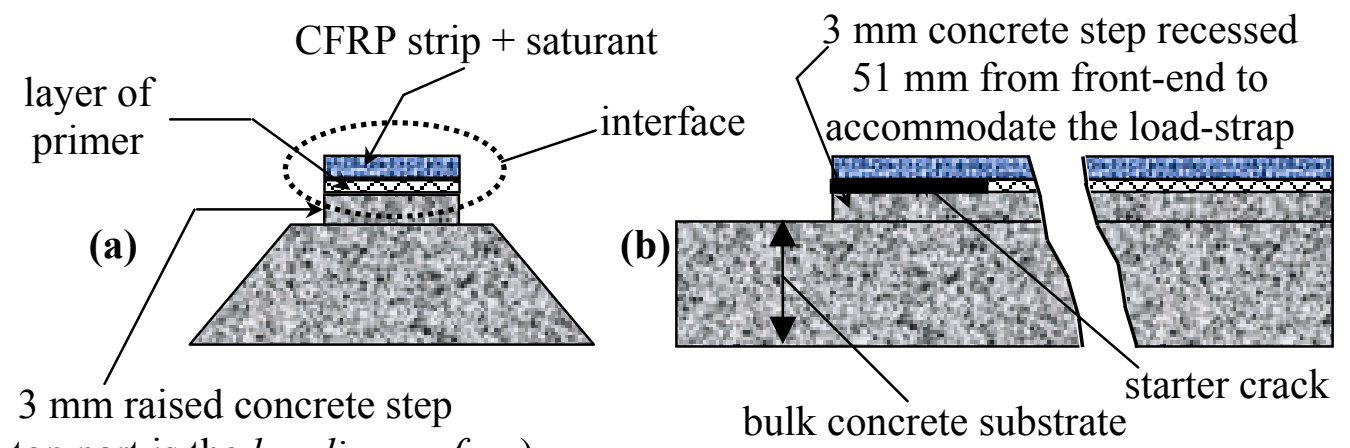

(the top part is the bonding surface)

Figure 7.1 CFRP-bonded concrete specimen-(a) cross-sectional view, and (b) side view (repeated from Fig. 3.19) 
also Table A.4 of Appendix A) were subjected to 10, 20, and 30 cycles of alternate wetting and drying in sodium sulfate -1 cycle $=3$ days of wetting (Figure 7.2) and 4 days of drying in an environmental chamber (Figure 7.3) maintained at $40 \mathrm{C}$ and $50 \%$ relative humidity. It should be noted here that of these twelve beams, conclusive fracture results were determined from nine specimens - the other three produced values of load that were outside the admissible range and, as such, stricken from consideration as shown in the following list (refer, once again, to Table A.4): (SS10): 2B3, 3B4, 5B4, 10B6; (SS20): 8B7, 3B8, 9B9, 9B10; (SS30): 7B11, 7B12, 8B13, 9B13; here, SS = sodium sulfate; 10 (e.g.) = number of cycles; concerning specimen-nomenclature, recall that the lead number designates the beam and the letter-and-number specifies the concrete batch (see Ch. 6).

The wetting-phase of conditioning was accomplished by placing the specimens in a zinc coated galvanized steel curing tank $\left(0.5 \mathrm{~m}^{3}=142\right.$ gal capacity $)$ lined with heavy plastic sheeting (see Fig. 7.2(a)). The corrosive solution used was made in a carboy (Fig. 7.2(b)) by dissolving anhydrous sodium sulfate pellets in distilled water and agitating thoroughly with a pneumatic stirrer (Fig. 7.2(c)). The $\mathrm{pH}$ of this solution, maintained between $6.2-6.5$, was monitored every two to three weeks and lowered appropriately, whenever needed, by the addition of sulfuric acid. As for the drying-phase, specimens were conditioned in a Cincinnati Sub-Zero ZH-16 environmental chamber (Fig. 7.3) having interior dimensions of $760 \mathrm{~mm}\left(30^{\prime \prime}\right) \times 760 \mathrm{~mm}\left(30^{\prime \prime}\right) \times 760 \mathrm{~mm}\left(30^{\prime \prime}\right)$. 


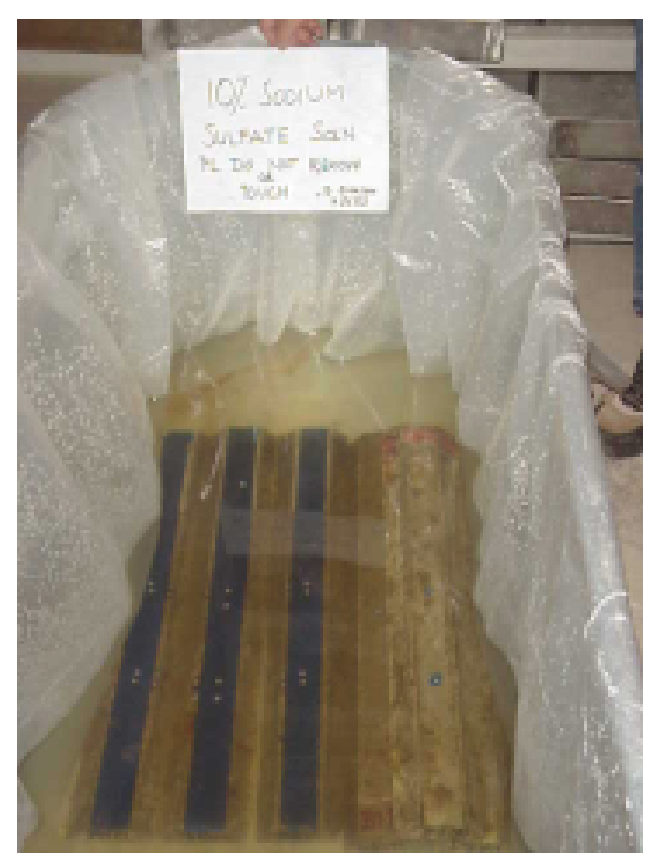

(a)

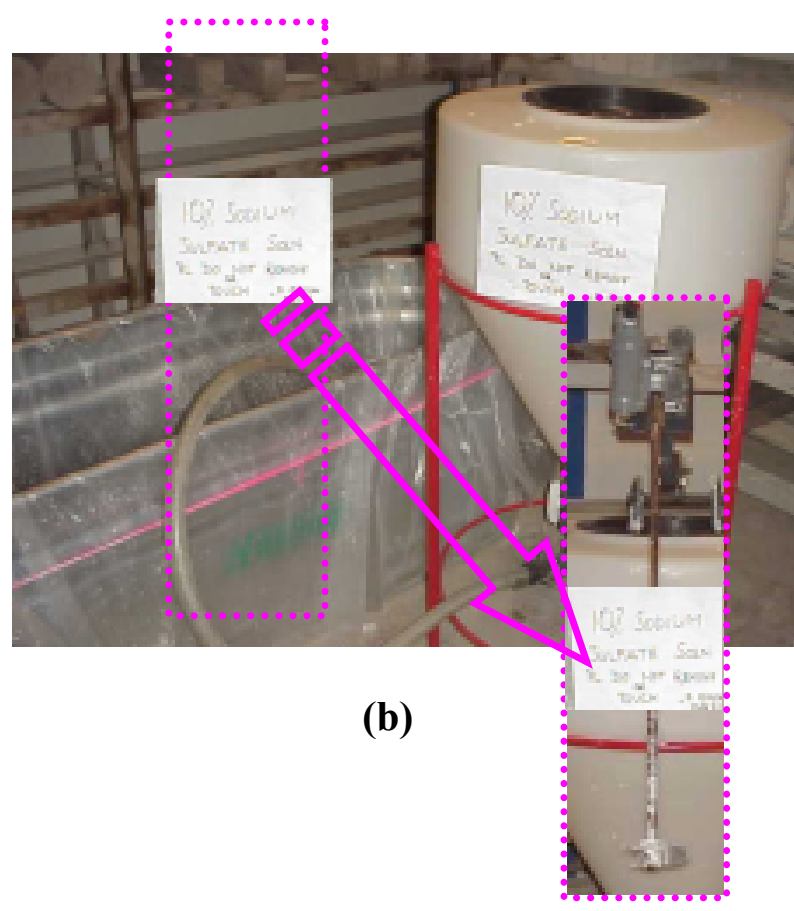

(c)

Figure 7.2 Pictures related to the wetting-phase-

(a) CFRP-bonded concrete specimens inside plasticlined tank filled with $10 \%$ sodium sulfate solution,

(b) carboy and tank, and

(c) pneumatic stirrer shown in front of carboy

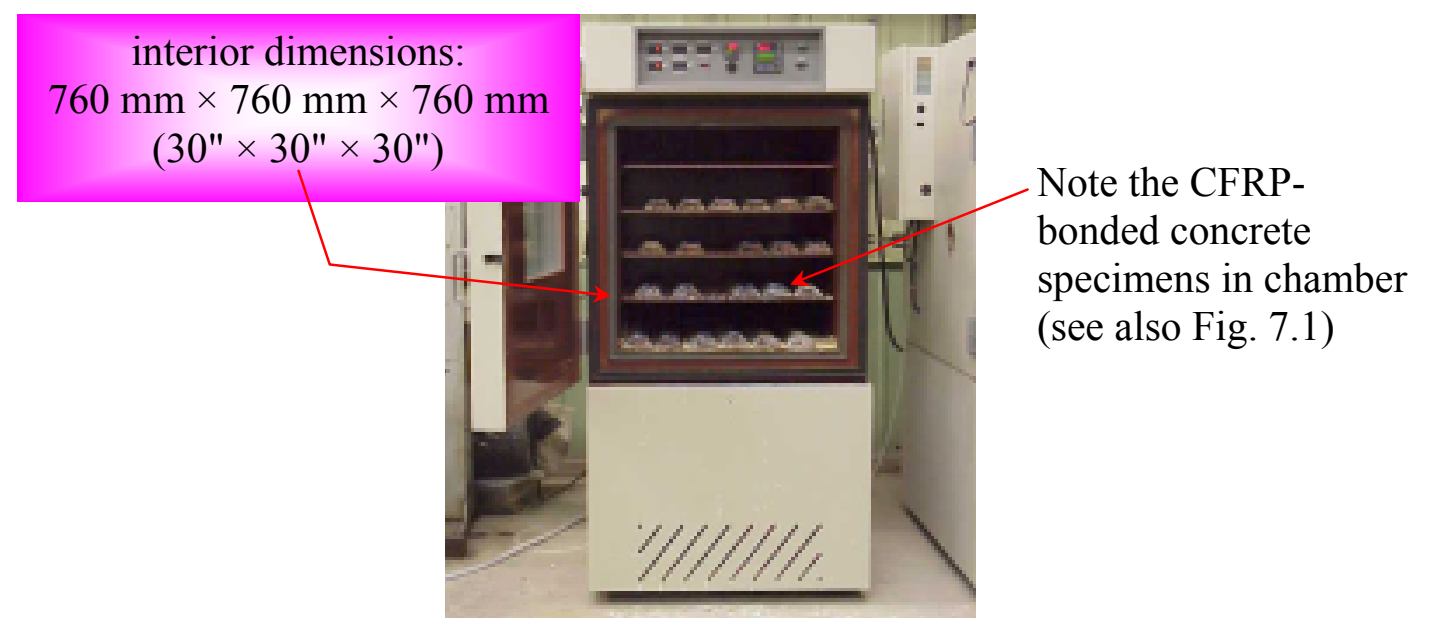

Figure 7.3 Picture related to the drying-phase - the Cincinnati Sub-Zero (CSZ), ZH-16, environmental chamber 
All of the concrete beams, with the exception of the pristine specimens (Table A.1), received a coating of MBrace primer on all surfaces, leaving open only the concrete raised-step and ledge (see Figs. 3.21 parts (c) and (d), and Fig. 7.4(b)) portions. This was done so that the bulk concrete substrate (Fig. 7.1(b)) might remain unscathed after being subjected to the variety of harsh environments. The sealing of the concrete beams in this fashion (Fig. 7.4) was designed, rather, to encourage attack on the interface by solely exposing it to the harmful effects of the various aggressive media-this is, after all, a necessary condition, if the central-issue of interface-reliability is to be investigated.

(a)

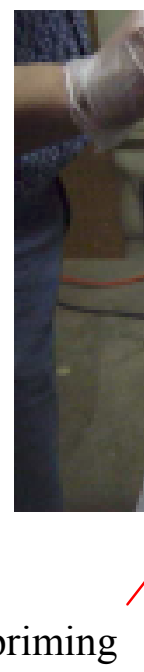

process of the concrete beam
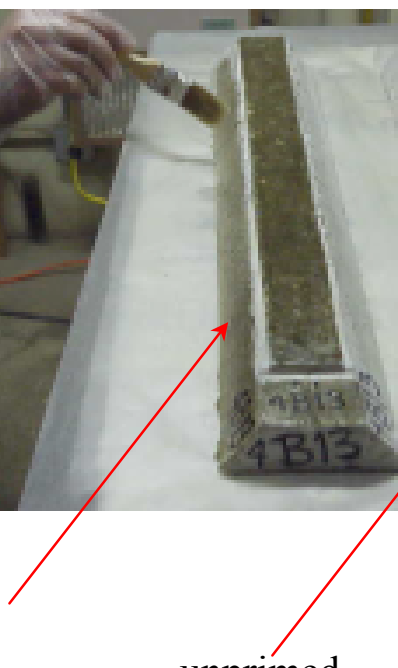

unprimed concrete beam

Note: only the concrete raised-step and ledge (see also Fig. 3.21 parts (c) and (d)) are left unprimed to enable aggressive-media penetration

the fully-primed concrete beam

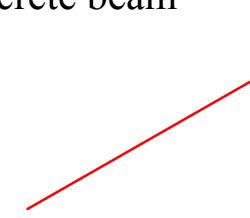

(b)

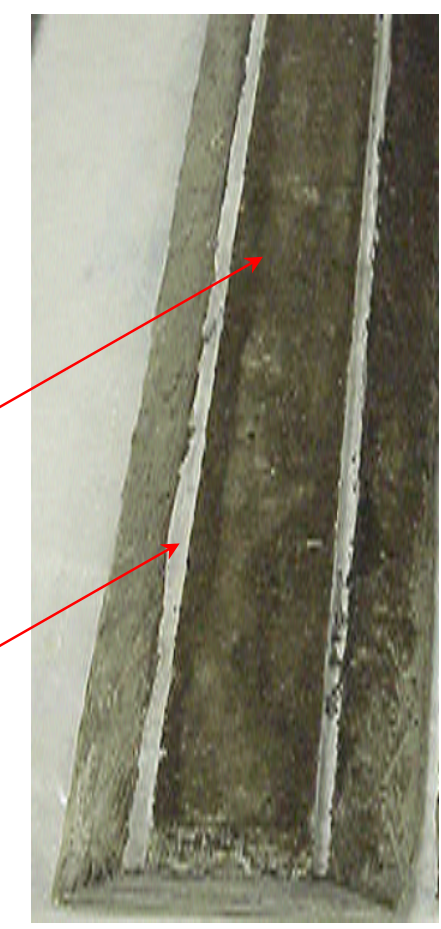

Figure 7.4 Pictures of the concrete beam-(a) the priming process (note contrast between the primed and unprimed beams (b) an example of a fully-primed concrete beam 


\section{SCCB Fracture (SS)}

The day before fracture-testing, i.e. following the end of every tenth wet-dry cycle, the CFRP layer of each beam (see Fig. 7.1) was lightly sanded and cleaned prior to the bonding of the LVL wooden contours (Fig. 3.21(a); see also Fig. 7.5 below) with the MBrace saturant. Also, to ensure proper bond between the wood and the CFRP layer,

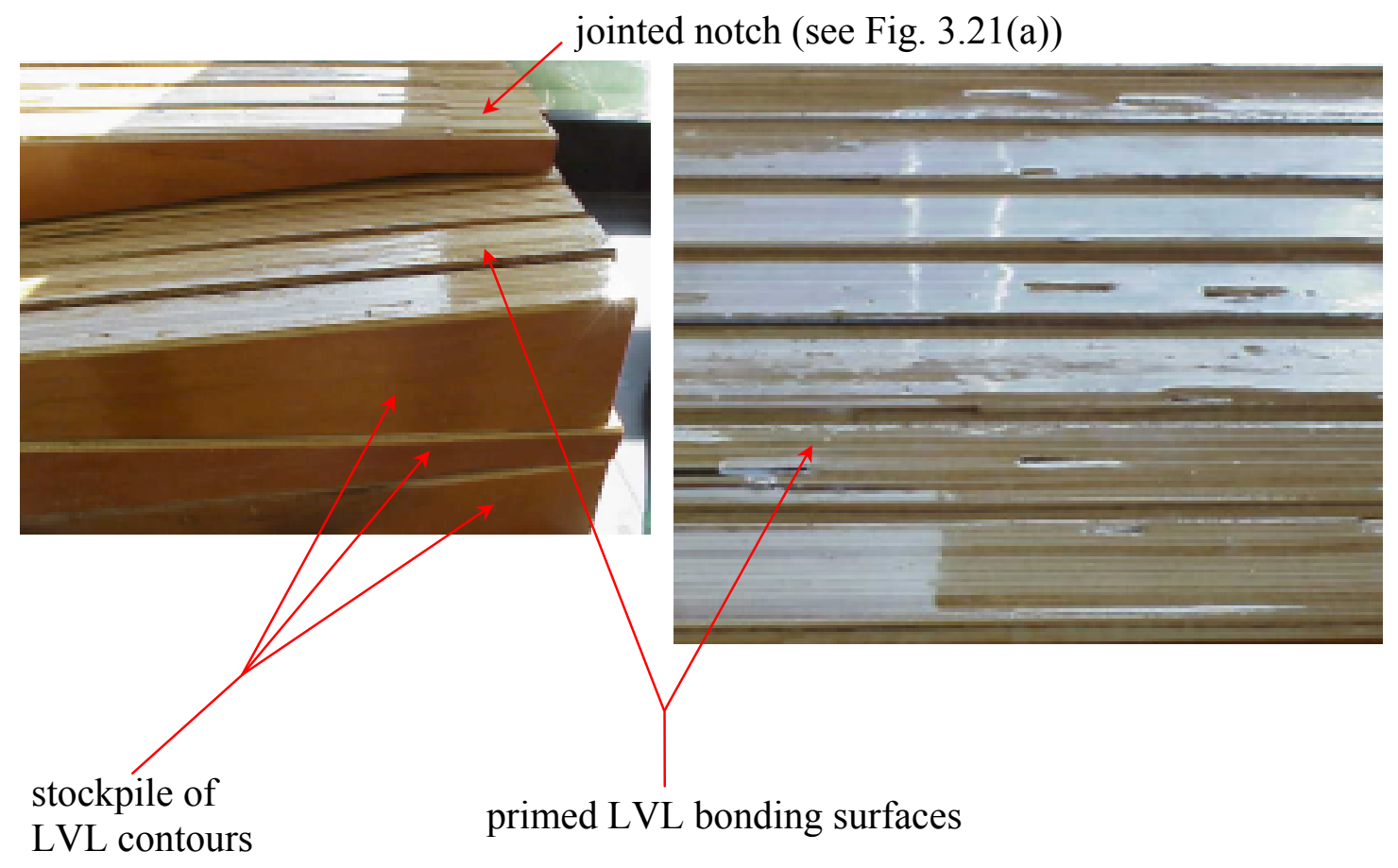

(a)

(b)

Figure 7.5 Pictures of the undersides of the LVL contours - (a) stockpiled specimens, and (b) close-up views of primed bonding surfaces

each of the bonding-surfaces of the wooden contours was prepared with a coating of the MBrace primer (Fig. 7.5). This single contoured-cantilever beam assembly was then set aside to cure for a 24-hour period after which the specimens were fractured. 
Adhering to the process of data-reduction presented in the previous chapter, all of the initiation and arrestment loads resulting from the wet-dry SCCB fracture tests were tabulated, similar to Table 6.1. Once again, this was accomplished by carefully inspecting the cracking-portions of the $P$ vs. COD graphs of each beam to establish the loads-ofinterest, i1 - i(n) and a1 - a(n-1), as was done in Figure 6.1 for the four pristine specimens; three select $P$ vs. COD graphs from each of the wet-dry target cycling-ages, specimens 3B4, 9B10, and 8B13, are shown in Figure 7.6. Next, the admissible loadranges $\left(R^{i}, R^{a}\right.$; see Eqs. (6.9) - (6.12)) were determined by using the statistical procedure outlined in Section 6.2, thus enabling the refinement of raw data by the designation of reduced head and set initiation- and arrestment- loads. By the averaging of these values, the four critical loads, $P_{c, \text { Head }}^{i}, P_{c, \text { Head }}^{a}, P_{c, \text { Set }}^{i}$, and $P_{c, \text { Set, }}^{a}$, were found, which when substituted, in turn, into Eq. (3.73 - also repeated in Ch. 6 as (6.1)), shown below, yielded the respective critical strain energy release rates:

$$
\text { (3.73) or (6.1): } \quad G_{I c}=\frac{P_{c}^{2}}{2 b} \frac{d C}{d a}
$$

—recall, once again, that $b=$ width of the specimen $=43.4 \mathrm{~mm}(1.71 ")$, and $d C / d a=$ compliance gradient $=1.78 \times 10^{-5} \mathrm{~N}^{-1}\left(7.92 \times 10^{-5} \mathrm{lb}^{-1}\right)$. These results are shown in Tables 7.1 - 7.3 and summarized with averaged head and set $G_{I c}$ values in Table 7.4. Also reported in these first three tables are the corresponding companion fracture energies and the resulting percentage fracture toughness reductions. The latter values are determined relative to the companion sample values of the same age (see Equation (7.1)) - the one exception being the $10^{\text {th }}$ cycle companion results. Apparently, the saturant that had been applied to the contour-CFRP layer of these specimens on the day prior to their fracture 

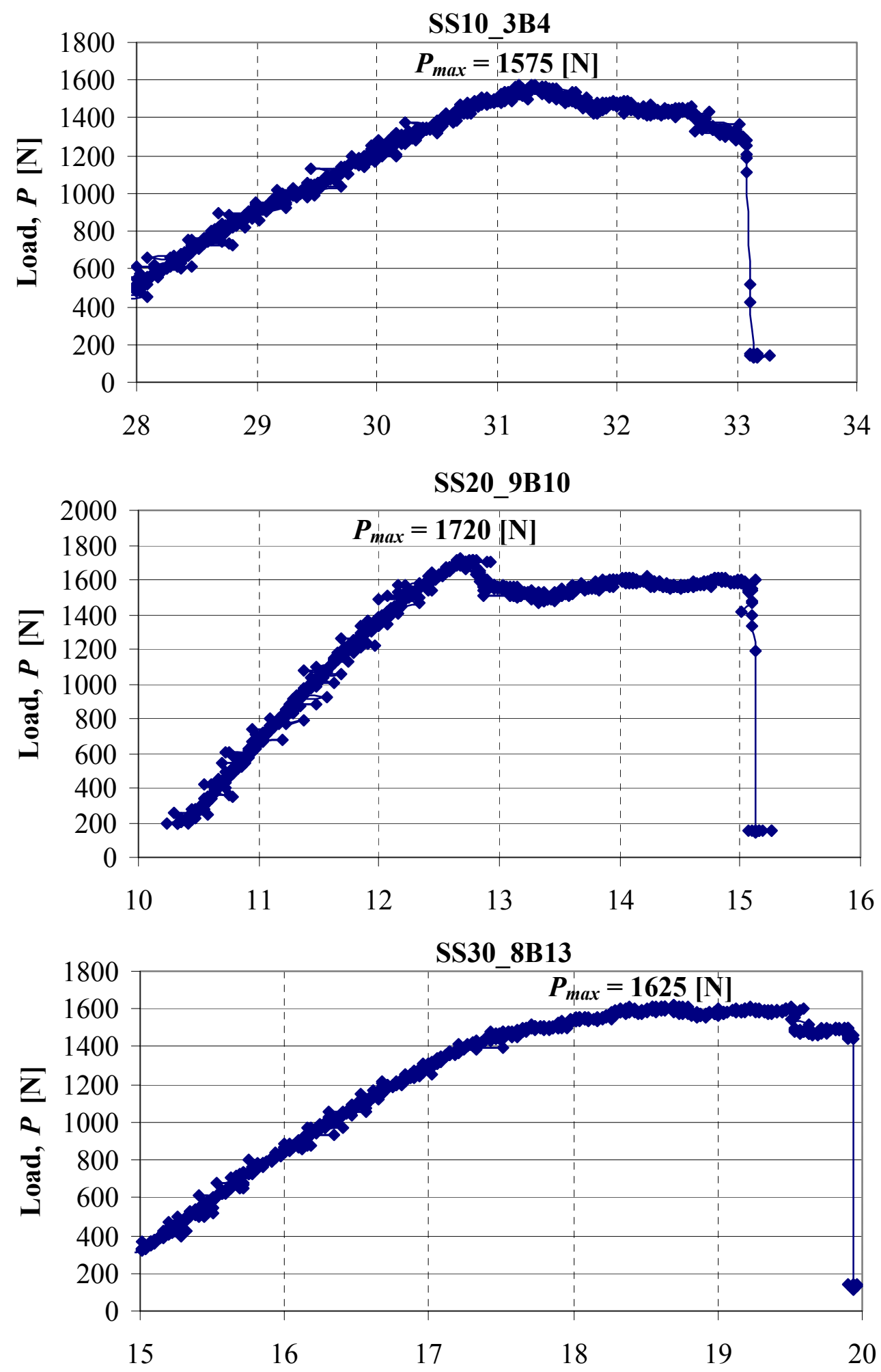

Crack Opening Displacement, COD [mm]

Figure 7.6 Fracture behavior of select WD-(a) 10-, (b) 20-, and (c) 30cycled beams in sodium sulfate (SS) 
had not fully cured, thus, culminating in the separation of this region during testing and not that of the, otherwise, customary CFRP-concrete interface layer. In an attempt to rectify this problem, then, it was decided that the four pristine dry fracture results of $\mathrm{Ch}$. 6 (see Table 6.3 ) be substituted in lieu of the erroneous $10^{\text {th }}$ cycle companion values, as indicated by asterisks in Figure 7.7. In this figure, $G_{I c}^{i}$ and $G_{I c}^{a}$ effect-based computations are reported on the top and bottom graphs (parts (a) and (b)), respectively. Moreover, corresponding head- and set- reductions are plotted side-by-side to facilitate in evaluating the range of variations encountered for a given age. Reductions are computed based on the following relationship:

$$
\% G_{I c} \text { Reduction }=\frac{G_{I c, \text { conditioned }(\# \text { cycles })}}{G_{I c, \text { companion }(\# \text { cycles })}} \times 100 \%
$$

where the critical strain energy release rate of the numerator may represent any one of the various conditioning schemes (i.e. wet-dry cycling under sodium -sulfate or -hydroxide, or freeze-thaw cycling under calcium chloride) for a specific age (i.e. 10, 20, or 30 cycles for the wet-dry cases, or 50,100, 150, 200, or 300 cycles for those of freeze-thaw), and the denominator is the corresponding $G_{I c}$ value of the companion beams at the same age.

Table 7.1 Reduced data results of wet-dry, SS10 fracture test specimens

\begin{tabular}{c|cc|cc}
\cline { 2 - 5 } & \multicolumn{3}{|c|}{ Head } & \multicolumn{2}{c}{ Set } \\
\hline $\mathbf{n}$ & $\mathbf{i}$ & $\mathbf{a}$ & $\mathbf{i}$ & $\mathbf{a}$ \\
\hline $\boldsymbol{G}_{\boldsymbol{I c}}\left[\mathbf{J} / \mathbf{m}^{2}\right]-\mathbf{S S 1 0} /$ pristine $^{*}$ & $\mathbf{5 3 1 / 6 1 7}$ & $\mathbf{5 0 8 / 5 8 2}$ & $\mathbf{4 9 1 / 6 2 0}$ & $\mathbf{4 6 9 / 5 8 1}$ \\
\hline $\boldsymbol{\sigma}\left[\mathbf{J} / \mathbf{m}^{2}\right]$ & 0.71 & 0.22 & 0.43 & 0.37 \\
\hline $\mathbf{C O V}$ & $3.67 \%$ & $2.07 \%$ & $2.92 \%$ & $2.82 \%$ \\
\hline$\% \boldsymbol{G}_{\boldsymbol{I} \boldsymbol{c}}$ Reduction & $86 \%$ & $87 \%$ & $79 \%$ & $81 \%$ \\
\hline
\end{tabular}

pristine values are taken from Table 6.3 
Table 7.2 Reduced data results of wet-dry, SS20 fracture test specimens

\begin{tabular}{|c|c|c|c|c|}
\hline & \multicolumn{2}{|c|}{ Head } & \multicolumn{2}{|c|}{ Set } \\
\hline & i & $\mathbf{a}$ & i & $\mathbf{a}$ \\
\hline $\mathbf{n}$ & 3 & 3 & 57 & 54 \\
\hline$G_{I c}\left[\mathrm{~J} / \mathrm{m}^{2}\right]-\mathrm{SS20} / \mathrm{WDC20}{ }^{*}$ & $614 / 744$ & $601 / 704$ & 548/828 & 530/804 \\
\hline$\sigma\left[\mathrm{J} / \mathbf{m}^{2}\right]$ & 0.30 & 0.43 & 0.92 & 0.93 \\
\hline COV & $2.19 \%$ & $2.67 \%$ & $4.09 \%$ & $4.20 \%$ \\
\hline$\% G_{I c}$ Reduction & $82 \%$ & $85 \%$ & $66 \%$ & $66 \%$ \\
\hline
\end{tabular}

"WDC20 = wet-dry companions at 20 cycles (see Tables 6.6 - 6.9)

Table 7.3 Reduced data results of wet-dry, SS30 fracture test specimens

\begin{tabular}{c|cc|cc}
\cline { 2 - 5 } & \multicolumn{2}{|c|}{ Head } & \multicolumn{2}{c}{ Set } \\
\hline $\mathbf{n}$ & 2 & 2 & $\mathbf{i}$ & $\mathbf{a}$ \\
\hline $\boldsymbol{G}_{\boldsymbol{I c}}\left[\mathbf{J} / \mathbf{m}^{2}\right]-\mathbf{S S 3 0} / \mathbf{W D C 3 0}$ & $\mathbf{5 3 2 / 7 8 2}$ & $\mathbf{5 2 1} / 764$ & $\mathbf{4 9 5 / 8 1 0}$ & $\mathbf{4 8 4 / 7 8 7}$ \\
\hline $\boldsymbol{\sigma}\left[\mathbf{J} / \mathbf{m}^{2}\right]$ & 0.07 & 0.07 & 3.50 & 3.88 \\
\hline $\mathbf{C O V}$ & $1.14 \%$ & $1.16 \%$ & $8.41 \%$ & $8.96 \%$ \\
\hline $\mathbf{\%} \boldsymbol{G}_{\boldsymbol{I c}}$ Reduction & $68 \%$ & $68 \%$ & $61 \%$ & $61 \%$ \\
\hline
\end{tabular}

"WDC30 = wet-dry companions at 30 cycles (see Tables 6.6 - 6.9)

Table 7.4 Sodium sulfate, wet-dry fracture results (unit: $\mathrm{J} / \mathrm{m}^{2}$ )

\begin{tabular}{|c|c|c|c|c|}
\hline & \multicolumn{2}{|c|}{ Initiation } & \multicolumn{2}{|c|}{ Arrestment } \\
\hline & $\boldsymbol{G}_{\boldsymbol{I}, \mathrm{H} \text {, ead }}$ & $\boldsymbol{G}_{I c, \text { Set }}$ & $\boldsymbol{G}_{I c, \text { Head }}$ & $\boldsymbol{G}_{\boldsymbol{I c}, \text { Set }}$ \\
\hline SS10 & 531 & 491 & 508 & 469 \\
\hline Mean: & \multicolumn{2}{|c|}{511} & \multicolumn{2}{|c|}{488} \\
\hline SS20 & 614 & 548 & 601 & 530 \\
\hline Mean: & \multicolumn{2}{|c|}{581} & \multicolumn{2}{|c|}{566} \\
\hline SS30 & 532 & 495 & 521 & 484 \\
\hline Mean: & \multicolumn{2}{|c|}{514} & \multicolumn{2}{|c|}{502} \\
\hline
\end{tabular}


(a) Initiation-Based Wet-Dry Sodium

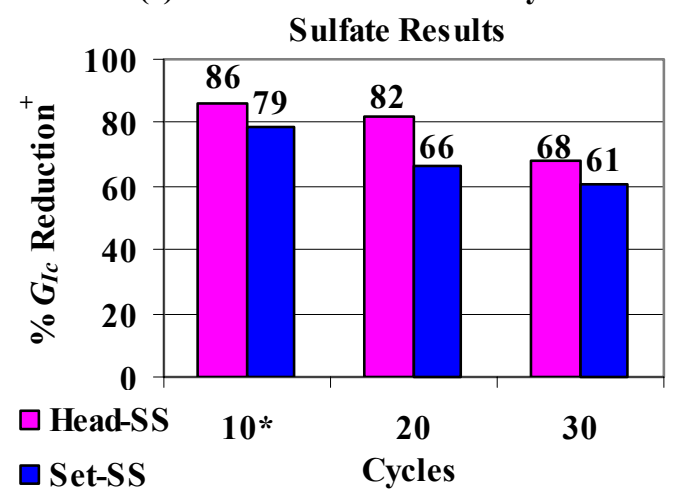

(b) Arres tment-Based Wet-Dry Sodium

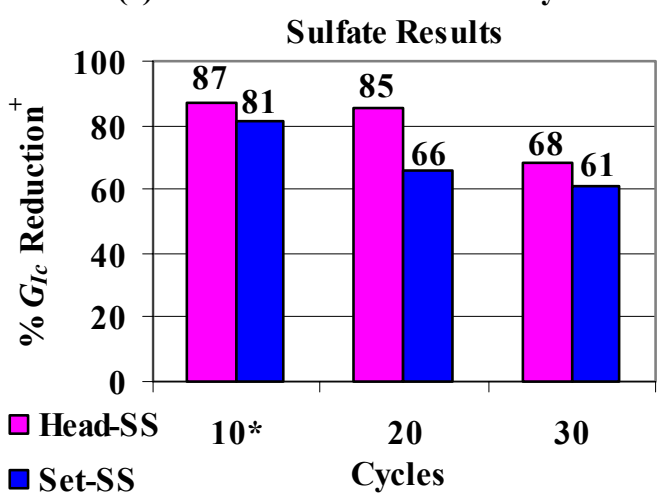

${ }^{+}$Reductions computed relative to companion values; " Pristine dry fracture results (see Table 6.3) substituted for $10^{\text {th }}$ cycle companion values

Figure 7.7 Percentage reductions in (a) $G_{I c}^{i}$ and (b) $G_{I c}^{a}$ resulting from 10, 20 , and 30 wet-dry cycles of sodium sulfate (SS) exposure

The average variation between head and set $G_{I c}$ percentage reductions for the sodium sulfate (SS) exposures was found to be $9 \%$, with maximum and minimum deviations of $14 \%$ and $6 \%$ for the $20^{\text {th }}$ and $10^{\text {th }}$ cycle arrestment-based cases, respectively. Interestingly, the effects of head-degradations in the first-half period of aging, i.e. between the $10^{\text {th }}$ and $20^{\text {th }}$ cycles, was not as pronounced as was the case in the 
latter-half of exposure-i.e. approximately $3 \%$ as opposed to $17 \%$. This large disparity may be indicative of the insufficient formation of gypsum or the underdevelopment of some of the other adverse products that are known to stem from exposure to sulfate attack, such as ettringite, at these comparatively early stages. After the complete duration of aging at 30 cycles, however, both the head and set initiation- and arrestment- based SS attack cases identically reduced the efficiency of the interface bond to $68 \%$ and $61 \%$, respectively, thus offering some verity with respect to the magnitude of deterioration that was sustained. Averaging these two values reveals that, under SS30/wet-dry cycling, the CFRP-concrete interface reliability may be characterized as being less than two-thirds as effectual were it, otherwise, not subjected to such attack.

\section{Patterns of Fracture (SS)}

Quantitatively (SS): Brittleness indices are once again investigated to gain a quantitative insight into the cracking patterns of SS/wet-dry cycled CFRP-concrete interfaces. Substitution of the critical strain energy release rates of Table 7.4 into Eq. (3.74—or 6.2),

$$
I=\frac{G_{I c}^{i}-G_{I c}^{a}}{G_{I c}^{i}}
$$

yields the following results (shown in tabular form):

Table 7.5 Brittleness indices of SS/wet-dry fracture test specimens

\begin{tabular}{c|c|c|c}
\cline { 2 - 4 } & SS10 & SS20 & SS30 \\
\hline$I$ & 0.04 & 0.02 & 0.02 \\
\hline
\end{tabular}


Each of the $I$ values of Table 7.5 correspond to stable forms of crack propagation as diagrammatically shown in Figures 3.14(a) and 3.15(b). The average of these values is approximately $I=0.03$, which reveals an even higher degree of cracking-stability than what was previously determined for the cases corresponding to the pristine $(I=0.06)$ and representative dry $(I=0.04)$ specimens.

Qualitatively (SS): The following three figures depict the fracture surfaces of each of the "refined" specimens (see Table A.4) that underwent SS exposure; Figure 7.8 displays the fracture results of beams after being conditioned for 10 wet-dry cycles, and Figs. 7.9 and 7.10 show, respectively, the effects after subjection to 20 and 30 such cycles.

Figure 7.8, parts (a) and (b), are top views of the concrete bonding surfaces of beams $2 \mathrm{~B} 3$ and $3 \mathrm{~B} 4$ after fracture - note the contrast in fracture patterns that exists between the two superficies. The former appears to be a more cohesive type of failure as evidenced by the coarse and jagged surface; the latter is considerably smoother and level, and, for the most part, the coat of primer still seems to be intact as revealed by the pervasively dark-gray areas visible amidst the whitish-speckles of the underlying concrete - as such, it may be characterized as an adhesive failure. The view in part (c) of beam 10B6 is somewhat of a mix between those of parts (a) and (b), though it more dominantly appears to be cohesive, as seen by the thin layer of concrete that was displaced on the underside of the LVL contour - contrast this with the dry companion surfaces of beams 4B12 and 8B12 of Figure 6.5, parts (c) and (d), for example. On the other hand, a comparison should be made with the pristine-dry beam 3B6 of Fig. 6.2, since both were cast from the same batch (\#6) of concrete. (This is significant from the 
(a)

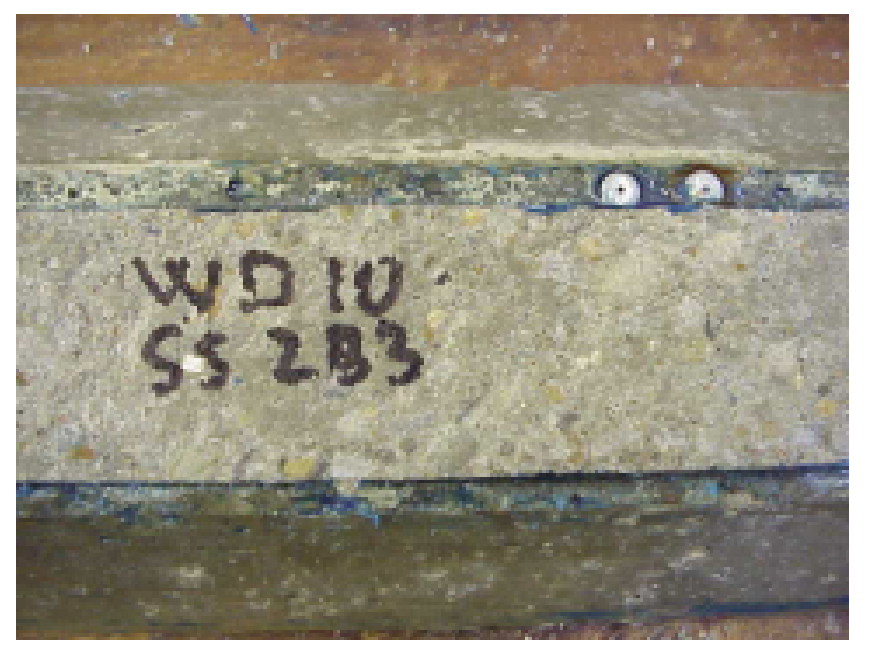

(b)

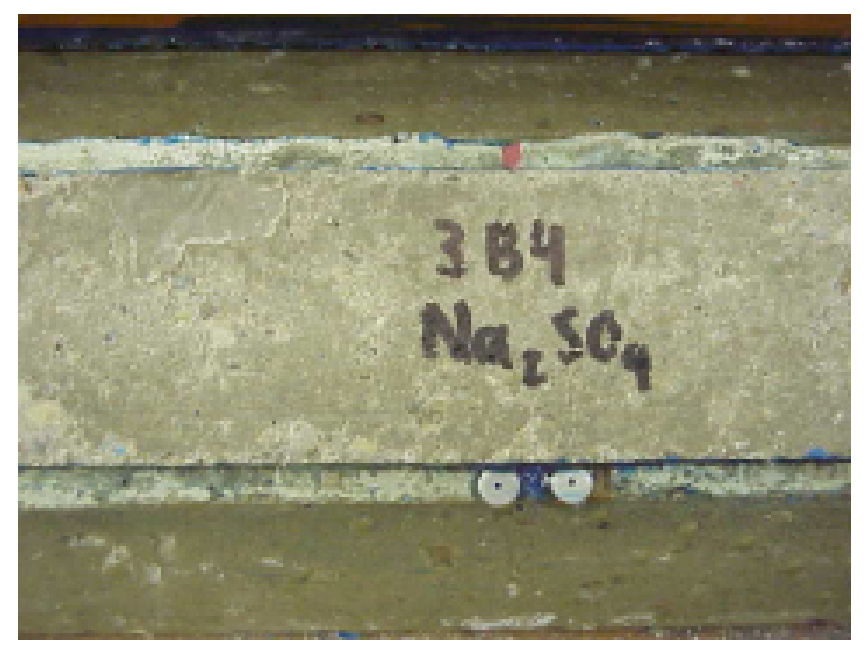

(c)

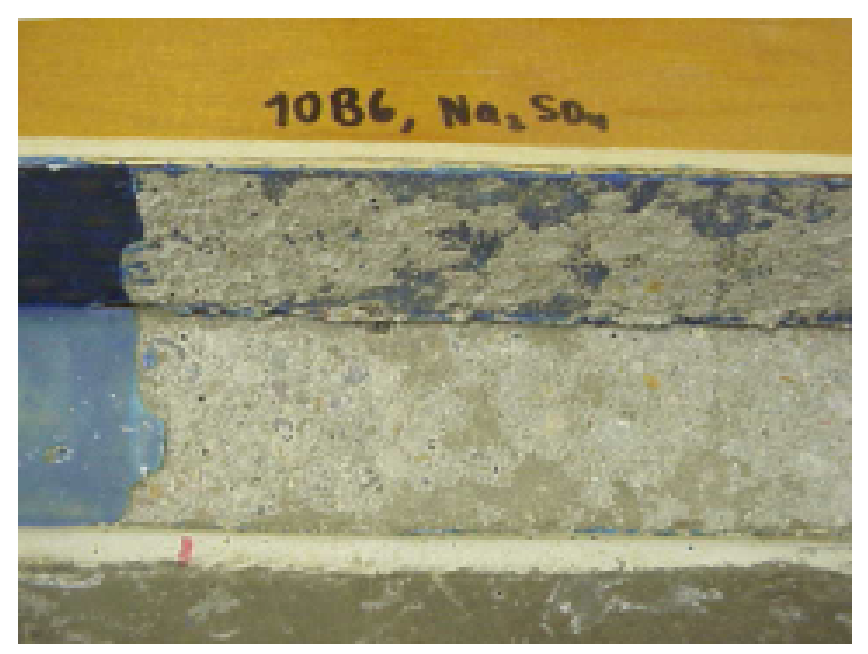

Figure 7.8 Pictures of the SS10/WD fracture surfaces showing beams(a) 2B3, (b) 3B4... see also Fig. 7.6(a), and (c) 10B6 
standpoint that dissimilarities in fracture patterns may arise from the use of different batches of concrete, and as such, elimination of this parameter may be afforded by studying the effects of interfaces from the same batch. Unfortunately, since ten separate batches were used in this study, accomplishing such an objective throughout is not only difficult to achieve, but in most cases, unpromising. It is with this latter point in mind that qualitative representations of fracture surfaces may, for this study, not yield a definitive conclusion - this, observed in hindsight.) A thin layer of concrete was also stripped away from the substrate onto the LVL in this case (i.e. the pristine-dry beam 3B6 of Fig. 6.2) as well, but it appears to be more cohesive overall, which may be due to the pristine condition of the interface.

Figure 7.9 pictures the surfaces of specimens $8 \mathrm{~B} 7,9 \mathrm{~B} 9$, and $9 \mathrm{~B} 10$ that underwent SS20/wet-dry cycles. Here again is some disparity in what is depicted as part (a) appears to be more adhesive, except for a central locale, while parts (b) and (c) seem to be almost entirely cohesive. (Apart from considering disparities that may arise from using distinct concrete batches, differences may also be attributed to an inconsistently spread saturant at the time of bonding (as what may have transpired in the case displayed by Fig. 7.10(c)), or due to an improperly prepared surface prior to bonding, though the latter situation is improbable here since great care was taken to preclude any doubts of such an occurrence, as fully described in Chapter 5.) Batch comparisons may be made by referring to Figures 6.4(b) - batch \#9, and 6.5, parts (a) and (b) — batch \#10. With respect to batch \#9, just the opposite of what was reported for batch \#6 above, is found to be the case, i.e. the WDC30 fracture surface is mixed, whereas the SS20/WD failure is cohesive. The batch \#10 
beams, on the other hand, are entirely cohesive, irrespective of being companion-dry (FTC200, in these cases) or SS20/WD specimens.

(a)
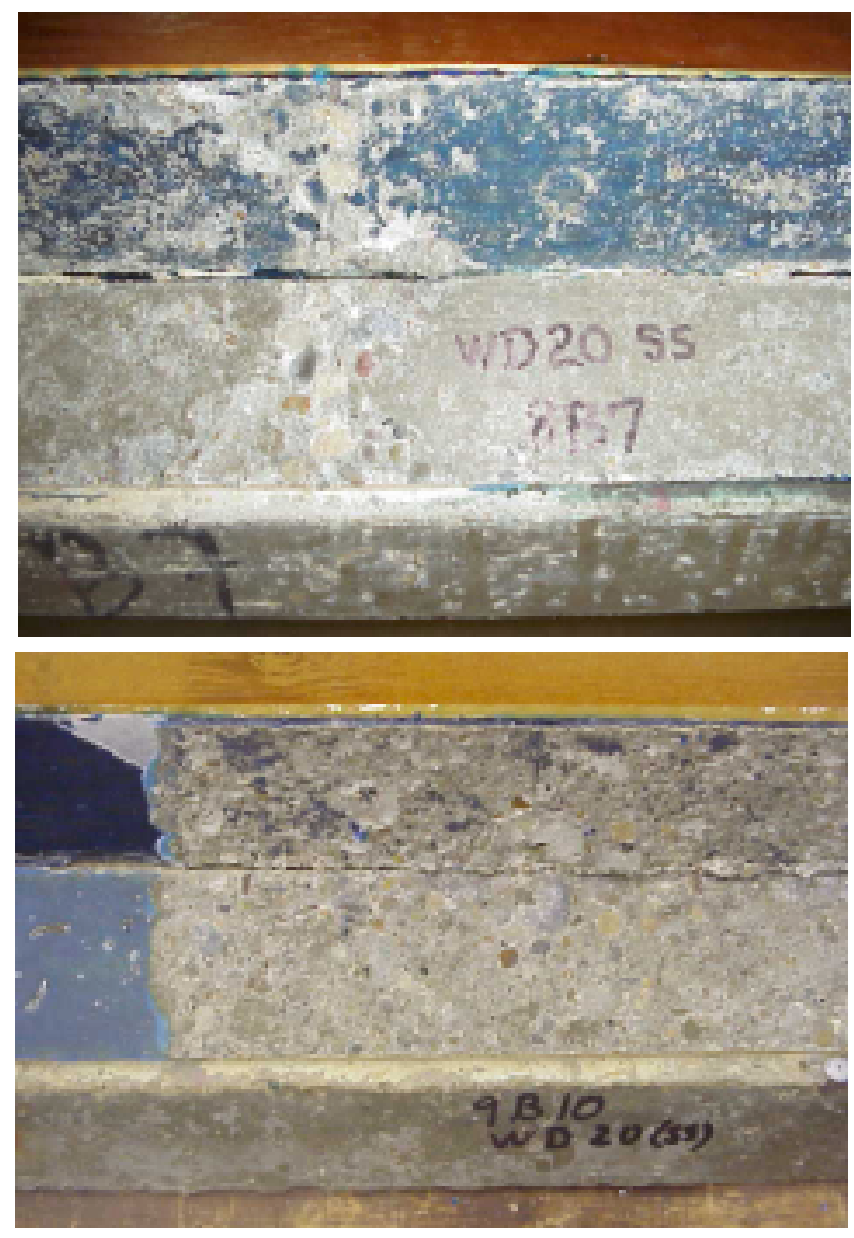

(b)

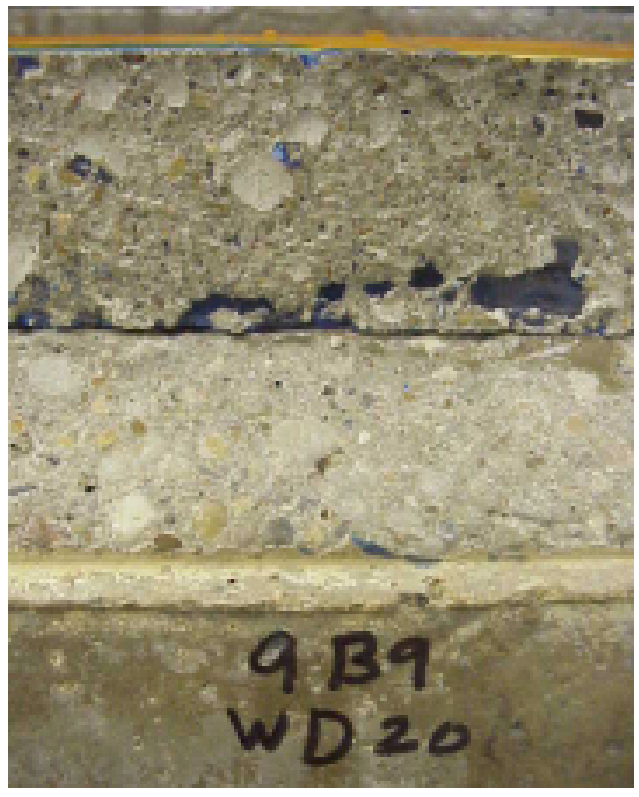

(c)

Figure 7.9 Pictures of the SS20/WD fracture surfaces showing beams(a) 8B7, (b) 9B9, and (c) 9B10... see also Fig. 7.6(b)

The last set of beams, 7B11, 7B12, and 8B13, underwent SS30/wet-dry conditioning and are shown in Figure 7.10. Parts (a) and (b) depict mostly cohesive 
(a)

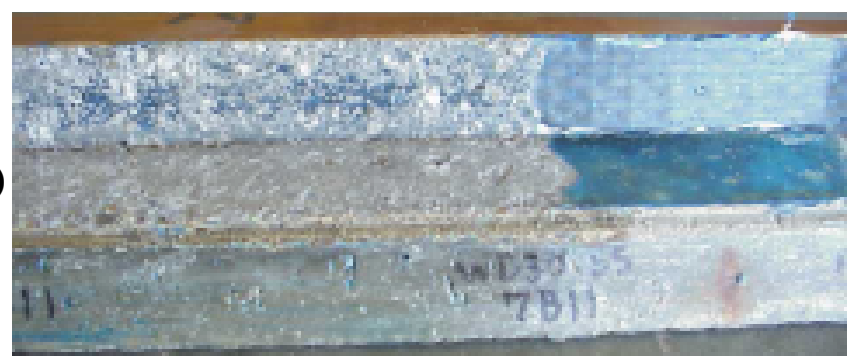

(b)

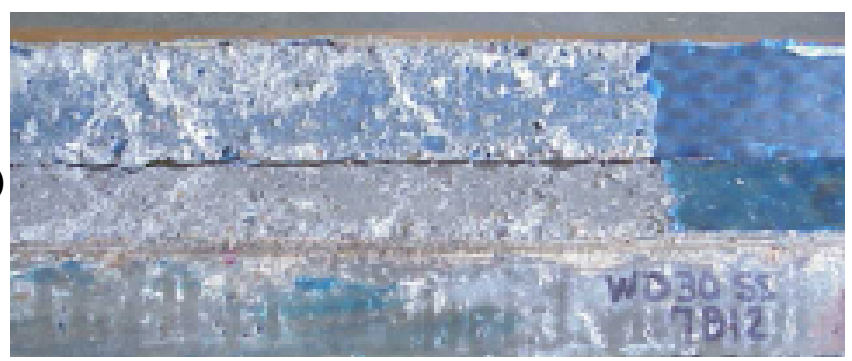

(c)

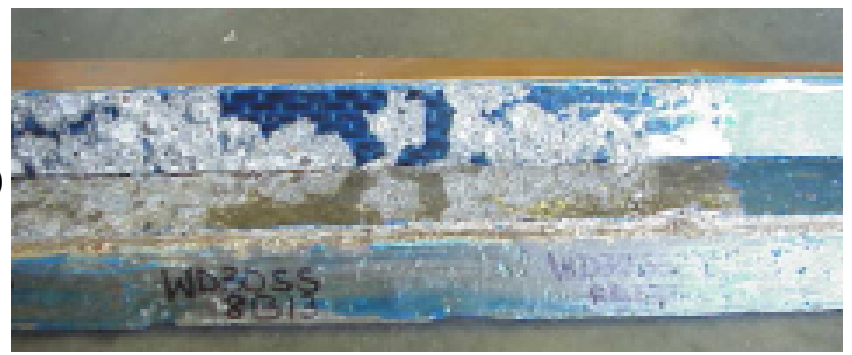

Figure 7.10 Pictures of the SS30/WD fracture surfaces showing beams-

(a) 7B11, (b) 7B12, and (c) 8B13... see also Figs. 7.6(a) and 7.12

failures with some adhesive characteristics exhibited in the central regions; part (c), already alluded to in the previous paragraph, has whole areas where the saturant appears not to have infiltrated properly_otherwise, it too displays similar failure patterns as described for parts (a) and (b). Batch comparisons of these two parts can, respectively, be made with Figures 6.4(a) — batch \#11, and 6.5, parts (c) and (d), along with 6.4(c) - batch $\# 12$. All four of the companion cases can dominantly be characterized as being cohesive 
failures, though the latter beam (Fig. 6.4(c)), in comparison, displays a somewhat mixed character. The weathered counterparts to these beams exhibit a much more mixed failure, the adhesive component of which, seems to occur down the middle of the bonding surface. This may be due to the concrete being sufficiently weakened at the outer edges due to gypsum formation, thus creating a disparity in the strengths of the concrete between the outer and inner (or central) regions with respect to the, more or less, uniform strength of the adhesive at these same locations.

With such variations arising from these qualitative evaluations, the only appropriate conclusions to be drawn are, at best, generalized conclusions. In this manner, then, it appears that the sodium sulfate/wet-dry cycled beams typically failed in a cohesive or else, mixed (i.e. cohesive-adhesive), mode with an emphasis still on the quality of cohesiveness. The first-half period of aging, i.e. ages 10 and 20, can perhaps be more strongly characterized as being cohesive- - just as their unconditioned (i.e. pristineand representative- dry) counterparts - whereas the 30-cycled specimens tended to be more cohesive at the outer-edges of the bonding surface and somewhat more adhesive down the middle. This may be due to the insufficient formation of gypsum (as noted previously through the fracture account) at the earlier ages. As for the latter-stages of weathering, on the other hand, it could be that the adverse effects of the sulfate attack weakened the concrete in the outer portions of the bonding surface while leaving the concrete of the central region intact. Thus, with such location-specific differences occurring with respect to the quality of the concrete, while it is believed that uniformity 
dictated the condition of the adhesive, it therefore makes sense that such a mixed-mode of failure should prevail.

Pre-Fracture Changes (SS)

A pre-fracture evolutionary look at the SS/wet-dry specimens is considered in this subsection, the organization of which is as follows: visual-, weight-, and strain- based changes.

Visual Changes (SS): Even prior to the fracturing of the CFRP-bonded concrete specimens (Fig. 7.1), certain visual changes occurred that are unmistakably attributable to sulfate attack. The most obvious of these is the formation of sulfate crystals which was seen with special clarity on top of the CFRP strips (see Figures 7.11(c) and 7.12, parts (b) and (c)), known technically as efflorescence. A yellow-discoloration, most likely as a consequence of efflorescence, could also be seen on all parts of the exposed concrete regions, i.e. the raised step, ledge, and just above the application-line of the primer on the bulk concrete substrate. The concrete at these locations has a gritty appearance and is somewhat susceptible to exfoliation when rubbed.

Figure 7.11(a) displays the effects of attack on one of the three plain specimens, 6B11-recall that these are aged specimens, fabricated without external CFRP reinforcement, and implemented expressly for the study of any possible changes that might be taking place within the concrete. Note that the primed surfaces, as shown, may be divided broadly into two categories, protected and unprotected. Regions falling under the former characterization are those that still have a shielding-screen of primer acting as a barrier to counter the adverse ingression by the sulfate ions. Visually, these areas can be 
differentiated by locales that are dark-gray in color. Zones pertaining to the latter case, instead, appear in discolored shades of white or yellow. It is believed that the whitish

(a)

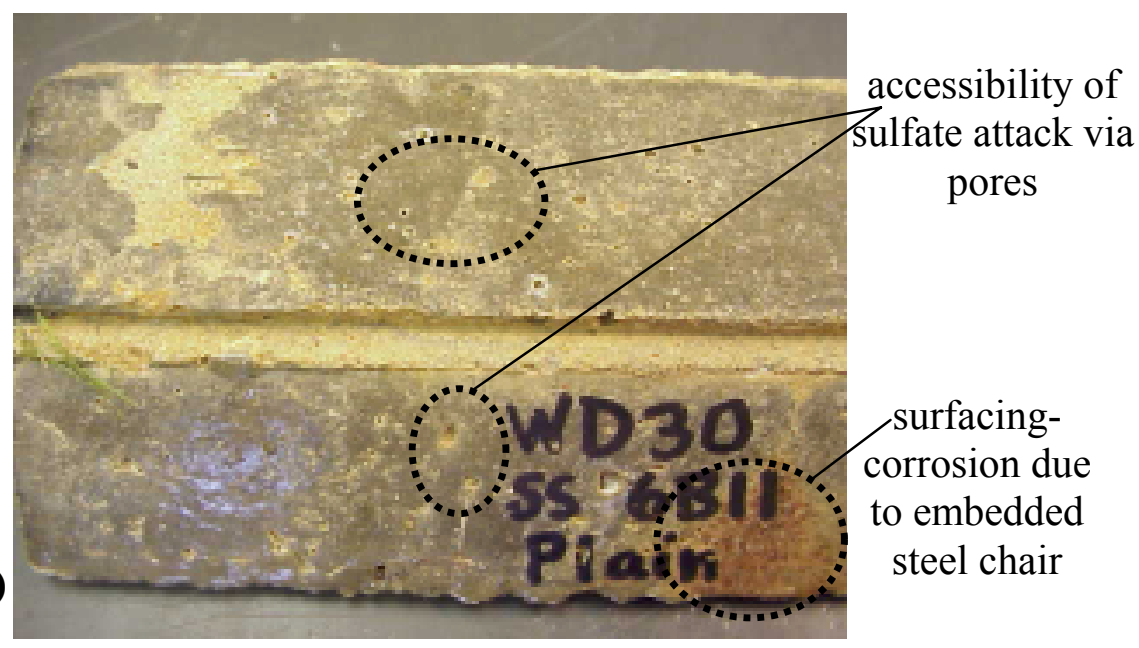

(b)

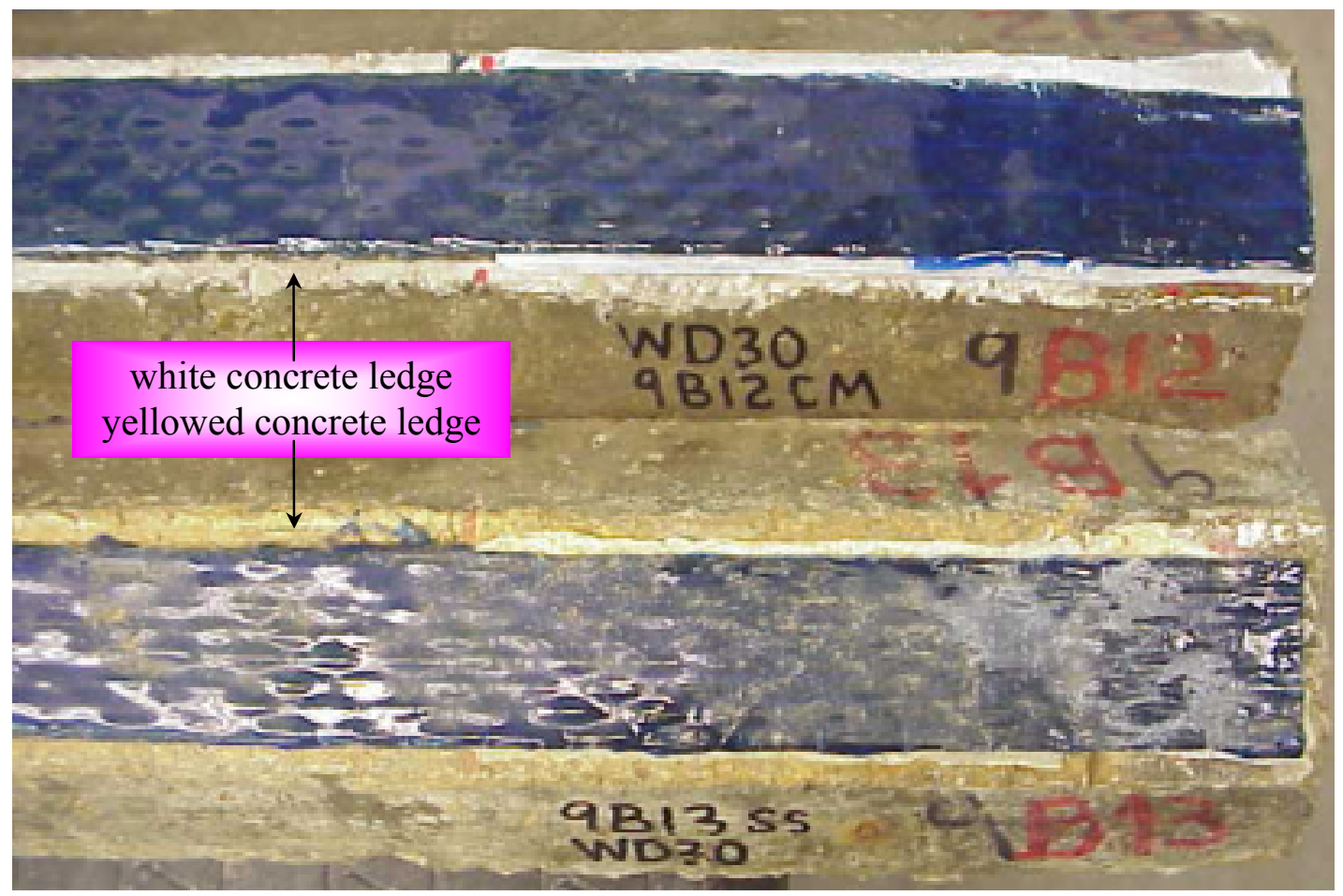

(c)

Figure 7.11 Pictures of visual SS30/WD changes taking place in specimens(a) 6B11 (plain SS30), (b) 9B12 (companion 30), and (c) 9B13 (actual SS30) 
areas are places that were more recently excoriated by the aggressive sulfate; yellowish locations, in contrast, likely sustained flaying for a longer duration of time. With the exception of voids and pores, places that received adequate coverage by the primer seemed to fare well against the deleterious sulfate effects for the extent of the conditioning protocol. Recall, too, that both the primer and saturant are epoxy-based resins, and therefore, if the sulfate attack is impeded by the one it is only logical that it should be further hindered when working in conjunction with the other, as is the case with the primer-saturant-CFRP strengthening system in use here. This is significant because it gives credence to the two claims made in the previous subsection asserting that: 1) a condition of uniformity prevails in the adhesive, i.e. the saturant is not prone to impairment from the aggressive sulfate solution, as is, for example, the concrete, and 2) the outer edges of the concrete become weakened as a result of this infiltration, as expected, but the central portion remains primarily intact since there is no direct route of transmission available for this media to gain access into this zone.

Figure 7.11, parts (b) and (c), contrasts top views of a WDC30 (i.e. a 30 week-old companion) beam, 9B12, with that of a SS30/wet-dry specimen, 9B13. Two things are immediately apparent—first, is the yellowed concrete ledge of the latter as contrasted with the white one of the former; second, in similar order, the effloresced CFRP strip as distinct from the bright blue sheen emanating from the unadulterated saturant. Note also the yellowish spots on the sides of the bulk concrete substrate of the weathered specimen wherever the coating of primer inadequately infiltrated the small pores and voids. These characteristics are perhaps even more clearly seen by analyzing the various views of 
beam 8B13, another SS30/wet-dry specimen, as shown in Fig. 7.12, parts (a) - (c). The first view shows the highly discolored concrete ledge, followed by the second, depicting concentrated areas of sulfate crystallization. An excellent close-up view of the raised

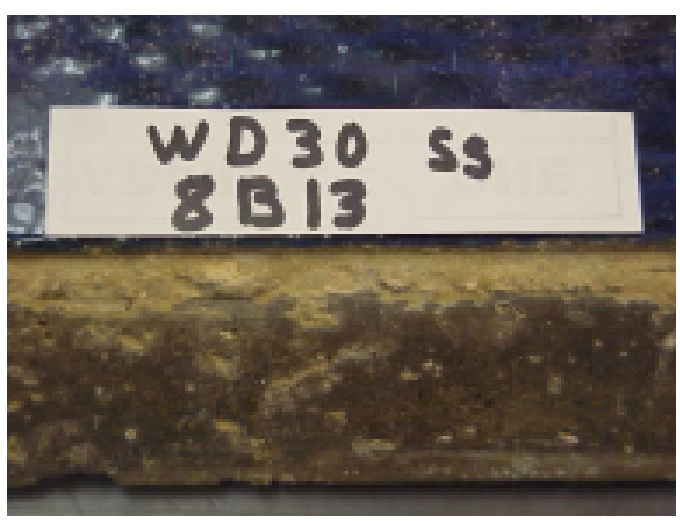

(a)

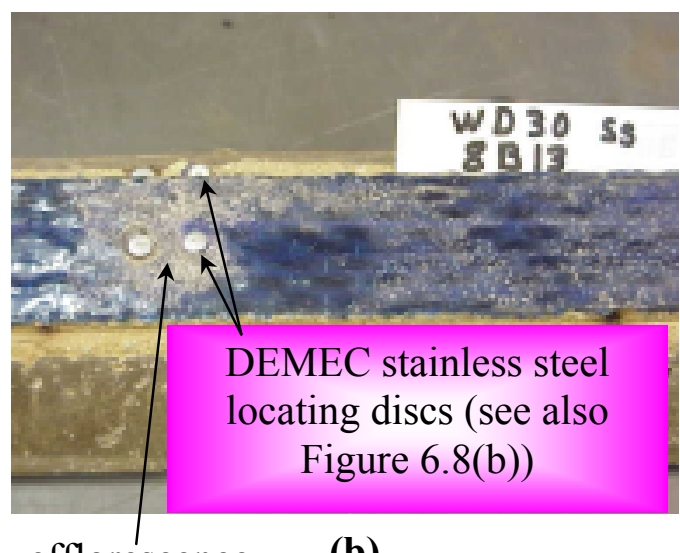

efflorescence

(b)

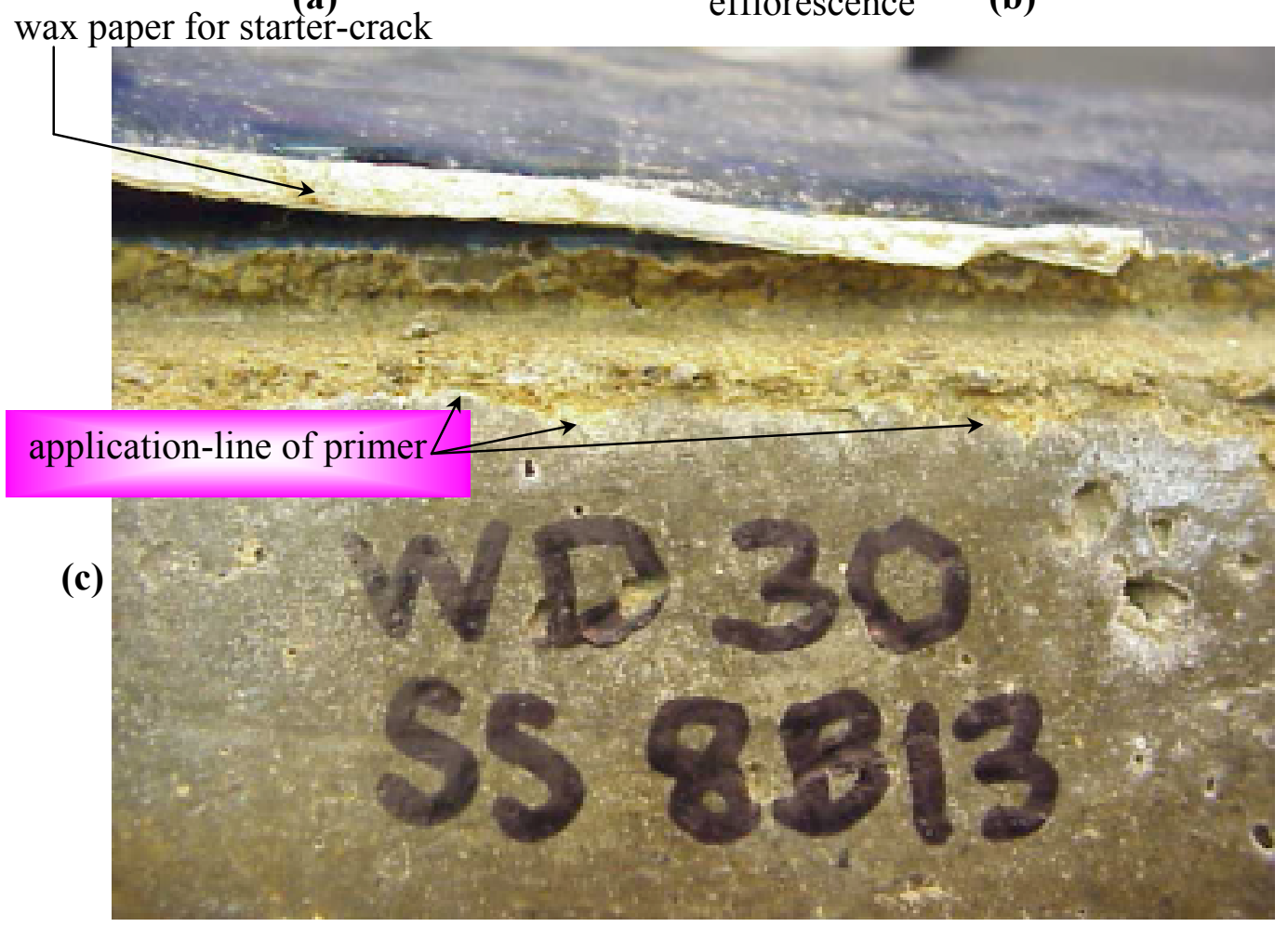

Figure 7.12 Pictures of pre-fracture SS30/WD changes in CFRP-bonded concrete specimen 8B13, displaying effects on the - (a) concrete ledge, (b) CFRP and concrete substrate, and (c) concrete raised step, ledge, and substrate (see also Figs. 7.6(a) and 7.10) 
concrete step is given in the third picture, detailing the deteriorated, gritty texture wrought on by the harmful sulfate solution under the cyclic-action of wetting and drying. Weight Changes (SS): As shown in Tables A.2 - A.4, weight changes corresponding to the SS/wet-dry conditioning protocol were recorded from a total of 12 specimens in the following three categories: Plain (Table A.2: 8B9, 5B10, and 6B11), Companion (Table A.3: 7B8, 9B11, and 9B12), and Actual WD (Table A.4: 5B4/10B6, 8B7/3B8, and 7B12/9B13). The companions' weight results were already presented separately in Chapter 6 (Fig. 6.6) where the dry specimens' weight changes were considered solely; these effects are again repeated here, as shown in Figure 7.13, along with the outcomes of the plain and actual wet-dry beams so that comparisons might easily be drawn between these different cases. The weighing-schedules of the SS/wet-dry beams were as follows: $0,2,5,10,15,20,25$, and 30 cycles - the $10^{\text {th }}$ cycle readings, however, were lost and so interpolated values are assumed in their stead.

Overall, the weight changes resulting from the specimens are quite slight, as expected. This is mainly due to the priming of the concrete substrate beams on all faces except for those of the raised step and ledge portions (see Fig. 3.21, parts (c) and (d), and Fig. 3.23). The trends of the submerged specimens, i.e. the actual and plain beams, are nearly identical for the first 20 cycles with net gains observed until week 15 (see Fig. 7.13). It is thought that around this time, proximate to such a local maximum, the destructive action of the sulfate initiated thus effecting some loss in the mass of concrete as evident in the downward trend that follows immediately thereafter. This disintegration of the concrete, arising from the salt-crystallization, was also perceptible from the change 
in appearance of the wet-bath solution which, at the start, was translucent, and toward the end became quite murky.

From cycle 20 and onwards, the weight-trends of the plain and actual specimens are seen to diverge. Previously, the weight gains of the plain specimens had been larger than those of the actual beams, attributable, most probably, to the more impervious bonding surface resulting from the saturant-and-CFRP layer of the latter. However, within a few days following the $20^{\text {th }}$ week of aging, a reversal of this trend occurs, as displayed by the intersection of these two graphs. Two explanations emerge in answer to this behavior, both of which are possible. Firstly, it is logical that the fiber reinforced beams had a relatively decreased incidence of concrete mass-loss. This is sensible not
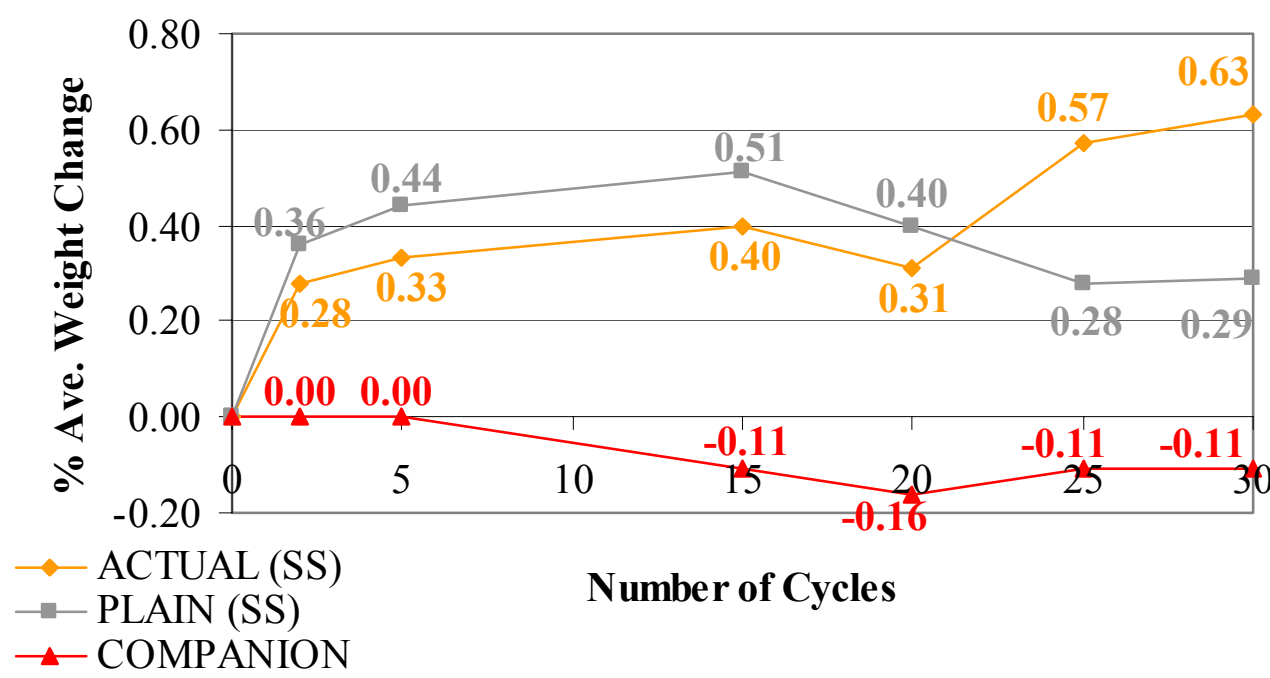

Figure 7.13 Percent average weight changes of the SS/wet-dry plain, companion, and actual beams

only from the standpoint that the saturant-and-CFRP layer acts to bar attack from the harmful media in much the same manner that a bandage is used in dressing a wound, but even more so, it actually fortifies the underlying concrete and thus enhances its 
durability. Secondly, it is probable that the saturant-and-CFRP constituent is responsible for some portion of moisture uptake- perhaps this phenomenon even occurs in a latent manner, i.e. it is not significantly observable in the earlier stages of weathering.

Nevertheless, when considering the preceding arguments, it should be borne in mind that the net weight changes are truly negligible, just as it was with the dry specimens' effects discussed in Chapter 6; the maximum percent average weight change was realized at age 30 for the actual specimens with a magnitude of only $0.63 \%$.

Strain Changes (SS): It is seen from Appendix A that the same three, weight change, plain specimens (Table A.2: 8B9, 5B10, and $6 \mathrm{~B} 11$ ) of the previous subhead have also been used for measuring strains; additionally, six companion (Table A.3: 4B3/2B6, 4B6/1B7, and 10B9/10B10) and six actual WD (Table A.4: 2B3/3B4, 9B9/9B10, and 7B11/8B13) beams were used for investigating strain changes. Measurements were taken using a DEMEC instrument as described in the previous chapter (see also Fig. 6.8), in accord with the weigh schedule, i.e. corresponding to weeks: $0,2,5,10,15,20,25$, and 30 - the $10^{\text {th }}$ cycle readings, however, were lost and so interpolated values are assumed in their stead.

Microstrains recorded at the top of the CFRP strip and the adjacent ledge of concrete (see Figs. 3.21(d) and 6.8(b)) as well as any resulting differential movement that occurred between the two are displayed in Figure 7.14. The average, absolute, differential movement that materialized between the CFRP and concrete is $26 \mu \varepsilon$, with absolute minimum and maximum differences of $15 \mu \varepsilon$ and $53 \mu \varepsilon$, respectively. By using the largest differential microstrain (i.e. $53 \mu \varepsilon$ ) coupled with the fact that $\mathrm{E}_{\mathrm{c}}=4.93 \times 10^{6} \mathrm{psi}$ 
(see Eq. 6.17), the maximum interfacial tensile stress that is developed can be computed as:

$$
\sigma_{\mathrm{t}, \mathrm{WD} / \mathrm{SS}}=261 \mathrm{psi}
$$

by Hooke's law (i.e. $\sigma_{\mathrm{t}}=\mathrm{E}_{\mathrm{c}} \cdot \varepsilon$, given as Eq. (6.15)), where the capitalized subscripts, $\mathrm{WD} / \mathrm{SS}$, indicate the condition of wet-dry cycling — the wetting-phase, of which, occurred

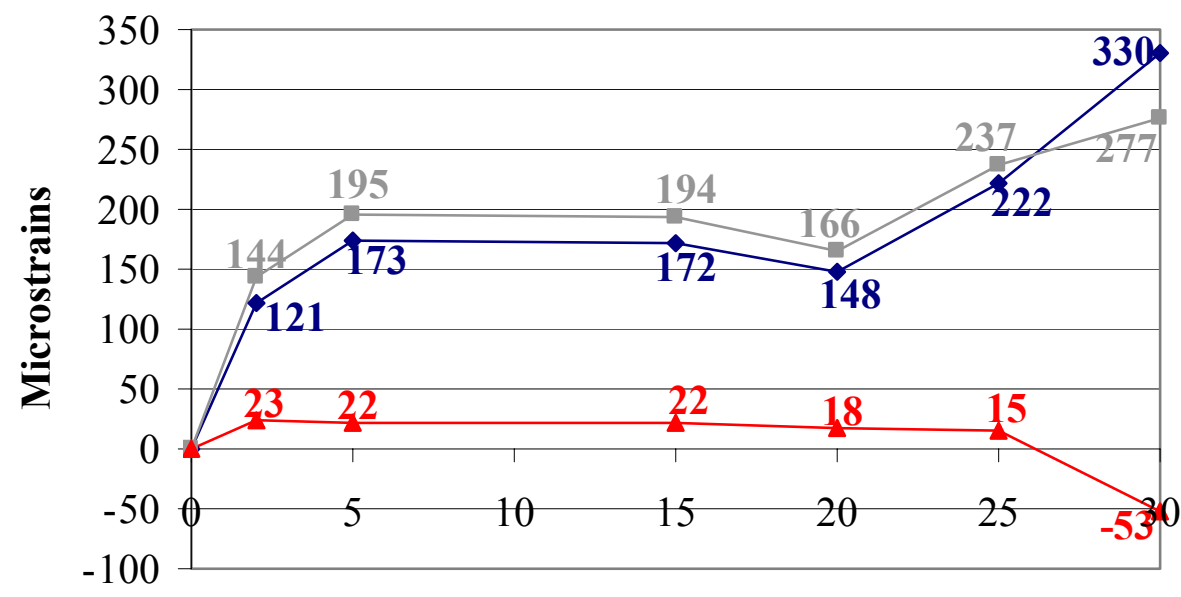

$\rightarrow$ CFRP (SS)

- Concrete (SS)

Number of Cycles

$\rightarrow$ Differential Movement

Figure 7.14 Microstrains of the SS/wet-dry beams in two locations:

(1) on the CFRP, and (2) on the adjacent concrete ledge (see Fig. 3.21(d)) - the differential movement between the two is shown as well

under a sodium sulfate solution. Comparison of this value $\left(\sigma_{\mathrm{t}, \mathrm{WD} / \mathrm{SS}}=261 \mathrm{psi}\right)$ with the corresponding tensile strengths of the previous chapter, i.e. Eqs. (6.13), (6.14), (6.13') and $(6.14 ')$, repeated here for convenience:

$$
\begin{aligned}
& \left(6.13,6.13^{\prime}\right): \quad f_{t}=0.08 \cdot f_{c}{ }^{\prime}=598 \mathrm{psi} \\
& \left(6.14,6.14^{\prime}\right): \quad f_{s p}=7.4 \cdot\left(f_{c^{\prime}}\right)^{1 / 2}[\text { in psi }]=640 \mathrm{psi}
\end{aligned}
$$


results in a factor greater than two between the level of an assumed tolerable interfacial strength $\left(f_{t}\right.$ or $\left.f_{s p}\right)$ and the actual measured stress, $\sigma_{t, W D / S s}(E q . ~(7.2))$. Therefore, under similar conditions and durations of exposure, no appreciable development of strain is expected to form at the WD/SS, CFRP-concrete interface.

\subsection{Sodium Hydroxide (NaOH-abbreviated: SH) Attack}

Preliminaries $(\mathrm{SH})$

A sodium hydroxide solution with a $\mathrm{pH}=12$ was used to simulate a condition of alkalinity that naturally arises from the hydrated cement, resulting in such deleterious effects as etching, spalling, and cracking of the resin (see also Chapter 2). As with the sodium sulfate protocol, twelve CFRP-bonded concrete specimens (see Figure 7.1, i.e. the SCCB without the LVL contour; see also Table A.4 of Appendix A) were subjected to 10,20 , and 30 cycles of alternate wetting and drying in sodium hydroxide -1 cycle $=3$ days of wetting (Figure 7.15) and 4 days of drying in an environmental chamber (Figure 7.16) maintained at $40 \mathrm{C}$ and $50 \%$ relative humidity. It should be noted here that of these twelve beams, conclusive fracture results were determined from nine specimens - the other three produced values of load that were outside the admissible range and, as such, stricken from consideration as shown in the following list (refer, once again, to Table A.4): (SH10): 1B3, 6B3, 2B4, 4B4; (SH20): 5B7, 2B8, 7B9, 6B10; (SH30): 3B11, 6B12, 3B13, 7B13; here, $\mathrm{SH}=$ sodium hydroxide; 10 (e.g.) = number of cycles; concerning specimen-nomenclature, recall that the lead number designates the beam and the letterand-number specifies the concrete batch (see Ch. 6). 


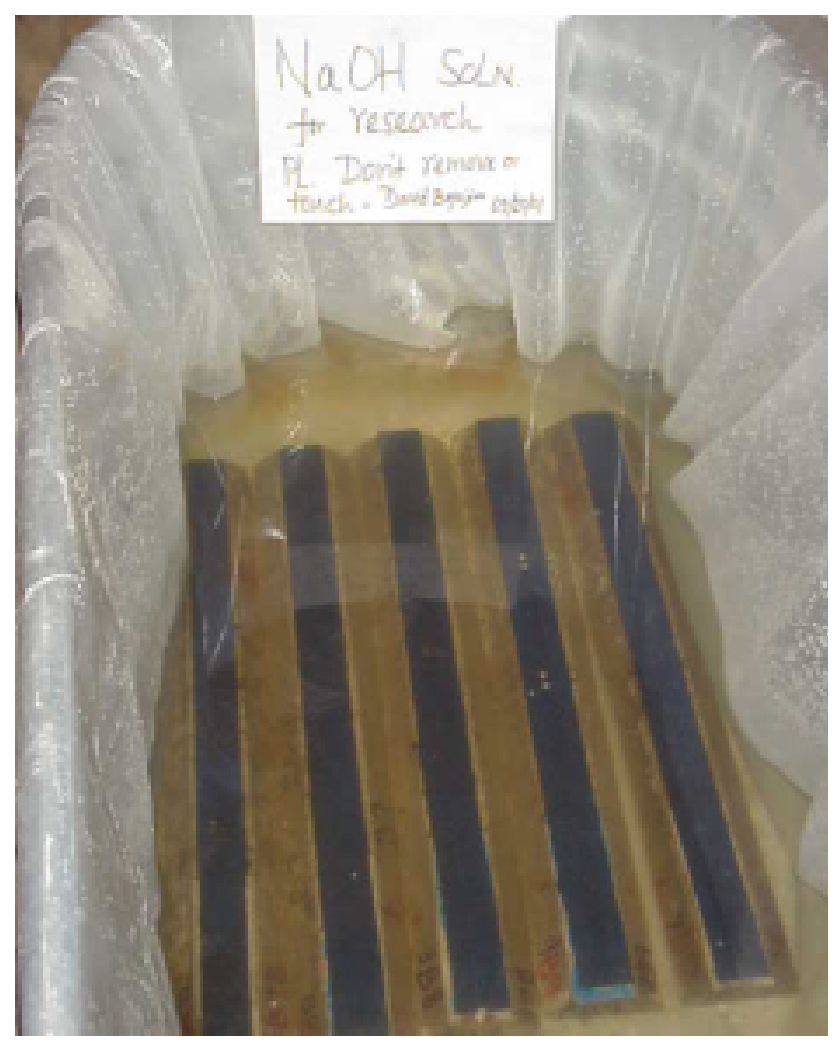

Figure 7.15 Pictures related to the wetting-phase-CFRP-bonded concrete specimens inside plastic-lined galvanized steel tank filled with sodium hydroxide solution $(\mathrm{pH}=12)$

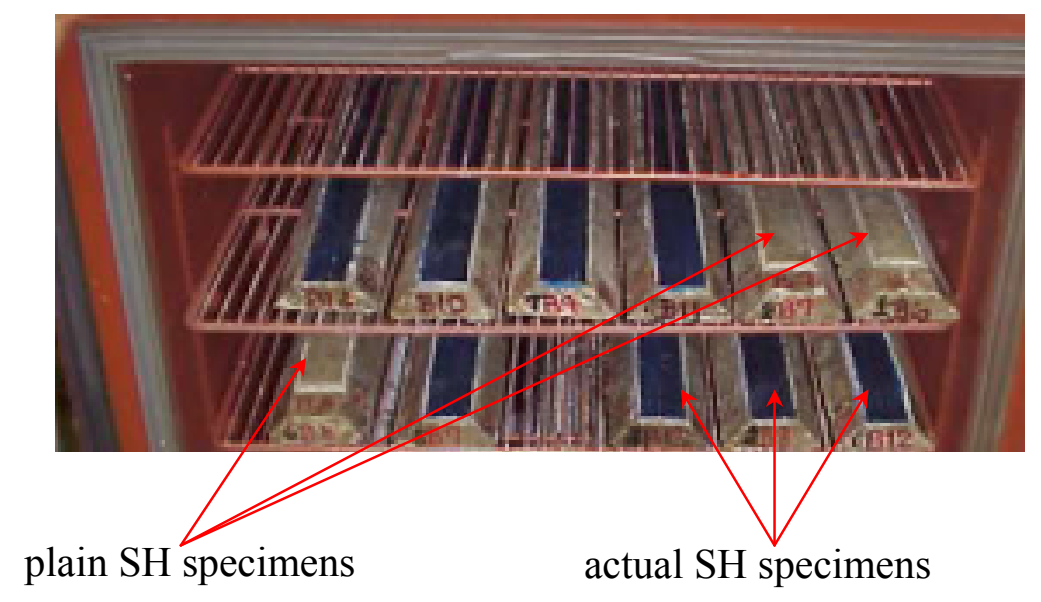

Figure 7.16 Picture related to the $S H$ drying-phase- specimens racked inside the Cincinnati Sub-Zero (CSZ), ZH-16, environmental chamber 


\section{SCCB Fracture (SH)}

Three select $P$ vs. COD graphs from each of the wet-dry target cycling-ages, specimens 6B3, 6B10, and 7B13, are shown in Figure 7.17. As in the sodium sulfate case, the admissible load-ranges $\left(R^{i}, R^{a}\right.$; see Eqs. (6.9) - (6.12)) were determined by using the statistical procedure outlined in Section 6.2, thus enabling the refinement of raw data by the designation of reduced head and set initiation- and arrestment- loads. By the averaging of these values, the four critical loads, $P_{c, \text { Head }}^{i} P_{c, \text { Head }}^{a}, P_{c, \text { Set }}^{i}$, and $P_{c, \text { Set }}^{a}$, were found, which when substituted, in turn, into Eq. (3.73-also repeated in Ch. 6 as (6.1)), shown below, yielded the respective critical strain energy release rates:

$$
\text { (3.73) or (6.1): } \quad G_{I c}=\frac{P_{c}^{2}}{2 b} \frac{d C}{d a}
$$

-recall, again, that $b=$ width of the specimen $=43.4 \mathrm{~mm}(1.71 ")$, and $d C / d a=$ compliance gradient $=1.78 \times 10^{-5} \mathrm{~N}^{-1}\left(7.92 \times 10^{-5} \mathrm{lb}^{-1}\right)$. These results are shown in Tables 7.6 - 7.8 and summarized with averaged head and set $G_{I c}$ values in Table 7.9. Also reported in these first three tables are the corresponding companion fracture energies and the resulting percentage fracture toughness reductions. The latter values are determined relative to the companion sample values of the same age (see Equation (7.1) shown again on the following page for ease of accessibility) - the one exception being the $10^{\text {th }}$ cycle companion results, as explained before in Section 7.2.

In Figure 7.18, $G_{I c}^{i}$ and $G_{I c}^{a}$ effect-based computations are reported on the top and bottom graphs (parts (a) and (b)), respectively. Moreover, corresponding head- and set- 
reductions are plotted side-by-side to facilitate in evaluating the range of variations encountered for a given age and environment. Reductions are computed based on:

$$
\text { (7.1): } \% G_{I c} \text { Reduction }=\frac{G_{I c, \text { conditioned }(\# \text { cycles })}}{G_{I c, \text { companion }(\# \text { cycles })}} \times 100 \%
$$

where the critical strain energy release rate of the numerator may represent any one of the various conditioning schemes (i.e. wet-dry cycling under sodium -sulfate or -hydroxide, or freeze-thaw cycling under calcium chloride) for a specific age (i.e. 10, 20, or 30 cycles for the wet-dry cases, or 50,100, 150, 200, or 300 cycles for those of freeze-thaw), and the denominator is the corresponding $G_{I c}$ value of the companion beams at the same age.

Table 7.6 Reduced data results of wet-dry, SH10 fracture test specimens

\begin{tabular}{c|cc|cc}
\cline { 2 - 5 } & \multicolumn{2}{|c|}{ Head } & \multicolumn{2}{c}{ Set } \\
\hline $\mathbf{n}$ & $\mathbf{i}$ & $\mathbf{a}$ & $\mathbf{i}$ & $\mathbf{a}$ \\
\hline $\boldsymbol{G}_{\boldsymbol{I c}}\left[\mathbf{J} / \mathbf{m}^{2}\right]-\mathbf{S H 1 0 / p r i s t i n e}$ & 2 & 2 & 24 & 26 \\
\hline $\boldsymbol{\sigma}\left[\mathbf{J} / \mathbf{m}^{2}\right]$ & $\mathbf{5 5 6 / 6 1 7}$ & $\mathbf{5 2 5 / 5 8 2}$ & $\mathbf{5 0 9 / 6 2 0}$ & $\mathbf{4 7 5 / 5 8 1}$ \\
\hline $\mathbf{C O V}$ & 0.01 & 0.05 & 0.53 & 0.66 \\
\hline$\% \boldsymbol{G}_{\boldsymbol{I c}}$ Reduction & $0.37 \%$ & $0.96 \%$ & $3.24 \%$ & $3.72 \%$ \\
\hline
\end{tabular}

"pristine values are taken from Table 6.3

Table 7.7 Reduced data results of wet-dry, SH20 fracture test specimens

\begin{tabular}{c|cc|cc}
\cline { 2 - 5 } & \multicolumn{3}{|c|}{ Head } & \multicolumn{2}{c}{ Set } \\
\hline $\mathbf{n}$ & $\mathbf{i}$ & $\mathbf{a}$ & $\mathbf{i}$ & $\mathbf{a}$ \\
\hline $\boldsymbol{G}_{\boldsymbol{I c}}\left[\mathbf{J} / \mathbf{m}^{2}\right]-\mathbf{S H 2 0} / \mathbf{W D C 2 0}$ & $\mathbf{5 3 0 / 7 4 4}$ & $\mathbf{5 0 6 / 7 0 4}$ & $\mathbf{4 7 5 / 8 2 8}$ & $\mathbf{4 5 0 / 8 0 4}$ \\
\hline $\boldsymbol{\sigma}\left[\mathbf{J} / \mathbf{m}^{2}\right]$ & 3.49 & 4.40 & 1.67 & 1.40 \\
\hline $\mathbf{C O V}$ & $8.12 \%$ & $9.32 \%$ & $5.93 \%$ & $5.59 \%$ \\
\hline$\% \boldsymbol{G}_{\boldsymbol{I c}}$ Reduction & $71 \%$ & $72 \%$ & $61 \%$ & $60 \%$ \\
\hline \multirow{2}{*}{ R } &
\end{tabular}

*WDC20 = wet-dry companions at 20 cycles (see Tables $6.6-6.9$ ) 
Table 7.8 Reduced data results of wet-dry, SH30 fracture test specimens

\begin{tabular}{|c|c|c|c|c|}
\hline & \multicolumn{2}{|c|}{ Head } & \multicolumn{2}{|c|}{ Set } \\
\hline & i & $\mathbf{a}$ & i & $\mathbf{a}$ \\
\hline $\mathbf{n}$ & 3 & 3 & 40 & 35 \\
\hline$G_{I c}\left[\mathrm{~J} / \mathrm{m}^{2}\right]-\mathrm{SH30} / \mathrm{WDC30}{ }^{*}$ & $460 / 782$ & $451 / 764$ & $465 / 810$ & 458/787 \\
\hline$\sigma\left[\mathrm{J} / \mathrm{m}^{2}\right]$ & 0.26 & 0.29 & 0.74 & 0.82 \\
\hline $\mathrm{COV}$ & $2.37 \%$ & $2.53 \%$ & $3.98 \%$ & $4.24 \%$ \\
\hline$\% G_{I c}$ Reduction & $59 \%$ & $59 \%$ & $57 \%$ & $58 \%$ \\
\hline
\end{tabular}

"WDC30 = wet-dry companions at 30 cycles (see Tables 6.6 - 6.9)

Table 7.9 Sodium hydroxide, wet-dry fracture results (unit: $\mathrm{J} / \mathrm{m}^{2}$ )

\begin{tabular}{|c|c|c|c|c|}
\hline & \multicolumn{2}{|c|}{ Initiation } & \multicolumn{2}{|c|}{ Arrestment } \\
\hline & $\boldsymbol{G}_{I c, \text { Head }}$ & $\boldsymbol{G}_{I c, \text { Set }}$ & $\boldsymbol{G}_{I c, \text { Head }}$ & $\boldsymbol{G}_{I c, \text { Set }}$ \\
\hline SH10 & 556 & 509 & 525 & 475 \\
\hline Mean: & \multicolumn{2}{|c|}{532} & \multicolumn{2}{|c|}{500} \\
\hline SH20 & 530 & 475 & 506 & 450 \\
\hline Mean: & \multicolumn{2}{|c|}{502} & \multicolumn{2}{|c|}{478} \\
\hline SH30 & 460 & 465 & 451 & 458 \\
\hline Mean: & \multicolumn{2}{|c|}{462} & \multicolumn{2}{|c|}{454} \\
\hline
\end{tabular}

The average variation between head and set $G_{I c}$ percentage reductions for the sodium hydroxide (SH) exposures (see Fig. 7.18) was found to be approximately 7\%, with maximum and minimum deviations of $12 \%$ and $1 \%$ for the $20^{\text {th }}$ and $30^{\text {th }}$ cycle arrestment-based cases, respectively. Interestingly, the set-based degradation effects occurring in the first-half period of aging, i.e. between the $10^{\text {th }}$ and $20^{\text {th }}$ cycles, was considerably more pronounced than what was experienced in the latter-half of exposure-i.e. approximately $26 \%-27 \%$ as opposed to $3 \%-7 \%$. This reveals the swift nature by which the harmful alkaline condition attacked the functionality of the resin. As 

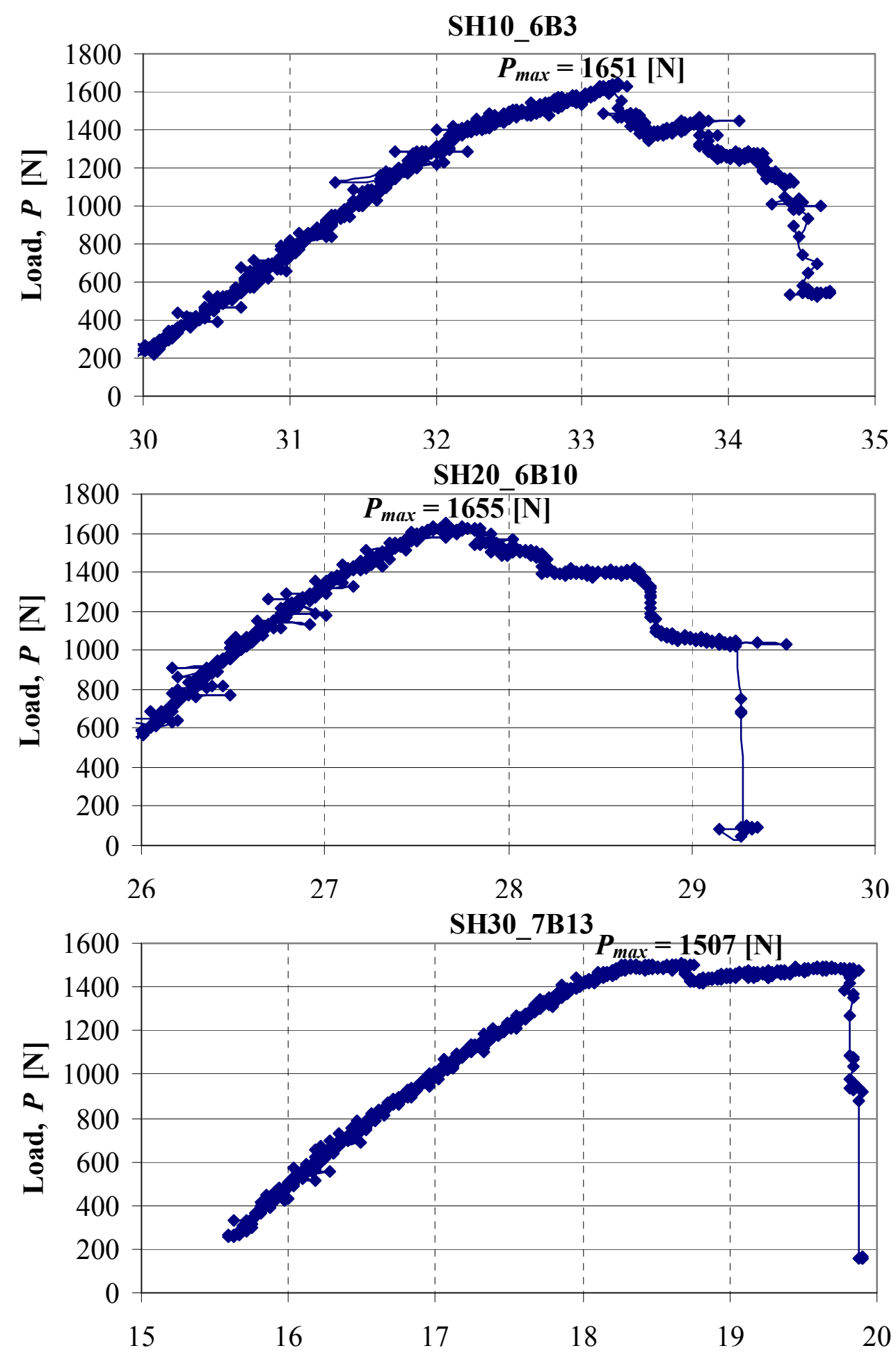

Crack Opening Displacement, COD [mm]

Figure 7.17 Fracture behavior of select WD-(a) 10-, (b) 20-, and (c) 30cycled beams in sodium hydroxide (SH) 
a matter of fact, the degradation experienced by the SS specimens at age 30 is equivalent to the harm sustained by those of the SH case at only age 20 .

After the complete duration of aging at 30 cycles, both the head and set initiationand arrestment- based SH attack events revealed nearly identical reductions in the interface bond efficiency of roughly 58\%. Thus under SH30/wet-dry cycling, the CFRP-

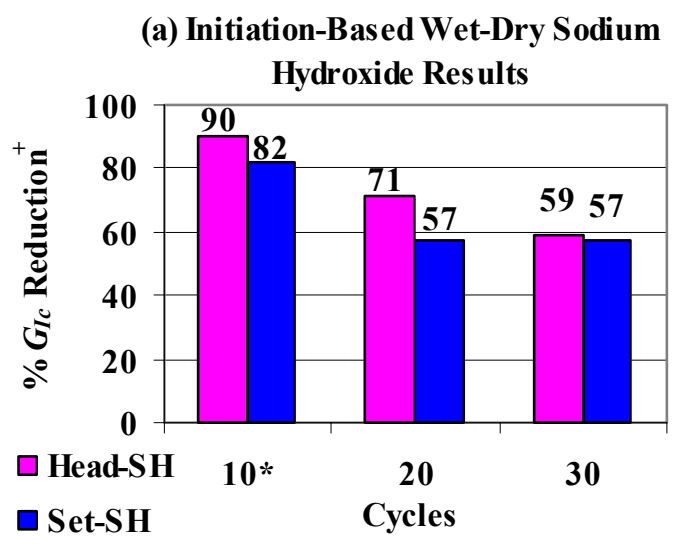

(b) Arrestment-Bas ed Wet-Dry Sodium

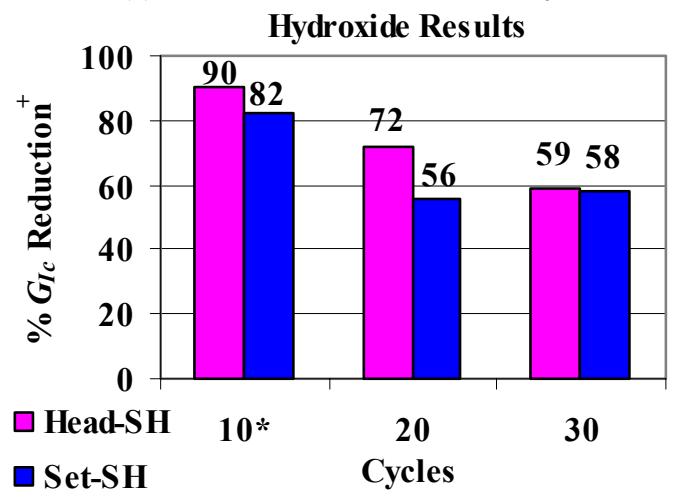

${ }^{+}$Reductions computed relative to companion values; " Pristine dry fracture results (see Table 6.3) substituted for $10^{\text {th }}$ cycle companion values

Figure 7.18 Percentage reductions in (a) $G_{I c}^{i}$ and (b) $G_{I c}^{a}$ resulting from 10, 20 , and 30 wet-dry cycles of sodium hydroxide $(\mathrm{SH})$ exposure 
concrete interface reliability may similarly be characterized as in the SS30/wet-dry case, in that it is less than two-thirds as effectual as it would have otherwise been were it not subjected to such attack.

\section{Patterns of Fracture (SH)}

Quantitatively (SH): Again, consideration of brittleness indices enables insights to be gained into the cracking patterns of SH/wet-dry cycled CFRP-concrete interfaces. Substitution of the critical strain energy release rates of Table 7.9 into:

$$
\text { (3.74) or (6.2): } \quad I=\frac{G_{I c}^{i}-G_{I c}^{a}}{G_{I c}^{i}}
$$

yields the following results (shown in tabular form):

Table 7.10 Brittleness indices of SH/wet-dry fracture test specimens

\begin{tabular}{c|c|c|c}
\cline { 2 - 4 } & SH10 & SH20 & SH30 \\
\hline$I$ & 0.06 & 0.05 & 0.02 \\
\hline
\end{tabular}

Each of the $I$ values of Table 7.10 correspond to stable forms of crack propagation as diagrammatically shown in Figures 3.14(a) and 3.15(b). The average of these values is approximately $I=0.04$-hence, the SH/wet-dry CFRP-concrete interface may also be characterized as being strain rate insensitive (see Sec. 3.4), as has been the case with all the specimens considered thus far.

Qualitatively (SH): Figure 7.19 depicts an evolutionary-sequence in the fracture surfaces of three "refined" specimens (see Table A.4) that underwent SH exposure; part (a) of the 
(a)

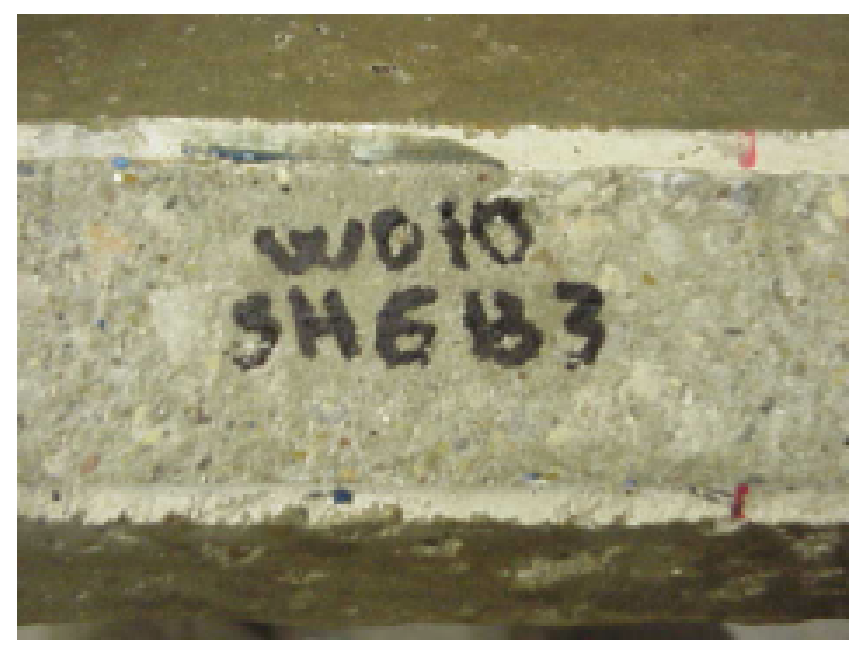

ellipses indicate darker regions of characteristically more adhesive failures

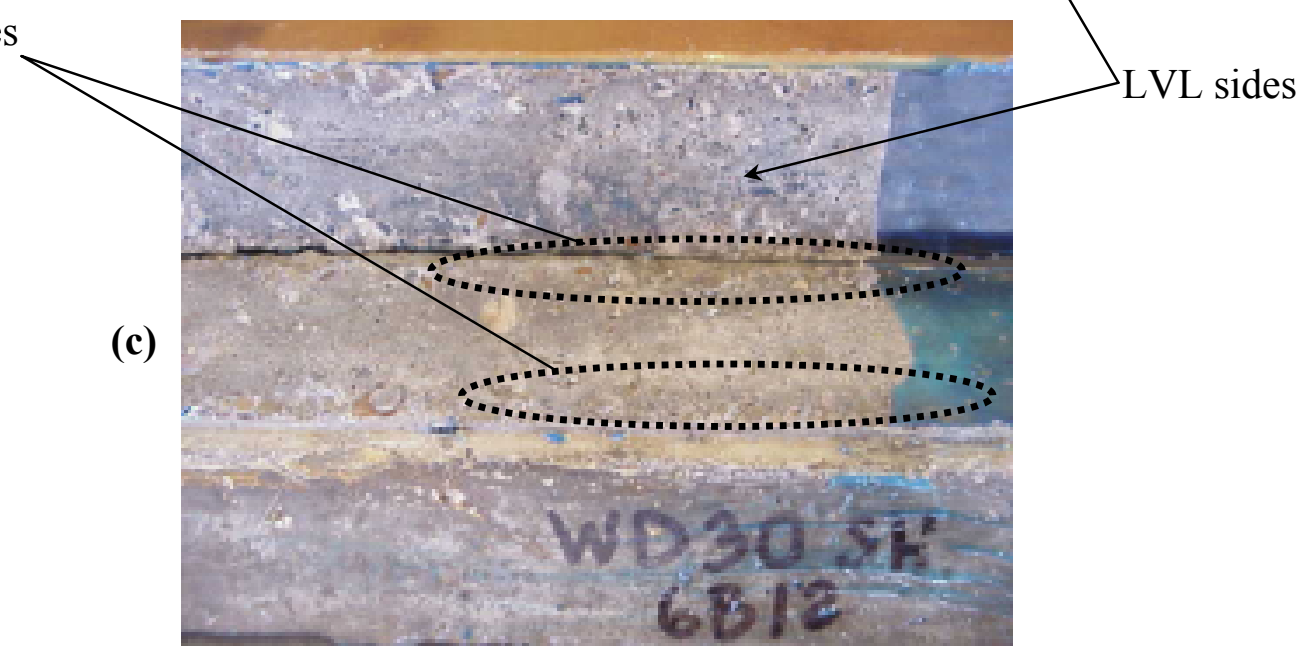

Figure 7.19 Fracture behavior of select SH10, 20, and 30/WD beams(a) 6B3, (b) 7B9, and (c) 6B12 
figure displays the fracture results on the concrete-side of beam $6 \mathrm{~B} 3$ after being conditioned for 10 wet-dry cycles, and parts (b) and (c) show, respectively, the toll sustained on both halves of the fracture surfaces for specimens 7B9 and 6B12 after subjection to 20 and 30 such cycles.

Note, first, that the fracture surface of part (a-SH10) is much more cohesive than its counterpart in part ( $\mathrm{b}-\mathrm{SH} 20$ ) as evidenced by the darker, i.e. primed, regions (see the areas indicated by the dotted ellipses) that have endured on the concrete bonding-faceincidentally, this is to be expected since the $10^{\text {th }}$ cycle fracture results were only reduced by between $10 \%$ and $18 \%$ relative to the companion dry values (see Fig. 7.18 ). By the same token, part (b) is considerably more cohesive than the fracture surface seen in (cSH30) - this latter view appears to be entirely adhesive (see the LVL side) with only a lean layer of cement residue shown to be displaced. This signifies the progressive degeneration that results at the adhesive-joint over both time and subjugation to the caustic, WD cycling-action.

From a qualitative standpoint, a reversal in cracking patterns can be seen to ensue. Not only does this appear to be true respecting the material that is being attacked, i.e. the concrete for the SS beams as opposed to the adhesive for the present SH samples, but the characteristic-location of attack seems to be transposed, as well. Recall that, with the latter-staged SS cases of the previous section, the cohesive failure issued mostly at the outer edges of the bonding surfaces - the exact opposite is displayed in parts (b) and (c) of Fig. 7.19, as depicted by the broken ellipses. Here, the outlying concrete regions suggest failure that was initiated by the adhesive whereas the midsection shows a greater 
degree of concrete-concrete retention-this behavior is more prominent in the view of part (b) than it is in (c), which may indicate that the degradative effects of the alkalineattack on the glue-line is more pervasive than is the dispersive-potency of the corresponding SS media with respect to the concrete.

\section{Pre-Fracture Changes (SH)}

Visual Changes (SH): In contrast with the clearly formed crystals of the SS cases, changes taking place in the SH specimens were quite insipid from a visual perspective. Figure 7.20 shows side views of two, 30-cycled specimens-one that underwent SS conditioning (part (a), beam 8B13), and beneath it, a SH aged counterpart (part (b), beam 3B13). An immediately obvious distinction of the latter is the color of the concrete ledge and raised-step, which have maintained the whitish tone that was present prior to any cycling - this is unmistakably perceived when compared with the unconditioned and unprimed beam shown in part (c). Note, in addition to the color, the texture and condition of the concrete as well. In the SS case, as previously mentioned, the exposed concrete regions appeared gritty and susceptible to exfoliation if rubbed; the same areas of the $\mathrm{SH}$ specimen (Fig. 7.20(b)) still look sound. Similar observations are discerned when contrasting the SS and SH interfaces just below the layer of CFRP (see Fig. 7.12(c) for a better view of this region in the SS case). As a final distinguishing feature, consider the condition of the protective shielding-screen of primer on the sides of the two specimens. Formerly, it was noted that this film had become flayed from within as the sulfate ions entered the concrete mass through small voids and pores that resisted, by and large, coverage from a single coating of the viscous primer. In the present case (Fig. 


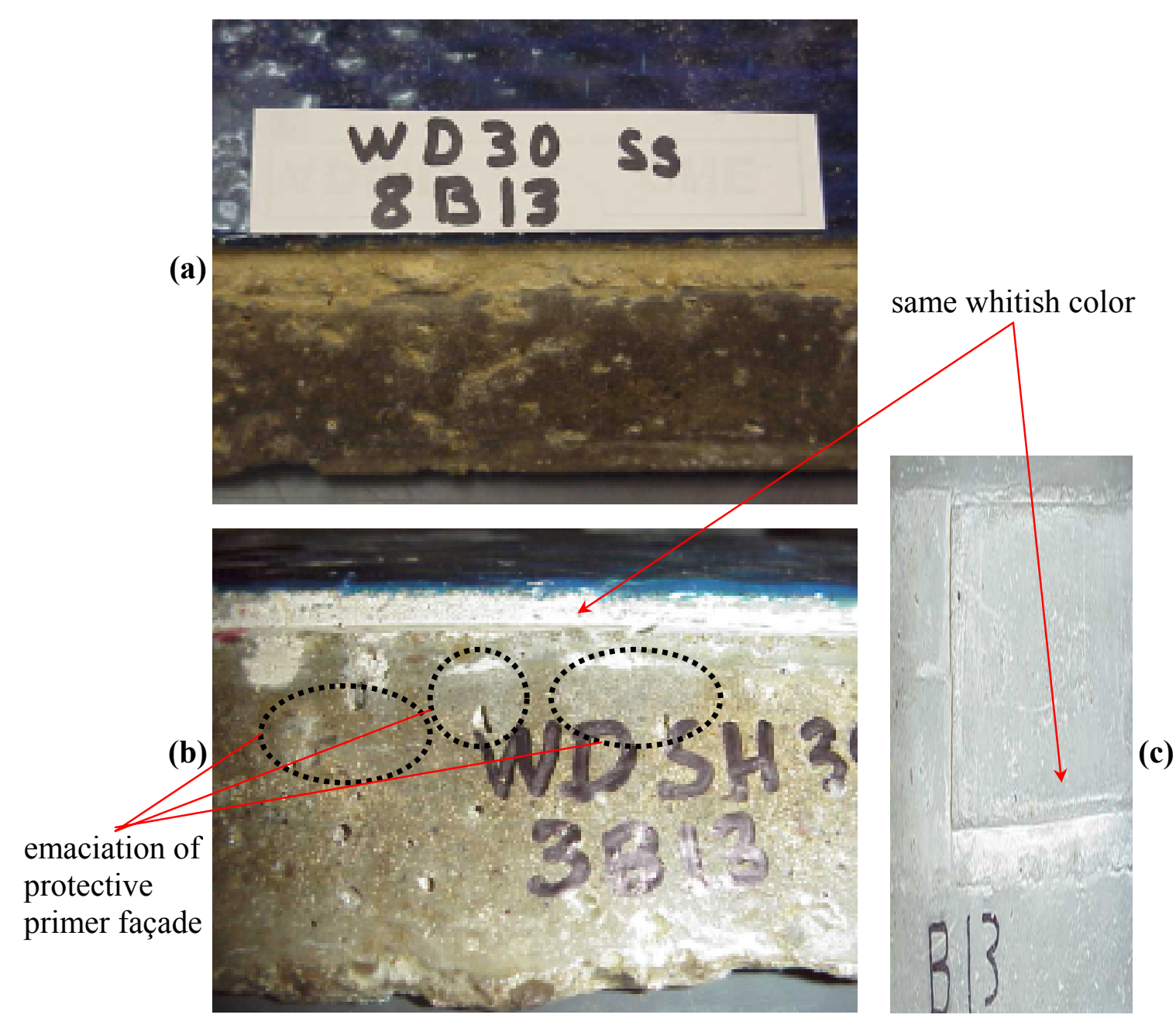

Figure 7.20 Pictures of two pre-fracture, 30-cycled beams-(a) SS30/WD, 8B13, and (b) SH30/WD, 3B13 — and (c) an unconditioned/unprimed concrete beam

7.20(b)), however, note how the façade of this epoxy-based protective skin has become emaciated from without by the caustic action of the SH media; this further corroborates the discussions of the previous subsection in which assertions of adhesive fractures were observed to dictate by subjecting the epoxy-based glue-line to cyclic SH/wet-dry conditions. 
Weight Changes (SH): As shown in Tables A.2 - A.4, weight changes corresponding to the SH/wet-dry conditioning protocol were recorded from a total of 12 specimens in the following three categories: Plain (Table A.2: 1B6, 2B7, and 6B8), Companion (Table A.3: 7B8, 9B11, and 9B12), and Actual WD (Table A.4: 6B3/4B4, 5B7/2B8, and 6B12/3B13). The companions' weight results were already presented separately in Chapter 6 (Fig. 6.6) where the dry specimens' weight changes were considered solely; these effects are again repeated here, as shown in Figure 7.21, along with the outcomes of the plain and actual wet-dry beams so that comparisons might easily be drawn between these different cases. The weighing-schedules of the SH/wet-dry beams were as follows: $0,2,5,10,15,20,25$, and 30 cycles - the $10^{\text {th }}$ cycle readings, however, were lost and so interpolated values are assumed in their stead.

Again, the overall weight changes resulting from the specimens are quite slight, as expected. This is mainly due to the priming of the concrete substrate beams on all faces except for those of the raised step and ledge portions (see Fig. 3.21, parts (c) and (d), and Fig. 3.23). Just as it was for the SS case, the trends of the submerged specimens, i.e. the actual and plain beams, are nearly identical for the first 20 cycles with net gains observed until week 15 (see Fig. 7.21). It is thought that around this time, proximate to such a local maximum, the adverse alkaline media effects initiated thus causing some mass-loss of the primer and saturant-similar to what was explained in the previous subhead as emaciation of the protective primer façade — and evidenced by a following, downward trend. Unlike the SS case, however, the plain specimens' weights are found to be consistently lower than those of their actual SH/wet-dry counterparts. The reason for this 
is that the actual beams are presumed to have a higher potential for water uptake within the saturant-and-CFRP layer in relation to the alkaline media. Recall that for the SS case, the opposite was noted since the ingress of the sulfate media into the concrete was thought to be hindered by the extra layer of saturant-and-CFRP.

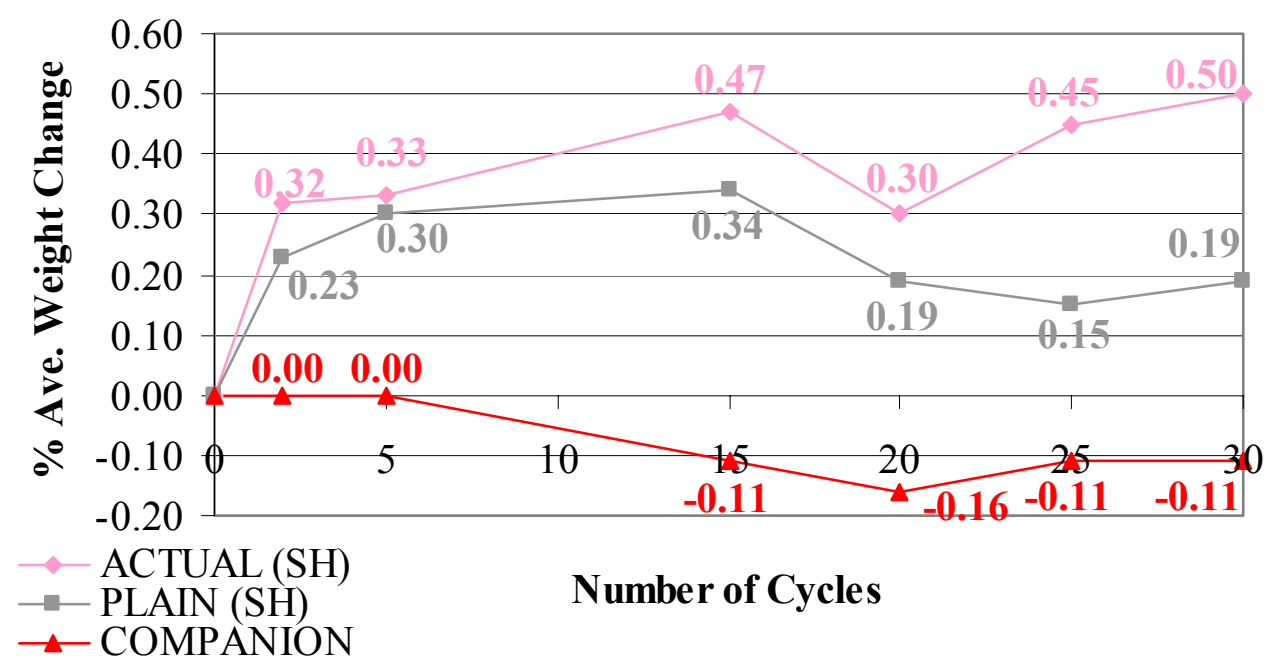

Figure 7.21 Percent average weight changes of the SH/wet-dry plain, companion, and actual beams

From cycle 20 and onwards, though, the weight-trends of the plain and actual specimens are seen to diverge; the actual SH/wet-dry specimens' trend continues to gain weight, whereas the plain beams' weights appear, for the most part, to stabilize. The explanation for this may be that while the façade of the primer coat becomes flayed, enough subsurface-primer remains to act as an effective barrier in preventing the ingress of moisture. With respect to the saturant-and-CFRP layer of the actual beams, on the other hand, the injurious outcomes sustained by the epoxy-based saturant, e.g. etching, spalling, pitting, and cracking, were, in all likelihood, sufficient to absorb moisture responsible for the weight gains seen - as stated before, this effect may also occur in a 
latent manner - one that is not significantly observable in the earlier stages of weathering.

It must, nevertheless, be reiterated that the preceding arguments should, at best, be considered as conjectures, since all the net weight changes are truly negligible and thus preclude both distinctive and definitive conclusions from being drawn; the maximum percent average weight change was realized at age 30 for the actual specimens with a magnitude of only one-half of one percent.

Strain Changes (SH): It is seen from Appendix A that the same three, weight change, plain specimens (Table A.2: 8B9, 5B10, and 6B11) of the previous subhead have also been used for measuring strains; additionally, six companion (Table A.3: 4B3/2B6, 4B6/1B7, and 10B9/10B10) and six actual WD (Table A.4: 1B3/2B4, 7B9/6B10, and 3B11/7B13) beams were used for investigating strain changes. Measurements were taken using a DEMEC instrument as described in the previous chapter (see also Fig. 6.8), in accord with the weigh schedule, i.e. at every $0,2,5,10,15,20,25$, and 30 cycles-the $10^{\text {th }}$ cycle readings, however, were lost and so interpolated values are assumed in their stead.

Microstrains recorded at the top of the CFRP strip and the adjacent ledge of concrete (see Figs. 3.21(d) and 6.8(b)) as well as any resulting differential movement that occurred between the two are displayed in Figure 7.22. The average, absolute, differential movement that materialized between the CFRP and concrete is $26 \mu \varepsilon$, just as it was for the SS case, with absolute minimum and maximum differences of $10 \mu \varepsilon$ and $48 \mu \varepsilon$, respectively. By using the largest differential microstrain (i.e. $48 \mu \varepsilon$ ) coupled with the 


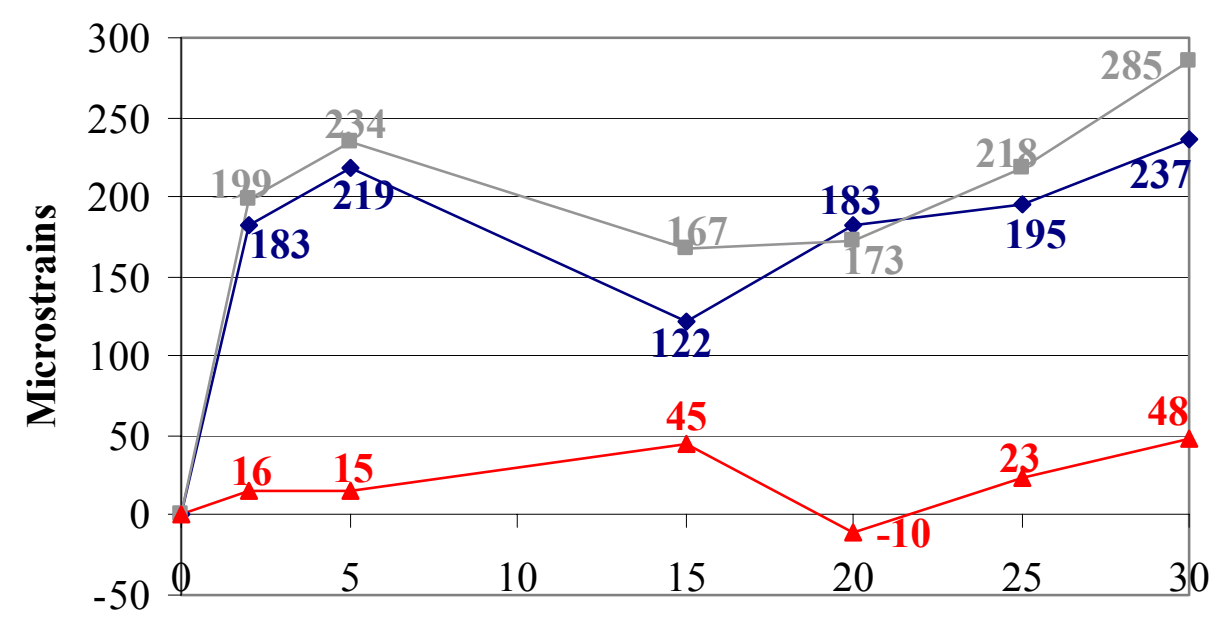

$\rightarrow$ CFRP (SH)

Number of Cycles

$\rightarrow$ Concrete $(\mathrm{SH})$

$\neg$ Differential Movement

Figure 7.22 Microstrains of the SH/wet-dry beams in two locations:

(1) on the CFRP, and (2) on the adjacent concrete ledge (see

Fig. 3.21(d)) - the differential movement between the two is shown as well

fact that $E_{c}=4.93 \times 10^{6}$ psi (see Eq. 6.17), the maximum interfacial tensile stress that is developed can be computed as:

$$
\sigma_{\mathrm{t}, \mathrm{WD} / \mathrm{SH}}=237 \mathrm{psi}
$$

by Hooke's law (i.e. $\sigma_{t}=E_{c} \cdot \varepsilon$, given as Eq. (6.15)), where the capitalized subscripts, $\mathrm{WD} / \mathrm{SH}$, indicate the condition of wet-dry cycling - the wetting-phase, of which, occurred under a sodium hydroxide solution. Comparison of this value with the corresponding tensile strengths of the previous chapter, i.e. Eqs. (6.13), (6.14), (6.13') and (6.14'), repeated here for convenience:

$$
\begin{aligned}
& \left(6.13,6.13^{\prime}\right): \quad f_{t}=0.08 \cdot f_{c^{\prime}}{ }^{\prime}=598 \mathrm{psi} \\
& \left(6.14,6.14^{\prime}\right): \quad f_{s p}{ }^{\prime}=7.4 \cdot\left(f_{c^{\prime}}\right)^{1 / 2}[\text { in psi }]=640 \mathrm{psi}
\end{aligned}
$$


results in a factor greater than 2.5 between the level of an assumed tolerable interfacial strength $\left(f_{t}\right.$ or $\left.f_{s p}\right)$ and the actual measured stress, $\sigma_{t, W D / S H}($ Eq. (7.3)). No appreciable development of strain is therefore expected to form at the WD/SH, CFRP-concrete interface.

\subsection{Conclusions}

Twenty four specimens were studied under two separate, 10, 20, and 30, cyclic wet-dry conditioning schemes; 1 cycle $=3$ days of wetting and 4 days of drying in an environmental chamber maintained at $40 \mathrm{C}$ and $50 \%$ relative humidity. Half of these were placed in a $10 \%$ sodium sulfate $\left(\mathrm{Na}_{2} \mathrm{SO}_{4}-\right.$ abbreviated throughout as: $\left.\mathrm{SS}\right)$ solution $(\mathrm{pH}=6.2-6.5)$ and half were soaked in a sodium hydroxide $(\mathrm{NaOH}-$ abbreviated: $\mathrm{SH})$ bath $(\mathrm{pH}=12)$ to simulate naturally occurring acidic and alkaline field exposureconditions, respectively. Sodium sulfate was used to simulate attack from ground water and wet soils in which gypsum predominantly forms, thus causing softening of the concrete surface and the ultimate loss of mass. Sodium hydroxide, on the other hand, poses a condition of alkalinity that naturally arises from the hydrated cement, resulting in such deleterious effects as pitting, etching, spalling, and cracking of the resin.

Organizationally, the chapter was broadly divided into two parts: Section 7.2, for the sodium sulfate exposure case, and Section 7.3, concerning the sodium hydroxide conditioning protocol. Each of these two sections was subdivided into the following four common categories: 


\section{Preliminaries}

\section{SCCB Fracture}

\section{Patterns of Fracture}

Quantitatively

Qualitatively

$>$ Pre-Fracture Changes

Visual Changes

Weight Changes

$\underline{\text { Strain Changes }}$

Qualitatively, pictures of both pre- and post- fracture beams were studied whereby the drawing of some generalized conclusions was possible. Considering first the latter category, it was noted that the SS/wet-dry specimens typically failed in a cohesive or else, mixed (i.e. cohesive/adhesive, with an emphasis still on the quality of cohesiveness) mode of failure. The inference to be drawn from this is that, as suspected, the interfacematerial identified as the weak-link when exposed to SS/wet-dry cycling, was the concrete. Inspection of fracture surfaces from among the first-half period of aging, i.e. ages 10 and 20 (Figs. 7.8 and 7.9), found that most specimen failures could be dominantly characterized as cohesive-just as their unconditioned (i.e. pristine- and representative- dry) counterparts - whereas the week-30 beams (Fig. 7.10) tended to be more cohesive at the outer-edges of the bonding surface and less so down the middle. For the younger-aged specimens, the described failure was presumed to be due to an insufficient formation of gypsum (as noted also through the fracture account). As for the latter-stages of weathering, it could be that the adverse effects of the sulfate attack weakened the concrete in the outer portions of the bonding surface while leaving the concrete of the central region intact and, otherwise, undiminished in strength. 
Attention was also focused on the use of different concrete batches, for the SS cases, as an afterthought to this research. It was asserted that dissimilarities in fracture patterns may arise from the use of different concrete batches. (In hindsight, it was suggested that future experimental programs, like this one, make an attempt to cast the concrete beams from the same batch to eliminate this parameter.) Unfortunately, since the beams of this study were cast in ten separate batches (really 14, of which, 4 were rejected from use-read Ch. 5), a superficial attempt at batch-comparisons was made for the SS conditioned specimens, only, the results of which found that some similarities in fracture patterns might be attributable to specimens having the same constitution.

Returning now back to the discussion on qualitative, post-fracture characterizations, pictures of the $\mathrm{SH} /$ wet-dry aged specimens, in contrast, revealed that the adhesive was the weak-link interface-material. A general evolutionary progression from typically cohesive-to-adhesive failures was seen to dictate when studying pictures displaying beam fractures from the $10^{\text {th }}-30^{\text {th }}$ cycles, respectively. A reversal in the pattern of failure from the above mentioned SS cases was found to govern in the SH cycled beams, such that, the outlying edges of the bonding surfaces fractured adhesively—and, this time, at only 20 weeks of exposure (see, e.g., Fig. 7.19(b)). (Incidentally, corroboration of this relatively precipitate-behavior of adhesivedegradation was also discovered independently from SCCB fracture testing, as will shortly be summarized.) By age 30, the fractures appeared to be, for the most part, adhesive in nature (see Fig. 7.19(c)), indicating, perhaps, that the degradative effects of 
the SH media on the glue-line is more pervasive than is the dispersive-potency of the SS condition with respect to the concrete.

Regarding, now, the former point of pre-fracture characterizations, pictures clearly revealed the presence of formed sulfate crystals atop the CFRP strips (Figs. 7.11(c) and 7.12, parts (b) and (c)) in the SS conditioned beams; technically, this phenomenon is known as efflorescence. Another result of this effect is the unmistakable yellow-discoloration (contrast Fig. 7.11, parts (b) and (c)) and gritty-texturing that was found to materialize wherever the concrete surfaces had been exposed (see Fig. 7.11) such areas are particularly susceptible to exfoliation when rubbed. On the other hand, those regions of the concrete that were adequately primed seemed to fare well against the deleterious sulfate effects for the extent of the conditioning-protocol. These aforementioned observations are significant for at least two reasons, where it concerns SS aging: 1) the integrity of the adhesive-bond (an epoxy-based resin, similar, in constitution, to the primer) can be assumed as being uniformly unimpaired, and 2) the outlying edges of the exposed concrete, namely, the ledge and raised-step areas, are what became weakened as a result of the sulfate attack; the central portions remained intact as there was no direct route of transmission available for this harmful media to gain access into this zone.

Unlike the marked changes visible in the SS cases, the SH specimens were quite insipid from a visual perspective. The exposed concrete regions maintained the whitish tone that was present under pristine conditions prior to any weathering (compare Fig. 7.20, parts (b) and (c)). The concrete-texture also felt and seemed sound, which is true of 
the interface region just below the CFRP layer (see, e.g. Fig. 7.12(c)). If anything did appear to have changed, it was the depth of the protective primer film that was applied to the various faces of the bulk concrete substrate. Formerly, with respect to the SS cases, it had been noted that this film became flayed from within as the sulfate ions entered the concrete mass through small voids and pores that resisted, by and large, coverage from a single coating of the viscous primer. Under SH conditioning, however, the façade of this epoxy-based protective skin became emaciated (Fig. 7.20(b)) from without by the caustic action of the alkaline media, thus corroborating the asserting conclusion that was stated above from the qualitative post-fracture surface-inspections - i.e., that more widespread damage was found to have affected the epoxy-based glue-line from $\mathrm{SH}$ conditioning than what appeared to have resulted, in contrast, from SS-exposures of the concrete. This is a significant point that should be regarded with seriousness, not only for reasons that are obvious, but because of the insidious nature by which the alkaline attack manifests itself-outwardly, little, if any, forewarning is discerned of the progressively decomposing adhesive-joint.

Weights and strains were two of the other pre-fracture parameters considered. Microstrains were recorded at the top of the CFRP strip and the adjacent ledge of concrete (see Figs. 3.21(d) and 6.8(b)) enabling the determination of any resulting differential movements that might have occurred between the two (see Figs. 7.14 and 7.22). The average, absolute, differential movement that materialized between the CFRP and concrete for the SS/wet-dry specimens was $26 \mu \varepsilon$, with absolute minimum and maximum differences of $15 \mu \varepsilon$ and $53 \mu \varepsilon$, respectively; similarly, these numbers for the 
SH beams were found to be: $26 \mu \varepsilon, 10 \mu \varepsilon$, and $48 \mu \varepsilon$. The largest measured differential microstrains in these two cases (i.e. $53 \mu \varepsilon$ and $48 \mu \varepsilon$ ), returned safety-factors in excess of 2 and 2.5, when respectively compared with the assumed corresponding tensile strengths $\left(f_{t}\right.$ or $\left.f_{s p}\right)$ of the previous chapter, i.e. Eqs. $(6.13),(6.14),\left(6.13^{\prime}\right)$ and $\left(6.14^{\prime}\right)$, repeated here for convenience:

$$
\begin{aligned}
& \left(6.13,6.13^{\prime}\right): \quad f_{t}=0.08 \cdot f_{c}{ }^{\prime}=598 \mathrm{psi} \\
& \left(6.14,6.14^{\prime}\right): \quad f_{s p}{ }^{\prime}=7.4 \cdot\left(f_{c}{ }^{\prime}\right)^{1 / 2}[\text { in psi }]=640 \mathrm{psi}
\end{aligned}
$$

Therefore, under similar conditions and durations of exposure, no appreciable development of strain is expected to form at either of the WD/SS or WD/SH, CFRPconcrete interfaces.

Percent average weight changes occurring between the SS/ and SH/ wet-dry plain, companion, and actual beams were displayed in Figures 7.13 and 7.21 , respectively. Similar trends were found to exist between the actual and plain beams for approximately 20 cycles with net gains observed until week 15, at which time, it is believed that the destructive SS and SH effects were initiated resulting in concrete and adhesive masslosses, respectively. As might be expected, the fiber reinforced beams of the SS protocol are believed to have had a relatively decreased incidence of concrete mass-loss relative to their plain counterparts. It is also felt, in both the SS and SH specimens, that the saturantand-CFRP constituent was responsible for some portion of moisture uptake, and this, perhaps, in a latent manner. The overall weight changes in both situations, however, were found to be negligible with maximum percent average changes of only $0.63 \%$ and $0.50 \%$, 
for the week-30, actual, SS and SH beams, respectively; thus, the aforementioned deductions are, at best, speculations and should not be mistaken for factual explanations.

The most significant information, of course, was gained through SCCB fracture testing, the results of which are presented below, compactly, in tabular (Table 7.11) and graphical (Figure 7.23) form. (Note that the brittleness indices obtained from these tests have also been provided in the table, giving quantitative evidence of characteristically stable crack-growth behavior throughout.) Inspecting the bar graphs of the figure (compiled from Figs. 7.7 and 7.18) reveals a declining trend of relative fracture toughness values as caused by the adverse wetting-drying regimes. The average variation between head and set $G_{I c}$ percentage reductions for the SS exposure scheme was found to be $10 \%$, with maximum and minimum deviations of $19 \%$ and $6 \%$ for the $20^{\text {th }}$ and $10^{\text {th }}$ cycle arrestment-based cases, respectively. As for the SH case, this average was found to be $8 \%$, with maximum and minimum deviations of $16 \%$ and $1 \%$ for the $20^{\text {th }}$ and $30^{\text {th }}$ cycle arrestment-based cases, respectively. Interestingly, the effects of SS head-degradations in the first-half period of aging, i.e. between the $10^{\text {th }}$ and $20^{\text {th }}$ cycles, was not as pronounced as was the case in the latter-half of exposure-i.e. approximately $3 \%$ as opposed to $16 \%$. This large disparity may be indicative of the insufficient formation of gypsum or the underdevelopment of some of the other adverse products that are known to stem from exposure to sulfate attack, such as ettringite, at these comparatively early stages. The performance of the SH beams, on the other hand, revealed first-half set-based degradation effects that were considerably more pronounced than what was experienced in the latterhalf of exposure-i.e. approximately $25 \%-26 \%$ as opposed to essentially $0 \%$. This 
indicates the swift nature by which the harmful alkaline condition succeeded in attacking the functionality of the resin. As a matter of fact, the degradation experienced by the SS specimens after 30 weeks of aging is basically equivalent to the harm sustained by those of the SH beams after only 20 weeks.

After the complete duration of aging at 30 cycles, head and set initiation- and arrestment- based reductions in fracture toughness values for the SS regime revealed identical results of $68 \%$ and $61 \%$, respectively; these values are $59 \%$ and $58 \%$ (averaged) for the SH beams. Averaging, in turn, each of these two cases (i.e. approximately, $64 \%$ and 58\%), shows that the CFRP-concrete interface reliability may be characterized as being less than two-thirds as effectual as it would have otherwise been were it not subjected to such attack. 
(a) Initiation-Based Wet-Dry Sodium Sulfate Results

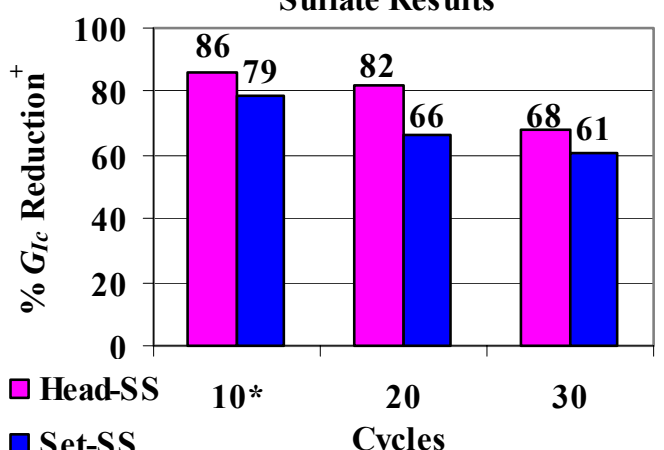

Set-SS Cycles

(c) Arrestment-Based Wet-Dry Sodium Sulfate Results

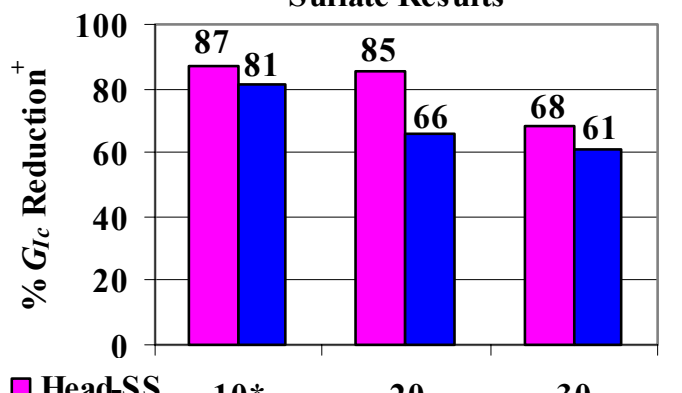

$\square$ Head-SS

10 *

20

30

Set-SS

Cycles (b) Initiation-Bas ed Wet-Dry Sodium Hydroxide Results

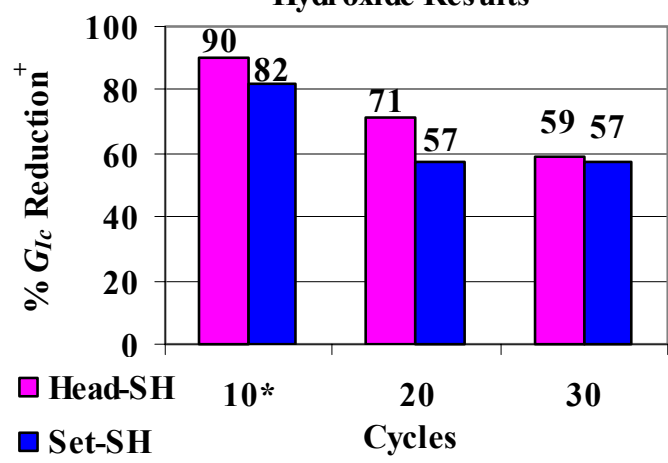

(d) Arres tment-Based Wet-Dry Sodium Hydroxide Results

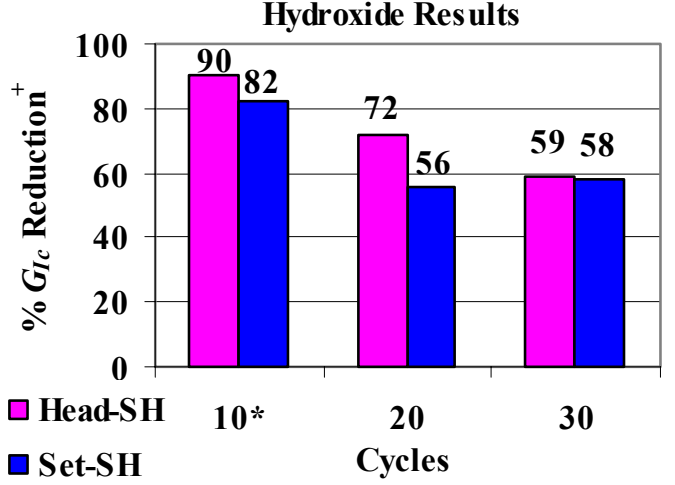

${ }^{\top}$ Reductions computed relative to companion values; "Pristine dry fracture results (see Table 6.3) substituted for $10^{\text {th }}$ cycle companion values

Figure 7.23 Conclusive percentage reductions in $\boldsymbol{G}_{\boldsymbol{I c}}^{\boldsymbol{i}} \boldsymbol{\&} \boldsymbol{G}_{\boldsymbol{I c}}^{\boldsymbol{a}}$ after 10, 20, and 30 WD cycles in $[(\mathbf{a}) \&(\mathbf{c})] \mathrm{SS}$, and [(b) \& (d)] SH media

Table 7.11 Averaged conclusive wet-dry fracture results (unit: $\mathrm{J} / \mathrm{m}^{2}$ ) (see also Tables 7.4/7.9 and 7.5/7.10)

\begin{tabular}{|c|c|c|c|c|c|c|}
\hline & \multicolumn{3}{|c|}{ 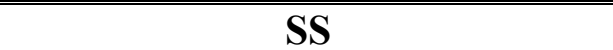 } & \multicolumn{3}{|c|}{$\overline{\text { SH }}$} \\
\hline & 10 weeks & 20 weeks & 30 weeks & 10 weeks & 20 weeks & 30 weeks \\
\hline$(1) G_{I c}^{i} / W D C^{*}$ & $511 / 618$ & $581 / 786$ & $514 / 796$ & $532 / 618$ & $502 / 786$ & $462 / 796$ \\
\hline (2) $G_{I c}^{a} / \mathrm{WDC}^{*}$ & $488 / 582$ & $566 / 754$ & $502 / 776$ & $500 / 582$ & $478 / 754$ & $454 / 776$ \\
\hline$\%(1),(2)$ & $83 \%, 84 \%$ & $74 \%, 75 \%$ & $64 \%, 65 \%$ & $86 \%, 86 \%$ & $64 \%, 63 \%$ & $58 \%, 58 \%$ \\
\hline$I$ [no unit] & 0.04 & 0.02 & 0.02 & 0.06 & 0.05 & 0.02 \\
\hline
\end{tabular}

${ }^{*} \mathrm{WDC}=$ ave. wet-dry companion results (week-10 values furnished by pristine beams) 


\section{CHAPTER 8}

\section{FREEZE-THAW FRACTURE}

\subsection{Introduction}

Elucidation, with respect to the question of permanence, was gained in the previous chapter concerning two actual, wet-dry, field exposure conditions found to distress the effective, long-term use of CFRP materials as an external strengthening system for concrete structures. This theme is once again reanimated in the present chapter to investigate the durability of this technology while subjected to an exposure protocol simulating wintry conditions experienced throughout many parts of the world.

Consideration is here given to specimens having encountered a maximum of 300 cycles of freezing and thawing while being immersed in a calcium chloride (i.e. salt) solution. In this context, a cycle is composed of 6 hours, divided into three equal frames of time. The first and last of these involve ramping from room temperature to a determined freeze state and vice-versa, respectively, and the middle segment acts as a connective between the two by maintaining the achieved condition of freezing, uniform, throughout its duration (see Figure 8.1 for a schematic representation). Outcomes of the test results are presented in Section 8.2, the topic-headlines, of which, adhere identically 


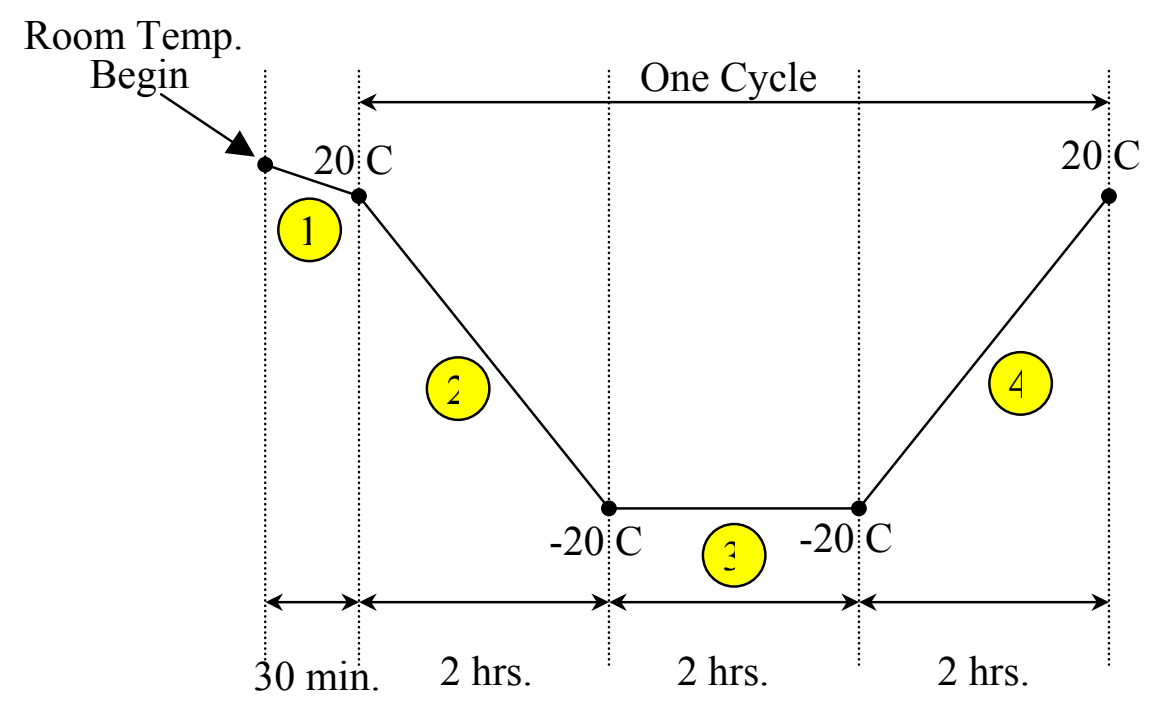

Figure 8.1 Freeze-thaw cycling scheme

to the past formats of Sections 7.2 and 7.3. As in these cases, the prefatory material is followed by the central issue of interpreting fracture data obtained through the employment of the SCCB test methodology. Cracking patterns are considered next from both, quantitative, as well as, qualitative, standpoints. And finally, any detectable visual, weight, and strain changes are analyzed. Degradative fracture results are computed at each of the five target ages, 50,100, 150, 200 and 300 cycles, relative to their companion values (see Tables $6.6-6.9$ ), and are charted as bar-graphs displaying percentage reductions in the values of $G_{I c}$ found with respect to the cycling-age. Also, interface fracture energies are computed using Eq. (3.73 - also repeated as (6.1)) by the two approaches discussed in Ch. 6, namely: the averaging, separately, of initiation and arrestment loads for the ascertaining of head- (i.e. topmost) and set- (i.e. all) loads. For a review of this procedure, read Section 6.2, and especially see the subsection titled "A Step-by-Step Example". Conclusive $G_{I c}$ values are then finally obtained by the averaging 
of such, correspondingly, admissible (see Eqs. $(6.9,6.10$; or $6.11,6.12)$ ) head and set results.

\subsection{Calcium Chloride ( $\mathrm{CaCl}_{2}$-abbreviated: $\left.\mathrm{CC}\right)$ Attack}

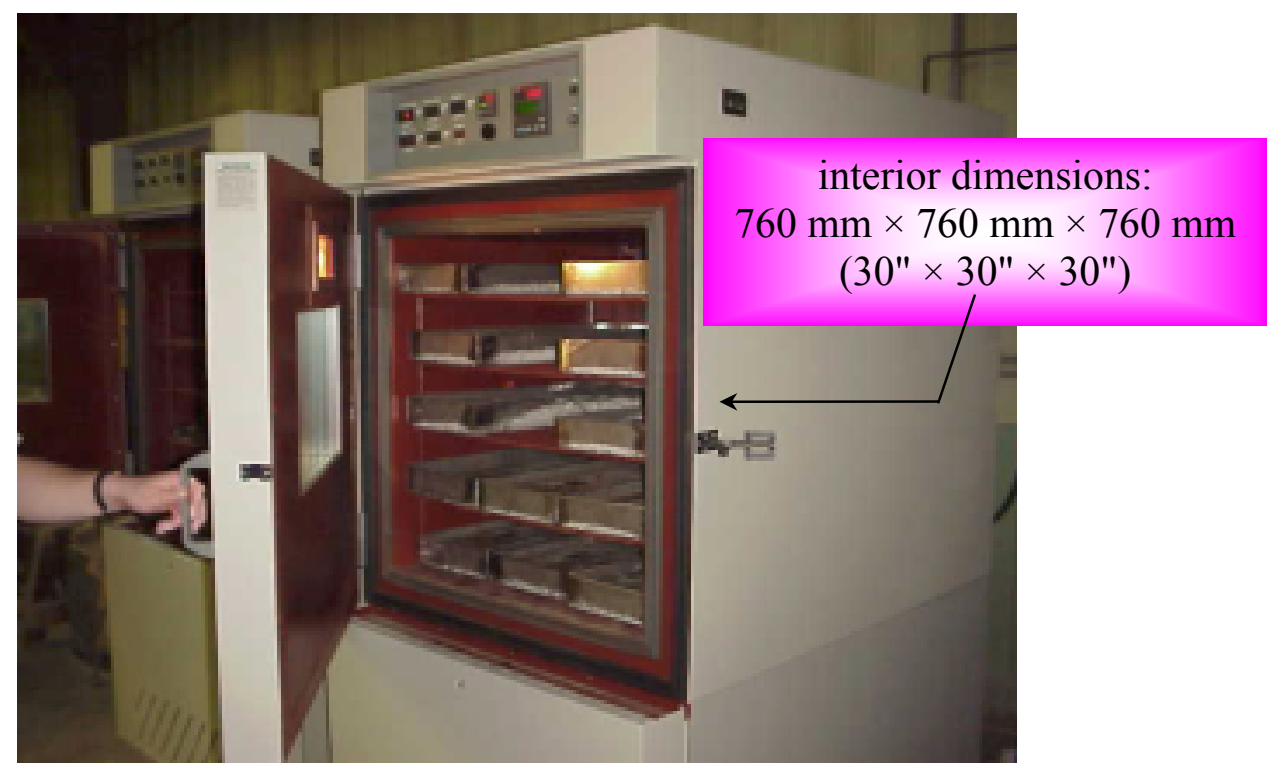

Figure 8.2 Pictures of the two CSZ (ZH-16) environmental chambersthe chamber of the FT conditioning protocol is shown in the foreground with a view of the racked stainless steel trays

\section{Preliminaries $(C C)$}

There is no available standard practice for the testing of concrete-FRP interfaces subjected to freeze-thaw conditioning under chloride solution. For this reason, ASTM C672 ("Standard Test Method for Scaling Resistance of Concrete Surfaces Exposed to Deicing Chemicals") and ASTM C666 ("Standard Test Method for Resistance of Concrete to Rapid Freezing and Thawing") were reviewed and modified appropriately in arriving at the formulation of the present protocol. The CFRP-bonded concrete specimens 
(without the LVL contour, Fig. 7.1) were subjected to a calcium chloride solution and underwent a total of 300 cycles of freezing and thawing within an environmental chamber (see Figure 8.2).

The CC solution used in this protocol was prepared in accordance with ASTM C672 by dissolving $4 \mathrm{~g}$ of anhydrous calcium chloride in $100 \mathrm{ml}$ of water. Special stainless steel trays (see Fig. 8.3(a)) were fabricated (Romisch, see Appendix D) with particular dimensions to fit inside the environmental chamber (see Figs. 8.2 and 8.3). These trays were produced out of AISI (i.e. American Iron and Steel Institute) Type 316 steel, possessing the following chief characteristics: "corrosion resistance superior to other stainless steels when exposed to sea water and many types of chemical corrosives". Each tray was made to contain the corrosive $\mathrm{CC}$ solution while accommodating two CFRP-bonded concrete beams. In order to prevent the bulk concrete substrate from becoming pulverized from the harsh FT conditioning scheme, each beam was placed in an inverted position within the stainless steel trays, as depicted in Figure 8.4. Moreover, the specimens were not placed flush atop the tray-bottoms, as this would possibly impede the ingress of the $\mathrm{CC}$ solution through the CFRP strip regions. Instead, each tray was furnished with two, $6.4 \mathrm{~mm}\left(1 / 4^{\prime \prime}\right)$ diameter plastic tubes (see Fig. 8.3(b)), one on either end, acting as raisers to sufficiently elevate the beams off the bottoms of the tray and, thus, permitting proper engulfment of the interface region by the solution.

The 5-age categories at which time 4 beams were destructively fractured (i.e. to obtain $G_{I c}$ values), are as follows: 50, 100, 150, 200, and 300 cycles. Each cycle (see schematic, Fig. 8.1) consisted of 2 hours of ramping from room temperature at $20 \mathrm{C}$ to 

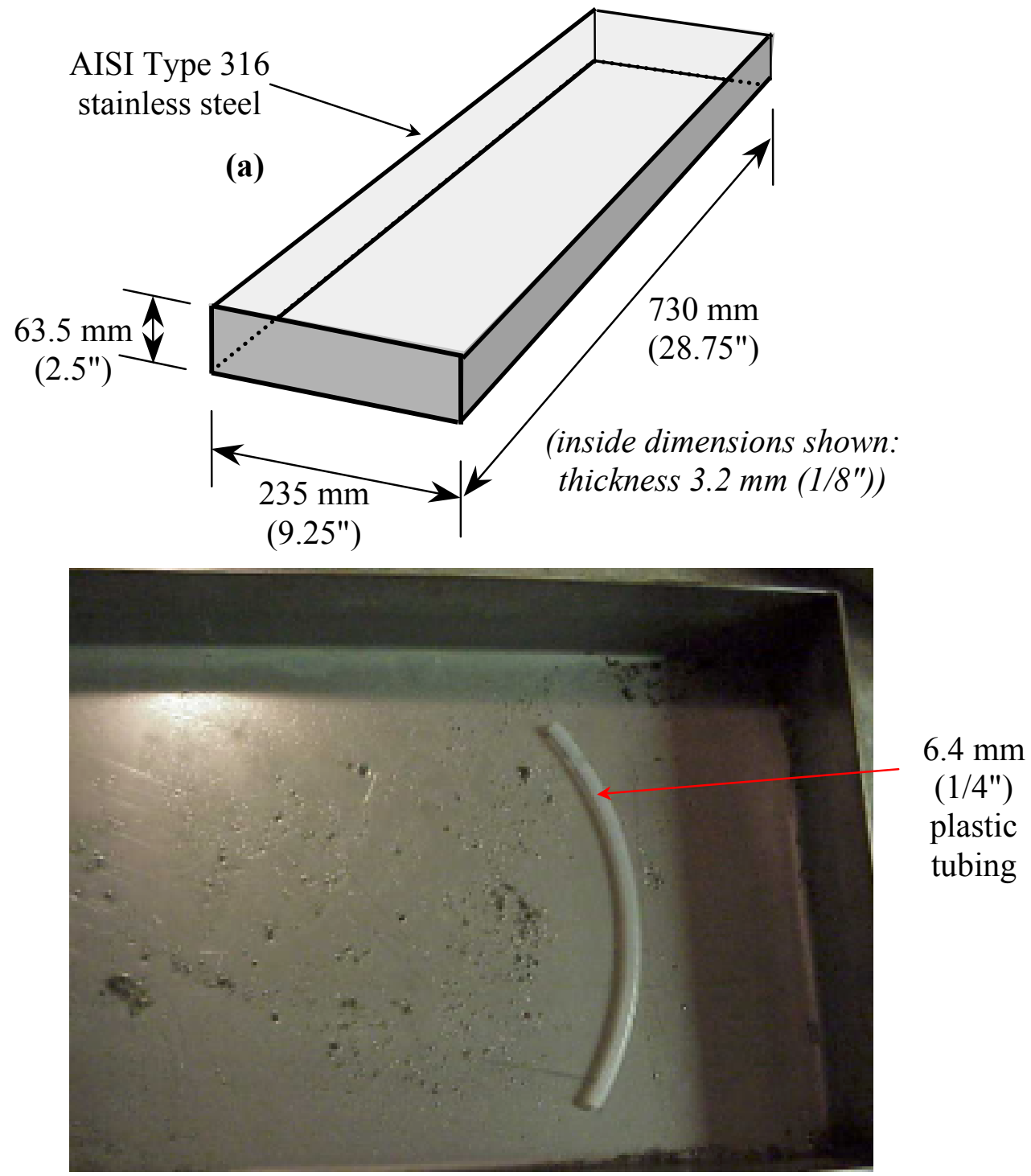

(b)

Figure 8.3 (a) Dimensions of stainless steel tray (not to scale), and

(b) plastic tubing used to elevate the CFRP-bonded specimens (one on each end-see Fig. 8.10)

freezing at $-20 \mathrm{C}, 2$ hours of constant freezing at $-20 \mathrm{C}$, and then, finally, ramping from a frozen state back to room temperature (i.e. thaw). A half-hour temperature-adjustmentsegment was programmed into the environmental chamber's computer to ensure an initial conditioning-temperature therein of $20 \mathrm{C}$ before commencing with the first cycle, as 
shown in Fig. 8.1 (see also the "Sample Freeze-Thaw Program" provided in Appendix C). As with the wet-dry cycling scheme, a total of 20 beams - here, four per each of the five ages - encountered this harsh aging protocol along with the use of 3 plain and 15 companion specimens (i.e. 3 for each of the 5-age categories) for the establishing of vital baseline data. From among the 20 actual FT (i.e. abbreviation for freeze-thaw) specimens, conclusive fracture results were determined from 15-the other five produced values of load that were outside the admissible range and, as such, stricken from consideration as shown in the following list (refer to Table A.5): (CC50): 5B3, 6B4, 7B6, 8B6; (CC100): 8B4, 5B6, 8B8, 1B9; (CC150): 3B7, 4B7, 2B9, 2B10; (CC200): 7B7, 5B9, 4B11, 2B13; (CC300) 2B11, 8B11, 4B13, 6B13; here, CC = calcium chloride; 150 (e.g.) $=$ number of cycles; concerning specimen-nomenclature, recall that the lead number designates the beam and the letter-and-number specifies the concrete batch (see Ch. 6).

Once again, all of the concrete beams, with the exception of the pristine specimens (Table A.1), received a coating of MBrace primer on all surfaces, leaving open only the concrete raised-step and ledge (see Figs. 3.21 parts (c) and (d), and Fig. 7.4(b)) portions. This was done so that the bulk concrete substrate (Fig. 7.1(b)) might remain unscathed after being subjected to the harsh environment. The sealing of the concrete beams in this fashion (Fig. 7.4) was designed, rather, to encourage attack on the interface by solely exposing it to the harmful effects of the various aggressive media - this is, after all, a necessary condition, if the central-issue of interface-reliability is to be investigated. 


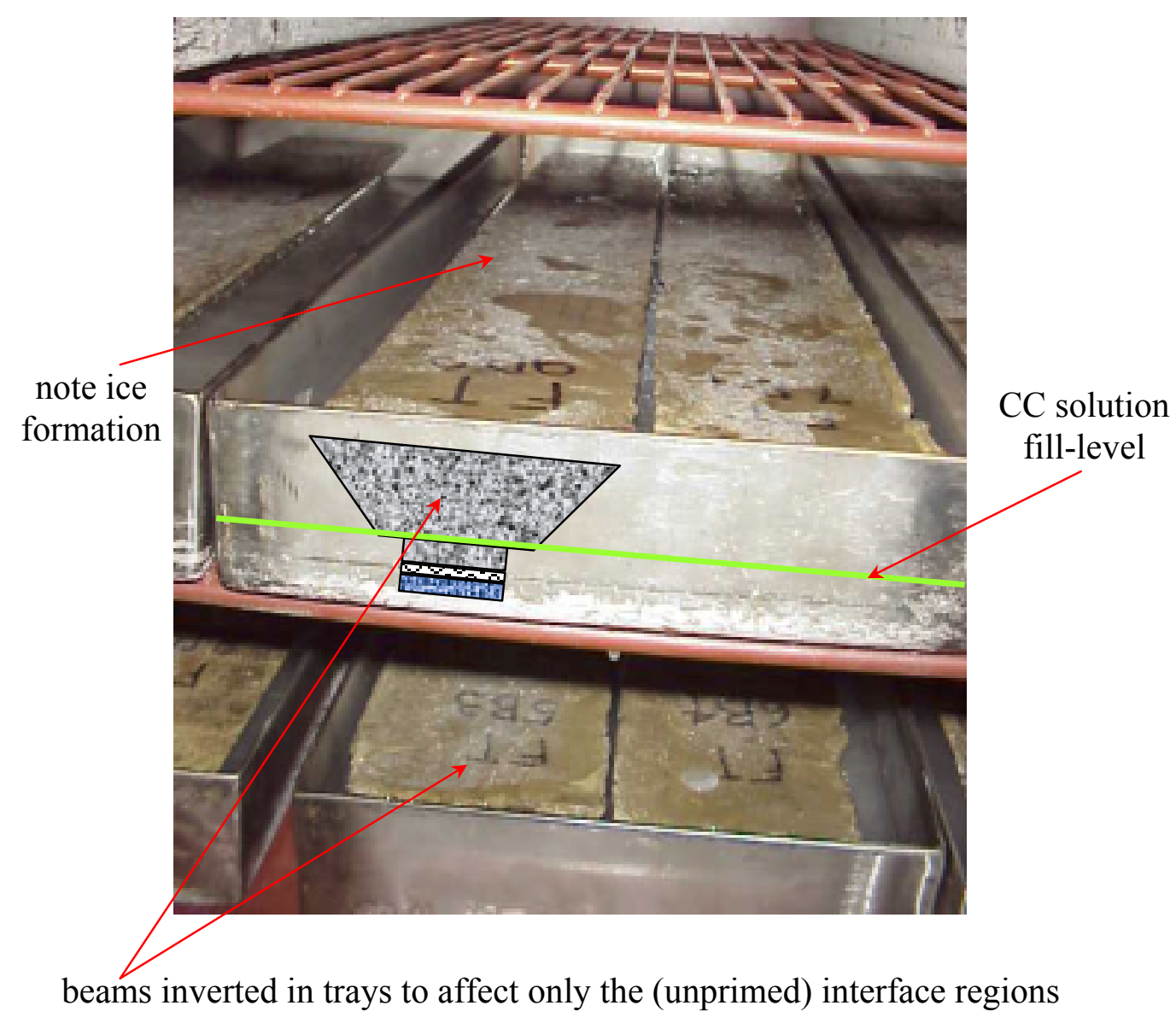

Figure 8.4 Picture of FT beams inverted in stainless steel trays

\section{SCCB Fracture (CC)}

The day before fracture-testing, i.e. following the end of each of the five targetages of weathering, the CFRP layer of each beam (see Fig. 7.1) was lightly sanded and cleaned prior to the bonding of the LVL wooden contours (Fig. 3.21(a); see also Fig. 7.5) with the MBrace saturant. Also, to ensure proper bond between the wood and the CFRP layer, each of the bonding-surfaces of the wooden contours was prepared with a coating 
of the MBrace primer (Fig. 7.5). This single contoured-cantilever beam assembly was then set aside to cure for a 24-hour period after which the specimens were fractured.

Adhering to the process of data-reduction presented in Chapter 6 , all of the initiation and arrestment loads resulting from the freeze-thaw SCCB fracture tests were tabulated, similar to Table 6.1. Once again, this was accomplished by carefully inspecting the cracking-portions of the $P$ vs. COD graphs of each beam to establish the loads-ofinterest, i1 - i(n) and a1 - a(n-1), as was done in Figure 6.1 for the four pristine specimens; five select $P$ vs. COD graphs from each of the freeze-thaw target cyclingages, specimens 5B3, 5B6, 2B10, 5B9, and 8B11, are shown in Figure 8.5. Next, the admissible load-ranges $\left(\mathrm{R}^{\mathrm{i}}, \mathrm{R}^{\mathrm{a}}\right.$; see Eqs. (6.9) - (6.12)) were determined by using the statistical procedure outlined in Section 6.2, thus enabling the refinement of raw data by the designation of reduced head and set initiation- and arrestment- loads. By the averaging of these values, the four critical loads, $P_{c, \text { Head }}^{i}, P_{c, \text { Head }}^{a}, P_{c, \text { Set }}^{i}$, and $P_{c, \text { Set }}^{a}$, were found, which when substituted, in turn, into Eq. (3.73-also repeated in Ch. 6 as (6.1)), shown below, yielded the respective critical strain energy release rates:

$$
\text { (3.73) or (6.1): } \quad G_{I c}=\frac{P_{c}^{2}}{2 b} \frac{d C}{d a}
$$

-recall, once again, that $b=$ width of the specimen $=43.4 \mathrm{~mm}(1.71 ")$, and $d C / d a=$ compliance gradient $=1.78 \times 10^{-5} \mathrm{~N}^{-1}\left(7.92 \times 10^{-5} \mathrm{lb}^{-1}\right)$. These results are shown in Tables $8.1-8.5$ and summarized with averaged head and set $G_{I c}$ values in Table 8.6. Also reported in these first five tables are the corresponding companion fracture energies and the resulting percentage fracture toughness reductions. The latter values are determined relative to the companion sample values of the same age (see Equation (7.1), repeated 

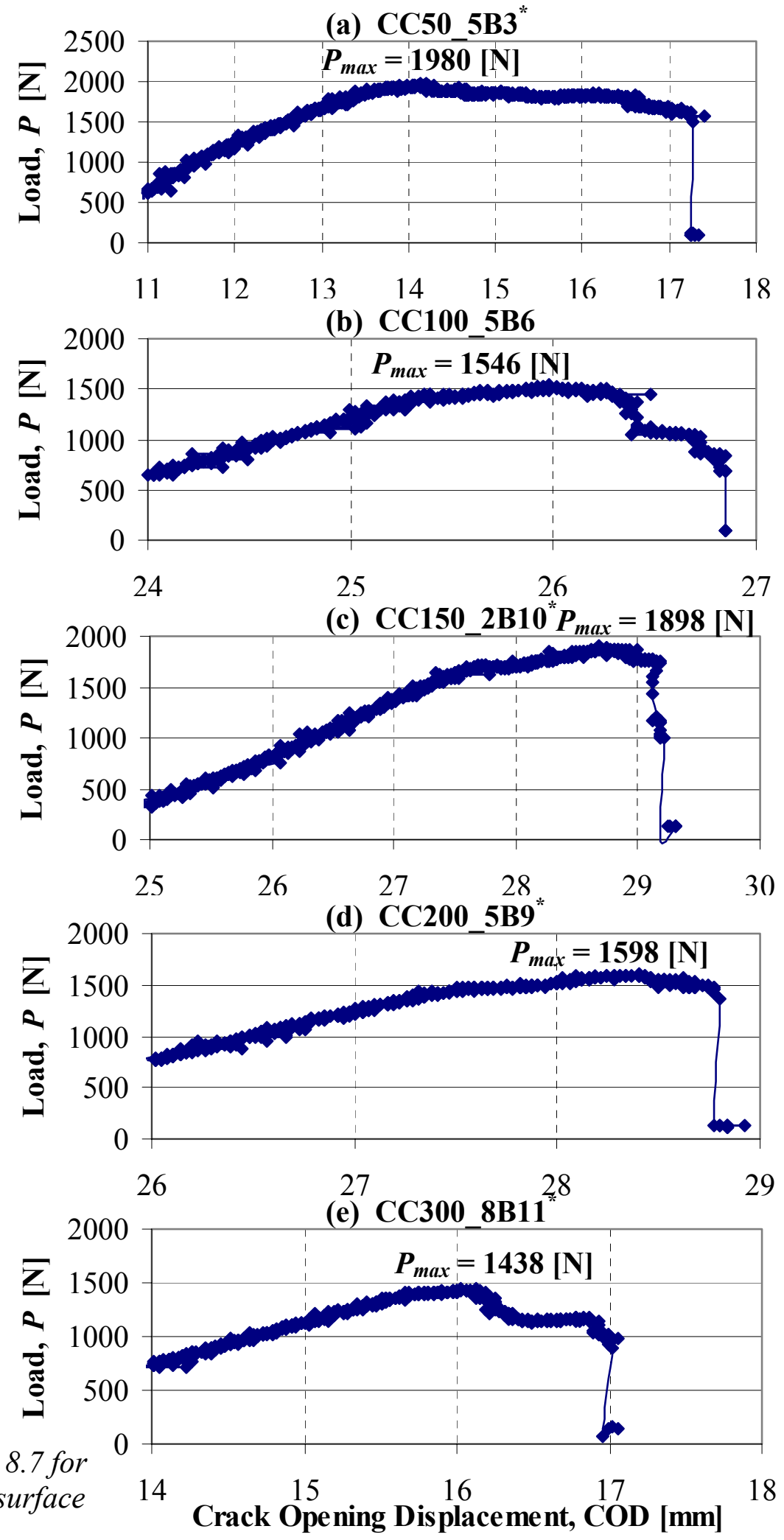

*See Fig. 8.7 for fracture surface Crack Opening Displacement, COD [mm]

Figure 8.5 Fracture behavior of select FT-(a) 50-, (b) 100-, (c) 150-, (d) 200-, and (e) 300- cycled beams in calcium chloride (CC) 
below for convenience). Bar graphs displaying $G_{I c}^{i}$ and $G_{I c}^{a}$ effect-based computations are shown in Figure 8.6, parts (a) and (b), respectively, with corresponding head- and setreductions plotted side-by-side to facilitate in evaluating the range of variations encountered for a given age. Reductions are computed based on the following relationship:

$$
\text { (7.1): } \% G_{I c} \text { Reduction }=\frac{G_{I c, \text { conditioned }(\# \text { cycles })}}{G_{I c, \text { companion }(\# \text { cycles })}} \times 100 \%
$$

where the critical strain energy release rate of the numerator may represent any one of the various conditioning schemes (i.e. wet-dry cycling under sodium -sulfate or -hydroxide (see Ch. 7), or freeze-thaw cycling under calcium chloride) for a specific age (i.e. 10, 20, or 30 cycles for the WD cases, or 50,100,150, 200, or 300 cycles for those of FT), and the denominator is the corresponding $G_{I c}$ value of the companion beams at the same age. (Note that the $200^{\text {th }}$-cycle set results are missing contributions from one of the three admissible specimen results, namely those of beam 4B11. The data acquisition system being used was of the type that would overwrite the former data file each time a test was run, i.e. data files were intermediately stored in the computer's random access memory (RAM) bank. For this reason, data had to be stored to a floppy disk prior to successive testing or it would be lost. The set-data file of specimen 4B11 was lost as a consequence of such a mishap - fortunately, however, head results were habitually collected from the display on the computer screen during each test-run, and are reported in Table 8.4. Correspondingly, the set outcomes that are shown were computed based on the data collected from specimens 5B9 and 2B13.) 
Table 8.1 Reduced data results of freeze-thaw, CC50 fracture test specimens

\begin{tabular}{c|cc|cc}
\cline { 2 - 5 } & \multicolumn{3}{|c|}{ Head } & \multicolumn{2}{c}{ Set } \\
\hline $\mathbf{n}$ & $\mathbf{i}$ & $\mathbf{a}$ & $\mathbf{i}$ & $\mathbf{a}$ \\
\hline $\boldsymbol{G}_{\boldsymbol{I c}}\left[\mathbf{J} / \mathbf{m}^{\mathbf{2}}\right]-\mathbf{C} \mathbf{5 0} / \mathbf{F T C 5 0} *$ & $\mathbf{5 7 2 / 6 0 2}$ & $\mathbf{5 5 8 / 5 8 5}$ & $\mathbf{5 6 3} / 605$ & $\mathbf{5 5 1 / 5 8 7}$ \\
\hline $\boldsymbol{\sigma}\left[\mathbf{J} / \mathbf{m}^{2}\right]$ & 15.42 & 14.15 & 8.51 & 7.69 \\
\hline $\mathbf{C O V}$ & $16.41 \%$ & $15.93 \%$ & $12.29 \%$ & $11.81 \%$ \\
\hline$\% \boldsymbol{G}_{\boldsymbol{I c}}$ Reduction & $95 \%$ & $95 \%$ & $93 \%$ & $94 \%$ \\
\hline
\end{tabular}

"FTC50 = freeze-thaw companions at 50 cycles (see Tables 6.6 - 6.9)

Table 8.2 Reduced data results of freeze-thaw, CC100 fracture test specimens

\begin{tabular}{c|cc|cc}
\cline { 2 - 5 } & \multicolumn{3}{|c|}{ Head } & \multicolumn{2}{c}{ Set } \\
\hline $\mathbf{n}$ & $\mathbf{i}$ & $\mathbf{a}$ & $\mathbf{i}$ & $\mathbf{a}$ \\
\hline $\boldsymbol{G}_{\boldsymbol{I c}}\left[\mathbf{J} / \mathbf{m}^{2}\right]-\mathbf{C 1 0 0} / \mathbf{F T C 1 0 0}$ & $\mathbf{5 4 7 / 6 2 2}$ & $\mathbf{5 3 0 / 5 9 6}$ & $\mathbf{5 3 8} / 643$ & $\mathbf{5 1 4 / 6 1 4}$ \\
\hline $\boldsymbol{\sigma}\left[\mathbf{J} / \mathbf{m}^{2}\right]$ & 2.56 & 3.36 & 1.23 & 1.32 \\
\hline $\mathbf{C O V}$ & $6.84 \%$ & $7.96 \%$ & $4.77 \%$ & $5.06 \%$ \\
\hline$\% \boldsymbol{G}_{\boldsymbol{I c}}$ Reduction & $88 \%$ & $89 \%$ & $84 \%$ & $84 \%$ \\
\hline
\end{tabular}

"FTC100 = freeze-thaw companions at 100 cycles (see Tables 6.6 - 6.9)

Table 8.3 Reduced data results of freeze-thaw, CC150 fracture test specimens

\begin{tabular}{c|cc|cc}
\cline { 2 - 5 } & \multicolumn{2}{|c|}{ Head } & \multicolumn{2}{c}{ Set } \\
\hline $\mathbf{n}$ & 3 & 3 & 39 & $\mathbf{a}$ \\
\hline $\boldsymbol{G}_{\boldsymbol{I c}}\left[\mathbf{J} / \mathbf{m}^{2}\right]-\mathbf{C} 150 / \mathbf{F T C 1 5 0}$ & $\mathbf{6 0 9 / 7 2 9}$ & $\mathbf{5 9 4 / 7 1 2}$ & $\mathbf{6 0 0} / 746$ & $\mathbf{5 9 0} / 723$ \\
\hline $\boldsymbol{\sigma}\left[\mathbf{J} / \mathbf{m}^{2}\right]$ & 5.00 & 4.69 & 1.85 & 1.70 \\
\hline $\mathbf{C O V}$ & $9.06 \%$ & $8.88 \%$ & $5.55 \%$ & $5.37 \%$ \\
\hline$\% \boldsymbol{G}_{\boldsymbol{I c}}$ Reduction & $68 \%$ & $68 \%$ & $80 \%$ & $82 \%$ \\
\hline
\end{tabular}

"FTC150 = freeze-thaw companions at 150 cycles (see Tables 6.6 - 6.9) 
Table 8.4 Reduced data results of freeze-thaw, CC200 fracture test specimens

\begin{tabular}{|c|c|c|c|c|}
\hline & \multicolumn{2}{|c|}{ Head } & \multicolumn{2}{|c|}{ Set } \\
\hline & $\mathbf{i}$ & $\mathbf{a}$ & $\mathbf{i}$ & $\mathbf{a}$ \\
\hline $\mathbf{n}$ & 3 & 3 & $17^{* \pi}$ & $14^{* \pi *}$ \\
\hline$G_{I c}\left[\mathrm{~J} / \mathrm{m}^{2}\right]-\mathrm{CC200/FTC200}{ }^{*}$ & $532 / 785$ & $577 / 757$ & $\mathbf{5 1 7 ^ { * * } / 7 6 6}$ & $\mathbf{5 1 1 ^ { * * * } / 7 7 0}$ \\
\hline$\sigma\left[\mathbf{J} / \mathbf{m}^{2}\right]$ & 5.13 & 5.03 & $1.15^{* * *}$ & $1.44^{* * 4}$ \\
\hline $\mathrm{COV}$ & $9.38 \%$ & $9.33 \%$ & $4.71^{* *} \%$ & $5.32^{* * *} \%$ \\
\hline$\% G_{I c}$ Reduction & $74 \%$ & $76 \%$ & $67^{* * *} \%$ & $66^{* * * 1 \%} \%$ \\
\hline
\end{tabular}

"FTC200 = freeze-thaw companions at 200 cycles (see Tables 6.6 - 6.9)

***Data file missing for Set-4B11 values

Table 8.5 Reduced data results of freeze-thaw, CC300 fracture test specimens

\begin{tabular}{c|cc|cc}
\cline { 2 - 5 } & \multicolumn{2}{|c|}{ Head } & \multicolumn{2}{c}{ Set } \\
\hline $\mathbf{n}$ & 3 & $\mathbf{a}$ & $\mathbf{i}$ & $\mathbf{a}$ \\
\hline $\boldsymbol{G}_{\boldsymbol{I c} \boldsymbol{c}\left[\mathbf{J} / \mathbf{m}^{2}\right]-\mathbf{C C 3 0 0 / F T C 3 0 0}}$ & $490 / 811$ & $476 / 775$ & $\mathbf{4 9 2 / 8 1 1}$ & $\mathbf{4 7 8} / 770$ \\
\hline $\boldsymbol{\sigma}\left[\mathbf{J} / \mathbf{m}^{2}\right]$ & 3.95 & 3.95 & 2.22 & 2.20 \\
\hline $\mathbf{C O V}$ & $8.98 \%$ & $9.11 \%$ & $6.72 \%$ & $6.79 \%$ \\
\hline $\mathbf{\%} \boldsymbol{G}_{\boldsymbol{I} \boldsymbol{c}}$ Reduction & $60 \%$ & $61 \%$ & $61 \%$ & $62 \%$ \\
\hline
\end{tabular}

${ }^{*}$ FTC300 $=$ freeze-thaw companions at 300 cycles (see Tables 6.6 - 6.9)

Table 8.6 Calcium chloride, freeze-thaw fracture results (unit: $\mathrm{J} / \mathrm{m}^{2}$ )

\begin{tabular}{|c|c|c|c|c|}
\hline & \multicolumn{2}{|c|}{ Initiation } & \multicolumn{2}{|c|}{ Arrestment } \\
\hline & $\boldsymbol{G}_{I c, \text { Head }}$ & $\boldsymbol{G}_{I c, S e t}$ & $\boldsymbol{G}_{I c, \text { Head }}$ & $\boldsymbol{G}_{\boldsymbol{I}, \text {,Set }}$ \\
\hline CC50 & 572 & 563 & 558 & 551 \\
\hline Mean: & \multicolumn{2}{|c|}{568} & \multicolumn{2}{|c|}{554} \\
\hline CC100 & 547 & 538 & 530 & 514 \\
\hline Mean: & \multicolumn{2}{|c|}{542} & \multicolumn{2}{|c|}{522} \\
\hline CC150 & 609 & 600 & 594 & 590 \\
\hline Mean: & \multicolumn{2}{|c|}{604} & \multicolumn{2}{|c|}{592} \\
\hline CC200 & 582 & $517^{* *}$ & 577 & $511^{* *}$ \\
\hline Mean: & \multicolumn{2}{|c|}{550} & \multicolumn{2}{|c|}{544} \\
\hline CC300 & 490 & 492 & 476 & 478 \\
\hline Mean: & \multicolumn{2}{|c|}{491} & \multicolumn{2}{|c|}{477} \\
\hline
\end{tabular}

"Data file missing for Set-4B11 values 
(a) Initiation-Bas ed Freeze-Thaw Calcium Chloride Results

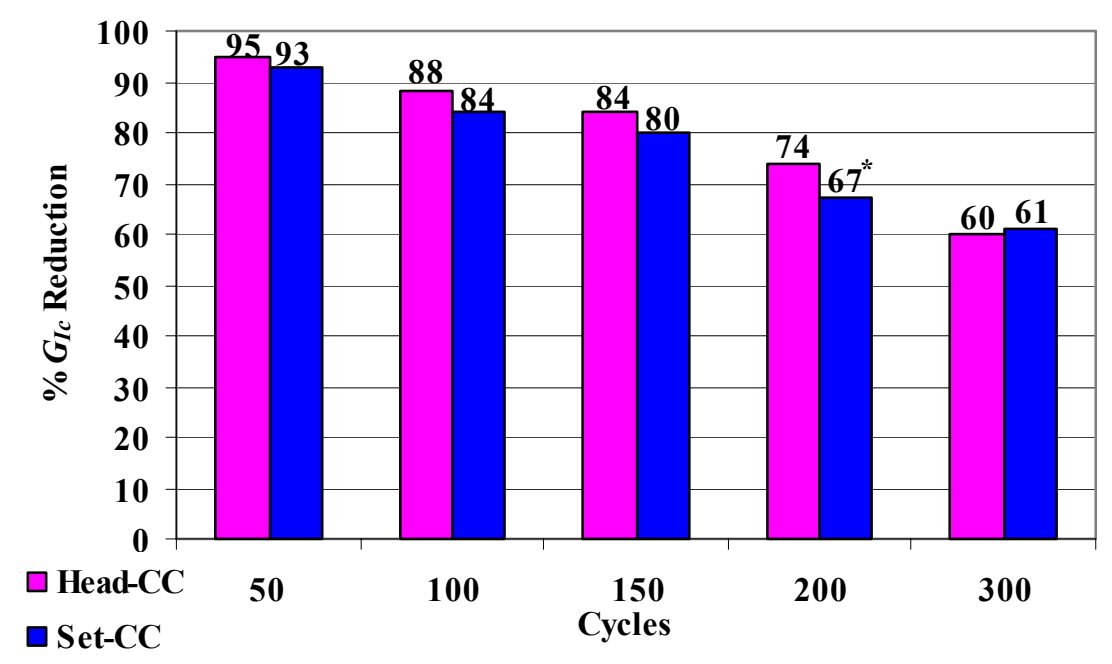

(b) Arrestment-Based Freeze-Thaw Calcium Chloride Results

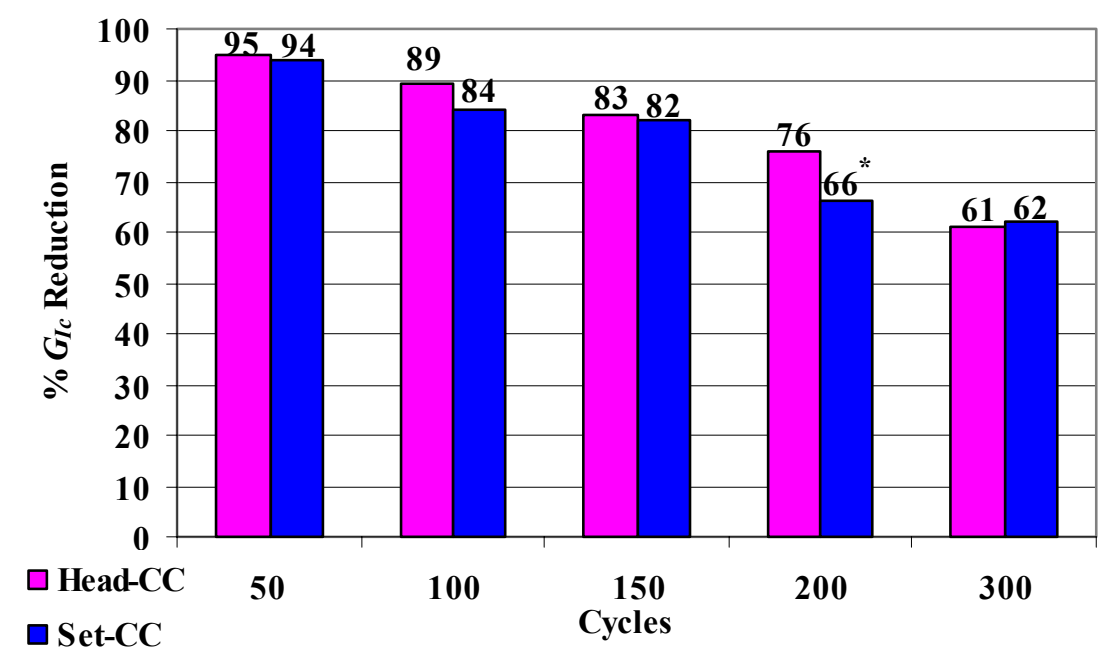

*Data file missing for Set-4B11 values

Figure 8.6 Percentage reductions in (a) $G_{I c}^{i}$ and (b) $G_{I c}^{a}$ resulting from $50,100,150,200$, and 300 freeze-thaw cycles of calcium chloride (CC) exposure

The absolute, average variation between head and set $G_{I c}$ percentage reductions for the calcium chloride (CC) exposures was found to be 5\%, with maximum and minimum deviations of $13 \%$ and $1 \%$ for the $200^{\text {th }}$ cycle and $100^{\text {th }} / 150^{\text {th }}$ cycle arrestment- 
based cases (Fig. 8.6(b)), respectively. As noted in the parenthetical statement preceding Table 8.1, the set-data file of one of the 200-cycled specimens, 4B11, was lost, and so the two asterisked percentage $G_{I C}$ reductions shown in Fig. 8.6 are believed to be slightly lower than what would have resulted had this information been available - the maximum percentage difference between head and set reductions would have otherwise probably been only $6 \%$ or $7 \%$, i.e. half as much as the (maximum) number just reported. After the complete duration of aging at 300 cycles, the net percentage reductions reveal some resemblance of the wet-dry cases of the previous chapter-here, these numbers are found to be between $60 \%-62 \%$. A similar conclusion may thus be drawn now, that, under CC300/freeze-thaw cycling, the CFRP-concrete interface reliability may be characterized as being less than two-thirds as effectual if it had, otherwise, not been subjected to such attack.

Patterns of Fracture (CC)

Quantitatively (CC): Brittleness indices are investigated to gain a quantitative insight into the cracking patterns of CC/freeze-thaw cycled CFRP-concrete interfaces. Substitution of the critical strain energy release rates of Table 8.6 into Eq. (3.74—or 6.2),

$$
I=\frac{G_{I c}^{i}-G_{I c}^{a}}{G_{I c}^{i}}
$$

yields the following results (shown in tabular form):

Table 8.7 Brittleness indices of CC/freeze-thaw fracture test specimens

\begin{tabular}{c|c|c|c|c|c}
\cline { 2 - 6 } & CC50 & CC100 & CC150 & CC200 & CC300 \\
\hline$I$ & 0.02 & 0.04 & 0.02 & 0.01 & 0.03 \\
\hline
\end{tabular}


Each of the $I$ values of Table 8.7 correspond to stable forms of crack propagation as diagrammatically shown in Figures 3.14(a) and 3.15(b). The average of these values is approximately $I=0.02$, which reveals an even higher degree of cracking-stability than what was reported, previously, for all the other cases encountered: the pristine $(I=0.06)$ and representative dry $(I=0.04)$ beams of Ch. 6 , and both cases of wet-dry $\left(I_{S S}=0.03\right.$, $\left.I_{S H}=0.04\right)$ specimens discussed in Ch. 7. Thus, the CFRP-concrete interface may be characterized as being strain rate insensitive (see Sec. 3.4), irrespective of the conditioning schemes considered herein.

Qualitatively (CC): The following figure (Fig. 8.7, parts (a) - (e)) depicts fracture surfaces of a "refined" specimen (see Table A.4) from each target-age, (a) 50, (b) 100, (c) 150, (d) 200, and (e) 300, that underwent CC/freeze-thaw weathering.

A defining trend of characterization for this grouping of specimens is that the failure pattern begins and ends cohesively (Fig. 8.7, parts (a) and (e)), whereas the midthree surfaces (parts (b) - (d)) appear to be more mixed and adhesive in the central regions, resembling, somewhat, the latter-staged SS/wet-dry beams of the previous chapter. As might be expected from being immaturely weathered, the first cohesive surface (i.e. 50-cycled; part (a)) corresponds more closely to the cohesive failures of the dry specimens (Ch. 6) in that the layer of concrete retained on the underside of the LVL is rather shallow. The final cohesive pattern (300-cycled) shown in part (e), on the other hand, exhibits deeper sections of concrete that became dislodged following fracture with a more prominent central adhesive zone, as evident by the dark-blue spots in this locale corresponding to the saturant. Interestingly, the bandwidth of this adhesive midsection 


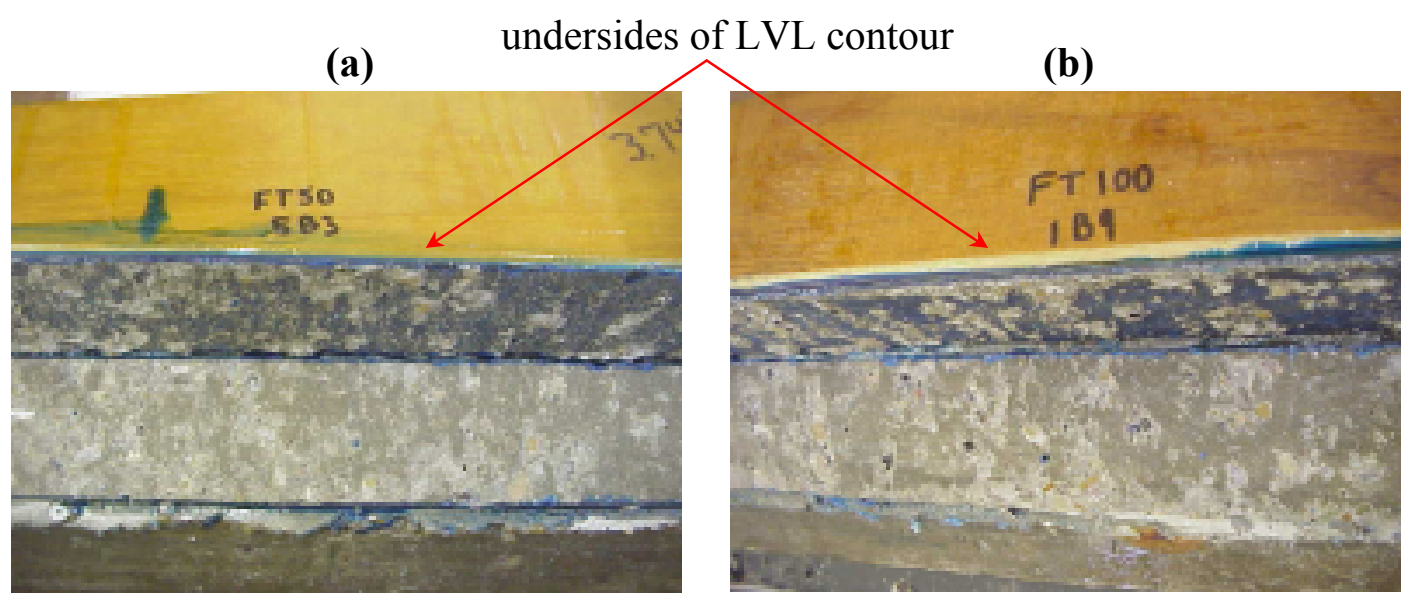

(c)

(d)

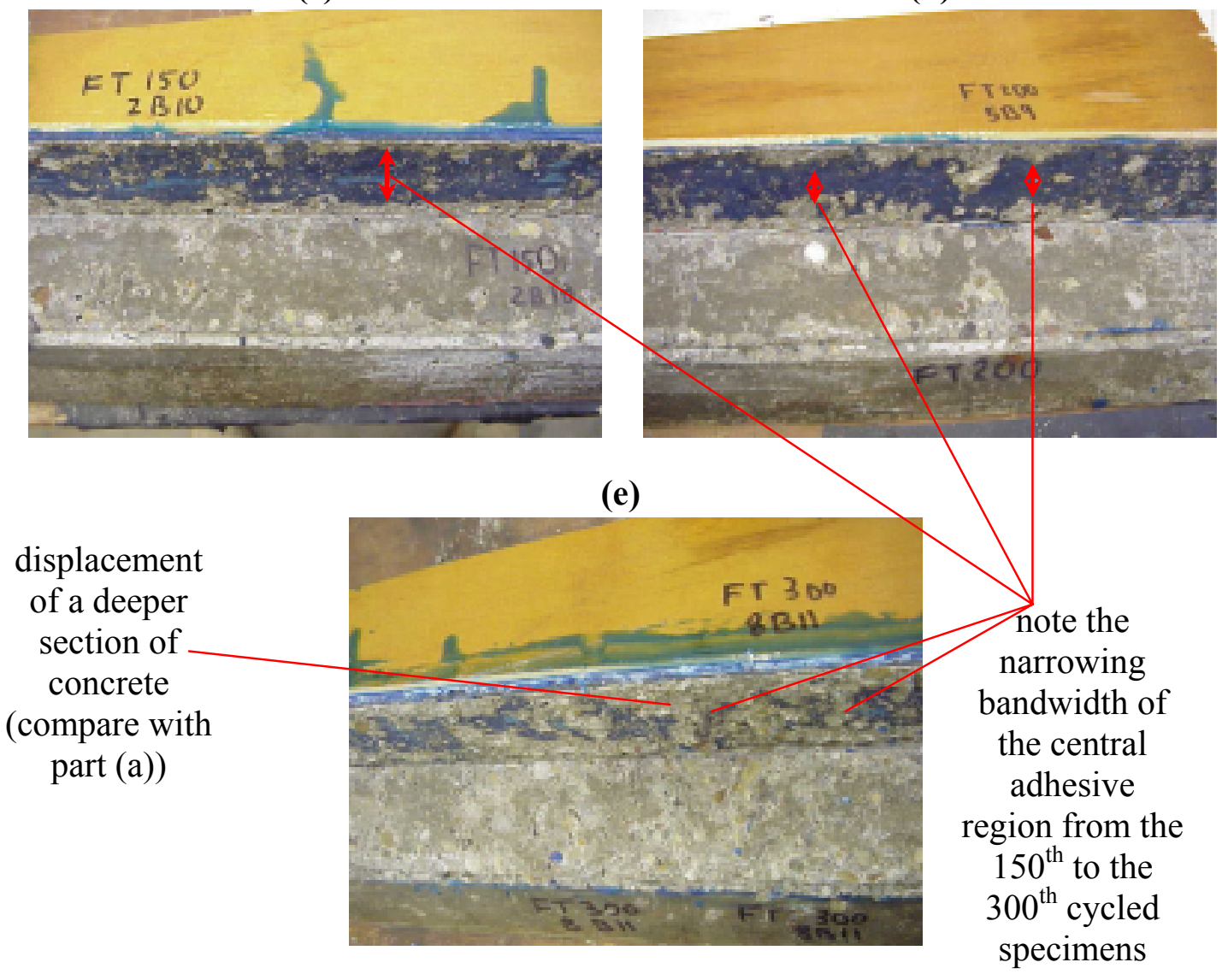

Figure 8.7 Pictures of CC/FT fracture surfaces displaying a specimen from each target-age (superscripted)-(a) $5 \mathrm{~B} 3^{50}$, (b) $1 \mathrm{~B} 9^{100}$, (c) $2 \mathrm{~B} 10^{150}$, (d) $5 \mathrm{~B} 9^{200}$, and (e) $8 \mathrm{~B} 11^{300}$ (NOTE: the fracture behavior of these specimens, with the exception of (b), are shown in Fig. 8.5) 
narrows progressively from about the time that the damaging $\mathrm{CC} /$ freeze-thaw conditions take effect-sometime around the $150^{\text {th }}$ cycle - till the end (see Fig. 8.7, parts (c) - (e)). This reveals the migratory history of deterioration.

To recapitulate, then, the early stages of conditioning culminated in shallowcohesive, or shallow-mixed-dominantly-cohesive failures (i.e. 100-cycled, part (b)), reminiscent of the dry fractures discussed in Ch. 6. Beginning around the middle of the targeting-ages (i.e. 150 cycles; part (c)), the interfacial-concrete at the outlying edges evidently became weakened due to the weathering it had sustained, and thus yielded more readily during fracture. The interfacial-core of concrete, on the other hand, had, as yet, not experienced such environmental-distress, and as such, resulted in a relatively adhesive type of failure at the midsection. With further aging and the inward migration of the disruptive $\mathrm{CC}$ solution, however, the outlying layers of concrete that became dislodged for specimens aged at 200- and 300- cycles, were found not only to be thicker (or deeper), but greater in width as well, i.e. the central adhesive zone was found to decrease with increased aging (as shown in Fig. 8.7, parts (c) - (e)).

\section{Pre-Fracture Changes (CC)}

Visual Changes (CC): One of the first observations evident from looking at the progressive pictures of freeze-thaw specimens in Figure 8.8, is the sharp contrast in appearance of the companion beam (Fig. 8.8(a)) with those of the others (parts (b) - (d)). The concrete ledge and raised-step of part (a) is in impeccable form. There is a certain defining smoothness and robustness of the concrete surfaces, both of which possess a whitish hue. Beneath this area, the epoxy-based primer is seen to fully cover the side of 

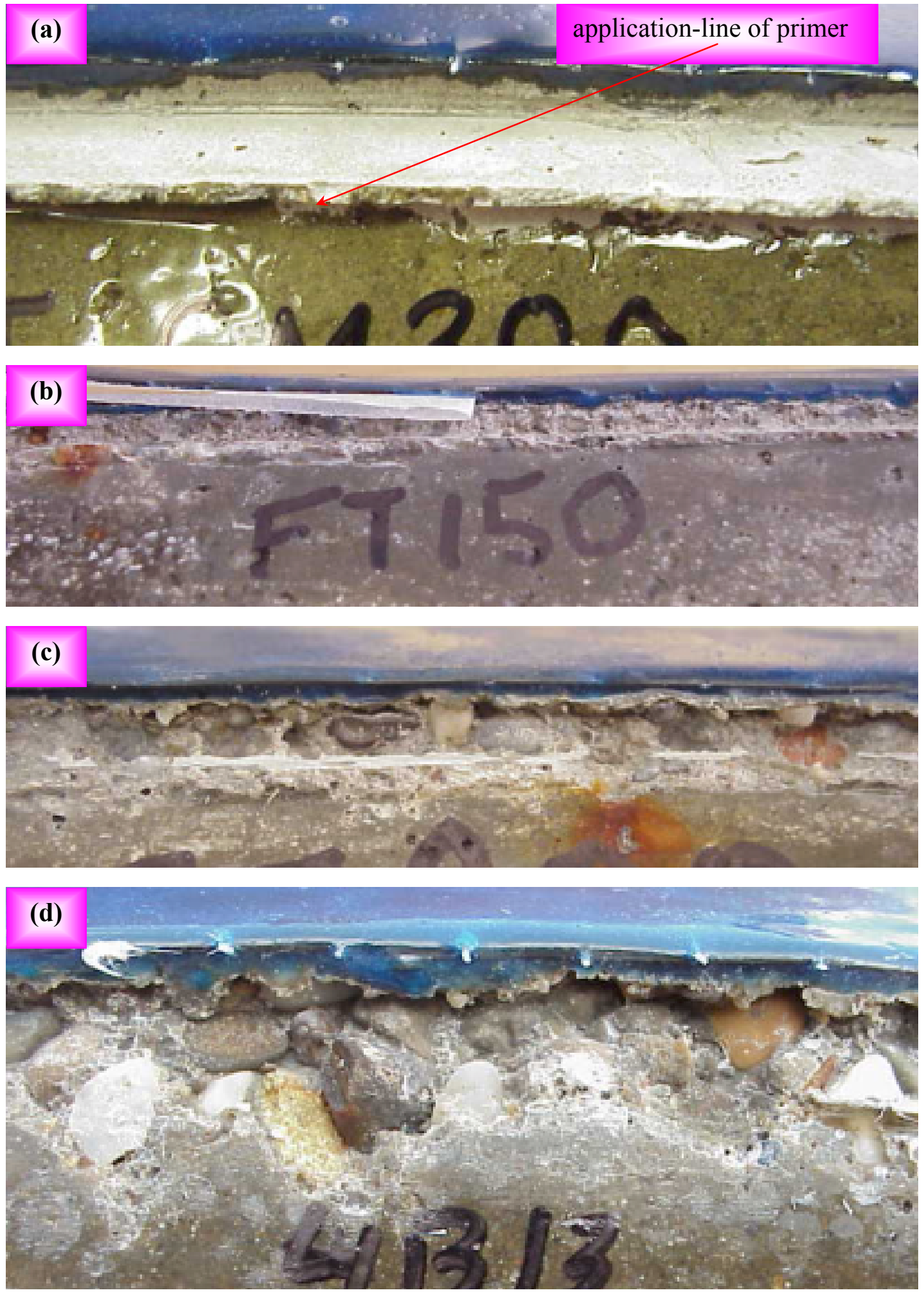

Figure 8.8 Pictures displaying progressive changes occurring at the CFRPconcrete interface of various CC/freeze-thaw specimens - (a) FT companion (300-cycle), (b) FT150, (c) FT200, and (d) FT300 
the concrete beam up until the brim of the ledge, as it was so designed (see the "Preliminaries (SS)" of Section 7.2) — not only does it cover the sides fully, but it also has a quality about it that makes it glisten. All of these characteristics are absent in the other three views of Fig. 8.8 (i.e. parts (b) - (d)). Notice that with age, the separation between the concrete ledge and raised-step regions have become indistinct. Moreover, what was once covered by concrete, is now seen to have gradually worn to such a degree, that the underlying coarse aggregates are left bare and vulnerable to further spalling. These areas have also become ash-colored and gray — in contrast, recall that for the wet-dry cycling cases, the sodium sulfate beams were yellowed while the alkaline samples retained their original whitish tones. The application-line of the primer is noted to have greatly receded, too, the cause of which is believed to have stemmed from within the deteriorating concrete substrate, as was the case with the SS/wet-dry beams of the previous chapter. A difference in these two cases, however, is the extent of retrogression issued. With the SS/wet-dry situations, it was found that the sulfate ions entered the concrete through small voids and pores (places where the primer-coat had not been adequately applied), and thus caused some local degradation; however, the application-line, was, for the most part, still viably left intact. An evolutionary process of erosion is seen to dictate the height of this epoxy barrier with respect to the $\mathrm{CC} /$ freeze-thaw specimens, under present consideration. Little or no wearing of this line is apparent at 150-cycles, which, subsequently, retreats to about a half-centimeter at 200-cycles, and finally, to approximately $0.5 \mathrm{~cm}$ to more than $1 \mathrm{~cm}$ at 300 -cycles (see parts (b), (c), and (d), respectively, of Fig. 8.8). As a final point of observation, note that the interface becomes 
partially separated, in a cohesive fashion within the concrete, of course, between cycles two- and three- hundred. This, therefore, further corroborates the theory described in the previous subsection regarding the narrowing central adhesive bandwidth.

Weight Changes (CC): A total of 23 specimens were used to periodically measure weight variations in the CFRP-bonded concrete beams (see Tables A.2, A.3, and A.5) for the following three categories: Plain (Table A.2: 9B3, 7B4, and 1B8), Companion (Table A.3: 7B3/8B3, 1B4/6B7, 5B8/6B9, 7B10/8B10, and 4B12/8B12), and Actual FT (Table A.5: 7B6/8B6, 8B8/1B9, 2B9/2B10, 4B11/2B13, and 4B13/6B13). The companions' weight results were already presented separately in Chapter 6 (Fig. 6.7) where the dry specimens' weight changes were considered solely; these effects are again repeated here, as shown in Figure 8.9, along with the outcomes of the plain and actual wet-dry beams so that comparisons might easily be drawn between these different cases. The weighingschedules of the $\mathrm{CC} /$ freeze-thaw beams were identical with the target-cycling ages, provided the initial-weighing is concatenated: $0,50,100,150,200$, and 300 cycles.

While the overall weight changes resulting from the specimens are quite slight, as expected, the maximum percentage increase now (i.e. 3.21\%; see Fig. 8.9) is, nevertheless, approximately 5 to (more than) 6 times as much as the respective SS and SH values cited in the previous chapter (Figs. 7.13 and 7.21). This is interesting for at least two reasons. Firstly, the beams here were partially submerged (see Fig. 8.4) unlike their wet-dry specimen counterparts which were completely immersed in a wet bath (see Fig. 7.2(a)). Secondly, the extent of mass loss resulting from these beams far exceeded those of the wet-dry protocol, as is seen in Figure 8.10(a), for example - the amount lost 
here may possibly be greater than what was fragmented in both the SS and SH cases combined, though losses in the latter specimens were never quantitatively measured.

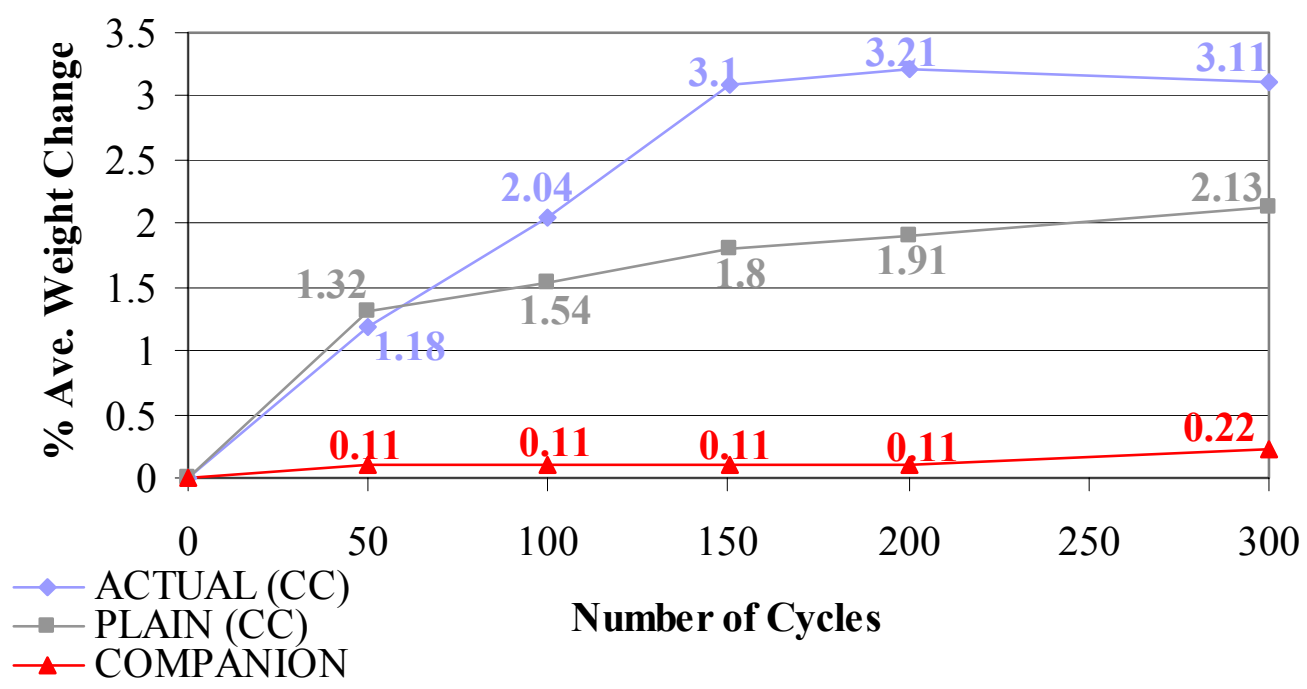

Figure 8.9 Percent average weight changes of the CC/freeze-thaw plain, companion, and actual beams

A simple answer to the first point above is that the primer coating truly fulfilled its intended objective by barring against moisture ingress. In other words, whether fully or partially submerged, the net moisture uptake effect remained practically the same. Answering the second question is somewhat more challenging, since logically these specimens should have lost weight over time, or at least, common sense would dictate that the gains made should not have been 5 - 6 times that of their wet-dry counterparts (again, see Fig. 8.10). The fact that gains did result suggests two outcomes. Undoubtedly, on the one hand, there was a considerable amount of salt crystallization and deposition that occurred within the specimens' open pores. On the other, the CC solution resulted in 
corrosion of the internal steel reinforcement—recall that each beam was reinforced with a pair of \#2 steel reinforcing bars. The fact that the beams were only partially submerged adds tenability that this should be the case, since with complete immersion, the specimens would have been deprived of vital oxygen, thereby stifling the corrodibility of the steel. (Note: the use of corrosion in this context is not to be confused with the variety that causes pitting.)

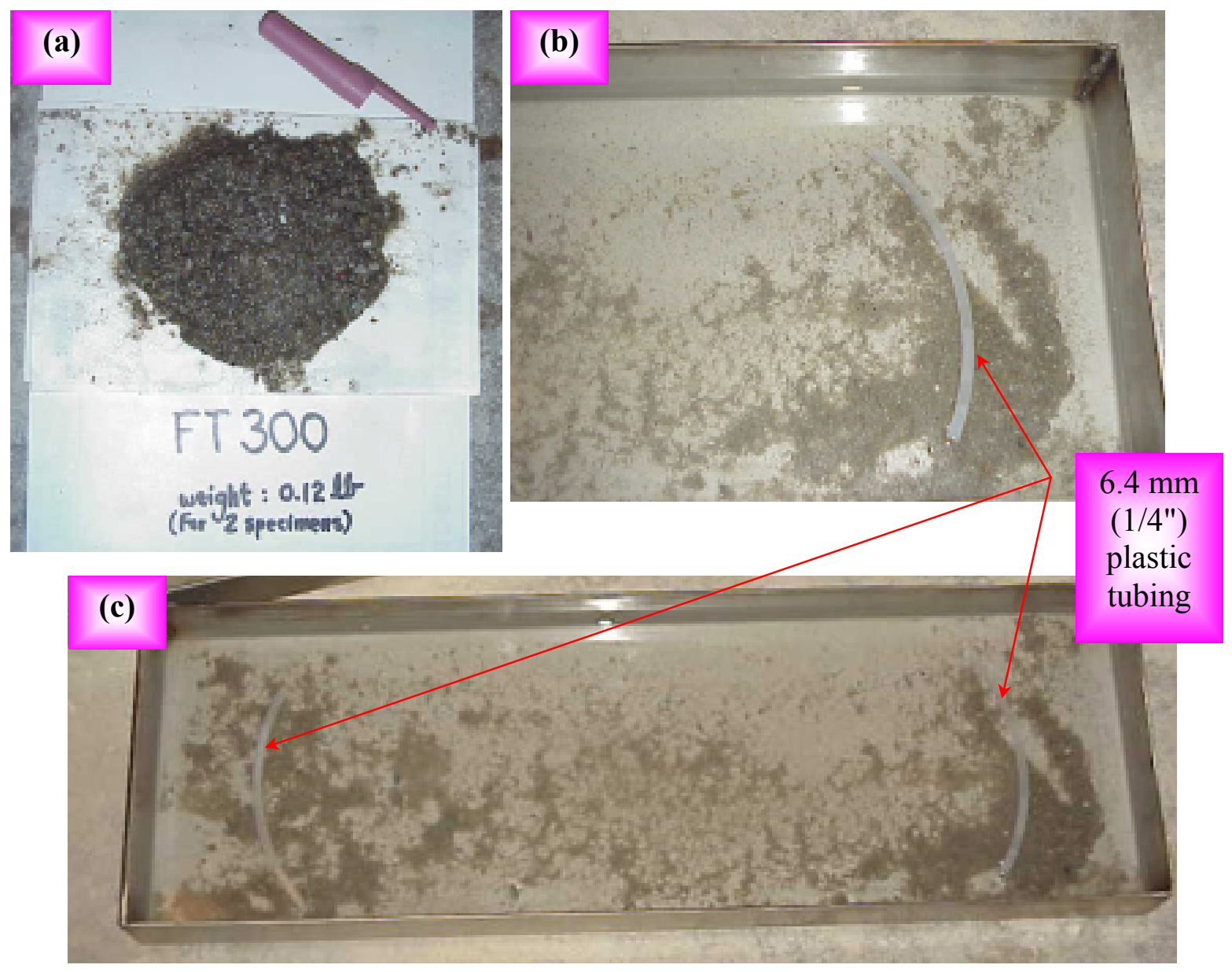

Figure 8.10 Pictures of concrete mass residue left behind in CC/freeze-thaw trays(a) mass lost for two specimens after 300 cycles was approx. $54 \mathrm{~g}$ $(0.12 \mathrm{lb}),(\mathbf{b}) \&$ (c) shows two views of the dispersed residue left behind (NOTE: dimensions of tray given in Fig. 8.3(a)) 
Considering now the trends displayed in Fig. 8.9, it is seen that the actual specimens' weight gains are greater than their plain counterparts. This is attributed to the actual beams' higher potential for water uptake within the saturant-and-CFRP layer. The rate of weight gain achieved by the actual beams is also unchanging for 150 cycles, after which, this trend essentially stabilizes. This conforms to both the qualitative post- and pre- fracture observations that asserted cycle-150 as a pivotal point of demarcation between specimen -soundness and -degeneration. It is speculated that in this first-half of aging, all the weight gains were due to moisture uptake and salt-crystallization within the exposed concrete regions and the saturant-and-CFRP layer. In the second-half, when the first of these effects is believed to have become equilibrated, however, the loss in concrete mass due to spalling and the initiating ferric oxide (responsible for causing reinforcement corrosion) coupled with a continuance of salt-crystallization, was seemingly found to be tantamount. (Note: the erosive, $\mathrm{CC} /$ freeze-thaw action apparently overpowered the fortifying effect of the CFRP reinforcement in the actual specimensrecall that it benefited the SS/wet-dry beams.) The same thing is noted for the plain specimens, except that the latter processes corresponding to weight-gains were more substantial than the spalling concrete, thus explaining the moderately increasing slope at cycle-300.

Strain Changes (CC): It is seen from Appendix A that the same three, weight change, plain specimens (Table A.2: 9B3, 7B4, and 1B8) of the previous subhead have also been used for measuring strains; additionally, five companion (Table A.3: 4B9, 1B10, 5B11, 10B12, and 5B13) and ten actual WD (Table A.5: 5B3/6B4, 8B4/5B6, 3B7/4B7, 


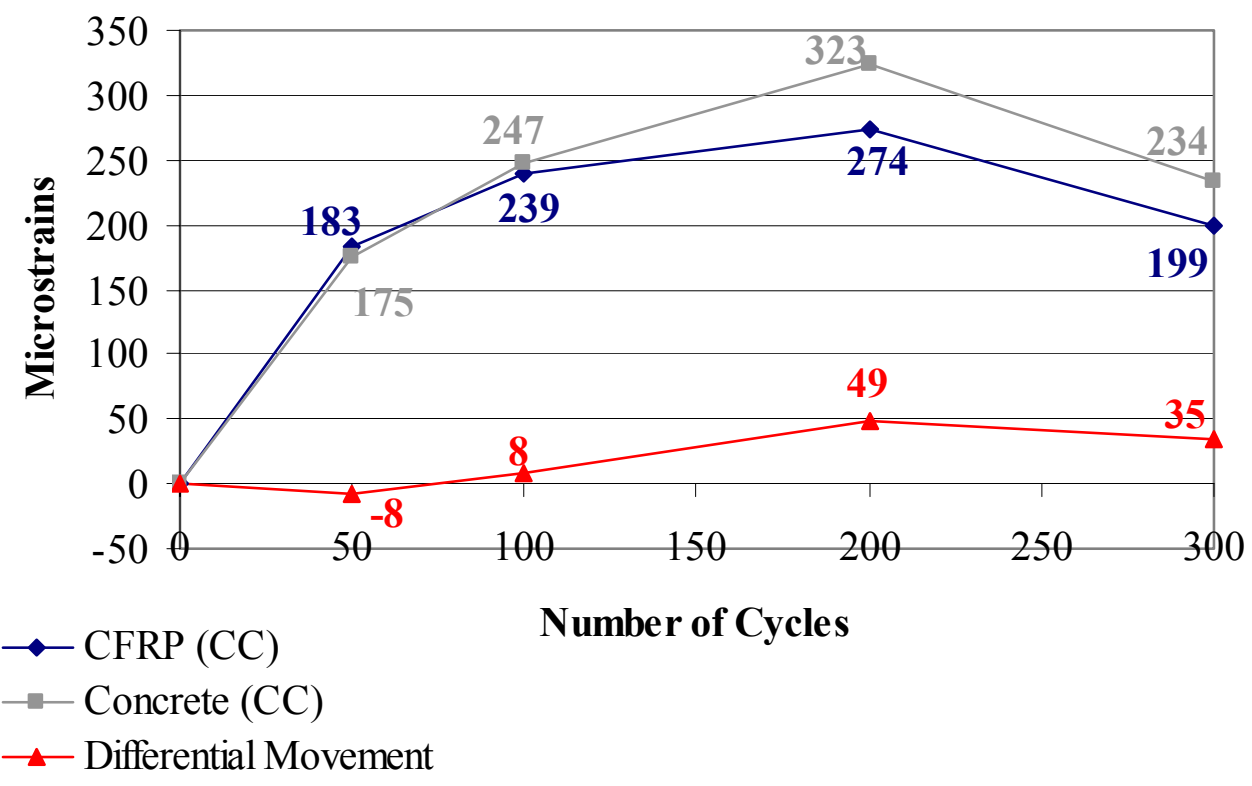

Figure 8.11 Microstrains of the CC/freeze-thaw beams in two locations: (1) on the CFRP, and (2) on the adjacent concrete ledge (see Fig. 3.21(d)) - the differential movement between the two is shown as well

7B7/5B9, and 2B11/8B11) beams were used for investigating strain changes. Measurements were taken using a DEMEC instrument as described in Chapter 6 (see also Fig. 6.8), in accord with the weigh schedule, i.e. at $0,50,100,150,200$, and 300 cyclesthe reading at cycle 150 contained an error and, as such, was omitted.

Microstrains recorded at the top of the CFRP strip and the adjacent ledge of concrete (see Figs. 3.21(d) and 6.8(b)) as well as any resulting differential movement that occurred between the two are displayed in Figure 8.11. The average, absolute, differential movement that materialized between the CFRP and concrete is $25 \mu \varepsilon$, with absolute minimum and maximum differences of $8 \mu \varepsilon$ and $49 \mu \varepsilon$, respectively. By using the largest 
differential microstrain (i.e. $49 \mu \varepsilon$ ) coupled with the fact that $\mathrm{E}_{\mathrm{c}}=4.93 \times 10^{6}$ psi (see Eq. 6.17), the maximum interfacial tensile stress that is developed can be computed as:

$$
\sigma_{\mathrm{t}, \mathrm{FT} / \mathrm{CC}}=242 \mathrm{psi}
$$

by Hooke's law (i.e. $\sigma_{\mathrm{t}}=\mathrm{E}_{\mathrm{c}} \cdot \varepsilon$, given as Eq. (6.15)), where the capitalized subscripts, FT/CC, indicate the condition of freeze-thaw cycling under a calcium chloride solution. Comparison of this value $\left(\sigma_{\mathrm{t}, \mathrm{FT} / \mathrm{CC}}=242 \mathrm{psi}\right)$ with the corresponding tensile strengths of Ch. 6, i.e. Eqs. (6.13), (6.14), (6.13') and (6.14'), repeated here for convenience:

$$
\begin{aligned}
& \left(6.13,6.13^{\prime}\right): \quad f_{t}=0.08 \cdot f_{c}{ }^{\prime}=598 \mathrm{psi} \\
& \left(6.14,6.14^{\prime}\right): \quad f_{s p}{ }^{\prime}=7.4 \cdot\left(f_{c}{ }^{\prime}\right)^{1 / 2}[\text { in psi }]=640 \mathrm{psi}
\end{aligned}
$$

results in a factor greater than 2.4 between the level of an assumed tolerable interfacial strength $\left(f_{t}\right.$ or $\left.f_{s p}\right)$ and the actual measured stress, $\sigma_{t, F T / C C}($ Eq. $(8.1))$. Therefore, under similar conditions and durations of exposure, no appreciable development of strain is expected to form at the FT/CC, CFRP-concrete interface.

\subsection{Conclusions}

A total of 35 (i.e. 20 actual and 15 companion) beams were fractured after being subjected to partial immersion (see Fig. 8.4) in a calcium chloride (abbreviated throughout as: CC) solution and undergoing 50, 100, 150, 200, and 300, alternate cycles of freezing and thawing. Each cycle (see Fig. 8.1) consisted of 2 hours of ramping from room temperature at $20 \mathrm{C}$ to freezing at $-20 \mathrm{C}, 2$ hours of constant freezing at $-20 \mathrm{C}$, and then finally, ramping from a frozen state back to room temperature (i.e. thaw). This durability scheme was chosen to simulate the harsh wintry effects experienced in many 
parts of the world when deicing salts are dispersed on highways and especially bridges to safeguard these surfaces from becoming frozen.

The organization of this chapter adhered to the following four categories of presentation (identical to Ch. 7):

$>$ Preliminaries

SCCB Fracture

$>$ Patterns of Fracture

Quantitatively

Qualitatively

\section{Pre-Fracture Changes}

Visual Changes

Weight Changes

$\underline{\text { Strain Changes }}$

Qualitatively, pictures of both pre- and post- fracture beams were studied whereby the drawing of some generalized conclusions was possible. Considering first the latter category, failure patterns advanced, from start to finish, in the following evolutionary sequence (numbers represent the age of cycling): (50): shallow-cohesive, (100): shallowmixed dominantly cohesive, (150): wide central adhesive bandwidth with outlying deepcohesive edges, (200): narrowed central adhesive bandwidth with outlying deep-cohesive edges, and (300): deep-cohesive with narrowed central adhesive bandwidth. This progressive record of failure reveals that the detrimental effects of the $\mathrm{CC} /$ freeze-thaw conditioning scheme initiated some time around cycle-150. It is here, at the outlying edges, that the interfacial concrete began to decompose first, after which it gradually started advancing inward from either end (see Fig. 8.7). 
Regarding, now, the former point of pre-fracture characterizations, the pictures of Fig. 8.8 revealed a sharp contrast between the appearance of the companion specimen and the actual FT conditioned beams. With age, the clearly defined concrete ledge and raised-step regions were found to become indistinct. Moreover, the hydrated cement paste cover had worn to such an extent that the underlying coarse aggregates became exposed and vulnerable to further spalling. These degenerated surfaces were also marked by an ashen-gray color, as opposed to the prominently whitish tone of the companion sample. Another effect realized as a consequence of $\mathrm{CC} /$ freeze-thaw aging that obviously stemmed from within the deteriorating concrete substrate, was the distinctly receding application-line of the primer (see, e.g., Fig. 8.8(a) in contrast to parts (b) - (d)). A final observation made from Fig. 8.8, parts (c) and (d), was that the interface was perceived to separate cohesively (i.e. within the concrete) even before the initiation of SCCB fracturetesting - this strictly conforms to the qualitative, post-fracture findings delineated in the previous paragraph.

Weights and strains were two of the other pre-fracture parameters considered. Microstrains were recorded at the top of the CFRP strip and the adjacent ledge of concrete (see Figs. 3.21(d) and 6.8(b)) enabling the determination of any resulting differential movements that might have occurred between the two (see Fig. 8.11). The average, absolute, differential movement that materialized between the CFRP and concrete for the $\mathrm{CC} /$ freeze-thaw specimens was $25 \mu \varepsilon$, with absolute minimum and maximum differences of $8 \mu \varepsilon$ and $49 \mu \varepsilon$, respectively. The resulting maximum interfacial tensile stress was: 


$$
\text { (8.1): } \quad \sigma_{\mathrm{t}, \mathrm{FT} / \mathrm{CC}}=242 \mathrm{psi}
$$

which is smaller than both the tensile strengths $f_{t}$ and $f_{s p}$ ' of Eqs. $(6.13) /\left(6.13^{\prime}\right)$ and $(6.14) /(6.14 ')$ —repeated below for convenience— by a factor in excess of 2.4.

$$
\begin{aligned}
& \left(6.13,6.13^{\prime}\right): \quad f_{t}=0.08 \cdot f_{c^{\prime}}{ }^{\prime}=598 \mathrm{psi} \\
& \left(6.14,6.14^{\prime}\right): \quad f_{s p}{ }^{\prime}=7.4 \cdot\left(f_{c^{\prime}}\right)^{1 / 2}[\text { in psi }]=640 \mathrm{psi}
\end{aligned}
$$

Thus, under similar conditions and durations of exposure, no appreciable development of strain is expected to develop at the CC/freeze-thaw CFRP-concrete interface.

Percent average weight changes occurring between the $\mathrm{CC} /$ freeze-thaw plain, companion, and actual beams were displayed in Figure 8.9. The maximum percentage increase achieved, while slight, was nevertheless approximately 5 to (more than) 6 times as much as the respective SS and SH values. Two things were attributed for such an increase in weight: 1) a considerable amount of salt crystallization and deposition occurring within the specimens' open pores, and 2) corrosion of the internal steel reinforcement (not of the pitting type). Further substantiation of the latter point arises from the fact that the beams were partially submerged, and as such, the vital oxygencomponent was present to initiate corrosion.

From Fig. 8.9 it was also noted that the actual specimens' weight gains exceeded that of their plain counterparts. This was attributed to the actual beams' higher potential for moisture uptake within the saturant-and-CFRP layer. The rate of weight gain achieved by the actual beams was also unchanging for 150 cycles, after which, this trend essentially stabilized. This conforms to both the qualitative post- and pre- fracture observations asserting cycle-150 as a pivotal point of demarcation between specimen 
-soundness and -degeneration. It was speculated that in the first-half of aging (i.e. until cycle-150), all of the weight gains were due to moisture uptake and salt-crystallization within the exposed concrete regions and the saturant-and-CFRP layer. For the second half, however, when the first of these effects was believed to have become equilibrated, the loss in concrete mass due to spalling and the initiating ferric oxide (responsible for causing reinforcement corrosion) coupled with a continuance of salt-crystallization, was seemingly found to be tantamount. The same thing was noted for the plain specimens, except that the latter processes corresponding to weight-gains were more substantial than the spalling concrete, thus explaining the moderately increasing slope at cycle-300.

The most significant information, of course, was gained through SCCB fracture testing, the results of which are presented below, compactly, in tabular (Table 8.8) and graphical (Figure 8.12) form. (Note that the brittleness indices obtained from these tests have also been provided in the table, giving quantitative evidence of characteristically stable crack-growth behavior throughout.) Inspecting the bar graphs of the figure (repeated from Fig. 8.6) reveals a declining trend of relative fracture toughness values as caused by the adverse $\mathrm{CC} /$ freeze-thaw regime. The average variation between head and set $G_{I c}$ percentage reductions for the $\mathrm{CC}$ exposure scheme was found to be $5 \%$, with maximum and minimum deviations of $13 \%$ and $1 \%$ for the $200^{\text {th }}$ cycle and $100^{\text {th }} / 150^{\text {th }}$ cycle arrestment-based cases (see Fig. 8.12(b), below), respectively. After the complete duration of aging at 300 cycles, head and set initiation- and arrestment- based reductions in fracture toughness values for the CC regime revealed results of $60 \%$ and $62 \%$ (averaged), respectively. This, once again, shows that the CFRP-concrete interface 
reliability may be characterized as being less than two-thirds as effectual as it would have otherwise been were it not subjected to such attack.

(a) Initiation-Bas ed Freeze-Thaw Calcium Chloride Results

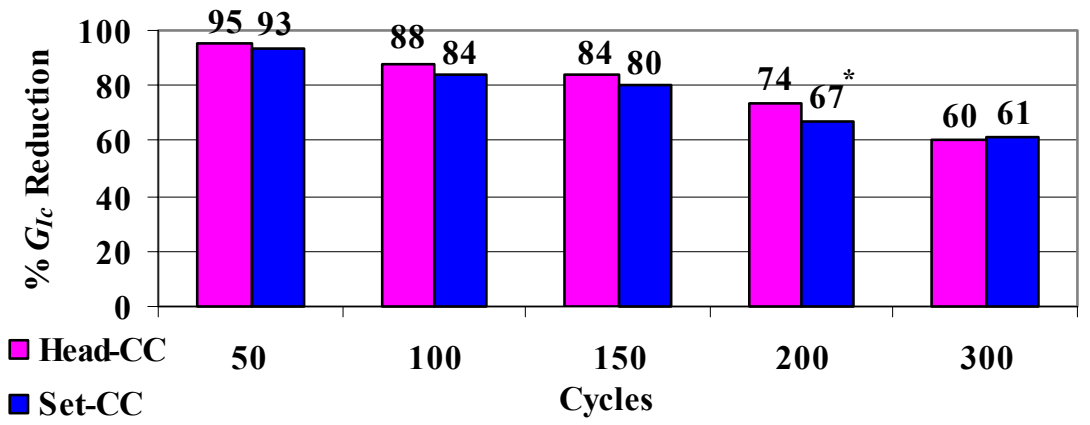

(b) Arres tment-Bas ed Freeze-Thaw Calcium Chloride Results

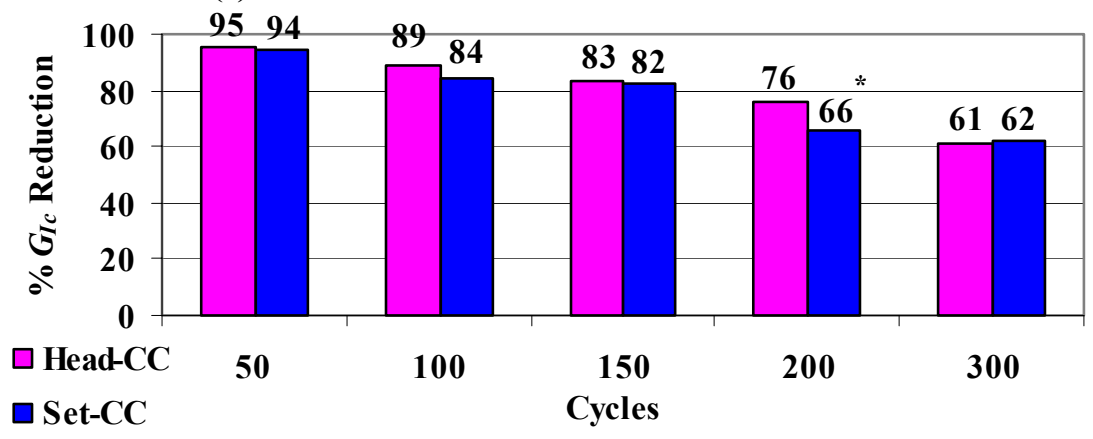

*Data file missing for Set-4B11 values

Figure 8.12 Conclusive percentage reductions in (a) $G_{I c}^{i}$ and (b) $G_{I c}^{a}$ resulting from 50,100, 150, 200, and 300 freeze-thaw cycles of calcium chloride (CC) exposure (repeated from Fig. 8.6)

Table 8.8 Averaged conclusive freeze-thaw fracture results (unit: $\mathrm{J} / \mathrm{m}^{2}$ ) (see also Tables 8.6 and 8.7)

\begin{tabular}{|c|c|c|c|c|c|}
\hline & \multicolumn{5}{|c|}{$\mathbf{C C}$} \\
\hline & 50 cycles & 100 cycles & 150 cycles & 200 cycles & 300 cycles \\
\hline$(1) G_{I c}^{i} / \mathrm{FTC}^{*}$ & $568 / 604$ & $542 / 632$ & $604 / 738$ & $550 / 776$ & $491 / 811$ \\
\hline (2) $G_{I c}^{a} /$ FTC $^{*}$ & $554 / 586$ & $522 / 605$ & $592 / 718$ & $544 / 764$ & $477 / 772$ \\
\hline$\%(1),(2)$ & $94 \%, 94 \%$ & $86 \%, 86 \%$ & $82 \%, 82 \%$ & $71 \%, 71 \%$ & $60 \%, 62 \%$ \\
\hline$I$ [no unit] & 0.02 & 0.04 & 0.02 & 0.01 & 0.03 \\
\hline
\end{tabular}

"FTC $=$ ave. freeze-thaw companion results 


\section{CHAPTER 9}

\section{CONCLUSIONS}

\subsection{Introduction...of the End}

The waves of future civil infrastructure applications are furiously surging toward the integration of FRP materials to (externally) shore the coasts of tomorrow's construction industry. In terms of bridges alone, about one-half of the approximately 600,000 U.S. overpasses in use, need to either be replaced or rehabilitated (Saadatmanesh and Ehsani, 1990). The absolutely central role played by the interface bond (no pun intended) in educing widespread acceptance of this technology should, by now, be exceedingly clear (e.g. read the last paragraph of p. 2).

Perhaps for the first time, this crucial objective was met here-through this research undertaking - by the implementing of the novel SCCB test specimen; secondary characterization methods were also used for corroborative purposes. CFRP-concrete beams were tested under dry (Ch. 6) and carefully planned environmental conditions (Chapters 7 and 8) in order to gauge relative effects on interface bond performance. Substantial degeneration of the aged-interfaces was discovered in all cases, i.e. WD/SS, $\mathrm{WD} / \mathrm{SH}$, and FT/CC, when compared with the companion beams of the same age. 
Organization of the End

This final chapter is basically divided into two portions: (1) Sections $9.2-9.5$, which consist of brief summarizations of the most pertinent discussions, relations, and results stemming from this work, and (2) Section 9.6, offering apropos recommendations. Section titles are as follows:

9.2 Summary of SCCB Relevancy and Appraisal

$>$ 9.3 Summary of Foremost Relevant Concepts and Relations

$>$ 9.4 Summary of Secondary Interface Characterizations

9.5 Summary of Primary Interface Characterizations

$>$ 9.6 Future Recommendations

\subsection{Summary of SCCB Relevancy and Appraisal of its Viability}

\section{Path of Relevancy}

(1) Oligatory requirement of an experimental Fracture Mechanics approach for interface bond characterization (read first paragraph on p. 3)

(2) Inexistence of such a device (until now, of course) to measure $G_{I_{c}}$ values for FRPconcrete interfaces (read Section 1.2)

(3) DCB, CDCB, or some other established variant thereof, being unviable as options due to the arm-breakoff (see Fig. 3.1 and the surrounding discussions) phenomenon

(4) Conception and birth of the novel SCCB approach - an outgrowth of the CDCB specimen (read Subsection of Ch.3 titled "Requisite of...", p. 128)

(5) SCCB testing enables:

(a) sensitivity for dry and aged interface characterization, unlike other large- or small- scaled tests previously used (consider, as an example, the case studies presented in Sec. 2.6)

(b) acquisition of fracture toughness data - a material constant (see Fig. 3.9 and the surrounding discussions)

(6) SCCB testing is an indispensable tool, rendering both quantitative and qualitative measures, the latter having immediate ramifications to the mainstreaming of FRPtechnology into civil infrastructure applications (Sec. 1.2) 
Appraisal of SCCB Viability

Unlike the well-established CDCB specimen, the novel single contouredcantilever beam was, from its very inception, shrouded in some confusion with respect to its viability. Particularly, it was speculated that the absence of symmetry accompanying this new specimen would impair its ability to behave purely under an opening, or cleavage, mode of loading due to a sizable degree of shearing stresses incurred at the interface. While this concern is not completely unfounded, it was (indirectly) discovered through the extensive course of calibrations conducted (Ch. 4), that this effect is much smaller than what was previously thought, and as such, can be neglected-even the CDCB specimen in all its perfect symmetry, was found to contain small discrepancies that never impeded its viability as a Mode I fracture tool. The reason for this is due, not so much to the size of the extra-Mode discrepancy itself-found to be between $1 \%-2 \%$ for the CDCB specimen and, likewise, $10 \%-11 \%$ for the SCCB - but to its relation with the type of material that is being tested. In other words, the determination of testappropriateness is largely a material-specific issue.

This is why, for example, that under the present setup - the design of which, can be improved (see Sec. 9.6) - the SCCB test would not be recommended for characterizing metallic materials. By way of contrast, however, the so-called CFRPconcrete interface "material" actually consists of three disparate constituents-the carbon fibers, the epoxy-based resins (saturant + primer), and of course the concrete. The latter material, itself, is single-handedly notorious for being the source of gross discrepancies. 
Consider the variance in concrete- $G_{I c}$ values, as reported by the following four researchers...

\section{Reported concrete- $G_{I c}$ values:}

(1) Moavenzadeh and Kuguel (1969): $G_{I c}=9.58 \mathrm{~J} / \mathrm{m}^{2}=0.0547 \mathrm{lb} / \mathrm{in}$

(2) Kaplan (1961): Reported values that ranged from: $G_{I c}=10.16 \mathrm{~J} / \mathrm{m}^{2}=0.0580 \mathrm{lb} / \mathrm{in}$ $G_{I c}=33.8 \mathrm{~J} / \mathrm{m}^{2}=0.1930 \mathrm{lb} / \mathrm{in}$

(3) J.H. Brown (1972): approximated from his reported $K_{I c}$ value assuming $\mathrm{E}_{\mathrm{c}}=24,800$ MPa and $v_{\mathrm{c}}=0.17$, and plane strain conditions (see Eq. (3.14)): $G_{I c}=8 \mathrm{~J} / \mathrm{m}^{2}=0.046 \mathrm{lb} / \mathrm{in}$

(4) Jenq and Shah (1985): Reported values that ranged from:

$G_{I c}=30.3 \mathrm{~J} / \mathrm{m}^{2}=0.1730 \mathrm{lb} / \mathrm{in}-G_{I c}=66.72 \mathrm{~J} / \mathrm{m}^{2}=0.3810 \mathrm{lb} / \mathrm{in}$

By (crudely) taking the mean of these values- $G_{I c}=26 \mathrm{~J} / \mathrm{m}^{2}=0.15 \mathrm{lb} / \mathrm{in}-$ for comparative purposes, absolute-discrepancies resulting from these experimental works are found to range between $15 \%-152 \%$ ! Of course, part of this wide-variation in error is due to the fact that different mixes of concrete were used; moreover, different experimental techniques were employed-thus, reemphasizing the importance for instituting a widely accepted experimental Fracture Mechanics methodology.

Apart from the grossly complex nature of the interface "material" that undoubtedly could account for most, if not all, of the trifling $10 \%-11 \%$ error itself, the discrepancies introduced as a consequence of the amount of skill required in manufacturing the SCCB specimen, must also be considered. A list of encountered human errors follows, as taken directly from the author's own experiences... 


\section{List of possible human errors incurred during SCCB manufacture:}

Insufficient surface preparation of the concrete bonding surface

$>$ Improper preparation of the primer or saturant

$>$ Insufficient impregnation (or wet-out) of the fibers

$>$ Misalignment of the contour during bonding (also of the specimen in the test fixture during loading)

A deficient quantity of saturant used in adhering the contour to the substrate resulting in air voids (gaps)

Excess saturant seeping over onto the concrete ledge and raised-step portions during bonding of the fiber-strip to the concrete beam, resulting in the altering of the true interface properties of the specimen

Excess saturant seeping over onto the concrete ledge and raised-step portions during the mating of the contour with the CFRP-bonded concrete beam, resulting in the altering of the true interface properties of the specimen

Why Mode I?: The Mode I $G_{c}$ value is of special importance in that it represents a practical lower limit of fracture toughness (see Figure 3.9 and the surrounding discussions), i.e. engineers are assured of conservatism in design. Its relevance is further illustrated by the conceptualized drawings of Figure 9.1 that show delaminations as starting (Fig. 9.1(a)) and progressing (Fig. 9.1(b)), in large part, due to an "opening" (not "sliding") behavior. Consider also the following three assertions...

\section{Mode I assertions}

Mode I loading is encountered in the overwhelming majority of actual engineering situations involving cracked components. Consequently, considerable attention has been given to both analytical and experimental methods designed to quantify Mode I stress-crack length relationships. Mode II is found less frequently and is of little engineering importance. ... Mode III may be regarded as a pure shear problem such as that involving a notched round bar in torsion. (Hertzberg, pp. $262 \& 263,1976)$ 
In isotropic materials, brittle fracture usually occurs in Mode I. Consequently,...Mode I [loading is used] in establishing fracture criteria for sudden fracture of flawed members when the materials in these members are loaded in the brittle state. Although fractures induced by sliding (Mode II) and tearing (Mode III) do occur, their frequency is much less than the opening mode [of] fracture (Mode I). ...we do note that improvement of fracture resistance for Mode I usually results in improved resistance to mixed mode crack extension. (Boresi, et al., pp. $610 \& 611,1993$ )

Since most materials are far less resistant to crack extension under the action of a normal load than under a shear load [or under a tearing load, for that matter,] Mode I fracturing has been of most interest in fracture mechanics. (Ripling, et al., p. 108, 1971)

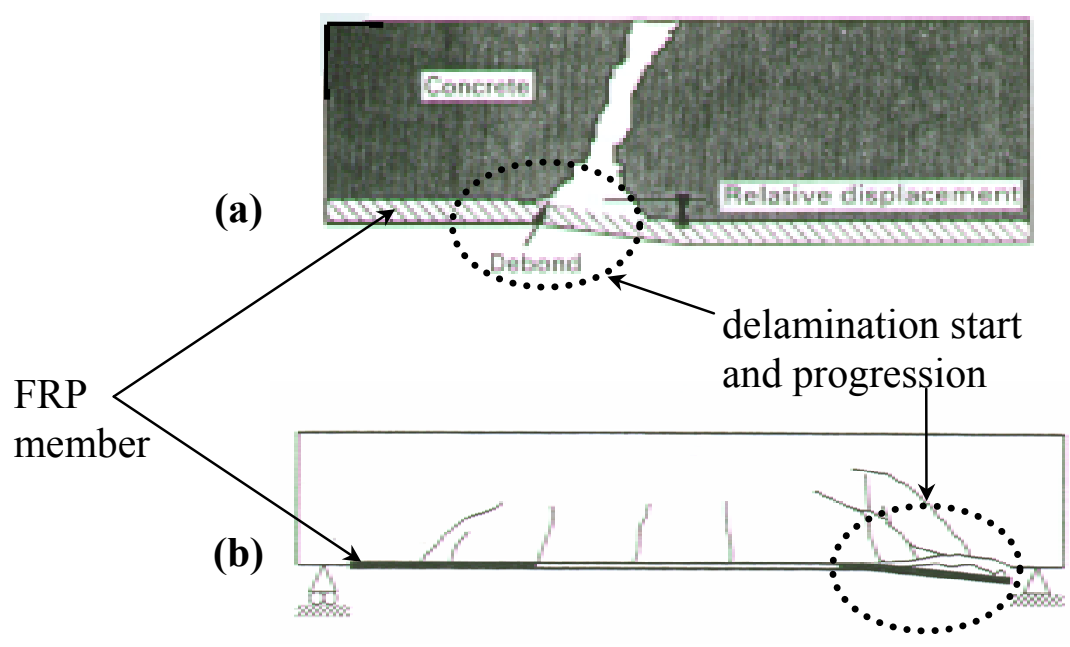

Figure 9.1 Conceptualized FRP-strengthened beam-structures showing delamination - (a) start (Karbhari et al. (1997)), and

(b) progression (Green et al. (1998)) 


\subsection{Summary of Foremost Relevant Concepts and Relations}

\section{Relevant Concepts}

Pertaining to Durability: Durability is defined as the capacity of a structural system to maintain its initial performance-properties over time. In this particular context, the

"structural system" pertains to the interface-bond, consisting largely of the FRP-strip and the underlying concrete substrate to which it is adhered.

Sulfate attack mechanisms were outlined by describing the damage that results from one of the following three most common salt-sources: sodium, calcium, and magnesium (see Sec. 2.3). Reactions arising from these elements with specific cement products (i.e. $\mathrm{Ca}(\mathrm{OH})_{2}$, calcium aluminate hydrate $\left(3 \mathrm{CaO} \cdot \mathrm{Al}_{2} \mathrm{O}_{3} \cdot 12 \mathrm{H}_{2} \mathrm{O}\right)$, and calcium silicate hydrates (previously known as tobermorite gel; see also p. 34)), were shown to precipitate gypsum and ettringite - agents known to expand and thereby disrupt the soundness of the concrete-structure. In defense of these actions, it was recommended that the concrete should be densely and with as low a permeability as possible. This can be achieved in one of three ways: (1) specify a maximum water-cement ratio, (2) a minimum-strength, or (3) a minimum cement content.

Efflorescence results from a leaching of lime compounds that leads to the formation of salt deposits (see Figs. 7.11(c) and 7.12(b)). This action typically affects the edges and corners of the structure first, and then ultimately culminates in the cracking and spalling-off of the concrete.

Salt weathering may occur under a variety of conditions such as: sea water splash, airborne salt deposits arising from marine or desert environments, and from applications 
of deicing agents in cold climate regions commonly referred to as scaling. Essentially, as pure water evaporates from the salt solution, a crystallized salt deposit remains which, upon subsequent wetting, grows and exerts an expansive force on the hardened cement paste.

Alkalinity emanates from the porewater environment of ordinary portland cement concrete and is characterized by a high $\mathrm{pH}$ - in the range of $10-13.5$. In order for alkaline attack to initiate, the porewater solution must move from the pores of the concrete and diffuse through the polymer matrix.

Freeze-thaw effects occur when moisture in concrete freezes and consequently expands in volume (approximately by $9 \%$ ). This then leads to the development of osmotic and hydraulic pressures and ultimately culminates in cracking and rupture of both the hardened cement paste as well as the aggregate particles. A number of suggestions for producing concretes to better endure the harmful effects of freeze-thaw cycling include...

\section{Steps to produce more durable concretes undergoing freezing and thawing}

$>$ Air-entrainment of $4 \%-6 \%$ with stable bubbles having low spacing factors

$>$ Use of aggregates with low-absorption

$>$ Minimization of the amount of mixing-water

$>$ Application of a surface-sealant, once the concrete has properly cured and dried 
Pertaining to Griffith: Griffith (1893 - 1963; see portrait in Fig. 3.4) is credited as being the father of the subject known today as Fracture Mechanics. Two separate and fundamentally new ideas were contained in his work $(1921,1924)$. One was the idea of "flaws" or minute defects which act as stress raisers and explain the difference between the observed, macroscopic and relatively low strength of solid materials versus the high values as expected from the known strengths of chemical bonds. Griffith's second idea postulated that an energy balance, responsible for governing the laws of crack propagation, existed between the surface energy (i.e. surface tension) of the newly created fracture surface and the elastic energy stored in the stress field (i.e. the balance of surface and mechanical energies). Later, it was demonstrated that the Griffith energy balance must not only be between the strain energy stored in the specimen and the surface energy, but the work done in plastic deformation as well.

Pertaining to Material Constancy of Fracture Toughness: The fracture toughness (i.e. $K_{c}$ ) of a material depends on the volume of the material that can deform plastically before fracture. Since this volume depends on the thickness, it follows that $K_{c}$ varies with thickness as shown in Figure 9.2. When the sample is thin (for example, with thickness $t_{1}$ of Figure 9.2 corresponding to parts (a) and (b)), plane stress conditions (i.e. $\sigma_{z}=0$; see also the aspect-ratio of Eq. (3.42)) prevail and the material exhibits maximum toughness. Note that if the sample were made any thinner, the toughness of the material would gradually decrease because less material would be available to absorb the energy of plastic deformation, as depicted by the slanted portions (a.k.a. shear lips) of the fractured surfaces in Figure 9.2 (a) and (b). 


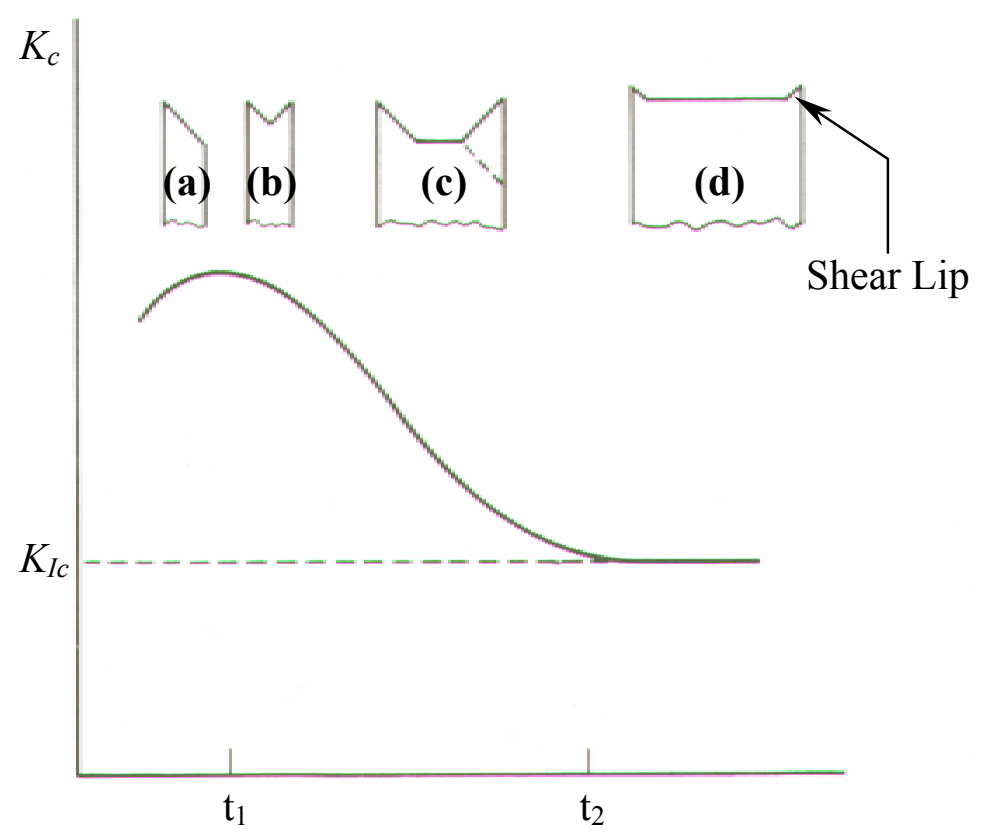

Figure 9.2 (repeated from Fig. 3.9) Fracture toughness $\left(K_{c}\right)$ as a function of specimen thickness (a) - (d) (Kanninen and Popelar, p. 179, 1985)

Alternately, when the thickness is increased ( $t_{2}$ corresponding to Figure $\left.9.2(d)\right)$ to bring about plastic constraint with plane strain conditions prevailing $\left(\sigma_{\mathrm{z}} \neq 0\right.$ and $\mathrm{r}_{\mathrm{y}}<<\mathrm{t}$; see also the aspect-ratio of Eq. (3.43)), the toughness drops to a level that may be onethird (or less) than that of the plane stress value (Hertzberg, p. 279, 1976). The approximate aspect-ratios for plane stress and plane strain conditions are as follows:

$$
\begin{array}{ll}
\frac{\mathrm{r}_{\mathrm{y}}}{\mathrm{t}} \geq 1 \quad \text { (in plane stress) } \\
\frac{\mathrm{r}_{\mathrm{y}}}{\mathrm{t}}<\frac{1}{10} \quad \text { (in plane strain) }
\end{array}
$$

For $\frac{r_{y}}{t} \in\left[\frac{1}{10}, 1\right)$, a mixed-mode of plane stress/strain is otherwise exhibited. 
One very important aspect of this lower level of toughness (i.e. the plane strain fracture toughness, $K_{I c}$ ) is that it does not decrease further with increasing thickness, thereby making it a conservative lower-limit and an especially useful parameter with regard to engineering applications. It is for this reason that $K_{I c}$ (or its energy counterpart, $G_{I c}($ Section 3.3)) is thought of as a material constant - a central issue to this dissertation. Pertaining to Cracking Behavior: All forms of cracking were divided into two broad classes: those of stable and unstable cracks. Generally, all structural adhesive systems display a form of elastic behavior prior to crack-initiation. In other words, the crack remains stationary with no measurable inelastic flow until a critical value, $G_{I c}$, is reached. The differences that exist between adhesive systems are made evident once this critical value is exceeded. Cracks proceeding with a nearly constant $G_{I c}$ value (i.e. stable crack growth) represent materials that are said to be strain rate insensitive (see Figs. 3.14(a) and 3.15, parts (b) and (d)). In contrast, strain rate sensitive materials display marked differences in the values of $G_{I c}$ that are responsible for initiating the stationary crack and those that sustain propagation (see Figs. 3.14(b) and 3.15(c)). Once the crack begins to move, the amount of crack extension force required to sustain crack-propagation decreases, thus accounting for this so-called "saw-toothed" behavior (see Fig. 3.14(b). At initiation, the crack "pops" ahead, running at a velocity higher than that as dictated by the rate of the actuator. At the end of the "pop", the crack stops from moving and is only reinitiated when the machine displacement increases sufficiently for the instantaneous load-level $(P)$ to once again reach a critical initiation-value, $P_{c}^{i}$. 
Pertaining to the Contoured-Cantilever: The height of the cantilevered member is made to vary linearly along its longitudinal axis (see Fig. 9.3 below) to render a constant compliance gradient (i.e. $d C / d a=$ constant), and thereby preclude the arduous task of measuring the location of the crack length (i.e. a) during fracture-testing. In other words, by designing the cantilevered member with a specific contour-shape (the subject of Chapter 4), it is possible to reduce the number of independent variables in the governing critical strain energy equation (Eq. (3.73) - a.k.a. the Irwin-Kies relation) from 2 to 1 :

$$
G_{I c}=G_{I c}\left(P_{c}, a\right) \rightarrow G_{I c}=\left.G_{I c}\left(P_{c}\right)\right|_{d C / d a=\text { const. }}
$$

For the case of the optimum shape (see Fig. 9.3), the value of the compliance gradient is:

$$
d C / d a=1.78 \times 10^{-5} \mathrm{~N}^{-1}=7.92 \times 10^{-5} \mathrm{lb}^{-1}
$$

with a coefficient of determination of $r^{2}=0.9956$ (see discussion on p. 166).

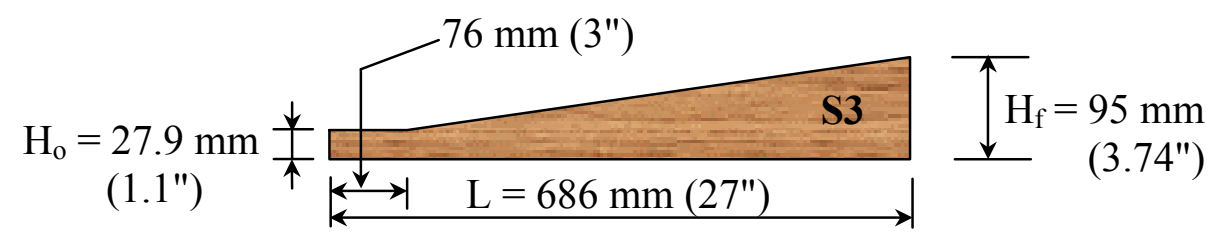

Figure 9.3 (repeated from Fig. 4.18) The optimum SCCB calibration specimen $\mathrm{S} 3$ - width (not shown), $\mathrm{b}=43.4 \mathrm{~mm}(1.71 ")$; contour angle $=6.3^{\circ}$

Pertaining to the Critical Load: A method was devised to answer the vitally important question, "Which load is critical?" in attempting to determine the CFRP-concrete value of fracture toughness (see Eq. (3.73)). The prescribed approach is statistically-based on a one- (may be higher, as deemed appropriate) standard deviation about the mean of the raw head loads (see Fig. 9.4 below); the raw-data is then refined in conformance to these admissible load-ranges (Eqs. (6.9), (6.10); or similarly, (6.11), (6.12)). Based on this 
refinement, two types of fracture energies are computed: one, is based on the refined mean head load, and the other, on the refined set load. These results are then averaged to determine a conclusive $G_{I C}$ value. A step-by-step example was provided in Section 6.2 (p. 236) to help elucidate this procedure.
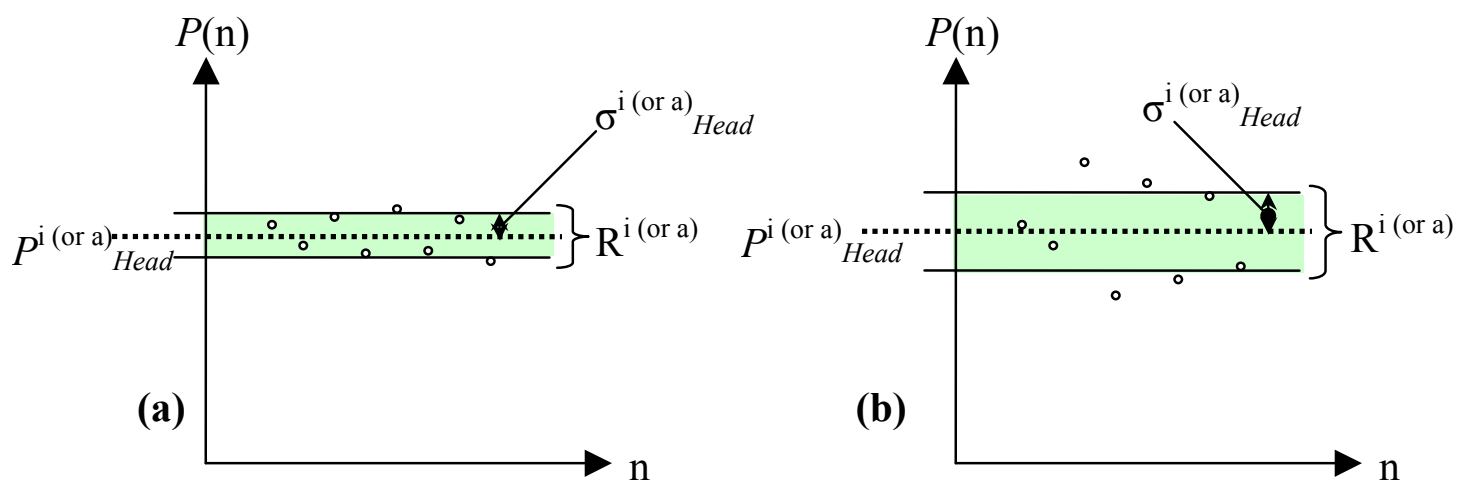

Figure 9.4 (repeated from Fig. 6.3) The statistical-based bandwidth for(a) compact, and (b) widespread data

\section{Relevant Relations}

$$
\begin{aligned}
& G_{I c}=G_{I c}\left(P_{c}, a\right) \rightarrow G_{I c}=\left.G_{I c}\left(P_{c}\right)\right|_{d C / d a=\text { const. }} \\
& d C / d a=1.78 \times 10^{-5} \mathrm{~N}^{-1}=7.92 \times 10^{-5} 1 \mathrm{~b}^{-1} \\
& G=\frac{K^{2}}{\mathrm{E}} \quad \text { (in plane stress) } \\
& G=\frac{K^{2}}{\mathrm{E}}\left(1-v^{2}\right) \quad \text { (in plane strain) }
\end{aligned}
$$


(3.44): $\quad \delta W_{e}=\delta U+\delta(\Delta K)-\delta Q+\delta F$

(3.45): $\quad G=\frac{\partial F}{\partial A}$

(3.73, 6.1): $\quad G_{I c}=\frac{P_{c}^{2}}{2 b} \frac{d C}{d a}$

(3.74): $\quad I=\frac{G_{I c}^{i}-G_{I c}^{a}}{G_{I c}^{i}}$

$P_{\text {Head }}^{\mathrm{i}}=\frac{\sum_{\mathrm{j}=1}^{\mathrm{n}} P_{\text {Head }}^{\mathrm{ij}}}{\mathrm{n}}$

(6.4):

$\sigma_{\text {Head }}^{\mathrm{i}}=\sqrt{\frac{\sum_{\mathrm{j}=1}^{\mathrm{n}}\left(P_{\text {Head }}^{\mathrm{ij}}-P_{\text {Head }}^{\mathrm{i}}\right)^{2}}{\mathrm{n}-1}}$

$P_{\text {Head }}^{\mathrm{a}}=\frac{\sum_{\mathrm{j}=1}^{\mathrm{n}} P_{\text {Head }}^{\mathrm{aj}}}{\mathrm{n}}$

(6.6):

$\sigma_{\text {Head }}^{\mathrm{a}}=\sqrt{\frac{\sum_{\mathrm{j}=1}^{\mathrm{n}}\left(P_{\text {Head }}^{\mathrm{aj}}-P_{\text {Head }}^{\mathrm{a}}\right)^{2}}{\mathrm{n}-1}}$

(6.7):

$$
\operatorname{COV}_{\text {Head }}^{\mathrm{i}}=\frac{\sigma_{\text {Head }}^{\mathrm{i}}}{P_{\text {Head }}^{\mathrm{i}}}
$$

(6.8):

$$
\mathrm{COV}_{\text {Head }}^{\mathrm{a}}=\frac{\sigma_{\text {Head }}^{\mathrm{a}}}{P_{\text {Head }}^{\mathrm{a}}}
$$

$$
\mathrm{R}^{\mathrm{i}}=\left[P_{\text {Head }}^{\mathrm{i}} \cdot\left(1-\operatorname{COV}_{\text {Head }}^{\mathrm{i}}\right), P_{\text {Head }}^{\mathrm{i}} \cdot\left(1+\operatorname{COV}_{\text {Head }}^{\mathrm{i}}\right)\right]
$$

$$
\mathrm{R}^{\mathrm{a}}=\left[P_{\text {Head }}^{\mathrm{a}} \cdot\left(1-\mathrm{COV}_{\text {Head }}^{\mathrm{a}}\right), P_{\text {Head }}^{\mathrm{a}} \cdot\left(1+\mathrm{COV}_{\text {Head }}^{\mathrm{a}}\right)\right]
$$

$$
\mathrm{R}^{\mathrm{i}}=\left[P_{\text {Head }}^{\mathrm{i}}-\sigma_{\text {Head }}^{\mathrm{i}}, P_{\text {Head }}^{\mathrm{i}}+\sigma_{\text {Head }}^{\mathrm{i}}\right]
$$




$\begin{array}{ll}\text { (6.12): } & \mathrm{R}^{\mathrm{a}}=\left[P_{\text {Head }}^{\mathrm{a}}-\sigma_{\text {Head }}^{\mathrm{a}}, P_{\text {Head }}^{\mathrm{a}}+\sigma_{\text {Head }}^{\mathrm{a}}\right] \\ (6.13): & \mathrm{f}_{\mathrm{t}}=0.08 \cdot \mathrm{f}_{\mathrm{c}}{ }^{\prime} ; \\ (6.14): & \mathrm{f}_{\mathrm{sp}}{ }^{\prime}=7.4 \cdot\left(\mathrm{f}_{\mathrm{c}}{ }^{\prime}\right)^{1 / 2} \quad[\text { in psi }] ; \quad\left(6.14^{\prime}\right): \mathrm{f}_{\mathrm{sp}}{ }^{\prime}=640 \mathrm{psi} \\ (6.15): & \sigma_{\mathrm{t}}=\mathrm{E}_{\mathrm{c}} \cdot \varepsilon \\ (6.16): & \mathrm{E}_{\mathrm{c}}=57,000 \cdot\left(\mathrm{f}_{\mathrm{c}}{ }^{\prime}\right)^{1 / 2} \quad\left[\mathrm{f}_{\mathrm{c}}{ }^{\prime} \text { in psi }\right] ; \quad(6.17): \mathrm{E}_{\mathrm{c}}=4.93 \times 10^{6} \mathrm{psi} \\ & \% G_{I c} \text { Reduction }=\frac{G_{\text {Ic, conditioned(\# } \text { cycles })}}{G_{\text {Ic, companion }(\# \text { cycles })}} \times 100 \%\end{array}$

\subsection{Summary of Secondary Interface Characterizations}

Visual-Based Pre-Fracture Inspections

Sodium Sulfate (SS)/PRE-VISUAL (30 Cycles):

Distinct efflorescence

$>$ Yellow-discoloration on the concrete ledge and raised-step regions

$>$ Gritty texture

$>$ Epoxy-based primer coat prone to flaying from within - a result of the ingression of the sulfate ions through improperly coated voids and exposed concrete regions

\section{Sodium Hydroxide (SH)/PRE-VISUAL (30 Cycles):}

$>$ Insipid outward appearance

$>$ Exposed concrete maintained original whitish tone

$>$ Concrete texture felt sound

$>$ On close inspection, the epoxy-based primer coat appeared emaciated - a result of the caustic action of the alkaline media

$>$ NOTE: The SH condition is an insidious type of attack as it does not offer any external forewarning of ensuing (glue-line) deterioration 


\section{Calcium Chloride (CC)/PRE-VISUAL (300 Cycles):}

Concrete ledge and raised-step regions became indistinct

$>$ Exposed concrete became ashen-gray in color

$>$ The hydrated cement paste had worn to the extent of exposing the aggregates

$>$ As with the SS case, the epoxy-based primer coat was flayed from within

\section{Visual-Based Post-Fracture Inspections}

\section{Dry Companion Beams (WD and FT)/POST-FRACTURE:}

In all of these companion beams, the post-fracture analysis revealed moderate- to highcohesive failures indicating a soundly intact adhesive-joint that is stronger than the underlying concrete

Sodium Sulfate (SS)/POST-FRACTURE (See Fig. 9.5 below):

10 Cycles: Deep-cohesive failure, indicative of a soundly intact adhesivejoint that is stronger than the underlying concrete

20 Cycles: Shallow-cohesive failure, indicating, in this case, some weakening (or softening) of the concrete

30 Cycles: Shallow-cohesive failure at outer edges/central portion exhibiting more of an adhesive failure, indicating sufficient weakening of the outer zones of concrete relative to the inner core concrete which is still intact

(a)

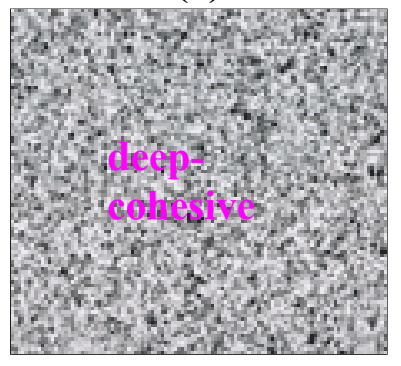

(b)

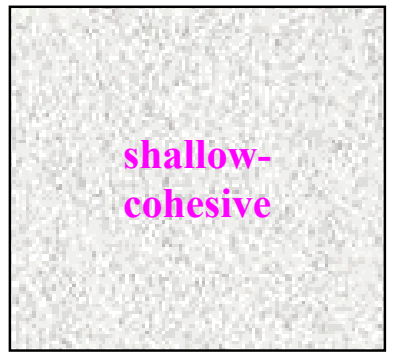

(c)

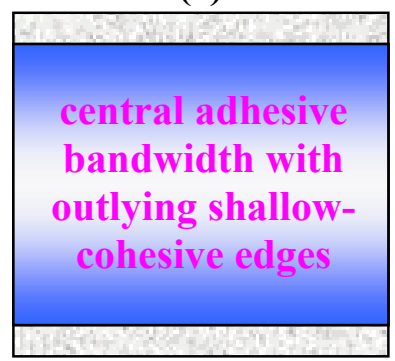

Figure 9.5 Conceptual fracture surfaces of beams undergoing:

(a) 10-, (b) 20-, and (c) 30- SS/wet-dry cycles 
Sodium Hydroxide (SH)/POST-VISUAL (See Fig. 9.6 below):

10 Cycles: Deep-cohesive failure, indicative of a soundly intact adhesivejoint that is stronger than the underlying concrete

20 Cycles: Centrally deep-cohesive with outer edges adhesive, indicating that the outer glue-joint has weakened

30 Cycles: Growth of outer adhesive edges inwardly with the central portion becoming a more shallow-cohesive failure, indicating a greater inward-penetration of the caustic SH solution into the glue-joint

(a)

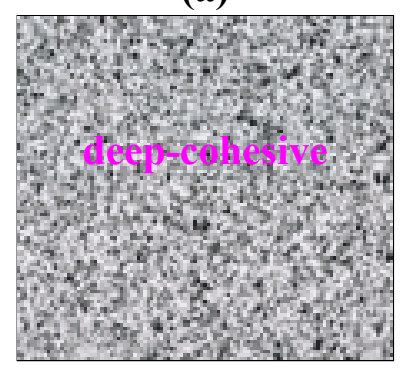

(b)

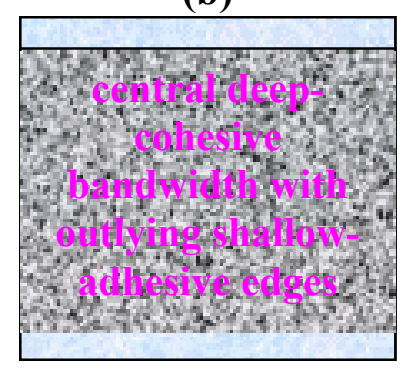

(c)

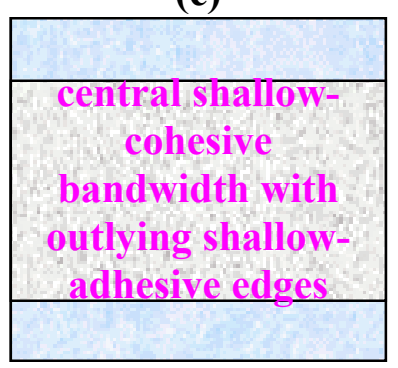

Figure 9.6 Conceptual fracture surfaces of beams undergoing:

(a) 10-, (b) 20-, and (c) 30- SH/wet-dry cycles 


\section{Calcium Chloride (CC)/POST-FRACTURE (See Fig. 9.7 below):}

50 Cycles: Shallow-cohesive, resembling in this case, little or no damage sustained by the concrete

100 Cycles: Shallow-mixed failure with more cohesive aspects, perhaps indicating some light or superficial weakening of the concrete

150 Cycles: Centrally adhesive with outer edges deep-cohesive, indicating the depth of concrete on the extreme zones becoming weakened

200 Cycles: Centrally adhesive, though now narrowed inwardly, with outer edges deep-cohesive, indicating a progressive action from 150 cycles

300 Cycles: Considerably narrowed central adhesive zone with outer edges deep-cohesive, indicating a progressive action from 200 cycles

(a)

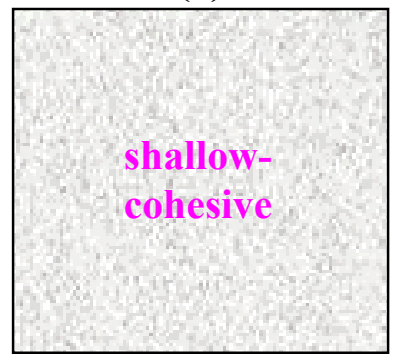

(d)

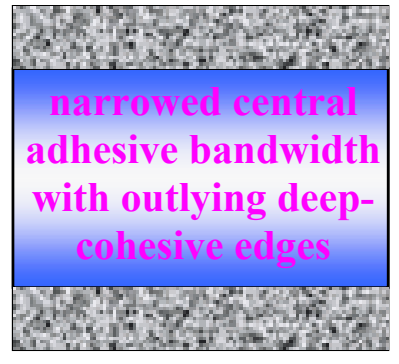

(b)

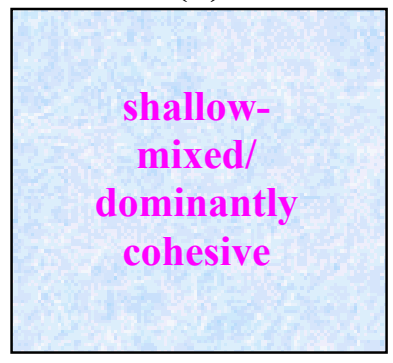

(e) (c)

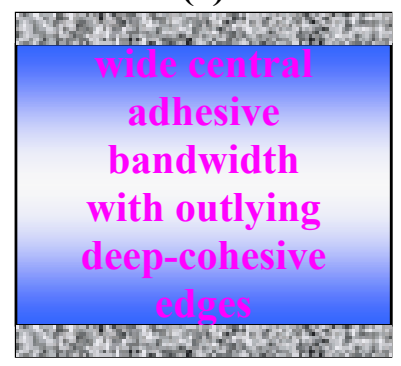

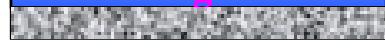

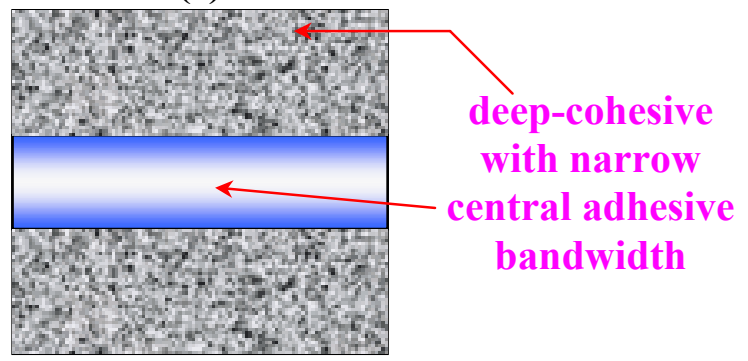

Figure 9.7 Conceptual fracture surfaces of beams undergoing: (a) 50-, (b) 100-, (c) 150-, (d) 200-, and (e) 300- CC/freeze-thaw cycles 


\section{Weight Changes}

Dry Weights(Figs. 6.6(WDC) and 6.7(FTC)): Negligibly small weight gains-the largest weight change occurred at the $300^{\text {th }}$ cycle of the freeze-thaw companion (FTC) specimen yielding only a $0.22 \%$ gain. The wet-dry companion beams showed net losses in weight - the largest occurring at week 20 with at only $-0.16 \%$. This may be attributed to changes of temperature, humidity, or both, temperature and humidity, that occurred in the curing-room at the different stages of specimen-storage. For instance, the wet-dry scheme was begun at the end of September, 2001, and so the environment of the curing-room was most probably different than at the time when the freeze-thaw regime began, approximately four months later, at the end of January, 2002. To reiterate, however, the magnitudes of these losses or gains are so slight that not much else may be commented here.

Wet-Dry Weights (Figs. 7.13(SS) and 7.21(SH)): Net weight gains were steadily realized until week 15 at which time it is believed that the destructive SS and SH effects were initiated in the concrete and adhesive, respectively. Again, overall weight changes were negligible: $0.63 \%$-SS and $0.50 \%$-SH. A couple additional observations include: (1) the SS cases had a relatively decreased incidence of concrete mass-loss as compared to their plain counterparts, most likely due to the reinforcing-action of the CFRP strip in the former case, and (2) the saturant-and-CFRP constituent is believed to be responsible for some of the moisture uptake, and this, perhaps in a latent manner. 
Freeze-Thaw Weights (Fig. 8.9(CC)): The weight changes were still slight here as well, though now, the maximum gain that occurred was between $5 \%-6 \%$ more than the gains of the wet-dry cases (for the actual beam, the maximum weight gain-occurring at cycle 200 - was found to be only $3.21 \%$ ). Two things are attributed for these increases in weight: (1) considerable salt crystallization and deposition in the specimens' open pores, and (2) corrosion of the internal steel reinforcement (not of the pitting type). To further elaborate on the latter point, recall that the beams were partially submerged (see Fig. 8.4) and so the necessary $\mathrm{O}_{2}$ (i.e. oxygen) component of rust $\left(\mathrm{FeO}_{2}\right)$ was present. As with the wet-dry beams, the saturant-and-CFRP constituent is here believed to also be responsible for some of the moisture uptake.

The rate of weight gains by the actual beams was also unchanging for 150 cycles, after which, this trend essentially stabilized. This especially conforms to the qualitative post-fracture observation, and may therefore be thought of as being a point of demarcation between specimen -soundness and -degeneration.

In this first half-period (i.e. until cycle-150), all of the weight gains are thought to have been due to moisture uptake and salt-crystallization within the exposed concrete and saturant-and-CFRP layer. For the second half, however, when moisture uptake is thought to have equilibrated, the loss in concrete mass due to spalling and the initiating corrosion, coupled with the continuance of salt-crystallization, appeared to be tantamount. The same thing was noted for the plain specimens, except that the processes of weight gain (i.e. salt-crystallization and corrosion) were more substantial than the spalling concrete, thus explaining the moderately increasing slope at cycle-300. 


\section{Strain Changes}

Dry Strains (Figs. 6.9(WDC) and 6.10(FTC)): The maximum differential microstrains of both the wet-dry and freeze-thaw companions were found to be $13 \mu \varepsilon$ and $38 \mu \varepsilon$, respectively—in terms of stresses, these numbers are: 64 psi and 187 psi. Comparison of these stresses with the more conservative of the two concrete tensile strengths (i.e. $\left(6.13^{\prime}\right): \mathrm{f}_{\mathrm{t}}=598 \mathrm{psi}$ ) reveals safety factors in excess of 9 and 3 , respectively. The other approach, as prescribed by the ACI, Eq. $(6.14): f_{\mathrm{sp}}{ }^{\prime}=7.4 \cdot\left(\mathrm{f}_{\mathrm{c}}{ }^{\prime}\right)^{1 / 2}[$ in psi $]=640$ psi, yields safety factors that are an order of magnitude larger, for the case of the WDC, and once again, in excess of 3 , for the FTC. Thus, it can safely be expected that, under dry conditions with temperatures near that of room and a variable relative humidity between approximately $50 \%-80 \%$, no appreciable development of strain will form at the CFRPconcrete interface.

Wet-Dry Strains (Figs. 7.14(SS) and 7.22(SH)): For the SS and SH cases, respectively, the largest differential movements were found to be $-53 \mu \varepsilon$ and $48 \mu \varepsilon$-yielding safety factors in excess of 2 and 2.5-after computing and comparing the stresses and strengths, as just described in the previous subhead. Note that for the SS case, the largest strain incurred is negative. Concern might be raised over any resulting interfacial compressive effects, here, however, engineering common sense should dictate that if the magnitude of the stress is less than half that of the concrete's tensile strength - a rather small number to begin with - the resultant stress itself must be too small to elicit any disrepair. Again, no appreciable development is therefore expected to form under this conditioning regime. 
Freeze-Thaw Strains (Figs. 8.11(CC)): For the CC case, the largest differential movement was found to be $49 \mu \varepsilon$-yielding a safety factor in excess of 2.4 -after computing and comparing the stress and strength. Once again, no appreciable development is therefore expected to form under this conditioning regime.

\subsection{Summary of Primary Interface Characterizations}

Fracture of Dry (Pristine and Representative) Specimens

The dry cracking behavior may be characterized as being stable due to the low brittleness indices: $I_{\text {pristine }}=0.06$ and $I_{\text {representative }}=0.04$ as such, the dry interface may thus be characterized as being strain rate insensitive. Averaged (conclusive) magnitudes of dry specimen, i.e. pristine and representative, critical strain energy release rates are shown in Figure 9.8. As expected, an upward trend exists between the pristine and representative specimen fracture values due to the strength-gains that are achieved by the concrete as the cement continues to hydrate. 
Table 9.1 (repeated from Table 6.11) Averaged conclusive dry fracture results

\begin{tabular}{c|c|c}
\cline { 2 - 3 } & Pristine & Representative \\
\hline $\boldsymbol{G}_{\boldsymbol{I C}}^{i}\left[\mathbf{J} / \mathbf{m}^{2}\right]$ & 618 & 720 \\
\hline $\boldsymbol{G}^{\boldsymbol{a}} \boldsymbol{I}\left[\mathbf{J} / \mathbf{m}^{2}\right]$ & 582 & 694 \\
\hline $\boldsymbol{I}$ & 0.06 & 0.04 \\
\hline
\end{tabular}

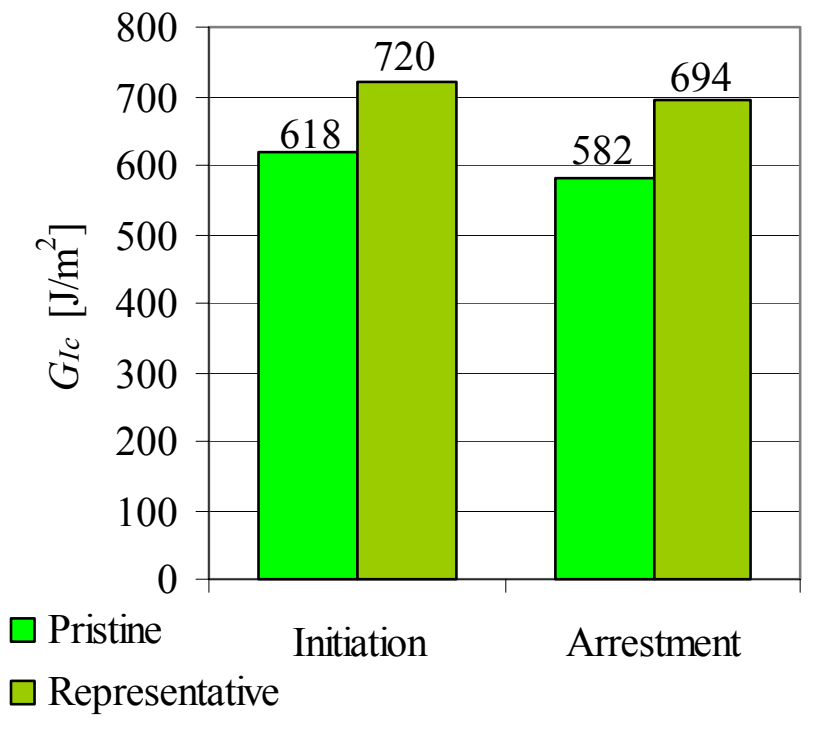

Figure 9.8 (repeated from Fig. 6.11) Averaged conclusive pristine- and representative- dry fracture results 


\section{Fracture of Wet-Dry (SS and SH) Specimens}

The wet-dry cracking behavior may be characterized as being stable due to the low brittleness indices (averaged, see Table 9.2) $-I_{S S}=0.03$ and $I_{S H}=0.04$. As such, the wet-dry interface may thus be characterized as being strain rate insensitive.

For the SS beams, the effects of head-degradations (See Fig. 7.23) in the first-half period of aging, i.e. between the $10^{\text {th }}$ and $20^{\text {th }}$ cycles, was not as pronounced as was the case in the latter-half of exposure-i.e. approximately $3 \%$ as opposed to $16 \%$. This large disparity may be indicative of the insufficient formation of gypsum or the underdevelopment of some of the other adverse products that are known to stem from exposure to sulfate attack, such as ettringite, at these comparatively early stages.

The performance of the SH beams, on the other hand, revealed first-half set-based degradation (See Fig. 7.23) effects that were considerably more pronounced than what was experienced in the latter-half of exposure-i.e. approximately $25 \%-26 \%$ as opposed to essentially $0 \%$. This indicates the swift nature by which the harmful alkaline condition succeeded in attacking the functionality of the resin. As a matter of fact, the degradation experienced by the SS specimens after 30 weeks of aging is basically equivalent to the harm sustained by those of the SH beams after only 20 weeks.

After the complete duration of aging at 30 cycles, head and set initiation- and arrestment- based reductions in fracture toughness values for the SS regime revealed identical results of $68 \%$ and $61 \%$, respectively; these values are $59 \%$ and $58 \%$ (averaged) for the SH beams. Averaging, in turn, each of these two cases (i.e. approximately, $64 \%$ 
and 58\%-see Figure 9.9), shows that the CFRP-concrete interface reliability may be characterized as being less than two-thirds as effectual as it would have otherwise been were it not subjected to such attack.

Table 9.2 (repeated from Table 7.11) Averaged conclusive wet-dry fracture results (unit: $\mathrm{J} / \mathrm{m}^{2}$ )

\begin{tabular}{|c|c|c|c|c|c|c|}
\hline & \multicolumn{3}{|c|}{ SS } & \multicolumn{3}{|c|}{ SH } \\
\hline & 10 weeks & 20 weeks & 30 weeks & 10 weeks & 20 weeks & 30 weeks \\
\hline (1) $G_{I c}^{i} / W D C^{*}$ & $511 / 618$ & $581 / 786$ & $514 / 796$ & $532 / 618$ & $502 / 786$ & $462 / 796$ \\
\hline (2) $G_{I c}^{a} / \mathrm{WDC}^{*}$ & $488 / 582$ & $566 / 754$ & $502 / 776$ & $500 / 582$ & $478 / 754$ & $454 / 776$ \\
\hline$\%(1),(2)$ & $83 \%, 84 \%$ & $74 \%, 75 \%$ & $64 \%, 65 \%$ & $86 \%, 86 \%$ & $64 \%, 63 \%$ & $58 \%, 58 \%$ \\
\hline$I$ [no unit] & 0.04 & 0.02 & 0.02 & 0.06 & 0.05 & 0.02 \\
\hline
\end{tabular}

"WDC = ave. wet-dry companion results (week-10 values furnished by pristine beams)

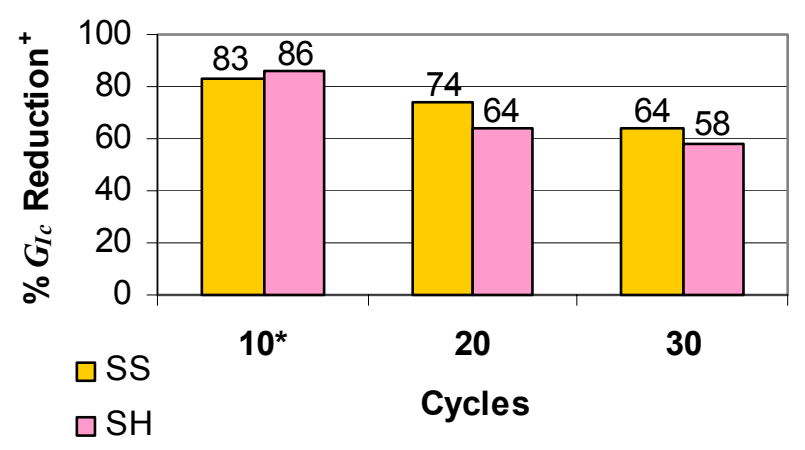

${ }^{+}$Reductions computed relative to companion values; "Pristine dry fracture results (see Table 6.3) substituted for $10^{\text {th }}$ cycle companion values

Figure 9.9 Averaged conclusive SS and SH wet-dry $\% G_{I c}$ reductions (based on Table 7.11) 
Fracture of Freeze-Thaw (CC) Specimens

The freeze-thaw cracking behavior may be characterized as being stable due to the low brittleness index (averaged, see Table 9.3) $-I_{C C}=0.02$. As such, the freeze-thaw interface may thus be characterized as being strain rate insensitive.

Inspection of the bar graphs (see Fig. 9.10) reveals a declining trend of relative fracture toughness values as caused by the adverse $\mathrm{CC}$ /freeze-thaw regime. The average variation between head and set $G_{I c}$ percentage reductions for the CC exposure scheme (see Fig. 8.12) was found to be 5\%, with maximum and minimum deviations of $13 \%$ and $1 \%$ for the $200^{\text {th }}$ cycle and $100^{\text {th }} / 150^{\text {th }}$ cycle arrestment-based cases (see Fig. 8.12(b)).

After the complete duration of aging at 300 cycles, head and set initiation- and arrestment- based reductions in fracture toughness values for the $\mathrm{CC}$ regime revealed results of $60 \%$ and $62 \%$ (averaged), respectively. This, once again, shows that the CFRPconcrete interface reliability may be characterized as being less than two-thirds as effectual as it would have otherwise been were it not subjected to such attack.

\section{Conclusive Magnitudes of Fracture: Dry - Weathered}

Figure 9.11 shows, conclusively, the relative toughness values of each of the cases studied. As expected, the beams with the greatest magnitudes of $G_{I c}$ were from the dry specimens. Of the aged instances, the SH condition was discovered to render the smallest critical strain energy release rate. In increasing sequence, the list of conclusive fracture energies is as follows (unit: $\mathrm{J} / \mathrm{m}^{2}$ ): $462^{\mathrm{SH} 30}, 491^{\mathrm{CC} 300}, 514^{\mathrm{SS} 30}, 618^{\text {Pris. }}, 720^{\text {Rep. }}$. 
Table 9.3 (repeated from Table 8.8) Averaged conclusive freeze-thaw fracture results (unit: $\mathrm{J} / \mathrm{m}^{2}$ )

\begin{tabular}{|c|c|c|c|c|c|}
\hline & \multicolumn{5}{|c|}{$\mathrm{CC}$} \\
\hline & 50 cycles & 100 cycles & 150 cycles & 200 cycles & 300 cycles \\
\hline$(1) G_{I C}^{i} / \mathbf{F T C}^{*}$ & $568 / 604$ & $542 / 632$ & $604 / 738$ & $550 / 776$ & 491/811 \\
\hline (2) $G_{I I}^{a}{ }_{I}$ FTC $^{*}$ & $554 / 586$ & $522 / 605$ & $592 / 718$ & $544 / 764$ & $477 / 772$ \\
\hline$\%(1),(2)$ & $94 \%, 94 \%$ & $86 \%, 86 \%$ & $82 \%, 82 \%$ & $71 \%, 71 \%$ & $60 \%, 62 \%$ \\
\hline$I$ [no unit] & 0.02 & 0.04 & 0.02 & 0.01 & 0.03 \\
\hline
\end{tabular}

"FTC $=$ ave. freeze-thaw companion results

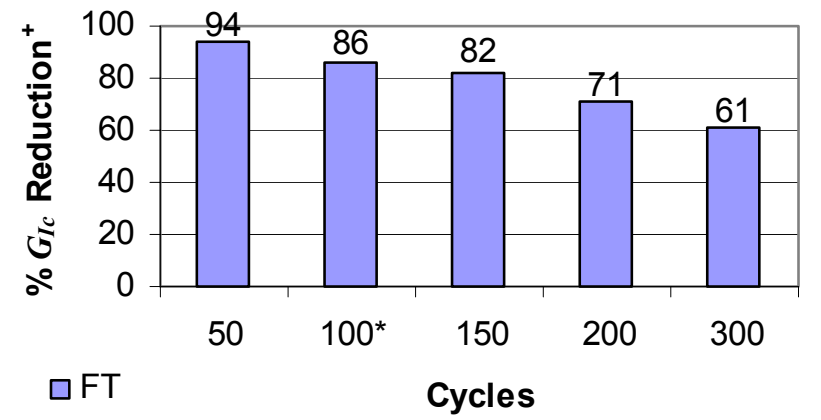

${ }^{+}$Reductions computed relative to companion values;

"Interpolated companion value for Cycle-100

Figure 9.10 Averaged conclusive $\mathrm{CC}$ freeze-thaw

$\% G_{I c}$ reductions (based on Table 8.8)

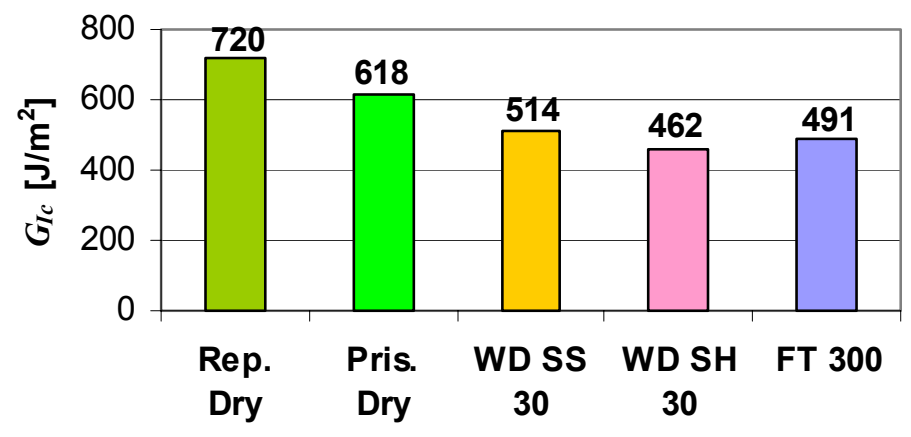

Figure 9.11 Conclusive magnitudes of fracture toughness:

Dry - Weathered (based on initiation energies) 


\subsection{Future Recommendations}

For the Enhancement of CFRP-strengthened Concrete Structures

Subject to Sulfate Attack: Source of attack: exposure with ground water and wet soils from which expansive-products, such as gypsum or ettringite, form, and thus cause the softening of the concrete surface and, ultimately, the loss of mass. The SS/wet-dry protocol of this research subjected 12-fracture and 3-plain specimens to 10, 20, and 30 cycles of attack in 10\% $\mathrm{Na}_{2} \mathrm{SO}_{4}$ solution; 9 beams were used as controls; 1 Cycle = 3 days $W$ and 4 days of $D$ at $40 C$ and $50 \%$ relative humidity.

Clearly, the chief source of deterioration in these situations is the attack of the concrete member. As such, ACI 201.2R-92 (1997) makes the following recommendations:

$>$ Use dense concretes with low water-cement ratios

$>$ Air-entrainment is of benefit insofar as it reduces the water-cement ratio and, hence, the permeability (Verbeck, 1968)

$>$ Use cements with limited $\mathrm{C}_{3} \mathrm{~A}$ content (i.e. Type V: $\leq 5 \%$; Type II: $\leq 8 \%$ ) —also, if

Type V cement is used, ASTM 150-02a (1994) specifies the following limit: $\mathrm{C}_{4} \mathrm{AF}+2 \mathrm{C}_{3} \mathrm{~A} \leq 25 \%$

$>$ Use some pozzolans and ground granulated iron blast-furnace slags - these increase the life expectancy of the concrete by reducing the potential for gypsum formation. (NOTE: some pozzolans and slags may improve the sulfate resistance of concretes made with Type I and II cements. On the other hand, others, especially Class $\mathrm{C}$ fly ashes, have been shown to counter this effect (ASTM C618, C989)

Subject to Alkaline Attack: Source of attack: naturally arises from the hydrated cement products themselves; has been found to cause etching, spalling and cracking of the resin. The SH/wet-dry protocol of this research subjected 12-fracture and 3-plain specimens to 10, 20, and 30 cycles of attack in a $p H=12 \mathrm{NaOH}$ solution; 9 beams were used as controls; 1 Cycle $=3$ days $\mathrm{W}$ and 4 days $D$ at $40 \mathrm{C}$ and $50 \%$ relative humidity.

Obviously, the most important measure to take in preventing this type of attack, is to develop and implement alkaline-resistant resins - the SCCB test method may play a central role to this end (read pp. 8 and 9). 
Subject to Freezing and Thawing in the presence of Deicing Chemicals: Source of attack: dispersal of deicing agents in many cold regions of the world to safeguard highways and bridges from becoming frozen. The CC/freeze-thaw protocol of this research subjected 20-fracture and 3-plain specimens to 50,100,150,200, and 300 cycles of attack in a CaCl2 solution; 20 beams were used as controls; cycling pattern shown in Figure 9.12.

It is critical to safeguard the concrete in this case as evident from the pictures in Figure 8.8, parts (c) and (d), and Figure 8.10. Use of a surface-sealant, if deemed appropriate by the circumstance, would be highly recommended.

Air-entrainment of $4 \%-6 \%$ with stable bubbles having low spacing factors

$>$ Use of aggregates with low-absorption

$>$ Minimization of the amount of mixing-water (i.e. low water-cement ratio- the less, the better)

Application of a surface-sealant, once the concrete has properly cured and dried

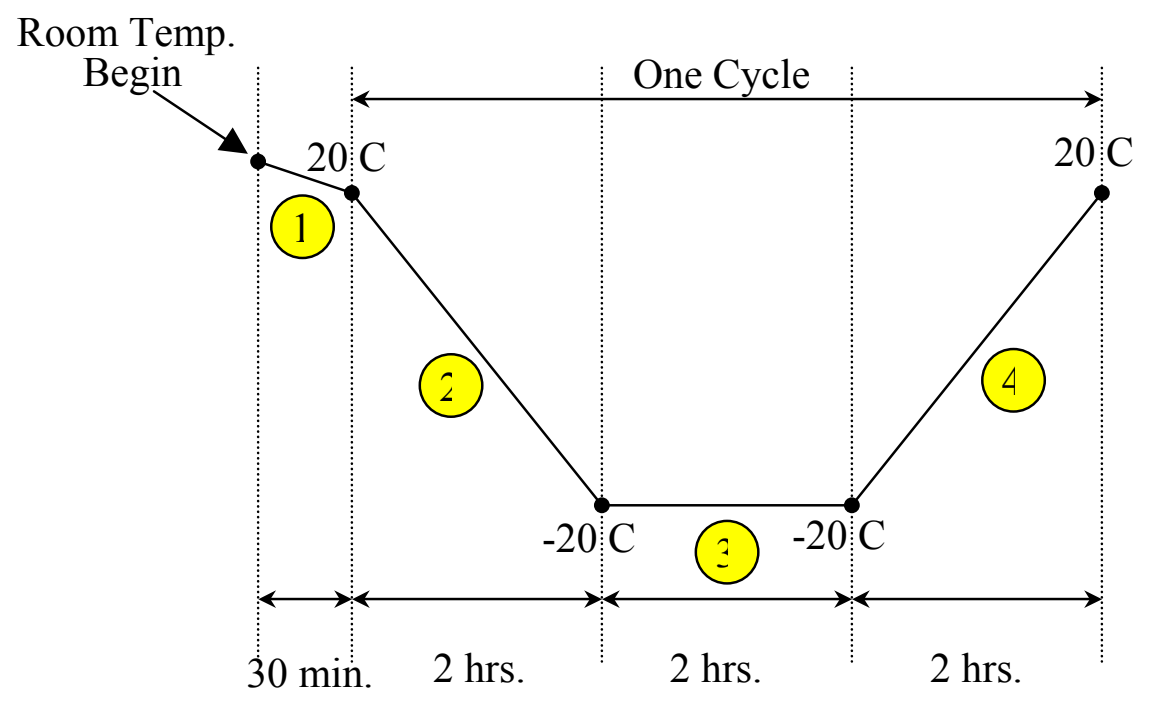

Figure 9.12 (repeated from Fig. 8.1) Freeze-thaw cycling scheme 
For Further Experimental Enhancement and Study

Test-Fixture Improvement:

(1) (This idea was just conceived by the author in the last month-October, 2002) It is possible to reduce the Mode II contribution, thereby enhancing the present setup, by adding a second steel jacket to encase the concrete beam and subsequently placing this assembly within the original gripmechanism with the provision of some type of roller-device (ball-bearings, perhaps) to act in between the two steel surfaces.

\section{Specimen Fabrication and Experimentation:}

(2) (a) Study the importance of surface-preparation of the concrete

(b) Study the effect of surface roughness (see Shen et al., 2002; Ebewele et al., 1980)

(3) Regulate the thickness of the resin at time of application (see Ebewele et al., 1979)

(4) Use more specimens per case - author's suggestion: 6-and limit the number of concrete batches (preferably to only 1 )

Qualification of Systems:

(5) Tests should be conducted on different:

(a) Fiber systems (glass, aramid, etc.)

(b) Resin systems (phenolics, vinylesters, etc.)

(c) Concretes (high/low strengths; high performance concretes, etc.)

Other Characterization-Tools:

(6) (a) X-ray diffraction (XRD)

(b) Scanning electron microscopy (SEM)

(c) Image analysis 


\section{$\underline{\text { Parameters and Correlations: }}$}

(7) (a) Fatigue (see Davalos and Jia, 2002)

(b) Stress corrosion cracking (see Marcus and Sih, 1971)

(c) Crack-position/velocity studies, from experimental, empirical, and analytical standpoints (see M.F. Kanninen's personal communication below)*

(d) Correlation between concrete-strength and interface fracture toughness

(e) Correlation between accelerated age-cycle with real-time weathering

*Personal Communication by M.F. Kanninen to D.M. Boyajian (2001)

(DEC. 26, 5:15 PM EST)

Mr. Boyajian,

I have looked in my files for a copy of my 1974 paper that attracted your attention, but could not find one. The background work that supported the approach that I described in that paper has long ago been discarded. So, to respond to your question, I will have to rely entirely upon my (fading) memory of that work.

There are, obviously, two major differences between the problem that I attacked three decades ago and your problem. The first is that I considered a constant height DCB specimen while your specimen is contoured. The second is that my specimen was monolithic so that there is no difference between the constitutive behavior of the bulk material and the fracture plane, while your fracture plane is a multimaterial set of layers that differ from the bulk material. Both of these make your analysis more complex than mine.

Another distinction, albeit one that does not much change the basic nature of the static compliance that is of immediate interest to you, is that my objective was a rapidly propagating fracture and dynamic crack arrest, while yours is, I assume, static crack growth initiation. I mention this distinction because at that time there was experimental work being done by Ed Ripling and Phil Crosley (now at the University of Illinois in Chicago) in which they used "constant $\mathrm{K}$ " contoured specimens to enable them to determine an arrest toughness. Because my colleagues responsible for the experimental portion of our work on nuclear power plant pressure vessel steel used constant height DCB's, I focused on this class of specimens. But, I was naturally interested in generalizing my model to specimens with arbitrarily varying heights just to see if it could be done. However, I was not successful. As best I remember, the reason was that the "slenderness" assumptions of beam theory can no longer be made, and a closed form solution cannot then be extracted.

At this point I should say that my analysis work was refined over the course of several years. I initially recognized that a beam on elastic foundation (BOEF) model would be much more physically appealing for crack run/arrest analysis than the simple built in beam model (in which the specimen grows as the crack grows!) that was then exclusively being used to analyze DCB (straight and contoured) specimens. The only difficulty that I had with this approach was properly assigning the spring constant, which is of course an arbitrary value in a BOEF model. 
This dilemma led me to later on back up and make a fresh start based on the $2 \mathrm{D}$ equations of the theory of elasticity, in which I introduced simplifying assumptions appropriate to the problem. This approach succeeded in identified an appropriate spring constant, and, although I never made much use of it, it also offered a way of accounting for an adhesive layer through a series of springs.

After I moved on to other things, Professor J. Gordon Williams picked up on this idea and wrote a number of papers on the use of a DCB specimen for adhesively bonded joints. His book, Fracture Mechanics of Polymers, published in 1984 has some information on his early work, which could be of some value to you. Gordon's book, also seems to have some simple compliance results for contoured specimens that could also be of use to you as will, I am sure, some of his later papers.

The bottom line is that, unless you are more clever than I was then, trying to analyze a contoured specimen using a BOEF model is probably not going to be fruitful. If your problem were my problem, I would instead develop a 2D finite element model, exercise it to obtain computational data, then develop an empirical curve fit which can be validated with your experimental compliance data.

I hope that this will be of some value and interest to you, and wish you good luck in your studies.

Regards,

Mel Kanninen 


\section{REFERENCES}

ACI 201.2R-92 (Revised 1997). Guide to Durable Concrete, ACI Manual of Concrete Practice, Part 1: Materials and General Properties of Concrete, American Concrete Institute, Farmington Hills, MI, 41 pp. (1997).

ACI 318R-92 (Revised 1992). Building Code Requirements for Reinforced Concrete, Am. Conc. Inst., Detroit, MI, 347 pp. (1992).

ACI 363R-92 (Reapproved 1997). State-of-the-Art Report on High-Strength Concrete, Am. Conc. Inst., Detroit, MI, 55 pp. (1992).

ACI 503R-93. Use of Epoxy Compounds with Concrete, Am. Conc. Inst., Detroit, MI, 28 pp. (1993).

ASTM B645-98 "Standard Practice for Plane-Strain Fracture Toughness Testing of Aluminum Alloys", American Society for Testing \& Materials, 4 pp., 1998.

ASTM C150-02a "Standard Specification for Portland Cement", Am. Soc. for Testing \& Matls., 7 pp., 1994.

ASTM C231-97e1 "Standard Test Method for Air Content of Freshly Mixed Concrete by the Pressure Method", Am. Soc. for Testing \& Matls., 8 pp., 1997.

ASTM C618-01 "Standard Specification for Coal Fly Ash and Raw or Calcined Natural Pozzolan for Use as a Mineral Admixture in Concrete", Am. Soc. for Testing \& Matls., 4 pp., 2001.

ASTM C666-97 "Standard Test Method for Resistance of Concrete to Rapid Freezing and Thawing", Am. Soc. for Testing \& Matls., 6 pp., 1997.

ASTM C672/C672M-98 "Standard Test Method for Scaling Resistance of Concrete Surfaces Exposed to Deicing Chemicals", Am. Soc. for Testing \& Matls., 3 pp., 1998.

ASTM C989-99 "Standard Specification for Ground Granulated Blast-Furnace Slag for use in Concrete and Mortars", Am. Soc. for Testing \& Matls., 5 pp., 1999.

ASTM D907-00 "Standard Terminology of Adhesives", Am. Soc. for Testing \& Matls., 11 pp., 2000. 
ASTM D1141-98e1 "Standard Practice for Substitute Ocean Water", Am. Soc. for Testing \& Matls., 3 pp., 1998.

ASTM D3433-99 "Standard Test Method for Fracture Strength in Cleavage of Adhesives in Bonded Metal Joints", Am. Soc. for Testing \& Matls., 7 pp., 1999.

ASTM D3762-98 "Standard Test Method for Adhesive-Bonded Surface Durability of Aluminum (Wedge Test)", Am. Soc. for Testing \& Matls., 4 pp., 1998.

ASTM E399-90 "Standard Test Method for Plane-Strain Fracture Toughness of Metallic Materials", Am. Soc. for Testing \& Matls., 31 pp., 1997.

ASTM E632-82 "Standard Practice for Developing Accelerated Tests to Aid Prediction of the Service Life of Building Components and Materials", Am. Soc. for Testing \& Matls., 6 pp., 1996.

ASTM E1290-99 "Standard Test Method for Crack-Tip Opening Displacement (CTOD) Fracture Toughness Measurement", Am. Soc. for Testing \& Matls., 12 pp., 1999.

ASTM E1820-01 "Standard Test Method for Measurement of Fracture Toughness", Am. Soc. for Testing \& Matls., 46 pp., 2001.

ASTM E1823-96e1 "Standard Terminology Relating to Fatigue and Fracture Testing", Am. Soc. for Testing \& Matls., 20 pp., 1996.

Anderson, Garron P., Bennett, S. John and DeVries, K. Lawrence. Analysis and Testing of Adhesive Bonds, Academic Press, New York, NY, 255 pp. (1977).

Andersson, K., Allard, A., Bengtsson, B. and Magnusson, B. "Chemical Composition of Cement Pore Solutions," Cement and Concrete Res., v19, pp. 327-332 (1989).

Bascom, W.D., Bullman, G.W., Hunston, D.L. and Jensen, R.M. "The Width-Tapered Double Cantilever Beam for Interlaminar Fracture Testing," 29th National SAMPE Symposium and Exhibition, v29, pp. 970-978 (1984).

Bascom, W.D., Boll, D.J., Hunston, D.L., Fuller, Bret and Phillips, P.J. "Fractographic Analysis of Interlaminar Fracture," Toughened Composites, ASTM STP 937, Norman J. Johnston, Ed., ASTM, pp. 131-149 (1985).

Bateson, S. "Critical Study of the Optical Properties of Glass Fibres," J. Applied Physics, v29, pp. 13-21 (1958). 
Bayer, P.D. and Cooper, R.E. "Size-Strength Effects in Sapphire and Silicon Nitride Whiskers at 2O[degrees]C," J. Mater. Sci., v2, pp. 233-237 (1967).

Bazant, Zdenek P. and Planas, Jaime. Fracture and Size Effect in Concrete and other Quasibrittle Materials, CRC Press LLC, Boca Raton, FL, 616 pp. (1998).

Benbow, J.J. "Stable Crack Propagation in Plastics," Proc. Phys. Soc., v78, pp. 970-978 (1961).

Berry, J.P. "Determination of Fracture Surface Energies by the Cleavage Technique," J. of Applied Physics, v34, n1, pp. 62-68 (1963).

Bokshtein, S.Z., Koshkin, S.T. and Svotkov, I.L. "Tensile Testing of Filament Crystals of Copper, Nickel and Cobalt to Failure," Soviet Phys. Solid State, v4, pp. 12711277 (1963).

Boresi, Arthur and Chong, K.P. Elasticity in Engineering Mechanics, Elsevier, New York, NY, 645 pp. (1987).

Boresi, Arthur, Schmidt, Richard J. and Sidebottom, Omar M. Advanced Mechanics of Material, John Wiley \& Sons, Inc., New York, NY, Fifth Edition, 811 pp. (1993).

Boyajian, David M. "Design of FRP-Strengthened Concrete Beams," Report submitted for partial fulfillment of the Ph.D. degree, Qualifying Examination Requirements, West Virginia University, 45 pp. (2002a).

Boyajian, David M., Davalos, Julio F. and Qiao, P. "Development of a Test Specimen for FRP-Concrete Mode-I Fracture," Proceedings of the Third International Conference on Advanced Composite Materials in Bridges and Structures (ACMBS), Ottawa, Canada, August 15-18, 2000, J.L. Humar and A.G. Razaqpur (editors), pp.445-452 (2000).

Boyajian, David M., Davalos, Julio F., Ray, Indrajit and Kodkani, Shilpa. "The CFRPConcrete Interface Subjected to Sodium -Sulfate and -Hydroxide Attack," $17^{\text {th }}$ Annual Technical Conference: American Society for Composites (ASC), West Lafayette, IN, October 21-23, 2002, C.T. Sun and Hyonny Kim (editors), Session WB5, paper 088, 9 pp. (2002b).

Boyajian, David M., Davalos, Julio F., Ray, Indrajit and Qiao, Pizhong. "Evaluation of Interface Fracture of Concrete Externally Reinforced with FRP," Proceedings of the Second International Conference on Durability of Fiber Reinforce Polymer (FRP) Composites for Construction (CDCC), Montréal, Québec, Canada, May 29-31, 2002, pp. 309-320 (2002c). 
Brenner, S.S. "Growth and Properties of Whiskers," Science, v128, pp. 569-575 (1958).

Brenner, S.S. "Tensile Strength of Whiskers," J. Appl. Phys., v27, pp. 1481-1491 (1956).

Brown, J.H. "Measuring the Fracture Toughness of Cement Paste and Mortar," Magazine of Concrete Research, v24, n81, pp.185-196 (1972).

Brown, Jr., William F. and Srawley, John E. "Plane Strain Crack Toughness Testing of High Strength Metallic Materials," ASTM, Special Technical Publication No. 410, pp. 1-65, with discussions pp. 66-129 (1966).

Buyukozturk, Oral and Hearing, Brian. "Failure Behavior of Precracked Concrete Beams," J. of Composites for Construction, v2, n3, pp. 138-144 (1998).

Carlsson, Leif A. and Pipes, Byron R. Experimental Characterization of Advanced Composite Materials, Prentice-Hall, Inc., Englewood Cliffs, NJ, 197 pp. (1987).

Carpinteri, Alberto. Structural Mechanics—A Unified Approach, E \& FN Spon, 1997.

Chajes, Michael J., Thomson, Jr., Theodore A. and Farschman, Cory A. "Durability of Concrete Beams Externally Reinforced with Composite Fabrics," Construction and Building Materials, v9, n3, pp. 141-148 (1995).

Cook, J. "Mechanical Testing of Whiskers," Composites, pp. 176-180 (1970).

Cordon, W.A. Freezing and Thawing of Concrete-Mechanisms and Control, American Concrete Institute/Iowa State University Press, Monograph No. 3, second printing, Detroit, Michigan, 99 pp. (1967).

Cotterell, B. and Chen, Z. "Buckling and Cracking of Thin Films on Compliant Substrates under Compression," Intl. J. of Fracture, v104, pp. 169-179 (2000).

Cousins, T.E., Lesko, J.J. and Carlin, B. "Tailored Performance and Durability of Reinforced Concrete Beams Strengthened with FRP Plates," Fiber Composites in Infrastructure, Proceedings of the Second International Conference on Composites in Infrastructure (ICCI), H. Saadatmanesh and M.R. Ehsani (editors), volume II, pp. 399-409 (1998).

Crosley, P.B. and Ripling, E.J. "A Thick Adherend, Instrumented Double-CantileverBeam Specimen for Measuring Debonding of Adhesive Joints," J. of Testing and Evaluation, v19, n1, pp. 24-28 (1991). 
Davalos, Julio, Madabhusi-Raman, Prabhu and Qiao, Pizhong. "Characterization of Mode-I Fracture of Hybrid Material Interface Bonds by Contoured DCB Specimens," Engineering Fracture Mechanics, v58, n3, pp. 173-192 (1997).

Davalos, Julio and Jia, Junhui. "Fatigue Behavior of FRP-Wood Bonded Interfaces," $2^{\text {nd }}$ Intl. CDCC conference, pp. 285-296 (2002).

Davies, P. and Benzeggagh, M.L. "Interlaminar Mode-I Fracture Testing," Chapter 3 of: Application of Fracture Mechanics to Composite Materials, pp. 81-112 (1989).

Devitt, D.F., Schapery, R.A. and Bradley, W.L. "A Method for Determining the Mode I Delamination Fracture Toughness of Elastic and Viscoelastic Composite Materials," J. of Composite Materials, v14, pp. 270-285 (1980).

Diamond, Sidney, "Effects of Two Danish Flyashes on Alkali Contents of Pore Solutions of Cement-Flyash Pastes," Cement and Conc. Res., v11, pp. 383-394 (1981).

Dull, D.L., Buch, J.D. and Raymond, L. "Compliance Calibrations of a Contoured and Face Grooved Double Cantilever Beam Specimen," Engineering Fracture Mechanics, v4, pp. 523-531 (1972).

Ebewele, Robert O., River, Bryan H. and Koutsky, James A. "Tapered Double Cantilever Beam Fracture Tests of Phenolic-Wood Adhesive Joints: Part I. Development of Specimen Geometry; Effects of Bondline Thickness, Wood Anisotropy and Cure Time on Fracture Energy," Wood and Fiber, v11, n3, pp. 197-213 (1979).

Ebewele, Robert O., River, Bryan H. and Koutsky, James A. "Tapered Double Cantilever Beam Fracture Tests of Phenolic-Wood Adhesive Joints: Part II. Effects of Surface Roughness, The Nature of Surface Roughness, and Surface Aging on Joint Fracture Energy," Wood and Fiber, v12, n1, pp. 40-65 (1980).

Ebewele, Robert O., River, Bryan H. and Koutsky, James A. "Relationship between Phenolic Adhesive Chemistry and Adhesive Joint Performance: Effect of Filler Type on Fracture Energy," J. of Polymer Science, v31, n7, pp. 2275-2302 (1986).

Felbeck, D.K. and Orowan, E. "Experiments on Brittle Fracture of Steel Plates," Welding Journal v34, pp. 570s-575s (1955).

Flashner, F., Kenig, S., Zewi, I.G. and Dodiuk, H. "Fracture Toughness of Adhesively Bonded Joints," Engineering Fracture Mechanics, v21, n5, pp. 997-1004 (1985).

FORCA TOW SHEET Technical Notes, Part 4: Applications List (as of March 1994), Tonen Corporation, Tokyo, Japan, 8 pp. (1995). 
Gallagher, J.P. "Experimentally Determined Stress Intensity Factors for Several Contoured Double Cantilever Beam Specimens," Engineering Fracture Mechanics, v3, pp. 27-43 (1971).

Gentry, T.R. "Conditioning of Fiber Reinforced Polymer Concrete Reinforcements in Basic Aqueous Solutions," Fiber Reinforced Polymer Reinforcement for Reinforced Concrete Structures (Selected Presentation Proceedings), Fourth International Symposium, ACI, pp. 81-91 (1999).

Gillis, Peter P. "Surface-Energy Determinations by Cleavage," J. of Applied Physics, v36, n4, pp. 1374-1376 (1965).

Gillis, Peter P. and Gilman, J.J. "Double-Cantilever Cleavage Mode of Crack Propagation," J. of Applied Physics, v35, n3, pp. 647-658 (1964).

Gilman, John J. "Direct Measurements of the Surface Energies of Crystals," J. of Applied Physics, v31, n12, pp. 2208-2218 (1960a).

Gilman, John J. "Physical Nature of Plastic Flow and Fracture," Plasticity, Proceedings of the Second Symposium on Naval Structural Mechanics, pp. 43-96 (1960b).

Gilman, John J. "Propagation of Cleavage Cracks in Crystals," J. of Applied Physics, v27, n11, pp. 1262-1269 (1956).

Glucklich, Joseph. "On Crack Stability in some Fracture Tests," Engineering Fracture Mechanics, v3, pp. 333-344 (1971).

Green, Mark F., Bisby, Luke A., Beaudoin, Yves and Labossiere, Pierre. "Effects of Freeze-Thaw Action on the Bond of FRP Sheets to Concrete," Proceedings of the First International Conference on the Durability of Fibre Reinforced Polymer (FRP) Composites for Construction (CDCC), pp. 179-190 (1998).

Griffith, A.A. "The Phenomena of Rupture and Flow in Solids," Philosophical Translations of the Royal Society of London, A221, pp. 163-198 (1921).

Griffith, A.A. "The Theory of Rupture," Proceedings of the First International Congress for Applied Mechanics, pp. 55-63 (1924).

Guo, Ya-Jun and Weitsman, Y. Jack. "A Modified DCB Specimen to Determine Mixed Mode Fracture Toughness of Adhesives," Engineering Fracture Mechanics, v68, pp. 1647-1668 (2001).

Han, Kyung S. and Koutsky, James. "The Interlaminar Fracture Energy of Glass Fiber Reinforced Polyester Composites," J. of Comp. Matls., v15, pp. 371-388 (1981). 
Harichandran, R.S. and Baiyasi, M.I. "Repair of Corrosion Damaged Columns using FRP Wraps", Michigan State University, Research Project No. R-1386 (2000).

Harrod, D.L., Hengstenberg, T.F. and Manjoine, M.J. "Fracture Toughness of Beryllium," J. of Materials, v4, n3, pp. 618-632 (1969).

Hertzberg, Richard W. Deformation and Fracture Mechanics of Engineering Materials, John Wiley \& Sons, New York, NY, 605 pp. (1976).

Holder, Leonard I. Calculus with Analytic Geometry, Wadsworth Publishing Company, Belmont, CA, 964 pp. (1988).

Holzer, Siegfried M. Computer Analysis of Structures: Matrix Structural Analysis Structured Programming, Elsevier Science Publishing Co., Inc., New York, NY, 426 pp. (1985).

Horrigmoe, G. "Effect of Pore Water and its Diffusion in Concrete," Mechanics of Geomaterials: Rocks, Concrete, Soils, John Wiley and Sons (1985).

Inglis, C.E. "Stresses in a Plate due to the Presence of Cracks and Sharp Corners," Transactions, Inst. Naval Architects, v55, n1, pp. 219-241 (1913).

Irwin, G.R. "Analysis of Stresses and Strains near the end of a Crack Traversing a Plate," J. Applied Mechanics, v79, pp. 361-364 (1957).

Irwin, G.R. "Fracture," Encyclopedia of Physics, Ed. S. Flugge, Springer, Berlin, Vol. VI, pp. 551-590 (1958).

Irwin, G.R. "Fracture Mechanics," Structural Mechanics, Proceedings of the First Symposium on Naval Structural Mechanics, Eds. J.N. Goodier \& N.J. Hoff, Pergamon Press, pp. 557-591 (1960).

Irwin, G.R. "Fracture Dynamics," Fracturing of Metals, American Society for Metals, Cleveland, pp. 147-166 (1948).

Irwin, G.R. and Kies, J.A. "Critical Energy Rate Analysis of Fracture Strength," The Welding Journal, Research Supplement, v33, pp. 193s-198s (1954).

Jenq, Y.S. and Shah, S.P. "A Fracture Toughness Criterion for Concrete," Engineering Fracture Mechanics, v21, n5, pp. 1055-1069 (1985). 
Jones, B.F. "Further Observations Concerning the Effect of Diameter on the Fracture Strength and Young's Modulus of Carbon and Graphite Fibers Made from Polyacrylonitrile," J. Mater. Sci., v6, pp. 1225-1227 (1971).

Jones, B.F. and Duncan, R.G. "The Effect of Fiber Diameter on the Mechanical Properties of Graphite Fibers Manufactured from Polyacrylonitrile and Rayon," J. Mater. Sci., v6, pp. 289-293 (1971).

Kanninen, Melvin F. "A Dynamic Analysis of Unstable Crack Propagation and Arrest in the DCB Test Specimen," Int. J. of Fracture, v10, n3, pp. 415-430 (1974).

Kanninen, M.F. "An Augmented Double Cantilever Beam Model for Studying Crack Propagation and Arrest," Int. J. of Fracture, v9, n1, pp. 83-92 (1973).

Kanninen, M.F. Personal E-mail Communication with D.M. Boyajian, Dec. 26 (2001).

Kanninen, Melvin F. and Popelar, Carl H. Advanced Fracture Mechanics, Oxford University Press, New York, NY, 563 pp. (1985).

Kaplan, M.F. "Crack Propagation and the Fracture of Concrete," Journal of the American Concrete Institute, Proceedings, v58, n5, pp. 591-610 (1961).

Karbhari, V.M. and Engineer, M. "Effect of Environmental Exposure on the External Strengthening of Concrete with Composites-Short Term Bond Durability," J. of Reinforced Plastics and Composites, v15, pp. 1194-1216 (1996a).

Karbhari, V.M. and Engineer, M. "Investigation of Bond between Concrete and Composites: Use of a Peel Test," J. of Reinforced Plastics and Composites, v15, pp. 208-227 (1996b).

Karbhari, V.M., Engineer, M. and Eckel II, D.A. "On the Durability of Composite Rehabilitation Schemes for Concrete: Use of a Peel Test," J. of Materials Science, v32, pp. 147-156 (1997).

Kargol, J.A. and Albright, D.L. "A Fracture Mechanics Method for Determining the Crack Propagation Resistance of Embrittled Aluminum Bicrystals," J. of Testing and Evaluation, v3, n3, pp. 173-178 (1975).

Karmarsch, K. Versuche uber die Festigkeit der zu Draht gezogenen Metallen. Jhhrb. Polytech. Inst. Wien, v18, pp.57-115 (1834).

Karmarsch, K. Uber die absolute Festigkeit der Metalldrahte. Mitt. Gew. Ver. Konigr. Hannover, pp.137-156 (1859). 
Keary, Paul E., Ilcewicz, Larry B., Shaar, Casey and Trostle, Jess. "Mode I Interlaminar Fracture Toughness of Composites Using Slender Double Cantilevered Beam Specimens." J. of Composite Materials, v19, pp. 154-177 (1985).

Kelsey, R.H. and Krock, R.H. "Tension Testing Alumina Whiskers," J. Mater. Sci., v2, pp. 146-159 (1967).

Kenny, P. and Campbell, J.D. "Fracture Toughness: An Examination of the Concept in Predicting the Failure of Materials," Prog. in Matls. Sci., v13, pp. 135-181 (1967).

Knott, J.F. Fundamentals of Fracture Mechanics, John Wiley \& Sons, New York, NY, 273 pp. (1973).

Kosmatka, Steven H. and Panarese, William C. Design and Control of Concrete Mixtures, $13^{\text {th }}$ ed. (revised $2^{\text {nd }}$ printing), Portland Cement Association (PCA), Skokie, IL, 205 pp. (1990).

Krafft, J.M. "Correlation of Plain-Strain Crack Toughness with Strain Hardening Characteristics of a Low, a Medium, and a High-Strength Steel," Applied Materials Research, v3, pp. 88-101 (1964).

Krafft, J.M. and Irwin, G.R. "Crack-Velocity Considerations," Fracture Toughness Testing and its Applications, American Society for Testing and Materials (ASTM), Special Technical Publication No. 381, pp. 114-128 (1964).

Krafft, J.M. and Sullivan, A.M. "Effects of Speed and Temperature upon Crack Toughness and Yield Strength in Mild Steel," Transactions, American Society for Testing and Materials (ASTM), v56, p. 160 (1963).

Lanczos, Cornelius. The Variational Principles of Mechanics, $3^{\text {rd }}$ Ed., University of Toronto Press, Toronto, 375 pp. (1966).

Lewis, D.W. and Dolch, W.L. "Porosity and Absorption," ASTM STP 169 (Significance of Tests and Props. of Conc. and Conc. Aggregates), Philadelphia, PA (1956).

MBrace Composite Strengthening System, Engineering Design Guidelines, $2^{\text {nd }}$ Ed., Master Builders, Inc., www.masterbuilders.com, Cleveland, OH (1998).

Marcus, H.L. and Sih, George C. "A Crackline-Loaded Edge-Crack Stress Corrosion Specimen," Engineering Fracture Mechanics, v3, pp.453-461 (1971).

McCormac, Jack C. Design of Reinforced Concrete, $3^{\text {rd }}$ Ed., Harper Collins College Publishers, New York, NY, 693 pp. (1993). 
Mendelson, Alexander. Plasticity: Theory and Applications, The Macmillan Company, $2^{\text {nd }}$ Printing, New York, NY, 353 pp. (1970).

Miller, A.G., Hertzberg, P.E. and Rantala, V.W. "Toughness Testing of Composite Materials," $12^{\text {th }}$ National SAMPE (Soc. for the Advancement of Material and Process Engineering) Technical Conference, Materials, v12, pp. 279-293 (1980).

Moavenzadeh, Fred and Kuguel, Roberto. "Fracture of Concrete," Journal of Materials, v4, n3, pp. 497-519 (1969).

Mostovoy, Sheldon, Crosley, P.B. and Ripling, E.J. "Note on the Dependence of Crack Velocity on Driving Force for an Epoxy Adhesive," Cracks and Fracture, ASTM STP 601, American Society for Testing and Materials, pp. 234-244 (1976).

Mostovoy, Sheldon, Crosley, P.B. and Ripling, E.J. "Use of Crack-Line-Loaded Specimens for Measuring Plane-Strain Fracture Toughness," J. of Materials, v2, n3, pp. 661-681 (1967).

Mostovoy, Sheldon and Ripling, E.J. "Effect of Temperature on the Fracture Toughness and Stress Corrosion Cracking of Adhesives," Applied Polymer Symposium, n19, pp. 395-408 (1972).

Mott, N.F. "Fracture of Metals: Theoretical Considerations," Engineering, v165, pp. 1618 (1948).

Mukhopadhyaya, P., Swamy, R.N. and Lynsdale, C.J. "Durability of Adhesive Bonded Concrete-GFRP Joints," Proceedings of the First International Conference on the Durability of Fibre Reinforced Polymer (FRP) Composites for Construction (CDCC), pp. 373-389 (1998).

Nairn, John A. "Energy Release Rate Analysis for Adhesive and Laminate Double Cantilever Beam Specimens Emphasizing the Effect of Residual Stresses," Int. J. of Adhesion \& Adhesives, v20, pp. 59-70 (2000).

Neuber, Heinz. The Theory of Notch Stresses, $2^{\text {nd }}$ ed., Julius Springer (Springer-Verlag), Berlin, Germany, 293 pp. (1958).

Neville, A.M. Properties of Concrete, $4^{\text {th }}$ ed., John Wiley \& Sons, Inc., London, England, 844 pp. (1996).

Noone, M.J. The Preparation, Structure and Mechanical Properties of Filamentary Forms of Silicon Carbide, Ph.D. Thesis, University of Leeds, U.K. (1967). 
Orowan, E. Die erhohte Festigkeit dunner Faden, der Joffe-Effekt und verwandte Erscheinungen vom Standpunkt der Griffitschen-Bruchtheorie. Z. Phys., v86, pp. 195-213 (1933).

Orowan, Egon. "Energy Criteria of Fracture," Weld. Journal, v34, pp. 157s-160s (1955).

Orowan, Egon. Fatigue and Fracture of Metals (MIT Symposium, 1950); John Wiley, New York (1950).

Padadakis, V.G., Fardis, M.N. and Vayenas, C.G. "Effect of Composition, Environmental Factors, and Cement-Lime Mortar Coating on Concrete Carbonation," Materials and Structures, v25, n149, pp. 293-304 (1992).

Paris, Paul C. and Sih, George C. "Stress Analysis of Cracks," Fracture Toughness Testing and its Applications, American Society for Testing and Materials (ASTM), Special Technical Publication No. 381, pp. 30-81 (1964).

Pereira, C.J., Rice, R.W. and Skalny, J.P. "Pore Structure and its Relationship to Properties of Materials," Pore Structure and Permeability of Cementitious Materials, Matls. Res. Soc., Symposium Proceedings, v137, pp. 3-21 (1989).

Perry, A.J., Phillips, K. and de Lamotte, E. "The Mechanical Properties of Carbon Fibres," Fibre Sci. Technol., v3, pp. 317-319 (1971).

Petch, N.J. "The Fracture of Metals," Progress in Materials Science, v5, pp. 1-52 (1954).

Phillips, H.B. "Stress Functions," J. of Math. and Physics, v13, pp. 421-425 (1934).

Polakowski, N.H. and Ripling, E.J. Strength and Structure of Engineering Materials, Prentice-Hall, Inc., Englewood Cliffs, New Jersey, 535 pp. (1966).

Qiao, P.Z., Wang, J.L. and Davalos, J.F. "Tapered Beam on Elastic Foundation Model for Compliance Rate Change of TDBC Specimen," submitted to Engineering Fracture Mechanics in 2001.

Quantrill, R.J., Hollaway, L.C. and Thorne, A.M. "Experimental and Analytical Investigation of FRP Strengthened Beam Response: Part I," Magazine of Concrete Research, v48, n177, pp. 331-342 (1996).

Rahman, A.H., Lauzier, C., Kingsley, C., Richard, J. and Crimi, J. "Experimental Investigation of the Mechanism of FRP Reinforcement for Concrete," Fiber Composites in Infrastructure, Proceedings of the Second International Conference on Composites in Infrastructure (ICCI), H. Saadatmanesh and M.R. Ehsani (editors), volume II, pp. 501-511 (1998). 
Ripling, E.J. Discussion of: "Plane Strain Crack Toughness Testing of High Strength Metallic Materials," original paper by Brown, Jr., William F. and Srawley, John E., American Society for Testing and Materials (ASTM), Special Technical Publication No. 410, p. 70 (1966).

Ripling, E.J., Crosley, P.B. and Wiersma, S.J. "A Review of Static Crack Arrest Concepts," Engineering Fracture Mechanics, v23, n1, pp. 21-33 (1986).

Ripling, E.J., Mostovoy, S. and Corten, H.T. "Fracture Mechanics: A Tool for Evaluating Structural Adhesives," J. Adhesion, v3, pp. 107-123 (1971).

Ripling, E.J., Mostovoy, S. and Patrick, R.L. "Measuring Fracture Toughness of Adhesive Joints," Materials Research \& Standards, v4, n3, pp. 129-134 (1964).

River, Bryan H. "Fracture of Adhesive-Bonded Wood Joints," Handbook of Adhesive Technology, $2^{\text {nd }}$ Ed., Eds. A. Pizzi and K.L. Mittal, Marcel Dekker Inc., New York, NY, (in press 2002).

River, Bryan H. and Okkonen, E.A. "Contoured Wood Double Cantilever Beam Specimen for Adhesive Joint Fracture Tests," J. of Testing and Evaluation, v21, n1, pp. 21-28 (1993).

Russell, A.J. and Street, K.N. "Factors Affecting the Interlaminar Fracture Energy of Graphite/Epoxy Laminates," Progress in Sci. and Engrg. of Composites, ICCMIV (Tokyo), pp. 279-286 (1982).

Rybicki, Edmund F., Hernandez, Jr., Tony D., Deibler, John E., Knight, Ronald C. and Vinson, Steven S. "Mode I and Mixed Mode Energy Release Rate Values for Delamination of Graphite/Epoxy Test Specimens," J. of Composite Materials, v21, pp. 105-123 (1987).

Saadatmanesh, Hamid and Ehsani, Mohammad R. "Fiber Composite Plates can Strengthen Beams," Concrete International, v12, n3, pp. 65-71 (1990).

Schardin, H. "Velocity Effects in Fracture," Fracture, Technology Press, New York, N.Y.pp. 297-330 (1959).

Schra, L., Boerema, P.J. and Van Leeuwen, H.P. "Experimental Determination of the Dependence of Compliance on Crack Tip Configuration of a Tapered Double Cantilever Beam Specimen," National Aerospace Institute of Amsterdam, Report TR 73025, 42 pp. (1973). 
Scott, C. Tim, River, Bryan H. and Koutsky, James A. "Fracture Testing Wood Adhesives with Composite Cantilever Beams," J. of Testing and Evaluation, v20, n4, pp. 259-264 (1992).

Shen, X., Myers, J., Maerz, N. and Galicki, G. "Effect of Surface Roughness on the Bond Performance Between FRP Laminates and Concrete," $2^{\text {nd }}$ Intl. CDCC conference, pp. 607-616 (2002).

Sloan, Forrest. "A Constant-G Double Cantilever Beam Fracture Specimen for Environmental Testing," J. of Comp. Materials, v27, n16, pp. 1606-1615 (1993).

Sneddon, I.N. "The Distribution of Stress in the Neighborhood of a Crack in an Elastic Solid," Proceedings, Royal Society of London, vA-187 (1946).

Soltis, P.J. "A New Method and Technique for Tension Testing Whiskers," J. Mater. Sci., v2, pp. 300-324 (1967).

Sommer, H. "Ein neues Verfahren zur Erzielung der Frost-Tausalz-Beständigkeit des Betons," Zement und Beton, v22, n4, pp. 124-129 (1977).

Springer, G.S. "Environmental Effects," Environmental Effects on Composite Materials, Technomic, Springer, G.S., ed., v3, pp. 1-34 (1988).

Srawley, John E. and Brown, Jr., William F. "Fracture Toughness Testing Methods," Fracture Toughness Testing and its Applications, ASTM, Special Technical Publication No. 381, pp. 133-198 (1964).

Sukley, R. and Howrylak, C., Pennsylvania Department of Transportation Research, Project No. 93-052 (1998).

Svensson, N.L. "The Variation of the Fracture Energy of Brittle Plastics with Temperature," Proc. Phys. Soc., v77, pp. 876-884 (1961).

Swanson, Gerald D. and Gross, Gordon E. "Range of Validity for Use of the Double Cantilever Fracture Energy Analysis," J. of Applied Physics, v40, n11, pp. 4684 \& 4685 (1969).

Tamuzs, V., Tarasovs, S. and Vilks, U. "Progressive Delamination and Fiber Bridging Modeling in Double Cantilever Beam Composite Specimens," Engineering Fracture Mechanics, v68, pp. 513-525 (2001).

Ting, R.Y. and Cottington, R.L. "Fracture Evaluation of High Performance Polymers as Adhesives," $12^{\text {th }}$ National SAMPE (Soc. for the Advancement of Material and Process Engineering) Technical Conference, Materials, v12, pp. 714-728 (1980). 
Toutanji, Houssam A. and El-Korchi, T. "Tensile Durability Performance of Cementitious Composites Externally Wrapped with FRP Sheets," Fiber Composites in Infrastructure, Proceedings of the Second International Conference on Composites in Infrastructure (ICCI), H. Saadatmanesh and M.R. Ehsani (editors), volume II, pp. 410-422 (1998).

Toutanji, Houssam A. and Gómez, William. "Durability Characteristics of Concrete Beams Externally Bonded with FRP Composite Sheets," Cement and Concrete Composites, v19, pp. 351-358 (1997).

Van Der Sluys, W.A. "Mechanisms of Environment Induced Subcritical Flaw Growth in AISI 4340 Steel," Engineering Fracture Mechanics, v1, pp. 447-462 (1969).

Verbeck, G.J. and Klieger, P. "Studies of 'Salt' Scaling of Concrete," Highway (Transportation) Research Board, Bulletin No. 150, pp. 1-13 (1957).

Verbeck, George J. and Landgren, Robert. "Influence of Physical Characteristics of Aggregates on Frost Resistance of Concrete," Proceedings, ASTM, v60, pp. 10631079 (1960).

Verbeck, G.J. "Field and Laboratory Studies of the Sulphate Resistance of Concrete," Performance of Concrete-Resistance of Concrete to Sulphate and Other Environmental Conditions, Thorvaldson Symposium, University of Toronto Press, Toronto, pp. 113-124 (1968). (Also reprinted as PCA Research Department Bulletin 227, Portland Cement Association, Skokie, IL (1969).)

Watson, R.J. "Case Histories of Structural Rehabilitation Utilizing Composites in Industrial and Marine Environments," Fiber Composites in Infrastructure, Proceedings of the $2^{\text {nd }}$ Intl. Conference on Composites in Infrastructure (ICCI), H. Saadatmanesh and M.R. Ehsani (editors), volume I, pp. 113-125 (1998).

Watson, R.J. "Field Condition Surveys of FRP Applications on Bridges," $2^{\text {nd }}$ Intl. CDCC conference, pp. 597-605 (2002).

Webster's Ninth New Collegiate Dictionary, Merriam-Webster Inc., Springfield, Massachusetts, 1563 pp. (1987).

Weibull, W. Proceedings of the Royal Swedish Inst. of Engineering Res. No. 151 (1939).

Weiss, V. and Yukawa, S. "Critical Appraisal of Fracture Mechanics," Fracture Toughness Testing and its Applications, American Society for Testing and Materials (ASTM), Special Technical Publication No. 381, pp. 1-22 (1964). 
Weitsman, Y. "Stresses in Adhesive Joints Due to Moisture and Temperature," J. Composite Materials, v11, pp. 378-394 (1977).

Westergaard, H.M. "Bearing Pressures and Cracks," J. Applied Mechanics, v61, pp. A49A53 (1939).

Whitney, J.M., Browning, C.E. and Hoogsteden, W. "A Double Cantilever Beam Test for Characterizing Mode I Delamination of Composite Materials," J. Reinforced Plastics and Composites, v1, pp. 297-313 (1982).

Williams, J.G. "Large Displacement and End Block Effects in the 'DCB' Interlaminar Test in Modes I and II," J. of Composite Materials, v21, pp. 330-347 (1987).

Williams, M.L. "On the Stress Distribution at the Base of a Stationary Crack," J. Applied Mechanics, pp. 109-114 (1957).

Yoffe, E.H. "The Moving Griffith Crack," The Philosophical Magazine, series 7, v42, pp. 739-750 (1951).

Yu, Hong-Hui and Hutchinson, John W. "Influence of Substrate Compliance on Buckling Delamination of Thin Films," International Journal of Fracture, v13, pp. 39-55 (2002). 


\section{APPENDIX A}

\section{SPECIMEN INVENTORY}

Table A.1 Pristine specimens

\begin{tabular}{|c|c|c|}
\hline Specimens & Total & Refined Total $^{*}$ \\
\hline 10B4, 3B3, 3B6, 4B8, 3B9, 3B10 & 6 & 4 \\
\hline
\end{tabular}

Table A.2 Plain specimens (i.e. no CFRP - these were non-fracture specimens used to gauge wt. \& strain changes in the concrete throughout the duration of aging)

\begin{tabular}{|c|c|c|c|}
\hline \hline \multicolumn{2}{|c|}{ Conditions } & Specimens & Totals \\
\hline \multirow{2}{*}{ WD } & $\mathrm{SS}$ & $8 \mathrm{~B} 9^{\mathrm{W}, \mathrm{S}}, 5 \mathrm{~B} 10^{\mathrm{W}, \mathrm{S}}, 6 \mathrm{~B} 11^{\mathrm{W}, \mathrm{S}}$ & 3 \\
\cline { 2 - 4 } & $\mathrm{SH}$ & $1 \mathrm{~B} 6^{\mathrm{W}, \mathrm{S}}, 2 \mathrm{~B} 7^{\mathrm{W}, \mathrm{S}}, 6 \mathrm{~B} 8^{\mathrm{W}, \mathrm{S}}$ & 3 \\
\hline \multirow{2}{*}{ FT } & $\mathrm{CC}$ & $9 \mathrm{~B} 3^{\mathrm{W}, \mathrm{S}}, 7 \mathrm{~B} 4^{\mathrm{W}, \mathrm{S}}, 1 \mathrm{~B} 8^{\mathrm{W}, \mathrm{S}}$ & 3 \\
\hline \multicolumn{2}{|l}{} & 9 \\
\hline
\end{tabular}

Table A.3 Companion specimens

\begin{tabular}{|c|c|c|c|c|c|c|c|}
\hline Conditions & \multicolumn{5}{|c|}{ Specimens at ages: } & Totals & Refined \\
\hline \multirow[b]{2}{*}{ WD } & 10 & & \multirow{2}{*}{$\frac{20}{4 \mathrm{~B} 6^{\mathrm{S}}, 1 \mathrm{~B} 7^{\mathrm{S}}}}$, & \multirow{2}{*}{\multicolumn{2}{|c|}{ 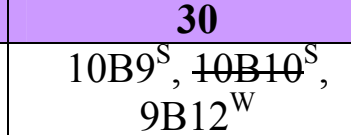 }} & \multirow{3}{*}{9} & \multirow[b]{2}{*}{4} \\
\hline & \multicolumn{2}{|c|}{$\begin{array}{c}4 \mathrm{~B} 3^{\mathrm{S}}, 2 \mathrm{~B} 6^{\mathrm{S}}, \\
7 \mathrm{~B}^{\mathrm{W}}\end{array}$} & & & & & \\
\hline \multirow[b]{2}{*}{ FT } & 50 & 100 & 150 & 200 & 300 & & \multirow[b]{2}{*}{12} \\
\hline & $\begin{array}{l}7 \mathrm{~B} 3^{\mathrm{W}} \\
8 \mathrm{~B} 3^{\mathrm{w}} \\
4 \mathrm{~B} 9^{\mathrm{s}}\end{array}$ & $\begin{array}{l}1 \mathrm{~B} 4^{\mathrm{W}} \\
6 \mathrm{~B} 7^{\mathrm{w}} \\
1 \mathrm{~B} 10^{\mathrm{S}}\end{array}$ & $\begin{array}{l}5 \mathrm{~B} 8^{\mathrm{W}} \\
6 \mathrm{~B} 9^{\mathrm{W}} \\
5 \mathrm{~B} 14^{\mathrm{s}}\end{array}$ & $\begin{array}{l}7 \mathrm{~B} 10^{\mathrm{W}}, \\
8 \mathrm{~B} 10^{\mathrm{w}} \\
10 \mathrm{~B} 12^{\mathrm{s}}\end{array}$ & $\begin{array}{l}4 \mathrm{~B} 12^{\mathrm{W}}, \\
8 \mathrm{~B} 12^{\mathrm{W}}, \\
5 \mathrm{~B} 13^{\mathrm{s}}\end{array}$ & 15 & \\
\hline & & & & & & 24 & 16 \\
\hline
\end{tabular}


Table A.4 Wet-Dry specimens

\begin{tabular}{|c|c|c|c|c|c|}
\hline \multirow[t]{2}{*}{ בConditions } & \multicolumn{3}{|c|}{ Specimens at ages: } & \multirow[t]{2}{*}{ Totals } & \multirow{2}{*}{$\begin{array}{l}\text { Refined } \\
\text { Totals }\end{array}$} \\
\hline & $\overline{10}$ & 20 & 30 & & \\
\hline SS & $\begin{array}{c}2 \mathrm{~B} 3^{\mathrm{S}}, 3 \mathrm{~B} 4^{\mathrm{S}} \\
5 \mathrm{~B} 4^{\mathrm{W}}, 10 \mathrm{~B} 6^{\mathrm{W}}\end{array}$ & $\begin{array}{l}8 \mathrm{~B} 7^{\mathrm{W}}, 3 \mathrm{~B} 8^{\mathrm{W}} \\
9 \mathrm{~B} 9^{\mathrm{s}}, 9 \mathrm{~B} 10^{\mathrm{s}}\end{array}$ & $\begin{array}{l}7 \mathrm{~B} 11^{\mathrm{S}}, 7 \mathrm{~B} 12^{\mathrm{W}} \\
8 \mathrm{~B} 13^{\mathrm{s}}, 9 \mathrm{gB} 13^{\mathrm{W}}\end{array}$ & 12 & 9 \\
\hline SH & $\begin{array}{l}1 \mathrm{~B} 3^{\mathrm{s}}, 6 \mathrm{~B} 3^{\mathrm{W}} \\
2 \mathrm{~B} 4^{\mathrm{s}}, 4 \mathrm{~B} 4^{\mathrm{W}}\end{array}$ & $\begin{array}{l}5 \mathrm{~B} 7^{\mathrm{W}}, 2 \mathrm{~B} 8^{\mathrm{W}}, \\
7 \mathrm{~B} 9^{\mathrm{s}}, 6 \mathrm{~B} 10^{\mathrm{s}}\end{array}$ & $\begin{array}{l}3 \mathrm{~B} 11^{\mathrm{s}}, 6 \mathrm{~B} 12^{\mathrm{W}}, \\
3 \mathrm{~B} 13^{\mathrm{W}}, 7 \mathrm{~B} 13^{\mathrm{S}}\end{array}$ & 12 & 9 \\
\hline & & & & 24 & 18 \\
\hline
\end{tabular}

Table A.5 Freeze-Thaw specimens

\begin{tabular}{|c|c|c|c|c|c|c|c|}
\hline Condition & \multicolumn{5}{|c|}{ Specimens at ages: } & Total & Refined \\
\hline & $\overline{50}$ & $\overline{100}$ & $\overline{150}$ & $\overline{200}$ & $\overline{300}$ & & \\
\hline $\mathrm{CC}$ & $\begin{array}{l}5 \mathrm{~B} 3^{\mathrm{S}}, \\
6 \mathrm{~B} 4^{\mathrm{s}}, \\
7 \mathrm{~B} 6^{\mathrm{W}}, \\
8 \mathrm{~B} 6^{\mathrm{W}}\end{array}$ & $\begin{array}{l}8 \mathrm{~B} 4^{\mathrm{S}}, \\
5 \mathrm{~B} 6^{\mathrm{s}}, \\
8 \mathrm{~B} 8^{\mathrm{W}}, \\
1 \mathrm{~B} 9^{\mathrm{W}}\end{array}$ & $\begin{array}{l}3 \mathrm{~B} 7^{\mathrm{S}}, \\
4 \mathrm{~B} 7^{\mathrm{S}} \\
2 \mathrm{~B} 9^{\mathrm{W}}, \\
2 \mathrm{~B} 10^{\mathrm{W}}\end{array}$ & $\begin{array}{c}7 \mathrm{~B} 7^{\mathrm{S}}, \\
5 \mathrm{~B} 9^{\mathrm{S}} \\
4 \mathrm{~B} 11^{\mathrm{W}} \\
2 \mathrm{~B} 13^{\mathrm{W}}\end{array}$ & $\begin{array}{l}2 \mathrm{~B} 11^{\mathrm{S}}, \\
8 \mathrm{~B} 11^{\mathrm{S}}, \\
4 \mathrm{~B} 13^{\mathrm{W}}, \\
6 \mathrm{~B} 13^{\mathrm{W}}\end{array}$ & 20 & 15 \\
\hline
\end{tabular}

"Refined totals are based on specimens that yielded admissible-loads during fracture testing (see Section 6.2 - especially Eqs. (6.9) - (6.12) and subsection titled "A Step-byStep Example")

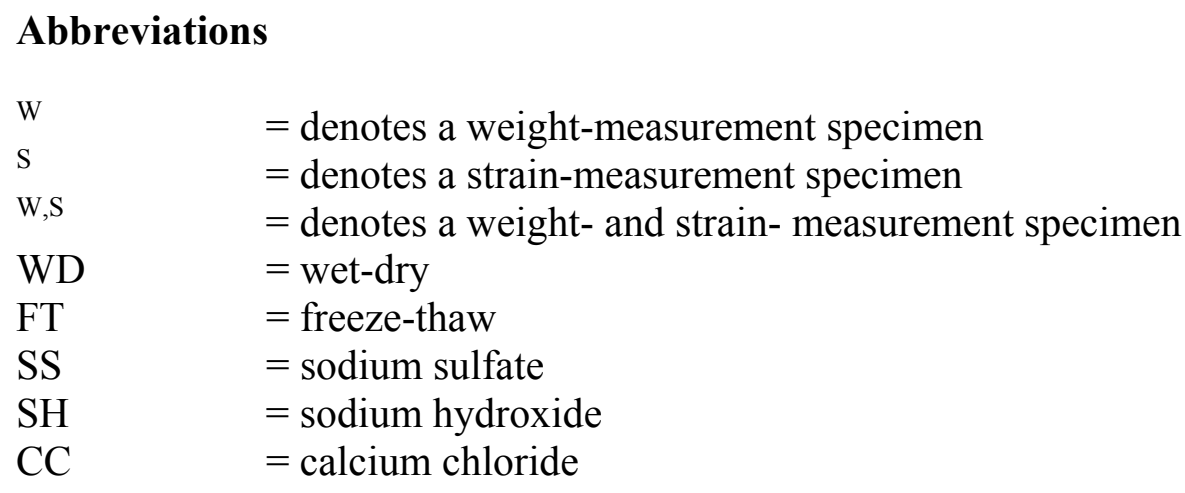




\section{APPENDIX B}

\section{RELEVANT PROPERTIES OF MATERIALS USED}

Table B.1 LVL (Laminated Veneer Lumber-used for the contours)

\begin{tabular}{|c|c|c|c|}
\hline \hline $\mathbf{n}$ & E [GPa] (Msi) & G [GPa] (Msi) & $\boldsymbol{\sigma}$ [GPa] (Msi) \\
\hline 20 & $13.22(1.917)$ & $0.43(0.063)$ & $1.21(0.175)$ \\
\hline
\end{tabular}

Note: $\mathrm{E}_{2} \approx(1 / 27) \cdot \mathrm{E}_{1}$ (assumed); Thus, $\mathrm{E}_{2} \approx 0.483 \mathrm{GPa}(0.070 \mathrm{Msi})$

Also, Poisson's ratio is assumed as: $v=0.3$

Table B.2 CFRP strip (MBrace CF130 high tensile, unidirectional carbon fiber)

\begin{tabular}{|c|c|c|c|c|c|}
\hline $\begin{array}{c}\mathbf{w} \\
\text { [fibers/mm] } \\
\text { (fibers/in) }\end{array}$ & $\begin{array}{c}\mathbf{t} \\
\text { [mm/ply] } \\
\text { (in/ply) }\end{array}$ & $\begin{array}{c}\text { E [GPa] } \\
\text { (Msi) }\end{array}$ & $\begin{array}{c}\mathbf{f}_{\mathbf{u}} \text { [GPa] } \\
\text { (Msi) }\end{array}$ & $\boldsymbol{\varepsilon}_{\mathbf{u}}$ & $\mathbf{V}_{\mathbf{f}}$ \\
\hline $\begin{array}{c}1700 \\
(43,200)\end{array}$ & $\begin{array}{c}0.16 \\
(0.0065)\end{array}$ & $\begin{array}{c}228 \\
(33)\end{array}$ & $\begin{array}{c}4.3 \\
(0.62)\end{array}$ & $1.67 \%$ & $20 \%-22 \%$ \\
\hline
\end{tabular}

Table B.3 MBrace primer (Repeated from Table 5.4)

\begin{tabular}{|c|c|c|c|}
\hline \hline E [GPa] (Msi) & $\begin{array}{c}\text { Yield Stress [MPa] } \\
(\mathbf{p s i})\end{array}$ & Yield Strain & Poisson's Ratio, v \\
\hline $0.72(0.104)$ & $14(2100)$ & $4 \%$ & 0.48 \\
\hline
\end{tabular}

Table B.4 MBrace saturant (Repeated from Table 5.5)

\begin{tabular}{|c|c|c|c|}
\hline \hline E [GPa] (Msi) & $\begin{array}{c}\text { Yield Stress [MPa] } \\
(\mathbf{p s i})\end{array}$ & Yield Strain & Poisson's Ratio, v \\
\hline $3(0.440)$ & $54(7800)$ & $2.5 \%$ & 0.40 \\
\hline
\end{tabular}


Table B.5 Concrete (made with locally available natural sand and \#8 pea-gravel aggregates; each beam furnished with a pair of \#2 steel reinforcing bars, Gr. 60, $\mathrm{f}_{\mathrm{y}}=60,000 \mathrm{psi}=413.7 \mathrm{MPa}$ ) (Repeated from Table 5.1)

\begin{tabular}{|c|c|c|c|c|}
\hline \hline $\mathbf{n}$ & $\begin{array}{c}\text { water-cement ratio, by } \\
\text { weight }\end{array}$ & Air content & $\begin{array}{c}\mathbf{f}_{\mathbf{c}}{ }^{\prime}[\mathrm{MPa}] \\
(\mathbf{p s i})\end{array}$ & $\begin{array}{c}\boldsymbol{\sigma}[\mathrm{MPa}] \\
(\mathbf{p s i})\end{array}$ \\
\hline 10 & 0.5 & $6 \%$ & $\begin{array}{c}51.6 \\
(7480)\end{array}$ & $\begin{array}{c}2.1 \\
(300)\end{array}$ \\
\hline
\end{tabular}

Note $_{1}$ : Refer also to Chapter 5 for further details on the materials used and their properties

Note $_{2}$ : All values in Tables B.2, B.3, and B.4 are taken from: MBrace Composite Strengthening System, Engineering Design Guidelines, $2^{\text {nd }}$ Ed., Master Builders, Inc., www.masterbuilders.com, Cleveland, OH, 1998, or by personal contact with the composites division manager at Master Builders.

\section{Notation}

$\begin{array}{ll}\mathrm{f}_{\mathrm{c}}{ }^{\prime} & =\text { compressive strength of concrete } \\ \mathrm{f}_{\mathrm{u}} & =\text { ultimate strength } \\ \mathrm{t} & =\text { thickness } \\ \mathrm{W} & =\text { width } \\ \mathrm{n} & =\text { number of specimens (i.e. sampling size) } \\ \mathrm{E} & =\text { modulus of elasticity } \\ \mathrm{G} & =\text { shear modulus } \\ \mathrm{V}_{\mathrm{f}} & =\text { fiber volume fraction } \\ \varepsilon_{\mathrm{u}} & =\text { ultimate strain } \\ \sigma & =\text { standard deviation }\end{array}$




\section{APPENDIX C}

INSTRUMENTATION (DEMEC, ZH-16, \& MTS)

\section{C.1 DEMEC (i.e. demountable mechanical) strain gauge (see also Ch. 6)}

\section{EXAMPLE:}

Suppose two strain readings are taken, e.g. the $1^{\text {st }}$ today, and the $2^{\text {nd }}$, a week later:

$$
\begin{array}{llll}
1^{\text {st }} \text { Reading: } & \mathrm{D}_{\mathrm{o}}=\text { Invar bar datum reading } & = & 2.600 \mathrm{~mm} \\
& \mathrm{R}_{\mathrm{o}}=\text { actual datum reading } & = & 2.656 \mathrm{~mm} \\
& & & \\
2^{\text {nd }} \text { Reading: } & \mathrm{D}_{\mathrm{i}}=\text { subsequent Invar bar reading } & = & 2.604 \mathrm{~mm} \\
& \mathrm{R}_{\mathrm{i}}=\text { subsequent actual reading } & = & 2.678 \mathrm{~mm}
\end{array}
$$

Then, the resulting change in microstrain (abbreviated, $\mu \varepsilon$ ) is computed as:

$$
\Delta(\mu \varepsilon)=\mathrm{G} \cdot\left\{\left(\mathrm{R}_{\mathrm{i}}-\mathrm{D}_{\mathrm{i}}\right)-\left(\mathrm{R}_{\mathrm{o}}-\mathrm{D}_{\mathrm{o}}\right)\right\}
$$

where $\mathrm{G}=$ the gauge factor $=5380$ (for this particular instrument). Therefore:

$$
\Delta(\mu \varepsilon)=5380 \cdot\{(2.678-2.604)-(2.656-2.600)\}=96.84 \mu \varepsilon
$$

Also, the actual change in length is simply:

$$
\Delta \mathrm{L}=\mathrm{L} \times \Delta(\varepsilon)
$$

where $\mathrm{L}=$ the gauge length $=($ for this particular instrument $)=150 \mathrm{~mm}$

$$
\Delta \mathrm{L}=150 \times\left(96.84 \times 10^{-6}\right)=0.01453 \mathrm{~mm}
$$




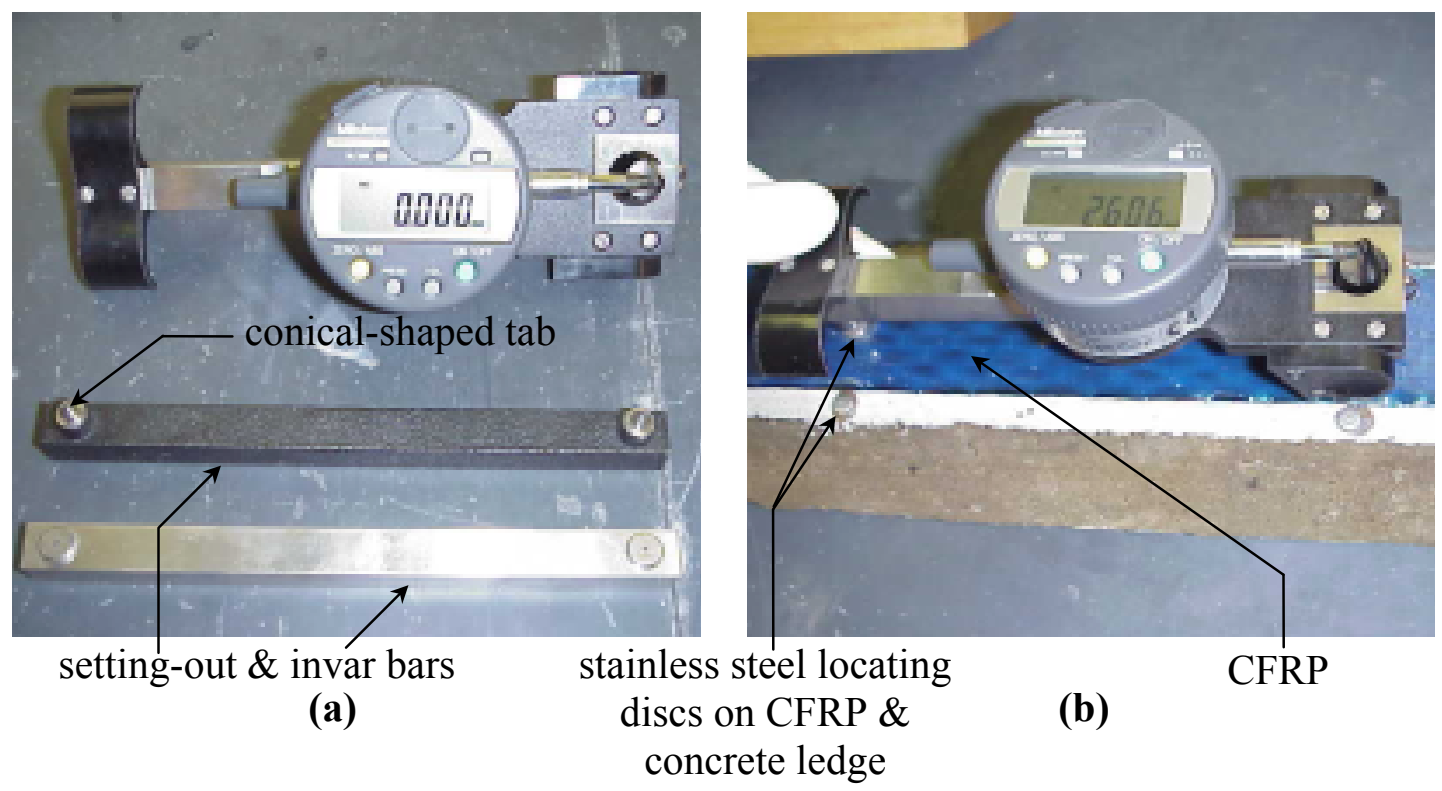

Figure C.1 The demountable mechanical strain gauge (DEMEC) -

(a) DEMEC with setting-out and invar bars

(b) DEMEC in use atop CFRP (repeated from Fig. 6.8)

\section{C.2 CSZ ZH-16 (Cincinnati Sub-Zero) environmental chamber}

\section{SAMPLE FREEZE-THAW PROGRAM:}

(1) -On the computerized keypad (Figure C.2(b)), bring-up: "Go to Profile" -Press the right-arrow key: $(\boldsymbol{\nabla})$ and enter the password: $\underline{9} \underline{1} 1$

(i.e. push the up-arrow key $(\boldsymbol{\Delta})$ to scroll through the letters and numbers and the right-arrow key to go to the next space.)

-When finished writing $\underline{9} \underline{1} \underline{1}$, press $(>)$ twice.

(2) -Next, bring-up: "Create Profile" and press ( $)$

-"Choose Name", say "yes" and press ( ) and then assign a name. Press ( )

(3) -Bring-up: "Ramp Time"

-"Step does not wait"

-Press the EVENT 1 button (see Fig. C.2(c)) to the right to turn it on

-Back on the computerized keypad, choose: "Condition System" and press ( )

-Make sure that the EVENT 2 button (see Fig. C.2) is off (i.e. in central position-

IMPORTANT: There should be no humidity with freezing) and press ( $)$ 
(4) -Enter the ramp time: 00:30:00 (i.e. 30 minutes - see Fig. C.3) ( )

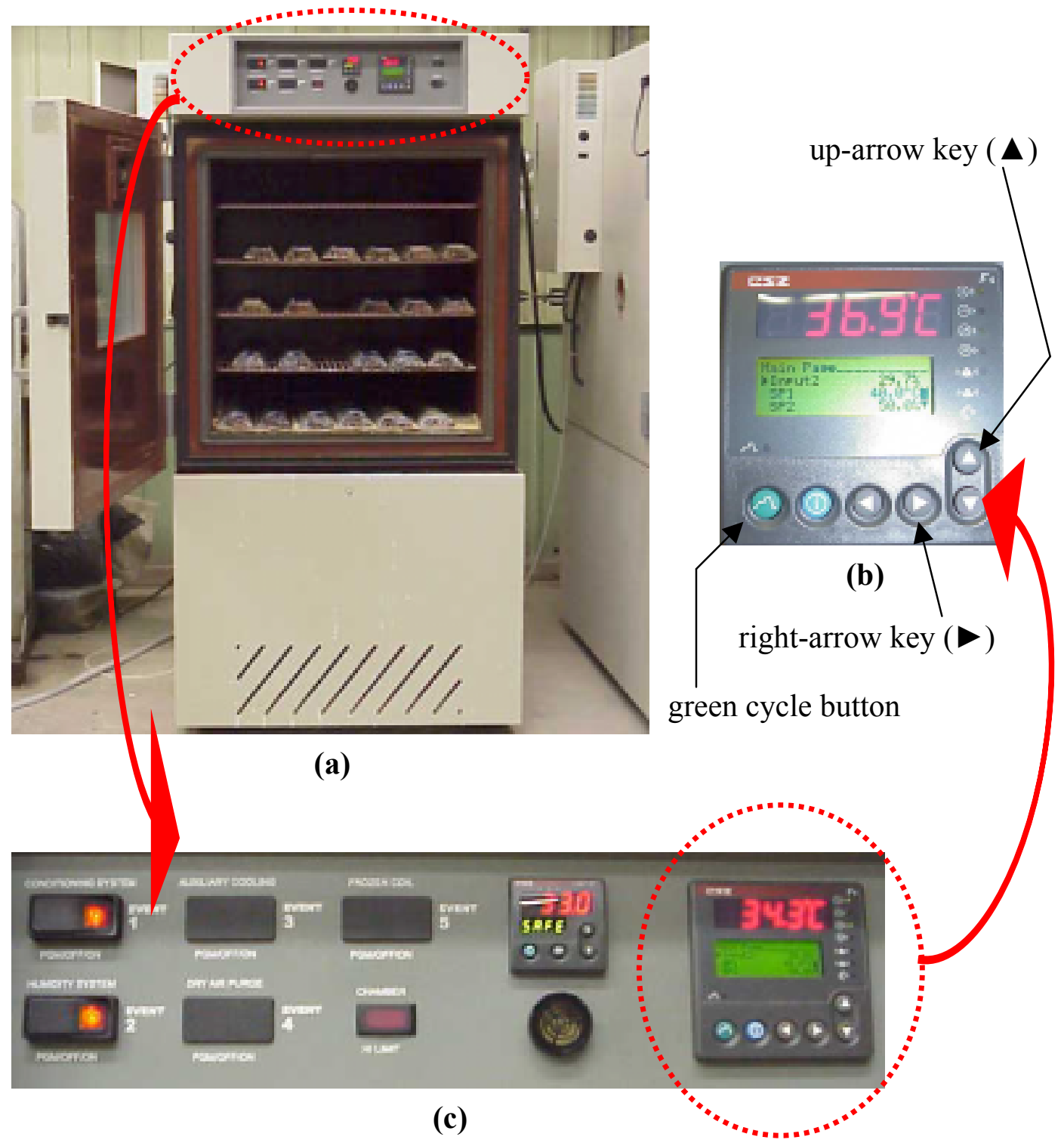

Figure C.2 The CSZ ZH-16 environmental chamber-(a) front view of chamber with wet-dry beams, (b) close-up of computerized keypad, and (c) close-up of control panel

(5) -Enter Channel 1 SP: Ramp to $20 \mathrm{C}$ in 30 minutes ( )

-"Next" ( ) (NOTE: the right-arrow key is implied in the steps following though it is no longer shown) 
(6) -Enter Ch. (i.e. channel) 2: It does not matter here as it was turned-off in step (3)

(7) -Choose Ch1: $\underline{\text { PID Set } 1}$

(8) -Choose Ch2: $\underline{\text { PID Set } 6}$ (for example, though it really does not matter here)

(9) -Choose: "Guar. Soak 1" $\quad \underline{\mathrm{NO}}$

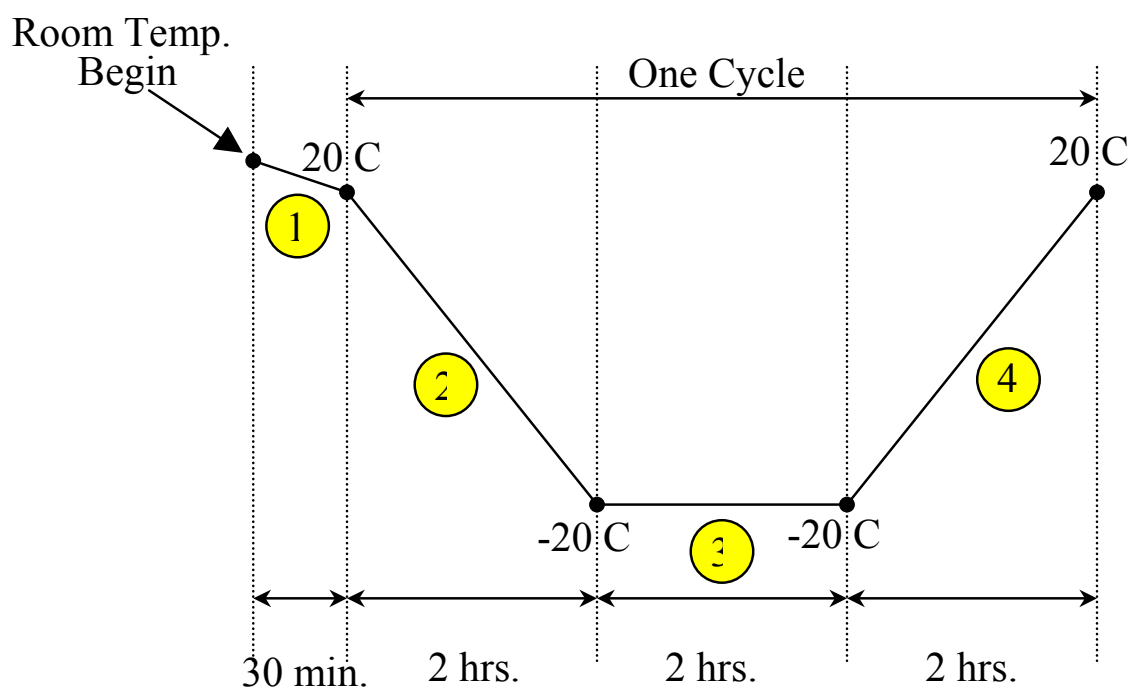

Figure C.3 Freeze-Thaw Cycling Scheme (repeated from Fig. 8.1)

(10) (SUMMARY of steps (4) - (9)):

-Choose: Step 1 (i.e. Initial 30 minute Ramp-Down-see Fig. C.3)

Ramp Time: 00:30:00 (i.e. 30 mins.-see Fig. C.3) ( $($ )

Press $(\boldsymbol{D})$ again

Ch1 (Set Temp.): 20 (i.e. -20 C)

Ch2 Humidity (Not important for this example since switch

PID Set 1 was turned-off-see step (3))

PID Set 6 (for example, though it really does not matter here as the switch was turned-off-see step (3))

Soak Guar. 1 $\underline{\mathrm{NO}}$ (i.e. Temp)

Soak Guar. 2 $\underline{\mathrm{NO}}$ (i.e. Humidity) 
-Choose: Step 2 (i.e. Actual cycle's 2 hour Ramp-Down-see Fig. C.3)

Ramp Time

Cond. Sys.

Humidity

Ramp Time:

Set Temp.:

Humidity

PID Set 1

PID Set 6

Soak Guar. 1

Soak Guar. 2

Choose Step does not wait

$\underline{\mathrm{ON}}$

$\underline{\mathrm{OFF}}$

02:00:00 (i.e. 2 hours)

-20 (i.e. -20 C)

(Not important for this example)

$\underline{\text { NO }}$ (i.e. Temp)

$\underline{\mathrm{NO}}$ (i.e. Humidity)

(12)

-Choose: Step 3 (i.e. Actual cycle's 2 hour Soak—see Fig. C.3)

Ramp Time

Cond. Sys.

Humidity

Choose Step does not wait

Ramp Time:

ON

Set Temp.

$\underline{\mathrm{OFF}}$

Humidity

02:00:00 (i.e. 2 hours)

PID Set 1

PID Set 6

Soak Guar. 1

-20 (i.e. -20 C)

(Not important for this example)

Soak Guar. 2

YES (i.e. Temp)

NO (i.e. Humidity)

(13)

-Choose: Step 4 (i.e. Actual cycle's 2 hour Ramp-Up-see Fig. C.3)

Ramp Time

Cond. Sys.

Humidity

Ramp Time:

Set Temp.:

Humidity

PID Set 1

PID Set 6

Soak Guar. 1

Choose Step does not wait

ON

Soak Guar. 2

$\underline{\mathrm{OFF}}$

02:00:00 (i.e. 2 hours)

20 (i.e. -20 C)

(Not important for this example)

NO (i.e. Temp)

$\underline{\mathrm{NO}}$ (i.e. Humidity)

(14) -Finally, bring-up: "Jump"

-Go to: "Name of Profile"

-Jump to Step 2 (i.e. Skip the Initial 30-minute Ramp-Down-see Fig. C.3-for the remainder of the conditioning scheme)

-How many repeats: $\underline{400}$ (i.e. the number of cycles)

-Step 6: $\quad$ END

-All Off

-press ( 4 ) (i.e. the left-arrow key, see Fig. C.2(c)) Save Profile: $\quad \underline{\text { YES }}$

NOTE 1: When turning machine on, press the: green cycle button (Fig. C.2(b)),

choose YES and choose "Profile Name" 
NOTE 2: $\quad$ Make sure that the EVENT 1 button (Fig. C.2(c)) is positioned to the extreme left (i.e. this is the program-mode) BEFORE starting machine

\section{C.3 MTS—458.10 Microconsole \& 418.91 Microprofiler}

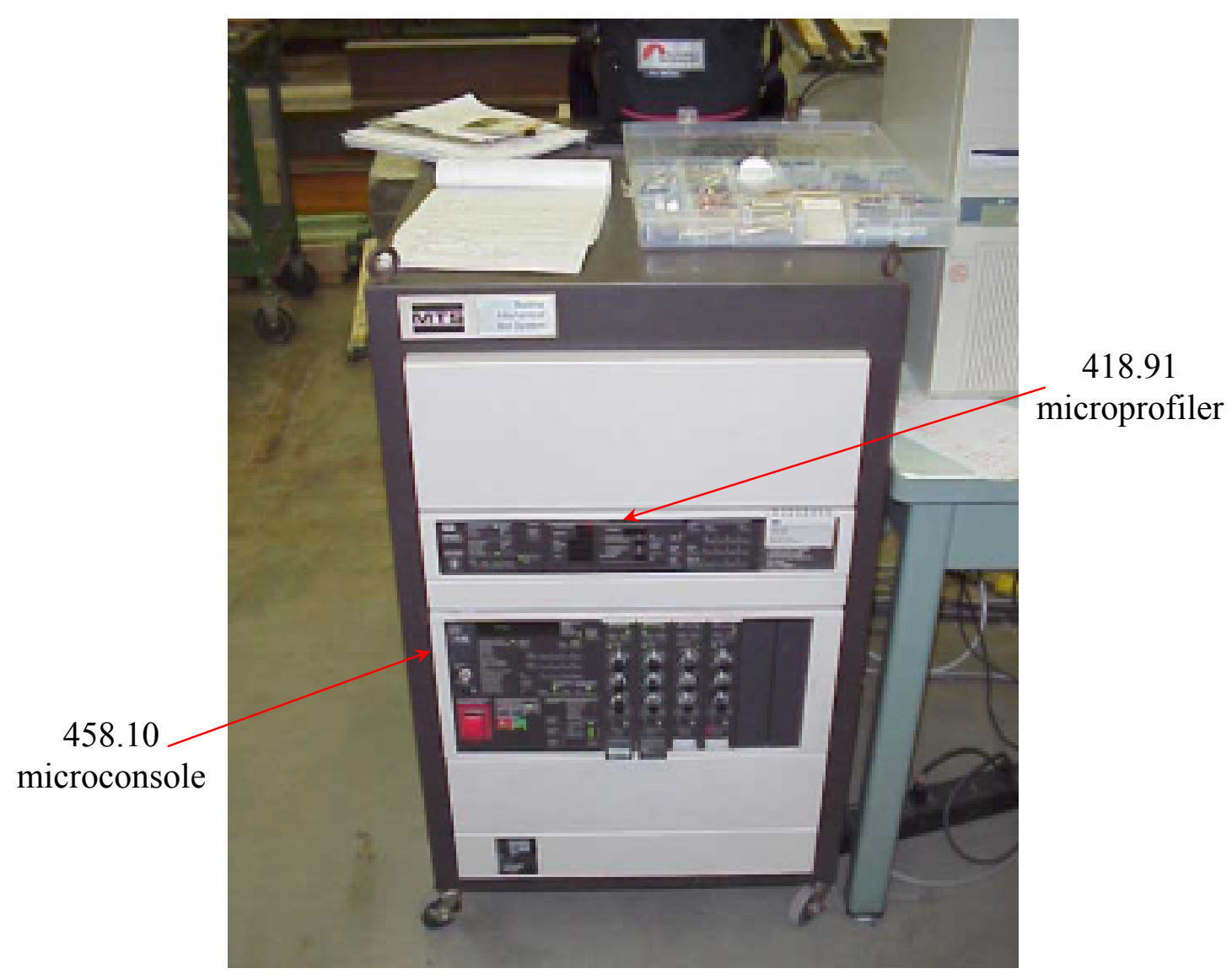

Figure C.4 MTS 418.91 microprofiler and 458.10 microconsole

\section{OPERATING GUIDELINES:}

\section{(a) 458.10 Microconsole:}

(1) -Turn on the MTS

-Hit Enter

-If a red light is lit, hit Reset

-Push the Disable/Enable button to Enable - a green light will appear

-On the Program Source, press Enable and, while keeping this depressed, press External 
(2) -To change cartridges:

-Turn Hydraulic Pressure OFF

-Put desired cartridge(s) in

-Hit Reset

-Put Display on correct cartridge column

-Go to Transducer Full Scale

-Type in correct number-example: 1000 for 1 kip load cartridge

-Check that the Upper \& Lower Limit Detects conform with the cartridges in use

-Check that the DC Error is zero or close to zero-if not, move Set Point until DC Error $=$ Set Point

(3) -To activate hydraulic pressure:

-Go from Off to Low to High-do not jump to High at once!

(Make sure that the interlocks are enabled-No red lights, only greenotherwise, hit Reset to clear the red lights)

\section{(b) 418.91 Microprofiler:}

(1) -On Function Select, go to Edit Program-Here the:

Segment $=1(0.001 \mathrm{in} / \mathrm{s}$, for this research $)$

2 (3 in/s, any value to promptly return the actuator to zero)

\begin{tabular}{|c|c|c|}
\hline Rate & $=$ & $\begin{array}{l}0.08 \text { (In Forestry Machine, i.e. } 0.08 \text { corresponds to } \\
0.001 \mathrm{in} / \mathrm{s} \text {, i.e. multiplied by a factor of } 80) \\
0.06 \text { (In Engrg. Lab Machine, i.e. } 0.06 \text { corr. to } 0.06 \\
\text { in } / \mathrm{min}=0.001 \mathrm{in} / \mathrm{sec})\end{array}$ \\
\hline Level & $=$ & $\begin{array}{l}100 \text { (In Forestry Machine, corr. to tension; -100 } \\
\text { corr. to comp.-i.e. a downward moving } \\
\text { actuator is Positive, and an upward moving } \\
\text { one, Negative) } \\
2.5 \text { (In Engrg. Lab Machine, i.e. for segment } 1: 2.5 \\
\text { corr. to 2.5", assuming that the } 2.5 \text { " Displ. } \\
\text { cartridge is being used) } \\
0 \text { (In Engrg. Lab Machine, i.e. for segment 2) }\end{array}$ \\
\hline
\end{tabular}

-Type the appropriate program block number (for this research: "22")

-Hit Enter; Hit Run/Enable; Hit Run Program

(2) -Back on the Microconsole: Hit Run

(3) -When finished with test run, hit Return to 0 


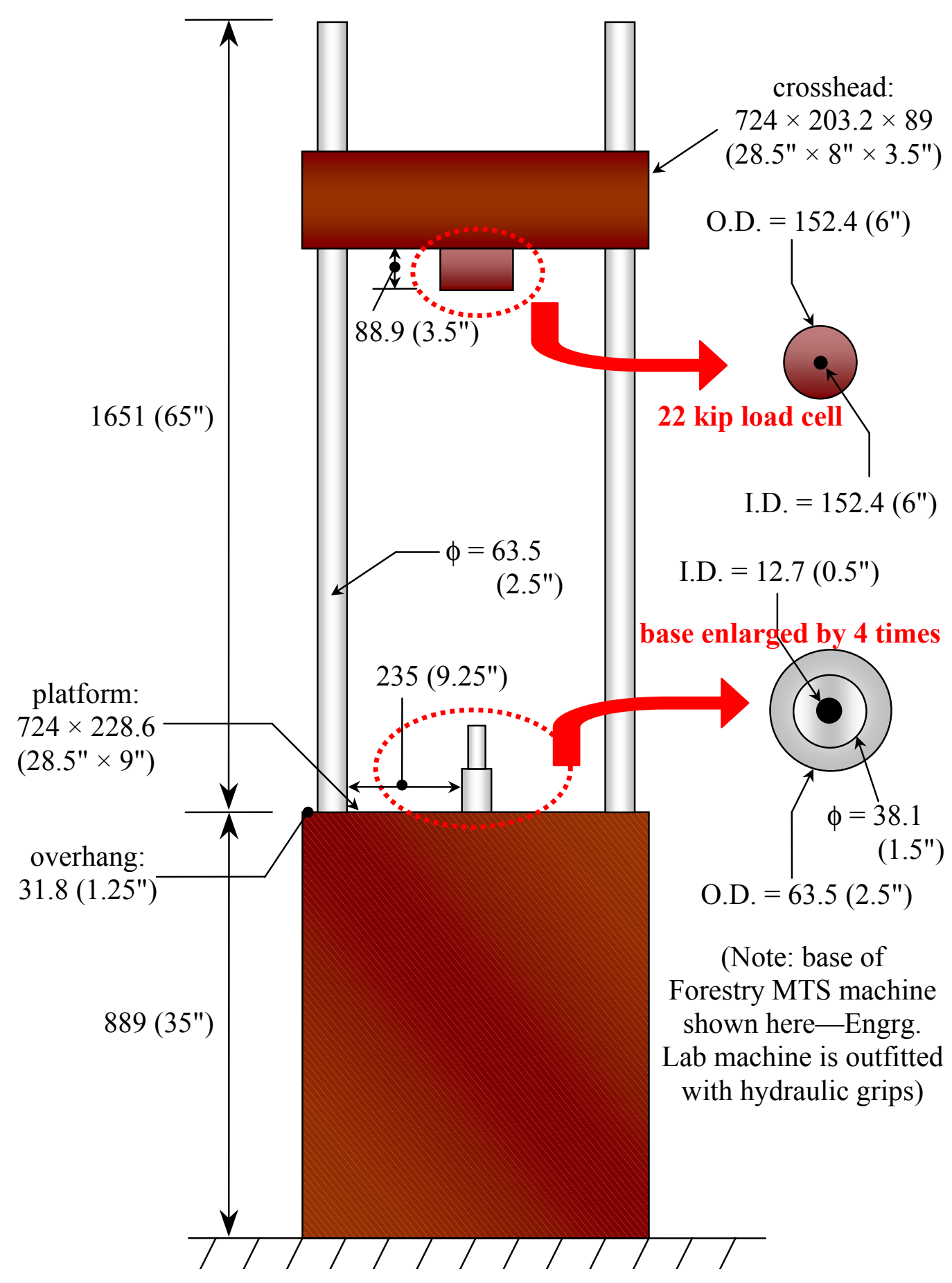

Figure C.5 MTS schematic (Unmarked dimensions in millimeters) 


\section{APPENDIX D}

\section{INDUSTRY CONTACTS}

\section{Cincinnati Sub-Zero:}

http://www.cszinc.com/

cszinc@cszinc.com [E-mail]

12011 Mosteller Road

Cincinnati, OH 45241-1528

(513) $772-8810$

(800) 989-7373 [Toll Free in North America]

(513) $772-9119$ [Fax]

\section{Fisher Scientific:}

https://www2.fishersci.com/

(800) 766-7000

(800) 926-1166 [Fax]

Sites in the U.S.:

\section{Atlanta \\ 3970 Johns Creek Court \\ Suite 500 \\ Suwanee, GA 30024 \\ Phone: 1-770-871-4500 \\ Fax: 1-770-871-4600}

\section{Chicago}

4500 Turnberry Dr.

Hanover Park, IL 60103

Phone: 1-630-259-1200

Fax: 1-630-259-4444

\author{
Houston \\ P.O. Box 1546 \\ 9999 Veterans Memorial Drive \\ Houston, TX 77251-1546 \\ Phone: 1-281-405-4000 \\ Fax: 1-281-878-2407

\section{Los Angeles} \\ 2761 Walnut Avenue \\ Tustin, CA 92780 \\ Phone: 1-714-669-4600 \\ Fax: 1-714-669-1613
}


3. Gilson Company, Inc.:

http://www.globalgilson.com/

techsupport@gisonco.com

P.O. Box 200

Lewis Center, OH 43035-0200

(740) 548-7298

(800) 444-1508

(740) 548-5314 [Fax]

4. Hoy Redi-Mix Co.:

http://www.hoymix.com/

(304) 598-0267

(304) 598-0268 [Fax]

5. MTS:

http://www.mts.com/

14000 Technology Drive

Eden Prairie, MN 55344-2290

(952) 937-4000

(952) 937-4515 [Fax]

6. Master Builders, Inc.:

http://www.masterbuilders.com/ askmbt@mbt.com

23700 Chagrin Rd.

Cleveland, $\mathrm{OH} 44122$

(800) 628-9990 (i.e. MBT-9990)

(216) 839-8821 [Fax] 


\section{Mastrad Quality and Test Systems:}

http://www.mastrad.com/

sales@mastrad.com

St. Pierre-Wetherby Road

Bardsey Leeds, England LS17 9BB

United Kingdom

(44) 1937574961

(44) 1937574450 [Fax]

\section{McMaster-Carr:}

http://www.mcmaster.com/

atl.sales@mcmaster.com

Sites in the U.S.:

\author{
Atlanta \\ 6100 Fulton Industrial \\ Blvd. \\ Atlanta, GA 30336-2852 \\ Phone: 1-404-346-7000 \\ Fax: 1-404-349-9091
}

\section{Cleveland}

200 Aurora Industrial Pkwy.

Aurora, OH 44202

Phone: 1-330-995-5500

Fax: 1-330-995-9600

\section{Chicago}

600 County Line Rd

Elmhurst, IL 60126-

2081

Phone: 1-630-833-0300

Fax: 1-630-834-9427

\section{Los Angeles}

9630 Norwalk Blvd.

Santa Fe Springs, CA 90670-2932

Phone: 1-562-692-5911

Fax: 1-562-695-2323

\section{Precision Tool \& Machine:}

http://www.plantfloor.com/wv/precisiontoolmachine.htm

400 Wheeling St.

Morgantown, WV 26505

(304) 291-3946 
10. Richmond Aircraft Products:

http://www.richmondaircraft.com/

info@richmondaircraft.com

13503 Pumice Street

Norwalk, CA 90650

(562) 404-2440

(562) 404-9011 [Fax]

11. Romisch Manufacturing \& Metals, Inc.:

http://www.plantfloor.com/wv/romischmanufacturingmetals.htm 421 Beechurst Ave.

Morgantown, WV 26505

(304) 296-4712

12. Trus Joist MacMillan:

100 TJM Drive

Buckhannon, WV 26201

(304) 472-2436

(304) 472-8564

(304) 472-7395 [Fax]

13. Vishay Measurements Group:

http://www.vishay.com/

measurementsgroup@vishay.com

P.O. Box 27777

Raleigh, NC 27611

(919) 365-3800

(919) 365-3945 [Fax] 
14. Watson Bowman Acme:

http://www.wbacorp.com/

info@wbacorp.com

stephan.tysl@wbacorp.com

julia.nichter@wbacorp.com

95 Pineview Dr.

Amherst, NY 14228

(716) 691-7566

(716) 691-9239 [Fax]

(216) 577-2812 [Steve Tysl's line]

(800) 677-4922 x205 [Julia Nichter's line]

15. Wilson Equipment:

45 Decker Ave.

Morgantown, WV 26505

(304) 296-7621

***Other pertinent industry contacts may be found through:

REINFORCED Plastics (published monthly by Elsevier)

http://www.reinforcedplastics.com/

$\underline{\text { RP@elsevier.co.uk }}$

P.O. Box 1656

New York, NY 10116-1656

(44) 1865843638 (telephone in UK)

(44) 1858434958 [Fax in UK] 